\title{
Common cyclical features in multiple time series and panel data
}

Citation for published version (APA):

Hecq, A. W. (2000). Common cyclical features in multiple time series and panel data: methodological aspects and applications. [Doctoral Thesis, Maastricht University]. Universiteit Maastricht. https://doi.org/10.26481/dis.20000928ah

Document status and date:

Published: 01/01/2000

DOI:

10.26481/dis.20000928ah

Document Version:

Publisher's PDF, also known as Version of record

\section{Please check the document version of this publication:}

- A submitted manuscript is the version of the article upon submission and before peer-review. There can be important differences between the submitted version and the official published version of record.

People interested in the research are advised to contact the author for the final version of the publication, or visit the DOI to the publisher's website.

- The final author version and the galley proof are versions of the publication after peer review.

- The final published version features the final layout of the paper including the volume, issue and page numbers.

Link to publication

\footnotetext{
General rights rights.

- You may freely distribute the URL identifying the publication in the public portal. please follow below link for the End User Agreement:

www.umlib.nl/taverne-license

Take down policy

If you believe that this document breaches copyright please contact us at:

repository@maastrichtuniversity.nl

providing details and we will investigate your claim.
}

Copyright and moral rights for the publications made accessible in the public portal are retained by the authors and/or other copyright owners and it is a condition of accessing publications that users recognise and abide by the legal requirements associated with these

- Users may download and print one copy of any publication from the public portal for the purpose of private study or research.

- You may not further distribute the material or use it for any profit-making activity or commercial gain

If the publication is distributed under the terms of Article $25 \mathrm{fa}$ of the Dutch Copyright Act, indicated by the "Taverne" license above, 


\title{
Common Cyclical Features in Multiple Time Series and Panel Data
}

Methodological Aspects and Applications

\author{
PROEFSCHRIFT
}

ter verkrijging van de graad van doctor aan de Universiteit Maastricht, op gezag van de Rector Magnificus,

Prof. dr. A.C. Nieuwenhuijzen Kruseman, volgens het besluit van het College van Decanen, in het openbaar te verdedigen op donderdag 28 september 2000 om 14.00 uur

door

Alain Willy HECQ 


\section{Promotor:}

Prof. dr. F. C. Palm

\section{Co-promotor:}

Dr. J-.P. Urbain

\section{Beoordelingscommissie:}

Prof. dr. G. A. Pfann (voorzitter)

Prof. dr. N. Haldrup (University of Aarhus, Denmark)

Prof. dr. P. Schotman 


\section{Contents}

Preface $\quad$ xiii

1 Introduction and Outline of this Thesis 1

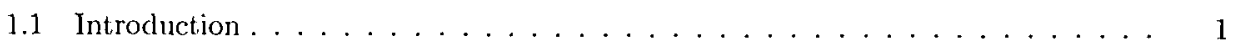

1.2 Typology of Common Factors . . . . . . . . . . . . . . . . 2

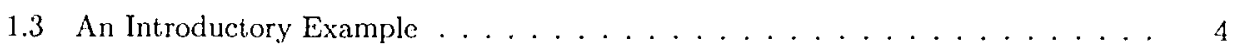

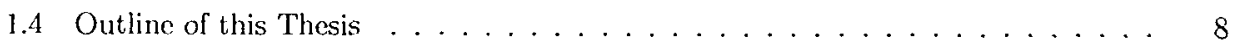

1.5 Out of the Line of this Thesis and Unfeatures . . . . . . . . . . . . 10

2 Codependence, Common Features \& Co. 13

2.1 Introduction and Motivations . . . . . . . . . . . . . . . . . . 13

2.2 Definition of Common Features . . . . . . . . . . . . . . . 15

2.3 Serial Correlation Common Feature . . . . . . . . . . . . . . 17

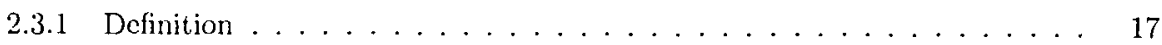

$2.3 .2 \mathrm{SCCF}$ and Unpredictability . . . . . . . . . . . . . 19

2.3 .3 Different Concepts of Elasticities . . . . . . . . . . . . . 20

2.3 .4 Conmon Cyclical Features and IRF . . . . . . . . . . . . . 22

2.3.5 Some Theoretical Common Feature Vectors . . . . . . . . . . . . . 25

2.3 .6 "Exogenous" Variables . . . . . . . . . . . . . . . . . . . . 27

2.4 Nested Reduced Rank . . . . . . . . . . . . . . . . . . . . . 27

2.5 Codependence. . . . . . . . . . . . . . . . . . . . 28

2.6 Non-synchronous Common Features . . . . . . . . . . . . . . . . . . 29

2.6.1 Non-synchronous VARMA Models ... . . . . . . . . . . . . . . 30

2.6 .2 Codependence in VAR Models . . . . . . . . . . . . . . . . . . 31

2.6 .3 Really Unsynchronized Models? . . . . . . . . . . . . . . . . 32

2.7 Scalar Component Models . . . . . . . . . . . . . . . . . . . . . . 32

2.8 Testing for Common Features Using Canonical Correlations . . . . . . . . . . 34

2.8 .1 Identification of VARMA Models . . . . . . . . . . . . 35

2.8.2 SCM in Practice and the Choice of Key Parameters . . . . . . . . . . 36

2.8 .3 The Canonical Correlation Test Statistic and its Duality . . . . . . . . 38 
2.8.4 Distribution and Test Statistics . . . . . . . . . . . . . . 38

2.9 Inference in Codependence and SCM: Monte Carlo Results . . . . . . . . 40

2.9.1 Codependence Test Statistics . . . . . . . . . . . . . . . 40

2.10 Applications in Common Features . . . . . . . . . . . . . . . . . . . . . . . .

2.10.1 Convergence within EC Economies . . . . . . . . . . . . . 45

2.10 .2 Once upon a Time Stationary Price Levels . . . . . . . . . . . . . . 47

2.10.3 Stability of Activity-Unemployment Relationship in a Codependent System 49

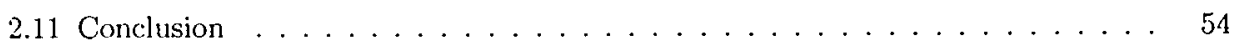

2.12 Appendix: Identifiability and Exchangeability . . . . . . . . 56

3 Testing for Common Features in Cointegrated VAR 61

3.1 Introduction and Motivation . . . . . . . . . . . . . . . . 62

3.2 Reduced Rank Structures . . . . . . . . . . . . . . . . . . 63

3.3 Testing Different Forms of Reduced Rank Structures . . . . . . . . . . . . . 67

3.3 .1 Reduced rank hypotheses . . . . . . . . . . . . . . 67

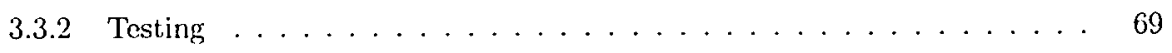

3.4 Monte Carlo Results . . . . . . . . . . . . . . . . . . . 71

3.5 Co-movements in US Macrocconomic Aggregates . . . . . . . . . . . . . . . . . . . . . . .

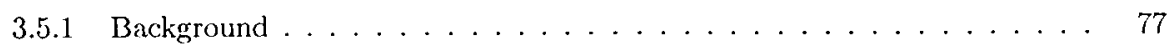

3.5 .2 Cointegration and Common Feature Analysis . . . . . . . . . . . 80

3.6 A Second Example: UK Equity, Gilt and Money Markets . . . . . . . . . . . . . . . . 82

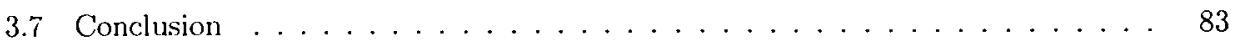

4 Permanent-Transitory Decomposition $\quad \mathbf{8 7}$

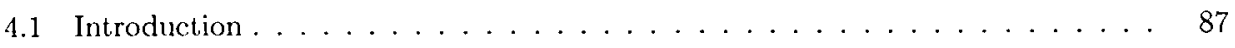

4.2 Permaneni-Transitory Decompositions . . . . . . . . . . . . . . . . . . . . . . . . . .

4.2 .1 Cointegrated VAR Models . . . . . . . . . . . . . . . . . . . . . . . . . . . . 90

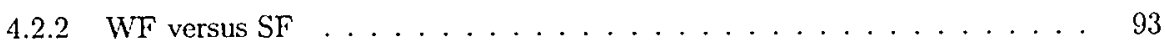

4.2 .3 Relation to other Decompositions . . . . . . . . . . . . 95

4.3 Applications . . . . . . . . . . . . . . . . . . . . . . . . . . . . . . . .

4.3 .1 US Business Cyclical Comovements . . . . . . . . . . . . . 96

4.3 .2 Comovements in International Stock Markets . . . . . . . . . . . . . . . . . 97

4.4 Conclusion . . . . . . . . . . . . . . . . . . 107

4.5 Appendix A: Proof of Proposition $4.4 \ldots \ldots \ldots \ldots$. . . . . . . . . 108

4.5 Appendix B: Summary of Partitioned Matrices Algebra . . . . . . . . . . . 109

4.6 Appendix C: Detailed Calculus for the $\operatorname{VAR}(2) \ldots \ldots \ldots \ldots$

4.7 Appendix D: Extension to the VAR(p) model . . . . . . . . . . . 111 
5 To Switch between Cointegration and Co-features 113

5.1 Introduction . . . . . . . . . . . . . . . . . . 113

5.2 Test Statistics and Switching Algorithms . . . . . . . . . 115

5.2.1 Usual Test Statistics and their Distributions. . . . . . . . . . . . 115

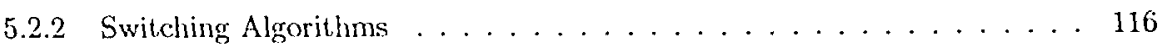

5.3 Monte Carlo Design and Test Statistics . . . . . . . . . . . . 118

5.3 .1 The Data Generating Process . . . . . . . . . . . . . . . . 118

5.3 .2 Simulation Results . . . . . . . . . . . . . . . . 118

5.4 Empirical Analyses . . . . . . . . . . . . . . . . . . . . 124

5.4 .1 About US Regional Income Dynamics . . . . . . . . . . . . . . . . . . . . . . . . . . . . . . . . . . . .

5.4 .2 Belgian Regional Employment Dynamics . . . . . . . . . . . 126

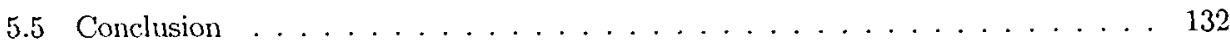

6 Assessing Forecast Performance 135

6.1 Introduction . . . . . . . . . . . . . . . . . 135

6.2 VAR Models with Cointegration and Common Features . . . . . . . . . 137

6.2 .1 Gencral Setting . . . . . . . . . . . . . . . . 137

6.3 Forecasting the US GDP: The Data and Long-Run Relationships . . . . . . . . 139

6.4 Common Cyclical Feature Propertics . . . . . . . . . . . . . . . 142

6.5 Forecast Evaluation . . . . . . . . . . . . . . . . 143

6.5.1 Construction of Forecasts . . . . . . . . . . . . . . 143

6.5 .2 Comparing Models . . . . . . . . . . . . . . . . . . 145

6.5.3 Comparing Spccifications . . . . . . . . . . . . . 145

6.5.4 Using Full Sample Error Correction Terms . . . . . . . . . . . . . . 148

6.5 .5 Using Information Critcria . . . . . . . . . . . . . 155

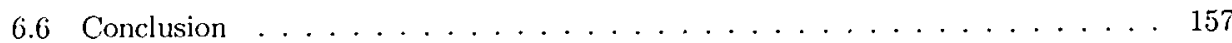

7 Does Seasonal Adjustment Induce Common Cycles? 161

7.1 Introduction and Motivation . . . . . . . . . . . . . . 161

7.2 Test Statistics . . . . . . . . . . . . . . . . . 163

7.3 The Data Gencrating Process and Simulation Results . . . . . . . . . . 163

7.4 The Japanese Consumption Function . . . . . . . . . . . . . . . . . 166

7.5 Assessing a Perfect European Optinum Currency Area . . . . . . . . . . 169

7.6 What can we do in Practice: Is there a Neat Solution? . . . . . . . . . . 171

7.6 .1 The Size Effect . . . . . . . . . . . . . . . . . . . . . . . . . . . . . . . . . . . . . .

7.6 .2 The Covariance Effect . . . . . . . . . . . . 173

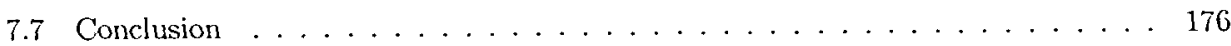


8 Separation in Common Features $\quad \mathbf{1 7 9}$

8.1 Introduction . . . . . . . . . . . . . . . . . . . . . . . . 179

8.2 Separation in Cointegration and in Cofcaturc . . . . . . . . . . . . 182

8.2 .1 Definitions . . . . . . . . . . . . . . . . . . 182

8.2.2 Relation between Cointegration Separation and Cofcature Separation . . 183

8.2 .3 Scparation and P-T Decomposition . . . . . . . . . . . . . . 186

8.3 Separated Cointegrated versus Partial Systems . . . . . . . . . . . . . 188

8.3.1 Weak Exogencity in Cointegrated VAR with Common Features . . . . . 189

8.3.2 Weak Exogeneity and Separability . . . . . . . . . . . . . . . . 190

8.3.3 Partial vs. Marginal Subsystem . . . . . . . . . . . . . . . . . 194

8.4 Inference on Cofeature Separation and Modeling Strategy . . . . . . . . . . 195

8.5 Empirical Analysis . . . . . . . . . . . . . . . . . . . . . . 198

8.5 .1 The Economic Model . . . . . . . . . . . . . . . . . . . . 199

8.5 .2 Empirical Results . . . . . . . . . . . . . . . . . . 200

8.6 Conchuding Remarks . . . . . . . . . . . . . . . . . . . . 210

9 Common Cyclical Features in Panel Data 213

9.1 Introduction . . . . . . . . . . . . . . . . . . . . . 213

9.2 Common Features in Time Series . . . . . . . . . . . . . . . . . . 214

9.3 Extension to Panel Data Models . . . . . . . . . . . . . . . . . 215

9.3 .1 A Pancl VECM Representation . . . . . . . . . . . . . . . . . 217

9.4 GMM Estimation . . . . . . . . . . . . . . . . . . . . 220

9.4 .1 Heterogeneous Independent Case . . . . . . . . . . . . . . . . 221

9.4 .2 Homogeneous and Heterogeneous Dependent Case . . . . . . . . . . 222

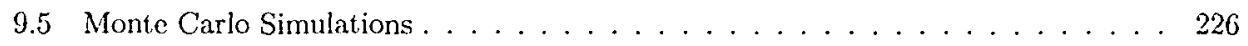

9.6 Empirical Analysis . . . . . . . . . . . . . . . . . . . . . . . . 229

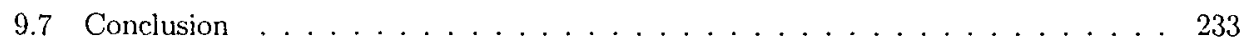

$\begin{array}{ll}10 \text { Postscript } & 237\end{array}$

$\begin{array}{ll}\text { Bibliography } & 243\end{array}$

$\begin{array}{lr}\text { Dutch Summary } & 259\end{array}$

$\begin{array}{ll}\text { French Summary } & 261\end{array}$

$\begin{array}{ll}\text { Curriculum Vitae } & 263\end{array}$ 


\section{List of Figures}

1.1 Per Capita US Real Regional Incomes and First Cointegrating Vector . . . . . . 6

1.2 Growth Rates of Regional Incomes . . . . . . . . . . . . . . . 6

1.3 First Common Cyclical Feature Relationship . . . . . . . . . . . . . 7

1.4 Spectral Densities of Income Growth Rates and of Co-feature Relationship . . . . 11

2.1 Basic Commodity Price Levels . . . . . . . . . . . . . . . . 48

2.2 The Four SCM $(0,0)$ Relationships for Prices . . . . . . . . . . . . 49

3.1 Log Levels of Macro Aggregates . . . . . . . . . . . . . . . . . . . . . . . . . . .

3.2 Growth Rates of Consumption, Output and Investment $\ldots \ldots \ldots \ldots \ldots$

3.3 Great Ratios . . . . . . . . . . . . . . . . . 79

4.1 Macro Aggregates and Permanent Component . . . . . . . . . . . . . . 98

4.2 Cyclical Parts for $y_{t} \ldots \ldots \ldots \ldots \ldots \ldots \ldots \ldots$

4.3 Output Cycles with WF for $y_{t}$ and $y_{\imath}^{*} \&$ NBER Contraction Periods . . . . . . 99

4.4 Unique Weak Form Common Cycle and NBER Contraction Periods . . . . . . . 99

4.5 Stock Market Indices (real US/logs/quarterly) . . . . . . . . . . . . . . . . 102

4.6 First two Cointegrating Vectors (not short-run adjusted) . . . . . . . . . . . 102

4.7 Time Series, Trend and Cycle Components . . . . . . . . . . . . . . . 105

4.8 Three Common Trends . . . . . . . . . . . . . . . . . . . . . . 106

4.9 Two SF Common Cycles . . . . . . . . . . . . . . . . . 106

5.1 A Rcalisation of the DGP. Data in Levels . . . . . . . . . . . . . . . . 119

5.2 A Realisation of the DGP, Cointegrating Vectors . . . . . . . . . . . . . 119

$5.3 \wedge$ Realisation of the DGP, Data in First Differences . . . . . . . . . . . 120

5.4 A Realisation of the DGP, Cofeature Relationships . . . . . . . . . 120

5.5 Belgian Provinces . . . . . . . . . . . . . . . . . 127

5.6 Two Step Approach, Cyclical Parts of Flemish Provinces . . . . . . . . . . . 129

5.7 Two Step Approach. Cyclical Parts of Walloon Provinces . . . . . . . . . . 130

5.8 Switching Method, Cyclical Parts of Flemish Provinces . . . . . . . . . . 132

5.9 Switching Method, Cyclical Parts of Walloon Provinces . . . . . . . . . 133 
6.1 Per-Capita Real and Real Private Output \& NBER Contractions . . . . . . . . 140

6.2 Model Comparison: 1-step ahead MSFE . . . . . . . . . . . . . . . 146

6.3 Model comparison, 4 -step ahead MSFE . . . . . . . . . . . . . . . . . . . . . . . . . . . .

6.4 MSFE for Model 1 with $\mathrm{p}=2, \mathrm{H}=1$ to $16 \ldots \ldots \ldots \ldots \ldots$

6.5 MSFE for Model 1 with $\mathrm{p}=6, \mathrm{H}=1$ to $16 \ldots \ldots \ldots \ldots \ldots$

6.6 MSFE for Model $2, \mathrm{H}=1$ to $16 \ldots \ldots \ldots \ldots \ldots \ldots \ldots$

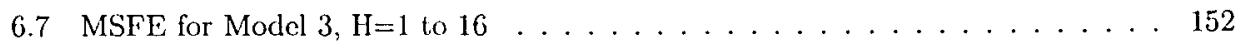

6.8 MSFE for Model $4, \mathrm{H}=1$ to $16 \ldots \ldots \ldots \ldots \ldots \ldots \ldots$

6.9 Stability Analysis / Model 3 / 1-step-ahead MSFE . . . . . . . . . . . . . . 156

6.10 Stability Analysis / Model 2 / 1-step-ahead MSFE . . . . . . . . . . . . . 157

6.11 MSFE, Comparison of $\operatorname{VAR}(1)$ and $\operatorname{VAR}(5)$ under $2 \mathrm{WF} \ldots \ldots \ldots \ldots$

7.1 Japanese Consumption and Income at Constant Prices (SA \& NSA) . . . . 168

7.2 Theoritical p-values of Ljung-Box Statistics in a SA White Noise . . . . . . . . 174

8.1 Cointegrating Relationships . . . . . . . . . . . . . . . . . . . 202

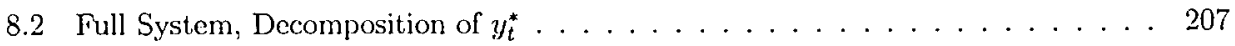

8.3 Full System, Decomposition of $y_{t} \ldots \ldots \ldots \ldots \ldots \ldots$. . . . . . . . . . . . . . . . . 208

8.4 Marginal Systems, Decomposition of $y_{t}^{*} \ldots \ldots \ldots \ldots \ldots \ldots$

8.5 Marginal Systems, Decomposition of $y_{t} \ldots \ldots \ldots \ldots \ldots \ldots \ldots$

8.6 Common Cycles in the Full System . . . . . . . . . . . . . 210

8.7 National Common Cycles in Marginal Systems . . . . . . . . . . . . 211

9.1 A Realization of the GDP for 10 Individuals . . . . . . . . . . . . 227

9.2 A Realization of the DGP with Additional Heteroscedasticity . . . . . . . . . 227

9.3 Consumption and Output Series for the 22 OECD Countries . . . . . . . . . 229 


\section{List of Tables}

2.1 Sizc and Power of Codependence Tests for DGP $(2.62) \ldots \ldots \ldots \ldots$. . . . . 41

2.2 Size and Power of Codependence Tests a MA(2) . . . . . . . . . . . . . 41

2.3 Size and Power of Codependence Tests in a ARMA $(1,1) \ldots \ldots \ldots \ldots$. . . . . . 41

2.4 Size of Codependence Test Statistics in a VAR . . . . . . . . . . . . 42

2.5 Power of Codependence Test Statistics in a VAR . . . . . . . . . . . . . 42

2.6 Power of Codependence Test in Omission of a Cointegrating Vector . . . . . . . . 43

2.7 Size of Codependence Tests in the Presence of GARCH . . . . . . . . . . . 43

2.8 Codependence Analysis for the Growth of Composite Leading Indicators . . . . 46

2.9 Values of Normalized SCM test statistics for Belgium/Germany . . . . . . . . . 46

2.10 Number of Zeros for Begium/Germany . . . . . . . . . . . . . . . . 47

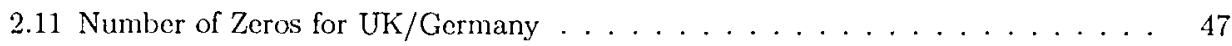

2.12 Exchangeable Models using SCM . . . . . . . . . . . . . . . . 59

3.1 Reduced Rank Hypotheses for $n=4 \ldots \ldots \ldots \ldots \ldots$

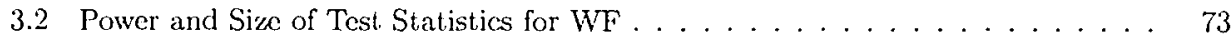

3.3 Power and Size of Test Statistics for SF . . . . . . . . . . . . . 74

3.4 Power and Size of Test Statistics for WF with Estimated $\beta \ldots \ldots \ldots \ldots$

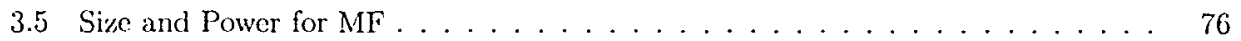

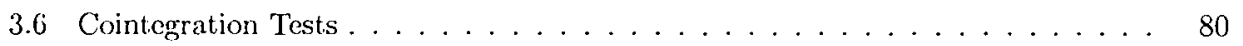

3.7 Common Feature Tests . . . . . . . . . . . . . . . . . . . . 80

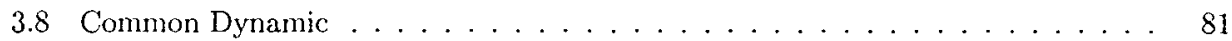

$3.9 \mathrm{WF}$ and SF Test Statistics without Dummies $(\mathrm{p}=2,3) \ldots \ldots \ldots$

$3.10 \mathrm{WF}$ and SF Test Statistics with Dummies $(\mathrm{p}=2,3) \ldots \ldots \ldots \ldots$

4.1 Common Features Test Statistics . . . . . . . . . . . . . . . . . . . . . . . . . . . . . .

4.2 Error Term Diagnostics . . . . . . . . . . . . . . . . . . . . . . . 101

4.3 Johansen Tests for Cointegration $(1975: 2-1999: 4) \ldots \ldots \ldots \ldots$. . . . . . . . 101

4.4 Common Cyclical Fcaturcs Tests $(1975: 2-1999: 4) \ldots \ldots \ldots \ldots$. . . . . . 103

5.1 Switching Cointegrating Trace Tests under WF restrictions . . . . . . . . . . 121

5.2 Two Step and Iterative WF Common Feature Tests, $p=2$ and $4 \ldots \ldots \ldots$. . . 122

5.3 Cointegration Trace Statistics, $\mathrm{p}=3,1947-1997 \ldots \ldots \ldots \ldots \ldots$. . . . . . . 124 
5.4 Two Step and Iterative WF Tests Statistics . . . . . . . . . . . 125

5.5 Iterative Cointegration Analysis . . . . . . . . . . . . . . . 125

5.6 Statistics on Provincial Employment Growth Rates . . . . . . . . . . . . 127

5.7 Statistics on Provincial Employment Cycles: the Switching Approach . . . . . . . 131

5.8 Statistics on Provincial Employment Cycles: the Two Step Approach . . . . . . . 131

6.1 p-values of Common Cyclical Features for Model 1 with $\mathrm{p}=2 \ldots \ldots \ldots$

6.2 p-values of Common Cyclical Features for Model 1 with $\mathrm{p}=6 \ldots \ldots \ldots$. . . . 142

6.3 p-values of Common Cyclical Features for Model $2 \ldots \ldots \ldots \ldots$. . . . . . 142

6.4 p-values of Common Cyclical Features for Model $3 \ldots \ldots$. . . . . . . . 143

6.5 p-values of Common Cyclical Features for Model $4 \ldots \ldots \ldots \ldots$

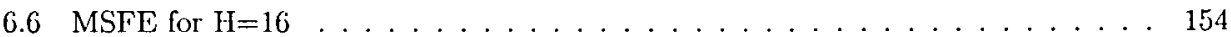

6.7 TMSFE for Model $2, \mathrm{H}=16 \ldots \ldots \ldots \ldots \ldots \ldots \ldots$

6.8 Determination of $\mathrm{p}$ and s Simultancously using Information Criteria $\ldots \ldots \ldots \ldots$

7.1 Empirical Sizes and Co-feature Vector Median and Spread for NSA . . . . . . . 165

7.2 Empirical Sizes and Co-feature vector Median and Spread for X-11 SA . . . . . 165

7.3 Empirical Sizes-Adjusted Power, Co-feature Vector Median and Spread . . . . . 166

7.4 HEGY Unit Root Test Statistic . . . . . . . . . . . . . . . . . . . . . 167

7.5 HEGY Seasonal Cointegration Tests . . . . . . . . . . . . . . . . . 167

7.6 Common Feature Test Statistics for Japanese Consumption Function . . . . . . . 169

7.7 Seasonal Monthly Unit Root Tests . . . . . . . . . . . . . . . . . . . 170

7.8 WF Tests Statistics for Raw Series $(\mathrm{p}=10) \ldots \ldots \ldots \ldots \ldots \ldots \ldots \ldots \ldots \ldots$

7.9 WF Tests Statistics with SA Series $(p=3,10) \ldots \ldots \ldots \ldots \ldots \ldots$

7.10 Empirical Sizes and Cofeature Vector Median and Interquartile Ranges for X-11 SA175

8.1 Weak Exogeneity Conditions . . . . . . . . . . . . . . . . . . . . 192

8.2 Analysis of Residuals of a VAR(4) (p-values) . . . . . . . . . . . . 201

8.3 Johansen's ML Tests Statistics . . . . . . . . . . . . . . . . . . . 201

8.4 Hypotheses Testing . . . . . . . . . . . . . . . . . . . . . 203

8.5 Common Feature Tests . . . . . . . . . . . . . . . . . . 204

8.6 FIML Estimates under Separation in Cointegration and in W F Common Features 206

8.7 Testing Short-Run Separation of Type At . . . . . . . . . . . . . . 206

8.8 FIML Estimates of the Common Factor Representation . . . . . . . . . . 206

8.9 Common Factors . . . . . . . . . . . . . . . . . . . . . 208

9.1 Monte Carlo Results: Separated vs. Marginal Systems . . . . . . . . . . . . . . 216

9.2 Monte Carlo Results: GMM Estimation and Test Statistics . . . . . . . . . . 228

9.3 Time Series Statistics: Individual Countries . . . . . . . . . . . . . . . 231

9.4 Common Features within 22 OECD Countries . . . . . . . . . . . . . . . 234

9.5 Common Features within G7 Countries . . . . . . . . . . . . 235 
10.1 Summary of Empirical Results . . . . . . . . . . . . . . 239 


\section{Preface}

I wish to thank the people and the events who helped me to accomplish this dissertation. Let me chronologically comment how I get here. I apologize if this preface is a little bit too long but I think it is the first time I've got the opportunity to thank these people. I'm not able to dissociate the people from the events because events are related to the fortune I have had to meet these people.

First of all, I would like to thank Jean-Pierre Urbain who is not only the co-supervisor of this thesis but also the person who guided me towards econometrics. When I was a student in economics at the Univcrsity of Liège, Jean-Pierre was a research assistant and gave, around 1987-1989, additional material for the econometric classes. Like most lazy, say normal, students I hated econometrics and mathematical economics in general. Indeed, I did economics because I wanted to be a journalist. I'm used to claim that Jean-Picrre was unbearably conceited (at that time) that I decided to do econometrics. This is only partly truc. I was also enthusiast to learn during Jean-Pierre's lectures how due to the presence of misspecifications in the residuals, it was casy to tell any story from an estimated regression. Models used by policy makers were so contaminated that. I was amazed how we all could still believe what it was said on TV news or written in newspapers. I then attended econometrics lectures and I wrote my bachelors thesis on a part of a Belgian econometric model. Fortunately, I have changed my mind vis-à-vis JeanPierre's behavior since that period and Jean-Pierre has also partly changed his mind vis-à-vis students. I have got to confess that we have a good friendly and working feeling for 10 years now.

After being graduated in economics, I felt unsatisfied with my four year studies or more preciscly with my knowledge: I had the fecling to know nothing actually. A plumber can repair something, a physician can save life. But as an economist, except those who believe they will save the world using an augmented Philips curve (I swear I knew some), I saw most of my former schoolmate working at banks via the indispensable experience at the wicket. However, the big problem when you put one foot into econometrics is that you catch the virus and you are not ready to accept to do anything else anymore. You are lost for the usual job market. I decided in 1989 to do a Masters degree in Louvain-La-Neuve with all the econometric courses I could find. I was there with Frederic Docquier who was a schoolmate of mine at the university and was also a research assistant in economics at the University of Liège. He introduced me to Professor Gadisscur from the Department of Quantitative History. He was seeking for someone to work on 
the availability of statistical data in Belgium. I started to work in 1990 at the University. I'm grateful to Frederic for that.

Of course my intellectual preference was still for cconometrics but I also learned a lot about the measurement, the building and the sources of statistical data. I had also the fortune to work with Eric Geerkens on these projects. I would like to thank him for these 5 or 6 years we worked together and for his friendship. Not only he helped me to slow down my elocution but I partly owe him to pass my Masters degree. Indeed, at many times Eric did my part of the job when we had to rush on the writing of a report while I had to study for an exam.

I decided in 1991 to work, in the beginning at part time, as an econometrician in the Department of Economics and Statistical Studies of the Ministry of the Walloon Region. I think I did that because I was proud to get the title of Econometrician. When I try to look at this period objectively, I even sce this experience as a positive one. I worked with nice people with whom I wrote some papers and had nice time. These are Frederic Docquier, Benoit Mahy and Michael Beine. This job has also showed me that I did not want to do this kind of work for this kind of pcople anymore (if you allow me to censor myself my vocabulary).

Consequently, when Jean-Pierre Urbain told me there existed a Ph.D. project about common cycles at Maastricht University, I was very excited to apply for this position. Here, I would like to thank (my vocabulary is too poor to find another expression) Delphine Baudoux, my partner. Her support was decisive to help me to make this jump in this new life, in this new job associates with a low salary and with an increase of uncertainty about the future. But whatever the comfortable position I lost and the associated material stuff I could not afford anymore, Delphine helped me to remember how life is short and that the most important thing is to enjoy it. There are so few people who have the fortune to do the things they like that I'm happy to have followed Delphine's advises and I hope I will still be indebted to her for a long time.

Next event was the interview I had to have with my future supervisor, Franz Palm. I had a paper (jointly with Michael Beine) at the ESEM96 in Istanbul and Jean-Pierre Urbain told me that it would be nice to get in touch with Franz Palm to obtain additional information about this AIO position. By chance we were at the same hotel but I was too shy to ring his door and I had decided to give up. The last day of the mecting; during breakfast, a person seated just near my table. I had the feeling he was Franz Palm even if I did not know what he looked like exactly because I caught only a glimpse of him a few years ago at Jean-Pierre Ph.D. defence. Michacl Beine convinced me to contact him and...He was Him. Franz proposed me to meet him later after having breakfast. I wore summer holiday clothes with a pair of shorts, I had wet hair after the morning shower and I was not ready at all for an interview. Actually, I did not know it was an interview and I was certainly less nervous than usual. A few weeks later, Franz Palm decided to give me the opportunity to work in this University with such a pleasant atmosphere and smart people around such as my colleagues Lars, Veronika, André and Dries. I wish also to thank Franz Palm for his experience I benefitted during these years and for his ability to be willing to lend a sympathetic ear to others. Actually I haven't realized that I was working under the supervision of Franz Palm and Jean-Pierre Urbain although I learned a lot from them. Instead, I've got the 
fecling that we have really worked together for four years and I hope this will be continued.

I have also had the luck to meet during my Ph.D. period nice people and friends. First of all, I must confess that I prefer Bertrand Candelon as a friend than as a discussant of a paper of mine as it was the case in Paris in early 1997. Beyond beers we had, we have spent many days in exchanging stimulating point of view and this from the time he was visiting Maastricht University for six months until now. We behave ourselves as a counter-example of what common features are. Both of us we make many linguistic errors in English but when we write down a paper together, something happens that amplifies our style to get something unreadable. I also want to thank my roommate for two years, Horst Zank, for his precious mathematical knowledgo as well as for his patience when he tried to teach me some dutch although he unfortunately failed (my feeling is that this was the first time Horst failed in doing something but maybe I'm wrong). I also want to thank Gianluca Cubadda for the stimulating discussion we had via c-mails, here in Maastricht and on his nice terrace in Roma about common features and wine.

The first versions of some of our papers have also benefitted from the comments and remarks of anonymous referces as well as from the comments of Michael Beine, Jocrg Breitung, Niels Haldrup, Tommaso Proietti and Bertrand Candelon. I also wish to thank the members of my Ph.D. committee, namely Peter Schotman, Nicls Haldrup and Gerard Pfaan who together with Jean-Pierre Urbain and Franz Palm have read carefully this work. They have improved this text through their questions and remarks as well as through the thousands of typos and linguistic errors they found. Obviously, I'm only responsible of remaining errors and the usual disclaimer applies. I'm not good in doing forecasts (otherwise I would certainly be an economists and I do not try to be an applied cconometrician) but I can already foresce that there are, in average, many more linguistic errors in this three pages preface nobody has corrected before than in the rest of this thesis.

I would like to thank Georges and Annette Blaise for the design of the cover. I also wish to thank my parents and my children Lucile and Achille. Even if these two 2 and 5 years old devils have frequently slowed down my work and decreased my efficiency, they have also showed me that something else exists in life.

Thank you all,

I have drawn extensively on our published articles and discussion papers: Chapter 2 reviews material in Hecq (1997) with elements from Beine and Hecq (1997, 1998, 1999) and Candelon and Hecq (2000); Chapter 3 draws on Hecq, Palm and Urbain (1998); Chapter 4 on Hecq, Palm and Urbain (2000a) and Hecq, Palm and Urbain (2000d); Chapter 5 on Hecq (1999) and partly on Candelon, Hecq and Lohest (2000); Chapter 6 on Cubadda and Hecq (2000); Chapter 7 on Hecq (1998) with elements from Beine, Candelon and Hecq (2000); Chapter 8 on Hecq, Palm and Urbain (2000c) and Chapter 9 on Hecq, Palm and Urbain (2000b).

I have tried to write up this thesis as a monograph. Once the concepts defined and the definitions given, I usually refer to those expressions in subsequent chapters. Consequently, the 
chapters included in this work do not clone their published version. Sometimes a chapter has been shortened due to the omission of redundant material, sometimes it has been extended because I have added additional applications (like in Chapters 3 and 7), I have mixed several papers (like in Chapters 2, 4 and 5) or I have kept an appendix (like in Chapter 4).

Equations are numbered consecutively within each chapter, with the first number referring to chapters. Definitions, corollaries, remarks...are also numbered consecutively throughout each chapter and are independent of the section in which they appear. That means that the first definition of Chapter 1 will be 1.1 and the next expression, for example a remark, will be 1.2 . Tables and figures are also numbered consecutively in each chapter but with their own numbering.

Alain W. Hecq

July 2000 


\section{Chapter 1}

\section{Introduction and Outline of this Thesis}

\subsection{Introduction}

Economic and financial time series exhibit many distinctive characteristics such as serial correlation, seasonality, volatility, trends, breaks, outliers or non-lincarities. However, in a multivariate analysis it is frequent to observe that one or more of these features detceted in the single series are common to several variables and thus disappear by some suitable combinations. Recognizing these common feature structures presents numerous advantages from an economic and a statistical point of view. Indeed, common features can be exploited to reduce the number of parameters to cstimatc and may increase efficiency since redundant factors can be removed. The summarization process helps to interpret the dynamies of the multiple series and may also improve forecast accuracy. The number of these common factors, their composition as well as the vectors that annihilate them are of main interest in economics because economic theory often predicts and explains such comovements.

For example, Keynes (1936)'s General Theory explains how consumption and real disposal income move together or how due to entrepreneurs' expectations, fluctuations in employment follow closely the fluctuations in real GDP. The European Union has imposed convergence criteria in terms of public debt, inflation or interest rates before countries may participate in European Monetary Union. Inflation pressures and indexation mechanisms force wages and prices to increase at similar rates. Interest rates for different maturities as well as spot and forward asset prices should not drift apart too far. Also the volatility of securities with the same risk is expected to be similar; there exists a comparable peak in the inflation rate for several countries after the first oil shock; there is a break in the national output growth for US and for most European countrics after the WWII; due to climatic conditions several commodity prices presents the same kind of seasonality. More generally, economic behavior, market forces and intervention mechanisms make economic variables tend to co-move. 
Consequently, it is observed in many empirical analyses that the whole is not necessarily equal to the sum of the parts. Obviously, only some aspects of this reduction process are analyzed in this monograph and the next sections of this chapter circumscribe its scope. We illustrate the distinction between exogenous/endogenous and between observable/latent factors. We also present an introductory analysis of long and short-run comovements.

$\Lambda$ word on notation used throughout the thesis: in the sequel $y_{t}=\left(y_{1 t}, \ldots, y_{n t}\right)^{\prime}$ denotes a $n$-dimensional process defined over a time scale $t=1 \ldots T$. Therefore the realization of a stochastic process (or the stochastic process itself) will be written as $\left\{y_{t}\right\}_{t=1}^{T}$ or $\left\{y_{t}, t=1 \ldots T\right\}$. However, it will often occur when there is no risk of confusion that we use solely $y_{t}$ to denote the stochastic process. $L$ and $\Delta$ are the common lag and first difference operators such that $L y_{t}=y_{t-1}$ and $\Delta y_{t}=y_{t}-y_{t-1}$. There may exist an ambiguity because in some empirical analyses $y$ also refers to national income or gross domestic product as in main macroeconomic textbooks. But usually the meaning should be obvious from the context and hence cause no confusion. When the same set of $n$ time series is considered for more then one individual or countries like in panel data analyses, $N$ refers to the number of individuals. $r$ and $s$ denote respectively the number of cointegrating and common feature vectors. When both long and short-run restrictions are involved, $\beta$ and $\tilde{\beta}$ refer respectively to cointegrating and common feature matrices of dimension $n \times r$ and $n \times s$ respectively. In some cases, like in Chapter 2 when we analyze common cyclical features in a stationary world, we often use $\delta$ instead of $\tilde{\beta}$ in order to simplify the notation.

\subsection{Typology of Common Factors}

The different aspects of this work can be introduced using the basic "Markowitz-Sharpe-Lintner" capital asset pricing model (CAPM). This equilibrium asset return model is widely used in financial literature and has given rise to numerous econometric evaluations. Denoting respectively $r_{i}, r_{f}$ and $r_{M}$ the returns on an asset $i=1 \ldots n$, on the risk-free asset and on the overall market portfolio, the CAPM can be analyzed empirically through the multivariate conditional regression model (sce for example Mills 1993, p150):

$$
y_{t}=\mathbf{a}+\mathbf{b} f_{t}+u_{t}, \quad t=1 \ldots T
$$

where $\mathrm{a}$ and $\mathrm{b}$ are $n \times 1$ vectors of parameters, $y_{t}=\left(r_{1}-r_{f}, \ldots, r_{n}-r_{f}\right)_{t}^{\prime}, f_{t}=\left(r_{M}-\right.$ $\left.r_{f}, \ldots, r_{M}-r_{f}\right)_{t}^{\prime}$ and the error $u_{t}$ is a $n$-dimensional zero mean NIID process with $E\left(u_{t} / I_{t-1}\right)=0$, $\operatorname{Cov}\left(u_{t}, f_{t} / I_{t-1}\right)=0, V\left(u_{t} / I_{t-1}\right)=\Omega$ independent of $I_{t-1}=\left\{y_{t-1}, f_{t-1}, y_{t-2}, f_{t-2} \ldots\right\}$.

This simple model illustrates the main concepts used in common feature analysis:

1. The overall market portfolio (or the basic portfolio) $f_{l}$, is (by some means or other) a linear combination of different asset returns, say $f_{t}=\mathbf{c}^{\prime} y_{t}$. Indeed, the column vector $\mathbf{c}$ gives the weights of different securities entering in the market index. The variables $f_{\ell}$ or alternatively 
c are called common factors or features. The disturbances $u_{t}$ are called specific factors. ${ }^{1}$

2. The factor loadings $b$ are the regression coefficients of the assets' payoffs on the payoffs of the market portfolio and collect the "betas" for the $n$ assets.

3. The orthogonal direction $\mathrm{d}$ with $\mathrm{d}^{\prime} \mathbf{b}=\mathbf{0}$ gives the linear combination of $y_{t}$ not infuenced by $f_{t}$. The column vector $\mathrm{d}$ or the associated linear combination $\mathrm{d}^{\prime} y_{t}$ are called the cofactors or the common feature vector/relationship. Some of these cofactors are associated with portfolios whose payoffs only depend on the idiosyncratic risk and are called zero-betas portfolios. ${ }^{2}$

In the CAPM (1.1), the common factor is observable because it is usually a market price index like the S\&P 500 for US or the CAC 40 for France. The common factor is also endogenous because the underlying price index is a weighted average of some selected individual stocks. Other kinds of factor models may be found in the literature. Before the formulation of the modern version of CAPM, Sharpe (1963) proposed a single index model. In this descriptive empirical model, asset, returns are influenced by an unobservable construct, a unique latent factor. Stacking assets $i, \forall i=1 \ldots n$, the following multivariate regression can be considered:

$$
\tilde{y}_{t}=\tilde{\mathbf{a}}+\tilde{\mathbf{b}} \tilde{f}_{t}+\tilde{u}_{t}
$$

where $\overline{\mathrm{a}}$ and $\tilde{\mathrm{b}}$ are $n \times 1$ vectors of parameters, $\tilde{y}_{t}=\left(r_{1}, \ldots, r_{n}\right)_{t}^{\prime}$ and the factor $\tilde{f}_{t}$ is a combination of economic indicators (gross domestic product, commodity prices, stock market indexes, interest rates...) that will influence assets' payoffs. In this case the factor is mainly exogenous becausc "cxternal" cconomic variables enter in $\tilde{f}_{t}$ and is unobservable because economic theory does neither formalize the relationship between these variables nor their weights in this construct. Both equations (1.1) and (1.2) stress the fourth important aspect in the common feature literature, namely:

4. The rank of $\overline{\mathrm{b}}$ and $\mathrm{b}$, or alternatively of $f_{t}$ and $\tilde{f}_{t}$ is equal to the number of common factors (sce Costa et al., 1997 for a more formal discussion about the CAPM and the APT models as well as their link to the reduced rank regression approach).

The CAPM (1.1) is an "ideal" model because elements of the common factor representation (1.1) have a direct economic meaning. In many applications, economic theory tells something about the cofactors (PPP as a cointegrating vector or the marginal propensity to consume are some examples) or about the loadings. But economic theory is rarely very helpful for determining the factors cxcept maybe their number like in applications on economic convergence among

\footnotetext{
${ }^{1}$ Notice that the CAPM model imposes the $n$ restrictions that the intercepts in each asset return equation are zero, i.e. $\mathbf{a}=0$.

'See for example Gourieroux, Monfort and Renault (1991) or Cuthbertson (1996) for further developments around the CAPM and Pastorello and Renault (1994) for more general factor models in finance.
} 
countries or regions. In other cases, stylized facts combined with economic theory propose clues for analyzing relationships: e.g. within macrocconomic aggregates, investment is more volatile than output which in turn is more volatile than consumption. In other applications the use of these methods will be considered as a multivariate descriptive data analysis issue. An example is the composite coincident indicator for business cycle measurement. (see inter alia Stock and Watson, 1991). This reduction analysis is also important to discover hidden common time functions within macrocconomic fluctuations and to help in uncovering masked common channels of transmission. This descriptive dircction is strongly connected with explanatory factor analyses applied for decades in biology, geology, psychology or social sciences. ${ }^{3}$

In this thesis we mainly focus on multivariate closed dynamic systems, that is to say on $\operatorname{VAR}(p), \operatorname{VMA}(q)$ or more generally $\operatorname{VARMA}(p, q)$ processes. Hence, the reduced number of dynamic factors that can summarize the behavior of a set of time series will mostly be a function of the previous observations of the dependent variables and the previous realizations of a noise. Consequently, the common features are endogenous and unobservable, a mixture of equations (1.1) and (1.2) in a sense. It will turn out that to know whether the factor analysis is explanatory or confirmatory will depend upon the talkativeness of economic theory for modeling economic relationships in that specific case.

\subsection{Cointegration \& Cyclical Feature: An Introductory Example}

A common feature analysis may be applied to anything that is present in univariate time series and which disappears by some appropriate combinations because more than one variable share this feature. For instance, the presence of common seasonality, common ARCH, common nonlinearities, common breaks or common outliers can be tested. However, due to the importance of the spurious regression issue (Granger and Newbold, 1974), the vast literature has mainly focussed on long-run comovements, namely the search for common stochastic trends (see Stock and Watson, 1988), through cointegration analyses. More recently, some authors have also analyzed the existence of short-run comovements between stationary time series or between first differences of cointegrated I(1) series (see Engle and Kozicki, 1993; Gouriéroux and Peaucelle, 1989; Tiao and Tsay, 1989), namely the presence of a common cyclical features. Among these approaches, the concept of serial correlation common features (henceforth SCCF) developed by Engle and Kozicki (1993) and previously, with different names, by Tiao and Tsay $(1985,1989)$, Velu, Reinsel and Wichern (1986) and Gouriéroux, Monfort and Renault (1991) appears to be useful. The existence of SCCF implies that stationary time series move together such that there exist linear combinations of these variables which yield whitc noise processes. ${ }^{4}$

This thesis focuses on the last two types of common features for macroeconomic analyses,

\footnotetext{
${ }^{3}$ See Basilevsky (1994) for a large spectrum of applications in different fields.

tNote that this thesis aims at investigating weaker notion where linear combinations exhibits a much less degree of serial correlation.
} 
i.e. on the treatment of both long-run and short-run comovements in an integrated setting. In order to sketch the main lines of this monograph, let us consider a subset of the time series we analyze in Chapter 5. These are the per capita real incomes for three (out of eight) regions of the United-States: New England (NE), Mideast (ME) and Great Lakes (GL). We can investigate the degree of convergence achieved by the three regions either in the long-run (Bernard and Durlauf, 1995) or in the short-run (Beine, Candelon and Hecq, 2000). Notice that the use of the past perfect in the previous sentence is meant to stress that both a common feature and a cointegration approach gives evidence of a realized process and not of the evolving process that would characterize an increasing integration between economics (see Beine and Hecq, 1998). The anmual variables run over 1948 to 1997. A VAR of order three seems to characterize quite well the covariance structure of the data. By reparametrization, the VECM reads:

$$
\Delta y_{t}=\mu+\alpha \beta^{* \prime} y_{t-1}^{*}+\Gamma_{1} \Delta y_{t-1}+\Gamma_{2} \Delta y_{t-2}+\varepsilon_{t}
$$

where $y_{t}=(\ln N E, \ln M E, \ln G L)_{t}^{\prime}, y_{t}^{*}=\left(y_{t}^{\prime}, \text { trend }\right)^{\prime}$ and $\mu$ is a vector of constants. The double scaled Figure 1.1 displays the log levels of these three variables and emphasizes the presence of upward trends that are maybe common and can thus be annihilated by taking some linear combinations of $y_{t}$. Indeed, Johansen's ML test statistics for the model with an unrestricted constant term and a restricted linear trend (see Johansen, 1995) reveal the presence of two common stochastic trends and thus one cointegrating vector given by (asymptotic standard errors in brackets):

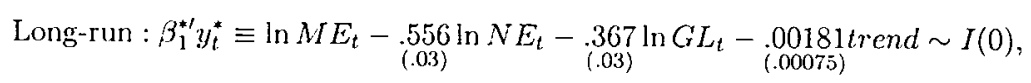

where the column vector $\beta_{1}^{*}$ is the first (significant) cointegrating vector for the model with a restricted deterministic trend in the long-run. The second part of Figure 1.1 plots this stationary relationship (non-adjusted for short-run dynamics). Figure 1.2 shows the first differences of regional incomes. It appears that the short-run fluctuations, namely the cyclical parts also secm to co-move. Searching for white noise directions or SCCF, that is searching for linear combinations of the first differences that are white noise processes (formally, we test using a canonical correlation analysis we see in next chapters whether there exists a matrix $\tilde{\beta}$ such as $\tilde{\beta}^{\prime} \Delta y_{t}=\tilde{\beta}^{\prime} \varepsilon_{t}$ ), we end up with the following relationship plotted in Figure 1.3.

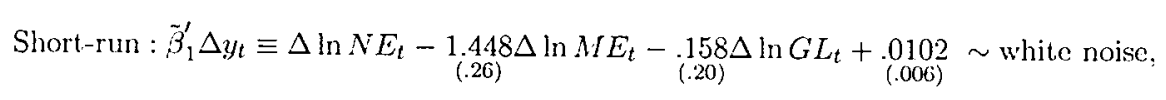

where Full Information Maximum Likelihood (FIML) estimates of standard errors are given in brackets and $\tilde{\beta}_{1}$ is the first cofeature vector. Both long and short-run relationships seem to be partially in accordance with a convergence hypothesis. Indeed while the signs favor this hypothesis, not as less as two independent common trends and two independent common cycles drive the system. 


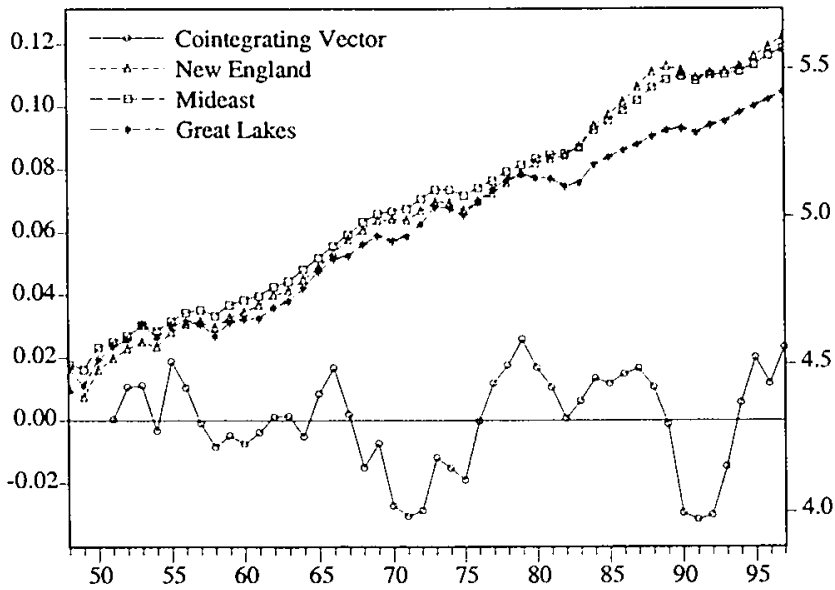

Figure 1.1: Per Capita US Real Regional Incomes and First Cointegrating Vector

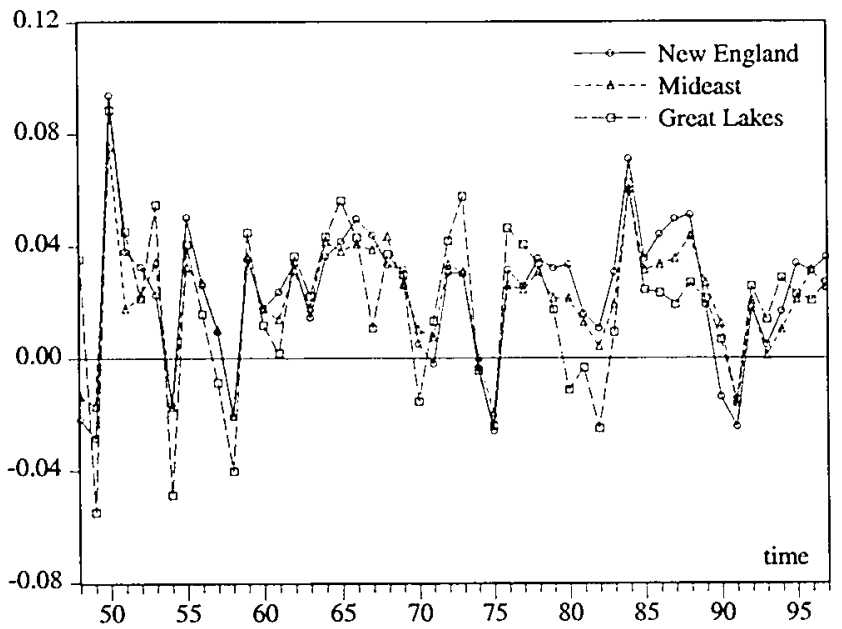

Figure 1.2: Growth Rates of Regional Incomes 


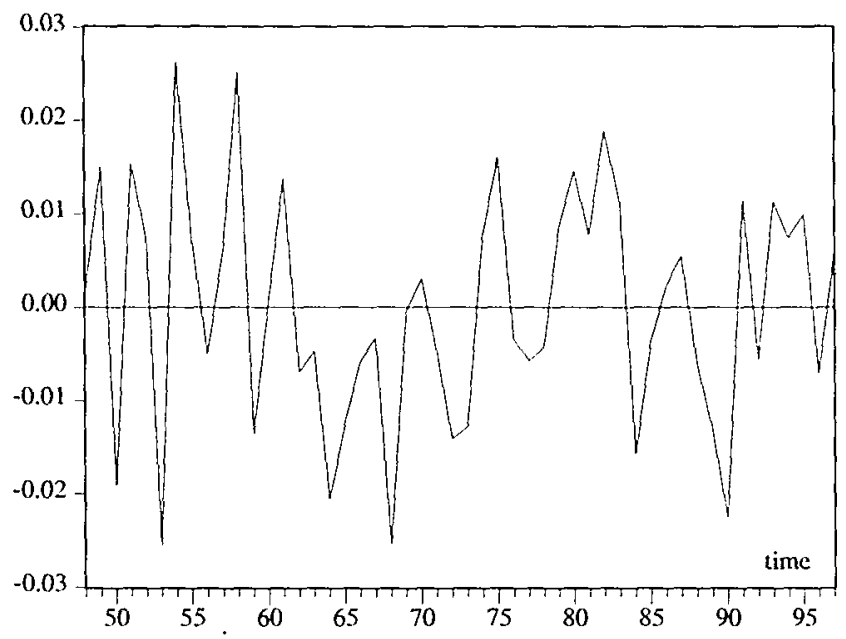

Figure 1.3: First Common Cyclical Feature Relationship

In order to avoid confusion it is worth noting from the outsct the place of a common cyclical feature approach in a general business cycle analysis. In the empirical economic literature, business cycle regularities are often presented as comovements of the deviations from a "trend" in different aggregated macroeconomic time series. Beyond this general intuitive definition, no consensus emerges about how to measure the trend (HP-trend, linear, quadratic or stochastic trends?) and how to analyze these so-called comovements. Moreover, no definitive answer exists about the primacy of using a theoretical framework over a more descriptive one. While comovements predict that expansions and downturns occur at about the same time in many industries and economic activities, even a clear definition of the duration of the business cycle is difficult to obtain: less than five years, between six and thirty-two quarters or between one and twelve years as in Burns and Mitchell (1946). Furthermore, it is not easy to determine the time series that are worth considering: national accounts, industrial production, prices, unemployment rates, sectorial data, monetary and financial variables, international indicators or regional ones. All these variables potentially explain and help measuring the business cycles.

Consequently, the statistical definition of the cycle used in this thesis fits well into Dicbold's (1998, p.128) more pragmatic view, namely that "cycles or cyclical fluctuations are any sort of dynamics (in the mean) not captured by trends or seasonals". 5 All that is required is that

\footnotetext{
${ }^{5}$ Notice that Diebold does not define what is meant by trend. In this thesis we mainly focus on (the reduce number of) stochastic trends for capturing the long-run behavior.
} 
there must be some dynamics, some persistence, some way by which the present is linked to the past, and the future to the present. In this framework, the common cyclical feature analysis is explicitly one of the approaches we may follow when studying comovements among cycles, anong macroeconomic short-run fluctuations. For instance, system (1.3) with both cointegrating relationship (1.4) and under the existence of one SCCF restrictions becomes:

$$
\Delta y_{t}=\mu+\tilde{\beta}_{1 \perp}\left(\Psi_{1}, \Psi_{2}, \Psi_{3}\right)\left(\begin{array}{c}
\beta_{1}^{* \prime} y_{t-1}^{*} \\
\Delta y_{t-1} \\
\Delta y_{t-2}
\end{array}\right)+\varepsilon_{t}
$$

where the $3 \times 2$ full column $\operatorname{rank} \tilde{\beta}_{1 \perp}$ is the orthogonal complement to $\tilde{\beta}_{1}$ in (1.5) such that $\tilde{\beta}_{1}^{\prime} \tilde{\beta}_{1 \perp}=0_{1 \times 3}$ and $\operatorname{rank}\left(\tilde{\beta}_{1}: \tilde{\beta}_{1 \perp}\right)=3 . \Psi_{1}, \Psi_{2}, \Psi_{3}$ are respectively $2 \times 1,2 \times 3$ and $2 \times 3$ matrices. The rank of $\Psi=\left(\Psi_{1}, \Psi_{2}, \Psi_{3}\right)$ is 2 and thus less than the number of variables. Therefore, we regard the system (1.6) as being capable of providing a reduced number of propagation mechanisms, of channels for the transmission of the information derived from the dynamics $\left(\beta_{1}^{* \prime} y_{t-1}^{*}, \Delta y_{t-1}, \Delta y_{t-2}\right)$ - sec Brillinger (1969) for this type of physical interpretation.

\subsection{Outline of this Thesis}

This thesis aims at investigating methods that incorporate both long-run and short-run restrictions in a single model. The introductory example presented above is convenient to outline the different aspects of the common cyclical features we will address. System (1.6) summarizes the behavior of three regional incomes within a finite VAR. In this framework there exists a linear combination that annihilates the matrices of the stationary process reparametrized by differencing and cointegration. The common dynamic factors are functions of lags of dependent variables and crror correction tcrms. While this widely used modeling framework is convenient for empirical studies, other forms of common dynamics have been proposed in the literature. Chapter 2 proposes to survey different approaches and especially the scalar component model (SCM) of Tiao and Tsay $(1985,1989)$ and the codependence framework by Gouriéroux and Pcaucelle (1989, 1993). We also evaluate by Monte Carlo simulations the finite sample properties of common cyclical feature test statistics in the presence of various kind of misspecification: non-Gaussian residuals, omission of a cointegrating vector. We present several empirical applications mainly for stationary time scries or for the first differences of non-cointegrated $\mathrm{I}(1)$ processes. This is also the place for briefly reviewing other types of common features than the dynamic ones.

In the illustration of the previous section, we have "extracted" only one short-run relationship, i.e. (1.5). Actually more than one cofeature vector was present and discovering a second relationship would be of great interest because in this case there would exist a higher degree of short-run convergence. However, the assumptions underlying the white noise direction model (i.e. SCCF) are often too strong to find further common structures. For example we cannot test for both a long and a short-run strong convergence hypothesis which will assume, in the 
previous three variables linear case, there exist either two, i.e. $n-1$, cointegrating and SCCF vectors. Therefore, we introduce in Chapter 3 a weaker form of reduced rank structure. When such a form exists, lincar combinations of the first differenced variables corrected for the long-run effects are white noise. We also consider the mixed form which combines SCCF and weak forms. We discuss the model selection issues which arise from this distinction and propose a simple approach to testing for these structures using a sequence of likelihood ratio test statistics. The finite sample behavior of the sequential approach is analyzed in a Monte Carlo experiment. Finally, we illustrate the relevance of the different forms of reduced ranks with empirical analyses of US macrocenomic fluctuations over the period 1954-1996 and of the UK equity, gilt and moncy markets.

System (1.6) stresses the common cyclical features, namely $\Psi \equiv\left(\Psi_{1}, \Psi_{2}, \Psi_{3}\right)$ times the lagged variables and the error correction terms. Another important issue is the determination of the common cycles, especially when there are only a few, for interpreting macroeconomic fluctuations. Consequently, Chapter 4 derives common trend-common cycle decompositions of non-stationary multiple times series generated by finite order Gaussian $\operatorname{VAR}(p)$ models with both cointegration and serial correlation common features. We extend existing analyses to the classes of reduced rank structures discussed in Chapter 3. Using the corresponding state space representation of cointegrated VAR models in vector error correction form we show how decompositions can be obtained even in the case where the number of common feature and cointcgration vectors is not equal to the number of variables. We consider the US macrocconomic fluctuations analyzed in Chapter 3 as well as a study of comovements between international stock market indices.

The aim of Chapter 5 is twofold. First, using Monte Carlo simulations we illustrate the usefulness of a small sample corrected version of common feature test statistics. This small sample correction is similar to the one proposed by Reinsel and Ahn (1992) for cointegration and gives for small $T$ (like in the introductory example where $T=50$ at most), better finite sample sizes properties than those obtained using the asymptotic $\lambda^{2}$ distribution. Secondly, we consider the small sample performance of cointegration and common cyclical features test statistics when both cointegrating and common feature vectors are unknown. We stress the advantages of using an iterative strategy to maximize the likelihood by imposing long and short-run restrictions until convergence is achicved instead of a two-step procedure.

A point that is also worth considering concerns the consequences of imposing common cyclical feature restrictions for forecast accuracy. Several papers have assessed the forecasting gains of imposing long-run constraints in VAR models. Monte Carlo studies reveal that these cointegrating restrictions help essentially for long-term and sometimes medium-term forecasts. However, policy makers and business cycle analysts are more interested in time horizons shorter than one year. The main goal of Chapter 6 is to examine whether short-term forccast accuracy is improved by also imposing different types of common cyclical features restrictions. This chapter is concerned with predicting the quarterly US real GDP using various information sets. While it cmerges from Monte Carlo experiments that co-feature restrictions reduce forecast errors (Vahid and Issier, 1999), it is less obvious to find on real data sets a specification which uniformly 
encompasses rival systems.

Thanks to using annual observations, we have succeeded in the introductory example to avoid an important issuc, namely the impact of seasonality and especially of seasonal adjustment. In Chapter 7 we examine how SCCF test statistics behave when X-11 seasonal adjusted data are encountered. We emphasize both size and power distortions. We illustrate the analysis on Japanese consumption/income relationship and on European industrial production indexes.

Until Chapter 7 we analyze small closed systems with only a few time series, say with two to seven variables. In the final two chapters we consider several practical issues that arise in empirical analyses with high dimensional vector time series. A typical cxample being the analysis of macroeconomic fluctuations, using $n$ variables, for different countries. When the number of countries or individuals becomes large, standard multivariate time series methods can hardly be applied and are likely to have poor behavior in finite samples.

In a first attempt to deal with this issue, Chapter 8 discusses the concept of separation in the context of the two different classes of cointegrated VAR models introduced in Chapter 3 . We show the connections between separation in cointegration and in common cyclical features and give their implications in terms of weak exogeneity conditions. An empirical analysis of the link between American and Canadian cyclical fluctuations shows the practical relevance of the approach proposed in this chapter. A simple switching algorithm allowing to test the block diagonality of the cofeature space is proposed.

In Chapter 9 we propose another framework for analyzing larger system. We extend the concept of SCCF to panel data models. This analysis is motivated both by the need to develop a methodology to systematically study and test for common structures and comovements in pancl data with autocorrclation present and by an increase in efficiency coming from pooling procedures. We propose sequential testing procedures and study their properties in a small scale Monte Carlo analysis. Finally, we apply the framework to the well known permanent income hypothesis for 22 OECD countries over the period 1950-1992.

Finally, chapter 10 gathers main conclusion.

\subsection{Out of the Line of this Thesis and Unfeatures}

On such a specific topic as the present one, there are obviously many things we cannot overview. For instance, other forms of reduced rank structures $\dot{a}$ la Ahn and Reinsel (1988) can be considered to further relax the weak form we propose in Chapter 3. Also, all kinds of common features can be integrated in i general framework. One can imagine to carry out an analysis within a common trend/common cycle/common ARCH/common break/common outlier modeling, together with non-linearities for instance. All of these features being estimated efficiently in one "shot"! More specifically, ARCH type crror processes invalidate usual inference based on canonical correlations and more robust procedure or pseudo-ML estimators could give better results. Other types of dynamic systems can also be considered instead of the finite order VAR that plays a central role in our analysis. In empirical studies, we often determine the order of 


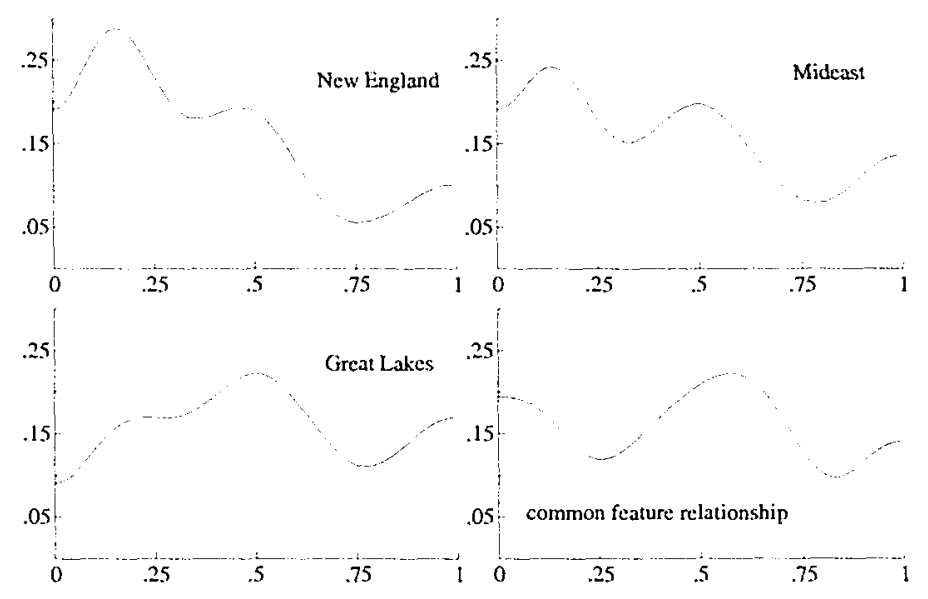

Figure 1.4: Spectral Densities of Income Growth Rates and of Co-feature Relationship

the lag length before we test for reduced ranks while a simultancous approach could be used (see also Chapter 6). We deal with closed systems while exogenous variables could help in explaining "idiosyncratic" parts. Dynamic cofactors can be developed. Also, except the application about Okun's law presented in Chapter 2, the cofeature relationship is assumed to be constant through time while structural breaks and regime shifts probably occur. The panel structure can be relaxed to get some additional effects. Structural time series models or Bayesian VAR can be considered, non-parametric methods can be used as well as other methods we have not even thought about.

But perhaps most importantly, we have restricted our analysis to tools coming from the time domain while the description of comovements at the business cycle frequencies is usually the main raison d'etre of the spectral analysis. Coming back to our example, Figure 1.4 displays the spectral densities scaled by $\pi$ of the growth rates of regional income scries as well as for the common cyclical feature relationships (1.5). ${ }^{6} \mathrm{~A}$ peak seems to exist with an average periodicity of around 13 ycars for New England and to a lesser extent for Mideast. It is not obvious to see if the sccond peak corresponding to cycle of 4 years is significant for the three series or whether some "waves" are due to small sample sizes. Indeed, important time series such as national income for most OECD countries do not have complex roots in their autoregressive part and consequently, do not have peaks at business cycle frequencies (Pagan, 1999).

\footnotetext{
${ }^{6} \mathrm{~A}$ Parzen window with a lag truncation parameter $M=12$ for 48 observations has been used. The usual rule of thumb is $\frac{T}{5} \leq M \leq \frac{T}{3}$.
} 
A frequency domain approach has some advantages. Such an analysis could be more flexible than the common cyclical feature one since it could test the presence of a common feature for a particular business cycle frequency and not for all frequencies jointly. Moreover a spectral analysis would be useful to overcome the problem of bias when using seasonally adjusted data (sec Cubadda, 1999 and Chapter 7). But some issues still need to be examined in more detail. For instance, Cubadda (1999) shows that in a set of differenced I(1) variables, the existence of SCCF does not imply high coherence at business cycle frequencies and vice versa. Neither the SCCF precludes absence of phase shifts between the first differenced time series. Some important problems like how to treat non stationarity (sec Maravall, 1993), how to test for the significance of the features at particular frequencies and how to deal with series that have a feature at different frequencies must be examined with care. Also, economic theory proposes relationships mainly in the time domain. $\Lambda$ first step towards the extension of common cyclical features in the frequency domain can be drawn from Stoffer (1999) and Breitung and Candelon (2000) in the case of stationary processes. But waiting for further developments we definitely stand in the time domain where numerous important issues are also worth considering. 


\title{
Chapter 2
}

\section{Codependence, Common Features \& Co. in Stationary Time Series: A Selective Survey}

\begin{abstract}
In this chapter, we start by reviewing the different approaches to common cyclical features for stationary time series. We show how they are nested within the Tiao and Tsay (1985, 1989) scalar component model (SCM). We emphasize the SCM because it constitutes a general framework for determining the presence of exchangeable structures, for selecting the lag order of a VARMA $(p, q)$ and for simplifying models. This survey focuses mainly on multivariate stationary processes whereas the joint treatment of long-run and short-run restrictions will form the core of the next chapters. Here, we warn against various kind of misspecifications by means of Monte Carlo experiments. We show the benefits of common cyclical features studies through several economic applications such as the convergence within EC economies, Okun's law, or the common fluctuations between seven primary commodity prices for the 17 th and 18 h century on the Roermond market.
\end{abstract}

\subsection{Introduction and Motivations}

Since the mid eighties time series econometrics has been subject to quite important theoretical and practical developments. Emphasis has been particularly placed on the statistical treatment of non stationary variables. This is reflected by the progress in asymptotic theory for integrated variables and in particular by the development of test statistics for the presence of unit roots and cointegration. Let us just name the seminal work by Dickey and Fuller (1979), Engle and Granger (1987), Johansen (1988), Stock and Watson (1988), Phillips (1987), to realize their considerable influence on the literature. This expansion towards a more refined modeling of

\footnotetext{
${ }^{0}$ The structure of this chapter is based on a revised version of Hecq (1997) with elements from Beine and Hecr $(1997,1998,1999)$ and Candelon and Hecg (2000).
} 
stochastic processes is associated with the appearance of numerous cconomic applications, new asymptotic results as well as studies of small sample properties of estimators via Monte Carlo experiments.

Cointegration is a special case of common features arising when there are some common stochastic trends among a group of economic time series. Amazingly, much less attention has been paid to other features for relationships involving stationary variables. This lack of interest may be due to the fact that the issue is seen as less crucial. Indeed, unlike for integrated processes, applications of traditional techniques do not suffer from spurious regression problems. Notice however that. Palm and Sneek (1984) and Granger et al. (1998) have shown evidence of spurious regressions when variables are asymptotically stationary but quite highly serially correlated.

In order to introduce common cyclical feature analyses, let us consider two different linear relationships between two stationary time series $x_{t}$ and $y_{t}$, in which only the first one is a MA(1) error process and the second one a white noise:

$$
\begin{aligned}
& x_{t}+\beta y_{t}=u_{t}, u_{t}=\varepsilon_{1 t}+\theta \varepsilon_{1 t-1}, \\
& x_{t}+\alpha y_{t}=e_{t}, e_{t}=\varepsilon_{2 t},
\end{aligned}
$$

where for $t=1 \ldots T, \alpha \neq \beta$ and $|\theta|<1, E\left(\varepsilon_{1 t}\right)=E\left(\varepsilon_{2 t}\right)=0, \operatorname{Var}\left(\varepsilon_{1 t}\right)=\sigma_{11}, \operatorname{Var}\left(\varepsilon_{2 t}\right)=\sigma_{22}$, $\operatorname{Cov}\left(\varepsilon_{1 t}, \varepsilon_{2 t}\right)=\sigma_{12}, \operatorname{Cov}\left(\varepsilon_{i t}, \varepsilon_{i t-j}\right)=0$ for $i=1,2, j \neq 0$. Solving the system for $x_{t}$ and $y_{t}$ yields

$$
\begin{aligned}
& x_{t}=\alpha(\alpha-\beta)^{-1} u_{t}-\beta(\alpha-\beta)^{-1} e_{t}, \\
& y_{t}=-(\alpha-\beta)^{-1} u_{t}+(\alpha-\beta)^{-1} e_{t} .
\end{aligned}
$$

Because $\left\{u_{t}\right\}_{t=1}^{T}$ is a moving average error process, $\left\{x_{t}\right\}_{t=1}^{T}$ and $\left\{y_{t}\right\}_{t=1}^{T}$ are moving averages too since they linearly depend on $u_{\ell}$. The process $\left\{x_{t}+\alpha y_{t}\right\}_{t=1}^{T}$ is however a white noise process by definition. Consequently, the vector $(1, \alpha)$ is, up to a scalar factor, the only one which can yicld this result.

Engle and Kozicki (1993), who formalized the idea of common features and proposed tools for analyzing them, have shown that the treatment of common features is useful for applied econometricians for both statistical and economic reasons. From a statistical point of view, common features mean a reduction in the number of redundant parameters, and imposing such restrictions will often yield a gain in estimation efficiency. From an economic perspective, common features mean that some series have something in common, a characteristic that does not immediately appear. Revealing this hidden framework should put forward that the variables are more strongly linked than we believed initially. This approach has been applied to the study of business cycle comovements or to address the question of similar volatility, and thus of same risk, among a set of financial variables.

In this thesis we concentrate ourselves on serial correlation and moving average common features for multivariate time series. We have already specified in Chapter 1 what is meant by cycle or cyclical fiuctuations. Consequently we use the expression common cyclical features 
as a generic one throughout this work. In this chapter, we study common features among a group of stationary time series or among non stationary variables that have been filtered (detrended, first differenced) in order to reach stationarity. Hence we assume here absence of cointegrating relationships while cointegrated systems will be considered in a later stage. We hope these simplifications will lead to a better understanding of the common features stakes whereas the next chapters aim at investigating the interaction between cointegration and common cyclical features in an integrated setting.

While the expression "common features" or "co-fcatures" stems from Engle and Kozicki's work, the concept of linear dependence which reduces some dynamic features among stationary time scrics was already put forward by Velu, Reinsel and Wichern (1986) through reduced rank in VAR models, by Gourićroux and Peaucelle (1989) via the codependence approach, by Ahn and Reinsel (1988) through nested reduced rank analysis. Vahid and Engle (1993) introduced the case of non-synchronous common features and Kugler and Neusser (1993) treat the codependence in a VAR framework. However, all these approaches may be seen as applications of reduced rank analyses and are "just" special cases of scalar component models proposed by Tiao and Tsay (1985, 1989). To clarify the discussion, a scalar component model or order $p_{1}, q_{1}$, denoted $\operatorname{SCM}\left(p_{1}, q_{1}\right)$, is a linear combination of a $n \times 1$ vector process $y_{t}$ in which at least $p_{1}<p$ or $q_{1}<q$ is verified, $p$ and $q$ being the order of the unconstrained VARMA $(p, q)$ generating $y t$.

This survey chapter is set up around the following points. Section 2.2 recalls the Engle and Kozicki (1993)'s definition of common features. Sections 2.3 to 2.7 summarize different formulations that have been experienced to simplify a linear multivariate dynamic system. It is shown how the Tiao and Tsay approach frames the other ones. Section 2.8 analyzes the canonical correlation tools for dynamic common factors. In Section 2.9 we investigate through Monte Carlo experiments how codependence test statistics behave in the presence of various types of misspecifications. Section 2.10 presents three economic applications. Finally Section 2.11 concludes and sketches some of the other type of common features than cyclical ones. Appendix stresses the identifiability issue and especially the exchangeability problem.

\subsection{Definition of Common Features}

In their 1993 paper, Engle and Kozicki (hereafter EK) introduced a class of statistical models and tests to analyzing whether a feature that is present in a set of time series is common to these. A feature is a characteristic of an cconomic time series, (such as serial correlation, heteroscedasticity, seasonality, trends), that must respect threc propertics:

Axiom 2.1 If $y$ has (docs not have) the feature, then $\lambda y$ will have (will not have) the feature for any $\lambda \neq 0$,

Axiom 2.2 If $y$ does not have the feature and $x$ does not have the feature, then $z=x+y$ will not have the feature, 
Axiom 2.3 If $y$ does not have the falture but $x$ have the feature, then $z=x+y$ will have the feature.

EK and Vahid and Engle (1993) define common features as follows:

Definition 2.4 A feature, which respects Axioms 2.1 to 2.3, that is present in each of a group of series is said to be common to those series if there exists a nonzero linear combination of the series that does not have the feature. Such a linear combination is called a cofeature combination and the vector which represents it is called a cofeature vector. For n-dimensional system $y_{t}=$ $\left(y_{11}, \ldots, y_{n t}\right)^{\prime}$ measured for $t=1 \ldots T$, there can exist $s<n$ linearly independent cofeature vectors. The collection of all linearly independent cofeature vectors form the $n \times s$ matrix $\delta$ where $\left\{\delta^{\prime} y_{t}\right\}_{t=1}^{T}$ does not have the feature. The range of the matrix $\delta$ which defines the subspace of all possiblc cofeature vectors is called the cofeature space.

An altcrnative interpretation of what "to have the feature" means has been mentioned by Granger (1993) in his comment to EK's paper (see also Sørensen, 1996), where the expression "to have the feature" is replaced by "to have a dominant statistical property" and such a property can be called a feature. If property $P_{1}$ dominates $P_{2}$, Axioms 2.1 to 2.3 can be reformulated such as

Axiom 2.5 If $y$ and $x$ have $P_{2}$, then $z=x+y$ will not have $P_{1}$,

Axiom 2.6 If $y$ has $P_{2}$ but $x$ has $P_{1}$, then $z=x+y$ will have $P_{1}$.

Definition 2.7 With respect to Axioms 2.5 and 2.6, we will talk about common features if all the elements of a set of time series have the dominant property $P_{1}$ and there exists a nonzero linear combination of them that has $P_{2}$ which is dominated by $P_{1}$.

The advantage of Definition 2.7 is that it clarifies what is meant by "not having a feature" like a nonseasonal property or a zero in the spectrum property.

As pointed out in Chapter 1, EK's idea is strongly related to the literature on dynamic factor models. From Definition 2.4, we do not have to specify the precise structure of the feature which could be a process with some degree of serial correlation, GARCH processes or some regime shifts. Consequently the type of common features studied by EK is widely driven by a misspecification framework and is based on regressions. Using some LM tests, say, to detect serial correlation, one ensures that the null is rejected for both variables $y_{t}$ and $x_{t}$. Afterwards, one seeks whether there exists a linear combination of the two time series which does not reject the null of absence of autocorrelation. Therefore, this type of procedure can lack of informative and statistical power because it does not emphasize the parameter restrictions that common features imply.

The class of models we consider in the sequel are multivariate linear time series, i.e. the class of VARMA models or more generally VARMAX processes. We review in the next section some of their important specifications. These are successively the serial correlation common features, nested reduced rank, codependence, non synchronous and scalar component models. 


\subsection{Serial Correlation Common Feature}

\subsubsection{Definition}

We consider the serial correlation common feature (SCCF) model in a multivariate framework. Actually, the approach originally developed by EK is based on regression analyses. In their comment to the EK's paper, both Hansen (1993) and Tsay (1993) point out that serial correlation common features restrictions are equivalent to some reduced-rank restrictions on parameter matrices of vector autoregressions. This multivariate extension has at least four advantages : $(i)$ the estimated coefficients do not depend on a particular choice of the dependent variable but only on a normalization, (ii) it allows to obtain more than one cofcaturing vector, (iii) it is easy to implement and (iv) yields estimates for the full model and not just of the common feature vector.

Consider a $n$-dimensional stationary zcro mean Gaussian VAR $(p)$ process for $y_{t}=\left(y_{1 t}, \ldots, y_{n t}\right)^{\prime}$

$$
\begin{aligned}
y_{t} & =\Phi_{1} y_{t-1}+\cdots+\Phi_{p} y_{t-p}+\varepsilon_{t}, \\
& =\Phi X_{t-1}+\varepsilon_{t} \quad t=1 \ldots T
\end{aligned}
$$

with $\Phi=\left(\Phi_{1}, \ldots, \Phi_{p}\right)$ a $n \times n p$ matrix of row rank $n-s, X_{t}=\left(y_{t}^{\prime}, \ldots, y_{t-p+1}^{\prime}\right)^{\prime}$ and where $\varepsilon_{t}$ is $n$-dimensional multivariate $N I I D(0, \Omega)$.

The idea behind the white noise direction (as labelled by Gourićroux el al., 1991) reduced rank models is that, the matrix $\Phi$ can be written as the product of two reduced rank matrices $\Phi=B\left(C_{1}, \ldots, C_{p}\right)=B C$, where $B$ is $n \times(n-s)$ and $C$ is $(n-s) \times n p$. Therefore, up to the multiplicative constants contained in $B$, there may be linear combinations of the $X_{t-1}$ vector that contain all the relevant information regarding $y_{t}$. The system may then be written as a dynamic endogenous factor model

$$
y_{t}=B f_{t}+\varepsilon_{t}
$$

with $f_{t}=C X_{t-1}$ being an $(n-s)$-dimensional vector time series of unobserved dynamic factors and $B$ a matrix of factor loadings. ${ }^{1}$ Furthermore, with $s$ independent cofeature vectors, there exists an $n \times s$ cofactor matrix $\delta=B_{\perp}$ orthogonal to $B$ such that $\delta^{\prime} y_{t}=\delta^{\prime} \varepsilon_{t}$ does not have any autocorrelation, or stated more correctly, is orthogonal to the past of $y_{t}$.

To determine the rank of $\Phi$, (and then also of $B$ and $C$ ), the variables in both $y_{t}$ and $X_{t-1}$ are first regressed on deterministic terms and on strictly exogenous variables if necessary. The coefficient matrices are then estimated by reduced rank regression (sec Anderson, 1998, Johansen, 1995 and Hendry, 1995 for cointegration) applied to the residuals of the first stage analysis, i.e.

\footnotetext{
${ }^{1}$ Note that these common cyclical features relationships do not have to be confused with common cycles for which an additional permanent-transitory decomposition must be assumed (see Chapter 4).
} 
by

$$
y_{t}=B C X_{t-1}+\varepsilon_{t}
$$

with parameter vector $\theta=\{B, C, \Omega\}, \theta \in \Theta$, with $\Theta$ the parameter space. The concentrated log-likelihood function of $(2.5)$, for $t=1 \ldots T$, is given by the expression

$$
\log L(B, C, \Omega)=-\frac{1}{2} T \log |\Omega|-\frac{1}{2} \sum_{t=1}^{T}\left(y_{t}-B C X_{t-1}\right)^{\prime} \Omega^{-1}\left(y_{t}-B C X_{t-1}\right) .
$$

Denoting $S_{00}=T^{-1} \sum_{t=1}^{T} y_{t} y_{t}^{\prime}, S_{01}=T^{-1} \sum_{t=1}^{T} y_{t} X_{t-1}^{\prime}, S_{11}=T^{-1} \sum_{t=1}^{T} X_{t-1} X_{t-1}^{\prime}$ and $S_{10}=S_{01}^{\prime}$ we can maximize the likelihood function with respect to $B$ and $\Omega$ for fixed $C$

$$
L_{\max }^{-2 / T}(\hat{B}(C), C, \hat{\Omega}(C))=L_{\max }^{-2 / T}(C)=\left|S_{00}-S_{01} C\left(C^{\prime} S_{11} C\right)^{-1} C^{\prime} S_{10}\right|,
$$

whose solution is given by the eigenvalues that solve $\left|\lambda S_{11}-S_{10} S_{00}^{-1} S_{01}\right|=0$. The first eigenvectors corresponding to the $n-s$ largest eigenvalues constitutes the $[(n-s) \times n] \times p$ rectangular matrix $\hat{C}$ from which an estimate of $\hat{B}$ can be calculated by OLS. Alternatively, the common fcature vectors correspond to the eigenvectors associated with the $s$ smallest eigenvalues that solve $\left|\lambda S_{00}-S_{01} S_{11}^{-1} S_{10}\right|=0$ and are such that $\hat{\delta}^{\prime} \hat{B}=0_{s \times(n-s)}$ (for a discussion of this dual problem, sec inter alia Section 2.8.3 of this thesis; Johansen, 1995; Gonzalo and Granger, 1995).

Once the rank, namely $n$ minus the number of statistically insignificant eigenvalues $s$, has been determined, nonlinear system least squares or constrained FIML estimators can be preferred to OLS to focus on the factorial representation (1.6) or (2.5). Indeed, if one wants to estimate the standard errors of cofeature vectors, the following pseudo-structural system can be easily implemented by full information maximum likclihood using widely available software such as PC-FIML for instance

$$
\left(\begin{array}{cc}
I_{s} & \delta_{s \times(n-s)}^{*} \\
0_{(n-s) \times s} & I_{n-s}
\end{array}\right) y_{t}=\left(\begin{array}{c}
0_{s \times n p} \\
\Phi_{1}^{*} \ldots \Phi_{p}^{*}
\end{array}\right)\left(\begin{array}{c}
y_{t-1} \\
\vdots \\
y_{t-p}
\end{array}\right)+v_{t}
$$

To obtain (2.8) the system (2.3) has been premultiplied by $\delta^{\prime}$ where this latter cofeature matrix has becn normalized and justidentified by putting an identity matrix on the first $s$ elements, namely $\delta^{\prime}=\left(I_{s}, \delta_{s \times(n-s)}^{* \prime}\right)$. Note we have assumed that the first block is full rank. Otherwise, another rotation of the co-feature matrix is necessary. The system is completed by the remaining $n-s$ unconstrained equation of the VAR in (2.3) such as $\Phi_{j}^{*}$ stands for the remaining $n-s$ coefficients matrices.

Consequently, we may be interested in the various aspects of common features recalled in Chapter 1 . We could be interested in the dimension and the estimates of the cofeature space $\delta^{\prime}$ 
in order to obtain some information on how the variables are linked. We may also be interested in the common cyclical features $f_{t}$ or the loadings in order to rewrite the whole system in a smaller dimension. This choice will be guided by the cconomic insights we focus on.

Remark 2.8 Factorizations (2.4) or (2.5) are not unique because for any non singular matrix, say $G, B f_{t}=B G^{-1} G f_{t}=B^{*} \int_{t}^{*}$. Hence some identifying restriction will be needed. However, although theory could help us in searching some restrictions on the common feature vectors $\delta$, it is more complicated to identify $B$ and $C$. The same duality also exists between cointegration and common trends for non stationary variables.

\subsubsection{SCCF and Unpredictability}

There obviously exists a strong link betwcen the common features and the principle of predictability relative to an available information set. For Fendry and Clements (1999), $\left\{\nu_{t}\right\}_{t=1}^{T}$ is an unpredictable process with regard to an information set $\mathcal{I}_{t-1}$ if

$$
D_{\nu_{l}}\left(\nu_{l} \mid \mathcal{I}_{l-1}\right)=D_{\nu_{l}}\left(\nu_{l}\right)
$$

so that the conditional and the unconditional distributions coincide (with non-degenerate density $\left.D_{\nu_{8}}().\right)$. Unpredicability presents the following properties:

1. Unpredictability is invariant undar non-singular contemporaneous transformation: for example, if $\nu_{t}$ is unpredictable, so is $\mathrm{B} \nu_{t}$ where $|\mathrm{B}| \neq 0$,

2. Unpredictability is not invariant under intertemporal transforms since if $\nu_{t}=\epsilon_{t}+\mathbf{A} h\left(\mathcal{I}_{t-1}\right)$, $D_{\nu_{t}}\left(\nu_{t} \mid \mathcal{I}_{t-1}\right)=D_{\nu_{t}}\left(\nu_{t}\right)$ when $\mathbf{A} h(\cdot) \neq 0$. This property explains why; although the change in the $\log$ of real equity prices may be unpredictable, the level is predictable: since $x_{t}=$ $\Delta x_{t}+x_{t-1}$, the prediction of the current levels is its immediate past value,

3. For two information sets with $\mathcal{I}_{t-2} \subset \mathcal{I}_{t-1}$, we can have

$$
D_{\nu_{t}}\left(\nu_{t} \mid \mathcal{I}_{t-2}\right)=D_{\nu_{t}}\left(\nu_{t}\right) \text { and } D_{\nu_{t}}\left(\nu_{t} \mid \mathcal{I}_{t-1}\right) \neq D_{\nu_{t}}\left(\nu_{t}\right)
$$

but not.

$$
D_{\nu_{t}}\left(\nu_{t} \mid \mathcal{I}_{t-1}\right)=D_{\nu_{t}}\left(\nu_{t}\right) \text { and } D_{\nu_{t}}\left(\nu_{t} \mid \mathcal{I}_{t-2}\right) \neq D_{\nu_{t}}\left(\nu_{t}\right)
$$

Serial correlation common feature modeling proposes a more restrictive definition of unpredictability where only unpredictability in mean is considered, namely

$$
\mathrm{E}\left(\nu_{t} \mid \mathcal{I}_{t-1}\right)=\mathrm{E}\left(\nu_{t}\right)=0
$$

where $\nu_{t}$ is under the assumption of SCCF given by $\delta^{\prime} y_{t}$ and the information set $\mathcal{I}_{t-1}=$ $\left\{y_{t-1}, \ldots, y_{0}\right\}$ so that by definition of SCCF we have $\mathrm{E}\left(\delta^{\prime} y_{t} \mid \mathcal{I}_{t-1}\right)=0$. 
It is important to note that SCCF implies that the linear combination cannot be forecasted from the multivariate information set including the past of all $n$ variables in the system, such as in $E\left(\delta^{\prime} y_{t} \mid \mathcal{I}_{t-1}\right)=0$. Comovement, as defined in this thesis, is a property of dependent dynamic systems presenting a structure for which it is possible to find a reduced number of channels for the transmission of the information. Knowing both common propagation mechanism (i.e. $f_{t}$ or $\left.C X_{t-1}\right)$ and the weights in $B$, series must be forecastable together.

But the SCCF model is not necessarily equivalent to a univariate white noise property where a linear combination could not be forecasted from its own past, i.c. $E\left(\delta^{\prime} y_{t} \mid \delta^{\prime} \mathcal{I}_{t-1}\right)=0$.

In order to illustrate this subtle distinction, let us consider the following example where the series have identical dynamics:

$$
\left(\begin{array}{l}
y_{1 t} \\
y_{2 t}
\end{array}\right)=\left(\begin{array}{ll}
\rho & 0 \\
0 & \rho
\end{array}\right)\left(\begin{array}{l}
y_{1 t-1} \\
y_{2 t-1}
\end{array}\right)+\left(\begin{array}{l}
\varepsilon_{1 t} \\
\varepsilon_{2 t}
\end{array}\right)
$$

where $\sigma_{12}=0$ in the extreme case where the variables are independent, $|\rho|<1$ and $\mathcal{I}_{t-1}=$ $y_{t-1}=\left(y_{1 t-1}, y_{2 t-1}\right)^{\prime}$. Graphically these series co-move but for the linear combination $y_{1 t}+y_{2 t}=$ $\rho\left(y_{1 t-1}+y_{2 t-1}\right)+\varepsilon_{1 t}+\varepsilon_{2 t}$ and either $\mathrm{E}\left(\delta^{\prime} y_{t} \mid y_{t-1}\right) \neq 0$ and $\mathrm{E}\left(\delta^{\prime} y_{t} \mid \delta^{\prime} y_{t-1}\right) \neq 0$.

Now consider another example of identical dynamics, with symmetric dynamics such that

$$
\left(\begin{array}{l}
y_{1 t} \\
y_{2 t}
\end{array}\right)=\left(\begin{array}{cc}
\rho & 0 \\
0 & -\rho
\end{array}\right)\left(\begin{array}{l}
y_{1 t-1} \\
y_{2 t-1}
\end{array}\right)+\left(\begin{array}{l}
\varepsilon_{1 t} \\
\varepsilon_{2 t}
\end{array}\right),
$$

where again $|\rho|<1$. The linear combination $y_{1 t}+y_{2 t}=\rho\left(y_{1 t-1}-y_{2 t-1}\right)+\varepsilon_{1 t}+\varepsilon_{2 t}$ still respect $\mathrm{E}\left(\delta^{\prime} y_{t} \mid y_{t-1}\right) \neq 0$ that is there docs not exist a SCCF vector but $\mathrm{E}\left(\delta^{\prime} y_{t} \mid \delta^{\prime} y_{t-1}\right)=0$ because $y_{1 t-1}-y_{2 t-1}$ is orthogonal to $\delta^{\prime} y_{t-1}=y_{1 t-1}+y_{2 t-1}$. Should we talk about comovements in this case? Ericsson (1993) and Granger (1993) criticize SCCF on the basis of this example and argue that SCCF docs not appear useful for important DGPs like (2.14).

We do not believe that we can talk about comovements if two series have only the same dynamics as in (2.13) or (2.14). It would otherwise imply that two independent random walks have some form of comovement. It would deny cointegration and spurious regression issue. At best we can guess that two series that have identical dynamics may co-move "in reality" but that the researcher has missed an important variable that has a driving influence on the joint observed dynamics. The problem obviously differs whether the innovations are correlated.

\subsubsection{Different Concepts of Elasticities}

Gouriéroux and Peaucelle $(1989,1993)$ introduced the concept of codependence, a form of common cyclical features for $\mathrm{MA}(q)$, to capture "long-run relationships" between stationary series. But this concept may have different meanings even for stationary processes. It is also important to make the distinction between the long-run elasticity which corresponds to the solution of an autoregressive distributed lag (ADL) model and the cofeature relationship. Consider a bivariate 
stationary zero mean $\operatorname{VAR}(1)$ process and let $y_{t}=\left(y_{1 t}, y_{21}\right)^{\prime}$ be generated by a joint normal distribution:

$$
\left(\begin{array}{l}
y_{1 t} \\
y_{2 t}
\end{array}\right) \mid \mathcal{I}_{t-1} \sim N I I D\left[\left(\begin{array}{ll}
\phi_{11} & \phi_{12} \\
\phi_{21} & \phi_{22}
\end{array}\right)\left(\begin{array}{l}
y_{1 t-1} \\
y_{2 t-1}
\end{array}\right),\left(\begin{array}{ll}
\sigma_{11} & \sigma_{12} \\
\sigma_{21} & \sigma_{22}
\end{array}\right)\right]
$$

We assume that $y_{2 t}$ is weakly exogenous for parameters of the conditional model. Taking the conditional expectation of $y_{1 t}$ given $y_{2 t}$ and $\mathcal{I}_{t-1}=\left\{y_{1 t-1}, y_{2 t-1}\right\}$ we obtain

$$
\mathrm{E}\left(y_{1 t} \mid y_{2 t}, \mathcal{I}_{t-1}\right)=\left(\phi_{11}-\omega \phi_{21}\right) y_{1 t-1}+\left(\phi_{12}-\omega \phi_{22}\right) y_{2 t-1}+\omega y_{2 t}
$$

where $\omega=\frac{\sigma_{12}}{\sigma_{22}}$.

By defining $\varepsilon_{t}=y_{t}-\mathrm{E}\left(y_{t} \mid \mathcal{I}_{t-1}\right)$ and by conditioning $y_{1 t}$ on $y_{2 t}$, the conditional model of $y_{1 t}$ given $y_{2 t}$ and $\mathcal{I}_{t-1}$ takes the form of a standard ADL(1,1) (see Hendry, 1995)

$$
y_{1 t}=\left(\phi_{11}-\omega \phi_{21}\right) y_{1 t-1}+\left(\phi_{12}-\omega \phi_{22}\right) y_{2 t-1}+\omega y_{2 t}+\varepsilon_{1 t}-\omega \varepsilon_{2 t}
$$

where $\varepsilon_{t}=\left(\varepsilon_{1 t}, \varepsilon_{2 t}\right)^{\prime}$.

Further, by considering the unconditional means $\mathrm{E}\left(y_{1 \ell}\right)=\bar{y}_{1}$ and $\mathrm{E}\left(y_{2 t}\right)=\bar{y}_{2}$ and taking the unconditional mean of $y_{1 t}=\mathrm{E}\left(y_{1 i} \mid y_{2 t}, \mathcal{I}_{t-1}\right)+\varepsilon_{i}$, with $\mathrm{E}\left[\mathrm{E}\left(y_{1 t} \mid y_{2 t}, \mathcal{I}_{t-1}\right)\right]=\mathrm{E}\left(y_{1 t}\right)$ by definition of conditional expectations, we obtain

$$
\begin{aligned}
\bar{y}_{1} & =\left(\phi_{11}-\omega \phi_{21}\right) \bar{y}_{1}+\left(\phi_{12}-\omega \phi_{22}\right) \bar{y}_{2}+\omega \bar{y}_{2}, \\
& \Rightarrow \\
\bar{y}_{1} & =\frac{\left(\phi_{12}-\omega \phi_{22}+\omega\right)}{\left(1-\left(\phi_{11}-\omega \phi_{21}\right)\right)} \bar{y}_{2}=\kappa_{1} \bar{y}_{2} .
\end{aligned}
$$

We assume for interpretation convenience that variables are in logs. In so doing, $\omega$ is the short-run elasticity (or the direct impact) in the ADL (2.17). $\kappa_{1}$ is the long-run elasticity, and by definition we have $\mathrm{E}\left(y_{1 t}-\kappa_{1} y_{2 t}\right)=0$. This long-run elasticity is the static solution of the dynamic equation (2.16) and always exists if the denominator in (2.19) is not equal to zero. However, the existence of SCCF relationships assumes additional orthogonality conditions, i.e. $\mathrm{E}\left(y_{1 t}-\kappa_{2} y_{2 t} \mid \mathcal{I}_{t-1}\right)=0$.

Lemma $2.9 S C C F$ is equivalent to the long-run solution of the $A D L(2.17)$ if $\phi_{12}=\omega \phi_{22}$ and $\phi_{11}=\omega \phi_{21}$. Moreover in this case the long-run solution is also the short-run elasticity.

Lemma 2.10 The prescnce of SCCF reduced rank for other solutions than $\phi_{12}=\omega \phi_{22}$ and $\phi_{11}=\omega \phi_{21}$ in (2.15) does not reproduce the long-run solution $\kappa_{1}$.

The proof of the Lemma 2.9 follows directly from (2.19). To illustrate Lemma 2.10 , let us assume that there exists another reduced rank structure, by substituting $\phi_{11}=\phi_{12} \phi_{21} \phi_{22}^{-1}$ in 
(2.15) such that

$$
\left(\begin{array}{l}
y_{1 t} \\
y_{2 t}
\end{array}\right) \mid \mathcal{I}_{t-1} \sim N I I D\left[\left(\begin{array}{cc}
\phi_{12} \phi_{21} \phi_{22}^{-1} & \phi_{12} \\
\phi_{21} & \phi_{22}
\end{array}\right)\left(\begin{array}{l}
y_{1 t-1} \\
y_{2 t-1}
\end{array}\right),\left(\begin{array}{ll}
\sigma_{11} & \sigma_{12} \\
\sigma_{21} & \sigma_{22}
\end{array}\right)\right]
$$

where $\left(1,-\phi_{12} \phi_{22}^{-1}\right)^{\prime}$ is the associated common feature vector. Using similar arguments as previously we obtain

$$
\begin{aligned}
\mathrm{E}\left(y_{1 t} \mid y_{2 t}, \mathcal{I}_{t-1}\right)=\left(\phi_{12} \phi_{21} \phi_{22}^{-1}-\omega \phi_{21}\right) y_{1 t-1}+\left(\phi_{12}-\omega \phi_{22}\right) y_{2 t-1}+\omega y_{2 t} \\
\bar{y}_{1}=\left(\phi_{12} \phi_{21} \phi_{22}^{-1}-\omega \phi_{21}\right) \bar{y}_{1}+\left(\phi_{12}-\omega \phi_{22}\right) \bar{y}_{2}+\omega \bar{y}_{2} \\
\quad \Rightarrow \\
\bar{y}_{1}=\frac{\left(\phi_{12}-\omega \phi_{22}+\omega\right)}{\left(1-\left(\phi_{12} \phi_{21} \phi_{22}^{-1}-\omega \phi_{21}\right)\right)} \bar{y}_{2}=\kappa_{2} \bar{y}_{2} .
\end{aligned}
$$

It turns out that SCCF and the long-run solution coincide only if $\phi_{12}=\omega \phi_{22}$ and in this case the unconditional expectation is equal to the conditional one. But the reduced rank (SCCF) given by $\phi_{11}=\phi_{12} \phi_{21} \phi_{22}^{-1}$ does not imply that $\kappa_{2}=\kappa_{1}$.

\subsubsection{Common Cyclical Features and Impulse Response Functions}

The presence of common cyclical features in the dynamics of multivariate time series has some natural implications for their impulse response functions (IRF). Firstly, there may be a decrease in their confidence intervals due to efficiency gains from excluding redundant parameters. Secondly, comovements imply commonality in IRF shapes since, by definition, IRFs are collinear for variables exhibiting SCCF. That means that an exogenous shock affecting the linear combination dies out in future periods. ${ }^{2}$

To explain some of the basic features, we have simulated two bivariate stationary $\operatorname{VAR}(1)$ for $y_{t}=\left(y_{1 t}, y_{2 t}\right)^{\prime}$. Remark that only $\mathcal{D G} \mathcal{G P}_{2}$ implies a SCCF structure with a cofeature vector given by $\delta=(1,1)^{\prime}$.

$$
\begin{aligned}
& \mathcal{D G} \mathcal{P}_{1}:\left(\begin{array}{l}
y_{1 t} \\
y_{2 t}
\end{array}\right)=\left(\begin{array}{c}
1.15 \\
-.25
\end{array}\right)+\left(\begin{array}{cc}
.7 & -.2 \\
-.2 & .7
\end{array}\right)\left(\begin{array}{l}
y_{1 t-1} \\
y_{2 t-1}
\end{array}\right)+\left(\begin{array}{l}
\varepsilon_{1 t} \\
\varepsilon_{2 t}
\end{array}\right), \\
& \mathcal{D G} \mathcal{P}_{2}:\left(\begin{array}{l}
y_{1 t} \\
y_{2 t}
\end{array}\right)=\left(\begin{array}{c}
1.15 \\
-.25
\end{array}\right)+\left(\begin{array}{cc}
.7 & -.2 \\
-.7 & .2
\end{array}\right)\left(\begin{array}{l}
y_{1 t-1} \\
y_{2 t-1}
\end{array}\right)+\left(\begin{array}{l}
\varepsilon_{1 t} \\
\varepsilon_{2 t}
\end{array}\right),
\end{aligned}
$$

\footnotetext{
${ }^{2}$ Note that with the codependence or the non-synchronous approaches we introduce in next sections, the IRFs are collinear after an adjustment delay given by the remaining MA part.
} 


$$
\left(\begin{array}{l}
\varepsilon_{1 t} \\
\varepsilon_{2 t}
\end{array}\right) \sim N I I D\left[\left(\begin{array}{l}
0 \\
0
\end{array}\right),\left(\begin{array}{ll}
16 & 14 \\
14 & 25
\end{array}\right)\right]
$$

For both DGPs the short-run elasticity is .56 while the long-run solutions of ADLs are respectively -.17 and -2.69 for $\mathcal{D G} \mathcal{P}_{1}$ and $\mathcal{D G} \mathcal{P}_{2}$. The SCCF elasticity is -1 . To compute the IRFs we consider the Choleski factorization of $\Omega$ such as $\Omega=P^{-1}\left(P^{-1}\right)^{\prime}$ with $P^{-1}=\left(\begin{array}{cc}4 & 0 \\ 3.5 & 3.5707\end{array}\right)$ or equivalently $I_{2}=P \Omega P^{\prime}$.

Figures show a realization for $T=100$ of both processes together with their IRFs. The first two figures illustrate quite well that it is not possible to discriminate graphically between systems where a common cyclical features structure is present from systems without this structure. This is why we need formal test statistics. The innovations in the IRFs analyses have been orthogonalized using the Choleski factorization and the shocks are $e_{1}=(1,0)^{\prime}$ and $e_{2}=(0,1)^{\prime}$. The last two graphs emphasize that shocks disappear when premultiplied by $\delta^{\prime}$ the IRFs obtained with the $\mathcal{D G} \mathcal{P}_{2}$, i.c. under a SCCF structure. This interpretation in terms of IRFs is interesting for the analysis of convergence processes between countries or rcgions.

We do not. want however to enter in the debate about the implications of common cyclical features for the identification of shocks in a structural VAR analysis (SVAR) although some authors (Gallo and Kempf, 1996a,b; Issler et al., 1994) have argued in favor of the usefulness of co-feature restrictions. In our opinion, their arguments, based on the same kind of analysis as for cointegrating restrictions (sce Mellander et al., 1992), are not fully convincing because correlation between shocks does not affect the forecasts (see EK's reply). See also Hoffman (1998) for a criticism of identification of shocks using cointegration restrictions. More fundamentally, we should note that economic theory is often helpless in describing innovation from transitory parts. Identification is indeed typically based on a priori assumptions and it is only in limited cases that the data reveal source of shocks. In our opinion, the important issue around the link between SCCF and IRFs is the delay of adjustment of the linear combination and not the series taken individually. For instance, in the analysis of US regions introduced in Chapter 1, we were interested in investigating to what extent regions are interconnected when hit by an exogenous shock. We have seen that some similarities occurred and that perhaps cconomic policy may concentrate on global measures instead of specific ones. But this is already another story. 

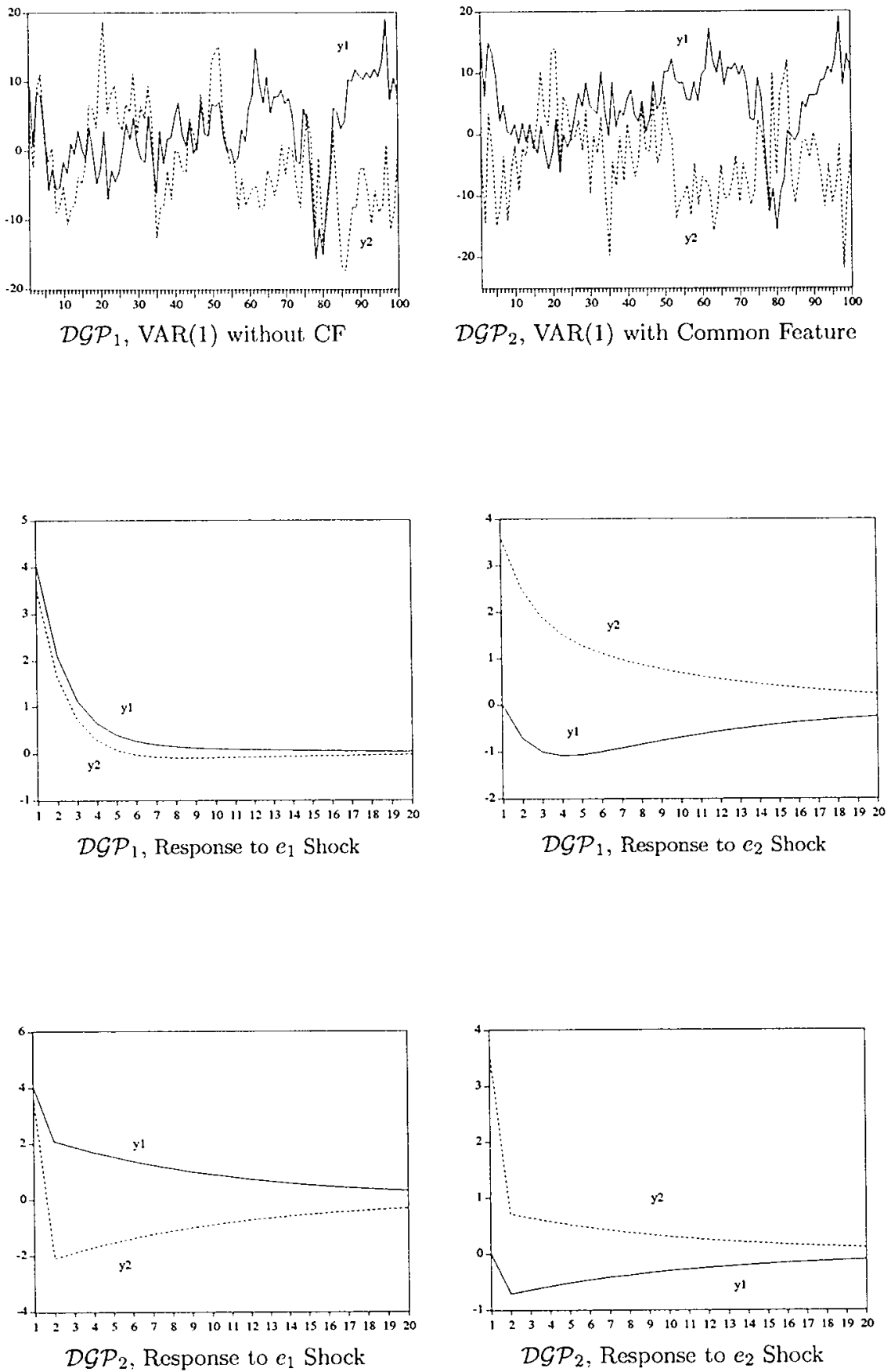


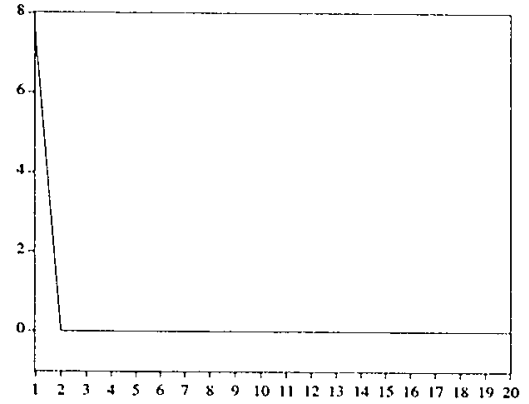

$\mathcal{D G} \mathcal{P}_{2}$, Response to $e_{1}$ Shock to the CF

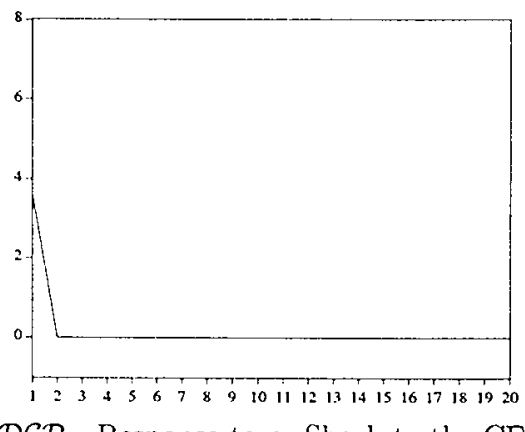

$\mathcal{D G} \mathcal{P}_{2}$, Response to $e_{2}$ Shock to the CF

\subsubsection{Some Theoretical Common Feature Vectors}

Very often empirical analyses consider economic relationships between stationary variables although without necessarily specifying that residuals must be white noise processes. For example a researcher can run a regression, perhaps using a instrumental variable estimator, to obtain the short-run propensity to consume, Okun's clasticity or estimated coefficients for the relative form of the purchasing power parity. Consequently, if one is not interested in potential efficiency gains, in improving forecast accuracy, in uncovering hidden structures, in summarizing information using a parsimonious model, in searching for directions that reduce the most the dynamics or by impulse response functions analyses...one may question the usefulness of common cyclical features restrictions.

$A$ notorious exception is the rational expectation literature whose "business", namely their optimality properties, implics some orthogonality conditions that ensure inter alia efficiency and unbiasness of the rational expectation scheme. ${ }^{3}$ For example, the efficient markets hypothesis and rational expectations give rise to cross-cquation parameter restrictions and to unpredictability conditions very similar to those used in the common features literature. Cuthbertson (1996) considers the two periods pure expectation hypothesis that implics that the long-short interest. rates spread $S p r_{t}=\left(R_{t}-r_{\ell}\right)$ provides an optimal forecast of future changes in the short rate such as $S p r_{t}=\frac{1}{2} \Delta r_{t+1}^{c}$, where $R_{t}$ and $r_{t}$ are respectively the long and the short term interest rates, $\Delta r_{t+1}^{c}=r_{t+1}^{e}-r_{t}$ and $R_{t}=\frac{1}{2}\left(r_{t}+r_{t+1}^{e}\right)$. Further assume an autoregressive scheme for $\Delta r_{t+1}$ given by (2.28) below and suppose that agents use the limited information set $\Lambda_{t}=$ $\left(\Delta r_{t}, \Delta r_{t-1}\right)$ to forecast future changes in interest rates, such that $\Delta r_{t+1}^{e}=\alpha_{3} \Delta r_{t}+\alpha_{4} \Delta r_{t-1}$ with the forecast error $\omega_{2 t+1}=\Delta r_{t+1}-\Delta r_{t+1}^{e}$. It can be shown that a joint test for both the pure expectation hypothesis and the rational expectation implies a cross-equation restriction and consists in formulating the system of equations

$$
\begin{aligned}
S p r_{t} & =\alpha_{1} \Delta r_{t}+\alpha_{2} \Delta r_{t-1}+\omega_{1 t+1}, \\
\Delta r_{t+1} & =\alpha_{3} \Delta r_{t}+\alpha_{1} \Delta r_{t-1}+\omega_{2 t+1},
\end{aligned}
$$

\footnotetext{
${ }^{3}$ See Pesaran (1989) for a clear presentation of the rational expectation hypothesis in economics.
} 
and in testing for $\alpha_{1}=\frac{\alpha_{3}}{2}$ and $\alpha_{2}=\frac{\alpha_{4}}{2}$ which means that $(2,-1)$ is a SCCF vector. Notice that an error term $\omega_{1 t+1}$ has been added to (2.27) either because the spread might be measured with error or because given the limited information set $\Lambda_{t} \subseteq \Omega_{t}$, the error term could also pick up could the difference between forecasts using these two information sets if $\Lambda_{t} \subset \Omega_{t}$.

Consider another example where a test for the efficiency market hypothesis (EMH) consists in running a regression from the future change in spot exchange rate $\Delta s_{t+1}$ on the forward premium $(f-s)_{t}$, or equivalently if we lag once

$$
\Delta s_{t}=\alpha+\beta(f-s)_{t-1}+\gamma^{\prime} \Lambda_{t-1}+\varepsilon_{t},
$$

where $\gamma$ is a vector of parameters. Equation (2.29) can be obtained from at least two ways. The first manner is to model the bivariate system $y_{\ell}=\left(\Delta s_{l}, \Delta f_{t}\right)^{\prime}$ in a VECM form where the left-hand side variables in both equations include lags of $\Delta s_{t}$ and $\Delta f_{t}$ plus an error correction term $(f-s)_{t-1}$. This framework can be summarized in the following VAR(2) example

$$
\begin{aligned}
& \Delta s_{t}=\alpha_{1}+\beta_{1}(f-s)_{t-1}+\gamma_{1} \Delta s_{t-1}+\gamma_{2} \Delta f_{t-1}+\varepsilon_{1 t}, \\
& \Delta f_{t}=\alpha_{2}+\beta_{2}(f-s)_{t-1}+\gamma_{3} \Delta s_{t-1}+\gamma_{4} \Delta f_{t-1}+\varepsilon_{2 t} .
\end{aligned}
$$

In this $\operatorname{VAR}(2)$ case the information set is $\Lambda_{t-1}=\left(\Delta s_{t-1}, \Delta f_{t-1}\right)^{\prime}$ and under the null hypothesis, the common feature vector we would obtain will be a weak form reduced rank structure (WF) whose common feature vector is $(1,0)^{\prime}$. We will see in details in next chapters, these type of models that jointly treat the presence of cointegrating and common feature relationships. But while this chapter focus on stationary time series, we can also formulate the problem underlying (2.29) within a stationary VAR model for $y_{t}=\left(\Delta s_{t},(f-s)_{t-1}\right)^{\prime}$ such that

$$
\begin{aligned}
\Delta s_{t} & =\mu_{1}+\delta_{1} \Delta s_{t-1}+\delta_{2}(f-s)_{t-2}+\eta_{1 \ell}, \\
(f-s)_{t-1} & =\mu_{2}+\delta_{3} \Delta s_{t-1}+\delta_{1}(f-s)_{t-2}+\eta_{2 t} .
\end{aligned}
$$

The information set $\Lambda_{t-1}$ may contain any relevant variables including lagged dependent variables (i.c. $\left.\Delta s_{t-1},(f-s)_{t-2}, \Delta s_{t-2},(f-s)_{t-3} \ldots\right)$ and lagged forecast errors. In this illustrative $\operatorname{VAR}(2)$ case it only allows for $\Lambda_{t-1}=\left(\Delta s_{t-1},(f-s)_{t-2}\right)^{\prime}$. If the EMH is true, then $\alpha=\left(\mu_{1}-\beta \mu_{2}\right)<0$, $\beta=1, \gamma_{1}=\left(\delta_{1}-\beta \delta_{3}\right)=0, \gamma_{2}=\left(\delta_{2}-\beta \delta_{4}\right)=0$ and $\varepsilon_{t}$ is serially uncorrelated. Consequently, it appears directly while estimating the latter stationary VAR that $(1,-1)$ is a common feature vector.

This latter example illustrates the differences and the similarities between the cointegration and the common cyclical feature literature. In testing for cointegration, one estimates some parameters, then one is interested to know whether the residuals of a relationship using these parameters are $\mathrm{I}(0)$ in order to avoid the spurious regression issue. In the common feature literature, we estimate a particular relationship using an IV estimator, then we check whether this relationship respect some orthogonality conditions for a specific information set $\Omega_{t}$. The 
answer will depend upon what we put in this information set. For instance, if we introduce multi-period ahead forward exchange rates, it is likely that the moving average component this operation implies will guide us to lag the information set. The relationship will not be a SCCF relationship anymore but a model which will keep a certain number of MA components like for the codependence model we analyze in Section 2.5 .

\subsection{6 "Exogenous" Variables}

In our presentation, the common cyclical feature $f_{t}$ is a lincar combination of the lagged dependent variables. A more general specification can be considered. When $f_{t}$ includes other elements than the lags of $y_{\ell}$, the VAR model is only an approximation of the multivariate process. To illustrate this point, replace the dynamic endogenous common factors $f_{t}$ by a $\operatorname{VAR}(p)$ of weakly exogenous variables $z_{t}$

$$
f_{l} \equiv z_{t}=\sum_{i=1}^{p} F_{i} z_{t-i}+u_{t}
$$

where the $F_{i}$ 's are $(n-s)$ dimensional squared matrices, $u_{t}$ is a $(n-s)$ dimensional zero mean white noise process independent of $\varepsilon_{t}$ with covariance matrix $\Sigma$. We can always find $(n-s) \times n$ matrices $A_{i}$ such that $A_{i} B=F_{i}$. Substituting in (2.30), we obtain

$$
z_{t}=\sum_{i=1}^{p} A_{i} B z_{t-i}+u_{\ell} .
$$

By definition $y_{t}-\varepsilon_{t}=B z_{t}$, and thus $y_{t-i}-\varepsilon_{t-i}=B z_{t-i}, \forall i=1$ to $p$. This yiclds

$$
z_{\ell}=\sum_{i=1}^{p} A_{i}\left(y_{t-i}-\epsilon_{t-i}\right)+u_{t} .
$$

Therefore these substitutions imply a $\operatorname{VARMA}(p, p)$ process for $y_{t}$ given by

$$
y_{t}=\sum_{i=1}^{p} B A_{i} y_{t-i}+B u_{t}+\varepsilon_{t}-\sum_{i=1}^{p} B A_{i} \varepsilon_{t-i} .
$$

This multivariate process can be represented as an infinite order VAR and only approximated by a finite order VAR. This is one of the reasons arguing in favor of VARMA models instead of VAR ones. VARMA models are however much more difficult to estimate and to identify.

\subsection{Nested Reduced Rank}

From a statistical point of view we may be interested in some linear combinations which reduce the order of a $\operatorname{VAR}(p)$ to a lower order $\operatorname{VAR}$ without reaching a white noise process. This is 
proposed by Ahn and Reinsel (1988), Reinsel (1993) and generalizes the earlier works of Velu, Reinsel and Wichern (1986) and thus also the SCCF. They consider a VAR $(p)$

$$
y_{t}=\Phi_{1} y_{t-1}+\cdots+\Phi_{p} y_{t-p}+\varepsilon_{t}
$$

but allow for a different structure on the $\Phi_{j}$ matrices. Their approach assumes that the $\Phi_{j}$ have a particular reduced rank structure, such that $\operatorname{rank}\left(\Phi_{j}\right)=r_{j} \geq \operatorname{rank}\left(\Phi_{j+1}\right)=r_{j+1}, j=1, \ldots p-1$. So the $\Phi_{j}$ can be represented in the form $\Phi_{j}=B_{j} C_{j}$, where $B_{j}$ is $n \times r_{j}$ and $C_{j}$ is $r_{j} \times n$, and it. is further assumed that $\operatorname{range}\left(B_{j}\right) \supset \operatorname{range}\left(B_{j+1}\right)$. Therefore, the model can be rewritten as

$$
y_{\ell}=\sum_{j=1}^{p} B_{j} C_{j} y_{t-j}+\varepsilon_{\ell} .
$$

This procedure generalizes SCCF because it does not require the strong hypothesis that a linear combination reduces a $\operatorname{VAR}(p)$ process to a white noise but uses the additional restriction that matrices are nested. However, this modeling strategy is more a statistical approach than an economic one. Statistically, it is worth reducing the number of parameters that should be estimated in order to get more gain in estimation efficiency or to improve forecasts. However that procedure may lack of economic meaning because such a reduction does not assume anything in terms of adjustment to shocks. Indeed, both a $\operatorname{VAR}(p)$ and a $\operatorname{VAR}(p-k)$ with $p>k$ have an infinite VMA representation. An approach with a more economic emphasis has been developed by Gouriéroux and Peaucelle $(1989,1993)$.

\subsection{Codependence}

Independently of the EK's works, Gourićroux and Peaucelle $(1989,1993)$ (GP hereafter) treat the common feature issue in a finite order vector moving average context, a framework called codependence. In order to define "a more stable linear relationship" as they call it, GP develop an analysis in terms of dynamic multipliers aimed to find a linear combination of a group of time series with a shorter memory than the individual series.

Let us consider $y_{t}$, a $n$ dimensional stationary vector process, admitting a multivariate moving average (VMA) representation of order $q$, such that

$$
y_{t}=\mu+\varepsilon_{t}+\Theta_{1} \varepsilon_{t-1}+\ldots+\Theta_{q} \varepsilon_{t-q}, \quad t=1 \ldots T,
$$

where $\mu$ is a vector of constants, $\varepsilon_{t}$ is a multivariate white noise with covariance matrix $\Omega_{\varepsilon}$; $\Theta_{j}, j=1 \ldots q$ are square matrices of size $n$. Given the stationarity hypothesis, a one time transitory shock on the innovation occurring at a given period, say $t=0$, will have effects $\Delta \varepsilon_{0}, \Theta_{1} \Delta \varepsilon_{0}, \ldots, \Theta_{q} \Delta \varepsilon_{0}, 0,0$ on the process $y_{t}$. The impact becomes equal to zero after $q$, which defines the order of persistence of the multivariate moving average process. 
In general, the order of persistence $q^{*}$ of a linear combination $z_{t}$, defined by

$$
z_{t} \equiv \delta^{\prime} y_{t}=\delta^{\prime} \mu+\delta^{\prime} \varepsilon_{t}+\delta^{\prime} \Theta_{1} \varepsilon_{t-1}+\ldots+\delta^{\prime} \Theta_{q} \varepsilon_{t-q}, \quad t=1 \ldots T
$$

is also at most $q$. The time series in $y_{t}$ are called codependent (Cod hereafter) if $q^{*}$ is less than $q$ and equal to $q-b$, where the degree of codependence is the integer $b$ with $0 \leq b \leq q$. Codependence holds for the VMA process $(2.36)$ if there exists a $(n \times s)$ matrix $\delta$ whose columns span the codependence space such that $\delta^{\prime} \Theta_{q-b} \neq 0$ but $\delta^{\prime} \Theta_{q-k}=0, \forall k<b$. In terms of reduced rank of some matrices, codependence implies that $\operatorname{rank}\left[\Theta_{1}: \ldots: \Theta_{q-b}\right]=n$ but $\operatorname{rank}\left[\Theta_{q-b+1}: \ldots: \Theta_{q}\right\}<n$.

For practical purposes, it is important to note that the codependence restrictions are also valid for the autocovariance matrices. Therefore, if one notes $\Gamma(i)=\mathrm{E}\left(y_{l}, y_{t-i}^{\prime}\right), i=0 \ldots q$, testing for codependence is equivalent to testing the null hypothesis

$$
H_{0}: \delta^{\prime} \Gamma(q-b+1)=\delta^{\prime} \Gamma(q-b+2)=\ldots=\delta^{\prime} \Gamma(q)=0 .
$$

Sec also Beine and Hecq $(1998,1999)$ for further details.

\subsection{Non-synchronous Common Features}

Given the strong assumptions underlying the SCCF modeling (sce Ericsson, 1993), Vahid and Engle (1997) develop a non-synchronous common cycle approach, namely a model where a time delay in the adjustment process is explicitly taken into account. This approach may be sensible when both empirical and theoretical considerations favor adjustment delays. To be more precise, while the SCCF framework assumes exactly collinear impulse responses, these impulse responses will, in the non-synchronous modeling, only be linearly dependent after a small number of period, say, one or two. In practice, the existence of non-synchronous common feature vectors means that linear combinations of, possibly highly correlated, variables are only MA(1) or MA(2) processes. Formally, we may stress the difference between synchronous (SCCF) and non-synchronous common cyclical features of order one by the two systems (2.39) and (2.40):

$$
\begin{gathered}
\text { Synchronous : }\left\{\begin{array}{l}
y_{1 t}=\gamma_{1} f_{t}+\varepsilon_{1 t} \\
y_{2 t}=f_{t}+\varepsilon_{2 t} \\
f_{t}=\alpha f_{t-1}+w_{t}
\end{array}\right. \\
\text { Non-synchronous : }\left\{\begin{array}{c}
y_{1 t}=\gamma_{2} f_{t}+\varepsilon_{1 t} \\
y_{3 t}=f_{t-1}+\varepsilon_{3 t} \\
f_{t+1}=\alpha f_{t}+w_{t}
\end{array}\right.
\end{gathered}
$$


where $y_{1 t}, y_{2 t}$ and $y_{33}$ are scalar $I(0)$ time series for $t=1 \ldots T, \varepsilon_{1 t}, \varepsilon_{2 t}$ and $\varepsilon_{3 t}$ are innovations and $f_{t}$ is the dynamic common factor. In (2.39), the presence of synchronous common cycies implies the existence of a normalized common feature vector $\delta^{\prime}=\left(1,-\gamma_{1}\right)$ such that $y_{1 t}-\gamma_{1} y_{2 t}=$ $\varepsilon_{1 t}-\gamma_{1} \varepsilon_{2 t}$ is a white noise process. In the non-synchronous case we obtain $y_{1 t}-\gamma_{2} \alpha y_{3 t}=$ $\varepsilon_{1 t}-\gamma_{2} \alpha \varepsilon_{3 t}+\gamma_{2} w_{t-1}$. That means that cven if the dynamic factor $f_{t}$ can be very complicated, a lincar combination yields only a MA(1). In the terminology of Tiao and Tsay (1985, 1989), we say that there exists a scalar component model of order $(0,1)$, denoted $\operatorname{SCM}(0,1) .{ }^{4}$

In order to define non-synchronous common features, Vahid and Engle (1997) start with the Wold decomposition of the stationary process $y_{t}$

$$
y_{t}=\varepsilon_{\ell}+\sum_{j=1}^{\infty} C_{j} \varepsilon_{t-j},
$$

with $\sum_{j=1}^{\infty} j\left|C_{j}\right|<\infty$ and they investigate the presence of linear combinations $\delta^{\prime} y_{t}$ such that $\delta^{\prime} C_{j}=0$ if $j>1$ and $\delta^{\prime} C_{j} \neq 0$ if $j=1$. Basically, non-synchronous common features of general order $q$ can be summarized by the following set of pseudo-structural equations,

$$
\begin{aligned}
y_{1 t}-\gamma_{2} y_{2 t} & =u_{t}, \\
u_{t} & =\delta^{\prime} \varepsilon_{t}+\delta^{\prime} C_{1} \varepsilon_{t-1}+\ldots \delta^{\prime} C_{q} \varepsilon_{t-q},
\end{aligned}
$$

with $\delta=\left(1,-\gamma_{2}\right)^{\prime}$.

Remark that three types of linear systems can give rise to this set of pseudo-structural equations: the $\operatorname{VMA}(q)$, the $\operatorname{VARMA}(p, q)$ and the $\operatorname{VAR}(p)$. We have already seen that an analysis of common features in a pure VMA framework has been proposed by Gourieroux and Peaucelle $(1989,1993)$ through the codependence analysis. In this framework, a codependence vector yields a system with a shorter memory than variables taken individually. However, this finite order vector moving average framework may be less attractive when we want to analyze cointegrated systems that are usually studied within $\operatorname{VAR}(p)$ and to a lesser extent for $\operatorname{VARMA}(p, q)$.

Consequently, we concentrate on the two other ways of obtaining non-synchronous common cycles.

\subsubsection{Non-synchronous VARMA Models}

Let us assume $y_{t}$ a $n$-dimensional vector of $I(0)$ time series following a $\operatorname{VARMA}(p, 1)$

$$
y_{t}=\Phi_{1} y_{t-1}+\cdots+\Phi_{p} y_{t-p}+\varepsilon_{t}+\Theta_{1} \varepsilon_{t-1}, \quad t=1 \ldots T .
$$

\footnotetext{
${ }^{4}$ In their working paper version, Vahid and Engle (1993) also used the terminology of Tiao and Tsay, i.e. they' search for scalar component models of order $\operatorname{SCM}(0,1)$. That seemed to be a better idea to avoid confusion than the term codependence but they rename their published 1997 paper "Codependent Cycles".
} 
Under $\operatorname{SCM}(0,1)$, we have $\delta^{\prime}\left(\Phi_{1} \ldots \Phi_{p}\right)=0$. A common feature analysis can hence be undertaken in order to investigate the presence of non-synchronous common cyclical features such that $\delta^{\prime} y_{t}=\delta^{\prime}\left(\varepsilon_{t}+\Theta_{1} \varepsilon_{t-1}\right)$ is a $s$ dimensional VMA(1) process.

Some remarks are in order:

Remark 2.11 There are no reasons for considering moving average processes of very low order. This order will depend on the data used, their frequency, and the degree of non-synchronous comovements.

Remark 2.12 Note that exogenous common factors entail MA components which could be confused with non synchronous comovements (see subsection 2.3.6).

\subsubsection{Codependence in VAR Models}

Testing for common features in $\operatorname{VARMA}(p, q)$ is the exception and not the rule, the most popular framework uses $\operatorname{VAR}(p)$ models as it is the case for cointegration. Kugler and Neusser (1993), Kugler and Schendener $(1991,1993)$ have extended the codependence approach, initially developed by Gouriéroux and Peaucelle for VMA(q), to VAR models. Note that (non exchangeable ${ }^{5}$ ) stationary VAR processes have an infinite VMA representation so that the concept of degree of codependence is infinite. To overcome this, they base their approach on the repeated substitution of a $\operatorname{VAR}(p)$ up to $\operatorname{lag} c-1$ as follows ${ }^{6}$

$$
\begin{gathered}
y_{t}=\Phi_{1} y_{t-1}+\ldots+\Phi_{p} y_{t-p}+\varepsilon_{t} \\
y_{t}=\varepsilon_{t}+A_{1} \varepsilon_{t-1}+\ldots+A_{c-1} \varepsilon_{t-c+1}+G_{1} y_{t-c}+\cdots G_{p} y_{t-c-p+1},
\end{gathered}
$$

where either the $A_{i}$ and the $G_{j}$ matrices are functions of the $\Phi$ matrices. By construction the lagged $y_{t}$ terms contain the remaining VMA $\sum_{i=0}^{\infty} A_{c+i} \varepsilon_{t-c-i}$. So the hypothesis they consider is

$$
H_{0}: \delta^{\prime} G_{1}=\cdots=\delta^{\prime} G_{p}=0
$$

A similar analysis is proposed by Vahid and Engle (1997) who show that a SCM(0,1), which is a non-synchronous common feature model, exists within a $\operatorname{VAR}(p)$ under some particular restrictions. Let us take the VAR(2) example of Vahid and Engle (1997) to simplify the algebra

$$
y_{l}=\Phi_{1} y_{t-1}+\Phi_{2} y_{t-2}+\varepsilon_{t}
$$

\footnotetext{
${ }^{5}$ See Appendix.

${ }^{6}$ There is a little notational difference between GP and Kugler and co-authors. Indeed, GP define the degree of codependence $b$ iss the reduction in a VMA $(q)$. Because this number is also infinite in a VMA $(\infty)$ Kugler and co-authors call codependence order $c$, namely the first zero autocovariance matrix.
} 
Because they search for a one lag length non-synchronous common features model, substituting for $y_{t-1}$ we obtain

$$
y_{\ell}=\left(\Phi_{1}^{2}+\Phi_{2}\right) y_{\ell-2}+\left(\Phi_{1} \Phi_{2}\right) y_{\ell-3}+\Phi_{1} \varepsilon_{t-1}+\varepsilon_{t}
$$

Non synchronous serial correlation common features implies $\delta^{\prime}\left(\Phi_{1}^{2}+\Phi_{2}\right)=0$ and $\delta^{\prime}\left(\Phi_{1} \Phi_{2}\right)=0$. Under these scalar component. restrictions the linear combination is $\delta^{\prime} y_{t}=\delta^{\prime} \Phi_{1} \varepsilon_{t-1}+\delta^{\prime} \varepsilon_{t}$.

The main difference between Vahid and Engle (1997) and Kugler and co-authors' framework is that the second one tests for the degree of persistence without any knowledge about what this degree should be and hence the MA order is not required to be very small as in Vahid and Engle.

\subsubsection{Really Unsynchronized Models?}

At this stage, we want to argue now that the model put forward by Vahid and Engle (1997) does not necessarily fulfil their requirement given by (2.40). Let us illustrate their model in terms of dynamic: factors in a $\operatorname{VAR}(2) y_{\ell}=\Phi_{1} y_{t-1}+\Phi_{1} y_{t-2}+\varepsilon_{t}$ with

$$
\left(\begin{array}{l}
y_{1 t} \\
y_{2 t}
\end{array}\right)=\left(\begin{array}{cc}
\phi_{1} & \phi_{2} \\
0 & 0
\end{array}\right)\left(\begin{array}{l}
y_{1 t-1} \\
y_{2 t-1}
\end{array}\right)+\left(\begin{array}{cc}
0 & 0 \\
\phi_{1} & \phi_{2}
\end{array}\right)\left(\begin{array}{l}
y_{1 t-2} \\
y_{2 t-2}
\end{array}\right)+\left(\begin{array}{l}
\varepsilon_{1 t} \\
\varepsilon_{2 t}
\end{array}\right)
$$

It is casily seen that a linear combination $y_{2 t}-y_{1 t-1}=\varepsilon_{2 t}-\varepsilon_{1 t-1}$ is a MA(1) but

$$
\left(\Phi_{1}^{2}+\Phi_{2}\right)=\left(\begin{array}{cc}
\phi_{1}^{2} & \phi_{2} \phi_{1} \\
\phi_{1} & \phi_{2}
\end{array}\right) \quad \text { and } \quad\left(\Phi_{1} \Phi_{2}\right)=\left(\begin{array}{cc}
\phi_{2} \phi_{1} & \phi_{2}^{2} \\
0 & 0
\end{array}\right)
$$

and while these matrices have both reduced rank equal to one, their left null spaces do not overlap, that is to say

$$
\operatorname{rank}\left(\begin{array}{cccc}
\phi_{1}^{2} & \phi_{2} \phi_{1} & \phi_{2} \phi_{1} & \phi_{2}^{2} \\
\phi_{1} & \phi_{2} & 0 & 0
\end{array}\right)=2
$$

This type of non synchronous comovements has to be considered within a polynomial common cyclical feature approach. In such a framework, a feature, that is present in each of a group of series $y_{t}=\left(y_{1 t}, \ldots, y_{n}\right)^{\prime}$ could be called dynamically common to those series if there exists a lag polynomial non zero linear combination of the series $\delta(L)$ such that $\delta(L)^{\prime} y_{t}$ does not have the feature. In the previous example $\delta(L)=(-L, 1)^{\prime}$.

We leave this extension of the non-synchronous framework for future rescarch.

\subsection{Scalar Component Models}

The Scalar Component Model (Tiao and Tsay, 1985, 1989) has already been introduced previously. In this section a more formal definition is given and we show that the scalar component 
model (SCM) approach nests most of the previous models. This section allows us to make the link with the issue of identification of VARMA we will see in next section.

By also considering contemporaneous linear transformations of a VARMA model, Tiao and Tsay's objectives are "(i) to reveal possibly hidden simplifying structure of the process, (ii) to achicve parsimony in parametrization and (iii) to identify the exchangeable models" (Tiao and Tsay, 1989). This latter point is analyzed in the Appendix.

Definition 2.13 For a $n$-dimensional process $y_{1}$ following a VARMA $(p, q)$ model, a non-zero linear combination $\nu_{0}^{\prime} y_{t}$ is said to follow a scalar component model $S C M\left(p_{1}, q_{1}\right)$ structure if there exist $p_{1} n$-dimensional vectors $\nu_{1}, \ldots, \nu_{p_{1}}$ such that:

(a) $\nu_{p_{1}}$ is non-zero when $p_{1}>0$, and

(b) the lincar combination of $y_{t}, \ldots, y_{t-p}$, that is $u_{t}=\sum_{j=0}^{p_{1}} \nu_{j}^{\prime} y_{t-j}$ satisfies:

$$
E\left(\varepsilon_{t-j} u_{t}\right)\left\{\begin{array}{l}
\neq 0 \text { if } j=q_{1} \\
=0 \text { if } j>q_{1}
\end{array}\right. \text {, }
$$

where $\varepsilon_{t}$ is a $n \times 1$ vector of innovations in the $y_{t}$ process.

This SCM structure can be written as

$$
\nu_{0}^{\prime} y_{l}=-\sum_{j=1}^{p_{1}} \nu_{j}^{\prime} y_{t-j}+\nu_{0}^{\prime} \varepsilon_{t}+\sum_{i=1}^{q_{1}} h_{i}^{\prime} \varepsilon_{t-i} .
$$

Starting from a VARMA $(p, q)$, SCM cntails that it is possible to find some linear combinations for which $p_{1} \leq p$ and $q_{1} \leq q$ with at least one strict inequality holds. Actually, we can see that their concerns are very similar to common features ones, even if Tiao and Tsay stress rather statistical aspects. It is interesting to note that all the previous common factor approaches are nested in scalar component models:

1. The serial correlation common feature approach corresponds to $\operatorname{SCM}(0,0)$ directions, i.c. t.esting for $p_{1}=0$, taking $q_{1}=0$,

2. The Vahid and Engle non-synchronous model test for $\operatorname{SCM}\left(0, q_{1}\right)$ with $q_{1}$ a small number,

3. Kugler and Neusser look for $\operatorname{SCM}\left(0, q_{1}\right)$ where $q_{1}$ should be determined and is not necessarily small.

4. Nested reduced rank framework search for $\operatorname{SCM}(p-k, 0)$ directions whilst codependence t.ests for $\operatorname{SCM}(0, q-b)$. Notice that we often prefer to use this latter type of notation instead of the Tiao and Tsay one. Indeed, writing the scalar component as $\operatorname{SCM}(p-k, q-b)$ allows to stress both the original model and the simplifying structure whilst writing $\operatorname{SCM}\left(p_{1}, q_{1}\right)$ cannot. 
In order to illustrate SCM, we consider a bivariate $\operatorname{VARMA}(1,1)$ model in which $y_{\iota}=$ $\left(y_{1 t}, y_{2 t}\right)^{\prime}, \varepsilon_{t}=\left(\varepsilon_{1 t}, \varepsilon_{2 t}\right)^{\prime}$

$$
\left(1-\Phi_{1} L\right) y_{t}=\left(I-\Theta_{1} L\right) \varepsilon_{t}
$$

To simplify matters we consider some linear combinations between the rows of both autoregressive and moving average matrices such as

$$
\Phi_{1}=\left(\begin{array}{ll}
\phi_{21} & \phi_{22} \\
\delta \phi_{21} & \delta \phi_{22}
\end{array}\right), \Theta_{1}=\left(\begin{array}{ll}
\theta_{21} & \theta_{22} \\
\lambda \theta_{21} & \lambda \theta_{22}
\end{array}\right)
$$

Consider now the lincar transformation $z_{t}=Q y_{t}$, where $Q=\left(\nu_{0}^{(1)}, \nu_{0}^{(2)}\right)^{\prime}, \nu_{0}^{(1)}=(\lambda, 1)^{\prime}$ and $\nu_{0}^{(2)}=$ $(-\delta, 1)^{\prime}$. Premultiplying $(2.53)$ by $Q$ we obtain

$$
Q y_{t}+G y_{t-1}=Q \varepsilon_{t}+H \varepsilon_{t-1}
$$

where

$$
G=\left(\begin{array}{ll}
\phi_{21}(\lambda-\delta) & \phi_{22}(\lambda-\delta) \\
0 & 0
\end{array}\right), H=\left(\begin{array}{ll}
0 & 0 \\
\theta_{21}(\lambda-\delta) & \theta_{22}(\lambda-\delta)
\end{array}\right)
$$

It is seen that both the second row of $G$ and the first row of $H$ contain only zero values. Inserting $Q^{-1} Q$ in front of $y_{t-1}$ and $\varepsilon_{t-1}$, the model for $z_{t}$ becomes $\left(1-\Phi_{1}^{*} L\right) z_{t}=\left(I-\Theta_{1}^{*} L\right) a_{t}$, with $a_{t}=Q \varepsilon_{t}, \Phi_{1}^{*}=Q \Phi_{1} Q^{-1}$ and $\Theta_{1}^{*}=Q \Theta_{1} Q^{-1}$. In this casc, $y_{1 t}$ follows a scalar component model of order $(1,0)$, denoted by $\operatorname{SCM}(1,0)$. This shows that, within a bivariate VARMA $(1,1)$ framework, $y_{1}$ should have both MA(1) and AR(1) terms, but there exists a vector which reduces this order to keep only an autoregressive process. Remark that a $\operatorname{SCM}(1,0)$ does not imply that $y_{1 t}$ follows an univariate $\operatorname{AR}(1)$ process since $y_{2 t-1}$ is present in the equation of $y_{1 t}$ (see Tiao and Tsay, 1989).

VARMA models are however not very attractive for empirical analysis due to the large number of parameters involved, the estimates of which are often highly correlated, and the existence of observationally equivalent models related to the notion of identifiability and exchangeability. By definition SCMs aim at reducing the number of estimated parameters. Moreover this modeling could give directions for further modeling strategy and show the price to pay if we choose either finite VAR or VMA processes. Testing for SCM also gives an indication of the existence of exchangeable models, which should be taken into account.

\subsection{Testing for Common Features Using Canonical Correlations}

All hypotheses considered in the preceding sub-sections can easily be investigated using a canonical correlation analysis. There are several advantages to test for common cyclical features in 
a whole system using canonical correlations, instead of limited information techniques such as IV or GMM. First of all, the estimated coefficients are, under the normality assumption, the maximum likelihood estimates (sce Johansen, 1995; Hendry, 1995 inter alia) and the likelihood ratio testing strategy can be applied. Second, cofeature vectors do not rely on the choice of a dependent variable but only on a particular normalization. Third, we can consider more than one common feature vector and we can test for their number. We define a common feature matrix in which some identification rules may be applied. Fourth, we can study either the vector which annihilates the feature or the factorial structure. Finally, reduced rank techniques are easy to implement, their distribution is well known in most cases and the degrees of freedom of LR-type tests are relatively casy to find.

In this section we recall the interest in a canonical correlation analysis for identifying VARMA models. But canonical correlation procedures are also routincly used in cointegration analyses (see Anderson, 1998; Johansen, 1995) and will be applied throughout this thesis for determining reduced rank restrictions on short-run parametcr matrices. Hence, usual test statistics and their distributions are also summarized in this section.

\subsubsection{Identification of VARMA Models}

Like information criteria, a canonical correlation analysis can be used in the identification stage of $\operatorname{VARMA}(p, q)$ models and is a generalization of sample autocorrelation and sample partial autocorrclation functions for univariate models (see Chapters 3 and 6 in Reinsel 1993). For a $n$-dimensional process $y_{t}$, consider the $n(m+1)$ vector

$$
Y_{m, t}=\left(y_{t}^{\prime}, y_{t-1}^{\prime}, \ldots, y_{t-m}^{\prime}\right)^{\prime}
$$

Tiao and Tsay propose to examine the squared canonical correlations between $Y_{m, t}$ and $Y_{h, t-j-1}=$ $\left(y_{t-j-1}^{\prime}, y_{t-j-2}^{\prime}, \ldots, y_{t-j-1-h}^{\prime}\right)^{\prime}$ with $h \geq m \geq 0$ and $j \geq 0$. Setting for example $m=h=0$, we may test for the significance of the autocovariance matrices by cxamining the squared canonical corrclations between $y_{t}$ and $Y_{t-j-1}$, for $j=0,1,2 \ldots$. These squared canonical correlations will be statistically zero in a $\mathrm{MA}(q)$ for $j \geq q$. We may also want to test for the significance of a set of autocovariance matrices by performing a canonical correlation analysis between $y_{t}$ and $Y_{h, t-j-1}$ with $h$ chosen equal to the order of the $\mathrm{MA}(q)$ for instance. This is the scheme which is examined by the codependence approach. In this case when $j=0$, we also test for SCCF.

On the other hand, it is also possible to analyze the order of $\operatorname{VAR}(p)$ models. Setting $m=h$ and $j=0$, one examines the partial canonical correlations between $y_{t}$ and $y_{t-m-1}$, filtered ${ }^{7}$ for $y_{t-1}, \ldots, y_{t-m}$. These correlations are equal to zero for $m \geq p$ in a $\operatorname{VAR}(p)$. Leaving $h>m$, wo can also test for the significance of a set of partial correlation matrices. This is the case of the "nested reduced-rank model" studied by Ahn and Reinsel (1988). The serial correlation common

\footnotetext{
'Notice that the canonical correlation (not trivialy equal to one) between two sets $\left(X_{1}, X_{3}\right)$ and $\left(X_{2}, X_{3}\right)$ are equal to the partial canonical correlations between $X_{1}$ and $X_{2}$ given $X_{3}$
} 
feature test statistic of EK and Velu et al. (1986) is also a test for $\operatorname{SCM}(0,0)$ assuming $m=0$ and $h \geq 0$.

Now, in order to select both MA and VAR order, one combines the analysis of correlation and partial correlation matrices. Recall that for an $\operatorname{ARMA}(p, q)$ process, $y_{t}-\sum_{j=1}^{p} \Phi_{j} y_{t-j}=$ $\varepsilon_{t}-\sum_{j=1}^{q} \Theta_{j} \varepsilon_{t-j}$, the variable $y_{t-k}$ is uncorrelated with $\varepsilon_{t-j}, j=0,1, \ldots q$, for all $k>q$. Therefore, if $m \geq p$, then there are (at least) $n$ linear combinations of $Y_{m, l}$ which are uncorrelated with $Y_{h, t-j-1}$. The first time the number of zero canonical correlations is equal to the number of variables allows one to determine the order of the VARMA process.

Tiao and Tsay $(1985,1989)$ however show that because simplifying reduced rank structures may exist among the coefficient matrices, there can be, due to the presence of scalar component models, some zcro canonical correlations between $Y_{m, t}$ and $Y_{h, t-j-1}$ even for $m<p$ and $j<q$. A careful study of these canonical correlations in a two way table depending on $m$ and $j$ can give all the relevant information.

\subsubsection{SCM in Practice and the Choice of Key Parameters}

Practical application of SCMs first require the investigator to fix some maximum lag orders for the autoregressive and the moving average components and especially to choose of $m$ and $h$. The selection of $h$ is important to show that the codependence approach is nested within the SCM. Tiao and Tsay (1985) as well as Reinsel (1993) take $h=m$. This technique allows to test for only one correlation and partial correlation matrix each time (when $j$ or $m$ equal zero). Indeed the following canonical correlation plan presents the case $h=m=2$.

$$
A_{1(m, h, j)}:\left(\begin{array}{llll} 
& j=0 & j=1 & j=2 \\
m=0 & y_{t} & y_{t} & y_{t} \\
& y_{t-1} & y_{t-2} & y_{t-3} \\
m=1 & y_{t} y_{t-1} & y_{t} y_{t-1} & y_{t} y_{t-1} \\
& y_{t-1} y_{t-2} & y_{t-2} y_{t-3} & y_{t-3} y_{t-4} \\
m=2 & y_{t} y_{t-1} y_{t-2} & y_{t} y_{t-1} y_{t-2} & y_{t} y_{t-1} y_{t-2} \\
& y_{t-1} y_{t-2} y_{t-3} & y_{t-2} y_{t-3} y_{t-1} & y_{t-3} y_{t-4} y_{t-5}
\end{array}\right)
$$

If we want to test for the significance of a set of covariance matrices, we prefer to let at each $(m, j)$ position, $h=m+(q-j)$, where $q$ is the last non zero moving average order we fix in the 
analysis. As a result, the previous scheme becomes with $q=2$.

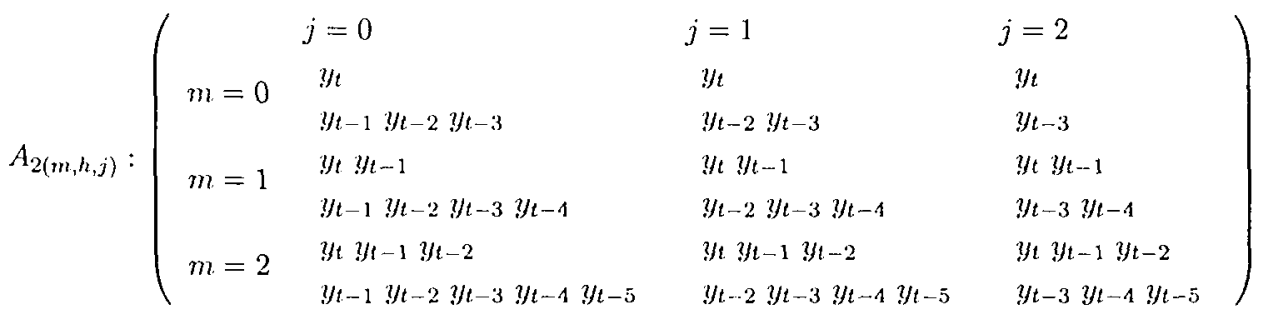

The matrix $A_{2(m, h, j)}$ is more informative about the possible simplifying structure although we loose some power when testing for the null hypothesis of reduced rank in partial correlation matrices since $A_{2(m, h, j)}$ requires additional lags of $y_{\ell}$. The study of the matrix $A_{2(m, h, j)}$ stresses several important things. The first row with $m=0$ gives the canonical correlation allowing to test for codependence. When $m=j=0$ we test for "perfect" codependence (i.e. $\operatorname{SCM}(0.0)$ ) which is also the SCCF test statistics. The study of partial correlation is performed by examining the first column, i.e. cases for which $j=0$. SCCF is also a special case of this column. ${ }^{8}$

$A_{1(m, h, j)}$ and $A_{2(m, h, j)}$ also show the importance of model identification, i.e. selection of lag orders for the initial VARMA. Indeed each position of $A_{2}$ can be viewed as a reduction of a more general process. For instance, if one tests for an $\operatorname{SCM}(0,1)$, given the initial model is an VARMA(2,1), one analyzes the zero canonical correlations between $y_{t}$ and $y_{t-2}, y_{t-3}$. In the matrix $A_{2}$, one moves from the position $(m, j)=(2,1)$ to $(m, j)=(0,1)$. However the canonical correlation between these two sets of variables also corresponds to a reduction from a pure $\mathrm{MA}(q)$ to a $\mathrm{MA}(1)$. This is also a codependence analysis which analyze the null hypothesis that the second and the third order autocovariance matrices are of reduced rank, given the first one is unconstrained.

Note finally that when we analyze both MA and AR components, the number of zero eigenvalues we test for, becomes $s=n \times(m+1)$, some of them are counted twice. Consequently, in order to determine the VARMA model and its SCM counterparts, Tiao and Tsay build a matrix $D_{1(m, h, j)}$ or $D_{2(m, h, j)}$ which are the diagonal increments of the number of zero cigenvalues of $A_{1(m, h, j)}$ or $A_{2(m, h, j)}$.

The number of degrees of freedom in scalar component models will depend on the number of restrictions on the selected VARMA and is equal $s \times\{(h-m) n+s\}$. For instance, testing for an $\operatorname{SCM}(0,0)$ will give the same eigenstructure if it comes from a $\operatorname{VAR}(1)$ or a $\operatorname{VARMA}(2,1)$, but. the number of restrictions is different.

\footnotetext{
${ }^{*}$ Note that it is not possible to control the lag augmentation both for codependence and for autoregressive nested reduced reduced rank, consequently we emphasize power of codependence test statistics with $A_{2(m, h, j)}$.
} 


\subsubsection{The Canonical Correlation Test Statistic and its Duality}

To simplify matters we assume that the unconditional mean has been substracted from all variables. Let us form the cross moments matrices of the two sets $Y_{m, t}$ and $Y_{h, t-1-j}$ and denote these by $S_{00}=\mathrm{E}\left(Y_{m, t} Y_{m, t}^{\prime}\right), S_{11}=\mathrm{E}\left(Y_{h, t-1-j} Y_{m, t-i-j}^{\prime}\right), S_{01}=\mathrm{E}\left(Y_{m, t} Y_{h, t-i-j}^{\prime}\right)$ and $S_{10}=\left(Y_{h, t-1-j} Y_{m, t}^{\prime}\right)$. The objective of the canonical correlation is to find vectors (or matrices) $a^{\prime}$ and $b^{\prime}$ such that the correlation between the lincar combinations $w_{1}=a^{\prime} Y_{m, \ell}$ and $w_{2}=b^{\prime} Y_{h, t-1-j}$ is maximum or minimum. The solution for $a_{s}$ (see inter alia Anderson 1984, Izeman 1980) consists in finding the eigensiructure of the following symmetric matrix

$$
\Lambda_{a}=S_{00}^{-1 / 2} S_{01} S_{11}^{-1} S_{10} S_{00}^{-1 / 2}
$$

The eigenvalues are the squared canonical correlations. The smallest measure the linear combinations of the components $Y_{m, t}$ and the components of $Y_{h, t-1-j}$ that are the least correlated. The cigenvector corresponding to these eigenvalues equals $a=S_{00}^{-1 / 2} e_{s}$ where $e_{s}$ are the eigenveciors associated with $s$ zero eigenvalues in (2.58). In the same way $b=S_{11}^{-1 / 2} u_{s}$ where $u_{s}$ are the eigenvectors associated with the $n-s$ nonzero eigenvalues in (2.59)

$$
\Lambda_{b}=S_{11}^{-1 / 2} S_{10} S_{00}^{-1} S_{10} S_{11}^{-1 / 2}
$$

Hence there exists the same duality between common cyclical features and common cycle as the one between cointegration and common trend. But in the opposite and sometimes less intuitive way. In the presence of, say, $r$ cointegrating vectors, it is possible to rewrite the whole system in a VECM form. The information on long run equilibria enters with one lag to show how the difference of dependent variables adjusts to them. By definition, there will remain $n-r$ common trends which govern the non-stationary stochastic trends. This information is sometimes more important than the estimates of long-run coefficients per se.

In the common cycle versus cofeature duality, the $s$ cofeature vectors, associated with some zcro eigenvalues, form a cofeature space $\delta^{\prime} . \delta^{\prime} y_{t}$ leads to a process that does no longer have the feature. The system may be rewritten as a function of the $n-s$ non zero common dynamic factors or common cycles using an additional common trend-common cycle decomposition (see Chapter 4).

\subsubsection{Distribution and Test Statistics}

Test statistics used to analyze the presence of zero eigenvalues are based on sequential reduced rank tests on the empirical counterparts of $\Lambda_{a}$ or $\Lambda_{b}$ matrices. Assuming that $y_{t}$ is a stationary Gaussian $\operatorname{VAR}(p)$ process, the likelihood ratio statistic for the null hypothesis that $s$ squared canonical correlation are equal to zero can be shown to be $\zeta_{1}$ (Anderson, 1984, Velu et al., 1986, 
Gourićroux et al., 1991a,b)

$$
\zeta_{1}=-T \sum_{i=1}^{s} \ln \left(1-\hat{\lambda}_{i}\right), \quad s=1 \ldots n,
$$

where $\hat{\lambda}_{i}$ are the squared canonical correlation coming from the solution of $\Lambda_{a}$ or $\Lambda_{b}$ with $s$ the potential number of zero cigenvalues. For instance, in the three variable case the sequential hypotheses are

$$
\begin{aligned}
& H_{0}^{1}: s \geq 1 \text { vs. } H_{A}^{1}: s=0 \\
& H_{0}^{2}: s \geq 2 \text { vs. } H_{A}^{2}: s<2 \\
& H_{0}^{3}: s=3 \text { vs. } H_{A}^{3}: s<3
\end{aligned}
$$

or alternatively

$$
\begin{aligned}
& H_{0}^{1}: s>0 \text { vs. } H_{A}^{1}: s=0 \\
& H_{0}^{2}: s>1 \text { vs. } H_{A}^{2}: s \leq 1 \\
& H_{0}^{3}: s>2 \text { vs. } H_{A}^{3}: s \leq 2
\end{aligned}
$$

$\mathrm{H}_{0}^{3}$ for example implies that we are testing for a 3 dimensional white noise process. Test statistic (2.60), which is appropriate to test for SCCF or autoregressive nested reduced rank, follows asymptotically a $\chi_{(\nu)}^{2}$ distribution with degrees of freedom equal to the number of restrictions imposed under the null, provided the null is true. In the $\operatorname{VAR}(p)$ for cxample, the unconstrained model has $n^{2} \times p$ parameters while the number of parameters of the common cyclical factor representation (2.8) is $n^{2} p+n s-s^{2}-s n p$, hence $\nu=s n p-s(n-s)$.

Notice that in the presence of MA components, (2.60) has to be adjusted. For instance if one tests the null hypothesis $\delta^{\prime} \Gamma(2)=\delta^{\prime} \Gamma(3)=0$, i.e. a reduced rank hypothesis in the second and the third autocovariance matrices, one carries out the multivariate regression $y_{t}=$ $\mu+\Upsilon_{1} y_{t-2}+\Upsilon_{2} y_{t-3}+u_{t}$. Thus, $y_{t}$ is not a whitc noise under the null because it includes an MA(1) component, making inference inefficient. To circumvent this problem, Tiao and Tsay (1985, 1989), Tsay $(1986,1989)$ proposed to adjust $(2.60)$ by an estimator of the estimated variance using Bartlett's formula. ${ }^{9}$ The LR test becomes

$$
\zeta_{2}=-T \sum_{i=1}^{s} \ln \left(1-\hat{\lambda}_{i} / \hat{d}_{i}\right) \quad s=1 \ldots n,
$$

with $\hat{d}_{i}=1+2 \sum_{\tau=1}^{j} \hat{\rho}_{\tau}\left(\hat{w}_{1 \ell}\right) \hat{\rho}_{\tau}\left(\hat{w}_{2 t}\right)$, where $\hat{\rho}_{\tau}\left(\hat{w}_{1 \ell}\right)$ and $\hat{\rho}_{\tau}\left(\hat{w}_{2 t}\right)$ are respectively the lag $\tau$ sample

\footnotetext{
${ }^{9}$ Note that other modification have been proposed in the literature like in Robinson (1973), West (1998), Velu and Reinsel (1994).
} 
autocorrelation of $\hat{w}_{1 t}$ and $\hat{w}_{2 t} . \hat{w}_{1 t}$ and $\hat{w}_{2 t}$ being the lincar combinations of $Y_{m, t}$ and $Y_{h, t-i-j}$ associated with the zero eigenvalue. $\zeta_{2}$ has the same asymptotic distribution as $\zeta_{1}$.

\subsection{Inference in Codependence and SCM: Monte Carlo Results}

In this section we present. Monte Carlo experiments in order to illustrate the behavior of codependence test statistics. While the method aims at discovering reduced rank in finite VMA parameter matrices, we consider the issue of determining the codependence order in $\operatorname{VMA}(q)$, $\operatorname{VARMA}(p, q)$ and $\operatorname{VAR}(p)$ models. We also investigate the impact of non-Gaussian residuals as well as the omission of a long-run relationship.

\subsubsection{Codependence Test Statistics}

Because of its appealing economic meaning in terms of adjustment to shocks we first evaluate the behavior of codependence procedures. Beine and Hecq (1999) have studied by Monte Carlo the impact of various form of misspecification on the size and power of test statistics $\zeta_{1}$ in (2.60). These include under or overestimation of the lag order, the presence of an infinite process instead of a pure $\mathrm{MA}(q)$ one, the omission of a cointegrating vector, the presence of GARCH errors and temporal aggregation. The simulations undertaken in Beine and Hecq (1999) were built under the white noise null hypothesis, consequently $\zeta_{1}$ could be used.

In this section we compare $\zeta_{1}$ and $\zeta_{2}$ with correlated errors. More precisely, we consider a MA(3) process $y_{t}=\varepsilon_{t}+\Theta_{1} \varepsilon_{t-1}+\Theta_{2} \varepsilon_{t-2}+\Theta_{3} \varepsilon_{t-3}$ with follows a $\operatorname{SCM}(0,3-2)$ under the null hypothesis with a cofeature vector equal to $(1,-1)$. The coefficient matrices are the following

$$
\begin{gathered}
\Theta_{1}=\left(\begin{array}{cc}
.6 & .2 \\
.4 & -.3
\end{array}\right), \Theta_{2}=\left(\begin{array}{cc}
.1 & -.5 \\
.1 & -.5
\end{array}\right) \Theta_{3}=\left(\begin{array}{cc}
-.32 & .16 \\
-.32 & .16
\end{array}\right), \\
\varepsilon_{t} \sim N\left[\left(\begin{array}{l}
0 \\
0
\end{array}\right),\left(\begin{array}{cc}
1 & .6 \\
.6 & 1
\end{array}\right)\right] .
\end{gathered}
$$

The simulations were carried out using a routine written in GAUSS 3.14. $T+50$ observations are generated but only last $T$ are used. We employ 10000 replications and the number of observations is 500. We report the following results: the percentage of rejection for $\zeta_{1}$ and $\zeta_{2}$ with a nominal size of $5 \%$. The median value of the potential common feature vector as well as the spread, i.e. the interquartile range. Notice that nonparametric summaries are more robust than parametric ones because one outcome may give very extreme results and can affect heavily the mean and the standard error of the estimates.

Table 2.1 reports that for the evaluation of size distortions in the reduced rank hypothesis, $\zeta_{1}$ test statistic does not reject the null more frequently than $\zeta_{2}$. The main difference is that $\zeta_{2}$ is better in determining the overall multivariate model, that is the VMA(3). It will hence be more 


\begin{tabular}{ccccccc}
\hline & $\zeta_{1}$ & \multicolumn{3}{c}{$\zeta_{2}$} & & \\
$j$ & $s \geq 1$ & $s=2$ & $s \geq 1$ & $s=2$ & $\hat{\beta}_{0.5}$ & $\hat{\beta}_{0.75-0.25}$ \\
\hline 0 & 100 & 100 & 100 & 100 & 2.486 & .808 \\
1 & 4.89 & 100 & 4.48 & 100 & 1.002 & .136 \\
2 & 4.13 & 91.5 & 3.82 & 87.63 & .996 & .303 \\
3 & 0.60 & 8.59 & 0.33 & 3.49 & .793 & 1.519 \\
4 & 0.58 & 8.47 & 0.36 & 4.08 & .773 & 1.510 \\
5 & 0.64 & 6.47 & 0.44 & 4.30 & .750 & 1.623 \\
\hline
\end{tabular}

Table 2.1: Size and Power of Codependence Tests for DGP (2.62)

\begin{tabular}{ccccccc}
\hline \hline & \multicolumn{1}{c}{$\bar{\zeta}_{1}$} & \multicolumn{1}{c}{$\zeta_{2}$} & & \\
$j$ & $s \geq 1$ & $s=2$ & $s \geq 1$ & $s=2$ & $\hat{\beta}_{0.5}$ & $\hat{\beta}_{0.75-0.25}$ \\
\hline 0 & 100 & 100 & 100 & 100 & 1.397 & 0.192 \\
1 & 4.83 & 100 & 4.98 & 100 & 0.999 & 0.098 \\
2 & 1.1 & 8.84 & 0.59 & 3.69 & 0.918 & 1.454 \\
\hline \hline
\end{tabular}

Table 2.2: Size and Power of Codependence Tests a MA(2)

likely to overestimate the dynamic structure with $\zeta_{1}$. An unbiased estimate of the cofeature vector is obtained under the null (when $j=1$ and 2 ) but the precision, measured by the inter-quartile range, is higher for $j=1$, the null hypothesis.

We have shown in the previous section that the orders of unconstrained models should be first determined, otherwise the SCM conclusions could be meaningless. In particular, because of the same canonical correlation plan a $\operatorname{SCM}\left(p-p, q_{1}\right)$ can be interpreted as an $\operatorname{SCM}(0, q-b)$, with $q_{1}=q-b$. We analyze this situation by comparing a MA(2) and an $\operatorname{ARMA}(1,1)$ both following a $\operatorname{SCM}(0,1)$. We simulate $y_{t}=\varepsilon_{t}+\Theta_{1} \varepsilon_{t-1}+\Theta_{2} \varepsilon_{t-2}$ and $y_{t}=\Phi_{1} y_{t-1}+\varepsilon_{t}+\Theta_{1} \varepsilon_{t-1}$ using the following coefficient matrices.

$$
\Theta_{1}=\left(\begin{array}{cc}
.6 & .2 \\
.4 & -.3
\end{array}\right), \Theta_{2}=\left(\begin{array}{ll}
.6 & .3 \\
.6 & .3
\end{array}\right), \Phi_{1}=\left(\begin{array}{cc}
.4 & -.12 \\
.4 & -.12
\end{array}\right)
$$

For $j=1$, the null hypothesis of interest, Tables 2.2 and 2.3 report that the empirical size is very similar is not different from the $5 \%$ nominal one.

\begin{tabular}{ccccccc}
\hline \hline & \multicolumn{1}{c}{$\zeta_{1}$} & \multicolumn{3}{c}{$\zeta_{2}$} & & \\
$j$ & $s \geq 1$ & $s=2$ & $s \geq 1$ & $s=2$ & $\hat{\beta}_{0.5}$ & $\hat{\beta}_{0.75-0.25}$ \\
\hline 0 & 100 & 100 & 100 & 100 & 1.801 & 0.223 \\
1 & 4.61 & 99.96 & 4.76 & 99.85 & 0.998 & 0.190 \\
2 & 2.02 & 20.57 & 1.43 & 9.24 & 0.928 & 0.954 \\
\hline \hline
\end{tabular}

Table 2.3: Size and Power of Codependence Tests in a ARMA(1,1) 


\begin{tabular}{ccccccc}
\hline & $\zeta_{1}$ & \multicolumn{3}{c}{$\zeta_{2}$} \\
$j$ & $s \geq 1$ & $s=2$ & $s \geq 1$ & $s=2$ & $\hat{\beta}_{0.5}$ & $\hat{\beta}_{0.75-0.25}$ \\
\hline 0 & 5.7 & 100 & 5.07 & 100 & .999 & 0.029 \\
1 & 4.91 & 100 & 4.86 & 100 & .999 & 0.033 \\
2 & 5.06 & 100 & 4.96 & 100 & .998 & 0.038 \\
\hline \hline
\end{tabular}

Table 2.4: Size of Codependence Test Statistics in a VAR

\begin{tabular}{rrccccc}
\hline & $\zeta_{1}$ & \multicolumn{3}{c}{$\zeta_{2}$} & & \\
$j$ & $s \geq 1$ & $s=2$ & $s \geq 1$ & $s=2$ & $\hat{\beta}_{0.5}$ & $\hat{\beta}_{0.75-0.25}$ \\
\hline 0 & 99.91 & 100 & 99.91 & 100 & .591 & 0.053 \\
1 & 26.56 & 100 & 21.18 & 100 & .541 & 0.071 \\
2 & 9.77 & 100 & 6.83 & 100 & .514 & 0.102 \\
\hline \hline
\end{tabular}

Table 2.5: Power of Codependence Test Statistics in a VAR

We want to show how codependence tests behave in the presence of various forms of misspecification. Successively, we brichy (for more details see Beine and Hecq, 1999) analyze the case where the DGP is a VAR instead of a pure $\operatorname{MA}(q)$ one, the presence of GARCH errors and the omission of a cointegrated vector.

\section{The MA $(\infty)$ Case}

This case considers a data generating process admitting an infinite VMA representation resulting from the inversion of a $\operatorname{VAR}(1), y_{t}=\Phi_{1} y_{t-1}+\varepsilon_{t}$. We consider the following values for the coefficient matrices $\Phi_{1}$ under the null $\left(H_{0}\right)$ and the alternative $\left(H_{A}\right)$. The results are reported in Tables 2.4 and 2.5 .

$$
H_{0}: \Phi_{1}=\left(\begin{array}{ll}
.6 & .3 \\
.6 & .3
\end{array}\right), \quad H_{A}: \Phi_{1}=\left(\begin{array}{cc}
.5 & .1 \\
.4 & .5
\end{array}\right) .
$$

The main results are that under $H_{0}$ there are no size distortions nor bias but under the alternative the power is very low. This lack of model identification may lead to misleading interpretation as we will see in the empirical section. This should imply some effort for a good selection of the VARMA orders. Notice that under the alternative, the wrong pseudo-true common feature vectors appear stable along $j$. See Beine and Hecq (1999) for a discussion of this point.

\section{Omission of a Cointegrating Vector}

Now we consider non-stationary processes and we investigate the effect of the omission of a cointegrating vector on codependence tests. We generated two $I(1)$ variables with a cointegrating vector equals to $(1,-2)$. The bivariate error process follows a $\operatorname{MA}(2) \varepsilon_{t}=\omega_{t}+\Theta_{1} \omega_{t-1}+$ 


\begin{tabular}{|c|c|c|c|c|c|c|}
\hline & $\zeta_{1}$ & & $\zeta_{2}$ & & & \\
\hline$j$ & $s \geq 1$ & $s=2$ & $s \geq 1$ & $s=2$ & $\hat{\beta}_{0.5}$ & $\hat{\beta}_{0.75-0.25}$ \\
\hline 0 & 100 & 100 & 100 & 100 & .004 & 0.441 \\
\hline 1 & 9.60 & 100 & 4.13 & 100 & 2.004 & 0.175 \\
\hline 2 & 1.67 & 10.4 & 0.96 & 5.6 & 2.280 & 2.433 \\
\hline
\end{tabular}

Table 2.6: Power of Codependence Test in Omission of a Cointegrating Vector

\begin{tabular}{ccccccc}
\hline \hline & $\zeta_{1}$ & \multicolumn{5}{c}{$\zeta_{2}$} \\
$j$ & $s \geq 1$ & $s=2$ & $s \geq 1$ & $s=2$ & $\hat{\beta}_{0.5}$ & $\hat{\beta}_{0.75-0.25}$ \\
\hline 0 & 100 & 100 & 100 & 100 & 1.617 & 0.559 \\
1 & 21.24 & 100 & 19.8 & 100 & 1.005 & 0.165 \\
2 & 6.08 & 32.63 & 4.13 & 20.35 & 0.806 & 1.500 \\
\hline \hline
\end{tabular}

Tabie 2.7: Size of Codependence Tests in the Presence of GARCH

$\Theta_{2} \omega_{l-2, \text { where }}$

$$
\Theta_{1}=\left(\begin{array}{cc}
.6 & .2 \\
.4 & -.3
\end{array}\right), \Theta_{2}=\left(\begin{array}{cc}
.3 & -.6 \\
-.1 & -.5
\end{array}\right)
$$

We assume that we do not take into account this long run relationship between the variables in levels and carry out the codependence analysis on the first differences of these $I(1)$ variables. Table 2.6 gives the power of the test statistic in this situation. The power is very low. For instance $\zeta_{1}$ has a power of $9.66 \%$ against the full rank alternative while the same test without the presence of a cointegrating vector has a power of $100 \%$ (results not reported here). We obtain somewhat intuitively plausible results. Indeed, due to a variable omission, test statistics spuriously conclude in favor of a codependence relationship.

\section{Presence of GARCH Errors}

In order to analyze the impact of non normal disturbances on codependence test statistics, we assume that both variables are conditionally heteroscedastic. ${ }^{10}$ More precisely, we assume IGARCH $(1,1)$ processes with normal conditional distributions and parameters values commonly estimated on financial data like intercst rates, exchange rates, i.c. a small $\alpha$ and a large $\beta$, both parametcrs adding to one. The pairs $(\alpha, \beta)$ are respectively equal to $(0.25,0.75)$ and $(0.1,0.9)$ for the first and the second variable. The DGP is a VMA(2) with parameters matrices given in (2.64).

The results of Table 2.7 point out that the use of the test statistics leads to an overestimation of the lag order. This is a multivariate extension of the results obtained by Diebold (1986) and Hecq (1996) for univariate time scries. Secondly, GARCH crrors are responsible for huge size

\footnotetext{
${ }^{10}$ Other forms of non normal disturbances, like outliers for instance, will give the same qualitative results.
} 
distortions in the sense that traditional critical values are too low for correctly rejecting the null. ${ }^{11}$ Note that the presence of leptokurtic unconditional distribution does not alter the results of the pure MA $(q)$ case. For instance Beine and Hecq (1999) have also considered a Student's t. non conditional distribution, and without any ARCH processes, there are no size or power distortions.

\subsection{Applications in Common Features}

Let us first briefly review a number of papers about cyclical features (maybe along with cointegration) as well as a review of the applications they consider.

References applying serial correlation common features techniques are Engle and Kozicki (1993) for real gross domestic products, Vahid and Engle (1993), Jobert (1995) for the consumption/income relationship for respectively US and US and France, Issler and Vahid (1998), Gallo and Kempf (1996a,b), Mills (1999) on key macroeconomic aggregates such as consumption, income and investment, Engle and Issler (1995), Caporale (1997) and Gervaz (1997) on respectively US, UK and Belgian sectoral production data, Romijm (1996) and Hock and Romijn (1996) for Dutch construction industry data, Variyam (1996) for US agricultural prices, Lucke (1994) for 21 West German macro data, Funke and Hall (1995) for the relationships between UK and German output and unemployment, Bai and Hall (1995) for quarterly European output, Parker (1997) for total payroll employment among Florida's cities, Beine, Candelon and Hecq (2000), Mills and Holmes (1999) and Parnisari (1999) for European convergence using industrial production or cconomic indicators, Pain and Thomas (1997) for real interest rates, Carlino and Sill (1998) for regional US per capita income, Mills (1998) for the relationships between equity, dividend index and interest rates, Cubadda, Savio and Zelli (1999) for sectoral Italian output data, Witt, Clarke and Fielding (1998) on regional crime rates in England.

Applications of codependence in VMA can be found in Gouriéroux and Peaucelle (1989, 1993) for relative PPP, in Beine and Hecq $(1997,1998)$ for bivariate real convergence among European countries in Beine and Hecq (1999) and in Candelon and Hecq (2000) for Okun's law.

Applications on non-synchronous common cyclical features and codependence in VAR include Rubin and Thygessen (1996) for convergence among European industrial production, Kugler and Schwendener (1993) and Kugler and Neusser (1993) for international real interest rates, Vahid and Engle (1997), Cubadda (2000) for the relationship between the consumption of durable and non-durable goods and disposable income, Sill (1997) for US regional employment data and Cubadda (1999) for consumption and income using Italian data.

Examples of applications on SCM are Tsay (1993) for multivariate GDP analysis: Tiao and Tsay $(1985,1989)$ for monthly flour prices in three US cities and Tiao, Tsay and Wang (1994)

\footnotetext{
${ }^{11}$ Moreover, Beine and Hecq (1999), Hecq (1995) have shown that size distorsions increase with the sample size. This is due to the fact that GARCH processes need time to spread out, especially in the case of a large value for $\beta$.
} 
on interest ratcs.

Because the next chapters treat common cyclical features in cointegrated $\operatorname{VAR}(p)$ models we focus in this section on codependence and SCMs through an analysis of convergence within EC economies, Okun's law and comovements between commodity prices.

\subsubsection{Convergence within EC Economies}

The first illustration we consider is the analysis of business cycle comovenents. Engle and Kozicki have already studied this issue in their introductory example. Using quarterly real gross national product growth rates, they find inter alia, that one could not reject the SCCF hypothesis between US and Canada. The hypothesis is rejected for the pair US/UK. In this section we first test for codependence and then for scalar components on monthly composite leading indicators (trend restored) for seven European countries, that is Germany, Belgium, France, United-Kingdom, Italy, the Netherlands and Denmark. These data come from the OECD database and span from 1979:m3 to 1994:m12.

We are going to test for bivariate common cyclical features between the growth rates of each country against Germany as a benchmark. We cannot reject the unit root hypothesis for variables in levels for cach country using Dickey Fuller test statistics and none of the pairs is cointegrated using either Engle Granger as well as Johansen's ML procedure (see Beine and Hecq 1998 for details). Before we carry out the analysis, an important hidden framework for those data is the way they have been built. We may imagine that the seasonal adjustment destroyed a part of the relevant information and has created some MA components (see Chapter 7). Nevertheless we take these data as given in this illustrative application. A second important remark is that the analysis would be more informative if it was carried out in a multivariate setting instead of a bivariate one (sce Chapter 8 ). But, we wanted to avoid the occurrence of cointegrating vectors in this application (see Chapter 3). Also the notion of convergence analyzed via codependence is similar to cointegration for $\mathrm{I}(1)$ times series, that is, a picture of a realized process and not a movement toward a direction per se. As a consequence, we divide the whole period of existence of the European Monetary System in two parts. The first subperiod ranges from the creation of the EMS (March 1979) to late 1986. This period is characterized by frequent central rates realignments, even if after 1983 coordination of economic policies was quite reinforced. The sccond subperiod starts in the beginning of 1987. This period, known as the "New EMS", is better characterized, at least until mid-1992, by a great stability of parities and a stronger credibility of national commitments. Table 2.8 summarizes the codependence results on monthly composite leading indicators indexes while further results for interest rates and unemployment. rates can be found in Beine and Hecq (1997, 1998). After analyzing the significance of the autocovariance matrices, the lag order of the VMA is fixed to 6 . Consequently Table 2.8 reports the codependence order written as a $\operatorname{SCM}(0,6-b)$, the value $6-b$ being the first significant lag.

It emerges that with the exception of the Netherlands during the global period, a codependence relationship is found. The degrees of codependence ranges from 5 to 2. For Belgium, 


\begin{tabular}{l|cc|cc|cc|}
\hline \hline Countries & $79 m 3-94 m .12$ & vector & $79 m 3-86 m 12$ & vector & $87 m 1-94 m 12$ & vector \\
\hline Ger/Au & $S C M(0,3)$ & $(1,-.72)$ & $S C M(0,2)$ & $(1,-.71)$ & $S C M(0,4)$ & $(1,-.19)$ \\
Ger/Be & $S C M(0,2)$ & $(1,-1.10)$ & $S C M(0,2)$ & $(1,-.93)$ & $S C M(0,1)$ & $(1,-1.73)$ \\
Ger/Fr & $S C M(0,2)$ & $(1,-1.35)$ & $S C M(0,2)$ & $(1,-1.17)$ & $S C M(0,1)$ & $(1,-1.82)$ \\
Ger/UK & $S C M(0,2)$ & $(1,-6.03)$ & $S C M(0,2)$ & $(1,-2.93)$ & $S C M(0,3)$ & $(1, .07)$ \\
Ger/Ita & $S C M(0,4)$ & $(1,-.48)$ & $S C M(0,2)$ & $(1,-.59)$ & $S C M(0,2)$ & $(1,-2.16)$ \\
Ger/Nl & $S C M(0,6)$ & - & $S C M(0,2)$ & $(1,-1.13)$ & $S C M(0,1)$ & $(1,-2.13)$ \\
Ger/Dk & $S C M(0,2)$ & $(1,-.83)$ & $S C M(0,1)$ & $(1,-.81)$ & $S C M(0,2)$ & $(1,-0.82)$ \\
\hline \hline
\end{tabular}

Table 2.8: Codependence Analysis for the Growth of Composite Leading Indicators

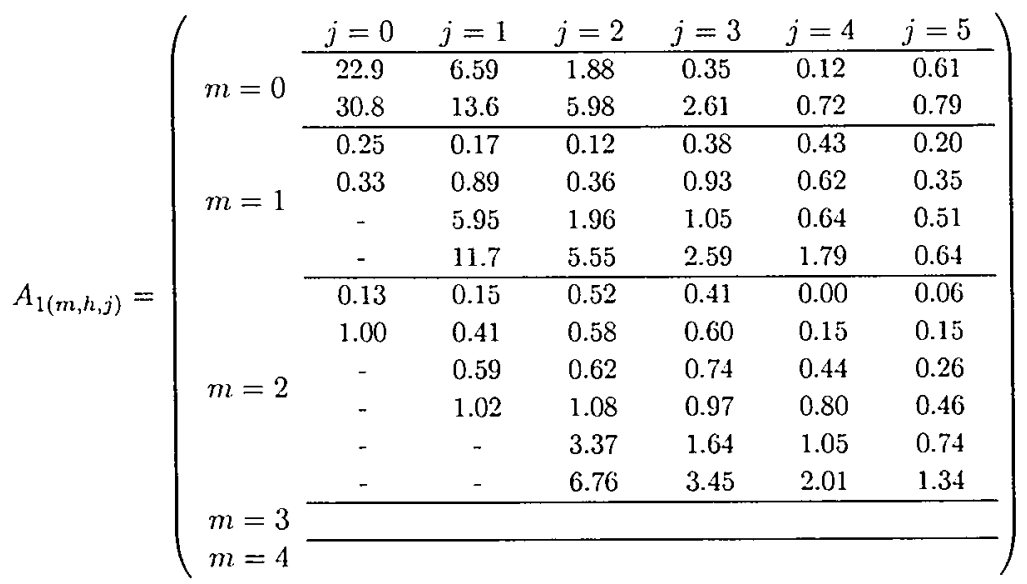

Table 2.9: Values of Normalized SCM test statistics for Belgium/Germany

France, Austria and Denmark, the values of the codependence vectors for the global period and the first subperiod seem quite close to the $(1,-1)$ vector, indicating well-market synchronization with Germany. With the exception of Denmark, the results relative to the second subperiod indicate a small divergence. Note however that for Belgium and France, the persistence of the shocks is lower in the last subperiod. This is also the case for the Netherlands. The evidence of relative divergence is somewhat clearer for Austria, though the sign of the components in the codependence vector remains unchanged. The results for the United Kingdom scem atypical. First, the value of the vector is quite different for the global period from the ones of the other countries. Secondly, as far as convergence is concerned, the UK scems to have diverged: the value of the vector in the last period has a sign in a direction indicating non synchronization.

Exception made of the UK/Germany relationship, the outcomes of the tests for SCM are fairly similar. So to save space, we show the details for the relationship Belgium/Germany only. Table 2.9 reports the matrix $A_{1(m, h, j)}$ (tests normalized by critical values) in order to select a parsimonious model. 


\begin{tabular}{lcccccc}
\hline & $j=0$ & $j=1$ & $j=2$ & $j=3$ & $j=4$ & $j=5$ \\
\hline$m=0$ & 0 & 0 & 0 & 1 & 2 & 2 \\
$m=1$ & 2 & 2 & 2 & 2 & 3 & 4 \\
$m=2$ & 2 & 4 & 4 & 4 & 4 & 5 \\
$m=3$ & 2 & 4 & 6 & 6 & 6 & 6 \\
$m=4$ & 2 & 4 & 6 & 8 & 8 & 8 \\
\hline
\end{tabular}

Table 2.10: Number of Zeros for Begium/Germany

\begin{tabular}{ccccccc}
\hline & $j=0$ & $j=1$ & $j=2$ & $j=3$ & $j=4$ & $j=5$ \\
\hline$m=0$ & 0 & 0 & 0 & 1 & 1 & 0 \\
$m=1$ & 1 & 2 & 2 & 2 & 3 & 4 \\
$m=2$ & 2 & 3 & 4 & 4 & 4 & 5 \\
$m=3$ & 2 & 4 & 4 & 5 & 5 & 5 \\
$m=4$ & 2 & 4 & 5 & 5 & 6 & 6 \\
\hline
\end{tabular}

Table 2.11: Number of Zeros for UK/Germany

Table 2.10 presents the number of zero eigenvalues of $A_{1(m, h, j)}$. We identify an VAR(1) model because it is the first time we obtain two zero eigenvalues. Going from $(m, j)=(1,0)$ to ( $m, j)=(0,0)$, we reject the scrial correlation common feature hypothesis. It scems that there is no common features in this case and the codependence degree of order 1 that one sees at the position $(m, j)=(0,3)$, is due to a decrease of power of codependence test statistic when the data generating process is in fact a VAR as our simulations have shown. The same results are detected for the other pairs, i.e. an identification of an unconstrained VAR(1) which does not, have serial correlation common features. The test statistic for the null hypothesis of $\operatorname{SCM}(0,0)$ is equal to 88.1 for $\mathrm{Be} / \mathrm{Ge}, 110$ for It/Ge, 96.7 for Fr/Ge, 114 for $\mathrm{Dk} / \mathrm{Ge}$ and 65.7 for Nl/Ge. These statistics follow a $\chi_{(1)}^{2}$ distribution under the null.

The UK/Germany relationship is different. The number of zero cigenvalues is reported in Table 2.11. There are two zero eigenvalues for both a VAR(2) and a VARMA $(1,1)$ model. We chose to start with the VARMA(1,1). The common feature restrictions are not rejected and we find one scalar component mociel $\operatorname{SCM}(1,0)$.

\subsubsection{Once upon a Time Stationary Price Levels}

In his seminal 1933 paper, Labrousse studies the empirical relationship between agricultural underproduction crisis and demographic crisis for the Old Regime. Driven by climatic or other exogenous factors like wars or speculative behavior, a huge increase of the whole primary commodity prices gave rise to famine, which dramatically allowed to restore the equilibrium at a lower price level. Labrousse (1933) and Appleby (1979) among others analyzed, what is called subsistence crisis using graphical analyses and bivariate static correlations between grain prices and demographic variables. 


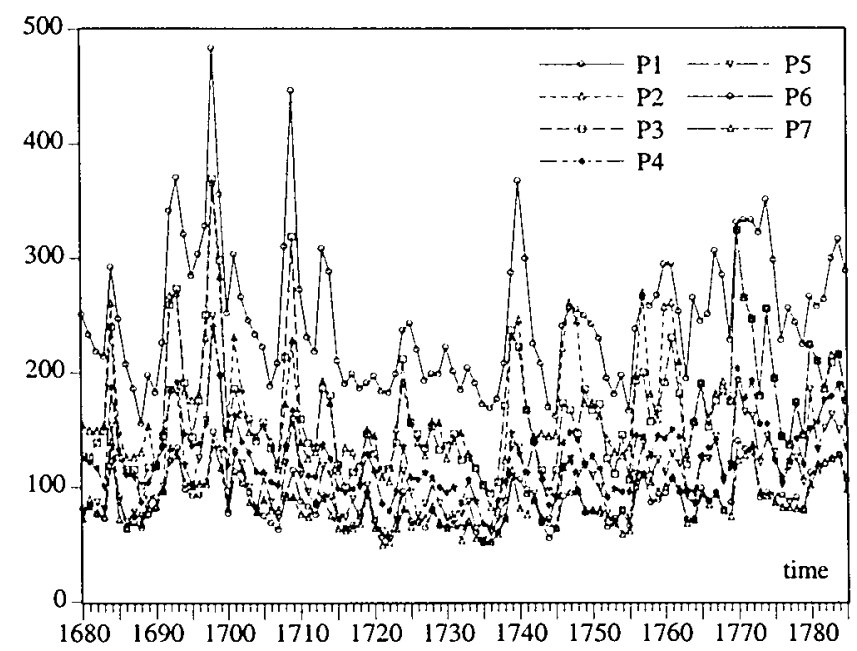

Figure 2.1: Basic Commodity Price Levels

We choose a multivariate time series approach in other to analyze the number of forces driving general price levels. Our data set consists in annual prices of six cereals and one leguminous plant for the period 1680 to 1785 . The data which have been observed on the Roermond market in the Spanish Low Countries are respectively the price levels of wheat, pea, rye, barley, buckwheat, spelt wheat and oats (series are from Ruwet, 1966). These series are respectively

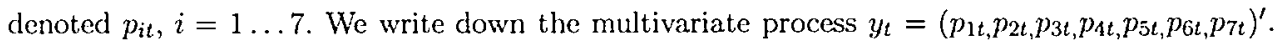
Note that we classify prices from wheat to oats along a nutritional schedule determined by the average of the prices on the whole period. The means of the variables are the following $\left(\bar{p}_{1,} \bar{p}_{2,} \bar{p}_{3}, \bar{p}_{4}, \bar{p}_{5}, \bar{p}_{6}, \bar{p}_{7}\right)=(248.92,173.04,166.69,126.91,108.75,87.55,86.75)$. In Figure 2.1 we can guess the general evolution of the data.

Graphically, the time series appear stationary in level. This is confirmed by Dickey-Fuller unit root tests (see Geerkens and Hecq, 1994). Such a property for price levels is quite different from the situation we know nowadays where prices are often $\mathrm{I}(1)$ or $\mathrm{I}(2)$.

We next try to identify relationships within the seven basic commodity prices. Using a bivariate VMA analysis only, Geerkens and Hecq (1994) were unable to reject the hypothesis that linear combinations reduce to a VMA(1). Because we suspect some stronger links, and especially some immediate shifts in the demanded quantities from better cereals to less nutritional and cheaper ones, we are seeking for $\operatorname{SCM}(0,0)$. In the first step of the analysis, it is very difficult to select a multivariate model for this data set. Indeed, the high number of variables may cause size 


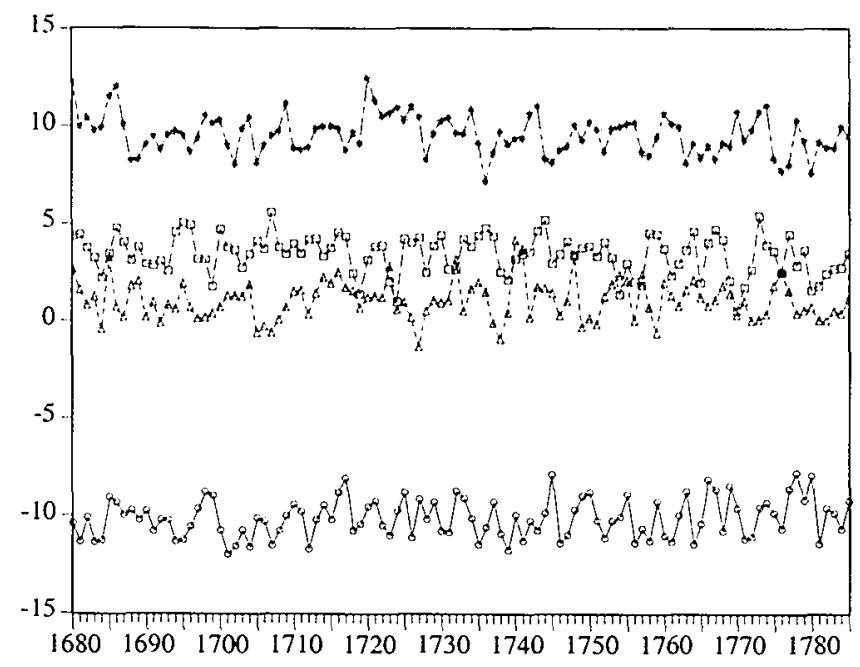

Figure 2.2: The Four $\operatorname{SCM}(0,0)$ Relationships for Prices

distortions and power problems. We have therefore just tricd to select a model by considering significant eigenvalues according to $\zeta_{1}$.

We select a VARMA $(1,1)$ model for the multivariate process while a VARMA $(3,0)$ or a VARMA (2.1) were also possible choices. We nevertheless prefer to take an VARMA $(1,1)$ not only because this is the more parsimonious model but also because we have seen that a exogenous common factor model can be rewritten in an endogenous $\operatorname{VARMA}(p, p)$ onc. We cannot reject the hypothesis of four $\operatorname{SCM}(0,0)$ : there exist four combinations which result in white noise processes.

\subsubsection{Stability of Activity-Unemployment Relationship in a Codependent System}

In the last empirical analysis of this chapter we consider for several OECD countries, the possible presence of a codependence relationship between the unemployment rate and the growth rate of economic activity. The strong persistence of the unemployment rate since the mid 70 's has led European policy makers and economists to seriously reconsider the link between economic activity and unemployment. In the empirical literature, most of the studies have considered this relationship within static linear models (see Okun, 1962, Smith, 1975 and Mossa 1997 inter alia) or VAR (c.g. Blanchard and Quah, 1989, Evans, 1989 and Henin and Jobert. 1993). 
Nevertheless, only few studies have simultaneously coped with two major weaknesses, namely the lack of dynamics within the joint (at least) bivariate process and the stability through time of this relationship. The codependence framework allows us to test for the shortest reaction time necessary for the activity-unemployment system to reach an equilibrium after a shock. This provides another useful tool to assess the efficiency of short-run stabilization mechanisms as well as economic policies. We add to this approach a recursive estimation of the codependence degree (and vector) in order to evaluate the potential deformation in output-unemployment short-run comovements.

Therefore, we conduct a codependence analysis applied to the changes in the unemployment rate and the growth rate of GNP for five OECD economies, that is Germany, USA, The Netherlands, Japan and France. ${ }^{12}$ As the first oil shock is likely to have altered the interaction between activity and unemployment, we conduct the analysis with scasonally adjusted quarterly data ranging from $74 \mathrm{Q} 1$ to $93 \mathrm{Q} 4$. We cannot, using ADF tests, reject the unit root hypothesis for all variables in levels and the level of output and the unemployment rate are not cointegrated for each country. ${ }^{13}$ Consequently the analysis can be carried out for first differences without the omission of useful information. Notice that Lagrange multiplier tests do not reject the absence of GARCH errors. As a consequence, we face a context appropriate to a codependence analysis in first differences and the problem can be rewritten as a pseudo-structural equation system:

$$
\begin{aligned}
\Delta U_{t} & =\delta_{1} \Delta y_{t}+u_{t} \\
u_{t} & =\delta^{\prime} \mu+\delta^{\prime} \varepsilon_{t}+\delta^{\prime} \Theta_{1} \varepsilon_{t-1}+\ldots+\delta^{\prime} \Theta_{q} \varepsilon_{t-q} .
\end{aligned}
$$

with $\delta=\left(1,-\delta_{1}\right)^{\prime} . U_{t}$ is the unemployment rate and $y_{t}=\ln \left(Y_{t}\right)$ is the $\log$ of output. It emerges that two indicators summarize some important features of the system: $\delta_{1}$ and $q-b$. Normalized on $\Delta U_{t}$, the codependence vector gives the "most stable elasticities", and provides an indication on the instantaneous reaction of the system after a shock as the famous Okun's coefficient. $q-b$ represents an indicator of "persistence" 14 of a shock affecting this dynamic system. These indicators are of major importance to gauge the effect of a short-run economic policy. The economy of a country with efficient short-run stabilization mechanisms and/or employment policies should exhibit a high negative short-run output-employment elasticity $\delta_{1}$ and a low delay of adjustment necessary to absorb exogenous shocks.

Our results illustrate the idea that the strength of the relationship is not reflected only by the value of the estimated elasticity (as in an OLS regression) but also by the estimated degree

\footnotetext{
${ }^{12}$ In order to save space we choose to illustrate our analysis by five countries that present different behaviors. A study spaning 11 OECD countries is proposed in Candelon (1998), Beine and Hecq (1999) and Candelon and Hecq (2000).

${ }^{13}$ Of course that does not indicate that these two variables are not cointegrated for a different sample. These results also depend upon the information set and do not imply that the variables are not cointegrated if we consider a larger number of variables. This is the case when estimating long-run wage equations for instance (see inter alia Hall, 1986, 1989; Hecq and Mahy, 1997).

${ }^{14}$ The term of MA-persistence would be better as we are in a stationary framework.
} 
of codependence and the remaining persistence of the shocks. While the smallest estimated clasticity is found in the case of Japan, it is worth noting that this vector reduces the process to a white noise. For Germany, no codependence relationship is found.

These results are based on a full sample analysis and hence assume constancy of the parameters. In order to evaluate the temporal stability of the rclationship (2.8), wo complete the full sample analysis by a recursive study. We first start with a sample of $t_{1} \leq T$ initial observations in order to obtain a first estimation. For this sample, we test the degree $b$ of codependence (or the value $q-b$ ) as well as the associated codependence vector. We use a GMM approach ${ }^{15}$ (in other words instrumental variables) to estimate the codependence vector and Hansen (1982)'s test for over-identifying restrictions for the codependence order. We expand one by one the sample size until the end of the sample $T$ and we calculate for each new sample the same statistics. We obtain $q \times\left(T-t_{1}+1\right)$ recursive coefficients and test statistics that can be represent graphically. In figures, codependence order refers to $q-b$. We summarize information by retaining, for cach sample size, the minimum of the $q-b$ order for which we do not reject the null, as well as the associated codependence vector. For recursive analyses, the first window, i.e. $t_{1}$, is set to 20 quarters. This choice allows to have sufficient recursive points in order to analyze deformations of activity-employment relationship. The basic model is chosen with $q=0$ lags which seems enough to whiten residuals.

\section{Full sample}

It may first be interesting to analyze the value of $\delta_{1}$ for the whole period, i.e. the last point on the graphs. Highest coefficients are for France $(-0.255)$, Germany $(-0.4)$, the Netherlands $(-0.45)$ and the United-States $(-0.475)$. It is possible to find for each of these economies, institutional or political factors that justify this classification. Kaufman (1988) cxplains that unemployment is more responsive in the United-States, because of the lack of job security pension and restrictions on layoffs. Employers are thus inhibited from reducing their workforce during recessions and hiring more people during expansions. Another reason is also proposed by Layard, Nickel and Jackman (1991). For these authors, countries whose wage negotiations are decentralized at the firm level cxhibit less rigidities: a shock in activity would modify wages and so labor demand immediately and fully. For Japan, the coefficient is negative but close to 0 , indicating a tight relation between activity and unemployment. This weak link between employment and real activity fluctuation could find its sources in cultural habits. For Europe, the relation is also tighter than in the North-American countries, mainly because of strict laws on firing and powerful unions. Those results are in accordance with those found by Moosa (1997).

\footnotetext{
${ }^{15}$ Together with Chapter 9 on panel data, this is the only case where we use a GMM approach instead of a canonical correlation framework. We use GMM to test for common features not only because it simplifies the computation but also because we have at most one codependence vector. Moreover, economic theory proposes to choose unemployment rate as the dependant variable. Finally, $\Delta y_{1}$ being more correlated with the set of instruments, GMM test statistics for the direction $\Delta U_{t}=f(\Delta y t)$ is more powerfull than for the opposite direction and also than a canonical correlation test statistic (see Candelon and Hecq, 1998).
} 
The second tool provided by our methodology is the delay of adjustment given by the codependence order. Its analysis for the whole period indicates two main types of countries: Those, in which the speed of adjustment is high (say less than 2 quarters), and those, for which adjustment speed is very low (more than 4 quarters for a full adjustment). Germany belong to this latter category. For these latter countries, it can be concluded that a stabilization mechanisms or employment policies take more than one year to have a full effect.

The Recursive Analysis

The previous partial conclusions for the whole period may be influenced by recent evolutions and do not give enough weights to periods of instability in the last 20 years. The recursive analysis provides some graphical clues for analyzing it. It appears clearly that crises (oil crisis in mid 70's or the 80 's business cycle) have increased the delay of adjustment. For most countries (the United-States, France and the Netherlands), the oil shock has slow down the adjustment mechanisms, phenomenon which reached a top level of five lags. This result is generally associated with an increase in $\delta_{1}$ (in absolute value). Economic grounds of such behaviors are linked to the rise of uncertainty induced by a severe recession phase of a cycle, inducing a higher wages rigidity, and consequently a disconnection between activity variations and change in unemployment. On the other side, some countrics do not seem to be particularly affected by these crises (Japan, Germany). In those countries, unemployment and output seem to be more affected by specific shocks rather than common ones, caused by crisis. The best example is provided by Germany, where both indicators are stable before the reunification and indicate a sharp increase in degree of adjustment afterwards.

In the recent past, the Netherlands exhibits simultaneously an increase of $\delta_{1}$ and of the speed of adjustment so that a quick and efficient reduction in unemployment after a short-run policy could be expected. The results for Japan do not indicate substantial modification in the joint activity/unemployment behavior. for the last ten years. The conclusion is less clear for the United-States and Germany where the adjustment speed decreases, indicating a possible delay between the shock and the full reaction of the system. In such countries, short-run employment policies appear inefficient and have probably to be replaced with structural policies. 

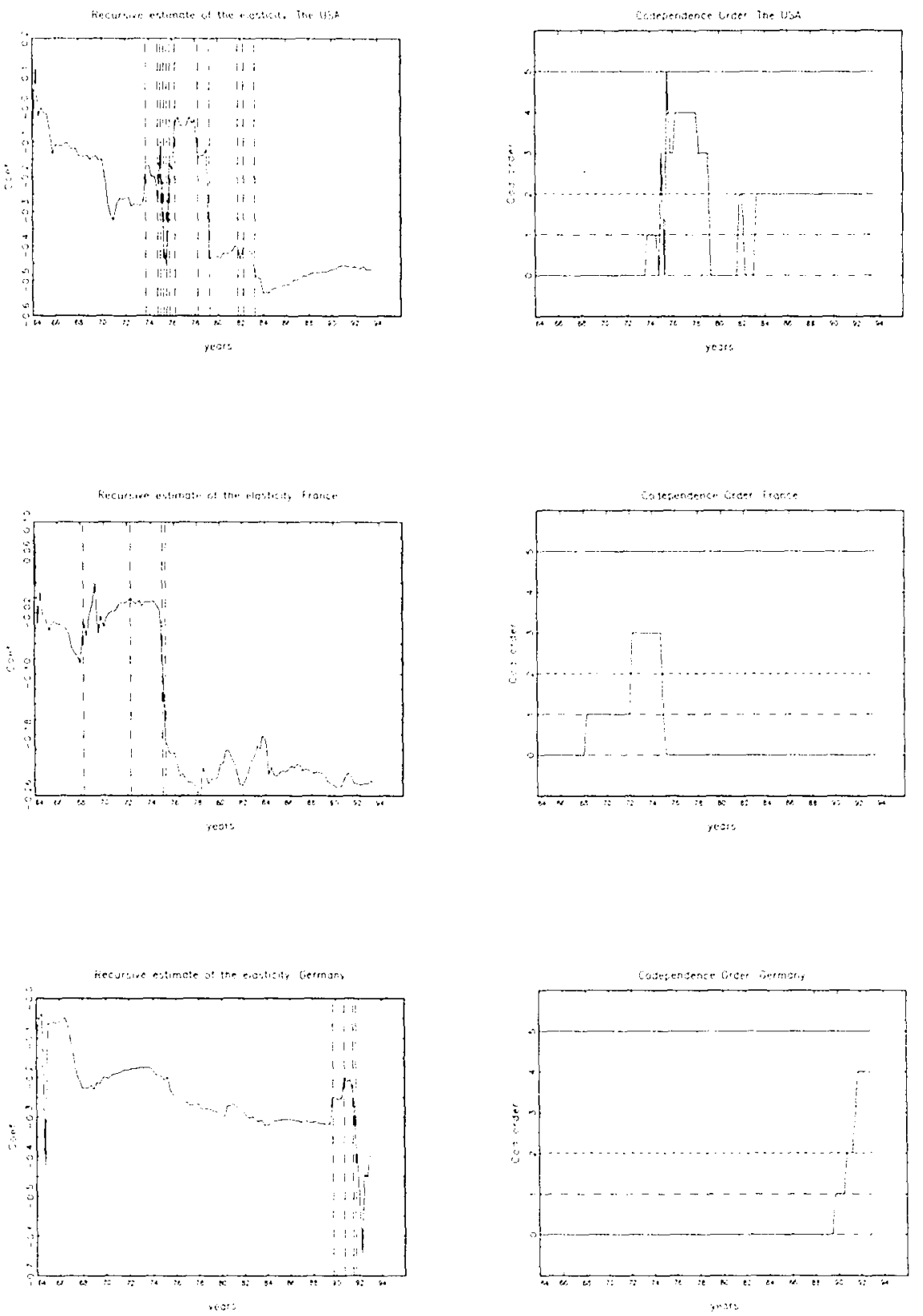

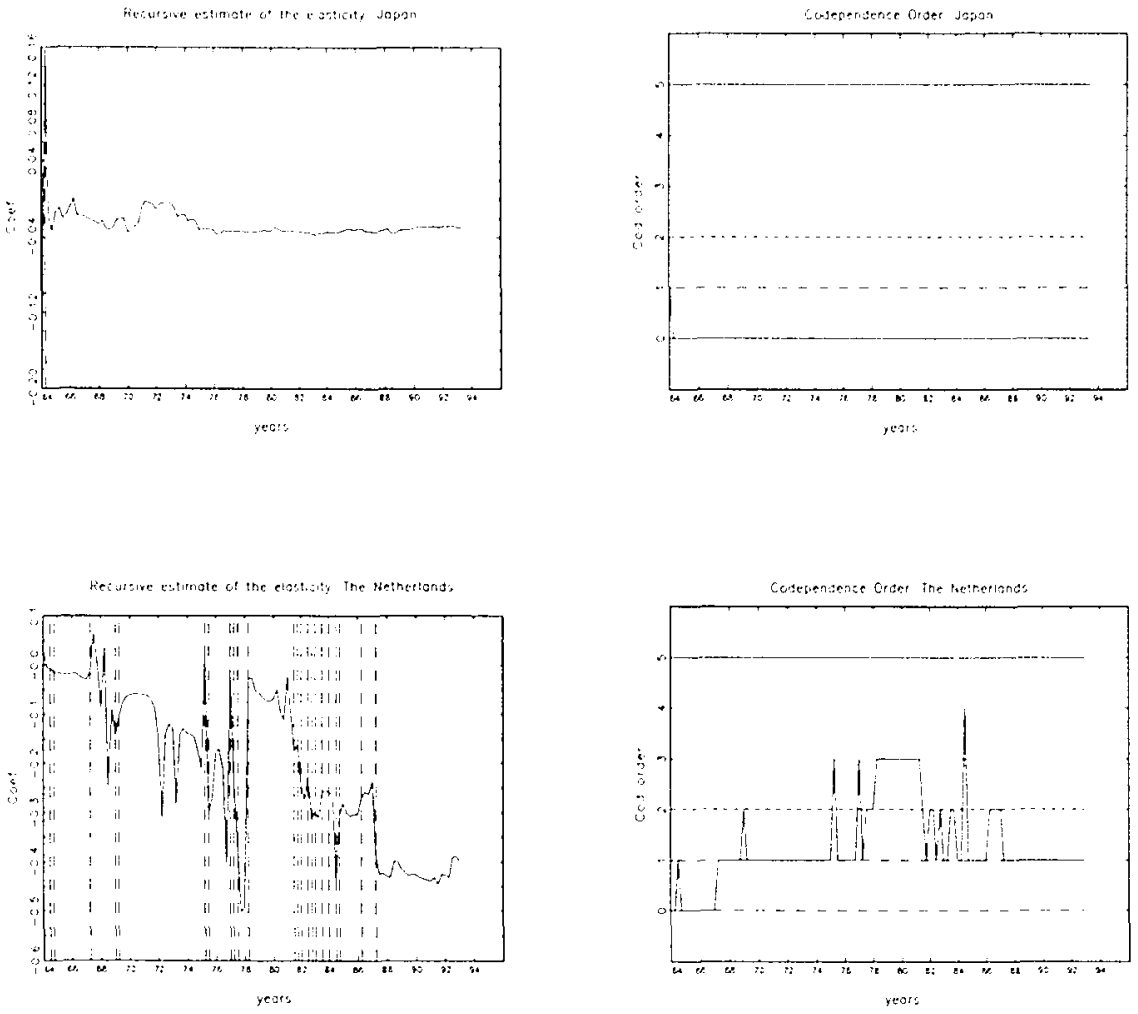

\subsection{Conclusion}

In this chapter we have reviewed different specifications aimed at analyzing short-run comovements between stationary time series. We have shown how these are nested within the scalar component model structure of Tiao and Tsay. We have also stressed the different aspects of the SCCF modeling. We emphasize the importance of the identification of the full model representing the data for two reasons. Firstly, the same canonical analysis can be performed as a reduction of two different models. Secondly, the choice of a wrong initial model seriously affects the power of co-feature test statistics and thus may erroneously lead to conclude of the existence of common features. The simulations also stressed the importance of Gaussian assumption for the size of tests statistics, as well as the need for taking into account the presence of cointegrating vectors. We illustrated these points both by Monte Carlo experiments and by empirical economic analyses. The remaining chapters of this thesis focus on relationships between cofeature and 
cointegration.

However, other types of common features than the cyclical ones have been considered in the literature. We end this subjective survey by summarizing some of the promising forms. No need to say, we do not, review papers about common stochastic trends or common stochastic seasonality, topies whercon there exists a huge number of theoretical and applied articles, survey and books. Instead, we focus on less frequently applied modeling such as common volatility, common breaks or common deterministic seasonality.

Engle and Hyllcberg (1996) propose an approach to testing for common deterministic seasonality, i.e. sines and cosines functions or equivalently seasonal dummies. They applied this framework to quarterly unemployment series and find that. Australia, Canada, Japan and the USA share common deterministic seasonal features. Sørensen (1996) has used this approach and find common deterministic seasonal features among wage, unemployment and exports quarterly series for a number of small European economies. Franses and Kunst (1999) generalize the common deterministic seasonality to pancl data. They apply this framework to 16 quarterly industrial production inclexes.

On high frequency data or even on monthly time series such as interest rates, stock prices or money stock, one observes volatility clustering. These phenomena can for instance be apprehended via ARCH type processes. Multivariate GARCH models however often contain too many parametcrs. But if one observes that assets with similar risk characteristics have similar volatility processes, a common ARCH modeling can solve this increase of parameters by testing and imposing a common volatile factor. Engle and Kozicki (1993) have proposed a LM strategy to cope with this issue and apply it on monthly interest rates for different maturities. Engle and Susmel (1993) have found some common volatility in international stock markets. Arshanapalli, Doukas and Lang (1997) test for common ARCH among security prices for US, Europe and the Pacific Rim capital markets. Multivariate factor modeling is also developed inter alia in Palm and Urbain (1995).

Anderson and Vahid (1998) investigate situations in which a multivariate nonlinear system contains common nonlinear components. The authors find some common nonlinear factors, such as logistic smooth transition autoregressive models (LSTAR), for US and Canadian output as well as within consumption, income and investment. This approach is applied by Harvey and Mills (1999) to income, consumption and investment for the G7 countries. A similar perspective is used by Paap and Franses (1999) to test for a common nonlinear cycle between US and Canadian unemployment variables and by Boswijk and Franses (1996) for common persistence within Belgian, German and UK industrial production. Gouriéroux and Jasiak (1999) introduce. the notion of copersistence in nonlinear processes.

Hendry (1996) and Chapter 9 in Clements and Hendry (1999) consider the removal of deterministic shifts in systems of equation using linear combinations of variables, a property they call co-breaking. They illustrate their approach on the moncy demand behavior. 


\subsection{Appendix: Identifiability and Exchangeability}

In order to explain the suitability of the codependence approach, Gouriéroux et al. (1991) argue that the restriction to vector moving average processes instead of VARMA models avoids the difficulty of exchangeable structures, i.e. when two different models give rise to the same covariance structure for the process $y_{\ell}$. In this Appendix, we recall some key concepts such as identifiability, exchangeability for $\operatorname{VARMA}(p, q)$ models and their relationships with scalar component models. To avoid confusion we use the concept of observational equivalence of models, a notion which nests identifiability and exchangeability issues. Indeed, we will not treat the general identification issue in econometrics. We also avoid the term identification because it is also used to determine a suitable order for univariate or multivariate $\operatorname{ARMA}(p, q)$ models. We prefer the expression of selection order in this latter problem.

Definition 2.14 If two models always generate identical outcomes, they are said to be observationally equivalent and data alone cannot distinguish between them. A sufficient condition is that all of their parameters are unidentifiable (identifiability issue), but this is not necessary and identified models can be observationally equivalent (exchangeability issue). Let $D_{x}\left(x_{t} \mid \theta\right)$ be the distribution generating a given set of observations $x_{t}$. If,$D_{x}\left(x_{t} \mid \theta\right)$ is unchanged under one-one transformations of the parameter from $\theta$ to $\psi=f(\theta)$ then $D_{x}\left(x_{t} \mid \theta\right)$ for $\theta \in \Theta$ and $D_{x}\left(x_{t} \mid \psi\right)$ for $\psi \in \Psi$ are observationally equivalent.

In multivariate time series modeling, let us consider the matrix operators $\Phi^{*}(L)$ and $\Theta^{*}(L)$ which are related to $\Phi(L)$ and $\Theta(L)$ by a common left matrix factor $C(L), \Phi^{*}(L)=C(L) \Phi(L)$ and $\Theta^{*}(L)=$ $C(L) \Theta(L)$. Consequently, if $C(L)$ exists, it is possible for two VARMA $(p, q)$ model representations, $\Phi(L) y_{t}=\Theta(L) \varepsilon_{t}$ and $\Phi^{*}(L) y_{t}=\Theta^{*}(L) \varepsilon_{t}$, to give rise the same coefficients in the infinite MA representation $y_{t}=\Psi(L) \varepsilon_{l}$, with $\Psi(L)=\Phi(L)^{-1} \Theta(L)=\Phi^{*}(L)^{-1} \Theta^{*}(L)$. They are observationally equivalent.

\subsubsection{Identifiability}

For a univariate ARMA model, the invertibility condition ensures the unique representation. The problem of identifiability for VARMA models is more complicated and has been studied by Hannan (1969, 1970). The books by Lütkepohl (1991) and especially Reinsel (1993), Hannan and Deistler (1988) give some insights on these topics.

Consider a $n$ dimensional $\operatorname{VARMA}(p, q)$

$$
\begin{aligned}
y_{t} & =\sum_{j=1}^{p} \Phi_{j} y_{t-j}+\varepsilon_{t}-\sum_{j=1}^{q} \Theta_{j} \varepsilon_{t-j} \\
\Phi(L) y_{t} & =\Theta(L) \varepsilon_{t} .
\end{aligned}
$$

Left-multiplying both sides by an arbitrary non singular matrix or by a matrix polynomial in $L$ yields a class of equivalent models that have identical covariance matrix structures. Therefore, without further restrictions, the model is not identifiable in the sense that we cannot uniquely determine the values of $p$ and 
$q$ and the coefficient matrices $\Phi_{j}$ and $\Theta_{j}$ from the covariance matrix of $y_{t}$. When the left multiplication increases the lag orders, we are confronted with the common factor problem (COMFAC), widely analyzed in univariate ARMA models. This is a rather trivial (conceptually) lack of identification. Less trivial cases are those for which the matrix operators are left coprime.

Treating the identifiability issue, Hannan $(1969,1970)$ began by supposing that the zeros of $|\Phi(L)|$ are outside the unit circle and the zeros of $|\Theta(L)|$ are on or outside the unit circle. Afterwards, Hannan considers the class of stationary processes satisfying the following sufficient conditions :

- The only common left divisors of $\Phi(L)$ and $\Theta(L)$ are unimodular ones, i.c., if $\Phi(L)=C(L) \Phi_{1}(L)$ and $\Theta(L)=C(L) \Theta_{\mathrm{I}}(L)$, then the determinant $|C(L)|$ is a constant. If this propriety holds, the operators $\Phi(L)$ and $\Theta(L)$ are said left coprime and the unique representation $\Psi(L)=\Phi(L)^{-1} \Theta(L)$ is said irreductible.

- from each class of equivalent models, choose one with minimal order $q$. Among these choose one with minimal order $p$. The resulting representation will be unique if the matrix $\left[\Phi_{p}: \Theta_{q}\right]$ is full rank $n$.

These two condition taken together ensure that $C(L)$ is a matrix of constants and in particularly an identity matrix since the MA and AR operators have leading coefficient matrices equal to the identity matrix. Indeed, because of the full rank hypothesis, the polynomial order will increase if the common $C(L)$ matrix relies on $L$.

In order to illustrate Hannan's conditions (see Reinsel $1993 \mathrm{p} 38$ ), we may consider a $n$-dimensional $\operatorname{ARMA}(1,1)\left(1-\Phi_{1} L\right) y_{t}=\left(1-\Theta_{1} L\right) \varepsilon_{t}$, and suppose $\operatorname{rank}\left[\Phi_{1}: \Theta_{1}\right]=n-s<n$. To keep our previous notations, there exists $s \times n$ matrix $\delta^{\prime}$ such that $\delta^{\prime}\left[\Phi_{1}: \Theta_{1}\right]=0$. So for any arbitrary $n \times s$ matrix $A$, we have

$$
\begin{aligned}
\left(I-\left(A \delta^{\prime}\right) L\right)\left(1-\Phi_{1} L\right) y_{\ell} & =\left(I-\left(A \delta^{\prime}\right) L\right)\left(1-\Theta_{1} L\right) \varepsilon_{t} \\
\left(I-\left(A \delta^{\prime}+\Phi_{1}\right) L\right) y_{\ell} & =\left(I-\left(A \delta^{\prime}+\Theta_{1}\right) L\right) \varepsilon_{t},
\end{aligned}
$$

which is of VARMA $(1,1)$ form $\left(1-\Phi_{1}^{*} L\right) y_{t}=\left(1-\Theta_{1}^{*} L\right) \varepsilon_{t}$, where $\Phi_{1}^{*}=A \delta^{\prime}+\Phi_{1}$ and $\Theta_{1}^{*}=A \delta^{\prime}+\Theta_{1}$ are arbitrary. Hence the parameters in such a VARMA $(1,1)$ are not unique and would not be identifiable unless appropriate reduced rank constraints are imposed.

\subsubsection{Exchangeability}

Exchangeability is a concept related to identifiability and means that two VARMA of different orders give rise to the same covariance structure for $y_{t}$ and therefore the same set of infinite coefficient matrices in the Wold decomposition. These different ARMA representations are also called observationally equivalent within the general class of all finite order ARMA models. We take up the example in Reinsel (1993) in order to illustrate exchangeability implications. Indeed, the existence of exchangeable models can, in particular, be caused by the presence of a unimodular matrix factor in a AR or MA matrix polynomial operator. For example, a VMA model $y_{t}=C(L) \Theta(L) \varepsilon_{t}$ can be rewritten in a finite $\operatorname{VARMA}(p, q)$, 
$C(L)^{-1} y_{l}=\Theta(L) \varepsilon_{t}$ if $C(L)$ is unimodular matrix. As a simple example, the bivariate MA(1) model $y_{t}=\varepsilon_{t}-\Theta_{1} \varepsilon_{t-1}$ and the bivariate $\operatorname{AR}(1)$ model $y_{t}-\Phi_{1} y_{t-1}=\varepsilon_{t}$, where

$$
\Theta_{1}=\left[\begin{array}{cc}
0 & 0_{12} \\
0 & 0
\end{array}\right] \text { and } \Phi_{1}=\left[\begin{array}{cc}
0 & -0_{12} \\
0 & 0
\end{array}\right]
$$

are easily seen to be exchangeable models since $\left(I-\Theta_{1} L\right)$ is unimodular with $\left(I-\Theta_{1} L\right)^{-1}=\left(I+\Theta_{1} L\right)$ here. Notice that in this case, either $\Phi_{1}$ and $\Theta_{1}$ are identifiable. Now, left-multiplying the previous MA(1) model by the following unimodular matrix

$$
C(L)=\left(I-\Phi_{1}^{*} L\right) \text { with } \Phi_{1}^{*}=\left[\begin{array}{ll}
0 & \alpha \\
0 & 0
\end{array}\right] .
$$

We obtain a non identifiable VARMA $(1,1)$ model $y_{t}-\Phi^{*} y_{t-1}=\varepsilon_{t}-\Theta^{*} \varepsilon_{t-1}$, where

$$
\Phi_{1}^{*}=\left[\begin{array}{cc}
0 & \alpha \\
0 & 0
\end{array}\right] \text { and } \Theta_{1}^{*}=\left[\begin{array}{cc}
0 & 0_{12}+\alpha \\
0 & 0
\end{array}\right] \text {. }
$$

The parameters of this model are not identifiable, at least if $\alpha$ is unknown, since $\alpha$ is arbitrary. Therefore, the parameters of an exchangeable model could or could not be identifiable. Using the same specification as in Section 2.9, we can illustrate the behavior of exchangeable models. We generated a $\operatorname{VAR}(1) y_{t}=\Phi_{1} y_{t-1}+\varepsilon_{t}$ with $\left(I-\Phi_{1} L\right)$ a unimodular matrix such as

$$
\Phi_{1}=\left[\begin{array}{ll}
0 & 8 \\
0 & 0
\end{array}\right] .
$$

Table 2.12 shows that we cannot reject the hypothesis of either an VMA(1) or a VAR(1) for the overall model. Note that the presence of a $\operatorname{SCM}(0,0)$ is also correctly found.

We already have seen that using a scalar component framework, results may depend of the identification, in the sense of order determination, of the initial VARMA $(p, q)$. A second problem arises with exchangeable models we must recover to avoid interpretation errors. For instance, the Vahid and Engle testing procedure may be misleading if both $\operatorname{SCM}(1,0)$ and $\operatorname{SCM}(0,1)$ are exchangeable. Consequently, we advise to start the reduced rank directions in a general VARMA model in order to find the common features both on AR and MA components. Since exchangeable scalar component models are synonymous with exchangeable model (Tiao and Tsay, 1989), that can help us to a better understanding of the hidden framework. 


$A_{1(m, h, j)}=\left(\begin{array}{ccccccc} & j=0 & j=1 & j=2 & j=3 & j=4 & j=5 \\\right.$\cline { 2 - 8 }$m=0 & 4.8 & 0.75 & 0.88 & 0.59 & 0.7 & 0.84 \\ & 100 & \mathbf{5 . 4 5} & 5.61 & 5.39 & 5.73 & 5.28 \\$\cline { 2 - 8 }$m=1 & 0.34 & 0.09 & 0.02 & 0.02 & 0.03 & 0.06 \\ & \mathbf{5 . 0 8} & 0.68 & 0.12 & 0.08 & 0.11 & 0.1 \\ & - & \mathbf{5 . 9 8} & 1.2 & 1.09 & 1.04 & 1.15 \\ & - & 100 & 8.44 & 8.29 & 8.2 & 8.26 \\ \hline & 0.33 & 0.06 & 0 & 0 & 0 & 0 \\ m=2 & 5.36 & 0.14 & 0 & 0 & 0.02 & 0.02 \\ & - & 1.14 & 0.22 & 0.04 & 0.07 & 0.05 \\ m=3 & - & 8.64 & 1.58 & 0.37 & 0.37 & 0.39 \\ m=4 & - & - & 10.26 & 2.61 & 2.52 & 2.39 \\$\cline { 2 - 7 } & - & - & 100 & 11.73 & 11.54 & 11.29 \\ \hline\end{array}

Table 2.12: Exchangeable Models using SCM 


\section{Chapter 3}

\section{Testing for Common Cyclical Features in VAR Models with Cointegration}

In this chapter we consider VAR models for variables exhibiting cointegration and common cyclical features. We focus on reduced rank structures involving both the long-run and the short-run dynamics of the system. While the presence of cointegration reduces the rank of the long run multiplier matrix, other types of common features learl to rank reduction of the short run dynamics. We distinguish between strong and weak form reduced rank structures. Strong form reduced rank structures analyzed by Engle and Kozicki (1993) arise when a linear combination of the first differenced variables in a cointegrated VAR is white noise whereas in the presence of a weak form reduced rank structure, linear combinations of the first differenced variables corrected for the long-run effects are white noise. We also consider the mixed form which combines strong and weak forms. We discuss the model selection issues which arise from this distinction and propose a simple approach to testing for these structures using a sequence of likelihood ratio test statistics. The finite sample behavior of the sequential approach is analyzed in a Monte Carlo experiment. Finally; we illustrate the relevance of the different forms of reduced ranks with empirical analyses of US business fluctuations over the period 1954-1996 and of the relationship between the UK equity; gilt and money markets.

\footnotetext{
${ }^{0}$ This chapter, except the additional example of Section 3.6, draws on Hecq, Palm and Urbain (1998).
} 


\subsection{Introduction and Motivation}

This chapter aims at analyzing common cyclical features ${ }^{1}$ in relation with cointegration. The strong assumption that some linear combination of the first differences of the variables in the model is white noise will be called a strong form reduced rank structure (SF). It corresponds to the case of serial correlation common features of the variables in first differences and assumes that the left null spaces of the short-run dynamic matrices and cointegrating matrix overlap. Of course, in line with other authors, when SCCF appears to be too strong, one could test for the existence of cofeatures in the form of linear combinations of the variables differenced once, that are not white noise but have lower order dynamics than the individual variables (see Chapter 2). It seems to be natural to consider an alternative weaker assumption under which the common cyclical part is reduced to a white noise by taking a linear combination of the variables in the first differences corrected for long-run effects. This case will be termed weak form reduced rank structures (WF). The WF is attractive as it allows for different common factors generating respectively the long-run and short-run dynamics of economic variables. Our framework is similar to that of Vahid and Engle (1993), but less restrictive as we explicitly consider the WF, that is lincar combinations of the first differenced $I(1)$ variables that are allowed to be predictable at low frequencies. In the presence of WF only, the lower bound to the number of common cycles is one whereas under SF, there have to be at least $r$ common cycles in the system, with $r$ being equal to the cointegration rank.

This chapter is organized as follows. In Section 3.2 we present different forms of reduced rank structures that arise in cmpirical work. We focus on the partially non-stationary vector autoregression that will be reparametrized as an Vector Error Correction Model (VECM). The relationships between the strong and weak form reduced rank structures will be analyzed. The mixed form (MF) combining SF and WF will also be considered. Our model representation follows the lines of Ahn (1997) and Reinsel and Ahn (1992) but focuses on the constraints between the number of cointegrating and common cyclical feature vectors. Section 3.3 presents a simple statistical procedure based on a two-step canonical correlation analysis that allows to test various kinds of reduced rank structures, in particular to check whether short and long-run matrices have a common left null space. In Section 3.4, we study the small sample behavior of common feature tests using Monte Carlo simulations. We show why the number of common feature vectors can be artificially bounded by a wrong assumption about the nature of the reduced rank structure. The results lead to a testing strategy that allows us to study cointegration and other common features of unknown order in an integrated framework. Finally, Section 3.5 illustrates the relevance of different forms of reduced structures, in particular of the WF for macroeconomic

\footnotetext{
${ }^{1}$ To avoid confusion, it should remembered that the term common cyclical features refers to a particular type of commonality leading to specific reduced rank structures. This concept should not be confused with the concept of cycle used in business cycle analyses (see the discussion in Cubadda, 1999). On the other hand, the concept of common cycles (in contrast to common cyclical features) refers to the common transitory component in particular permanent-transitory decompositions (see Vahid and Engle, 1993 and Chapter 4).
} 
applications. It also demonstrates the use of the tests in the search for long-run and short-run relationships among real consumption, investment and gross domestic product in the US, in the period 1954-1996 as well as within the UK money markets. A final section concludes.

\subsection{Reduced Rank Structures}

Let us consider a Gaussian Vector Autoregression of finite order p model for an $n$-vector time series $\{y \iota, t=1, \ldots, T\}$ :

$$
y_{t}=\sum_{i=1}^{p} \Phi_{i} y_{t-i}+\varepsilon_{t}, \quad t=1, \ldots, T
$$

for fixed values of $y_{-p+1}, \ldots, y_{0}$ and where $\varepsilon_{t}$ is a $n$-dimensional homoskedastic Gaussian mean innovation process relative to $\Im_{t}=\left\{y_{t-1}, y_{t-2}, \ldots, y_{1}\right\}$ with nonsingular covariance matrix $\Omega$. Let $L$ denote the lag operator and define $\Phi(L)=I_{n}-\sum_{i=1}^{p} \Phi_{i} L^{i}$. We make the following assumption

Assumption 1 (Cointegration): In the VAR model (3.1), we assume that

1. $\operatorname{rank}(\Phi(1))=r, 0<r<n$, so that $\Phi(1)$ can be expressed as $\Phi(1)=-\alpha \beta^{\prime}$, with $\alpha$ and $\beta$ both $(n \times r)$ matrices of full column rank $r$;

2. the characteristic equation $|\Phi(\xi)|=0$ has $n-r$ roots equal to 1 and all other roots outside the unit circle.

Assumption 1 implies (see Johansen, 1995) that the process $y_{t}$ is cointegrated of order $(1,1)$. The columns of $\beta$ span the space of cointegrating vectors, and the elements of $\alpha$ are the corresponding adjustment coefficients or factor loadings. Decomposing the matrix lag polynomial $\Phi(L)=\Phi(1) L+\Phi^{*}(L)(1-L)$, and defining $\Delta=(1-L)$, we obtain the vector error correction model:

$$
\Delta y_{t}=\alpha \beta^{\prime} y_{t-1}+\sum_{j=1}^{p-1} \Phi_{j}^{*} \Delta y_{t-j}+\varepsilon_{t}, \quad t=1, \ldots, T,
$$

where $\Phi_{0}^{*}=I_{n}, \Phi_{j}^{*}=-\sum_{k=j+1}^{p} \Phi_{k}(j=1, \ldots, p-1)$. Note that for notational convenience, deterministic terms (constants, trends, ...) are omitted at this level of presentation. Throughout this chapter we will also assume that $p$ is known. Serial correlation common feature (SCCF, see Engle and Kozicki, 1993) holds for the VECM (3.2), if there exists a $(n \times s)$ matrix $\tilde{\beta}$, whose columns span the cofeature space, such that $\tilde{\beta}^{\prime} \Delta y_{t}=\tilde{\beta}^{\prime} \varepsilon_{t}$ is a $s$-dimensional vector mean innovation process with respect to the information available at time $t, \Im_{t}$. 
Consequently, serial correlation common features arise if there exists a cofeature matrix $\ddot{\beta}^{\prime}$ such that the following two conditions are satisfied:

$$
\begin{array}{ll}
\text { Assumption 2: } & \tilde{\beta}^{\prime} \Phi_{j}^{*}=0_{(s \times n)}, \quad j=1 \ldots p-1 \\
\text { Assumption 3: } & \tilde{\beta}^{\prime} \Phi(1)=-\tilde{\beta}^{\prime} \alpha \beta^{\prime}=0_{(s \times n)}
\end{array}
$$

Assumption 2 implies that $\tilde{\beta}^{\prime}$ must lie in the intersection of the left null spaces of the matrices describing the short-run dynamies. Given that $\Phi_{j}^{*}=-\sum_{k=j+1}^{p} \Phi_{k}, j=1, \ldots, p-1$ and $\Phi_{p}^{*}=-\Phi(1)=-\left(I_{n}-\sum_{j=1}^{\prime} \Phi_{j}\right)$, Assumption 3 implies that $\beta^{\prime}\left(I_{n}-\Phi_{1}\right)=0_{(s \times n)}$, e.g. $\Phi_{1}$ must have eigenvalues equal to one with multiplicity $s$ and the corresponding eigenvectors must lie in the intersection of the left null spaces of the $\Phi_{j}^{*}$ matrices. Note that if the ranges of the $\Phi_{j}^{*}$ 's matrices are nested, i.c. if $\operatorname{range}\left(\Phi_{j+1}^{*}\right) \subseteq \operatorname{range}\left(\Phi_{j}^{*}\right)$, a nested reduced rank structure arises (sce e.g. $\Lambda \mathrm{hn}$ and Reinsel, 1988). We consider the restrictions implied by (3.3) or by (3.3) and (3.4) without imposing further nesting of the ranges of the $\Phi_{j}^{*}$ 's. This leads us to distinguish the following two concepts:

Definition 3.1 (Strong Form Reduced Rank Structure): If in addition to Assumption 1 (cointegration) both Assumptions 2 and 3 hold, the implied reduced rank structure of the VECM (3.2) will be labelled a strong form reduced rank structure (SF). Under SF, there exists a $(n \times s)$ matrix $\tilde{\beta}$, whose columns span the cofeature space, such that $\tilde{\beta}^{\prime} \Delta y_{t}=\tilde{\beta}^{\prime} \varepsilon_{t}$ is a $s$-dimensional vector moan innovation process with respect to the information available at time $t$.

Definition 3.2 (Weak Form Reduced Rank Structure): If in addition to Assumption 1 (cointegration) only Assumption 2 holds, the implied reduced rank structure of the VECM (3.2) will be labelled a weak form reduced rank structure (WF). Under WF, there exists a $(n \times s)$ matrix $\tilde{\beta}$, whose columns span the cofeature space, such that $\tilde{\beta}^{\prime}\left(\Delta y_{t}-\alpha \beta^{\prime} y_{t-1}\right)=\tilde{\beta}^{\prime} \varepsilon_{t}$ is a $s$-dimensional vector mean innovation process with respect to the information available at time $t$.

Remark 3.3 The SF is usually considered in the literature [sce inter alia Engle and Kozicki, 1993, Vahid and Engle, 1993 among others]. It leads to serial correlation common features. Define a $(n(p-1)+r) \times 1$ vector $X_{t-1}^{*}=\left[\Delta y_{t-1}^{\prime}, \ldots, \Delta y_{t-p+1}^{\prime}, y_{t-1}^{\prime} \beta\right]^{\prime}$ and $a n \times(n(p-1)+r)$ matrix $\Phi^{*}=\left\{\Phi_{1}^{*}, \ldots, \Phi_{p-1}^{*}, \alpha\right\}$, so that the (3.2) is written as

$$
\Delta y_{t}=\Phi^{*} X_{i-1}^{*}+\varepsilon_{t}, \quad t=1, \ldots, T .
$$

Under the assumption of a $S F, \Phi^{*}$ is of reduced rank $n-s$ and can be uritten as $\Phi^{*}=$ $A^{*}\left[C_{1}^{*}, \ldots, C_{p-1}^{*}, C_{p}^{*}\right]=A^{*} C^{*}$, where $A^{*}$ is $n \times(n-s)$ full column rank matrix and $C^{*}$ is $(n-s) \times(n(p-1)+r)$ and $\tilde{\beta}^{\prime} A^{*} C^{*} X_{t-1}^{*}=0$, c.g. $\tilde{\beta} \in s p\left(A_{\perp}^{*}\right)$ where $A_{\perp}^{*}$ is the orthogonal complement ${ }^{2}$ of $A^{*}$. Consequently, as pointed out by Vahid and Engle (1993), in a $n$-dimensional

\footnotetext{
${ }^{2}$ In the sequel, space will be denoted by sp. We shall always denote the orthogonal complement of any $n \times s-$
} 
$I(1)$ vector process $y_{t}$ with $r<n$ cointegrating vectors, if the elcments of $y_{t}$ have common cyclical features (given by $f_{t}=C^{*} X_{t-1}^{*}$ ) there can be at most $n-r$ linearly independeni cofcature vectors that eliminate the common cyclical features since the cofeature matrix must ${ }^{3}$ lie in $s p\left(\alpha_{\perp}\right)$. The $S F$ implies that $s \leq n-r$ and that the common dynamic factors $f_{t}$ consist of linear combinations of the elements of $X_{i-1}^{*}$. The implications of the SF can be stated more formally as:

Lemma 3.4 For the $S F, s p(\alpha) \subseteq s p\left(\tilde{\beta}_{\perp}\right)$.

The proof follows directly from the linear independence between the vectors $\beta$ and $\tilde{\beta}$ (sec Vahid and Engle, 1993) so that $\operatorname{rank}[\beta: \tilde{\beta}]=r+s \leq n$. Hence we have that $\operatorname{dim}[\operatorname{sp}(\alpha)] \leq$ $\operatorname{dim}\left[\operatorname{sp}\left(\tilde{\beta}_{\perp}\right)\right]$ or that $\operatorname{rank}(\alpha) \leq \operatorname{rank}\left(\tilde{\beta}_{\perp}\right)$ implying that $r \leq n-s$.

Remark 3.5 In the case of $W F$, we analogously define a $n(p-1) \times 1$ vector $X_{t-1}=\left[\Delta y_{t-1}^{\prime}, \ldots, \Delta y_{t-p+1}^{\prime}\right]^{\prime}$ and the $n \times n(p-1)$ matrix $\Phi=\left[\Phi_{1}^{*}, \ldots, \Phi_{p-1}^{*}\right]$, so that (3.2) becomes

$$
\Delta y_{t}=\alpha \beta^{\prime} y_{t-1}+\Phi X_{t-1}+\varepsilon_{t}, \quad t=1, \ldots, T .
$$

Under the assumption of a WF, $\Phi$ is of reduced rankn-s and can be written as $\Phi=A\left[C_{1}, \ldots, C_{p-1}\right]=$ $A C$, where $A$ is $n \times(n-s)$ full column rank matrix and $C$ is $(n-s) \times n(p-1)$ such that $\tilde{\beta}^{\prime} A C X_{t-1}=0$. The cofeature matrix $\tilde{\beta}$ must lie in space $\left(A_{\perp}\right)$ but not necessarily in space $\left(\alpha_{\perp}\right)$.

It is important to stress the difference between SF and WF. Firstly, the assumption of a SF reduced rank rules out predictability at any frequency and hence implies common cycles at all frequencies. On the contrary, by allowing for linear combinations that are predictable in the long-run, the WF reduced rank structure focuses on the remaining dynamics. Secondly, in the WF case, both the possible number and the nature of the common cyclical features change: $s$ may be greater than $n-r$ but has to remain $\leq n-1$ and the corresponding $n-s$ common dynamic factors consist of linear combinations of the elements of $X_{t-1}, f_{t}=C X_{t-1}$, which only contain lagged first differences of the process. It is important to notice that the existence of $s$ weak form common feature vectors with $s>r$, implies the existence of $s-r$ strong form common features as is shown in Lemma 3.6.

Lemma 3.6 In the VAR model (3.1) under Assumption 1 with $s>r$, Assumption 2 implies the existence of $s-r$ SF common feature vectors.

Proof. Denote by $\tilde{\beta}$ the $n \times s$ matrix of linearly independent WF common feature vectors. Any nonsingular transformation of $\bar{\beta}, \widetilde{\beta} A$, with $A$ being an $s \times s$ nonsingular matrix, also forms

dimensional matrix $B$, with $n>s$ and $\operatorname{rank}(B)=s$, by the $n \times(n-s)$ matrix $B_{\perp}$ such that $B^{\prime} B_{\perp}=0$ with $\operatorname{mank}\left(B_{\perp}\right)=n-s$ and $\operatorname{rank}\left(B: B_{\perp}\right)=n$. We then say that $B_{\perp}$ spans the null space of $B$ and $B^{\prime}$ spans the left null space of $B_{\perp}$.

${ }^{3}$ Consider the VAR(2) model $\Delta y_{t}=-\left(I-\Phi_{1}-\Phi_{2}\right) y_{t-1}-\Phi_{2} \Delta y_{t-1}+\varepsilon_{t}$ unth $n=4$, and $\operatorname{rank}\left(I-\Phi_{1}-\Phi_{2}\right)=$ $r=2$. In this case, $\operatorname{rank}\left(\Phi_{2}\right)$ should be necessarily equal to 2,3 or 4 . Otherwise in the strongly nested structure, $\operatorname{mank}\left(\Phi_{2}\right)=1$ would mean that $s+r=5>n$ which is not possible. 
a basis of the space spanned by the columns of $\bar{\beta}$ and therefore is also a basis of the WF common feature space. The matrix $\widetilde{\beta}^{\prime} \Phi(1)=\widetilde{\beta}^{\prime} \alpha \beta^{\prime}$ has rank $\min (r, s)$. Therefore, if $s>r$, there are $s-r$ linearly independent column vectors such that there is an $n \times(s-r)$ matrix $B$ with full column rank such that $B^{\prime} \alpha=0$. $B$ can be constructed as $B=\tilde{\beta} A^{*}$ by choosing the $s \times(s-r)$ matrix $A^{*}$ with rank $s-r$ such that $B$ forms a basis for the left null-space of $\alpha$. Note that we can always normalize $B$ such that the upper part equals $I_{s-r}$.

Remark 3.7 The WF restrictions are generally not invariant to altemative vector error correction representations such as that where $y_{t-p}$ appears in levels instead of $y_{t-1}$. The implications of the lack of invariance are that the results from a reduced rank analysis of short-run dynamics are parametrization-specific. ${ }^{4}$ Invariance may be obtained at the price of assuming a SCCF or that the ranges of $\Phi_{j}^{*}$ 's are nested (see c.g. Ahn and Reinsel, 1988). The methods put forward in this chapter can be applied to any of these alternative parametrizations. We present the analysis for the VECM (3.2) with $y_{t-1}$ appearing in levels, first, because this parametrization is frequently used in empirical work; sccond because if a reduced rank structure is found it will imply a lower order SCM than for other parametrizations; third, the WF are more likely to be appropriate as it applies to the coefficients of the higher order lags of $\triangle y_{t}$ in the VECM, which are usually of less significance than those of small order lags of $\Delta y_{\ell}$ (for non-seasonal processes). That also means that the absence of a WF structure defined above does not preclude the existence of an other reduced rank structure with the error correction terms at $t-i, i>1$.

When $n>2$, besides the $s-r$ SF common features implied by $s$ WF common features when $s-r>0$, the mixed form (MF) reduced rank restrictions may arise. They combine the SF and the WF in the following way.

Definition 3.8 (Mixed Form Reduced Rank Structure): If in addition to Assumption 1 (cointegration) Assumption 2 holds for $s$ common feature vectors $\tilde{\beta}$, Assumptions 2 and 3 hold for $s_{1}$ common feature vectors $\widetilde{\beta}_{1}$ with $s>s_{1}$ and $n-r>s_{1}>\max (0, s-r)$, then the implicd reduced rank structure of the VECM (3.2) will be labelled a mixed form reduced rank structure (MF). Under $M F$, the $(n \times s)$ matrix $\left[\widetilde{\beta}_{1}: \widetilde{\beta}_{2}\right]$, with $\widetilde{\beta}_{2}$ being a $\left(n \times s_{2}\right)$ matrix of full rank and $s_{2}=s-s_{1}$, with column vectors of $\tilde{\beta}$ for which Assumption 3 does not hold spans the co-feature space, such that $\widetilde{\beta}_{1}^{\prime} \Delta y_{t}=\widetilde{\beta}_{1}^{\prime} \varepsilon_{t}$ is a $s_{1}$-dimensional vector mean innovation process with respect to the information available at time $t$ and $\widetilde{\beta}_{2}^{\prime}\left(\Delta y_{t}-\alpha \beta^{\prime} y_{t-1}\right)=\widetilde{\beta}_{2}^{\prime} \varepsilon_{t}$ is a $s_{2}$-dimensional vector mean innovation process with respect to the information available at time $t$.

Remark 3.9 Under the $M F$, there are $s_{1}-\max (0, s-r)>0 S F$ common feature vectors which are not implied by the WF and yield restrictions on the parameters of the VECM (3.2) that can be tested. The matrix $\tilde{\beta}_{1}$ consists of $s-r$ columns which are linear combinations of $\tilde{\beta}$ and $s_{1}-\max (0, s-r)$ columns of $\tilde{\beta}$ which satisfy Assumption 3.

\footnotetext{
${ }^{4}$ For instance, for the VAR(2) model in footnote 3 , the WF is implied by ${ }^{-1} \Phi_{2}=0$, whereas when $y_{t-2}$ is included in the error-correction term, WF restrictions require $\tilde{\beta}^{\prime}\left(I-\Phi_{1}\right)=0$.
} 
Remark 3.10 Note that in the mixed case $s_{1}$ and $s_{2}$ have to satisfy the inequalities $s_{1}+s_{2} \leq n-1$ and $s_{1} \leq n-r$. Also, along the lines of Lemma 3.4, we get $s p(\alpha) \supseteq \operatorname{sp}\left(\tilde{\beta}_{\perp}\right)$.

Notice that we could easily extend these representations in order to analyze models in which only a part of short-run components disappears.

\subsection{Testing Different Forms of Reduced Rank Structures}

\subsubsection{Reduced rank hypotheses}

The difference between the SF and the WF can be illustrated in terms of two competing models where we assume both cointegration and the existcnce of a $(n \times s)$ common feature matrix $\tilde{\beta}$. Under the assumption of SF the following model holds

$$
\tilde{\beta}^{\prime} \Delta y_{t}=\tilde{\beta}^{\prime} \varepsilon_{t}, \quad t=1, \ldots, T,
$$

while under WF we have

$$
\tilde{\beta}^{\prime}\left(\Delta_{y_{1}}-\alpha \beta^{\prime} y_{t-1}\right)=\tilde{\beta}^{\prime} \varepsilon_{t}, \quad t=1, \ldots, T .
$$

Let us first assume that the cointegrating rank $r$ is known and fixed. The resulting structure of the various hypotheses of interest. is summarized in Table 3.1, where $\subset$ indicates the direction of the nesting between the different implied models. For a given maintained reduced rank structure (WF or SF), we may consider the sequence of hypotheses (or models) in each column separately in order to test $H_{0}: \operatorname{rank}(\widetilde{\beta}) \geq s$ against $H_{a}: \operatorname{rank}(\tilde{\beta})<s$ for the different values of $s$ starting with $s=0$ for the model without. common features. In the SF case, the maximum number of common feature vectors is $n-r$. For the WF $s$ has an upper bound ${ }^{5}$ of $n-1$.

For each value of $s(\leq n-r)$ we can also compare the SF against the nesting alternative of a WF. Table 3.1 shows that the hypotheses are nested "horizontally" and "vertically". In empirical work, one will usually start by considering "vertical" sequences of nested hypotheses for the WF and the SF respectively and for each sequence determine the value of $s$ for which the null hypothesis is not rejected. Denote these values by $s$ and $s_{1}$ respectively and by $H_{s_{1}, s}$ the hypothesis that the number of SF and WF common features is $s_{1}$ and $s$ respectively. Next, for the values of $s$ larger than $\max (1, s-r+1)$ and for which the SF is not rejected, one will usually test horizontally the SF against the WF. All other "diagonal" comparisons, such as $H_{0, s}$ versus $H_{1,1}$ for instance, involve non-nested hypotheses. The table is easily extended if we also consider the cointegrating rank as unknown. Table 1 presents the structure of reduced rank hypotheses for $s_{1}=0, \ldots, n-r ; s=0, \ldots, n$ and $r=1, \ldots, n-1$ and $n=4$.

\footnotetext{
${ }^{5} s=n$ implies that $\Delta y_{t}-\alpha \beta^{\prime} y_{t-1}$ is already a $n$ dimensional vector white noise process.
} 


\begin{tabular}{|c|c|c|c|c|c|c|c|c|c|c|}
\hline$r=1$ & $\begin{array}{l}s 1=0 \\
s 1=1 \\
s 1=2 \\
s 1=3\end{array}$ & $\begin{array}{c}H_{0,0} \\
H_{1,1}\end{array}$ & $\begin{array}{l}\supset \\
=\end{array}$ & $\begin{array}{c}H_{0,1} \\
\cup \\
H_{1,1} \\
\cup \\
H_{2,2}\end{array}$ & $\begin{array}{l}= \\
\supset \\
=\end{array}$ & $\begin{array}{c}H_{0,1} \\
\cup \\
H_{1,2} \\
\cup \\
H_{2,2} \\
\cup \\
H_{3,3}\end{array}$ & $\begin{array}{l}= \\
\supset \\
=\end{array}$ & $\begin{array}{c}H_{1,2} \\
\cup \\
H_{2,3} \\
\cup \\
H_{3,3}\end{array}$ & $\begin{array}{l}= \\
\supset\end{array}$ & $\begin{array}{c}H_{2,3} \\
\cup \\
H_{3,4}\end{array}$ \\
\hline$r=2$ & $\begin{array}{l}s 1=0 \\
s 1=1 \\
s 1=2 \\
s 1=3\end{array}$ & $\begin{array}{c}H_{0,0} \\
\cup \\
H_{1,1}\end{array}$ & $\begin{array}{l}\supset \\
=\end{array}$ & $\begin{array}{c}H_{0,1} \\
\cup \\
H_{1,1} \\
H_{2,2}\end{array}$ & $\begin{array}{l}\supset \\
\supset \\
=\end{array}$ & $\begin{array}{c}H_{0,2} \\
\cup \\
H_{1,2} \\
\cup \\
H_{2,2}\end{array}$ & $\begin{array}{l}= \\
\supset \\
\supset\end{array}$ & $\begin{array}{c}H_{0,2} \\
\cup \\
H_{1,3} \\
\cup \\
H_{2,3}\end{array}$ & $\begin{array}{l}= \\
\supset\end{array}$ & $\begin{array}{c}H_{1,3} \\
\cup \\
H_{2,4}\end{array}$ \\
\hline$r=3$ & $\begin{array}{l}s 1=0 \\
s 1=1 \\
s 1=2 \\
s 1=3\end{array}$ & $\begin{array}{c}H_{0,0} \\
\cup \\
H_{1,1}\end{array}$ & $\begin{array}{l}\supset \\
=\end{array}$ & $\begin{array}{c}H_{0,1} \\
\cup \\
H_{1,1}\end{array}$ & $\begin{array}{l}\supset \\
\supset\end{array}$ & $\begin{array}{c}H_{0,2} \\
\cup \\
H_{1,2}\end{array}$ & $\begin{array}{l}\supset \\
\supset\end{array}$ & $\begin{array}{c}H_{0,3} \\
\cup \\
H_{1,3}\end{array}$ & $\begin{array}{l}= \\
\supset\end{array}$ & $\begin{array}{c}H_{0,3} \\
\cup \\
H_{1,4}\end{array}$ \\
\hline
\end{tabular}

Table 3.1: Reduced Rank Hypotheses for $n=4$ 


\subsubsection{Testing}

Given that the hypotheses to be tested are nested, we rely on ML estimation of the underlying models following the approaches by Reinsel and Ahn (1992), Ahn (1997), Ahn and Reinsel (1988), Rcinsel (1993) among others. Usually, when $r$ and $s$ are unknown, it appears impossible to find an explicit solution for the likelihood equations [see Johansen (1995), Ahn (1997)]. There are essentially two approaches to the determination of $r, s$ and to the estimation of the parameters of interest. The first approach proposed and investigated by Ahn (1997), Ahn and Reinsel (1988) is to exploit the nested reduced rank structures and to compute numerically a Gaussian reduced-rank estimator based on iterative solution of approximate Newton-Raphson equations. Alternatively, one may follow a two-step approach in which $r$ is first determined, while ignoring restrictions on the short-run dynamics of the model. Once $r$ is determined and $\beta$ (the cointegrating matrix) assumed known, $s$ may be determined using the approach proposed by Vahid and Engle (1993) for example. The rationale behind this simple two-step analysis is that the determination of $r$ and the estimation of $\beta$ are not afected in terms of asymptotic efficiency by the presence of the nested reduced rank structure (see also Ahn, 1997; Phillips, 1991).

We use the two-stcp approach, although one may reasonably suspect small sample efficiency losses compared to using a full information estimation method. As pointed out by various authors, a convenient way to test for reduced rank structures within the VECM is based on canonical correlation analysis. Let us first assume that $r$ and $\beta$ are known or that super consistent estimates are available so that we may now essentially consider them to be fixed and given.

Define the $T \times n$ matrices $W_{1}=\Delta Y=\left(\Delta y_{1}, \ldots, \Delta y_{T}\right)^{\prime}, Y_{-1}=\left(y_{0}, \ldots, y_{T-1}\right)^{\prime}, Z_{1}=\Delta Y^{*}$ with $\Delta Y^{*}$ being the LS residuals from the multivariate regression of $\Delta Y$ on $Y_{-1} \beta$ and the $T \times$ $(n(p-1)+r)$ matrix $W_{2}=\left[Z_{2}, Y_{-1} \beta\right]$ with $Z_{2}$ being the $T \times n(p-1)$ matrix $\left(\Delta Y_{-1}^{*}, \ldots \Delta Y_{-p+1}^{*}\right)$. Under the maintained hypothesis of a SF reduced rank structure, the sequence of common feature Gaussian likelihood ratio test statistics for $H_{0}: \operatorname{rank}\left(\Phi^{*}\right) \leq n-s$ against $H_{a}: \operatorname{rank}\left(\Phi^{*}\right)>n-s$, where $\Phi^{*}$ is defined in (3.6), or equivalently for $H_{0}: \operatorname{rank}(\tilde{\beta}) \geq s$ against $H_{a}: \operatorname{rank}(\tilde{\beta})<s$ can be shown [see Lïtkepohl (1991), Velu et al., (1986)] to be

$$
\xi_{S}=-T \sum_{i=1}^{s} \log \left(1-\lambda_{i}\right), \quad s=1, \ldots, n-r,
$$

where $0 \leq \lambda_{1} \leq \lambda_{2} \leq \ldots \leq \lambda_{n-r}<1$ are the ordered eigenvalues of the symmetric matrix $\left(W_{1}^{\prime} W_{1}\right)^{-1 / 2} W_{1}^{\prime} W_{2}\left(W_{2}^{\prime} W_{2}\right)^{-1} W_{2}^{\prime} W_{1}\left(W_{1}^{\prime} W_{1}\right)^{-1 / 2}$. The test statistic $(3.9)$ can also be interpreted as the minimum of the objective function of the GMM estimator of $\tilde{\beta}$ subject to the normalized $(1 / T) \hat{\beta}^{\prime} W_{1}^{\prime} W_{1} \bar{\beta}=I_{s}$ (see Anderson and Vahid, 1998). For known $r$ and $\beta$, under the null the test statistic $\xi_{S}$ is asymptotically $\chi^{2}$-distributed with $s(n(p-1)+r)-s(n-s)$ degrees of freedom (Vahid and Engle, 1993).

In the case of WF reduced rank structure, this likelihood ratio test for $H_{0}: \operatorname{rank}(\tilde{\beta}) \geq s$ against $H_{a}: \operatorname{rank}(\tilde{\beta})<s$ reads as 


$$
\xi_{W^{\prime}}=-T \sum_{i=1}^{s} \log \left(1-\tilde{\lambda}_{i}\right), \quad s=1, \ldots, n-1,
$$

where $0 \leq \tilde{\lambda}_{1} \leq \tilde{\lambda}_{2} \leq \ldots \leq \tilde{\lambda}_{n-1}<1$ are the ordered cigenvalues of the symmetric matrix $\left(Z_{1}^{\prime} Z_{1}\right)^{-1 / 2} Z_{1}^{\prime} Z_{2}\left(Z_{2}^{\prime} Z_{2}\right)^{-1} Z_{2}^{\prime} Z_{1}\left(Z_{1}^{\prime} Z_{1}\right)^{-1 / 2}$. This statistic has an asymptotic $\chi^{2}$-distribution with $s(n(p-1))-s(n-s)$ degrees of freedom under the null.

A MF reduced rank structure hypothesis $H_{0}: \operatorname{rank}\left(\tilde{\beta}_{1}\right) \geq s_{1}$, for $n-r \geq s_{1}>\max (0, s-r)$ and $\operatorname{rank}\left(\tilde{\beta}_{2}\right) \geq s_{2}$ against $H_{a}: \operatorname{rank}\left(\tilde{\beta}_{1}\right)<s_{1}$ or $\operatorname{rank}\left(\tilde{\beta}_{2}\right)<s_{2}$ can be tested in several ways. One way is to test SF restrictions for $s_{1}=1, \ldots, n-r$, using the statistic $\xi_{S}$ in (3.9). As this test ignores the restrictions implied by the existence of $s_{2}$ weak form common features, some power might be lost as it will be illustrated in the following section.

Alternatively, the canonical correlation between $\tilde{\beta}^{\prime} \Delta y_{t}$ and $\beta^{\prime} y_{t-1}$ corrected for the short-run dynamics can be obtained, for given $\tilde{\beta}$ and $\beta$, where $\tilde{\beta}$ denotes the $n \times s$ matrix of WF common features such that $\tilde{\beta}^{\prime}\left(\Delta y_{t}-\alpha \beta^{\prime} y_{t-1}\right)=\tilde{\beta}^{\prime} \varepsilon_{t}$. This test aims at determining the rank of $s \times r$ matrix $\tilde{\beta}^{\prime} \alpha$. If $s-r>0, \tilde{\beta}^{\prime} \alpha$ has rank $r$ at most and as stated in Lemma 3.6, the WF implies $(s-r) \mathrm{SF}$ common features. The emergence of SF common features vectors beyond the $(s-r)$ implied by the WF requires that $\operatorname{rank}\left(\tilde{\beta}^{\prime} \alpha\right)<r$. The ordered squared canonical correlations $\lambda_{i}^{*}$ between $\tilde{\beta}^{\prime} \Delta y_{t}$ and $\beta^{\prime} y_{t-1}$ could be used in the statistic

$$
\xi_{M}=-T \sum_{i=1}^{s_{1}} \log \left(1-\lambda_{i}^{*}\right)
$$

for $s_{1}=\max (1, s-r+1), \ldots \min (n-r, s)$ to test the MF. The number of degrees of freedom involved is equal to the number of elements of $\tilde{\beta}^{\prime} \alpha, s r$, minus the number of unknown parameters $\left(s-s_{1}\right)\left(s_{1}+r\right)$ in the restricted system (i.e. the matrices $A$ and $C$ in (3.12))

$$
\left[\begin{array}{cc}
I_{s_{1}} & A \\
0_{\left(s-s_{1}\right) \times s_{1}} & I_{\left(s-s_{1}\right)}
\end{array}\right] \tilde{\beta}^{\prime} \Delta y_{t}=\left[\begin{array}{c}
0_{s_{1} \times r} \\
C_{\left(s-s_{1}\right) \times r}
\end{array}\right] \beta^{\prime} y_{t-1}+\nu_{\ell} .
$$

The eigenvectors associated with the zero eigenvalues (the zero squared canonical correlations) are the coefficients used to form the linear combinations of $\bar{\beta}$ which are SF common features. The values of these eigenvectors allow to see whether there is an identification problem. If these cigenvectors have only one nonzero element there is no identification problem as the "linear combination" of $\tilde{\beta}$ is simply a vector from $\tilde{\beta}$.

The above approach however requires $\tilde{\beta}$ to be given to avoid the generated regressors problem. When $\tilde{\beta}$ is unknown, the parameters $\tilde{\beta}$ and $\alpha$ from the WF can be estimated jointly by FIML for given $\beta$, e.g. by maximizing the likelihood function based on the $(s \times 1)$ subsystem $(3.8)$, normalized on the first $s$ variables of $\Delta y_{t}$ by setting $\tilde{\beta}^{\prime}=\left(I_{s}, \tilde{\beta}^{* \prime}\right)$, and completed by adding $(n-s)$ "reduced form" equations for the remaining $(n-s)$ variables in $\Delta y_{t}$. The MF can then 
be tested against the WF by testing for the validity of the additional parameter restrictions implied by (3.12) using LR test statistics. No efficiency loss arises if superconsistent estimate are substituted for the cointegrating vectors $\beta$. This cstimation procedure has been used in the empirical analysis reported in Section 3.5. ${ }^{6}$

For given $r$, a likelihood ratio test statistic for the null hypothesis of a SF against the alternative of a WF, for each possible common feature rank $s=\max (1, s-r+1) \ldots n-r$, is given by

$$
\xi_{S W}=-T \sum_{i=1}^{s} \log \left\{\frac{\left(1-\lambda_{i}\right)}{\left(1-\tilde{\lambda}_{i}\right)}\right\}
$$

where the $\tilde{\lambda}_{i}$ 's and the $\lambda_{i}$ 's are defined as above. Conditional on known $r$ and $\beta$, all variables involved are weakly stationary both under the null and the alternative, so that standard asymptotic theory applies. $\xi_{S W}$ has an asymptotic $\chi^{2}$-distribution with degrees of freedom equal to the number of restrictions rs imposed under the $H_{0}$. If the null hypothesis is rejected, one can proceed further in determining $s$ by testing the number of zero squared canonical correlations between $Z_{1}$ and $Z_{2}$. Note that the test statistics (3.9),(3.10),(3.11) and (3.13) only enable to formally compare the nested models from Table 3.1. For "diagonal" type of model comparisons involving non-nested hypotheses, wc propose to select the model which minimizes, for given $p, r$ and $\beta$, one of the well-known model selection criteria (AIC, SBC, HQC) where, given that we have omitted deterministic terms, the number of parameters is $n(n(p-1)+r)-s(n(p-1)+r)+s(n-s)$ under the SF and $n(n(p-1)+r)-s(n(p-1))+s(n-s)$ under the WF. ${ }^{7}$

\subsection{Monte Carlo Results}

In this section we present evidence on the finite sample behavior of the sequential test procedure put forward in Section 3.3. One should indeed be carcful when interpreting the outcome of the three sequences of LR tests $\xi_{S}, \xi_{W}$ and $\xi_{S W}$. Given that $s$ is unknown, and given the sequential nature of the testing procedure, the significance levels of the individual tests in the sequence must be distinguished from the overall Type I error of the sequential testing procedure. Also, the above sequential procedures are essentially based on asymptotic properties such as the irrelevance of the reduced rank structure for the optimal estimation of $\beta$ and the determination of $r$. An illustrative Monte Carlo experiment should shed some light on the finite sample behavior of the sequences of common features LR tests presented in the preceding section. We concentrate on two issues which we believe are particularly relevant for applications:

\footnotetext{
${ }^{6}$ Alternalively, the subsystem (3.12) normalized as above could be estimated by GMM, to get unrestricted estimates of the matrices $\bar{\beta}^{* \prime}$ and $\bar{\beta}_{\alpha}^{\prime}$ (for given $\beta$ ) and testing the rank of the matrix $\bar{\beta}_{\alpha}^{\prime}$ (see Hecc, Palm and Urbain, 1999).

${ }^{7}$ These model selection criteria can be also used to select the optimal values for $\tau$ and $s$ given $p$ (as we axsumed in the preceding section) and have also recently been considered for common feature analysis by Vahid and Issler (1999) for unkown $s$ and $p$ (see also Chapter 6).
} 
1. the size and power in finite samples of the common feature LR tests,

2. the possible effect of incorrectly specifying the number of cointegrating vectors in the first step.

In order to address these issues we consider a simple trivariate data generating process (DGP) where we assume the existence of two common feature vectors, i.e. $s=2$. Throughout the simulations, $p$ is assumed to be given and equal to its truc value $p=2$. Strong and weak form reduced rank structures are considered. The DGP is a Gaussian VAR of order two written in VECM form. In order to provide some motivation for the choice of the DGP, we label the three variables $c_{\ell}, i_{l}, y_{l}$ as for consumption, investment and real output. In line with a simple form of a neo-classical model, we assume the existence of two long-run relationships: $c_{t}-y_{t}$ and $i_{t}-y_{t}$. The covariance matrix has been calibrated on quarterly real US data for consumption, investment and value added for the period 1950-1996.

$$
\begin{aligned}
{\left[\begin{array}{l}
\Delta c_{t} \\
\Delta i_{t} \\
\Delta y_{t}
\end{array}\right]=} & {\left[\begin{array}{lll}
0.2 & 0.1 & 0.1 \\
0.8 & 0.4 & 0.4 \\
0.4 & 0.2 & 0.2
\end{array}\right]\left[\begin{array}{c}
\Delta c_{t-1} \\
\Delta i_{t-1} \\
\Delta y_{t-1}
\end{array}\right]+\alpha \beta^{\prime}\left[\begin{array}{c}
c_{t-1} \\
i_{t-1} \\
y_{t-1}
\end{array}\right]+\left[\begin{array}{l}
\varepsilon_{1 t} \\
\varepsilon_{2 t} \\
\varepsilon_{3 t}
\end{array}\right], } \\
& {\left[\begin{array}{l}
\varepsilon_{1 t} \\
\varepsilon_{2 t} \\
\varepsilon_{3 t}
\end{array}\right] \sim N\left(\left[\begin{array}{l}
0 \\
0 \\
0
\end{array}\right],\left[\begin{array}{ccc}
1.0 & 0.6 & 0.6 \\
0.6 & 1.0 & 0.6 \\
0.6 & 0.6 & 1.0
\end{array}\right]\right) . }
\end{aligned}
$$

The cofeature matrix associated with the DGP in (3.14) is given by

$$
\tilde{\beta}^{\prime}=\left(\begin{array}{ccc}
1 & -0.25 & 0 \\
1 & 0 & -0.5
\end{array}\right) .
$$

It yields two linear combinations of the variables in the model that annihilate the short-run dynamics. In our experiments, the nature of the reduced rank structure depends on the choice of the values for $\alpha$ and $\beta$. Table 3.2 illustrates the outcome of the simulations when the DGP has a WF reduced rank structure. The upper panel of Table 3.2 presents the results for $r=2$, both in the DGP and in the estimated model. The second panel analyzes the case of over-specification of the cointegration rank while the third panel presents the under-specification case. In Tablc 3.3 we report the results when the DGP displays a SF reduced rank structure. In this case the number of cointegrating vectors is by definition bounded to be equal to one in the DGP. We therefore present simulation results for models with the correct specification of the cointegrating rank as well as over-specification of $r$.

In each case, we use 10,000 replications and a sample size of $T=1000$ and 100 respectively. The cointegration coefficients $\beta$ are set equal to their true values while $\alpha$ and $\bar{\beta}$ are estimated by ML as described in Section 3.3. If $r$ is assumed to be equal to $1, \beta$ is set equal to the values of the first cointegrating vector. All simulations have been performed with GAUSS and the first 50 


\begin{tabular}{|c|c|c|c|c|c|c|c|}
\hline DGP : WF $s=2$ & Estim & ted $\mathrm{N}$ & del & & & & \\
\hline Coint, vector $r=1$ & $r=1$ & & & & & & \\
\hline$[-.5]$ & & & $=10$ & & & $T=100$ & \\
\hline$\alpha=\mid .1$ & & $\xi_{S}$ & $\xi_{W}$ & $\xi_{S W}$ & $\xi_{S}$ & $\xi_{W}$ & $\xi_{S W}$ \\
\hline$[.2]$ & $s \geq 1$ & 5.12 & .31 & 10.84 & 5.75 & .37 & 11.89 \\
\hline & $s \geq 2$ & 100 & 4.92 & 100 & 100 & 6.03 & 100 \\
\hline$\beta^{\prime}=\mid \begin{array}{lll}1 & 0 & -1\end{array}$ & $s=3$ & 100 & 100 & 100 & 100 & 100 & 100 \\
\hline Coint. vector $r=1$ & $r=2$ & & & & & & \\
\hline$[-.5]$ & & & $T=1$ & & & $T=10$ & \\
\hline$\alpha=1$ & & $\xi_{s}$ & $\xi_{w}$ & $\xi_{S W}$ & $\xi_{S}$ & $\xi_{W}$ & $\xi_{S W}$ \\
\hline$[2$ & $s \geq 1$ & 10.68 & .33 & 17.4 & 12.68 & .44 & 19.17 \\
\hline & $s \geq 2$ & 100 & 4.9 & 100 & 100 & 6.76 & 100 \\
\hline$\beta^{\prime}=\left[\begin{array}{lll}1 & 0 & -1\end{array}\right]$ & $s=3$ & 100 & 10 & 100 & 100 & 100 & 100 \\
\hline Coint. vector $\mathrm{r}=2$ & $r=2$ & & & & & & \\
\hline$\alpha=\left[\begin{array}{cc}-.5 & -.2 \\
1 & -3\end{array}\right]$ & & & $=10$ & & & $T=100$ & \\
\hline$\alpha=\left|\begin{array}{cc}.1 & -.3 \\
2 & 2\end{array}\right|$ & & $\xi_{S}$ & $\xi_{W}$ & $\xi_{S W}$ & $\xi_{S}$ & $\xi_{W}$ & $\xi_{S W}$ \\
\hline $\begin{array}{lll}.2 & -2\end{array}$ & $s \geq 1$ & 100 & .4 & 100 & 99.84 & .4 & 99.92 \\
\hline$\Rightarrow\left[\begin{array}{lll}1 & 0 & -1\end{array}\right]$ & $s \geq 2$ & 100 & 5.11 & 100 & 100 & 6.09 & 100 \\
\hline$\beta^{\prime}=\left[\begin{array}{lll}1 & 0 & -1 \\
0 & 1 & -1\end{array}\right]$ & $s=3$ & 100 & 100 & 100 & 100 & 100 & 100 \\
\hline Coint. vector $\mathrm{r}=2$ & $r=1$ & & & & & & \\
\hline$\alpha=\left[\begin{array}{cc}-.5 & -.2 \\
.1 & -.3\end{array}\right]$ & & & $=10$ & & & $T=100$ & \\
\hline $\begin{array}{l}-.3 \\
.2\end{array}$ & $s \geq 1$ & $\begin{array}{c}\xi_{S} \\
100\end{array}$ & $\begin{array}{l}\xi_{W} \\
5.05\end{array}$ & $\begin{array}{c}\xi_{S W} \\
100\end{array}$ & $\begin{array}{c}\xi_{S} \\
95.34\end{array}$ & $\begin{array}{l}\xi_{W} \\
4.8\end{array}$ & $\begin{array}{c}\xi_{S W} \\
97.02\end{array}$ \\
\hline$\quad\left[\begin{array}{lll}1 & 0 & -1\end{array}\right]$ & $s \geq 2$ & 100 & 100 & 100 & 100 & 93.32 & 100 \\
\hline$\beta^{\prime}=\left[\begin{array}{lll}1 & 0 & -1 \\
0 & 1 & -1\end{array}\right.$ & $s=3$ & 100 & 100 & 100 & 100 & 100 & 100 \\
\hline
\end{tabular}

Table 3.2: Power and Size of Test Statistics for WF

observations initialize the processes. The empirical (size unadjusted) power and size are given in percentage rejection frequencies. The nominal size used to obtain these rejection frequencies is fixed at $5 \%$ for each individual test.

We draw some conclusions from Table 3.2:

- When the DGP has a WF reduced rank structure and the number of cointegrating vectors is correctly (first and third block) specified, $\xi_{W}$ determines without size distortions the correct number of common feature vectors.

- The statistic $\xi_{S}$ detects a SF reduced rank structure implied by a WF reduced rank structure $(s-r>0)$ with a rejection frequency of approximately $5 \%$ when $r$ is correctly specified (panel one). When $r$ is overestimated, the size of $\xi_{S}$ is larger than the nominal size of $5 \%$ (panel two). The power of $\xi_{S w}$ is very high even for a sample of 100 observation.

- In the second panel of Table 3.2 the results are computed under the assumption that the 


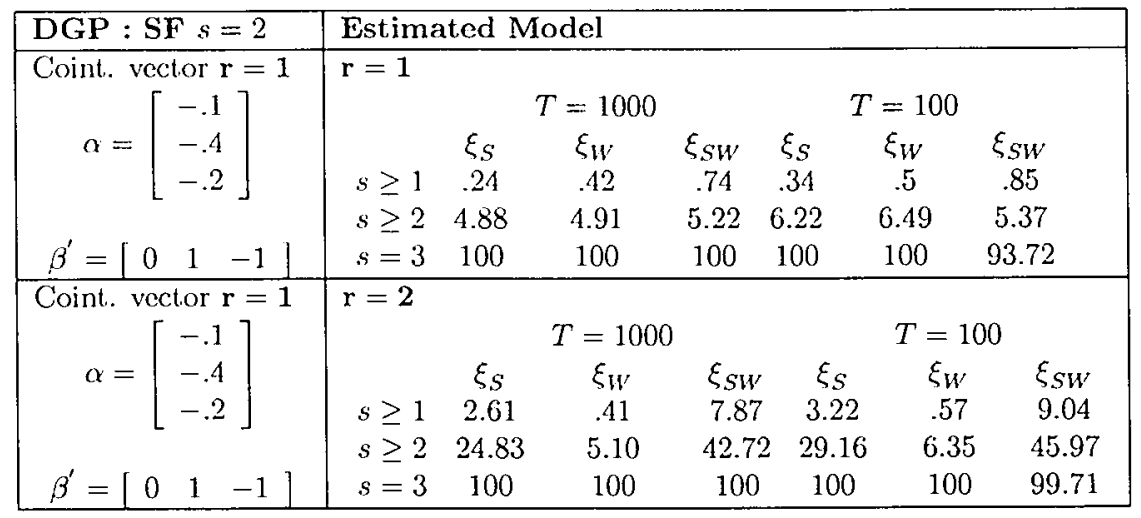

Table 3.3: Power and Sizc of Test Statistics for SF

retained number of cointegrating vectors exceeds the true number of cointegrating vectors in the DGP. It is interesting to note that the sequence of $\xi_{W}$ still selects the correct number of common feature vectors without size distortions although we have overspecified the number of cointegrating vectors. This is not surprising since the coefficient of a non significant $I(1)$ variable in a $I(0)$ model converges in probability to zero. $\xi_{S}$ still rejects the presence of any cofeature vector since this case excludes the existence and an implied MF $(s-r=0)$.

- When the number of cointegrating vectors is under-specified (fourth panel), $\xi_{W}$ and $\xi_{S}$ however clearly fail in detecting two common feature vectors. Excluding some cointegrating vectors lcads in fact to an omitted variables problem. It this therefore not surprising to see that the properties of $\xi_{W}$ and $\xi_{S}$ are affected.

- Overall, the likelihood ratio statistics $\xi_{S W}$ for the null of SF against the WF has high power close to one in most cases. When $s-r>0$ in the DGP, there are $(s-r)$ implied $\mathrm{SF}$ common features vectors and the rejection frequencies for $s=1$ in panels 1 and 2 of Table 3.2 have to interpreted as an empirical size of the test. In these cases, the statistic $\xi_{S W}$ rejects too frequently the (impled) null hypothesis.

Table 3.3 reports some simulation results for a DGP with SF:

- When the DGP has a SF and the number of cointegrating vectors has been correctly specified, both $\xi_{S}$ and $\xi_{W}$ behave fairly well in detecting the two cofeature vectors. Note also the behavior of the sequence of the LR tests $\xi_{S W}$ that does not show any significant size distortion.

- If we overestimate the number of cointegrating vectors, (second panel) the size of $\xi_{S}$ is slightly distorted. $\xi_{W}$ still behaves very well in detecting the correct number of common 


\begin{tabular}{|ll|lcc|rrr|}
\hline DGP & Model & $p=2$ & & & & & \\
WF & WF & $\beta_{\text {knoun }}$ & $\hat{\beta}_{2-\text { steps }}$ & $\hat{\beta}_{\text {iter }}$ & $\beta_{\text {knoum }}$ & $\hat{\beta}_{2-\text { steps }}$ & $\hat{\beta}_{\text {iter }}$ \\
\hline$r=1$ & $r=2$ & $6.76(5.90)$ & $7.56(6.84)$ & $7.14(6.29)$ & $9.38(5.10)$ & $11.40(6.60)$ & $10.20(5.94)$ \\
$r=2$ & $r=2$ & $6.09(5.43)$ & $6.98(6.25)$ & $6.50(5.76)$ & $9.13(4.88)$ & $11.57(6.56)$ & $10.26(5.64)$ \\
\hline
\end{tabular}

Table 3.4: Power and Size of Test Statistics for WF with Estimated $\beta$

feature vectors. However the LR tests for SF versus WF display significant size distortions reaching $40 \%$ instead of the $5 \%$ chosen nominal level.

Although not reported, the use of finite sample corrections $a$ la Ahn and Reinsel (1992) does not significantly reduce this size distortion problem in the case of $p=2$. Note however that small sample corrections are worth considering for smaller sample sizes and/or longer lag length and/or higher dimensional systems (sec Chapter 5 ).

We complete the previous simulation results by considering cases where the cointegrating vectors are unknown and have to be estimated in a first step. We concentrate on the small sample propertics, c.g. $T=100$. Results for $T=1000$ do not differ from the case with known cointegrating vectors. We illustrate the behavior of the common feature test within the WF for $H_{0}: s \geq 2$ using threc approaches: two step estimation of $\tilde{\beta}$ with known $\beta$, two step estimation of $\tilde{\beta}$ with $\beta$ estimated by Johansen's (1995) MLE and the iterative method proposed in Chapter 5 where $\tilde{\beta}$ and $\beta$ are jointly cstimated by MLE using an iterative switching approach similar to that proposed by Hansen and Johansen (1998). Results are reported for the correct choice of $r$ and for the case where $r$ is overestimated. Finite sample results in the case of underestimation of $r$ are not reported but again they suffer from important size distortions. We compare results obtained both for the statistics presented above and for small sample corrected versions (in parentheses) thereof where $\xi_{W}$ and $\xi_{S}$ are respectively premultiplied by the factors $(T-n(p-1)) / T$ and $(T-n(p-1)-r) / T$, a similar small sample correction than the one proposed by Ahn and Reinsel (1992) for cointegration analyses (see Chapter 5 for further details). Since the outcome does not differ significantly from the previous ones with $p=2$, we also report simulation results for the case of overfitting, that is the case where $p=4$ in the estimation and construction of the test statistics.

We may first conclude that the small sample corrections are worth considering for $p=4$. The over-estimation of the cointegrating rank does again not affect the results. The following inequality is observed for the empirical sizes: $\operatorname{size}\left(\beta_{\text {known }}\right)<\operatorname{size}\left(\hat{\beta}_{i t e r}\right)<\operatorname{size}\left(\hat{\beta}_{2-s t e p s}\right)$, although these differences may be small in some particular cases but may depend upon the DGP (see Chapter 5).

Table 3.5 contains some illustrative simulation results for a DGP with a MF reduced rank. For this purpose, the DGP is slightly modificd and extended in order to account for a MF. The selected DGP is a VAR(2) with $n=4, r=2$ and $s=3$. From Lemma 3.6 there is one implied cofeature vector $(s-r=1)$. The loading matrix $\alpha$ is chosen such that the DGP displays one 


\begin{tabular}{|cc|c|c|c|c|}
\hline \hline$T$ & Size & Power: & Power: & Power: \\
& $\alpha_{3,1}=-0.4$ & $\alpha_{3,1}=-0.45$ & $\alpha_{3,1}=-0.5$ & $\alpha_{3,1}=-0.35$ \\
\hline \multirow{3}{*}{$\xi_{S}$} & 100 & 7.60 & 13.94 & 26.98 & 48.96 \\
& 250 & 5.70 & 24.94 & 55.68 & 95.66 \\
& 1000 & 5.18 & 78.12 & 99.34 & 100 \\
\hline \multirow{3}{*}{$\xi_{M}$} & 100 & 7.76 & 19.84 & 40.12 & 63.42 \\
& 250 & 5.64 & 39.86 & 74.90 & 97.84 \\
& 1000 & 5.14 & 92.44 & 100 & 100 \\
\hline \multirow{3}{*}{$\xi_{M}^{*}$} & 100 & 8.84 & 21.60 & 41.50 & 65.86 \\
& 250 & 6.80 & 39.76 & 74.16 & 99.00 \\
\hline \hline
\end{tabular}

Table 3.5: Size and Power for MF

additional cofeature vectors, i.e. $s_{1}=2$. The following matrices are retained:

$$
\tilde{\beta}=\left(\begin{array}{ccc}
1 & 1 & 1 \\
-.25 & 0 & 0 \\
0 & -.5 & 0 \\
0 & 0 & -.4
\end{array}\right), \tilde{\beta}_{\perp}=\left(\begin{array}{c}
-.1 \\
-.4 \\
-.2 \\
-.25
\end{array}\right), \alpha=\left(\begin{array}{cc}
-.2 & .2 \\
-.8 & 0 \\
-.4 & .8 \\
-.5 & 0
\end{array}\right), \beta=\left(\begin{array}{cc}
1 & 0 \\
0 & 1 \\
1.2 & -.8 \\
-1 & -1
\end{array}\right)
$$

This particular choice of $\alpha$ implies the existence of $\tilde{\beta}_{1}^{\prime}$ satisfying $8 \tilde{\beta}_{1}^{\prime} \alpha=0$. As discussed in the preceding section, we report results for $\xi_{S}$ and $\xi_{M}$. In the latter case we compute two versions of the statistics. The first one $\xi_{M}$ assumes a known $\tilde{\beta}^{\prime}$, while $\xi_{M}^{*}$ is computed using the estimated $\tilde{\beta}^{\prime}$ obtained from a first step canonical correlation analysis as explained in Section 3.3. The latter statistics suffers theoretically from the generated regressors problem and is hence strictly speaking not $\chi^{2}$ distributed unless an appropriate correction is used. The results are however reported for both versions of the test for the sake of comparison.

In Table 3.5 we report rejection frequencies, based on 5,000 replications, under the correct assumption of a mixed form with $s_{1}=2$ (size of the tests) as well as those obtained when we let the parameter $\alpha_{3,1}$ successively take the values $-0.45,-0.5$ which implies the existence of a weak form. ${ }^{9}$ In the last column, we report the rejection frequency obtained for $\alpha_{3,1}=-0.35$ which implies an explosive system. with a root inside of the unit circle. In all the cases, the empirical power is not size adjusted.

From Table 3.5, we observe that $\xi_{S}$ and $\xi_{M}$ do not suffer from serious size distortion. The slightly higher rejection rate observed for $\xi_{M}^{*}$ stems from the preliminary estimation of $\tilde{\beta}$. With respect to the empirical powers, it appears that both versions of $\xi_{M}$, that allow to detect.

\footnotetext{
${ }^{8}$ The columns of the $4 \times 2$ matrix $\tilde{\beta}_{1}$ may simply be constructed by adding the first and second column of $\tilde{\beta}$ on the one hand and by adding the second and the third one on the other hand.

${ }^{9}$ Remark that the values of $\alpha_{3,1}$ chosen for the computation of the empirical powers only imply small deviation from the mixed form. For other values the empirical power rapidly reaches 1 .
} 
identification problems, perform substantially better than $\xi_{S}$. Remark that $r$ is assumed known and thus the cointegrating rank is correctly specified.

This limited Monte Carlo evidence leads us to propose the following model selection strategy.

1. Start by determining the number of cointegrating vectors, trying to avoid underestimation of $r$. In practice, Johansen's ML statisties complemented by a visual inspection may prove useful to determine an upper bound for $r$,

2. compute the sequences of common feature LR tests $\xi_{S}$ and $\xi_{W}$ and select $s$ for the SF and WF respectively, check whether the number of WF common features exceeds $r$, in which case the WF implies $s-r$ SF common features,

3. for the cases where $s_{1}=\max (1, s-r+1), \ldots, n-r$ compute $\xi_{S W}$ to sclect the appropriate reduced rank structure,

4. for the cases where $s_{1}=\max (1, s-r+1), \ldots, \min (n-r, s)$ compute MF tests $\xi_{M}$,

5. repeat the analysis with $r-i$ cointegrating vectors instead of $r-i+1$, for $i=1, \ldots, r$. For cach case compute the various information criteria.

\subsection{First Application: Co-movements in US Macroeconomic Ag- gregates}

\subsubsection{Background}

A vast amount of empirical macroeconomic literature has studied the long-run implications of the real business cycle models, see e.g. Neusser (1991), King et al. (1991), Kunst and Neusser (1991). With the exception of the work of Issler and Vahid (1996), little work has however been done on short-run co-movements in neoclassical growth models. As in Issler and Vahid (1996) we analyze a simple form of the real business cycle (RBC) model which assumes common trends and common cycles between U.S. per capita real consumption, investment and output. We relax the hypotheses about the number of common feature and cointegrating vectors. More formally, consider the following trivariate system for the logarithms of income $y_{t}$, consumption $c_{t}$ and investment $i_{t}$ put forward by King, Plosser and Rebelo (1988) and analyzed by Issler and Vahid (1996):

$$
\begin{aligned}
c_{t} & =x_{t}^{p}+\bar{c}+\pi_{c} \hat{k}_{t}, \\
i_{t} & =x_{t}^{p}+\bar{\imath}+\pi_{i} \hat{k}_{t}, \\
y_{t} & =x_{t}^{p}+\bar{y}+\pi_{y} \hat{k}_{t},
\end{aligned}
$$

where $x_{t}^{p}=x_{t-1}^{p}+\varepsilon_{t}^{p}$ is the common trend, that is a random walk measuring among other the impact of technology processes, $\bar{y}, \bar{c}$ and $\bar{\imath}$ are the constant steady state values, $\hat{k}_{t}$ is the common 


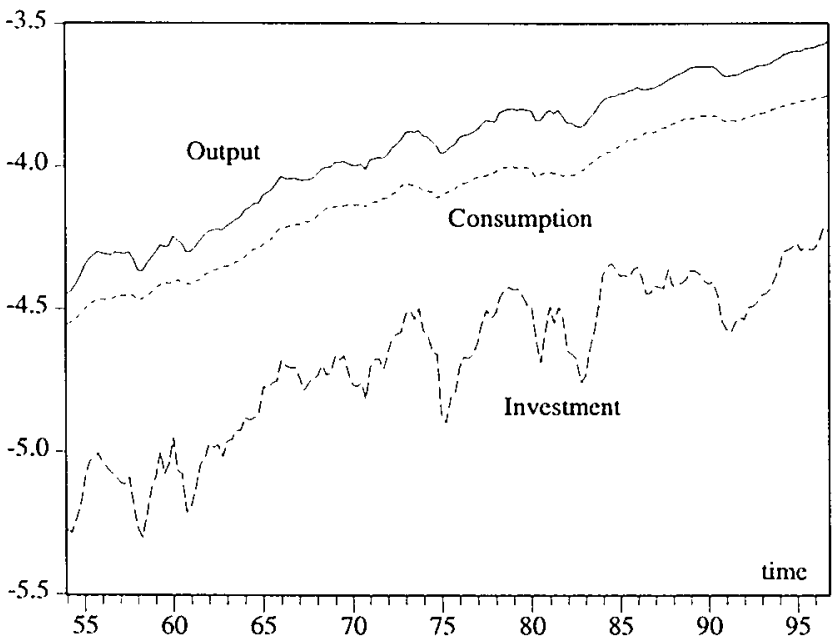

Figure 3.1: Log Levels of Macro Aggregates

cycle, that is the stationary transitory deviation of the capital stock from its steady state value and $\pi_{c}, \pi_{i}$ and $\pi_{y}$ are constant parameters. $\varepsilon_{t}^{p}$ and $\hat{k}_{t}$ may be correlated.

We analyze the period 1954:1 - 1996:4, that is 172 quarterly observations. Data prior to 1954:1 were used as initial observations in regressions that contain lags. Notice that we had the observations from 1948:1 onwards but we preferred, as King et al. (1991) suggested, to exclude turbulent periods during Korean War, price control and Treasury-Fed agreement. The data used are the revised (May 1997) series from the Survey of Current. Business national account for the United States (source BEA). The variables are $c_{t}$ : personal consumption expenditures, $i_{t}$ : gross private domestic investment and the output $y_{t}$ is the GDP less the government expenditures. The three variables have been divided by the size of the civilian population above sixteen years of age. The series are seasonally adjusted and transformed into natural log. Figures $3.1,3.2$ and 3.3 display respectively the log levels, ${ }^{10}$ the growth rates of aggregates and the great ratios with cointegrating vectors $(1-1)$. Figure 3.2 indicates the existence of an outlier in the first quarter of 1975 in the investment variable. While that can alter test statistics, we do not correct for this aberrant value in this chapter (see results in Chapter 4 and 6 where we consider an impulse dummy in 1975:Q1).

\footnotetext{
${ }^{10}$ We add 1 to the log of investment to scale series.
} 


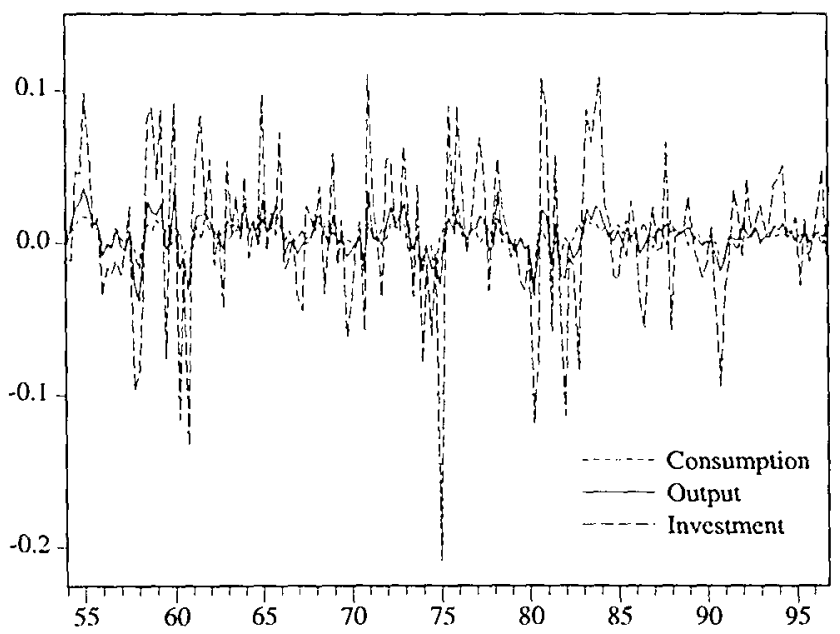

Figure 3.2: Growth Rates of Consumption, Output and Investment

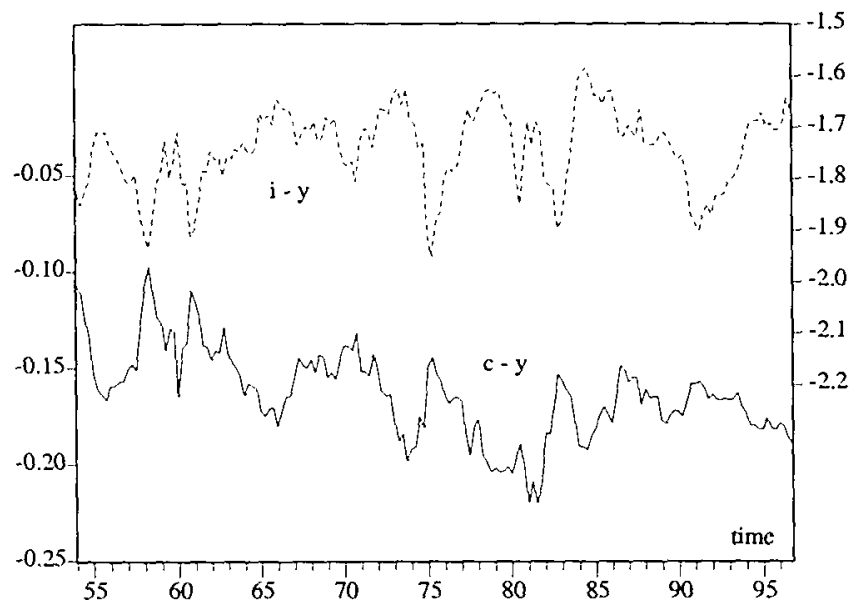

Figure 3.3: Great Ratios 


\begin{tabular}{l|cccc}
\hline \hline & Max.Eig. Test & 95\% cv & Trace Test & $95 \% c v$ \\
\hline$r=0$ & $28.08^{*}$ & 21.0 & $45.96^{*}$ & 29.7 \\
$r \leq 1$ & $14.39^{*}$ & 14.1 & $17.88^{*}$ & 15.4 \\
$r \leq 2$ & 3.48 & 3.8 & 3.48 & 3.8 \\
\hline
\end{tabular}

Table 3.6: Cointegration Tests

\begin{tabular}{c|cccccccc}
\hline \hline$r=2$ & \multicolumn{2}{|c}{$-T \ln \left(1-\lambda_{i}\right)$} & \multicolumn{2}{c}{$d f$} & \multicolumn{2}{c}{$P b>\chi_{d f}^{2}$} & \multicolumn{2}{c}{$P b>\chi_{d f}^{2}$} \\
\hline & $\xi_{S}$ & $\xi_{W}$ & $\xi_{S}$ & $\xi_{W}$ & $\xi_{S}$ & $\xi_{W}$ & $\xi_{S}^{\text {Cor }}$ & $\xi_{W}^{\text {cor }}$ \\
$s \geq 1$ & 20.51 & 10.44 & 12 & 10 & .058 & .402 & .092 & .466 \\
$s \geq 2$ & $(53.54)$ & 33.83 & $(26)$ & 22 & $(.001)$ & .051 & $(.004)$ & .087 \\
$s=3$ & $(150.1)$ & 117.3 & $(42)$ & 36 & $(<.001)<.001$ & $(<.001)<.001$ \\
\hline \hline
\end{tabular}

Table 3.7: Common Feature Tests

\subsubsection{Cointegration and Common Feature Analysis}

The model that best characterizes the covariance structure of the data is a VAR of order 5 (using LR statistics) with an unrestricted intercept in the short-run. Table 3.6 presents the test statistics for the Johansen (1995) rank test of the number of cointegrating relationships with small sample correction and the $5 \%$ critical values.

The results in Table 3.6 indicate that we cannot reject the presence of two cointegrating vectors whose coefficients are not far from the theoretical ones i.e. $c_{t}-y_{t}$ and $i_{t}-y_{t}$ are both $I(0) .{ }^{11}$ The cstimated cointegrated relationships are respectively $c_{t}-\underset{(0.016)}{0.958 y_{t}}$ and $i_{t}-\underset{(0.045)}{1.103 y_{t}}$ where asymptotic standard errors are reported in parentheses. The likelihood ratio test for unit long-run elasticities in both vectors (e.g. stationarity of the great ratios), $\chi_{(2)}^{2}$ distributed under the null, yiclds a value of 3.07 so that these restrictions are not rejected at the $5 \%$ level. For the determination of the number of common feature vectors, we use the sequential likelihood ratio tests presented in Section 3.3. For $p=5$ and $r=2$ fixed we obtain the tests statistics and the $p$-values reported in Table 3.7. $\xi_{S}$ and $\xi_{W}$ respectively denot.c likelihood ratio tests for the number of common features for given cointegrating rank $r$ and a given reduced rank structure. $\xi_{S}^{c o r}$ and $\xi_{W}^{c o r}$ are the small sample corrected version. $\xi_{S W}=\xi_{S}-\xi_{W}$ yields the likelihood ratio statistic for the null of SF against WF for given cointegrating and cofeature rank. For $s=1$, the $\xi_{S W}$ tcst statistic has a value of 10.06 for a $\chi_{(2)}^{2}$ null distribution which yields a rejection probability of .0065 . We reject the SF model in favor of the WF one. The common feature relation we would retain in the SF case is $\Delta c_{t}+0.106 \Delta i_{t}-.959 \Delta y_{t}$. In the class of WF we still have to choose between $s=1$ and $s=2$. The test statistic $\xi_{W}=33.83$ does not reject the null hypothesis of $s \geq 2$, i.e. the presence of at least two common features vectors. Notice that information criteria also favor the WF assumption with $s=2$. With $s=2$ and $r=2$, the WF

\footnotetext{
${ }^{11}$ The traditional Augmented Dickey Fuller unit root tests statistics also strongly reject the null hypothesis. For instance for and $\mathrm{ADF}(4)$ we get the values $\tau_{\mu}=-3.31, \tau_{\tau}=-3.97$ for the variable $c_{t}-y_{t}$ and $\tau_{\mu}=-4.14$, $\tau_{\tau}=-4.23$ for the variable $i_{\ell}-y_{k}$.
} 


\begin{tabular}{|l|cc|cc|cc|}
\hline \multicolumn{2}{|c|}{ Depend.var. } & \multicolumn{2}{c|}{$\Delta c_{t}$} & \multicolumn{2}{|c|}{$\Delta i_{t}$} & \multicolumn{2}{c|}{$\Delta y_{t}$} \\
\hline regressors & coef. & t-value & coef. & t-valuc & coef. & t-valuc \\
\hline common-factor & -0.350 & $(-4.59)$ & -3.392 & $(-7.34)$ & -0.706 & $(-5.65)$ \\
constant & 0.045 & $(2.48)$ & -0.074 & $(-.670)$ & 0.053 & $(1.76)$ \\
$(c-y)_{t-1}$ & 0.103 & $(3.59)$ & 0.399 & $(2.29)$ & 0.192 & $(4.07)$ \\
$(i-y)_{t-1}$ & 0.014 & $(1.60)$ & -0.083 & $(-1.58)$ & 0.010 & $(.672)$ \\
\hline$R^{2}$ & \multicolumn{2}{|c|}{0.17} & \multicolumn{2}{|c|}{0.30} & \multicolumn{2}{c|}{0.24} \\
$D W$ & \multicolumn{2}{|c|}{2.18} & \multicolumn{2}{|c|}{1.92} & \multicolumn{2}{c|}{1.79} \\
\hline Tests & test & p-value & test & p-value & test & p-valuc \\
$B J$ & 7.86 & $(.019)$ & 15.49 & $(.00)$ & 26.38 & $(.00)$ \\
$A R C H\{1: 2]$ & .47 & $(.625)$ & .81 & $(.446)$ & 6.10 & $(.00)$ \\
$L M-A R[1\}$ & 2.55 & $(.111)$ & .37 & $(.544)$ & 2.54 & $(.113)$ \\
$L M-A R[1: 4]$ & 1.97 & $(.101)$ & .13 & $(.971)$ & .94 & $(.442)$ \\
$L M-A R\{1: 12]$ & 1.64 & $(.086)$ & .83 & $(.616)$ & .85 & $(.596)$ \\
$R E S E T$ & .16 & $(.686)$ & .19 & $(.659)$ & .07 & $(.786)$ \\
\hline \hline
\end{tabular}

Table 3.8: Common Dynamic

does not imply a SF common feature since $s-r=0$.

FIML estimates of the WF model, under $s=2$ and permuting the columns of $\tilde{\beta}$ in order to find the vectors with the meaningful economic interpretation, results in the following two cofeature relationships $\Delta c_{t}^{*}-\underset{(0.087)}{0.501} \Delta y_{i}^{*}$ and $\Delta i_{i}^{*}-\underset{(0.481)}{4.776} \Delta y_{i}^{*}$ where asymptotic standard crrors are reported in parentheses and where a ${ }^{*}$ indicates that the corresponding variables are expressed in deviation from long-run effects. ${ }^{12}$ It is seen that these two vectors fit pretty well business cycle stylized facts, c.g. that consumption is smoother than output, investment is more volatile than output and there is a single synchronous cycle which can be extracted using the Stock-WatsonBeveridge-Nelson decomposition developed in Chapter 4.

We can also extract the common 'factor' series $f_{t}$ which is a linear combination of 12 variables: the three variables in the system (3.15)-(3.17) in first differences lagged one up to four periods. Because every growth rate is a function of the same set of variables, the common dynamic factor, a constant and the two lagged cointegrating vectors, we can compute the weights of the common factor by OLS in each regression to see which variable is the most affected by the common dynamic factor. The results are reported in Table 3.8. The usual $t$-statistics are reported in brackets. It is easily seen that the slope coefficients of the common factor form an orthogonal complement of $\tilde{\beta}$. It is interesting to realize that investment is most strongly related to the

\footnotetext{
${ }^{12}$ To check whether the series $\widetilde{\beta}^{\prime}\left(\triangle y_{t}-\alpha \beta^{\prime} y_{t-1}\right)$ are white noise, the series and their autocorrelation functions were analyzed. Approximate standard-errors for the estimated autocorrelations are .077 . All estimated autocorrelations were found to be smaller than .2 in absolute value. Although in some instances, an autocorrelation coefficient lies outside the \pm 2 standard-error band, the results suggest that the series are approximately white noise. The LM-test of autocorrelation takes values 1.115 (.351) and .855 (.661) respectively at lag 12 and 21 for the first series, with $p$-values given between parentheses. For the second series, the corresponding values are 1.256 $(.249)$ and $1.338(.149)$.
} 
common factor, a finding that is quite plausible given that in the WF case, the common factor reflects common short-run fluctuations in the series. The presence of GARCH and non-normality of the disturbances as indicated by the model diagnostics in Table 3.8 result from a few outliers. Notice that non-normality does not modify the size and power of common feature tests while GARCH residuals lead to over-rejection of the null hypothesis (see Chapter 2 and Beine and Hecq, 1999). This does not seem to be the case in our application.

The final step is to investigate the potential presence of MF common features. The test statistic $\xi_{M}^{*}$ for $s_{1}=1$ and $s_{1}=2$, takes the values 2.077 and 21.723 respectively. If $\tilde{\beta}$ was known, these statistics would respectively be $\chi_{(1)}^{2}$ and $\chi_{(2)}^{2}$ distributed under the null hypothesis and we would hence not reject the existence of one mixed form cofeature vector. Notice that from the eigenvalues (and their associated eigenvectors) used to calculate $\xi_{M}$ we may easily obtain the matrices entering the restricted representation (3.12). In our particular case, $A$ becomes a scalar and equals $0.02384 \approx 0$ so that identification problems are avoided (see Section 3.3). Assuming that the first vector is of a strong form, FIML estimation of the MF model with unknown $\tilde{\beta}$ leads to the following two cofeature linear combinations $\Delta c_{t}-\underset{(0.191)}{0.639} \Delta y_{t}+\underset{(0.044)}{0.039} \Delta i_{t}$ and $\Delta i_{t}^{*}-\underset{(0.475)}{4.866} \Delta y_{t}^{*}$. The likelihood ratio test of the MF against the WF is distributed $\chi^{2}(1)$ under the null of the MF. Its value is 1.472 . The likelihood ratio test for the additional restriction that the coefficient of $\Delta i_{t}$ in the first cofeature vector is zero has a value of 0.592 . It is also $\chi^{2}(1)$ distributed. Consequently, we may reestimate the MF model by FIML which leads to the following two cofeature relations $\Delta c_{t}-\underset{(0.067)}{0.485} \Delta y_{t}$ and $\Delta i_{t}^{*}-\underset{(0.467)}{4.816} \Delta y_{t}^{*}$. Notice that a MF model that assumes that the first vector is of a weak form is rejected at any reasonable significance level ( $p$-value less than 0.001).

At this stage it may be interesting to note the sharp reduction in the number of parameters that have to be estimated once we impose (valid) reduced rank structures. The unrestricted trivariate VAR(5) model contains 45 unknown parameters. Under cointegration with $r=2$, the number of unknown parameters reduces to 44 . With one SF cofeature vector it reduces to 32 while with two weak form cofeature vectors the number becomes 22 . Note finally that the mixed form only contains 20 unknown parameters.

\subsection{A Second Example: UK Equity, Gilt and Money Markets}

We do not want to end this chapter in giving the impression that the SCCF model must be thrown away. Instead, we claim that "lighter" specifications are worth considering when restrictions under the strong form are rejected. We illustrate the techniques developed in this chapter by means of a neat application where it is sensible to test the WF versus the SF due to the fulfillment of the condition $r+s \leq n$ for both specifications. We consider the relationship between the UK equity, gilt and money markets already analyzed in Mills $(1998,1999)$. In his analysis, Mills tests for cointegration and SCCF among four time series: FTA 500 equity index $(P)$, the associated dividend index $(D)$, the 20 year gilt yield $(R 20)$ and the yield on treasury bills $(R S)$ for the period 
from January 1969 to December 1995, i.e. 324 monthly observations. ${ }^{1.3}$ After an evaluation of different specifications, the best. model retained by Mills is a VAR or order two in log levels with two cointegrating vectors and a deterministic trend constrained in the long-run. We refer to Mills for details as well as for figures of time series and long-run relationships.

Beyond the presence of two cointegrating vectors, no SCCF relations are found and Mills (1998, p295) explains that this result is due to the fact that the linear combination is still correlated with the error correction terms. This argument exactly matches our previous observation on different data sets and pleas in favor of a weak form direction.

We can not therefore miss the opportunity to test for the SF against the WF on Mills' data. We choose either $p=2$ or $p=3$ to assess the stability of the outcome to the lag length selection. We condition the co-feature analysis upon two cointegrating vectors that we estimate in a first step (obviously they are reestimated under either $p=2$ and $p=3$ ) and we keep a linear deterministic trend in the long-run. In Chapter 2 we have seen that inference is sensitive to phenomena such as the presence of ARCH processes or simply aberrant values. Using a BoxPlot analysis we have detected huge aberrant values in $1975 \mathrm{~m} 1$ and $1987 \mathrm{~m} 10$ for $\Delta \ln P_{t}$ and in $1979 \mathrm{~m} 7$ and $1993 \mathrm{~m} 3$ for $\Delta \ln D_{t}$. We thus add unrestricted impulse dummies in the system for these months. The outliers for $P_{t}$ are due to the strong rebound of equities in 1975 and, of course, the crash of 1987 . The 1979 outlier for $D_{t}$ is related to the Budget of that year.

Tables 3.9 reports outcomes for the system without dummies. It emerges that we do not find any SCCF vector (in accordance with Mills, 1998) but we do not reject the presence of one and maybc two WF relationships. Table 3.10 reports the same statistics computed with four impulse dummics. Both for $p=2$ and $p=3$, we do not reject the presence of at most one co-feature vector. Testing SF versus WF, we obtain values of 4.38 and 3.09 for respectively $p=2$ and $p=3$. This test statistics follows a $\chi^{2}(2)$ under the null and consequently we retain one SCCF vector given by (FML estimates with standard errors in brackets) $\Delta \ln P_{t}=-.76 \Delta \ln R 20_{t}+$ $.35 \Delta \ln D_{t}+\underset{(.12)}{.08} \Delta \ln R S_{t}$ for $p=2$ and $\Delta \ln P_{t}=-. .79 \Delta \ln R 20_{t}+\underset{(.069)}{.06} \Delta \ln D_{t}+\underset{(.12)}{.10} \Delta \ln R S_{t}$ for $p=3$. With both lags there scems to exist a SCCF relation between equities growth rate and long term interest rates.

\subsection{Conclusion}

In this chapter, we studied a linear Gaussian VAR model with nonstationary but cointegrated variables that have common cyclical features.

We introduced the concepts of strong, weak and mixed form reduced rank structures and discussed their implications for the VAR. SF reduced rank structures arise when the common features are such that there exists one or several linear combinations of the set of variables under investigation expressed in first differences which are white noise. The existence of a WF reduced

\footnotetext{
${ }^{13}$ Data are available on Mills' website http://info.lboro.ac.uk/departments/ec/staff/index.hlml. I would like to thank Terence Mills who has kindly allowed me to use his data set.
} 


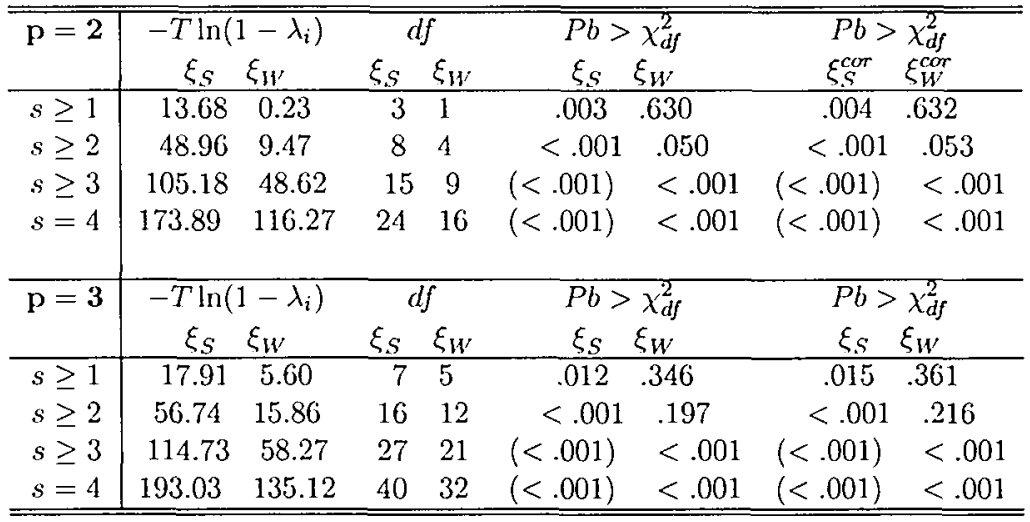

Table 3.9: WF and SF Test Statistics without Dummies $(p=2,3)$

\begin{tabular}{|c|c|c|c|c|c|c|c|c|}
\hline$p=2$ & $\begin{array}{c}-T \ln ( \\
\xi_{S}\end{array}$ & $\begin{array}{l}\left.1-\lambda_{i}\right) \\
\xi_{W}\end{array}$ & $\xi_{S}{ }^{a}$ & $\xi_{W}$ & $\begin{array}{c}P b> \\
\xi_{S}\end{array}$ & $\begin{array}{l}\chi_{d f}^{2} \\
\xi_{W}\end{array}$ & $\begin{array}{l}P \mathrm{~Pb}> \\
\xi_{S}^{c o r} \\
\end{array}$ & $\begin{array}{l}\chi_{d f}^{2} \\
\xi_{W}^{c o r}\end{array}$ \\
\hline$s \geq 1$ & 5.45 & 1.07 & 3 & 1 & .141 & .299 & .147 & .302 \\
\hline$s>2$ & 43.67 & 21.36 & 8 & 4 & $<.001$ & $<.001$ & $<.001$ & $<.001$ \\
\hline$s \geq 3$ & 109.28 & 63.08 & 15 & 9 & $<.001)$ & $<.0$ & $<.001)$ & $<.0$ \\
\hline$s=4$ & 178.60 & 131.00 & 24 & 16 & $(<.001)$ & $<.001$ & $(<.001)$ & $<.001$ \\
\hline$p=3$ & $\begin{array}{c}-T \ln ( \\
\xi_{S}\end{array}$ & $\begin{array}{l}\left.1-\lambda_{i}\right) \\
\xi_{W}\end{array}$ & $\xi_{S}$ & $f_{w}$ & $\begin{array}{c}\mathrm{Pb} \\
\xi_{S}\end{array}$ & $\begin{array}{l}\chi_{d f}^{2} \\
\xi_{W}\end{array}$ & $\begin{array}{l}P b> \\
\xi_{S}\end{array}$ & $\begin{array}{l}\chi_{d f}^{2} \\
\xi_{W}\end{array}$ \\
\hline$s \geq 1$ & 12.61 & 9.52 & 7 & 5 & .082 & .089 & .093 & .098 \\
\hline$s \geq 2$ & 53.95 & 31.27 & 16 & 12 & $<.001$ & .002 & $<.001$ & .002 \\
\hline$s \geq 3$ & 120.94 & 74.74 & 27 & 21 & $<.001)$ & $<.00$ & $(<.001)$ & $<.001$ \\
\hline$s=4$ & 197.94 & 151.03 & 40 & 32 & $(<.001)$ & $<.001$ & $(<.001)$ & $<.001$ \\
\hline
\end{tabular}

Table 3.10: WF and SF Test Statistics with Dummies $(p=2,3)$ 
rank structure implies that linear combinations of the first differences of the variables in the model in deviation from the long-run relationships are white noise. We showed that the constraint that the number of common features plus the number of cointegrating relationships should be less than or equal to the number of variables no longer applies when the WF is analyzed. This allows to consider more significant long-run relationships between the variables in first differences. It. also yiclds an efficiency increase for the estimates, resulting from the reduction in the number of free parameters to be estimated.

We designed a modeling strategy and proposed likelihood ratio tests for the three types of reduced rank structures. Wo studied the small sample properties of the test using Monte Carlo simulations. It appeared that in particular under SF it is of great importance to correctly determine the cointegrating rank before testing SF against WF. An empirical analysis of the relationship between the consumption, investment and real GDP leads to the conclusion that the existence of a WF reduced rank structure with one common trend and one weak form common cycle is not rejected by the information in the series. The presence of two common feature vectors means that the short-run dynamics of the system is governed by a single weak form common cycle as it will be shown in Chapter 4 where we present the Stock-Watson-Beveridge-Nelson decompositions of $y_{t}$ into permanent and transitory components for the SF and the WF reduced rank structures. 


\title{
Chapter 4
}

\section{Permanent-Transitory}

\section{Decomposition in VAR Models with Cointegration and Common Cycles}

\begin{abstract}
In this chapter we derive permanent-transitory decompositions of non-stationary multiple times series generated by finite order Gaussian VAR(p) models with both cointegration and serial correlation common features. We extend existing analyses to the two classes of reduced rank structures discussed in Chapter 3. Using the corresponding state space representation of cointegrated VAR models in vector error correction form we show how decompositions can be obtained even in the case where the number of common feature and cointegration vectors is not equal to the number of variables. Empirical analyses of US business fluctuation and of comovements in international stock markets show the practical relevance of the approach we propose.
\end{abstract}

\subsection{Introduction}

The purpose of this chapter is to derive permanent-transitory decompositions of nonstationary multiple times series generated by a finitc order Gaussian VAR $(p)$ model with both cointegration and serial correlation common features. For cointegrated processes (without common cyclical features restrictions), several permanent-transitory decompositions have been extensively used in empirical and theoretical analyses: these include the multivariate extension of the BeveridgeNelson (B-N) decomposition proposed by Stock and Watson (1988), the observable permanenttransitory decomposition of Gonzalo and Granger (1995) where the components are identified as being combinations of the observable series. Engle and Kozicki (1993) introduced the concepts of serial correlation common features in cointegrated VAR models and Vahid and Engle (1993) gave

${ }^{0}$ This chapter draw's on Hecq, Palm and Urbain (2000a) and on Hecq, Palm and Urbain (2000d) for the Section 4.3.2. 
conditions under which the eyclical component of multiple time series (三 transitory component) can be represented by a reduced number of common stochastic cycles. The decompositions into permanent and transitory components in the latter framework were initially obtained under the strong assumption that the number of common cycles arising from common cyclical features and the number of common trends arising from cointegration add up to the number of variables in the system. In this case, the Stock-Watson and the Granger-Gonzalo decompositions coincide (Proietti, 1997). An interesting extension was recently obtained by Proietti (1997) who showed how feasible decompositions could be obtained even in the case where the sum of the number of common feature vectors and the number of cointegrating vectors is less than the dimension of the system.

In this chapter we extend the aforementioned analyses to the finite order Gaussian VAR models with two cointegration and common cyclical features structures analyzed in Chapter 3. In the standard case both long run and short run dynamics matrices are nested in such a way that linear combinations of the variables in first differences are innovation processes w.r.t. to past information set (SCCF or SF). The second class of structures arises when linear combinations of the variables in first differences corrected for the long run (cointegration) relations are innovation processes w.r.t. to past information (WF). The framework is similar to that of Vahid and Engle (1993), but less restrictive as they explicitly consider linear combinations of the first differenced I(1) variables that are allowed to be predictable at low frequencies. We have seen in Chapter 3 the implications of both cases for the number of common cycles are quite different. In the standard case, the upper bound on the number of common feature vectors is given by the number of common trends. In the latter case the upper bound is simply the number of time series minus one. We believe that in applications such as business cycle analysis it is important to dispose of the strong assumption that long-run relationships between variables and short-run dynamics are interrelated. In particular, an interesting issue is concerned with the question whether the observed variables in first differences corrected for the long-run relationships exhibit common features. Macroeconomic applications will illustrate the usefulness of our theoretical results for time series modeling.

We propose a permanent-transitory decomposition which satisfies three criteria. Firstly, the decomposition we study is expressed in terms of observable variables and only involves quantitics already available from the vector error correction model (VECM) and the estimation of common features and cointegrating vectors. The approach we follow is similar to that of Proietti (1997). Secondly, the decomposition takes into account cointegrating and common cyclical feature restrictions. An important case is when the number of common feature vectors is equal to or smaller than the number of common trends in the SF framework. We extend this analysis to the case where the number of common feature vectors has as upper bound the number of time series minus one in the WF structure. Thirdly, this decomposition should not only be a permanent-transitory decomposition but also a common trend-common cycle decomposition. Intuitively this means that common trends disappear when premultiplying the vectors of time series by the cointegrating matrix and the common feature matrix annuls the common cycles. 
This Chapter is organized as follows. In Section 4.2 we introduce the state space representation of the cointegrated process and we derive a permanent-transitory decompositions of SCCF and WF. We present two empirical analyses in Section 4.3.

\subsection{Permanent-Transitory Decompositions}

Let us consider a VECM with the properties given in Chapter 3

$$
\Delta y_{t}=m+\alpha \beta^{\prime} y_{t-1}+\sum_{i=1}^{p-1} \Gamma_{i} \Delta y_{t-i}+\varepsilon_{t}, \quad t=1, \ldots, T .
$$

Throughout this chapter we will again assume that $p$ is known. We consider both the SCCF (or SF) and the weak form reduced rank structure, i.c. models such that respectively $\tilde{\beta}^{\prime}(\Delta y / t-$ $m)=\tilde{\beta}^{\prime} \varepsilon_{t}$ or $\tilde{\beta}^{\prime}\left(\Delta y_{t}-m-\alpha \beta^{\prime} y_{t-1}\right)=\tilde{\beta}^{\prime} \varepsilon_{t}$ are $s$-dimensional vector mean innovation process with respect to the information set $\Im_{t}$ containing the past of the series. We refer to Assumptions 1,2 and 3 of Chapter 3 in the sequel.

We consider the following multivariate version of the Beveridge-Nelson (B-N) permanenttransitory (P-T) decomposition:

Definition 4.1 (B-N Permanent-Transitory Decomposition) Let $y_{t}$ be a n-dimensional integrated process of order one generated by (4.1). A permanent-transitory decomposition of $y_{t}$ is a pair of processes $\left(\mu_{t}, \psi_{t}\right)$, such that: $(i) \mu_{t}$ is a random walk process while $\psi_{t}$ is a covariance stationary process, (ii) $\operatorname{Var}\left(\Delta \mu_{t}\right)$ and $\operatorname{Var}\left(\Delta \psi_{t}\right)$ are strictly positive, (iii) $y_{t}=\mu_{t}+\psi_{t}$, (iv) both $\mu_{t}$ and $\psi_{t}$ should be functions of the observable variables, $(v)$ if there exists a cointegrating matrix $\beta$ satisfying Assumption 1 and a cofeature matrix $\tilde{\beta}$ satisfying either both Assumption 2 and 3 of Chapter $3(S F)$ or Assumption 2 only $(W F)$, then $\tilde{\beta} \psi_{t}=0$ and $\beta^{\prime} \mu_{t}=0$.

The definition is in line with those used by Quah (1991) and Gonzalo and Granger (1995) with the exception that we require $\mu_{t}$ to be a random walk while Quah (1991) and Gonzalo and Granger (1995) assume $\mu_{t}$ to be differenced stationary. $\mu_{t}$ is then called the (common) permanent component of $y_{\ell}$ while $\psi_{t}$ represents the (common) transitory covariance stationary component of $y_{t}$. In line with the existing related literature the latter component will often be labelled the common cycle although one should recognize that situations may occur where this terminology is not appropriate. ${ }^{1}$ The decomposition may also be labelled orthogonal if we add the requirement that $\Delta \mu_{i}$ is uncorrelated with $\psi_{t}$ at all leads and lags. Contrary to Quah (1991) we will not require the last property to hold in general. It is interesting however to contrast this definition with that underlying the Gonzalo-Granger (1995) definition of a P-T decomposition. The major difference comes from the random walk nature of the permanent component and from $(v)$ which requires that the cointegrating matrix annihilates the common stochastic trends of

\footnotetext{
${ }^{1}$ See footnote 1 of Chapter 3.
} 
the multivariate process $y_{t}$, while the cofeature matrix should annihilate the transitory (cyclical) component. The Gonzalo and Granger (1995) definition requires the common factors to be linear in the observable variables and also requires the change in the permanent component not to be Granger-caused by the transitory component. This last requirement is satisfied under $(i)-(v)$. Notice also under $(i)-(v)$ the decomposition is not only a permanent-transitory decomposition but also a common permanent-transitory decomposition.

\subsubsection{Cointegrated VAR Models}

Let us first rewrite the VECM (4.1) in a state space form such that

$$
\begin{aligned}
\Delta y_{t} & =Z f_{t}, \\
f_{t} & =c+Q f_{t-1}+Z^{\prime} \varepsilon_{t} \quad t=1, \ldots, T,
\end{aligned}
$$

with

$$
f_{t}=\left[\begin{array}{l}
\Delta y_{t} \\
\Delta y_{t-1} \\
\vdots \\
\Delta y_{t-p+2} \\
\beta^{\prime} y_{t-1}
\end{array}\right], Q=\left[\begin{array}{ccccc}
\Gamma_{1}+\alpha \beta^{\prime} & \Gamma_{2} & \cdots & \Gamma_{p-1} & \alpha \\
I_{n} & 0_{n \times n} & \cdots & 0_{n \times n} & 0_{n \times r} \\
0_{n \times n} & \ddots & \ddots & \vdots & \vdots \\
\vdots & \ddots & \ddots & \vdots & \vdots \\
\beta^{\prime} & 0_{r \times n} & \cdots & \cdots & I_{r}
\end{array}\right]
$$

where $f_{t}$ a $(n(p-1)+r) \times 1$ vector, $Z=\left[I_{n}, 0_{n \times n}, \ldots, 0_{n \times r}\right]$ a $(n \times(n(p-1)+r))$ matrix, $Q$ a $(n(p-1)+r) \times(n(p-1)+r)$ matrix, and $c^{\prime}=\left[m^{\prime}, 0_{(1 \times n)}, \cdots, 0_{(1 \times n)}, 0_{(1 \times r)}\right]$ a vector of dimension $1 \times(n(p-1)+r) \cdot(4.2)$ is the measurement equation while (4.3) is the transition equation. ${ }^{2}$ Stability conditions for the state-space representation (4.2)-(4.3) are satisfied under Assumption 1 and the condition that $\Gamma(1)-\alpha \beta^{\prime}$ and $\beta^{\prime}\left(\Gamma(1)-\alpha \beta^{\prime}\right)^{-1} \alpha$ have rank $n$ and $r$ respectively. The nonsingularity of $\Gamma(1)-\alpha \beta^{\prime}$ is satisfied under the assumption that the variables are at most integrated of order one and hence these rank conditions play the same role as the condition that. $\alpha_{\perp}^{\prime} \Gamma(1) \beta_{\perp}$ is of rank $n-r$ in Johansen (1995), see also Proietti (1997).

Consider the non-zero drift $\operatorname{case}^{3}$ (i.e. when the unconditional mean $E\left(\Delta y_{t}\right) \neq 0$ ) of the multivariate Beveridge-Nelson (B-N) decomposition where the trend ( $\mu_{t} \equiv$ permanent component) is represented by a random walk and the cycle $\left(\psi_{t} \equiv\right.$ transitory component) by a weakly stationary process :

$$
y_{t}=\mu_{t}+\psi_{t}
$$

\footnotetext{
${ }^{2}$ Note that Proietti (1997) writes the VAR in an interim multiplier representation system while we keep the form with the error correction term lagged of one period.

${ }^{3}$ The most interesting cases arise when the drift is non-zero, as it allows for an autonomous upward or downward movement in the data which is often encountered in empirical work. The driftless model is thus just a particular case of the more general one.
} 
where

$$
\mu_{\ell}=y_{l}+\left\{\lim _{l \rightarrow \infty} \sum_{i=1}^{l} \Delta \hat{y}_{t+i \mid t}-E\left(\Delta y_{l}\right)\right\},
$$

is the trend component, that is the value the series would take if it were on its long run path. $\Delta \hat{y}_{t+i \mid t}$ denotes the $i t h$-step ahead best lincar unbiased forecast of $\Delta y_{t}$ based on the information set $\Im_{\ell}$ that is the optimal prediction in the minimum mean square sense. Following Proietti (1997) and Harvey (1989) we assume that the mean rate of the drift is constant over the sample. By successive substitution of (4.3) we get the expected value of the drift $c_{i}^{*}=\left(\sum_{0}^{T-1} Q^{i}\right) c$ which under the stability conditions is equal to $\left(I_{(n(p-1)+r)}-Q\right)^{-1} c=c^{*}$ when $T \rightarrow \infty$. We then transfer both $c$ and $c^{*}$ to the measurement equation to get:

$$
\begin{aligned}
\Delta y_{t} & =Z \int_{t}^{*}+Z c^{*}, \\
f_{t}^{*} & =Q f_{t-1}^{*}+Z^{\prime} \varepsilon_{t,} \quad t=1, \ldots, T,
\end{aligned}
$$

where $f_{t}^{*}=f_{t}-c^{*}$. Since $\Delta \hat{y}_{t+i \mid t}=Z Q^{i} f_{l \mid}^{*}$, where $f_{t \mid l}^{*}$ is the updated estimate of the statc vector yiclded by the Kalman filter, we know that under the stability conditions the expression $\sum_{i=1}^{\infty} Q^{i}$ converges to $\left(I_{(n(p-1)+r)}-Q\right)^{-1} Q$ and $\psi_{t}=-Z\left(I_{(n(p-1)+r)}-Q\right)^{-1} Q f_{t \mid t}^{*}$. Since the components of $f_{t}^{*}$ have already been observed at time $t$, we have $f_{t \mid t}^{*}=f_{t}^{*}$. After some straightforward but tedious matrix manipulations ${ }^{5}$ we may state the following proposition for the VECM representation of a VAR. The proposition restates Proietti's (1997) result for the interin multiplier representation.

Proposition 4.2 Under Assumption 1 and for the VECM (4.1) case, the decomposition: $y_{1}=$ $\mu_{t}+\psi_{t}$, provides a $B$-N permanent $\left(\mu_{t}\right)$ - transitory $\left(\psi_{t}\right)$ decomposition of $y_{t}$ where

$$
\begin{aligned}
\psi_{t}= & -(I-P)\left(\Gamma(1)-\alpha \beta^{\prime}\right)^{-1} \Gamma^{*}(L) \Delta y_{t}+P y_{t}, \\
= & \psi_{2 t}+\psi_{1 t}, \\
& \mu_{t}=(I-P)\left(\Gamma(1)-\alpha \beta^{\prime}\right)^{-1} \Gamma(L) y_{\ell},
\end{aligned}
$$

where $\Gamma(L)=I_{n}-\Gamma_{1} L-\ldots-\Gamma_{p-1} L^{p-1}=\Gamma(1)+\Delta \Gamma^{*}(L)$, with $\Gamma(1)=\left(I_{n}-\Gamma_{1}-\ldots-\Gamma_{p-1}\right)$.

\footnotetext{
${ }^{4}$ Notice that the only difference between the drift and the driftless case is that the updated estimate of the state at time $t$ is equal to $f_{t \mid t}^{*}=\int_{t}-c^{*}$. That means that the unconditional mean, which is constant over the sample, is substracted from the observations.

${ }^{5} A$ detailed proof for a VAR(2) is presented in Appendix.
} 


$$
\begin{gathered}
\Gamma^{*}(L)=\Gamma_{0}^{*}+\Gamma_{1}^{*} L+\ldots+\Gamma_{p-2}^{*-2} L^{p \cdots 2}, \Gamma_{j}^{*}=\sum_{i=j+1}^{p-1} \Gamma_{i} \text { and where } \\
P=\left(\Gamma(1)-\alpha \beta^{\prime}\right)^{-1} \alpha\left[\beta^{\prime}\left(\Gamma(1)-\alpha \beta^{\prime}\right)^{-1} \alpha\right]^{-1} \beta^{\prime}
\end{gathered}
$$

is a $(n \times n)$ matrix satisfying the following set of properties

$$
\begin{array}{llll}
\text { 1. } & \beta^{\prime} P=\beta^{\prime} & \text { 2. } & P\left(\Gamma(1)-\alpha \beta^{\prime}\right)^{-1} \alpha=\left(\Gamma(1)-\alpha \beta^{\prime}\right)^{-1} \alpha \\
\text { 3. } & P^{i}=P & \text { 4. } & \alpha_{\perp}^{\prime} \Gamma(1) P=0 \\
\text { 5. } & P \beta_{\perp}=0 & \text { 6. } & \operatorname{rank}(P)=r \text { and } \operatorname{rank}(I-P)=n-r
\end{array}
$$

The proof is similar to the one proposed in Proietti (1997) and omitted here to save space. Using the expressions for $c^{*}=\left(I_{(n(p-1)+r)}-Q\right)^{-1} c$ we obtain:

$$
\begin{gathered}
E\left(\Delta y_{t}\right)=E\left(\Delta y_{t-1}\right)=\cdots E\left(\Delta y_{t-p+1}\right)=Z c^{*}=(I-P)\left(\Gamma(1)-\alpha \beta^{\prime}\right)^{-1} m, \\
E\left(\beta^{\prime} y_{t-1}\right)=-\left[\beta^{\prime}\left(\Gamma(1)-\alpha \beta^{\prime}\right)^{-1} \alpha\right]^{-1} \beta^{\prime}\left(\Gamma(1)-\alpha \beta^{\prime}\right)^{-1} m,
\end{gathered}
$$

so that the trend/cycle decomposition for the non-zero drift case is obtained by replacing $\Delta y_{t}$ by $\Delta y_{t}-Z c^{*}$ in equation (4.8). We can either substract the empirical mean of $P y_{t}$ in equation (4.8) or use the expression $\left(\Gamma(1)-\alpha \beta^{\prime}\right)^{-1} \alpha\left[\beta^{\prime}\left(\Gamma(1)-\alpha \beta^{\prime}\right)^{-1} \alpha\right]^{-1} E\left(\beta^{\prime} y_{t-1}\right)$, where $E\left(\beta^{\prime} y_{t-1}\right)$ is evaluated in (4.11). In order to make the common trends appear more explicitly, we use the fact that $\alpha\left(\alpha^{\prime} \alpha\right)^{-1} \alpha^{\prime}+\alpha_{\perp}\left(\alpha_{\perp}^{\prime} \alpha_{\perp}\right)^{-1} \alpha_{\perp}^{\prime}=I_{n}$, insert this expression in front of $\Gamma(L) y_{t}$ in $(4.9)$ and by property 2 of the $P$ matrix given in Proposition 4.2 we obtain:

$$
\begin{aligned}
\mu_{t} & =(I-P)\left(\Gamma(1)-\alpha \beta^{\prime}\right)^{-1}\left[\alpha\left(\alpha^{\prime} \alpha\right)^{-1} \alpha^{\prime}+\alpha_{\perp}\left(\alpha_{\perp}^{\prime} \alpha_{\perp}\right)^{-1} \alpha_{\perp}^{\prime}\right] \Gamma(L) y_{t} \\
& =(I-P)\left(\Gamma(1)-\alpha \beta^{\prime}\right)^{-1} \alpha_{\perp}\left(\alpha_{\perp}^{\prime} \alpha_{\perp}\right)^{-1} \alpha_{\perp}^{\prime} \Gamma(L) y_{t}
\end{aligned}
$$

Hence the common trends are given by $\alpha_{\perp}^{\prime} \Gamma(L) y_{t}$ (see inter alia Johansen, 1995) and the corresponding loadings are $(I-P)\left(\Gamma(1)-\alpha \beta^{\prime}\right)^{-1} \alpha_{\perp}\left(\alpha_{\perp}^{\prime} \alpha_{\perp}\right)^{-1}$. The form of the cyclical component $\psi_{t}$ also allows us to distinguish between the part linked to the process of adjustment to equilibrium $\psi_{1 \ell}=P y_{t}$ and $\psi_{2 t}=-(I-P)\left(\Gamma(1)-\alpha \beta^{\prime}\right)^{-1} \Gamma^{*}(L) \Delta y_{t}$. The former part is specific to cointegrated processes and has the cointegrating relationships as cycle generators while the latter are the short run fluctuations in a stricter sense. If we take the first difference of $\mu_{t}$ and substitute (4.1) for $\Gamma(L) \Delta y$ we obtain

$$
\begin{aligned}
\Delta \mu_{t} & =(I-P)\left(\Gamma(1)-\alpha \beta^{\prime}\right)^{-1} \alpha_{\perp}\left(\alpha_{\perp}^{\prime} \alpha_{\perp}\right)^{-1} \alpha_{\perp}^{\prime} \Gamma(L) \Delta y_{t} \\
& =(I-P)\left(\Gamma(1)-\alpha \beta^{\prime}\right)^{-1} \alpha_{\perp}\left(\alpha_{\perp}^{\prime} \alpha_{\perp}\right)^{-1} \alpha_{\perp}^{\prime}\left(\alpha \beta^{\prime} y_{t-1}+m+\varepsilon_{t}\right), \\
& =(I-P)\left(\Gamma(1)-\alpha \beta^{\prime}\right)^{-1} \alpha_{\perp}\left(\alpha_{\perp}^{\prime} \alpha_{\perp}\right)^{-1} \alpha_{\perp}^{\prime}\left(m+\varepsilon_{t}\right),
\end{aligned}
$$

which shows that the trend component of the decomposition is a random walk with $(m \neq 0)$ or 
without $(m=0)$ drift. This last expression also shows that any perturbation of the transitory component $\psi_{t}$ does not have any long-run effects on the permanent component.

In the next subsection we take the common cyclical feature restrictions into account and distinguish between the two models introduced in the previous section.

\subsubsection{WF versus $\mathrm{SF}$}

The previous section restated the results of Proietti (1997) which allow to get a B-N type decomposition where the permanent and the transitory parts are linear combinations of observable variables. Now we show how to take into account common feature restrictions both in SF and WT cases.

In order to impose common feature restrictions, consider the orthogonal projection of the transitory component $\psi_{t}$ into the space spanned by $\tilde{\beta}$ and its orthogonal complement. Wc therefore postmultiply the orthogonal matrix $\tilde{\beta}\left(\tilde{\beta}^{\prime} \tilde{\beta}\right)^{-1} \tilde{\beta}^{\prime}+\tilde{\beta}_{\perp}\left(\tilde{\beta}_{\perp}^{\prime} \tilde{\beta}_{\perp}\right)^{-1} \tilde{\beta}_{\perp}^{\prime}=I_{n}$ by the transitory part $\psi_{t}=\psi_{2 t}+\psi_{1 t}$. This transitory part is now formed by four elements, i.c. $\psi_{t}=\psi_{2 A t}+\psi_{1 A t}+$ $\psi_{2 B t}+\psi_{1 B t}$ with $\tilde{\beta}$ and $\tilde{\beta}_{\perp}$ being involved in the $A$ and $B$ parts respectively. Propositions 4.3 and 4.4 show which part may be simplified in the SF and the WF cases respectively.

Proposition 4.3 If Assumptions 1, 2 and 3 of Chapter 3 hold (SF), the B-N decomposition of $y_{t}$ is given by $y_{t}=\mu_{S, t}+\psi_{S, t}$ where

$$
\begin{aligned}
\psi_{S, t}= & \psi_{2 B t}+\psi_{1 B t}, \\
= & -\tilde{\beta}_{\perp}\left(\tilde{\beta}_{\perp}^{\prime} \tilde{\beta}_{\perp}\right)^{-1} \tilde{\beta}_{\perp}^{\prime}(I-P)\left(\Gamma(1)-\alpha \beta^{\prime}\right)^{-1} \Gamma^{*}(L) \Delta y_{t} \\
& +\tilde{\beta}_{\perp}\left(\tilde{\beta}_{\perp}^{\prime} \tilde{\beta}_{\perp}\right)^{-1} \tilde{\beta}_{\perp}^{\prime} P y_{t}, \\
\mu_{S, t}= & y_{t}-\psi_{S, t}^{\prime}
\end{aligned}
$$

Proof. To show that the first two parts disappear, notice that by Assumption 2, $\tilde{\beta}^{\prime}(\Gamma(1)-$ $\left.\alpha \beta^{\prime}\right)=\tilde{\beta}^{\prime}, \tilde{\beta}^{\prime}\left(\Gamma(1)-\alpha \beta^{\prime}\right)^{-1}=\tilde{\beta}^{\prime}\left(\Gamma(1)-\alpha \beta^{\prime}\right)\left(\Gamma(1)-\alpha \beta^{\prime}\right)^{-1}=\tilde{\beta}^{\prime}$ and $\tilde{\beta}^{\prime} P=0$, so that $\tilde{\beta}^{\prime} \psi_{2 A t}=0$ and $\tilde{\beta}^{\prime} \psi_{1 A t}^{\prime}=0$.

The decomposition presented in Proposition 4.3 satisfies all of the conditions in Definition 4.1, since the common cyclical feature restrictions are explicitly taken into account and $\tilde{\beta}^{\prime}{ }^{\prime}{ }_{S, t}=0$ by definition of orthogonal complements.

Proposition 4.4 If Assumptions 1 and 2 of Chapter 3 hold (WF), the B-N decomposition of $y_{t}$ is given by $y_{t}=\mu_{W, t}+\psi_{W, t}$ where

$$
\begin{aligned}
& \psi_{W, t}=\psi_{2 B t}+\psi_{1 B t}+\psi_{2 A t}^{*}+\psi_{1 A t}^{*}, \\
& \mu_{W, t}=y_{t}-\psi_{W, t}
\end{aligned}
$$


where

$$
\begin{aligned}
& \psi_{2 B t}=-\tilde{\beta}_{\perp}\left(\tilde{\beta}_{\perp}^{\prime} \tilde{\beta}_{\perp}\right)^{-1} \tilde{\beta}_{\perp}^{\prime}(I-P)\left(\Gamma(1)-\alpha \beta^{\prime}\right)^{-1} \Gamma^{*}(L) \Delta y_{t}, \\
& \psi_{1 B t}=\tilde{\beta}_{\perp}\left(\tilde{\beta}_{\perp}^{\prime} \tilde{\beta}_{\perp}\right)^{-1} \tilde{\beta}_{\perp}^{\prime} P y_{t}, \\
& \psi_{2 A t}^{*}=-\tilde{\beta}\left(\tilde{\beta}^{\prime} \tilde{\beta}\right)^{-1} \tilde{\beta}^{\prime} \alpha\left[\beta^{\prime}\left(\Gamma(1)-\alpha \beta^{\prime}\right)^{-1} \alpha\right]^{-1} \beta^{\prime}\left(\Gamma(1)-\alpha \beta^{\prime}\right)^{-1} \Gamma^{*}(L) \Delta y_{t}, \\
& \psi_{1 A t}^{*}=+\tilde{\beta}\left(\tilde{\beta}^{\prime} \tilde{\beta}\right)^{-1} \tilde{\beta}^{\prime}\left[\alpha\left[\beta^{\prime}\left(\Gamma(1)-\alpha \beta^{\prime}\right)^{-1} \alpha\right]^{-1} \beta^{\prime}+\alpha \beta^{\prime}\right] y_{t} .
\end{aligned}
$$

The proof follows directly from the properties of the $P$ matrix and the results reported in the Appendix. This decomposition is in agreement with our conditions $(i)-(i v)$ from Definition 4.1 and does moreover explicitly take WF common feature restrictions into account. However, from (4.16), we see that the decomposition of $y_{t}$ into $\psi_{W, l}$ and $\mu_{W, l}$ does not decompose $y_{t}$ into common trends-common cycles due to the presence of two additional stationary terms in $\psi_{W, t}$, that is $\psi_{2 A t}^{*}$ and $\psi_{1 A t}^{*}$. These two terms do not disappear when premultiplied by $\tilde{\beta}$ so that condition $(v)$ which is required to obtain a common permanent-transitory decomposition of $y_{t}$ is not satisfied. In this case, $y_{t}$ is the sum of three different components: a common stochastic trend component $\left(\mu_{t}\right)$, a (weak form) common stochastic cycles component $\left(\psi_{2 B t}+\psi_{1 B t}\right)$ and an additional "uncommon" transitory component that is not annihilated by the cofeature combinations due to the long-run predictability of the linear combinations under the WF.

In order to obtain a B-N type decomposition similar to those presented in Proposition 4.3 satisfying condition $(v)$ of Definition 4.1 , we have to consider a new variable defined as

$$
y_{t}^{*}=y_{t}-\left(\psi_{2 A t}^{*}+\psi_{1 A t}^{*}\right)
$$

We then state the following result which follows directly from the definition of $y_{t}^{*}$ :

Proposition 4.5 If Assumptions 1 and 2 of Chapter 3 hold (WF), the B-N decomposition of

$$
y_{t}^{*}=y_{t}-\left(\psi_{2 A t}^{*}+\psi_{1 A t}^{*}\right)
$$

is given by $y_{i}^{*}=\mu_{W, L}+\psi_{W, t}^{*}$ where:

$$
\begin{aligned}
\psi_{W, t}^{*} & =\psi_{2 B t}+\psi_{1 B t}, \\
& =-\tilde{\beta}_{\perp}\left(\tilde{\beta}_{\perp}^{\prime} \tilde{\beta}_{\perp}\right)^{-1} \tilde{\beta}_{\perp}^{\prime}(I-P)\left(\Gamma(1)-\alpha \beta^{\prime}\right)^{-1} \Gamma^{*}(L) \Delta y_{t}+\tilde{\beta}_{\perp}\left(\tilde{\beta}_{\perp}^{\prime} \tilde{\beta}_{\perp}\right)^{-1} \tilde{\beta}_{\perp}^{\prime} P y_{t}, \\
\mu_{W, t}^{*} & =y_{t}^{*}-\psi_{W, t}^{*}=(I-P)\left(\Gamma(1)-\alpha \beta^{\prime}\right)^{-1} \alpha_{\perp}\left(\alpha_{\perp}^{\prime} \alpha_{\perp}\right)^{-1} \alpha_{\perp}^{\prime} \Gamma(L) y_{t}, \\
& =\mu_{W, t} .
\end{aligned}
$$

It is easily seen that the common cyclical component $\psi_{W, t}^{*}$ disappears if we consider $\tilde{\beta}^{\prime} y_{t}^{*}$. Remark that the form of the common trends is identical in Propositions 4.4 and 4.5 and is given by $\alpha_{\perp}^{\prime} \Gamma(L) y_{t}$. In order to interpret $y_{i}^{*}$, let us consider $\tilde{\beta}^{\prime} \Delta y_{i}^{*}$. According to the definition of the weak form, we have that $\tilde{\beta}^{\prime}\left(\Delta y_{t}-\alpha \beta^{\prime} y_{t-1}-m\right)=\tilde{\beta}^{\prime} \varepsilon_{t}$. From (4.18), (4.16) and using results 
from Appendix A, we have

$$
\begin{aligned}
\tilde{\beta}^{\prime} \Delta y_{t}^{*} & =\tilde{\beta}^{\prime} \Delta y_{t}-\tilde{\beta}^{\prime}\left(\Gamma(1)-\alpha \beta^{\prime}\right) P\left(\Gamma(1)-\alpha \beta^{\prime}\right)^{-1} \Gamma(L) \Delta y_{t}, \\
& =\tilde{\beta}^{\prime} \Delta y_{t}-\tilde{\beta}^{\prime}\left(\Gamma(1)-\alpha \beta^{\prime}\right) P\left(\Gamma(1)-\alpha \beta^{\prime}\right)^{-1}\left(\alpha \beta^{\prime} y_{t-1}+m+\varepsilon_{t}\right), \\
& =\tilde{\beta}^{\prime} \Delta y_{t}-\tilde{\beta}^{\prime}\left(\alpha \beta^{\prime} y_{t-1}+m^{*}+\varepsilon_{t}^{*}\right),
\end{aligned}
$$

where the last equality follows from the property of the $P$ matrix and where

$$
\begin{aligned}
m^{*} & =\left(\Gamma(1)-\alpha \beta^{\prime}\right) P\left(\Gamma(1)-\alpha \beta^{\prime}\right)^{-1} m, \\
& =-\alpha\left[\beta^{\prime}\left(\Gamma(1)-\alpha \beta^{\prime}\right)^{-1} \alpha\right]^{-1} \beta^{\prime}\left(\Gamma(1)-\alpha \beta^{\prime}\right)^{-1} m, \\
& =\alpha E\left(\beta^{\prime} y_{t-1}\right), \\
\varepsilon_{t}^{*} & =\left(\Gamma(1)-\alpha \beta^{\prime}\right) P\left(\Gamma(1)-\alpha \beta^{\prime}\right)^{-1} \varepsilon_{t} .
\end{aligned}
$$

Consequently, it appears that substracting $\psi_{2 A t}^{*}$ and $\psi_{1 A t}^{*}$ from the level is equivalent to adjusting the first differences $\Delta y /$ for the long run relationships as was done in Definition 4.1. Because the common cycles are for $y_{t}^{*}$ and not for $y_{t}$ we call them weak form common cycles. Further, it is worth mentioning that these different decompositions are expressed in terms of observable variables and only involve quantitics already available from the VECM form and the estimation of common features and cointegrating vectors.

\subsubsection{Relation to other Decompositions}

It may be of some interest to briefly relate or contrast the decompositions discussed until now with some of those existing in the literature. One may first notice that a factor decomposition in the sense of Gonzalo-Granger (1995) is easily obtained, see also Proietti (1997), by adding the first part of $\psi_{t}$ in (4.8) to $\mu_{t}$ in (4.9), which yields:

$$
\begin{aligned}
\mu_{t}^{G-G} & =(I-P)\left(\Gamma(1)-\alpha \beta^{\prime}\right)^{-1}\left(\Gamma(L)-\Delta \Gamma^{*}(L)\right) y_{t}, \\
& =(I-P) y_{t},
\end{aligned}
$$

which gives the decomposition $x_{t}=P y_{t}+(I-P) y_{t}$, the first and the second r.h.s. parts being respectively the Gonzalo-Granger's Transitory and Permanent components. Since $\Gamma(L)-$ $\Delta \Gamma^{*}(L)=\Gamma(1)$, it appears that the common trends are given by $\alpha_{\perp}^{\prime} \Gamma(1) y_{t}$ and not by $\alpha_{\perp}^{\prime} y_{t}$ as in Gonzalo-Granger (1995). It is also worth mentioning that this decomposition only exists under the strict restrictions that the matrix $\left(\beta: \alpha_{\perp}\right)$ is full rank. While this always holds in a VAR(1) cointegrated models, it generally does carry over to higher order cointegrated VAR systems (sec for cxample Exercise 4.3 in Johansen, 1995). Under the SF with $s+r=n$, the Gonzalo-Granger permanent component is given by $\mu_{t}^{G-G}=(I-P)\left(\Gamma(1)-\alpha \beta^{\prime}\right)^{-1} \Gamma(1) y_{t}$ and the Gonzalo-Granger transitory component is then given by $\psi_{t}^{G-G}=P y_{t}$, with $y_{t}=\mu_{t}^{G-G}+\psi_{t}^{G-G}$, $\tilde{\beta}_{t}^{\prime} \psi_{t}^{G-G}=0$ and $\tilde{\beta}^{\prime}(I-P)\left(\Gamma(1)-\alpha \beta^{\prime}\right)^{-1} \Gamma(1) y_{t}=\tilde{\beta}^{\prime}\left(\Gamma(1)-\alpha \beta^{\prime}\right)^{-1} \Gamma(1) y_{t}=\tilde{\beta}^{\prime} \Gamma(1) y_{t}=\tilde{\beta}^{\prime} y_{t}$. 


\begin{tabular}{ccccccccc}
\hline \hline $\mathrm{r}=2$ & $-\mathrm{T} \ln \left(1-\lambda_{i}\right)$ & \multicolumn{2}{c}{ df } & \multicolumn{2}{c}{ Prob $>\chi_{d f}^{2}$} & \multicolumn{2}{c}{ Prob $>\chi_{d f}^{2}$} \\
\hline & $\xi_{S}$ & $\xi_{W}$ & $\xi_{S}$ & $\xi_{W}$ & $\xi_{S}$ & $\xi_{W}$ & $\xi_{S}^{c o r}$ & $\xi_{W}^{c o r}$ \\
$s \geq 1$ & 23.36 & 12.06 & 12 & 10 & 0.02 & 0.28 & 0.04 & 0.34 \\
$s \geq 2$ & $(54.04)$ & 33.10 & $(26)$ & 22 & $(.001)$ & 0.06 & $(.001)$ & 0.10 \\
$s=3$ & $(145.02)$ & 108.53 & $(42)$ & 36 & $(<.001)$ & $<.001$ & $(<.001)<.001$ \\
\hline \hline
\end{tabular}

Table 4.1: Common Features Test Statistics

Consequently the B-N and Gonzalo-Granger (1995) decompositions coincide under SF and when $s+r=n$. In this case the space that generates the common trends is such that $s p(\tilde{\beta})=s p\left(\alpha_{\perp}\right)$ and so we obtain the Gonzalo-Granger common trends $\alpha_{\perp}^{\prime} y_{t}$. This decomposition always trivially meets condition $(v)$ of Definition 4.1 since any matrix belonging to the space generated by the columns of $\alpha_{\perp}^{\prime} \Gamma(1)$, (or $\alpha_{\perp}^{\prime}$ if $s+r=n$ ) annihilates the transitory component $\psi_{t}^{G-G}$ without any further rank restrictions that are required to satisfy the definition of common feature used in definitions of the SF and the WF. It is indeed easily seen that the "feature" underlying the Gonzalo-Granger decomposition is nothing but the presence of the error correction term of the VECM which corresponds to the case where only Assumptions 1 and 3 of Chapter 3 hold.

The common factor decompositions of Escribano and Peña (1994) or Kasa (1992) such as $y_{t}=\beta\left(\beta^{\prime} \beta\right)^{-1} \beta^{\prime} y_{t}+\beta_{\perp}\left(\beta_{\perp}^{\prime} \beta_{\perp}\right)^{-1} \beta_{\perp}^{\prime} y_{t}$ have a similar property since again any matrix belonging to the column space of $\beta_{\perp}$ is a common feature matrix with respect to those decompositions withou : generally satisfying the standard definition of serial correlation common feature. ${ }^{6}$

\subsection{Applications}

\subsubsection{US Business Cyclical Comovements}

We derive a permanent-transitory decomposition for the three dimensional system of the previous chapter. We have seen in Chapter 3 that there exist two cointegrating vectors whose cocfficients are not far from the theoretical ones i.c. $c_{t}-y_{t}$ and $i_{t}-y_{t}$ are both $I(0)$. A sub-sample analysis however reveals that these restrictions may not be supported for the whole sample period and caused a slight shift in the transitory component. These restrictions are henceforth not imposed in the sequel. For $p=5$ and $r=2$ fixed, ${ }^{7}$ we obtain the tests statistics and the $p$-values reported in Table 4.1 .

For $s=1$, the $\xi_{S W}$ test statistic has a value of 11.20 for a $\chi_{(2)}^{2}$ null distribution which yields a rejection probability of 0.0037 . We reject the SF model in favor of the WF one. The

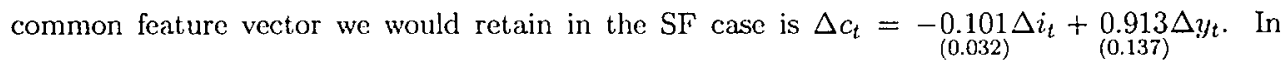
the class of WF we still have to choose betwcen $s=1$ and $s=2$. The test statistic $\xi_{W}=$

\footnotetext{
${ }^{6}$ Notice that these decomposition are morevoer not P-T decompositions in the sense of Gonzalo and Granger (1995)

${ }^{7}$ A dummy was introduced in 1975:Q1 to capture the effect of a sharpe decrease in the investment growth rate (see Figure 3.2).
} 
33.10 do not reject the null hypothesis of $s \geq 2$. The presence of two common feature vectors means that the short-run dynamics of the system is governed by a single weak form common cycle. As it is the case for cointegration, the data have determined the space spanned by the columins of the common feature space. We can uniquely identify the parameters of the cofeature vectors by imposing zero restrictions and normalizations (Johansen, 1995). FIML estimates of the WF model, under $s=2$ and permuting the columns of $\tilde{\beta}$ in order to find the vectors with the meaningful economic interpretation, results in the following two cofeature relationships $\Delta c_{t}^{*}-0.498 \Delta y_{i}^{*}$ and $\Delta i_{t}^{*}-4.764 \Delta y_{i}^{*}$ where asymptotic standard errors are reported in parentheses (0.087) $(0.483)$

and where $\mathrm{a}$ * indicates that the corresponding variables are expressed in deviation from long-run effects. It is scen that these two vectors match pretty well business cycle stylized facts, e.g. that consumption is smoother than output, investment is more volatile than output and there is a single synchronous cycle.

In Figure 4.1 the log of the variables and their permanent components are given. The cycles in these graphs are computed using Proposition 4.4 that is to say, all WF common feature restrictions are imposed. However this decomposition is not a Common trend-Common cycle decomposition stricto sensus as condition $(v)$ of the B- $N$ decomposition is not satisfied .

Figure 4.2 compares for each time scries the cyclical/transitory part of the series. Figure 4.3 presents the $\mathrm{BN}$ cycles of the original output $\left(y_{t}\right)$ series and of the deviation of output from the long run effects $\left(y_{t}^{*}\right)$. In Figure 4.4 the latter is compared with the transitory component. obtained from the GG decomposition. The shaded areas indicate the NBER peak to trough periods. The unique (weak form) common cyclical component is given in Figure 4.5 with the shaded arcas again indicating the NBER peak to trough periods. These graphs show the business cycles where fluctuations of investment, consumption and output are in agreement with the real business cycle model introduced above. These graphs could be used for example to date expansion and contractions periods of business cycles. ${ }^{8}$ Notice that the trends extracted for consumption and income in Figure 4.1 do not appear smoother than the observed series. Engle and Issler (1995) report a similar finding for quarterly per capita disposable income and per capita total consumption for 1953.2-1984.4. This result is due to the strong negative correlation between the permanent and transitory parts of these series.

\subsubsection{Comovements in International Stock Markets}

For a long time, researchers, investors and speculators have been interested in understanding the interrelationship between international stock markets. The international capital asset pricing model (ICAPM, see e.g. Adler and Dumas, 1983) has provided a theoretical basis for portfolio

\footnotetext{
${ }^{8}$ Vahid and Issler (1996) have shown that using common feature restrictions, the percentage of variance attributed to the permanent innovation is less than from the previous work by King et al. (1991) for instance. As already mentioned, these authors only allow for one common feature vector.
} 


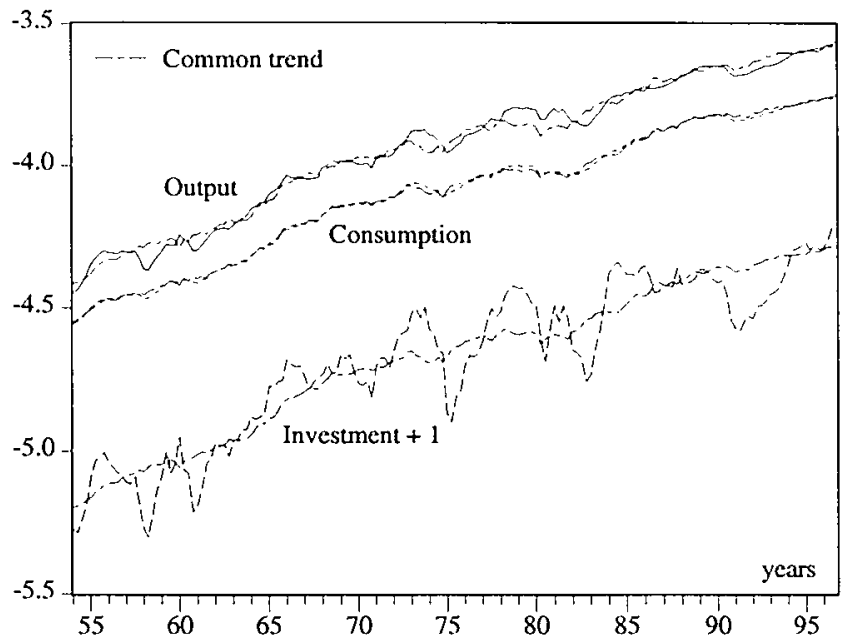

Figure 4.1: Macro Aggregates and Permanent Component

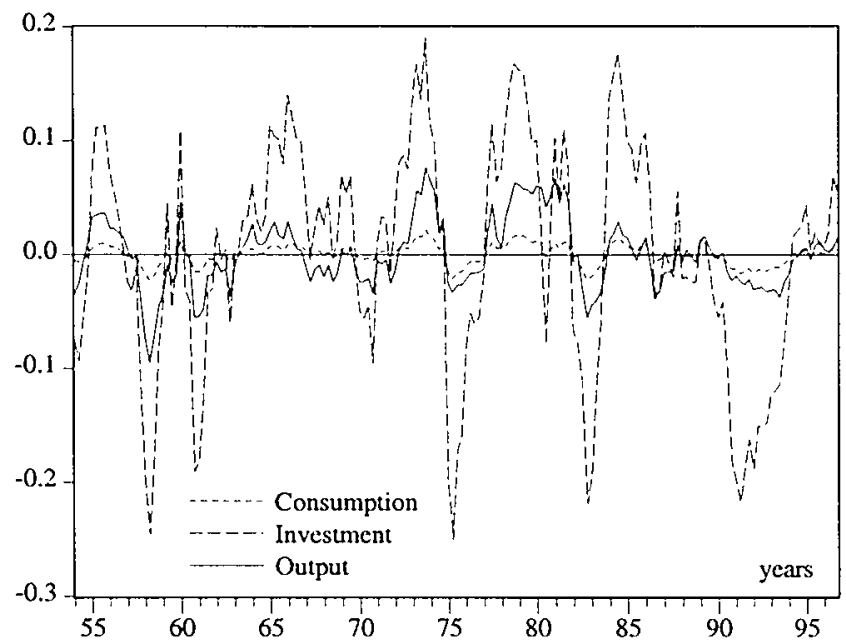

Figure 4.2: Cyclical Parts for $y_{t}$ 


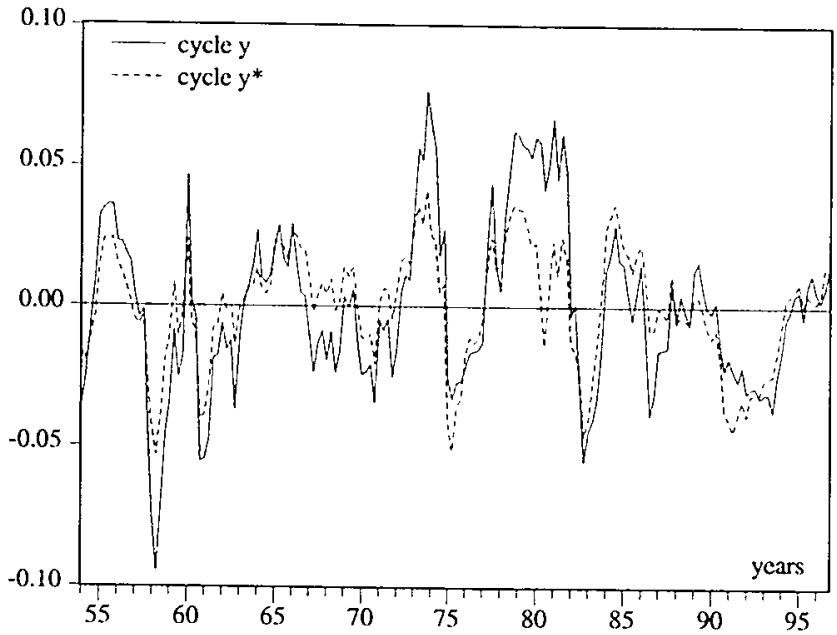

Figure 4.3: Output Cycles with WF for $y_{t}$ and $y_{i}^{*} \&$ NBER Contraction Periods

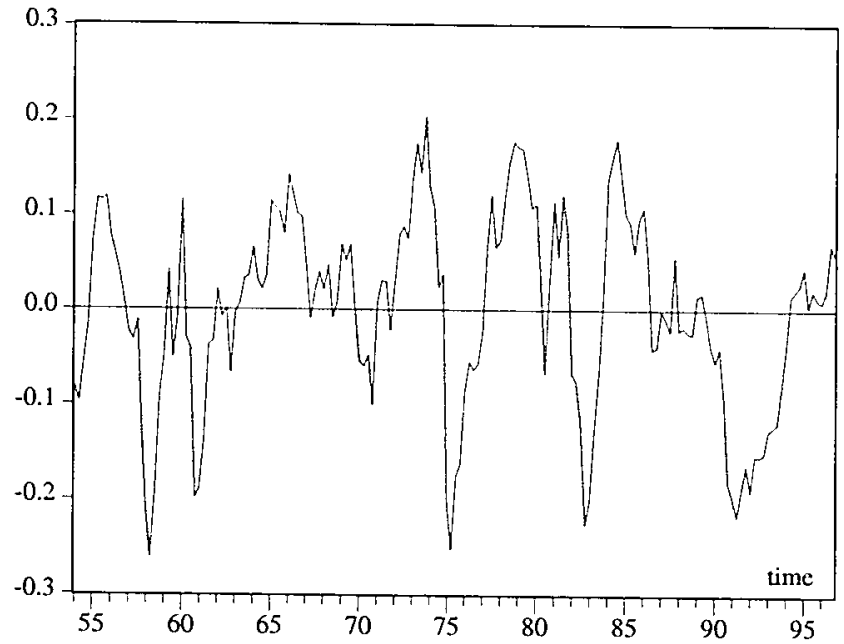

Figure 4.4: Unique Weak Form Common Cycle and NBER Contraction Periods 
selection in an international context and through that for the links between international financial markets. The arbitrage pricing theory (APT), a generalization of the domestic CAPM, has also been extended to apply to international finance.

In many empirical studies market efficiency has been analyzed by measuring the degree of integration of international financial markets, by assessing the size and speed of the transmission of news across markets and by testing the explanations provided by theory. Empirical studies have established the strong role which arbitrage plays in international financial markets. Efficiency studies suggest that international narkets react quickly to news, are volatile and difficult to predict, though more recent evidence suggests that excess returns on equity and foreign exchange markets contain predictable components (see e.g. Bekacrt and Hodrick, 1992).

The objective of this second application is to study the nature of the relationship between five major international stock market indices. Using the VAR methodology, we shall allow for separate short-run and long-run interactions and we pay special attention to features that are common to single stock market indices but disappear when a suitable combination of these scries is taken. Such common features arise when the series under study exhibit comovements, that is when the series are generated by common factors. The analysis which makes use of canonical correlations allows us to empirically determine the number and nature of the common factors driving the stock market indices. Therefore, the analysis not only leads to a more parsimoniously parametrized model, it also yields information that is crucial from an economic point of view. For instance, the information on the common factors can be incorporated in an ICAPM or APT model which require that the number and the properties of factors to be specified.

The common feature analysis is applied to quarterly time series from June 1974 to December 1999 for real stock price indices for the US, UK, Canada, Germany and Japan. In line with Fama and French (1988) and Kasa (1992) among others, we shall model the log-price series, instead of return series. The starting point will be the assumption that a log-price series is the sum of a random walk component and a stationary component. This assumption is in agreement with empirical evidence that stock prices are close to being random walks with a stationary component that is partly predictable and therefore can be used by investors in winner-loser and momentum strategies. We shall investigate whether the stationary and non-stationary components can be described and modelled in terms of common factors, namely respectively common trends and common cyclical components.

As Kasa (1992) and Richards (1995) we analyze quarterly stock market indices for UK, US, Germany, Japan and Canada. All data are taken from Datastream. The stock price data are the (monthly) Datastream Price Index (code TOTMKi, $i=\mathrm{US}$, UK, BD, JP, CN). The series are converted to real US $\$$ using end of month exchange rates (code USX $\$ i, i=$ GBP, DMK, JPY,CN\$) and the US consumer price index (CPI, seasonally adjusted, code USCP....E). The choice of the US $\$$ as a unit of measurement means that we look at the series from the point of view of a US investor. This choice allows us to compare the results with those of Kasa (1992) and Richards (1995). In order to allow a comparison with the above studies and to avoid having to deal with heavy short-run noise and conditional heteroskedasticity, we use every third monthly 


\begin{tabular}{lcccc}
\hline Eq. & B-P Q(12) & LM-AR(1-4) & ARCH(1-2) & J-B normal \\
\hline US & 19.9 & 0.498 & 1.11 & .05 \\
Can & 18.07 & 1.919 & .52 & .73 \\
Ger & 7.88 & 0.175 & 0.36 & 6.21 \\
Jap & 5.36 & 0.593 & 1.19 & 2.03 \\
UK & 8.16 & 1.215 & 2.15 & 1.86 \\
\hline
\end{tabular}

Table 4.2: Error Term Diagnostics

\begin{tabular}{lcccccccc}
\hline$H_{0}$ & $\hat{\lambda}$ & Log-Lik & $\lambda_{\max }$ & $\lambda_{\text {Inax }}^{\text {small }}$ & $\lambda_{\text {tnax }}^{5 \%}$ & Trace & Trace $^{\text {small }}$ & Trace $^{5 \%}$ \\
\hline$r=0$ & .31 & 1388.86 & 36.67 & 29.26 & 37.5 & 95.81 & 76.46 & 87.3 \\
$r \leq 1$ & .25 & 1403.26 & 28.79 & 22.97 & 31.5 & 59.14 & 47.2 & 63.0 \\
$r \leq 2$ & .15 & 1411.67 & 16.82 & 13.43 & 25.5 & 30.36 & 24.23 & 42.4 \\
$r \leq 3$ & .09 & 1416.33 & 9.32 & 7.44 & 19.0 & 13.53 & 10.8 & 25.3 \\
$r \leq 4$ & .04 & 1418.44 & 4.20 & 3.35 & 12.3 & 4.20 & 3.35 & 12.3 \\
\hline
\end{tabular}

Table 4.3: Johansen Tests for Cointegration (1975:2-1999:4)

observation for the period June 1974 to December 1999. To compare the five time series they have been rescaled such that June $1983=100$ and then logarithms were taken. Figure 4.5 presents the five time series.

The model that best characterizes the covariance structure of the data is a VAR $(4)^{9}$ with a restricted trend in the long-run and an unrestricted constant. ${ }^{10}$ We add two unrestricted impulse dummies for the October 1987 crash and the small crash in the beginning of September 1998. As we use four lags, the estimation period starts in June 1975 and $T=99$. To save space, we do not report estimates of the unrestricted VAR(4). From Table 4.2 where some model diagnostics are given for the VAR(4) we do not find an indication of misspecification in the residuals. ${ }^{11}$

Using Johansen's (1995) ML approach, the results in Table 4.3 indicate that one would formally retain one cointegrating vector at a $5 \%$ level. However the value of the test statistic for the presence of a second vector is quite close to the critical value. Moreover a graphical analysis of the long-run relationships also suggests the presence of two long-run relationships. Therefore we decided to set $r$ equal to 2 . Figure 4.6 reproduces the first two cointegrating vectors.

None of the variables can be excluded from the two cointegrating vectors using statistics

\footnotetext{
${ }^{9}$ The fourth lag is strongly significant for UK and Japan.

${ }^{10}$ The significant linear deterministic trend in the cointegrating space likely captures the effect of missing variables such as other stock prices, real economic activity, interest rates... (see also Chapter 8 for the case of the consumption function in US).

${ }^{11}$ Note: Jarque-Bera normality (J-B) test distributed as a $\chi^{2}(2)$, Box-Pierce' $Q$-statistic (B-P) distributed as a $\chi^{2}(12)$. LM test for autocorelation from 1 to 4 distributed as a $F(4,71)$ and LM test for ARCH process from 1 to 2 distributed as a $F(2,71)$.
} 


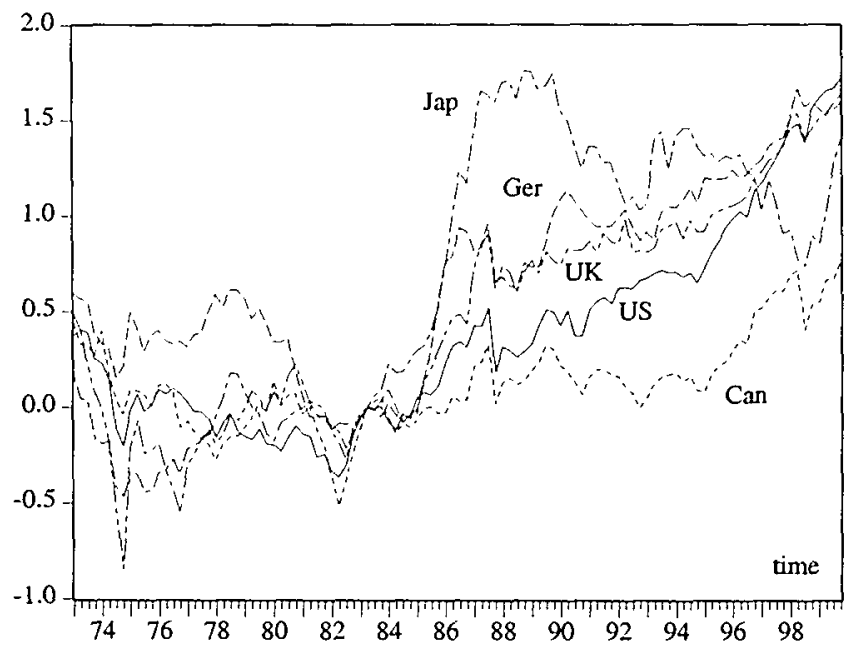

Figure 4.5: Stock Market Indices (real US/logs/quarterly)

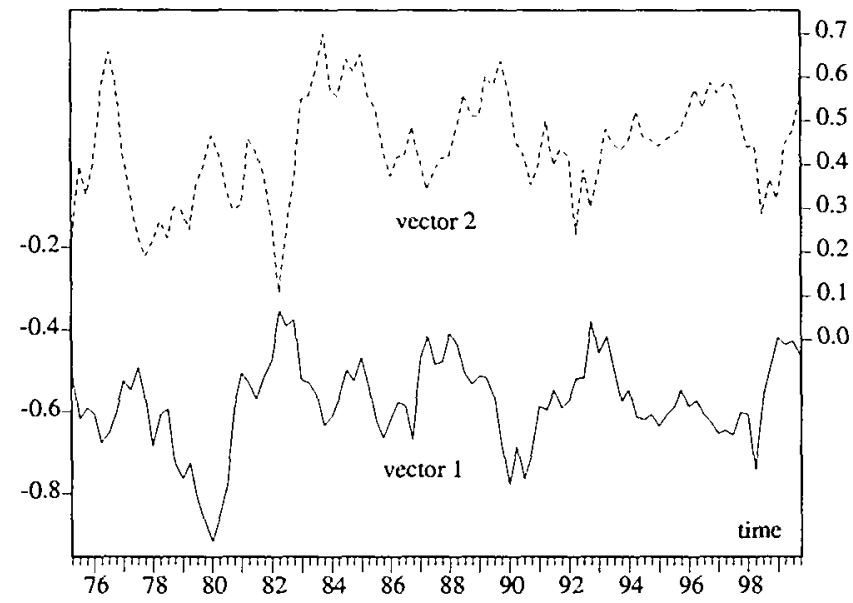

Figure 4.6: First two Cointegrating Vectors (not short-run adjusted) 


\begin{tabular}{lcccccccc}
\hline$H_{0}$ & $\hat{\tilde{\lambda}}_{S F}$ & Log-Lik & $p$-val.SF & $p$-val.SF & $\hat{\tilde{\lambda}}_{W F}$ & Log-Lik & $p$-val.WF & $p$-val. ${ }_{W F}^{\text {cor }}$ \\
\hline$s \geq 1$ & .08 & 1398.78 & .77 & .88 & .06 & 1400.19 & .86 & .92 \\
$s \geq 2$ & .11 & 1392.64 & .81 & .93 & .11 & 1394.38 & .81 & .91 \\
$s \geq 3$ & .27 & 1376.87 & .19 & .52 & .21 & 1382.77 & .38 & .66 \\
$s \geq 4$ & .40 & 1351.5 & $(.001)$ & $(.03)$ & .33 & 1362.76 & .02 & .12 \\
$s=5$ & .52 & 1314.4 & $(<.001)$ & $(<.001)$ & .43 & 1334.35 & $<.001$ & .001 \\
\hline
\end{tabular}

Table 4.4: Common Cyclical Features Tests ( 1975:2-1999:4)

distributed as $\chi^{2}(2)$. The cointegrating relationships are

$$
\begin{aligned}
& \hat{\beta}_{1}^{\prime} y_{t} \equiv U S_{t}-\underset{(.178)}{.45} \operatorname{Can}_{t}-\underset{(.091)}{.75 \operatorname{Ger}_{t}}+\underset{(.048)}{.26} \mathrm{Jap}_{t}-\underset{(.0015)}{.0062 \text { trend } \sim I(0)} \text {, }
\end{aligned}
$$

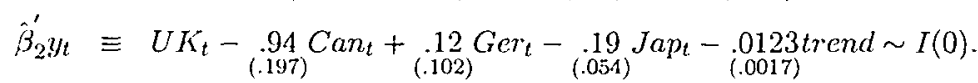

The first differences of the stock price indices also reveal some potential short-run comovements. Table 4.4 reports the statistics for testing the significance of canonical correlations (eigenvalues are denoted by $\hat{\lambda}_{\text {. }}$ ), the log-likelihood value and $p$-values of the SF and WF common cyclical feature test, both using the asymptotic and a small sample corrected version. Numbers in parentheses for the SF are those for which the constraint $r+s \leq n$ that ensures the linear independence of the cointegrating and the common feature space is not respectcd. Using both asymptotic and small sample version test statistics we cannot reject the presence of three SF vectors. For the WF, asymptotic tests do not reject three co-feature vectors while the small sample corrected version would even retain a fourth one. For the third co-feature vector, a LR test of the SF null hypothesis against the WF has a value of 11.79 and is distributed as a $\chi^{2}(6)$ under the null. While the $p$-value of 0.06 is small, in the sequel to condense the presentation, we only consider the SF with $s=3$, implying the existence of two stationary common factors.

Using a suitable rotation (normalization of the common feature matrix), the three SF cofeature vectors $\beta^{\prime} \Delta y_{t}$ are:

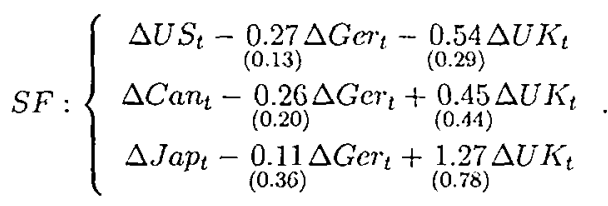

FIML estimates of the standard errors are given in brackets. A word of caution is in order here. As is obvious from the estimation results, several coefficients in the common feature vectors are not significantly different from zero. These vectors could therefore be restricted by deleting insignificant variables. Hence, some cofeature vectors could be trivial ones such that perhaps $(1,0,0)^{\prime}$ for the first equation, which is meant that some stock prices are pure random walk (with drift). We have not pursued this path as we think that the evidence suggests 
that the value of some coefficients can not be determined with sufficient precision. The most important relationship is the first one with a positive relationship between US and Germany and UK respectively suggesting that for a US investor the German and the UK stock markets are complementary to the US stock market. Canada does not seem to be influenced by Germany and UK in the second equation while in the third equation UK and Japan appear to be substitute markets for a US investor.

Next, we extract common trend and common cycle components. We concentrate on the SF because under $r+s=n$ as in this application, the multivariate Stock-Watson-Beveridge-Nelson (SWBN) decomposition with common features is identical to those proposed by Vahid and Engle (1993) and by Gonzalo and Granger (1995) respectively. In Figure 4.7, we give the individual series and their trend and cycle components (the cycle components are given in the lowest righthand-side graph) using the multivariate SWBN decomposition imposing the restrictions implied by cointegration and the choice of the three SF vectors made in our analysis. The three common trends and the two SF common cyclical components are given in Figures 4.8 and 4.9.

The position of a series with respect to its common stochastic trend could reveal some changes in the process. For instance, at the end of the period, the UK stock price index lies above its stochastic trend, meaning the market could be over-valued and the stock price is expected fall. For Canada, Germany and Japan, the analysis suggests undervaluation of the stock market at the end of the observation period. Notice also that a comparison of Figures 4.7 and 4.8 suggests that one of the common trends is closely related with the trend components of Japan and possibly reflects the structural changes in Japan and their impact on the other four stock markets.

The main conclusions from the empirical analysis are as follows. First, there are three common stochastic trends driving the long-run movements of the five stock markets. These stochastic trends can be interpreted as the reflecting structural economic movements in the US, Europe and Japan respectively. These results are at variance with the findings by Kasa (1992) for the period 1974-1990 that there are four cointegrating relations between these indices, that is these indices are driven by a single common trend. Kasa's finding is likely to be duc to an overparametrization of his model. He used a tenth order VAR for the quarterly series. We have also analyzed the data for the period 1974-1990. We found that there is little reason for including lags beyond the fourth order. For the fourth order VAR for the subperiod 1974-1990, the evidence confirms our conclusion of two cointegrating relations. That conclusion therefore seems to be fairly robust. It does not seem to be affected by the increase in financial market integration which took place in the last decade. On the basis of the finding of three region-specific common trends, international financial markets appear to be less integrated than suggested by Kasa's analysis and US investors are likely to have gained from international diversification.

Second, our findings from the canonical correlation analysis indicate that the strong form reduced rank structure implying two common cyclical movements is supported by the empirical evidence. This means that there are short-run predictable components in the series considered, a finding that is in agreement with the results from the literature on the performance of value, momentum or winner-loser based investment strategies. In particular, the negative short-run 

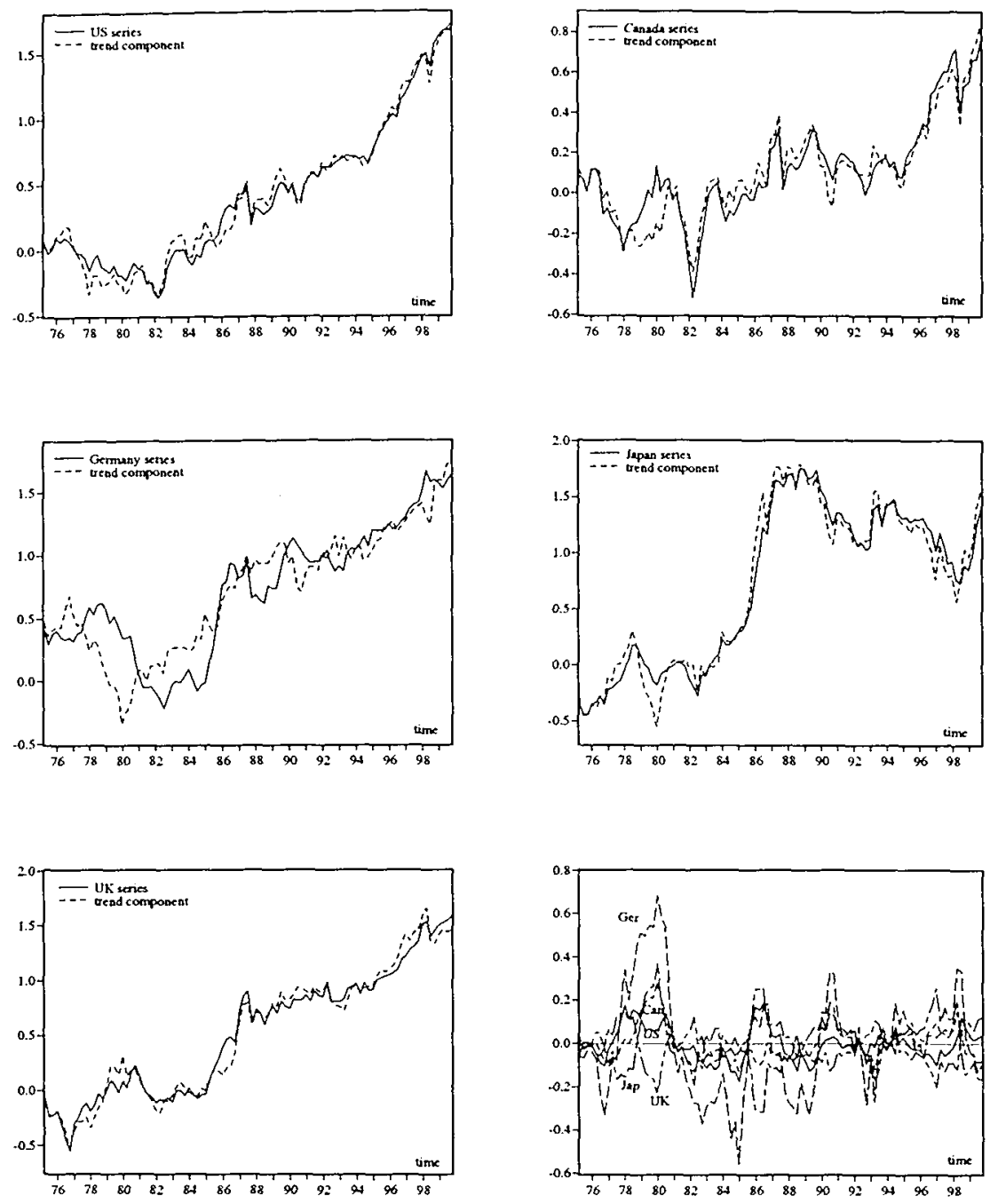

Figure 4.7: Time Series, Trend and Cycle Components 


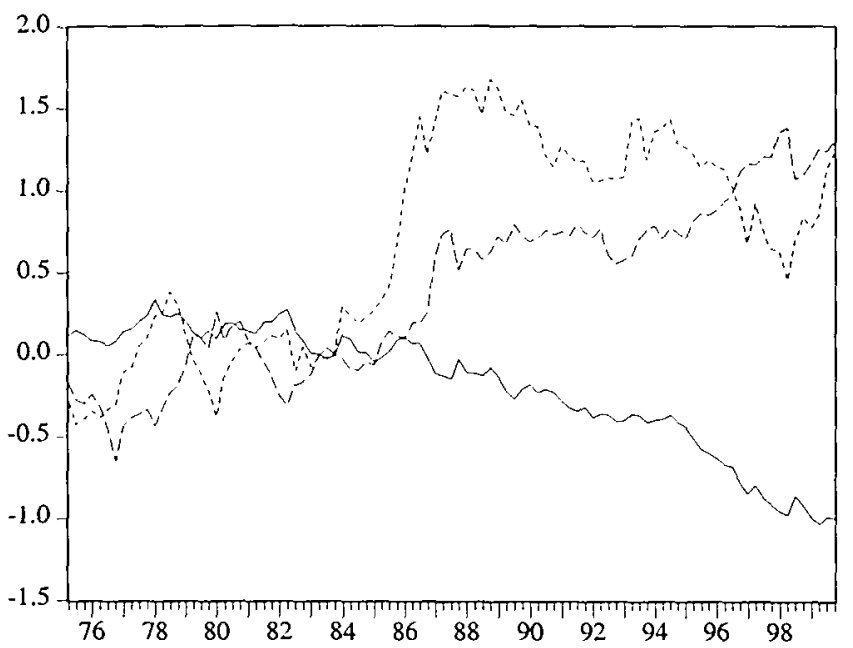

Figure 4.8: Three Cormmon Trends

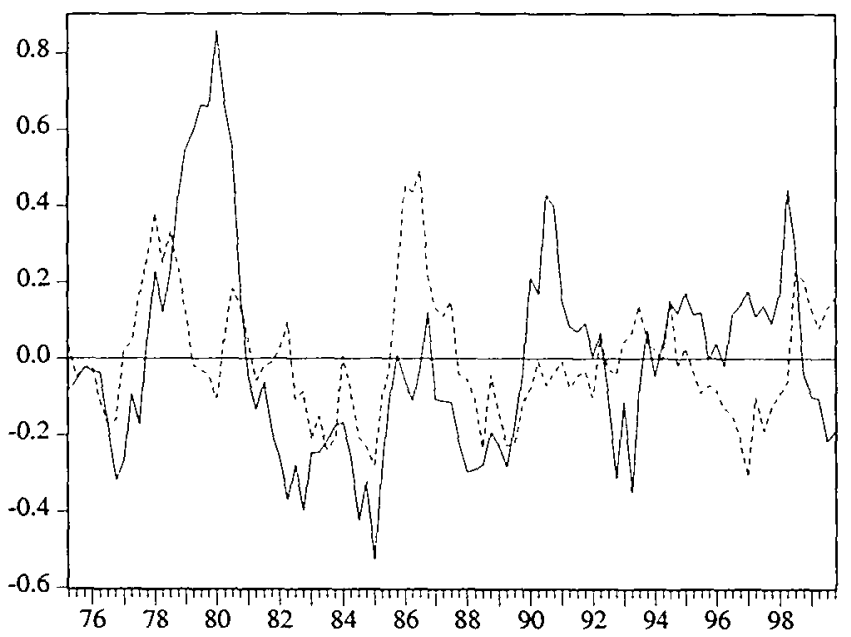

Figure 4.9: Two SF Common Cycles 
correlation between the UK market and the Canadian and Japanese stock markets respectively sugrests short-run opportunities for international risk diversification for US investors.

Third, these predictable parts are interrelated in the sense that they appear to be driven by two underlying common cyclical components. Our findings are in line with but more general than findings by Fama and French (1988), who conclude that for return horizons beyond a year, the evidence suggests predictable price variation due to mean reversion. However, our analysis is more general as it explores the existence and nature of the relationships between long-run and short-run predictable components of different national stock market indices. Our findings are at variance with those of Richards (1995) who concludes that national stock market indices include a common world component and two country-specific components; one permanent and one transitory. The evidence supporting the conclusion of two common SF components is in favor of using models which go beyond the domestic CAPM in the direction of international CAPM or APT models. For instance, the empirical evidence for the presence of three region-specific common trends has implications for the form of the factor model structure.

Fourth, additional analyses carried out for subperiod but not reported here indicate that our conclusions are fairly robust. One might have expected that increased integration of world financial markets would have affected and changed the interrelationships. There is little cvidence of major structural changes in the transmission mechanisms over the period that we have studied.

\subsection{Conclusion}

In this chapter we extend the Proietti (1997) Beveridge-Nelson decomposition to the case in which combinations of the first differenced variables corrected for long run effects are white noise. In this framework, (labeled weak form reduced rank structure), it is possible to have a larger number of common feature vectors than in the standard serial correlation common feature case studied initially by Vahid and Engle (1993). Similarly to the Gonzalo-Granger decomposition, the different decompositions considered in this paper are expressed in terms of observable variables and only involve quantitics already available from the VECM form and the estimation of common features and cointegrating vectors. The decompositions are hence computationally easy to obtain. Two applications show the feasibility of the approach and its potential value. 


\subsection{Appendix A: Proof of Proposition 4.4}

Under WT, from (4.8)-(4.9) we have

$$
\begin{aligned}
\psi_{W, t}= & -\tilde{\beta}_{\perp}\left(\tilde{\beta}_{\perp}^{\prime} \tilde{\beta}_{\perp}\right)^{-1} \tilde{\beta}_{\perp}^{\prime}(I-P)\left(\Gamma(1)-\alpha \beta^{\prime}\right)^{-1} \Gamma^{*}(L) \Delta y_{t}+\tilde{\beta}_{\perp}\left(\tilde{\beta}_{\perp}^{\prime} \tilde{\beta}_{\perp}\right)^{-1} \tilde{\beta}_{\perp}^{\prime} P y_{t} \\
& -\tilde{\beta}\left(\tilde{\beta}^{\prime} \tilde{\beta}\right)^{-1} \tilde{\beta}^{\prime}(I-P)\left(\Gamma(1)-\alpha \beta^{\prime}\right)^{-1} \Gamma^{*}(L) \Delta y_{t}+\tilde{\beta}(\tilde{\beta} \tilde{\beta})^{-1} \tilde{\beta}^{\prime} P y_{t} .
\end{aligned}
$$

We shall now derive the expression $(4.16)$ for $\psi_{2 A t}^{*}$ and $\psi_{1 A t}^{*}$. First note that $\tilde{\beta}^{\prime}\left(\Gamma(1)-\alpha \beta^{\prime}\right)=$ $\tilde{\beta}^{\prime}-\tilde{\beta}^{\prime} \alpha \beta^{\prime}$ so that $\tilde{\beta}^{\prime}=\tilde{\beta}^{\prime}\left(\Gamma(1)-\alpha \beta^{\prime}\right)+\tilde{\beta}^{\prime} \alpha \beta^{\prime}$, hence, $\tilde{\beta}^{\prime}\left(\Gamma(1)-\alpha \beta^{\prime}\right)^{-1}=\tilde{\beta}^{\prime}+\tilde{\beta}^{\prime} \alpha \beta^{\prime}\left(\Gamma(1)-\alpha \beta^{\prime}\right)^{-1}$. From the definition of $P$, we also have

$$
\begin{aligned}
\tilde{\beta}^{\prime} P & =\tilde{\beta}^{\prime} \alpha\left[\beta^{\prime}\left(\Gamma(1)-\alpha \beta^{\prime}\right)^{-1} \alpha\right]^{-1} \beta^{\prime}+\tilde{\beta}^{\prime} \alpha \beta^{\prime}\left(\Gamma(1)-\alpha \beta^{\prime}\right)^{-1} \alpha\left[\beta^{\prime}\left(\Gamma(1)-\alpha \beta^{\prime}\right)^{-1} \alpha\right]^{-1} \beta^{\prime} \\
& =\tilde{\beta}^{\prime} \alpha\left[\beta^{\prime}\left(\Gamma(1)-\alpha \beta^{\prime}\right)^{-1} \alpha\right]^{-1} \beta^{\prime}+\tilde{\beta}^{\prime} \alpha \beta^{\prime}
\end{aligned}
$$

Since the columns of $\tilde{\beta}^{\prime}$ span the cofeature space it holds that

$$
\begin{aligned}
& \tilde{\beta}^{\prime}(P-I)\left(\Gamma(1)-\alpha \beta^{\prime}\right)^{-1} \Gamma^{*}(L) y_{t} \\
= & \left.\tilde{\beta}^{\prime} \alpha \mid \beta^{\prime}\left(\Gamma(1)-\alpha \beta^{\prime}\right)^{-1} \alpha\right]^{-1} \beta^{\prime}\left(\Gamma(1)-\alpha \beta^{\prime}\right)^{-1} \Gamma^{*}(L) y_{t}+ \\
& \tilde{\beta}^{\prime} \alpha \beta^{\prime}\left(\Gamma(1)-\alpha \beta^{\prime}\right)^{-1} \Gamma^{*}(L) y_{t}-\tilde{\beta}^{\prime} \Gamma^{*}(L) \Delta y_{t}-\tilde{\beta}^{\prime} \alpha \beta^{\prime}\left(\Gamma(1)-\alpha \beta^{\prime}\right)^{-1} \Gamma^{*}(L) \Delta y_{t} \\
= & \tilde{\beta}^{\prime} \alpha\left[\beta^{\prime}\left(\Gamma(1)-\alpha \beta^{\prime}\right)^{-1} \alpha\right]^{-1} \beta^{\prime}\left(\Gamma(1)-\alpha \beta^{\prime}\right)^{-1} \Gamma^{*}(L) \Delta y_{t}
\end{aligned}
$$

Multiplying respectively (4.22) and (4.23) by $\tilde{\beta}\left(\tilde{\beta}^{\prime} \tilde{\beta}\right)^{-1}, \psi_{2 A t}^{*}$ and $\psi_{1 A t}^{*}$ as given in (4.16) can be directly obtained.

Using (4.22)-(4.23) we obtain a compact expression that can be used to compute the sum of these two components.

$$
\begin{aligned}
& \tilde{\beta}^{\prime}(P-I)\left(\Gamma(1)-\alpha \beta^{\prime}\right)^{-1} \Gamma^{*}(L) \Delta y_{t}+\tilde{\beta}^{\prime} P y_{t} \\
= & \tilde{\beta}^{\prime} \alpha\left[\beta^{\prime}\left(\Gamma(1)-\alpha \beta^{\prime}\right)^{-1} \alpha\right]^{-1} \beta^{\prime}\left(\Gamma(1)-\alpha \beta^{\prime}\right)^{-1} \Gamma^{*}(L) \Delta y_{t} \\
& +\tilde{\beta}^{\prime} \alpha\left[\beta^{\prime}\left(\Gamma(1)-\alpha \beta^{\prime}\right)^{-1} \alpha\right]^{-1} \beta^{\prime} y_{t}+\tilde{\beta}^{\prime} \alpha \beta^{\prime} y_{t} \\
= & \tilde{\beta}^{\prime}\left(\Gamma(1)-\alpha \beta^{\prime}\right) P\left(\Gamma(1)-\alpha \beta^{\prime}\right)^{-1}\left[\Gamma^{*}(L) \Delta y_{t}+\Gamma(1) y_{t}\right] \\
& -\tilde{\beta}^{\prime}\left(\Gamma(1)-\alpha \beta^{\prime}\right) P\left(\Gamma(1)-\alpha \beta^{\prime}\right)^{-1} \alpha \beta^{\prime} y_{t}+\tilde{\beta}^{\prime} \alpha \beta^{\prime} y_{t}
\end{aligned}
$$

Since $P\left(\Gamma(1)-\alpha \beta^{\prime}\right)^{-1} \alpha=\left(\Gamma(1)-\alpha \beta^{\prime}\right)^{-1} \alpha$, (see property 2 of Proposition 4.2), (4.24) finally becomes

$$
\begin{aligned}
& \tilde{\beta}^{\prime}(P-I)\left(\Gamma(1)-\alpha \beta^{\prime}\right)^{-1} \Gamma^{*}(L) \Delta y_{t}+\tilde{\beta}^{\prime} P y_{t} \\
= & \tilde{\beta}^{\prime}\left(\Gamma(1)-\alpha \beta^{\prime}\right) P\left(\Gamma(1)-\alpha \beta^{\prime}\right)^{-1} \Gamma(L) y_{t} \\
= & \left(\tilde{\beta}^{\prime}-\tilde{\beta}^{\prime} \alpha \beta^{\prime}\right) P\left(\Gamma(1)-\alpha \beta^{\prime}\right)^{-1} \Gamma(L) y_{t}
\end{aligned}
$$




\subsection{Appendix B: Summary of Partitioned Matrices Algebra}

- Determinant of a Partitioned Matrix

$$
\left|\begin{array}{ll}
A & B \\
C & D
\end{array}\right|=\left|A-B D^{-1} C\right||D|=|A|\left|D-C A^{-1} B\right|
$$

- Inversion of a Partitioned Matrix

$$
\left[\begin{array}{cc}
A & B \\
C & D
\end{array}\right]^{-1}=\left[\begin{array}{cc}
A^{-1}+A^{-1} B R^{-1} C A^{-1} & -A^{-1} B R^{-1} \\
-R^{-1} C A^{-1} & R^{-1}
\end{array}\right]
$$

with $R=\left(D-C A^{-1} B\right)$

$$
\begin{gathered}
{\left[\begin{array}{ll}
A & 0 \\
0 & D
\end{array}\right]^{-1}=\left[\begin{array}{cc}
A^{-1} & 0 \\
0 & D^{-1}
\end{array}\right]} \\
{\left[\begin{array}{ll}
A & 0 \\
C & D
\end{array}\right]^{-1}=\left[\begin{array}{cc}
A^{-1} & 0 \\
-A^{-1} C D^{-1} & D^{-1}
\end{array}\right]}
\end{gathered}
$$

- Sherman-Morrison-Woodbury formula

$$
\left(W \pm U V^{\prime}\right)^{-1}=W^{-1} \mp W^{-1} U\left(I \pm V^{\prime} W^{-1} U\right)^{-1} V^{\prime} W^{-1}
$$

- Multiplication of Partitioned Matrices

$$
\left[\begin{array}{ll}
A & B \\
C & D
\end{array}\right]\left[\begin{array}{ll}
E & F \\
G & H
\end{array}\right]=\left[\begin{array}{ll}
A E+B G & A F+B H \\
C E+D G & C F+D H
\end{array}\right]
$$

\subsection{Appendix C: Detailed Calculus for the VAR(2)}

For easy of exposition, we focus on the driftless case:

$$
\begin{gathered}
{\left[\begin{array}{c}
\Delta y_{t} \\
\beta^{\prime} y_{t-1}
\end{array}\right]=\left[\begin{array}{cc}
\Gamma_{1}+\alpha \beta^{\prime} & \alpha \\
\beta^{\prime} & I_{r}
\end{array}\right]\left[\begin{array}{c}
\Delta y_{t-1} \\
\beta^{\prime} y_{t-2}
\end{array}\right]+Z^{\prime} \varepsilon_{t}} \\
I-Q=\left[\begin{array}{cc}
I_{n}-\Gamma_{1}-\alpha \beta^{\prime} & -\alpha \\
-\beta^{\prime} & 0_{r \times r}
\end{array}\right]=\left[\begin{array}{cc}
A & B \\
C & D
\end{array}\right]
\end{gathered}
$$


- Computation of $(I-Q)^{-1}$

First note some intermediate results

$$
\begin{aligned}
R & =\left(D-C A^{-1} B\right)=-\beta^{\prime}\left(\Gamma(1)-\alpha \beta^{\prime}\right)^{-1} \alpha \\
A^{-1} B & =-\left(\Gamma(1)-\alpha \beta^{\prime}\right)^{-1} \alpha \\
C A^{-1} & =-\beta^{\prime}\left(\Gamma(1)-\alpha \beta^{\prime}\right)^{-1} \\
A^{-1} B R^{-1} C A^{-1} & =-\left(\Gamma(1)-\alpha \beta^{\prime}\right)^{-1} \alpha\left[\beta^{\prime}\left(\Gamma(1)-\alpha \beta^{\prime}\right)^{-1} \alpha\right]^{-1} \beta^{\prime}\left(\Gamma(1)-\alpha \beta^{\prime}\right)^{-1}
\end{aligned}
$$

$(I-Q)^{-1}=$

$\left[\begin{array}{cc}(I-P)\left(\Gamma(1)-\alpha \beta^{\prime}\right)^{-1} & -\left(\Gamma(1)-\alpha \beta^{\prime}\right)^{-1} \alpha\left[\beta^{\prime}\left(\Gamma(1)-\alpha \beta^{\prime}\right)^{-1} \alpha\right]^{-1} \\ -\left[\beta^{\prime}\left(\Gamma(1)-\alpha \beta^{\prime}\right)^{-1} \alpha\right]^{-1} \beta^{\prime}\left(\Gamma(1)-\alpha \beta^{\prime}\right)^{-1} & -\left[\beta^{\prime}\left(\Gamma(1)-\alpha \beta^{\prime}\right)^{-1} \alpha\right]^{-1}\end{array}\right]$

where $P=\left(\Gamma(1)-\alpha \beta^{\prime}\right)^{-1} \alpha\left[\beta^{\prime}\left(\Gamma(1)-\alpha \beta^{\prime}\right)^{-1} \alpha\right]^{-1} \beta^{\prime}$.

- Computation of $Z(I-Q)^{-1} Q$ with $Z=\left[I_{n}: 0_{n \times r}\right\}$

$$
\begin{aligned}
& Z(I-Q)^{-1} Q= \\
& {\left[(I-P)\left(\Gamma(1)-\alpha \beta^{\prime}\right)^{-1}\left(\Gamma_{1}+\alpha \beta^{\prime}\right)-P-\left(\Gamma(1)-\alpha \beta^{\prime}\right)^{-1} \alpha\left[\beta^{\prime}\left(\Gamma(1)-\alpha \beta^{\prime}\right)^{-1} \alpha\right]^{-1}\right]}
\end{aligned}
$$

- Computation of $-Z(I-Q)^{-1} Q f_{t}$

$$
\begin{aligned}
& \text { where } f_{t}=\left[\begin{array}{c}
\Delta y_{t} \\
\beta^{\prime} y_{l-1}
\end{array}\right] \\
& \psi_{t}=-Z(I-Q)^{-1} Q f_{t}=-(I-P)\left(\Gamma(1)-\alpha \beta^{\prime}\right)^{-1}\left(\Gamma_{1}+\alpha \beta^{\prime}\right) \Delta y_{t}+P \Delta y_{t}+P y_{t-1} \\
& =-(I-P)\left(\Gamma(1)-\alpha \beta^{\prime}\right)^{-1}\left(\Gamma_{1}+\alpha \beta^{\prime}\right) \Delta y_{t}+P y_{t} \\
& =-(I-P)\left(\Gamma(1)-\alpha \beta^{\prime}\right)^{-1}\left(\Gamma_{1}\right) \Delta y_{t}+P y_{t}
\end{aligned}
$$

- Computation of $y_{t}-\left(-Z(I-Q)^{-1} Q f_{t}\right)$

$$
\begin{aligned}
\mu_{t} & =y_{t}-Z(I-Q)^{-1} Q f_{t}=y_{t}+(I-P)\left(\Gamma(1)-\alpha \beta^{\prime}\right)^{-1}\left(\Gamma_{1}\right) \Delta y_{t}-P y_{t} \\
& =(I-P) y_{t}+(I-P)\left(\Gamma(1)-\alpha \beta^{\prime}\right)^{-1}\left(\Gamma_{1}\right) \Delta y_{t} \\
& =(I-P)\left(\Gamma(1)-\alpha \beta^{\prime}\right)^{-1}\left(\Gamma(1)-\alpha \beta^{\prime}\right) y_{t}+(I-P)\left(\Gamma(1)-\alpha \beta^{\prime}\right)^{-1}\left(\Gamma_{1}\right) \Delta y_{t} \\
& =(I-P)\left(\Gamma(1)-\alpha \beta^{\prime}\right)^{-1}\left(\Gamma(1) y_{t}-\alpha \beta^{\prime} y_{t}+\Gamma_{1} \Delta y_{t}\right) \\
& =(I-P)\left(\Gamma(1)-\alpha \beta^{\prime}\right)^{-1}\left(\Gamma(1) y_{t}+\Gamma_{1} \Delta y_{t}\right) \\
& =(I-P)\left(\Gamma(1)-\alpha \beta^{\prime}\right)^{-1}\left(I-\Gamma_{1} L\right) y_{t} \\
& =(I-P)\left(\Gamma(1)-\alpha \beta^{\prime}\right)^{-1} \Gamma(L) y_{t}
\end{aligned}
$$


- Computation of $\mathrm{E}(\Delta y / t)$

$$
\begin{aligned}
E\left(\Delta y_{t}\right) & =Z c^{*} \\
& =Z(I-Q)^{-1} c \\
& =(I-P)\left(\Gamma(1)-\alpha \beta^{\prime}\right)^{-1} m
\end{aligned}
$$

Consequently $Z c^{*}$ should be substracted from $\Delta y_{t}$ in the fourth step. The mean of $P y_{t}$ can also be substracted so as to yield a zero mean cyclical process.

\subsection{Appendix D: Extension to the $\operatorname{VAR}(p)$ model}

We can easily extend the VAR(2) state space representation to the VAR(P) such that:

$$
\left[\begin{array}{c}
\Delta y_{t} \\
\Delta y_{t-1} \\
\vdots \\
\vdots \\
\Delta y_{t-p+2} \\
\beta^{\prime} y_{t-1}
\end{array}\right]=\left[\begin{array}{ccccc}
\Gamma_{1}+\alpha \beta^{\prime} & \Gamma_{2} & \cdots & \Gamma_{p-1} & \alpha \\
I_{n} & 0_{n \times n} & \cdots & 0_{n \times n} & 0_{n \times r} \\
0_{n \times n} & \ddots & \ddots & \vdots & \vdots \\
\vdots & \ddots & \ddots & \vdots & \vdots \\
\beta^{\prime} & 0_{r \times n} & \cdots & \cdots & I_{r}
\end{array}\right]\left[\begin{array}{c}
\Delta y_{t-1} \\
\Delta y_{t-2} \\
\vdots \\
\vdots \\
\Delta y_{t-p+1} \\
\beta^{\prime} y_{t-2}
\end{array}\right]+Z^{\prime} \varepsilon_{t}
$$

Then form the matrix partitioned matrix $(I-Q)$ such as

$$
(I-Q)=\left[\begin{array}{ll}
A & B \\
C & D
\end{array}\right]
$$

with

$$
\begin{aligned}
& A=\left[\begin{array}{cccc}
I_{n}-\Gamma_{1}-\alpha \beta^{\prime} & -\Gamma_{2} & \cdots & -\Gamma_{p-1} \\
-I_{n} & I_{n} & \cdots & 0_{n \times n} \\
0_{n \times n} & -I_{n} & \ddots & 0_{n \times n} \\
\vdots & \vdots & \ddots & \vdots \\
\vdots & \vdots & \ddots & \vdots
\end{array}\right] \\
& B^{\prime}=\left[\begin{array}{lllll}
-\alpha & 0_{n \times r} & \cdots & \cdots & 0_{n \times r}
\end{array}\right], C=\left[\begin{array}{lllll}
-\beta^{\prime} & 0_{r \times n} & \cdots & \cdots & 0_{r \times n}
\end{array}\right], D=\left[0_{r \times r}\right\}
\end{aligned}
$$

The only difference between $\operatorname{VAR}(2)$ and $\operatorname{VAR}(\mathrm{p})$ is that matrix $A$ have been inflated and therefore it is not so easy to compute its inverse as in A ppendix C. Nevertheless, let us define $U^{\prime}=\left[I_{n} \vdots 0_{n \times n} \vdots \ldots \vdots 0_{n \times n}\right], V^{\prime}=$ $\left[\Gamma_{1}-\alpha \beta^{\prime}: \Gamma_{2} \vdots \ldots \Gamma_{p-1} \vdots 0_{n \times n}\right]$ and $W$ a lower triangular matrix with $I_{n}$ on the main diagonal, $-I_{n}$ below the main diagonal and zero elsewhere. So we can apply the Sherman-Morrison-Woodbury formula. 


\title{
Chapter 5
}

\section{To Switch between Common Features and Cointegrating Restrictions}

\begin{abstract}
The aim of this chapter is twofold. First we introduce and evaluate through Monte Carlo simulations a small sample version of common feature test statistics similar to the one proposed by Reinsel and Ahn (1992) for cointegration. This correction was already used in Chapters 3 and 4. Using Monte Carlo simulations, it is shown in this chapter that this correction improves the small sample behavior of test statistics. Secondly, we consider the small sample performances of cointegration and common cyclical features LR test statistics when both cointegrating and common feature vectors are unknown. For rather small samples, we stress the advantages of using an iterative strategy to maximize the likelihood by both imposing long and short-run restrictions until convergence is achieved. Studies of US regional per capita incomes and Belgian employment cycles illustrate this analysis.
\end{abstract}

\subsection{Introduction}

We consider the $n$-dimensional vector autoregressive model of order $p$ with $r$ cointegrating relationships:

$$
\Delta y_{\ell}=\mu+\alpha \beta^{\prime} y_{t-1}+\sum_{i=1}^{p-1} \Gamma_{i} \Delta y_{t-i}+\varepsilon_{t}, \quad t=-p+1, \ldots 0,1, \ldots T
$$

where $\varepsilon_{t} \sim N I I D(0, \Omega)$. We assume that $y_{t}$ is $I(1)$ and then that $\Delta y_{t}$ and $\beta^{\prime} y_{i}$ are $I(0)$ with $\operatorname{rank}(\beta)=r$. The first $p$ presample values initialize the process. Chapter 2 has reviewed some of the potential problems for common feature test statistics in the presence of various types of

\footnotetext{
${ }^{0}$ This chapter is based on Hecq (1999) and partly on Candelon, Hecq and Lohest (2000) for the Section 5.4.2.
} 
misspecification. In this chapter we propose to evaluate through Monte Carlo experiments two additional small sample issues.

The first point we address is the practical relevance of a small sample corrected version of common feature test statistics. For cointegration, some small sample properties of Johansen's ML testing procedures are documented in Cheung and Lai (1993), Söderlind and Vredin (1996) and Jacobson et al. (1998) inter alia. It emerges that LR tests are often too liberal: the small sample "implied "critical values are higher than the asymptotic critical values in the published tables. This leads to overestimate $r$ in empirical work. Some small sample corrections ( $\dot{a}$ la Reinsel and Ahn, 1992, for instance) may be helpful but they often lead in practice to underestimate $r$ (Söderlind and Vredin, 1996). An alternative solution is to obtain critical values by the use of bootstrap methods. However, this is not the common empirical practice maybe because the routines are not casily available. The effect of size distortions may in some cases be attenuated by the fact that in empirical work, test statistics at a particular rejection frequency are only one tool researchers use. A priori information, but also graphical analyses of cointegrating relationships, the roots of the companion matrix and a sensitivity analysis (to $p$ and $n$ ) are of great help.

The second issue we want to investigate is the small sample performance of cointegration and common cyclical features test statistics when both cointegrating and common feature vectors are estimated. Indeed, under serial correlation common feature restrictions, there exists a common feature space spanned by the columns of $\tilde{\beta}$ such that $\tilde{\beta}^{\prime}\left(\Delta y_{t}-\mu\right)$ is a $s$ dimensional white noise in (5.1); and under the weak form reduced rank structure $\tilde{\beta}^{\prime}\left(\Delta y_{t}-\mu-\alpha \beta^{\prime} y_{t-1}\right)$ is a $s$ dimensional white noisc. Consequently, it is easy to reparametrize the VECM in (5.1) to impose the common feature restrictions (e.g. Vahid and Engle, 1993) and then to take these short-run restrictions into account for forecasting purposes or to get more accurate estimates. For instance, a VAR(2) with SCCF restrictions can be reparamatrized such that

$$
\Delta y_{t}=\mu+\tilde{\beta}_{\perp} \Psi_{1}^{\prime} \beta^{\prime} y_{t-1}+\tilde{\beta}_{\perp} \Psi_{2}^{\prime} \Delta y_{t-1}+\varepsilon_{t}
$$

where $\tilde{\beta}_{\perp}$ is the orthogonal complement of the cofeature matrix, with $\tilde{\beta}^{\prime} \tilde{\beta}_{\perp}=0_{s \times n}$ and $\operatorname{rank}[\tilde{\beta}$ : $\left.\tilde{\beta}_{\perp}\right]=n$. In the two step approach we have used in previous chapters, the cointegrating matrix is estimated first for a specific rank $r$, and then assumed known for the determination of the common feature rank. Indeed, the superconsistency principle pleas for first estimating the cointegrated matrix, say $\hat{\beta}$. If just-identifying restrictions are imposed, $T(\hat{\beta}-\beta)$ and $\sqrt{T}(\hat{\alpha}-\alpha)$ converge in distribution at rates $T$ and $\sqrt{T}$ respectively. Notice that without identifying restrictions on $\hat{\beta}$, consistent and asymptotically normal estimates of $\Pi=\alpha \beta^{\prime}$ can be obtained by LS estimation. We also have $\sqrt{T}\left(\left[\hat{\Gamma}_{1, \ldots}, \hat{\Gamma}_{p-1}\right]-\left[\Gamma_{1, \ldots}, \Gamma_{p-1}\right]\right) \stackrel{d}{\rightarrow} N\left(0, \Sigma_{\dot{\Gamma}}\right)$ (see Johansen, 1995 or Lütkepohl, 1991 for details). But for small sample sizes, more accurate estimates could be obtained by switching or iterating between long-run and short-run restrictions until the convergence is reached yiclding the ML estimator as proposed by Hansen and Johansen (1998) for SCCF.

The structure of this chapter is as follows. Section $\mathbf{5 . 2}$ describes the test statistics and the switching algorithms. Section 5.3 reports evidence from Monte Carlo experiments. While the 
results of this chapter can be carried out with SCCF restrictions, we mainly focus on the WF structure. Indeed, imposing WF restrictions is convenient because it. allows to consider both cointegration and common feature test statistics without the constraint $r+s \leq n$. Section 5.4 proposes two cconomic illustrations.

\subsection{Test Statistics and Switching Algorithms}

\subsubsection{Usual Test Statistics and their Distributions}

As a shortcut, let us use the expression $\operatorname{Can} C o r\left(\Delta y_{t}, y_{t-1} \mid 1, \Delta y_{t-1}\right)$ to summarize the program that leads, for a $\operatorname{VAR}(2)$, to the computation of squared canonical correlations used in the Johansen's ML procedure. That means that in the VECM, one extracts the squared canonical correlations betwcen $\Delta y_{t}$ and $y_{t-1}$, both terms being regressed on a constant (model with an unconstrained constant) and here on the first lag of $\Delta y_{t}$, i.c. $\Delta y_{t-1}$. In order to test for the significance of the $r$ largest eigenvalues, one can rely on Johansen's trace statistics or one of the versions adjusted for small sample effects:

$$
\begin{aligned}
L R_{r} & =T \sum_{i=1}^{T} \ln \left(1-\lambda_{i}\right), \quad r=1 \ldots n, \\
L R_{r}^{c o r} & =(T-n p) \sum_{i=1}^{r} \ln \left(1-\lambda_{i}\right) \quad r=1 \ldots n .
\end{aligned}
$$

Under $H_{0}$, LR test statistics follow a functional of Brownian motions and asymptotic critical values can be found in Johansen (1995) or in Osterwald-Lenun (1992). Once $\beta$ has been estimated in a first step we can implement the common feature test statistics. We make again the distinction between the $\mathrm{SF}$ and the WF structures. These are respectively based on the following programs $S F: \operatorname{CanCor}\left(\Delta y_{t},\left\{\Delta y_{t-1}, \hat{\beta}^{\prime} y_{t-1}\right\} \mid 1\right)$ and $W F: \operatorname{CanCor}\left(\Delta y_{t}, \Delta y_{t-1} \mid 1, \hat{\beta}^{\prime} y_{t-1}\right)$. For the VECM of order $p-1$, the significance of the first $s$ eigenvalues is evaluated through:

$$
L R_{s}=T \sum_{i=1}^{s} \ln \left(1-\tilde{\lambda}_{i}\right) \sim \chi^{2}\left(v_{1}\right), \quad s=1 \ldots n,
$$

with $v_{1}=s \times n(p-1)-s \times(n-s)$ for the weak form and $v_{1}=s \times(n(p-1)+r)-s \times(n-s)$ for the strong form.

We investigate the behavior of a correction for small sample sizes in (5.5). We have chosen a similar small sample correction to the one proposed by Reinsel and Ahn (1992) for cointegration 
in (5.4), i.e.

$$
\begin{aligned}
& L R_{s}^{c o r}=(T-n(p-1)) \sum_{i=1}^{s} \ln \left(1-\tilde{\lambda}_{i}\right), \\
& L R_{s}^{c o r}=(T-n(p-1)-r) \sum_{i=1}^{s} \ln \left(1-\tilde{\lambda}_{i}\right),
\end{aligned}
$$

for respectively the weak form and the strong form. ${ }^{1}$ We have also computed the usual Bartlett correction by replacing respectively $(T-n(p-1))$ and $(T-n(p-1)-r)$ by $\left(T-\frac{1}{2}(n+n(p-1)+3)\right)$ and $\left(T-\frac{1}{2}(n+n(p-1)+r+3)\right)$. We do not present the results of this last corrections and will only comments the differences. Notice that Johansen (1999) has also developed a Bartlett type correction for cointegration.

More importantly and in order to avoid confusion, we do not compute an incorrect approximate $F$-test by dividing $L R_{s}$ by the number of degrees of freedom $v_{1}$ and multiplying by, e.g. for the WF, $(T-n(p-1)) / T$. Indeed, in the context of testing for the number of zeros eigenvalues, $\chi^{2}$ test statistics are asymptotic approximation of ratios of Whishart distributions in case, say, where $n>2$. The appropriate non standard F-version of this test is described in Rao (1973, p.556) and Anderson (1984, section 8).

\subsubsection{Switching Algorithms}

For the SCCF or the SF model with unknown $\beta$, Hansen and Johansen (1998, p.95) show how to impose common feature restrictions. Let us rewrite the cointegrated VAR(2) such as

$$
\Delta y_{t}=\mu+\tilde{\beta}_{\perp} \Psi_{1}^{\prime} \beta^{\prime} y_{t-1}+\tilde{\beta}_{\perp} \Psi_{2}^{\prime} \Delta y_{t-1}+\varepsilon_{t}
$$

where $\tilde{\beta}_{\perp}$ is again the orthogonal complement of the cofeature matrix, namely $\tilde{\beta}^{\prime} \tilde{\beta}_{\perp}=0_{s \times n}$ and $\operatorname{rank}\left[\tilde{\beta}: \tilde{\beta}_{\perp}\right]=n$. Hansen and Johansen (1998) impose SCCF restrictions by premultiplying the cointegrated VAR in (5.7) by the partitioning matrix $\mathrm{B}$ where

$$
\mathbf{B}=\left(\begin{array}{c}
\underbrace{\left(\tilde{\beta}_{\perp}^{\prime} \tilde{\beta}_{\perp}\right)^{-1} \tilde{\beta}_{\perp}^{\prime}}_{(n-s) \times n} \\
\underbrace{\tilde{\beta}^{\prime}}_{s \times n}
\end{array}\right)
$$

\footnotetext{
${ }^{1}$ The difference is due to the fact that in the WF, one concentrates on cointegrating vectors. Also remark from (5.1) that $T$ is the real number of observation after the deduction of initial observations in regression containing lags.
} 
to obtain:

$$
\begin{aligned}
\left(\tilde{\beta}_{\perp}^{\prime} \tilde{\beta}_{\perp}\right)^{-1} \tilde{\beta}_{\perp}^{\prime} \Delta y_{t} & =\mu^{*}+\Psi_{1}^{\prime} \beta^{\prime} y_{t-1}+\Psi_{2}^{\prime} \Delta y_{t-1}+\left(\tilde{\beta}_{\perp}^{\prime} \tilde{\beta}_{\perp}\right)^{-1} \tilde{\beta}_{\perp}^{\prime} \varepsilon_{t} \\
\tilde{\beta}^{\prime} \Delta y_{t} & =\mu^{* *}+\tilde{\beta}^{\prime} \varepsilon_{t}
\end{aligned}
$$

where $\mu^{*}=\left(\tilde{\beta}_{\perp}^{\prime} \tilde{\beta}_{\perp}\right)^{-1} \tilde{\beta}_{\perp}^{\prime} \mu$ and $\mu^{* *}=\tilde{\beta}_{\mu}^{\prime}$ are vector column of size respectively $(n-s)$ and $s$. System (5.9) can be solved such that

$$
\left(\tilde{\beta}_{\perp}^{\prime} \tilde{\beta}_{\perp}\right)^{-1} \tilde{\beta}_{. \perp}^{\prime} \Delta y_{t}=\left(\mu^{*}-\omega \mu^{* *}\right)+\Psi_{1}^{\prime} \beta^{\prime} y_{t-1}+\Psi_{2}^{\prime} \Delta y_{t-1}+\omega \tilde{\beta}^{\prime} \Delta y_{t}+\left(\tilde{\beta}_{\perp}^{\prime} \tilde{\beta}_{\perp}\right)^{-1} \tilde{\beta}_{\perp}^{\prime} \varepsilon_{t}-\omega \tilde{\beta}^{\prime} \varepsilon_{t},
$$

where $\omega=\operatorname{Cov}\left(\left(\tilde{\beta}_{\perp}^{\prime} \tilde{\beta}_{\perp}\right)^{-1} \tilde{\beta}_{\perp}^{\prime} \varepsilon_{l}, \tilde{\beta}^{\prime} \varepsilon_{l}\right) \operatorname{Var}\left(\tilde{\beta}^{\prime} \varepsilon_{t}\right)^{-1}$

Cointegrating vectors under common feature restrictions can be obtained by solving the program $\operatorname{CanCor}\left(\left(\tilde{\beta}_{\perp}^{\prime} \tilde{\beta}_{\perp}\right)^{-1} \tilde{\beta}_{\perp}^{\prime} \Delta y_{t}, y_{t-1} \mid 1, \Delta y_{t-1}, \tilde{\beta}^{\prime} \Delta y_{t}\right)$. As a result, it is possible to estimate $\beta$ without constraints in a first step. Then, fixing the matrix $\beta$ to its estimated value, one obtains the estimates of the $n-s$ dynamic common factors $\Psi^{\prime}=\left(\Psi_{1}^{\prime}, \Psi_{2}^{\prime}\right)$ through (5.7), i.e. using the duality principle (see Chapter 2). We can restimate $\beta$ in (5.9) and go on iterating until convergence is reached.

In the WF case, the constrained model is simply:

$$
\Delta y_{t}=\mu+\alpha \beta^{\prime} y_{t-1}+\tilde{\beta}_{\perp} \Psi_{2}^{\prime} \Delta y_{t-1}+\varepsilon_{t}
$$

Imposing WF restrictions is convenient because it allows to consider both cointegration and common feature test statistics without the constraint $r+s \leq n$. To solve (5.10), we start by estimating $\beta$ by ML and we fix it to estimate the number of common feature vectors $s$. We estimate the $n-s$ dynamic common factors forming $\Psi_{2}^{\prime}$ in (5.10) and we use this constraint to reestimate $\beta$ using the program $\operatorname{CanCor}\left(\Delta y_{t}, y_{t-1} \mid 1, \hat{\Psi}_{2}^{\prime} \Delta y_{t-1}\right)$. This sequence is itcrated until convergence is reached. ${ }^{2}$ Note however that in both cases (SCCF and WF) there is no guarantee that the global maximum is reached but still the likelihood is increased in each iteration.

In both cases, tests for common features and cointegration can be obtained by comparing maxima for each $r=0 \ldots n$ and $s=0 \ldots n$. In general, convergence is attained after a few iterations.

\footnotetext{
${ }^{2}$ In our simulations, convergence is defined when the difference in the value of log-likelihood between two iterations is less then $10^{-6}$.
} 


\subsection{Monte Carlo Design and Test Statistics}

\subsubsection{The Data Generating Process}

The underlying data generating process in the simulation design is a stylized second order VAR with four $I(1)$ variables and two cointegrating vectors. There are three wcak form common feature vectors, i.e. $\operatorname{rank}\left(\Gamma_{1}\right)=1$. The three normalized linearly independent co-feature vectors

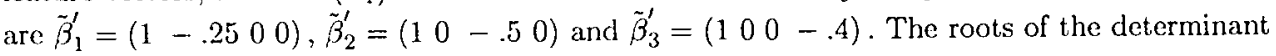
of the characteristic equation are $1,1,-1.55,1.02 \pm .35 i$. From Lemma 3.6 we know that $s W F$ vectors imply $s-r$ strong form or SCCF vectors. ${ }^{3}$ In this casc that means that one SCCF vector cxists. But this vector suffers from identification problem (sec Chapter 3).

$$
\begin{aligned}
\left(\begin{array}{l}
\Delta y_{1 t} \\
\Delta y_{2 t} \\
\Delta y_{3 t} \\
\Delta y_{4 t}
\end{array}\right)= & \left(\begin{array}{c}
.25 \\
-.15 \\
-.1 \\
.5
\end{array}\right)+\left(\begin{array}{cc}
-.2 & .2 \\
-.8 & -.4 \\
-1 & .8 \\
-.5 & 0
\end{array}\right)\left(\begin{array}{llll}
1 & 0 & 1.2 & -1 \\
0 & 1 & -.8 & -1
\end{array}\right)\left(\begin{array}{l}
y_{1 t-1} \\
y_{2 t-1} \\
y_{3 t-1} \\
y_{4 t-1}
\end{array}\right) \\
& +\left(\begin{array}{c}
-.1 \\
-.4 \\
-.2 \\
-.25
\end{array}\right)\left(\begin{array}{llll}
2 & 1 & 1 & 1
\end{array}\right)\left(\begin{array}{l}
\Delta y_{1 t-1} \\
\Delta y_{2 t-1} \\
\Delta y_{3 t-1} \\
\Delta y_{4 t-1}
\end{array}\right)+\left(\begin{array}{l}
\varepsilon_{1 t} \\
\varepsilon_{2 t} \\
\varepsilon_{3 t} \\
\varepsilon_{4 t}
\end{array}\right)
\end{aligned}
$$

where the $\varepsilon_{i t}$ follow a zero mean Gaussian distribution with all variances equal to 1 and all covariances equal .7. Three sample sizes are considered, namely $T=50,100,200$. A sample size of 50 observations roughly corresponds to that in macrocconomic studies on annual post WWII data sets (a good example for SCCF is Engle and Issler, 1995). 100 and 200 observations are common with quarterly time series. ${ }^{4}$ Computations have been carried out using Gauss version 3.2.32. 10.000 replications are used, the first 50 observations are discarded to remove dependence on initial observations. Figures 5.1 to 5.4 portray a typical realization of such a four dimensional process with 100 obscrvations.

\subsubsection{Simulation Results}

The main results are summarized in Table 5.1 and Table 5.2. Table 5.1 analyzes the empirical size and power of Johansen trace statistics (model with an unconstrained constant) with and without WF common feature restrictions. The nominal size has been fixed to $5 \%$ with asymptotic critical values given in Osterwald-Lenun (1992). Rows labelled $s=0$ report the rejection frequencies

\footnotetext{
${ }^{3}$ This is a direct extension of Vahid and Engle (1993)'s lemma which shows that in a cointegrated VAR(1), i.e. a model with $n$ WF vectors, there exist $n-r$ SCCF vectors. We did not implement a mixed form, i.e. the case where more then the $s-r$ implied SF vectors exists.

${ }^{4}$ Notice that the use of seasonally adjusted data dramatically alters size and power of common feature test statistics (Cubadda, 1999 and Chapter 7 of this monograph). At the opposite, time aggregation causes lack of power (Beine and Hecq, 1999).
} 


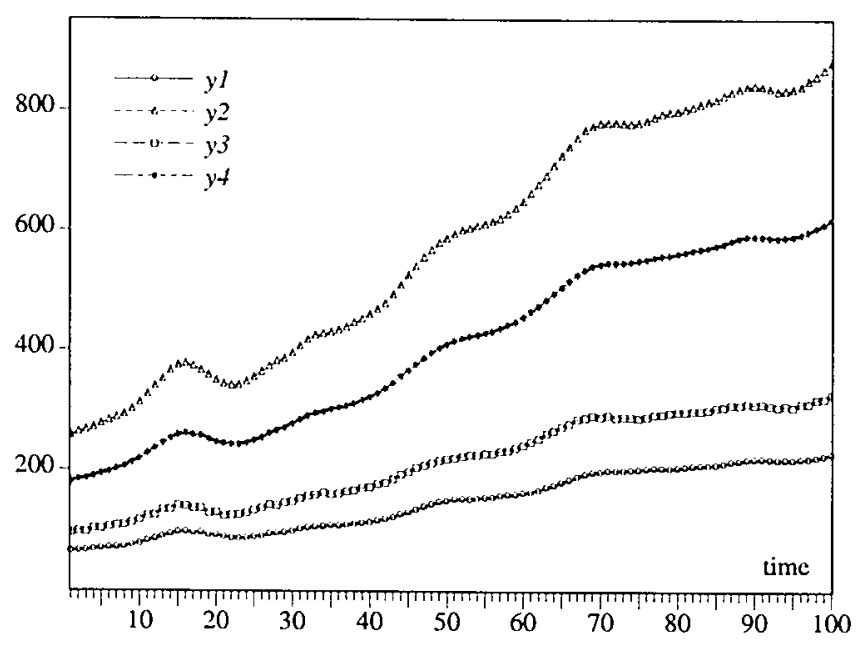

Figure 5.1: A Realisation of the DGP, Data in Levels

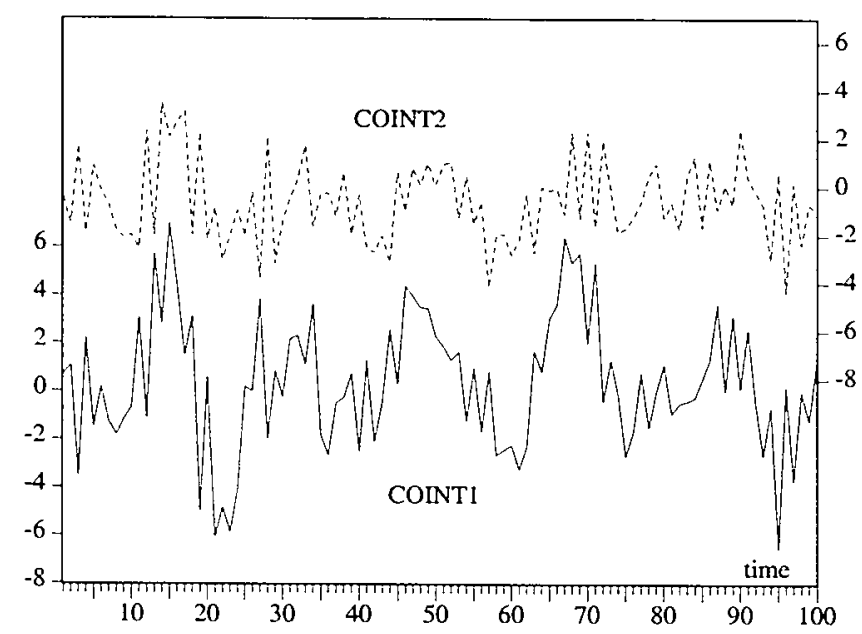

Figurc 5.2: A Rcalisation of the DGP, Cointegrating Vectors 


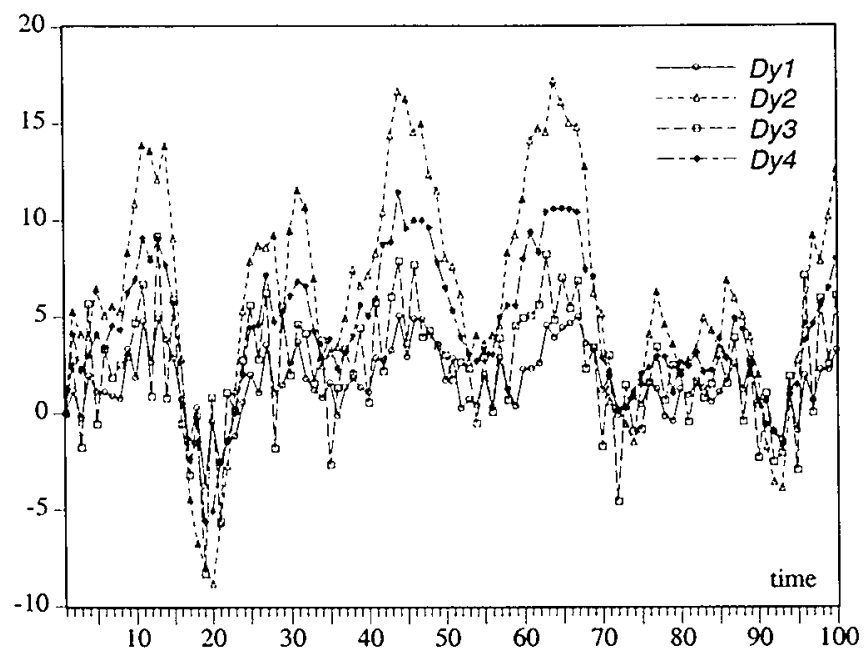

Figure 5.3: A Realisation of the DGP, Data in First Differences

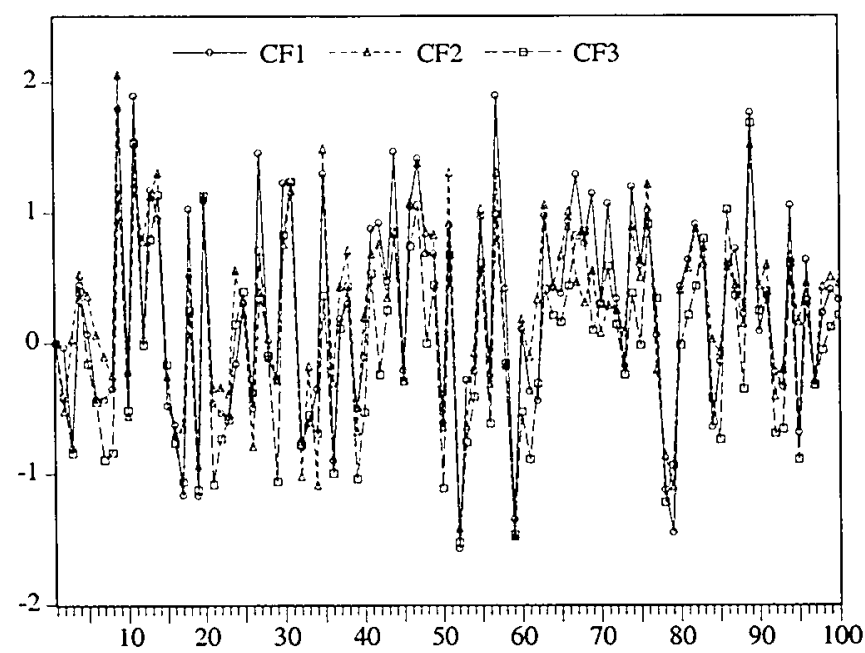

Figure 5.4: A Realisation of the DGP, Cofeature Relationships 


\begin{tabular}{|c|c|c|c|c|c|c|c|c|c|}
\hline$T$ & $\begin{array}{l}I_{0}: \\
\text { Fixed }\end{array}$ & $\begin{array}{l}\tau^{*}=0 \\
\gamma=2\end{array}$ & $r \leq 1$ & $\boldsymbol{r} \leq 2$ & $r \leq 3$ & $\begin{array}{l}r=0 \\
p=4\end{array}$ & $\tau^{*} \leq 1$ & $r \leq 2$ & $r \leq 3$ \\
\hline \multirow[t]{5}{*}{50} & $s=0$ & $100 \quad 100$ & $00.5 \cdot 496.33$ & $15.41 \quad 7.08$ & $7.03 \quad 4.74$ & $98.45 \quad 53.03$ & $\begin{array}{lll}68.16 & 10.07\end{array}$ & $16.98 \quad 1.52$ & $10.49 \quad 3.83$ \\
\hline & $s=1$ & $100 \quad 100$ & 99.5896 .52 & 15.327 .16 & 6.974 .6 & $98.90 \quad 60.14$ & $71.74 \quad 12.52$ & $16.68 \quad 1.90$ & $9.15 \quad 3.26$ \\
\hline & $s=2$ & $100 \quad 100$ & 99.6397 .46 & 14.947 .05 & 6.78 .4 .36 & $90.84 \quad 82.98$ & $81.84 \quad 24.77$ & $15.45 \quad 2.66$ & $7.66 \quad 2.50$ \\
\hline & $s=3$ & $100 \quad 100$ & $100 \quad[06)$ & $13.1 \quad 6.04$ & $5.78 \quad 3.59$ & $100 \quad 100$ & $06.54 \quad 63.27$ & $13.33 \quad 1.69$ & $5.92 \quad 1.8 x$ \\
\hline & $s=4$ & $100 \quad 100$ & 70.5067 .62 & $4.4 .75 \quad 31.16$ & $13.910 . \%$ & $100 \quad 100$ & $79.59 \quad 50.32$ & $44.75 \quad 16.55$ & $13.91 \quad 5.85$ \\
\hline \multirow[t]{5}{*}{100} & $s=0$ & $100 \quad 100$ & $100 \quad 100$ & $8.35 \quad 5.86$ & $-1.48 \quad 3.42$ & $100 \quad 100$ & $97.01 \quad 84.70$ & $9.66 \quad 4.25$ & $5.97 \quad 3.55$ \\
\hline & $s=1$ & $100 \quad 100$ & 100100 & 8.35 .89 & 4.443 .45 & $100 \quad 100$ & $97.94 \quad 87.72$ & $9.66 \quad 4.19$ & $\begin{array}{ll}5.61 \quad 3.38\end{array}$ \\
\hline & $s=2$ & $100 \quad 100$ & 100100 & 8.255 .63 & 4.463 .47 & $100 \quad 100$ & $98.80 \quad 93.16$ & $8.87 \quad 3.81$ & $5.06 \quad 3.10$ \\
\hline & $s=3$ & $100 \quad 100$ & $100 \quad 100$ & 7.895 .28 & 4.083 .03 & $100 \quad 100$ & $100 \quad 99.08$ & $7.89 \quad 3.29$ & $4.06 \quad 2.36$ \\
\hline & $s=4$ & $100 \quad 100$ & $07.39 \quad 05.46$ & 64.0159 .86 & 13.3711 .28 & $100 \quad 100$ & $97.30 \quad 92.13$ & $64.91 \quad 53.52$ & $13.27 \quad 0.20$ \\
\hline \multirow[t]{5}{*}{200} & $s=0$ & $100 \quad 100$ & $100 \quad 100$ & $6.26 \quad 4.90$ & $2.74 \quad 2.35$ & $100 \quad 100$ & $100 \quad 100$ & $6.67 \quad 4.1$ & $3.03 \quad 2.23$ \\
\hline & $s=1$ & 100100 & $100 \quad 100$ & 6.34 .92 & 2.722 .34 & $100 \quad 100$ & $100 \quad 100$ & $6.4 \quad 4.07$ & $\begin{array}{ll}3.03 & 2.29\end{array}$ \\
\hline & $s=2$ & 100100 & 100100 & 6.174 .69 & 2.742 .3 .4 & $100 \quad 100$ & $100 \quad 100$ & 6.33 .8 .4 & $2.75 \quad 2.20$ \\
\hline & $s=3$ & 100100 & $100 \quad 100$ & 5.804 .62 & $2.72 \quad 2.35$ & $100 \quad 100$ & $100 \quad 100$ & $5.78 \quad 3.76$ & $2.68 \quad 2.02$ \\
\hline & $s=4$ & 100100 & $100 \quad 100$ & 71.6360 .31 & $12.44 \quad 11.41$ & $100 \quad 100$ & $100 \quad 100$ & $71.63 \quad 66.97$ & $12.44 \quad 10.76$ \\
\hline
\end{tabular}

Table 5.1: Switching Cointegrating Trace Tests under WF restrictions

when no common feature restrictions are imposed. For $s=1,2,3,4$ we report rejection frequencies obtained using an iterative procedure to reach the maxima. $s=3$ is the correct number of restrictions. When one overestimates $s$, i.e. $s=4$, the estimated system reduces to a VAR(1). For the dynamics, we consider a structure with the correct choice of the lag order, i.c. $p=2$. We compare these results with the one we obtain when we overestimate the lag order, i.e. $p=4$. In the DGP, we have fixed $r=2$. Consequently, the rejection frequencies under the hypotheses $r=0$ and $r \leq 1$ indicate the power when detecting the presence of respectively at least one and more than one cointegrating vector. For the size properties we focus on the column $H_{0}: r \leq 2$. In each cell we report the rejection frequencies of the usual asymptotic trace test and the small sample corrected version.

Some comments are in order:

- For $s=0$, we note some size distortions for small samples, especially for $T=50$ or 100 . Empirical sizes for the asymptotic tests are about $16 \%$ for $T=50$ and $9 \%$ for $T=100$ whatever the choice of $p$. The small sample correction behaves quite well when the true lag order $p=2$ is used but behaves poorly when $p$ increases because of the strong correction $T-n p$ lead to underestimate the number of cointegrating vectors. ${ }^{5}$

- Taking into account the common feature restrictions always slightly reduces (mainly for

\footnotetext{
${ }^{5}$ An alternative strategy is proposed by Gonzalo and Pitarakis (1999) in the cointegration context. They show the usefulness of information criteria in determining jointly the lag order $p$ and the number of cointegrating vectors $r$. We have already proposed this approach for a common feature analysis in Chapter 3 and we will use it in Chapter 6.
} 


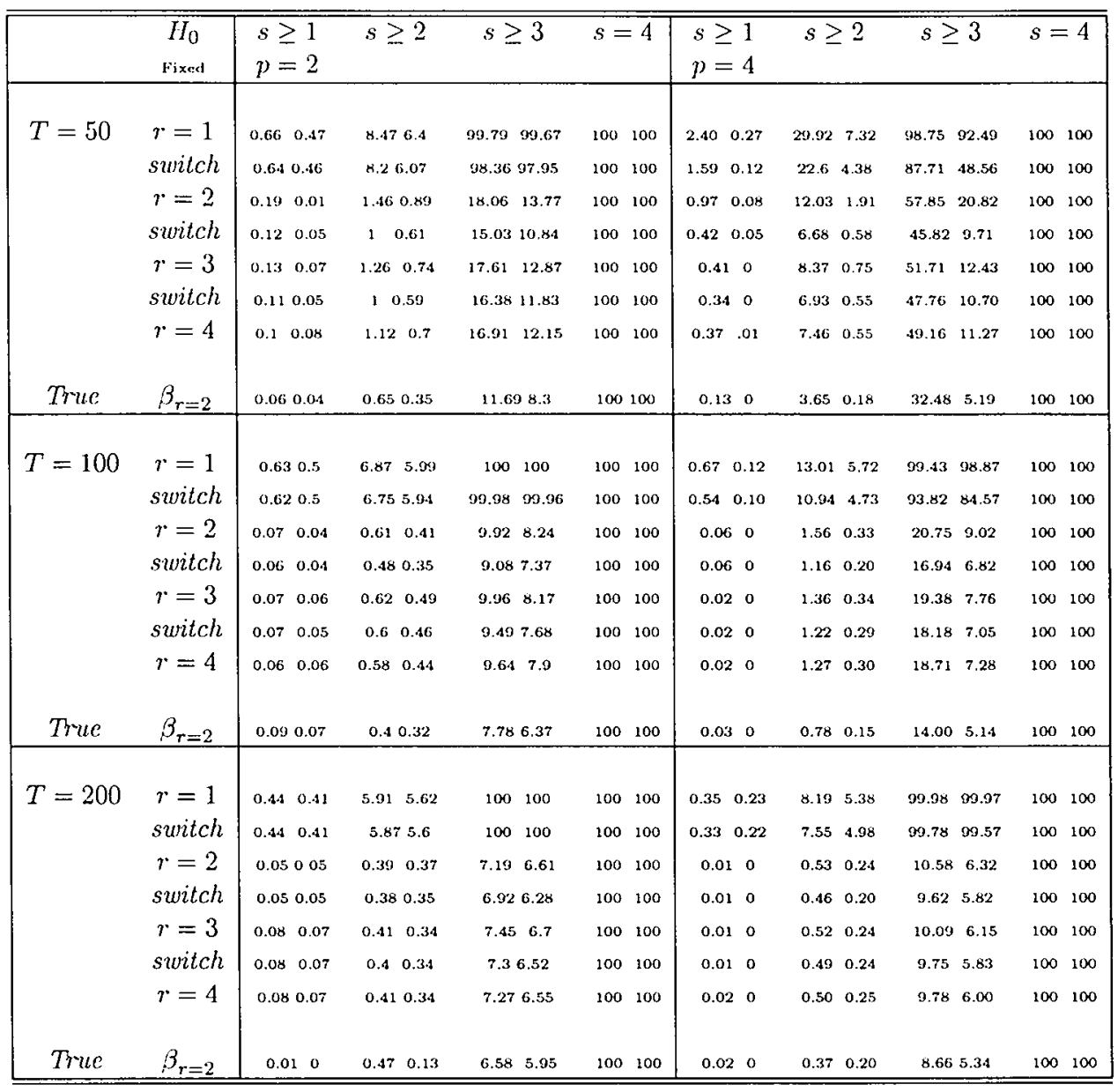

Table 5.2: Two Step and Iterative WF Common Feature Tests, $\mathrm{p}=2$ and 4 
$T=50)$ size distortions with the reduction being maximum with the correct number of restrictions, i.e. $s=3$. But an overestimation of the number of common feature restrictions leaves some dynamics in the system. This huge size increase is also observed by Boswijk and Franses (1992) for cointegration tests.

- The most important aspect when testing for cointegration under WF restrictions is maybe the increase of power when the lag length is large. With $T=50$ and for $s=0$, we determine in only $10 \%$ the presence of at least a second cointegrating vector for the small sample corrected statistics while this proportion increases to $63 \%$ with $s=3$.

Table 5.2 presents WF common feature test statistics. For each $r$, we compare the rejection frequencies with and without adopting a switching procedure. For $r=4$, it does not make sense to switch because the cointegrating space spans $\mathbb{R}^{n}$. Results with $\beta$ known are also given for the purpose of comparison.

The following results emerge:

- The switching procedure reduces considerably the size distortions. However, these size distortions are larger than the ones obtained with known cointegrating vectors.

- The behavior of the test statistics is "optimal "when the cointegrating rank is fixed to its true value. Test statistics marginally suffer when $r$ is overestimated but are dramatically altered, in the sense of discovering less common feature vectors, when $r$ is underestimated. See Chapter 3 on this point.

- Small sample corrections seem to work well, especially when the lag order is relatively large. For instance, under $r=2$ when $p=4$ and $T=50$, the empirical size is $9.7 \%$ with the small sample correction and $45.82 \%$ without it. Notice that for the same specification, a two steps strategy would respectively yield empirical sizes of $20.82 \%$ and $57.85 \%$.

We do not report the results of the SCCF test statistics since we want to let $r$ and $s$ vary freely. The underlying DGP implies one SF common feature vector but to detect it is better to rely on the mixed form framework developed in Chapter 3. To simplify the analysis, we consider the same DGP with $\Gamma_{1}=0_{n \times n}$. In this case, we have a $\operatorname{VAR}(1)$ and the space spanned by $\tilde{\beta}$ is actually the space spanned by the columns of $\alpha_{\perp}$. We consider the case with $T=50$ and $p=2$ in the estimated model and compare the SCCF empirical sizes $\left(H_{0}: s \geq 2\right)$ when $\beta$ is known and estimated. In the latter case we can analyze the effect of an overestimation of the number of cointegrating vector $r$. The following pairs refer to $L R_{s}$ and $L R_{s}^{c o r}$ respectively. When $\beta$ is fixed to its true value we obtain the rejection frequencies $[9.65 \% 5.22 \%]$. When $\beta$ is estimated, the two step approach gives respectively $[14.63 \% 8.83 \%]$ and $[47.43 \% 30.09 \%]$ for $r=2$ and $r=3$. A switching procedure reduces these percentages. But the important point here is the large size distortion when overestimating $r$. This explains why we have proposed in Chapter 3 to start with the WF in order to know if there exists some implied $n-r$ SF. In the case where this makes 


\begin{tabular}{l|ccccc}
\hline \hline $\mathrm{H}_{0}$ & Eigv. & Loglik & $-T \sum \ln \left(1-\lambda_{i}\right)$ & $-(T-n p) \sum \ln \left(1-\lambda_{i}\right)$ & $95 \% \mathrm{cv}$ \\
\hline$r=0$ & .837 & 1404.41 & $208^{*}$ & $130^{*}$ & 114.9 \\
$r \leq 1$ & .562 & 1424.23 & $120.8^{*}$ & 75.52 & 87.3 \\
$r \leq 2$ & .500 & 1440.89 & $81.19^{*}$ & 50.74 & 63.0 \\
$r \leq 3$ & .401 & 1453.23 & $47.87^{*}$ & 29.92 & 42.4 \\
$r \leq 4$ & .296 & 1461.68 & 23.19 & 14.5 & 25.3 \\
$r \leq 5$ & .122 & 1464.83 & 6.28 & 3.92 & 12.3 \\
\hline \hline
\end{tabular}

Table 5.3: Cointegration Trace Statistics, p=3, 1947-1997

sense, i.c. $s+r \leq n$, a LR test with the SF null hypothesis against the WF alternative can be casily undertaken.

Remark 5.1 Other small sample corrections. For our DGP, Bartlett small sample correction behave better for $p=2$, is equivalent to the one proposed in this paper for $p=3$ and the correction $\grave{a}$ la Reinsel and Ahn is far better when $p=4$. Also the "crude" F-test approximation (i.e. not the Rao (1973) one) of the asymptotic $\chi^{2}$ appears to work quite well for $p=2$. However, when increasing the lag order, the empirical size approaches zero, being less than $1 \%$ even when $T=200$. This leads in practice to overestimating the number of common feature vectors.

\subsection{Empirical Analyses}

\subsubsection{About US Regional Income Dynamics}

The first application illustrates the usefulness of the proposed procedure for analyzing the longrun and short-run comovements among per capita real incomes of six regions of the United States. These are New England (NE), Mideast (ME), Great Lakes (GL), South East (SE), South West (SW) and Far West (FW). ${ }^{6}$ The annual time series, ranging from 1947 to 1997, are from the Burcau of Economic Analysis (BEA); US GDP deflator is used to transform the data in real terms. In order to save space we do not plot the series because part of them have been introduced in Chapter 1. On similar variables but for another period, Vahid and Engle (1993) obtain one cointegrating relationship and two serial correlation common feature vectors among NE, ME, GL, FW. Carlino and Sill (1998) find out two cointegrating vectors and four SCCF vectors among seven regions (all regions minus $\mathrm{RM}$ ).

As it is often the case, different criteria determine different lag orders. In our six dimensional system, SBC sclects one lag, the likelihood ratio favors three lags while AIC and HQC would select up to six lags! This is clearly a small sample problem. We consider $p=3$ which was enough to ensure the absence of any vector and/or individual misspecification in the residuals.

\footnotetext{
${ }^{6}$ We did not consider the last to regions, i.e. Rocky Mountains (RM) and Plains (PL) for practical reason of lack of degrees of freedom.
} 


\begin{tabular}{l|ccc|ccc}
\hline \hline$\chi^{2}$ adj p-valuc & \multicolumn{3}{|c|}{ 2 step } & \multicolumn{3}{|c}{ Iterative } \\
$\mathrm{H}_{0}$ & $r=2$ & $r=3$ & $r=4$ & $r=2$ & $r=3$ & $r=4$ \\
\hline$s \geq 1$ & .703 & .875 & .869 & .786 & .899 & .895 \\
$s \geq 2$ & .721 & .928 & .956 & .792 & .943 & .969 \\
$s \geq 3$ & .417 & .609 & .903 & .567 & .790 & .938 \\
$s \geq 4$ & .197 & .105 & .261 & .380 & .402 & .336 \\
$s \geq 5$ & .027 & .006 & .021 & .091 & .072 & .031 \\
$s=6$ & $<.001$ & $<.001$ & $<.001$ & $<.001$ & $<.001$ & $<.001$ \\
\hline
\end{tabular}

Table 5.4: Two Stcp and Iterative WF Tests Statistics

\begin{tabular}{l|ccccc}
\hline \hline $\mathrm{H}_{0}$ & \multicolumn{2}{|c}{$-T \sum \ln \left(1-\lambda_{i}\right)$} & $-(T-n p)$ & $\ln \left(1-\lambda_{i}\right)$ & $95 \% \mathrm{cv}$ \\
& $s=4$ & $s=5$ & $s=4$ & $s=5$ & \\
\hline$r=0$ & $193.69^{*}$ & $182.33^{*}$ & $121.06^{*}$ & 113.96 & 114.9 \\
$r \leq 1$ & $110.45^{*}$ & $96.24^{*}$ & 69.03 & 60.15 & 87.3 \\
$r \leq 2$ & $68.50^{*}$ & 62.11 & 42.81 & 38.82 & 63.0 \\
$r \leq 3$ & 34.48 & 30.86 & 21.55 & 19.28 & 42.4 \\
$r \leq 4$ & 11.98 & 12.97 & 7.49 & 8.10 & 25.3 \\
$r \leq 5$ & 4.35 & 5.24 & 2.71 & 3.27 & 12.3 \\
\hline
\end{tabular}

Table 5.5: Itcrative Cointegration Analysis

Table 5.3 reveals that asymptotic Johansen's trace statistics (with a constrained deterministic trend in the cointegrating space) detect four and almost. five cointegrating vectors at $95 \%$ level and even six at $85 \%$. Even if these results can be motivated by cconomic convergence arguments, previous studies on the same kind of data stress the presence of only a small number of cointegrating vectors. A graphical inspection tells us that the number of cointegrating vectors may be up to four but more probably three. The moduli of the largest roots of the companion matrix $(1, .967, .967, .856, .856)$ favor three common trends.

Using this information, we conduct WF common feature tests with successively $r=2,3$ or 4 . Table 5.4 presents $p$-values for a two-step and the iterative approach (small sample corrected version). The two step approach selects $s=4$ while the switching approach reinforces the probability of non rejecting the null that $s \geq 4$, but does not reject the presence of a fifth common feature vector. Using $s=4$ and $s=5$ it is possible to compute cointegration test statistics under these constraints. It emerges from Table 5.5 that one would not accept more than three cointegrating vectors but $r=1$ or 2 are also possible choices. Actually, a plot of these graphs does not even give a clear cut between $r=1,2$ or 3 . We do not go further because under the assumption of only three cointegrating vectors, exclusion test statistics do not reject the null that SW income does not belong to the cointegrating space. It is then possible to reduce the dimension of the system (see Harbo et al., 1998) but this is beyond the scope of this illustrative examplc.

Finally, even if a large number of common trends seems to govern the long-run behavior 
of the six US per capita real incomes, the short-run dynamies can be summarized by only one common factor. This unique weak form common cycle may be extracted using methods proposed in Chapter 4 to give information about regional macroeconomic fluctuations.

\subsubsection{Belgian Regional Employment Dynamics}

Candelon, Hecq and Lohest (2000) investigate the relationship between employment dynamics and migration in Europe. They consider the case of Belgium, a very interesting country to forecast the degree of labor mobility in Europe. First, Belgium presents a variate industrial profiles. Old industrial regions mainly based on metallurgy (Hainaut, Liege) or weaver (like in Verviers in the province of Liege), and recent districts growing with high-tech industries (Antwerp). Sccond, Belgium is composed of multicultural groups with their own language and their own economic dynamics. The French speaking region and the German speaking region form the Walloon Region and Flemish speaking region is called Flanders. Brussels is the third region and is partly French and Flemish speaking. Nevertheless, the federal government imposes the same employment legislation and the same social laws but delegates a high level of autonomy to local governments. The role played by the federal government can be thus compared to the European commission. Lastly, wage disparities within Belgium are inexistent and can not be considered as a determinant of migration. Consequently, the major economic factor leading people to migrate could be the employment dynamics.

Candelon, Hecq and Lohest (2000) have applied the permanent-transitory decomposition proposed in Chapter 4 for studying regional employment dynamics. They also analyze the role of the trend and cycle innovations on migration flows in a causality analysis. In this subsection we analyze the cyclical parts of provincial employment data with and without taking into account the connections between cointegration and common feature restrictions. Our analysis covers the period 1948-1997 and is performed for 10 provinces Antwerp, Flemish Brabant, Walloon Brabant, Eastern Flanders, Western Flanders, Hainaut, Liege, Limburg, Luxemburg, Namur and Brussels, which is considered as a province and a region at the same time. Provinces of Hainaut, Liege, Luxemburg, Namur, Walloon Brabant form Wallonia. Flanders includes the provinces of Anvers, Flemish Brabant, Eastern Flanders, Western Flanders and Limburg. Figure 5.5 specifies the spatial localization of the 10 provinces. We consider employment series as defined in the ONSS (Office National de Sécurité Sociale) database.

From Table 5.6, a simple statistical analysis (median, mean, variance and skewness) of employment growth rates reveals important disparities among provinces. The Flemish provinces, in particular Limburg, have the highest average employment growth. Using the median in order to get a more robust analysis to outliers, Flanders Brabant have the highest employment growth rate. Disparities are also noticeable between provinces: Hainaut and Liege, which are early industrialized provinces based on mining, metallurgy or weaver, have a low employment growth rate, East Flanders, West Flanders, Namur and Antwerp have a moderate one, whereas Walloon Brabant, Flanders Brabant, Limburg and Luxemburg have a fast positive evolution of 


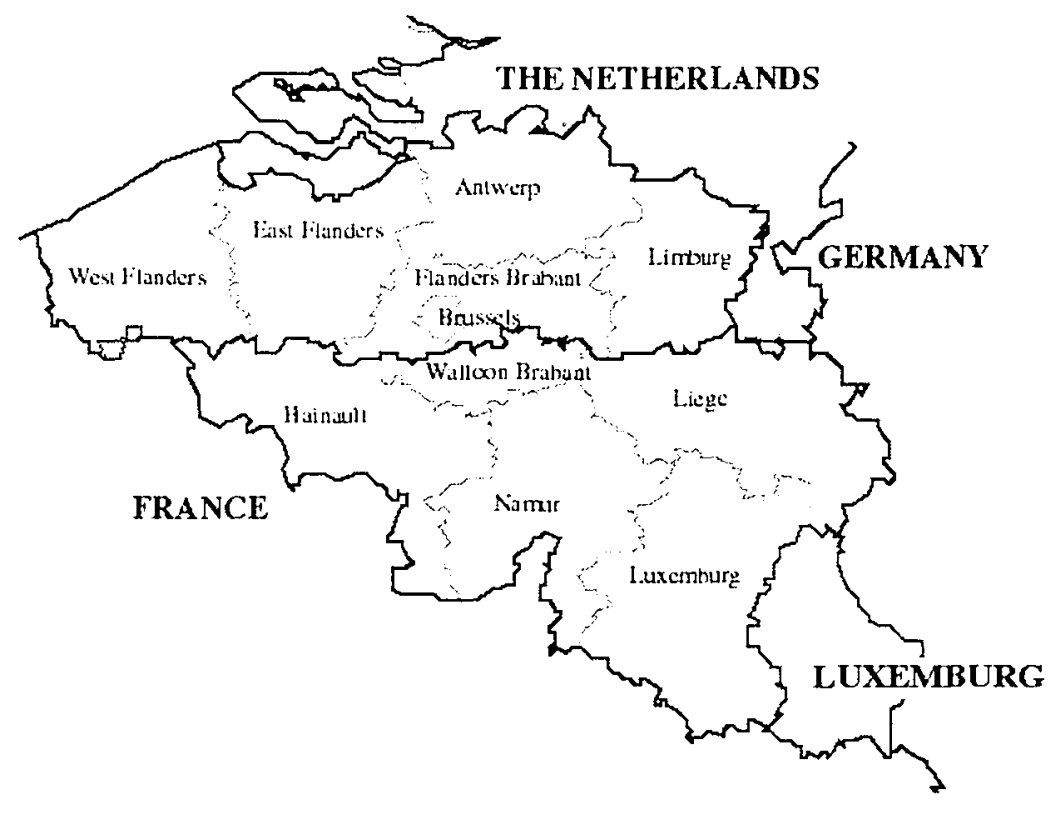

Figure 5.5: Belgian Provinces

\begin{tabular}{lcrrrrc}
\hline \hline Provinces & Nedian & Nean & Stdv & Min & Max & Skewness \\
\hline Antwerp & .0097 & .0120 & .0299 & .0299 & .0890 & -1.314 \\
Flanders Brabant & .0201 & .0200 & .0247 & -.0258 & .0965 & .479 \\
West Flanders & .0126 & .0146 & .0257 & -.0478 & .0748 & .151 \\
East Flanders & .0106 & .0083 & .0254 & -.0699 & .0680 & -.599 \\
Limburg & .0141 & .0207 & .0277 & -.0321 & .1005 & 1.096 \\
\hline Walloon Brabant & .0145 & .0150 & .0314 & -.0468 & .1338 & 1.003 \\
Hainaut & -.0058 & -.0041 & .0283 & -.0643 & .1017 & 1.128 \\
Licge & -.0044 & -.0026 & .0211 & -.0441 & .0533 & .374 \\
Luxemburg & .0200 & .0214 & .0478 & -.1519 & .1762 & .461 \\
Namur & .0096 & .0096 & .0324 & -.0483 & .1355 & 1.552 \\
\hline \hline
\end{tabular}

Table 5.6: Statistics on Provincial Employment Growth Rates 
cmployment. Most of these latter provinces are located in the Flemish region, except Luxemburg. This exception can be explained by the strong economic link of this province with Luxemburg country, which has a typical employment dynamics. We can also analyze the asymmetry of these "growth rates cycles" using the skewness statistics $\hat{\mu}_{3}$. The conventional stand is that downturns are faster than upturns. Asymmetry is expected to result in negative skewness for procyclical variables because fewer but larger negative growth rates dominate over the more numerous but smaller positive growth rates. Under the null of no skewness $\frac{\sqrt{ } T}{\sqrt{6}} \hat{\mu}_{3} \sim N(0,1)$. Having a look at these statistics we can infer that Limburg, Walloon Brabant, Hainaut, Namur provinces are asymmetric and countercyclical, while Antwerpen, East Flanders are asymmetric and procyclical using a one sided test at a $5 \%$ nominal level.

The methodology defined in Chapter 4 is been applicd to provincial employment in order to characterize the common employment dynamies both with and without an iterative approach. We use series in levels and not in logs in order to get comparable results when we come from a region to its subregional entities. Two different analyses are performed: one for Flanders and another one for Wallonia. Both of these studies are performed integrating Brussels as an additional variable resulting in six dimensional systems. This choice is motivated by the important part of the Walloon (Flemish) population, who is working in Brussels Capital (especially civil servants). ${ }^{7}$

We have retained a $\operatorname{VAR}(2)$ for Flemish provinces and a VAR(3) for Walloon ones. ${ }^{8}$ For Flanders, two cointegrating vectors, hence four common trends, are retained. This result suggests that employment dynamics in Flanders is heterogeneous in the long-run. In the short-run, two SF common feature vectors and three WF are found. We retain the structure with three WF vectors. Cyclical employment dynamics in Flanders highly depends on the specific condition of the provincial labor market, hence only a slight part of this dynamics can be considered as common. In Wallonia, three or four cointegrating vectors and hence three or two common trends are retained. Long-run employment dynamics is thus more heterogeneous in Flanders than in Wallonia. For short-run comovements, three WF common feature vectors and hence three weak form common cycles are accepted. This result induces that short-run employment dynamics in Wallonia has the same kind of homogencity compared to as Flanders provinces. Detailed results can be found in Candelon, Hecq and Lohest (2000).

Once we have determined $r$ and $s$ in a two step analysis, we compare this two step procedure with the one that "switches" or iterates between cointegrating and common feature restrictions until the convergence of the (local) likelihood. A fourth WF cofeature vector is found in Wallonia and to a lesser extent within Flemish provinces. However, we retain the same number $r$ and $s$ for both methods to dissociate the increase in estimation accuracy due to the iterative procedure from a modification of the number of comovements. From these analyses, it is now possible to

\footnotetext{
${ }^{7}$ Nevertheless, we do not integrate the other region, arguing that the attractiveness of the Flemish region (Walloon) for the Walloon population is not important because of cultural and linguistic barriers. The consequences of this hypothesis of separability are analyzed in Chapter 8.

${ }^{8}$ In both systems, there is a deterministic linear trend constrained in the long-run and two additional impulse dummies (1964 and 1969) unconstrained in the short-run.
} 


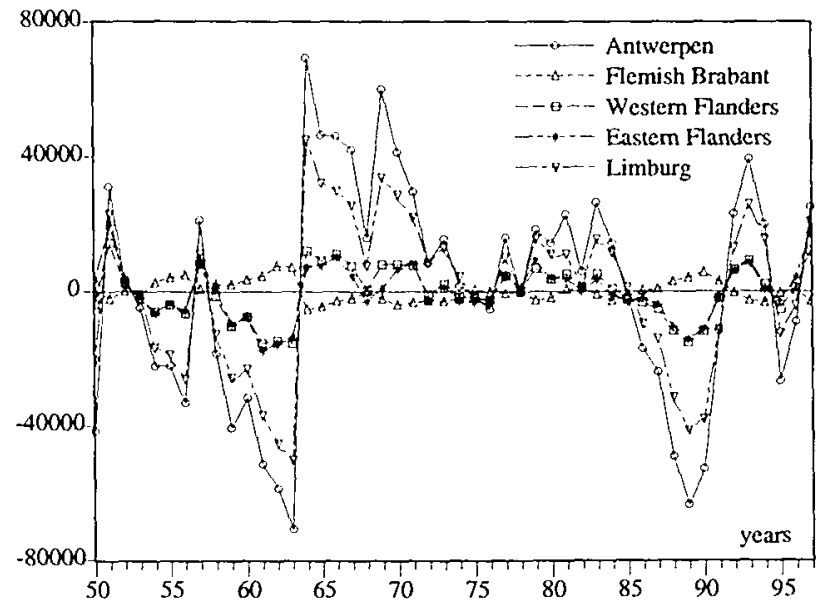

Figure 5.6: Two Step Approach, Cyclical Parts of Flemish Provinces

extract the cyclical parts of employment at a provincial level. Figures 5.6 and 5.7 present these results for the two step approach while Figures 5.8 and 5.9 are for the switching approach. Inside the same region, the same scale $(Y$-axe) is used for both methods.

Using the iterative approach, we notice one major cycle (with a through in 63, a peak in 70 and a through in 89) within Flemish provinces. Two provinces seem to dominate this evolution: Antwerp and Limburg. This can find its origin in the fact that both are old industry provinces (shipyards, automobile industries,...) and are natural trade ways to the Netherlands (imports arrive in Antwerp and transit via Limburg). They thus have been affected by oil shocks (in the 70's) but also by the favorable evolution in the labor market in the Netherlands (especially Limburg since the $80^{\circ} \mathrm{s}$ ). They are morcover the most important Flemish provinces in terms of population. It is also noticcable that. West Flanders and East Flanders share the same evolution, whereas Flanders Brabant has nearly no short-run dynamics. The shape is a little bit different using the two step estimator: the peak appears in 1964 and Flemish Brabant looks like countercyclical.

With the itcrative approach, employment cycle is unsynchronized within Wallonian provinces (through in 67, peak in 74 and through in 86). It scems that Wallonian provinces have been more affected by the industrial restructuring in the mid 60's (first mines closed) and the oil shocks in the mid 70's. Liege and Hainaut are the two Wallonian provinces that have been the most affected, because they gather firms dealing with the metallurgy of iron and steel and mines (La Louviere for example). Namur and the Walloon Brabant have specific evolutions. This is mainly due to their proximity with the federal capital Brussels and to the decision to settle the regional 


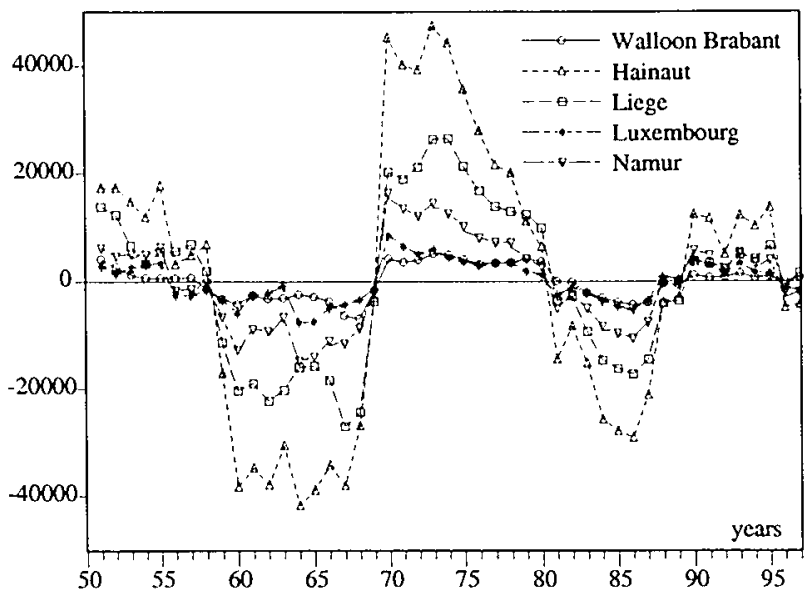

Figure 5.7: Two Step Approach, Cyclical Parts of Walloon Provinces

administration in Namur (now capital of Wallonia). The province of Luxemburg has a specific cyclical dynamics because of the strong link with the country of "Luxemburg", and its labor market, mainly affected by the banking sector. With the two step approach, the amplitude of the cycle is higher. A second peak exists in 1971 and Luxembourg is now procyclical.

Some of the differences could appear clearer using descriptive statistics reported in Tables 5.7 and 5.8. Beyond the median, the standard deviation, the max, the min and the skewness of the series we have also reported the Bera and Jarque normality test and the first two estimated partial autocorrelation coefficients. Some comments are in order: with the two step method, we observe more extreme values, the skewness is higher but only significant for Flanders Brabant, the volatility of the cycles increases in Wallonia both with respect to the standard deviation critcria and the partial autocorrelations.

Retaining the cycles obtained from the switching approach, Candelon et al. (2000) perform a Granger-causality analysis between migration flows and the innovations of the trends and cycles of the employment dynamics. It appears that only a permanent shock in Flemish provinces (except East. Flanders) affects migration flows in Belgium (in Wallonia and Flanders). This result is not surprising since people accepting to move only when the favorable (or unfavorable) employment perspectives are expected to go on in the future. Thus, the choice to migrate is not a static decision but a long term arbitrage between the mobility costs (mainly cultural and linguistic changes) and the expected benefits. 


\begin{tabular}{lcrrrrrrc}
\hline \hline Provinces & Median & Stdv & Min & Max & Skewness & BJ & $\hat{\phi}_{11}$ & $\hat{\phi}_{22}$ \\
\hline Antwerp & 3356 & 36648 & -65671 & 64985 & -.054 & 2.39 & .63 & .05 \\
Flanders Brabant & 95 & 980 & -2104 & 2018 & -.082 & 1.07 & .35 & -.01 \\
West Flanders & 683 & 8314 & -15670 & 14581 & -.115 & 1.67 & .56 & -.03 \\
East Flanders & 302 & 10951 & -22219 & 22536 & -.075 & .58 & .55 & -.10 \\
Limburg & 1879 & 27201 & -51328 & 48802 & -.088 & 2.08 & .65 & 0 \\
\hline Walloon Brabant & -261 & 2001 & -5472 & 3788 & -.174 & .29 & .68 & -.29 \\
Hainaut & 86 & 17210 & -29987 & 42790 & .336 & 1.09 & .84 & -.30 \\
Liege & 226 & 8951 & -21747 & 20599 & -.045 & .03 & .75 & -.36 \\
Luxemburg & 527 & 3097 & -6873 & 6676 & .013 & .84 & .75 & .05 \\
Namur & 19 & 2759 & -7345 & 5023 & -.181 & .34 & .73 & -.21 \\
\hline \hline
\end{tabular}

Table 5.7: Statistics on Provincial Employment Cycles: the Switching Approach

\begin{tabular}{lcrrrcccc}
\hline \hline Provinces & Median & Stdv & Min & Max & Skewness & BJ-test & $\hat{\phi}_{11}$ & $\hat{\phi}_{22}$ \\
\hline Antwerp & 2598 & 33769 & -70935 & 69435 & -.21 & 1.15 & .54 & .01 \\
Flanders Brabant & -192 & 3050 & -5345 & 7570 & .62 & 3.19 & .63 & -.22 \\
West Flanders & 484 & 7816 & -15442 & 14450 & -.29 & 1.42 & .51 & -.06 \\
East Flanders & 282 & 7930 & -17503 & 19827 & -.03 & .03 & .50 & -.16 \\
Limburg & 1843 & 22752 & -50104 & 45214 & -.35 & 1.63 & .56 & -.04 \\
\hline Walloon Brabant & 572 & 3150 & -6987 & 5109 & -.20 & 1.71 & .84 & -.32 \\
Hainaut & 4878 & 25511 & -41757 & 47474 & .03 & 1.72 & .88 & -.28 \\
Liege & 2456 & 14557 & -26996 & 26606 & -.06 & 1.93 & .88 & -.43 \\
Luxemburg & 473 & 3814 & -7658 & 8624 & -.01 & 1.00 & .76 & -.06 \\
Namur & -210 & 8239 & -14768 & 16480 & .12 & 1.69 & .84 & -.14 \\
\hline \hline
\end{tabular}

Table 5.8: Statistics on Provincial Employment Cycles: the Two Step Approach 


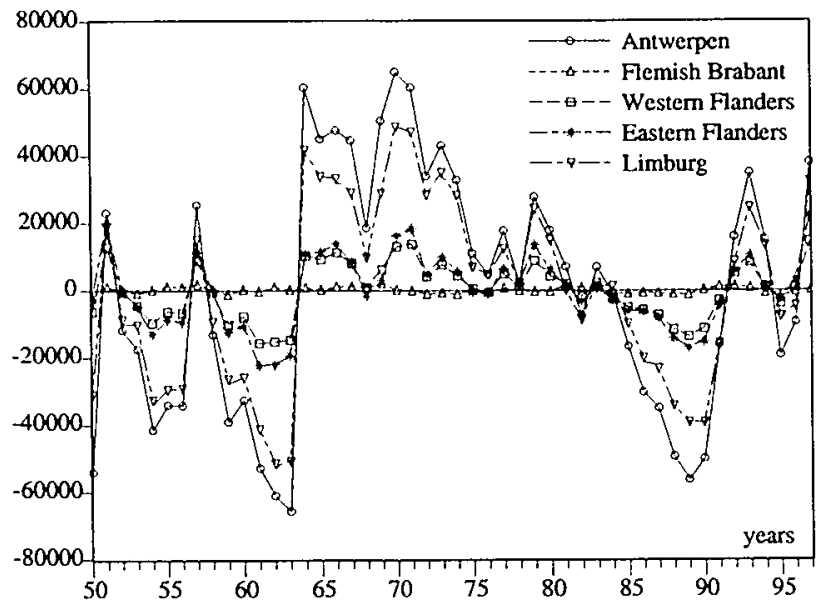

Figure 5.8: Switching Method, Cyclical Parts of Flemish Provinces

\subsection{Conclusion}

To summarize this chapter, a switching procedure that takes into account restrictions coming from short and long-run comovements, is worth considering in small samples. This procedure give better size properties for cointegration but especially for common features test statistics. The power of Johansen 's trace test for cointegration is considerably improved when imposing common dynamic factor restrictions. However, this iterative strategy does increase the computation complexity ${ }^{9}$ and the chance of imposing wrong restrictions that may alter the result instead of improving it. We show the relevance of these tools both on US and Belgian time series.

\footnotetext{
${ }^{9} \mathrm{~A}$ Gauss program may be obtained upon request.
} 


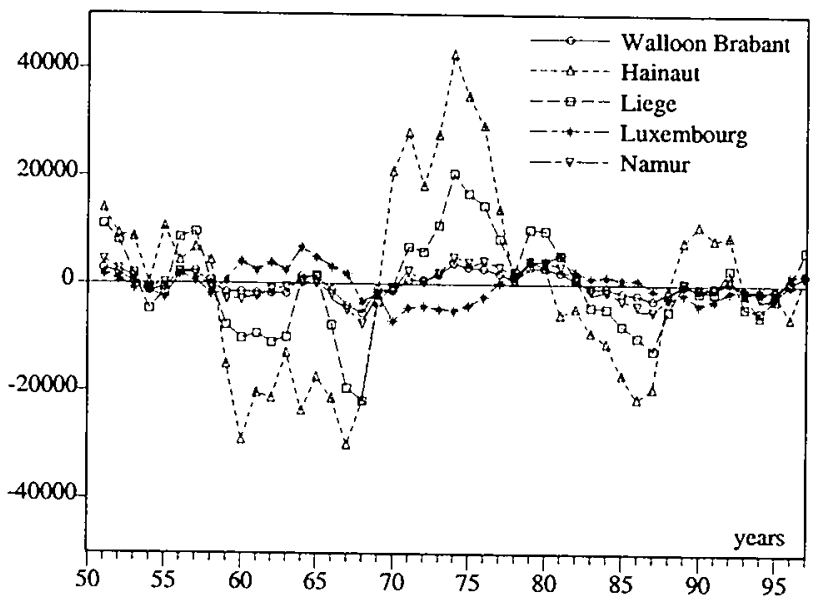

Figure 5.9: Switching Method, Cyclical Parts of Walloon Provinces 


\title{
Chapter 6
}

\section{Assessing Forecast Performance of Common Cyclical Features Restrictions in Cointegrated Systems}

\begin{abstract}
Several papers have assessed the gains in forecast precision due to imposing long-run constraints in VAR. Monte Carlo studies reveal that these cointegrating restrictions help only for long-term and sometimes medium-term forecasts. However, policy makers and business cycle analysts are more interested in time horizons less than one year. The main goal of this chapter is to examine whether short-term forecast accuracy is improved by also considering common cyclical features restrictions. In particular, we consider the two forms of co-features developed in previous chapters, namely the serial correlation common feature and the weak form reduced rank structure. The empirical analysis is concerned with predicting the quarterly US real GDP using various information sets.
\end{abstract}

\subsection{Introduction}

Several papers have assessed the forecasting gains of imposing cointegrating vectors in VAR. Monte Carlo studies provide evidence that these cointegrating restrictions help long-term and sometimes medium-term forecasts. This is especially true when the model is correctly specified with respect to the number of unit roots, the lag length order and when the cointegrating vectors are known (see among others Engle and Yoo, 1987; Reinsel and Ahn, 1992; Clements and Hendry, 1998, 1999¹ Lin and Tsay, 1996; Söderlind and Vredin, 1996). Moreover, forecasting gains are doubtful for short horizons and for the first differences of the variables. For seasonal modeling, Kunst (1993), Reimers (1996) do not find strong forecast improvements from imposing seasonal

\footnotetext{
${ }^{0}$ This Chapter is based on Cubadda and Hecq (2000).

'We will refer to the two books by Clements and Hendry $(1998,1999)$. Indeed, these books gather the main authors' contributions from articles that have been published a few years ago in different journals.
} 
cointegrating vectors. Also, the message is less clear cut when working with real data sets (Hoffman and Rashe, 1996). Note also that Christoffersen and Diebold (1998) have recently given evidence against the usefulness of cointegrating restrictions even for long-run forecasts.

Many practitioners, like policy makers or business cycle analysts, are however more interested in short or medium-term forecasts. One of the most difficult and most often asked practical queries is the evaluation of key macrocconomic variables like output growth, inflation or unemployment, at a time horizon less than one year. It is thus of great interest to cxamine whether forecast accuracy is improved by considering, in addition to long-run constraints coming from cointegration, common cyclical features restrictions. Indeed, whereas cointegration implies longrun comovements, common-cyclical features emphasizc short-run macrocconomic fluctuations. Hence, these are potentially more relevant when the goal is short-term forecasting.

We have seen in Chapter 2 that common cyclical features are particular cases of the Scalar Component Model (SCM) which was proposed by Tiao and Tsay $(1985,1989)$ in order to achieve a parsimonious statistical model. And it is well known that a (valid) reduction of the number of parameters improves efficiency of estimates (sec e.g. Lütkepohl, 1991). Vahid and Issler (1999) have also shown, in an extensive Monte Carlo exercise, that imposing SCCF restrictions can also yield improvements in prediction performances.

Contrary to Vahid and Issler (1999), we limit ourselves in this chapter to an empirical analysis. We investigate the point forecasts of the growth rate of the quarterly US real gross domestic. To this aim, we consider several models which include different key macroeconomic variables such as sectorial employment, interest rates, consumption or investment. Hence, we will not develop theoretical arguments as in Clements and Hendry $(1998,1999)$ for cointegration. Neither will we evaluate through Monte Carlo experiments the ability of co-feature restrictions in improving forecasts (see Vahid and Issler, 1999).

The plan of this chapter is as follows. Section 2 recalls common cyclical features definitions. Section 3 presents the data, the different models retained for analyzing the comovements with real US GDP, and the long-run relationships. Section 4 presents the common feature analysis and Section 5 is devoted to the forecast evaluation. Section 6 concludes. 


\subsection{VAR Models with Cointegration and Common Cyclical Fea- tures}

\subsubsection{General Setting}

For expositional purpose, let us consider a zero mean Gaussian VAR of order two for a $n$-vector time series $\left\{x_{t}, t=1, \ldots, T\right\}:^{2}$

$$
x_{t}=A_{1} x_{t-1}+A_{2} x_{t-2}+\varepsilon_{t},
$$

where the conditions given in previous chapters are assumed (see e.g. Chapter 3, Section 3.2). Let us further assume that the process $x_{\imath}$ is $\mathrm{I}(1)$ and is cointegrated of order $(1,1)$. Hence, $\operatorname{rank}\left(A_{1}+A_{2}-I\right)=r$, and satisfies $0<r<n$ and $(6.1)$ can be reparametrized as a VECM,

$$
\Delta x_{t}=\alpha \beta^{t} x_{t-1}-A_{2} \Delta x_{t-1}+\varepsilon_{t},
$$

where $\alpha$ and $\beta$ are both $(n \times r)$ matrices of full column rank $r$. The columns of $\beta$ span the space of cointegrating vectors, and the elements of $\alpha$ are the corresponding adjustment coefficients or factor loadings. We add to (6.2) the two types of common cyclical feature restrictions arising from the SCCF and the WF models. Let us denote the corresponding potential cofeature matrices by respectively $\tilde{\beta}_{1}^{\prime}$ and $\tilde{\beta}_{2}^{\prime}$.

- The presence of SCCF implies there exists a common feature matrix $\tilde{\beta}_{1}^{\prime}$ such that $\tilde{\beta}_{1}^{\prime} \Delta x_{t}=$ $\tilde{\beta}_{1}^{\prime} \varepsilon_{t}$ is a $s$-dimensional white noise process in (6.2) and consequently that $\tilde{\beta}_{1}^{\prime} A_{2}=0$ and $\bar{\beta}_{1}^{\prime} \alpha \beta^{\prime}=\tilde{\beta}_{1}^{\prime}\left(A_{1}-I\right)=0$.

- In the WF framework, only the assumption $\tilde{\beta}_{2}^{\prime} A_{2}=0$ is valid but $\tilde{\beta}_{2}^{\prime}\left(A_{1}-I\right) \neq 0$. There cxists a common feature matrix $\tilde{\beta}_{2}^{\prime}$ such that $\tilde{\beta}_{2}^{\prime}\left(\Delta x_{t}-\alpha \beta^{\prime} x_{t-1}\right)=\tilde{\beta}^{\prime} \varepsilon_{t}$ is a s-dimensional white noise process. Consequently, the common feature vector does not lie in the intersection of the long-run and the short-run matrices anymore. As a result, the WF allows to consider linear combinations of the first differenced $I(1)$ variables that are predictable in the long-run, i.c. at low frequencies.

Under the SCCF and the WF restrictions, the $n$ equations of the system do not have the same set of explanatory variables and consequently FIML estimates can be obtained in estimating the systems formulated as follows:

$$
S C C F:\left(\begin{array}{cc}
I_{s} & \tilde{\beta}_{1}^{* \prime} \\
0 & I_{n-s}
\end{array}\right) \Delta x_{t}=\left(\begin{array}{cc}
0 & 0 \\
\Phi_{1} & \Phi_{2}
\end{array}\right)\left(\begin{array}{c}
\Delta x_{t-1} \\
\hat{\beta}^{\prime} x_{t-1}
\end{array}\right)+\nu_{t},
$$

\footnotetext{
${ }^{2}$ Contrary to previous chapters $x_{t}$ represent the $n$-vector time series and in Section 6.5 to $6.6, y_{t}$ denotes the real output variable.
} 


$$
W F:\left(\begin{array}{cc}
I_{s} & \tilde{\beta}_{2}^{* \prime} \\
0 & I_{n-s}
\end{array}\right) \Delta x_{\ell}=\left(\begin{array}{cc}
0 & \Phi_{3}^{*} \\
\Phi_{1}^{*} & \Phi_{2}^{*}
\end{array}\right)\left(\begin{array}{c}
\Delta x_{\ell-1} \\
\hat{\beta}^{\prime} x_{t-1}
\end{array}\right)+\eta_{t},
$$

where $\Phi_{1}, \Phi_{2}, \Phi_{1}^{*}, \Phi_{2}^{*}$ and $\Phi_{3}^{*}$ are rectangular matrices, of size $(n-s) \times n,(n-s) \times r,(n-s) \times n$, $(n-s) \times r$ and $s \times r$ respectively, to be estimated. $\tilde{\beta}_{1}^{\prime}=\left(I_{s}: \tilde{\beta}_{1}^{* \prime}\right)$ and $\tilde{\beta}_{2}^{\prime}=\left(I_{s}: \tilde{\beta}_{2}^{* \prime}\right)$ are the normalized co-feature vectors for respectively the strong and the weak form.

Remark 6.1 $A$ variant of the SCCF is the notion of non-synchronous common cycles (henceforth, NSCC) or codependent cycles introduced by Vahid and Engle (1997). We have already presented this model for stationary time series in Chapter 2. The presence of such non-synchronous cycles relaxes the SCCF requirements by allowing the impulse response functions to be collincar only after a certain step, permitting adjustment delays. Moreover, NSCC are relevant for analyzing cyclical comovements among seasonally cointegrated time series (sce Cubadda, 1999). ${ }^{3}$ One can see that the presence of a WF structure is a necessary condition to observe a NSCC model. Indeed, in order to introduce the NSCC model, let us take (6.1) in first differences. We obtain the differenced non invertible model

$$
\Delta x_{t}=A_{1} \Delta x_{t-1}+A_{2} \Delta x_{t-2}+\varepsilon_{l}-\varepsilon_{t-1},
$$

lagging both side of (6.2) and substituting $\Delta x_{t-1}$ in (6.5) we obtain

$$
\Delta x_{t}=\left(-A_{1}+A_{1}^{2}+A_{1} A_{2}\right) x_{t-2}+\left(A_{2}-A_{1} A_{2}\right) \Delta x_{t-2}+\varepsilon_{t}-\left(I-A_{1}\right) \varepsilon_{t-1} .
$$

A non-synchronous common feature vector emerges if there exists a matrix $\tilde{\beta}_{3}^{\prime}$ such that $\tilde{\beta}_{3}^{\prime} \Delta x_{t}=$ $\tilde{\beta}_{3}^{\prime} \varepsilon_{t}-\tilde{\beta}_{3}^{\prime}\left(I-A_{1}\right) \varepsilon_{t-1}$ and implies that both conditions $\tilde{\beta}_{3}^{\prime}\left(-A_{1}+A_{1}^{2}+A_{1} A_{2}\right)=0$ and $\tilde{\beta}_{3}^{\prime}\left(A_{2}-\right.$ $\left.A_{1} A_{2}\right)=0$ are met. ${ }^{4}$ From the latter condition we have $\tilde{\beta}_{3}^{\prime}\left(I-A_{1}\right) A_{2}=0$ which shows that the necessary condition for having a NSCC is the presence of WF structure, i.e. that rank $\left(A_{2}\right)<n$. Otherwise if $A_{2}$ was a square matrix of full rank, $\tilde{\beta}_{3}^{\prime}\left(I-A_{1}\right)$ must yield a zero matrix in order to maintain the property that $\tilde{\beta}_{3}^{\prime}\left(I-A_{1}\right) A_{2}=0$. This would rule out the definition under NSCC.

We have already shown in Chapter 3 that it is a good idea to start with the WF because it is then easier to interpret the nature of the SF: implied vectors or mixed form. When finding a $W F$ and rejecting the $S F$, an additional implication of the previous result is that we should also test for the non-synchronous common cycle model, given that the necessary condition for NSCC is satisfied.

\footnotetext{
${ }^{3}$ We will only consider seasonally adjusted time series in this chapter. An evaluation of the forecast performances on unadjusted industrial production indices can be found in Cubadda and Hecq (2000).

${ }^{4}$ Note that what works for VAR(2) is also valid for general $V A R(p) A(L) x_{t}=\varepsilon_{\mathrm{t}}$, with $A(L)=I-A_{\mathrm{i}} L^{i}, i=1 \ldots p$ with $\operatorname{det}(A(\xi))$ having $n-r$ roots on the unit circle and $r$ rools outside the unil circle. Recursive substitution gives $\Delta x_{t}=A_{1} \alpha \beta^{\prime} x_{t-2}+A_{i}^{*} \Delta x_{t-i-1}+\varepsilon_{t}-\left(I-A_{1}\right) \varepsilon_{t-1}, i=1 \ldots p$ with with $A_{i}^{*}=A_{i+1}-A_{1} \sum_{i=1}^{p} A_{i+1}, i=1 \ldots p$.
} 


\subsection{Forecasting the US GDP: The Data and Long-Run Relation- ships}

The central role of this analysis is played by the per-capita US real gross domestic product. Following several studies and similarly to Chapters 3 and 4 , we have substracted from the real output, the amount of real government expenditures of goods and services in order to obtain a per-capita real private output. ${ }^{5}$ Figure 6.1 plots the logs of the quarterly data, seasonally adjusted, over 1947:Q1 to 1998:Q4. ${ }^{6}$ We have added the official NBER contraction periods. It emerges that while NBER period of contractions obviously match the evolution of the real GDP, it is not fully the case for the real private output series. Indeed, the huge increase of public expenditures during the Korean War induces in the private GDP series an additional contraction around 1951 and 1953. A small contraction also appears around 1966 and 1967. Because of the big differences between these two series, especially during to Korean War, and in order to avoid the observations occurring during the period of price control (extreme inflation variability) and Treasury-Fed accord, our analysis runs over 1954:Q1-1998:Q4. The last 16 observations from 1995:Q1 to 1998:Q4 are retained as the forecast period.

In order to obtain point forecasts for the real GDP, we have analyzed output comovements together with some key macroeconomic variables including national accounts, sectorial employment, money stock, interest rates and prices. While it was not easy to incorporate all these variables in a single model and also because we wanted to compare the merits of different modeling, we consider four alternative information sets denoted by $\mathcal{M}_{1}, \mathcal{M}_{2}, \mathcal{M}_{3}$ and $\mathcal{M}_{4}$. We present briefly the content of these four models. We also report the cointegrating vectors obtained from Johansen's ML test statistics.

Model $1\left(\mathcal{M}_{1}\right)$ proposes to link the per-capita real private GDP $\left(y_{t}\right)$ to total employment in four economic activities: Manufacturing (Man), Services (Serv), Transports \& Public Utilities (Tran) and Government (Gov). The quarterly seasonally adjusted employment time series are released by the US Bureau of Labor Statistics (BLS) ${ }^{7}$ Because it has not been obvious to discriminate between models with lags order $p=2$ and $p=6$, we consider both a second $\left(\mathcal{M}_{1}^{p=2}\right)$ and a sixth $\left(\mathcal{M}_{1}^{p=6}\right)$ order VAR. Results of Johansen's ML test statistics (model with a deterministic trend restricted in the long-run), provide evidence of two cointegrating vectors in both systems with the following just-identified cointegrating vectors (asymptotic standard errors

\footnotetext{
${ }^{5}$ Note that some authors propose more precise definitions by substracting only a part of government expenditures and not the total amount.

${ }^{6}$ Data are from the Bureau of Economic Analysis (BEA). We have divided series by the size of the civilian population above sixteen years of age in order to get a per-capita variable.

${ }^{7}$ Wi have considered all aggregative series for the number of employees in non-farm industries, but from the analysis of cointegration, only these sectors were significant in the cointegrating space.
} 


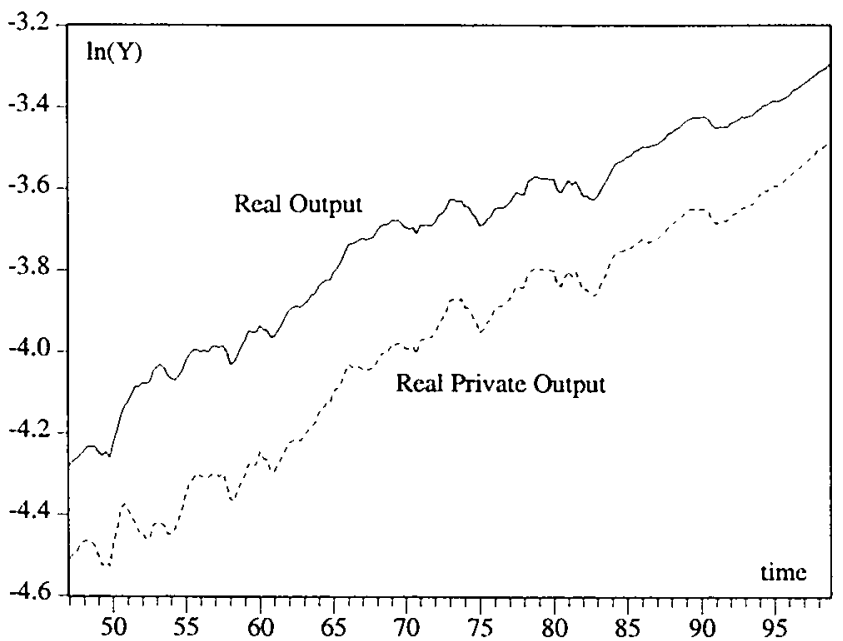

Figure 6.1: Per-Capita Real and Real Private Output \& NBER Contractions

in brackets):

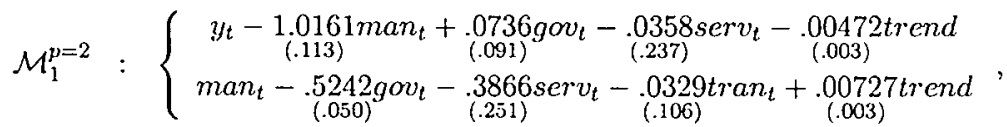

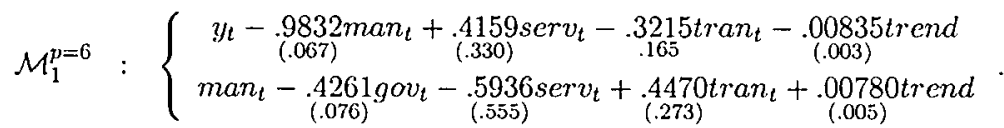

Model $2\left(\mathcal{M}_{2}\right)$ is a trivariate system for investment, consumption and income similar to the one already considered in Chapters 3 and 4. A fifth order VAR characterizes quite well the covariance structure of the data. There is no deterministic trend but an unrestricted impulse dummy has been added in 1975:Q1 to capture the decrease of investment of 20\% that quarter (see Figure 3.2 in Chapter 3). We end up with the two following cointegrating vectors:

$$
\mathcal{M}_{2}:\left\{\begin{array}{c}
c_{t}-.9441 y_{t} \\
(.022) \\
i_{t}-1.0976 y_{t} \\
(.058)
\end{array}\right.
$$

Model $3\left(\mathcal{M}_{3}\right)$ links the private GDP to long-term and short-term interest rates. Several 
papers have considered the significant influence of the spread on the business cycle (see inter alia Lahiri and Wang (1996) and references quoted). Both interest rates are from Citibase. The long term interest rate is the "Bond Yield: Moody 's AAA Corporate (FYAAC)" while the shortterm is the "US Treasury Bills in the Secondary Market at 3 Months (FYGM3)". We select data corresponding to March, June, September and December of each year to obtain quarterly series. Within a VAR(3), there exists one cointegrating vector. We have added two unrestricted impulse dummies in 1980:Q2 and in 1980:Q4 to capture the large variations in the short-term interest rate.

$$
\mathcal{M}_{3}: R_{t}^{s}-\underset{(.080)}{1.1146 R_{t}^{l}}+\underset{(.010)}{.0425 y}
$$

Model $4\left(\mathcal{M}_{1}\right)$ encompasses the two previous information sets. It is a seven dimensional system in which we have added inflation, money stock and the two interest rates to the small RBC model $\left(\mathcal{M}_{2}\right)$ for real consumption, investment and income. This is a very similar approach to the one followed by King et al. (1991) except that we consider two interest rates instead of only the short-term one. Inflation is the quarterly growth rate of the implicit GDP deflator (source BEA). Money stock is the sum of "Currency held by the public" and "Total deposit (demand and time) to commercial banks". For monetary aggregates, the seasonally adjusted data from 1959 come from the Federal Bank of St-Louis. The series from 1954 until 1958 are from Friedman and Schwartz (1963) statistical appendix. Notice that similarly to Friedman and Schwartz (1963), the concept of money we retain is in between M1 and M2 but closer to M2. The analysis is carried out with a VAR(4); two additional dummies in 1981:Q1 and 1983:Q1 are added. Using Johansen's trace ticst, we do not reject the presence of four cointegrating vectors. It is not necessary to normalize the cointegrating matrix to obtain point forecasts. However, it is convenient to report normalized vectors in order to give an economic meaning to the long-run relationships. For $\mathcal{M}_{1}$ there cxist. some additional non significant parameters in the cointegrating vectors after imposing just-identifying restrictions. In order to display more informative cointegrating vectors for $\mathcal{M}_{A}$, we test the significance of three additional restrictions. The system becomes overidentified. A test statistic for the additional overidentifying restrictions has a value of 2.423 for a $\chi^{2}(3)$ distribution under the null ( $p$-value is .489).

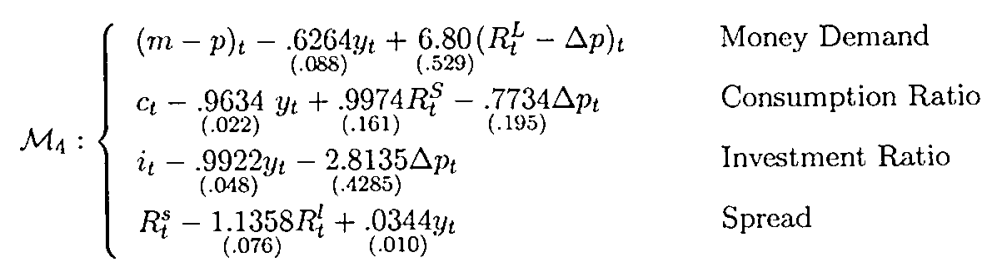




\begin{tabular}{ccccccc}
\hline \hline$H_{0}:$ & $\lambda^{S F}$ & Test $^{S F}$ & Small $^{S F}$ & $\lambda^{W F}$ & Test $^{W F}$ & Small $^{W F}$ \\
\hline$s \geq 1$ & .021 & .315 & .335 & .012 & .153 & .159 \\
$s \geq 2$ & .074 & .041 & .052 & .013 & .388 & .406 \\
$s \geq 3$ & .356 & $<.001$ & $<.001$ & .063 & .097 & .111 \\
$s \geq 4$ & .377 & $(<.001)$ & $(<.001)$ & .085 & .022 & .028 \\
$s=5$ & .517 & $(<.001)$ & $(<.001)$ & .399 & $<.001$ & $<.001$ \\
\hline \hline
\end{tabular}

Table 6.1: p-values of Common Cyclical Features for Model 1 with $\mathrm{p}=2$

\begin{tabular}{ccccccc}
\hline \hline$H_{0}:$ & $\lambda^{S F}$ & Test $^{S F}$ & Small $^{\text {SF }}$ & $\lambda^{W F}$ & Test $^{W F}$ & Small $^{W F}$ \\
\hline$s \geq 1$ & .179 & .118 & .307 & .105 & .679 & .835 \\
$s \geq 2$ & .231 & .012 & .108 & .196 & .186 & .476 \\
$s \geq 3$ & .435 & $<.001$ & $<.001$ & .229 & .027 & .202 \\
$s \geq 4$ & .535 & $(<.001)$ & $(<.001)$ & .294 & $<.001$ & .025 \\
$s=5$ & .564 & $(<.001)$ & $(<.001)$ & .522 & $<.001$ & $<.001$ \\
\hline \hline
\end{tabular}

Table 6.2: $\mathrm{p}$-values of Common Cyclical Features for Model 1 with $\mathrm{p}=6$

\subsection{Common Cyclical Feature Properties}

Once we have determined the number of cointegrating vectors, we fix the parameters of these vectors to their estimated values. Afterwards, we test for common cyclical features for the five models. Tables 6.1 to 6.5 report eigenvalues and $p$-values for the two different common cyclical feature test statistics. $P$-values for the SCCF model when the number of co-feature vectors does not respect the inequality $r+s \leq n$ are reported within brackets. Columns labelled "Small" gives small sample corrected version test statistics (see Chapter 5 ).

From the tables we retain three, two, one and four WF vectors for respectively $\mathcal{M}_{1}, \mathcal{M}_{2}$, $\mathcal{M}_{3}, \mathcal{M}_{4}$. Concerning the SCCF model, LR tests do not reject the presence of two vectors for $\mathcal{M}_{1}$, one vector for $\mathcal{M}_{2}$ and $\mathcal{M}_{3}$ (p-values quite close to $\left.5 \%\right)$ and two vectors for $\mathcal{M}_{4}$. For $\mathcal{M}_{3}$ both SCCF and WF test statistics do not reject the hypothesis of one co-feature vector. Using the test statistics proposed in Chapter 3 , we obtain $\xi_{S W}=-2(2423.96-2428.47)=9.02$. This test statistics is distributed as a $\chi^{2}(1)$ under the null of a SCCF structure and consequently the strong form is rejected at usual significance level.

Notice that restrictions coming from co-feature vectors reduce much more drastically the number of redundant parameters than the ones obtained from cointegration. In $\mathcal{M}_{1}^{p=6}$ for ex-

\begin{tabular}{ccccccc}
\hline \hline$H_{0}:$ & $\lambda^{S F}$ & Test $^{S F}$ & Small $^{\text {SF }}$ & $\lambda^{W F}$ & Test $^{W F}$ & Small $^{W F}$ \\
\hline$s \geq 1$ & .122 & .052 & .087 & .059 & .462 & .530 \\
$s \geq 2$ & .151 & $(.007)$ & $(.020)$ & .113 & .149 & .224 \\
$s=3$ & .428 & $(<.001)$ & $(<.001)$ & .372 & $<.001$ & $<.001$ \\
\hline \hline
\end{tabular}

Table 6.3: p-values of Common Cyclical Features for Model 2 


\begin{tabular}{ccccccc}
\hline \hline$H_{0}:$ & $\lambda^{S F}$ & Test $^{S F}$ & Small $^{S F}$ & $\lambda^{W F}$ & Test $^{\text {WF }}$ & Small $^{W F}$ \\
\hline$s \geq 1$ & .062 & .064 & .076 & .008 & .846 & .855 \\
$s \geq 2$ & .166 & $<.001$ & $<.001$ & .145 & .003 & .004 \\
$s=3$ & .380 & $(<.001)$ & $(<.001)$ & .244 & $<.001$ & $<.001$ \\
\hline \hline
\end{tabular}

Table 6.4: p-values of Common Cyclical Features for Model 3

\begin{tabular}{ccccccc}
\hline \hline$H_{0}:$ & $\lambda^{S F}$ & Test $^{S F}$ & Small $^{\text {SF }}$ & $\lambda^{W F}$ & Test $^{W F}$ & Small $^{W F}$ \\
\hline$s \geq 1$ & .096 & .637 & .798 & .071 & .688 & .799 \\
$s \geq 2$ & .204 & .082 & .280 & .140 & .283 & .499 \\
$s \geq 3$ & .311 & $<.001$ & .005 & .152 & .129 & .350 \\
$s \geq 4$ & .318 & $(<.001)$ & $(<.001)$ & .217 & .012 & .092 \\
$s \geq 5$ & .463 & $(<.001)$ & $(<.001)$ & .268 & $<.001$ & .007 \\
$s \geq 6$ & .581 & $(<.001)$ & $(<.001)$ & .460 & $<.001$ & $<.001$ \\
$s=7$ & .671 & $(<.001)$ & $(<.001)$ & .552 & $<.001$ & $<.001$ \\
\hline \hline
\end{tabular}

Table 6.5: p-values of Common Cyclical Features for Model 4

ample, the unrestricted five dimensional VAR needs to estimate 150 mean parameters (except. deterministic components). The presence of two cointegrating vectors yields a VECM with 141 mean parameters. With two SCCF and three WF vectors there are respectively only 93 and 72 parameters, i.e. more than the ha'f of the unrestricted model. It would be still possible to increase the number of constraints using the mixed form that combines SCCF and WF (see Chapter 3).

\subsection{Forecast Evaluation}

\subsubsection{Construction of Forecasts}

Imposing both common cyclical features and cointegrating restrictions, the different models are estimated by FIML over 1954:Q1+ $p$ to 1994:Q4. The coefficient estimates we obtain are not updated over the forecast period. That means that we do not reestimate the model each time when an additional observation is available. These models are then used to generate point forecasts of $1,2,4$, and 8 periods in the future for a maximum forecast horizon varying from 1 to 16 quarters.

More formally, denote $h$ the number of steps-ahead we want to obtain (here $1,2,4,8$ ) and $H$ the maximum forecast horizon or the forecast period. The common practice (see for example Clements and Hendry, 1998, 1999) consists in fixing $H$ to some value, say 16 in our analysis. The forecasts evaluation criteria are calculated by averaging the 16 squared 1-step errors, the 15-step errors... This procedure leads the evaluation of forecast accuracy to be arbitrary dependent of a fixed forecast horizon $H$. Moreover that can hide the consequences of breaks and parameter non-constancies occurring within the forecast period. In order to avoid these potential pitfalls, 
we allow the forecast horizon $H$ to vary from 1 to 16 quarters and, for $H \geq h$, we calculate for $\Delta y$, the growth rate of the GDP, the mean-square forecast error (MSFE) such as

$$
\operatorname{MSFE} E_{h, H}=\frac{1}{H-h+1} \sum_{i=h}^{H-h+1}\left(\Delta \widehat{y}_{T+i, h}-\Delta y_{T+i}\right)^{2},
$$

with for $H \geq h, h=1,2,4,8$ and $H=1,2, \ldots 16$.

We denote by $\Delta x_{t}=\left(\Delta x_{1, t}, \ldots \Delta x_{n-1, t}, \Delta y_{t}\right)^{\prime}, t=1 \ldots T$, the $n$-dimensional process for a model $\mathcal{M}_{j}$ which includes the growth rate of private GDP $\left(\Delta y_{t}\right)$ and $n-1$ remaining variables. Also denote by $\Delta \hat{x}_{T+i, h}$ the $h$-step ahead forecast made for period $T+i(i \geq h)$ and based on parameter estimation up to $T$. The $h$-step ahead forecasts use actual observed values for lagged $x_{\imath}$ 's which go back to $h$ periods. Fixing the forecast horizon at $H=1$, we obtain one value for the 1-stcp-ahead forccast. If $H=2$, we can average over two values for the 1-step-ahcad forecast crrors and we have one 2-step-ahead forecast error, ... with $H=4$ we can average over four 1-step-ahead forecast errors, three 2-step errors...and we have one value for the 4-step error.

Notice that we will not assess the forecast accuracy using multivariate criteria like the trace of the mean square forecast errors (TMSFE) or the Clements and Hendry (1995)'s GFESM ${ }^{8}$ since our main goal is to evaluate the behavior of different models whose common component is $y_{t}$, the output variable. Moreover, the most important variable for business cycle analysis is the growth rate of national product (see Harding and Pagan, 1999 and their interpretation of Burns and Michel original purpose).

The performance of the different information sets (models) $\mathcal{M}_{j}, j=1,2,3,4$, will be investigated by four specifications. These are:

- VECM: the vector error correction which takes into account of cointegrating vectors,

- SCCF (or SF): the VECM with additional SCCF (or SF) restrictions,

- WF: the VECM with additional WF restrictions,

- DV: the VAR in first differences which is misspecified due to the omission of long-run relationships.

We have added the misspecified model DV because it has been shown (see inter alia Clements and Hendry, 1999) that DV behaves sometimes better than a VECM for forecasting purposes. For example, DV forecasts appear to provide some immunity against parameter non-constancies that could seriously bias VECM forecasts.

\footnotetext{
${ }^{8}$ Only this last criterion is invariant to linear transformations which is the case if we consider both levels and in differences.
} 


\subsubsection{Comparing Models}

The first type of results we report is a comparison between the different models $\mathcal{M}_{1}^{p=2}, \mathcal{M}_{1}^{p=6}, \mathcal{M}_{2}, \mathcal{M}_{3}$ and $\mathcal{M}_{4}$. We construct the ratios of the MSFE of the first four models over $\mathcal{M}_{4}$, namely we obtain the MSFE ratios $\mathcal{M}_{1}^{p=2} / \mathcal{M}_{4}, \mathcal{M}_{1}^{p=0} / \mathcal{M}_{4}, \mathcal{M}_{2} / \mathcal{M}_{4}$ and $\mathcal{M}_{3} / \mathcal{M}_{4}$. Figures 6.2 and 6.3 present these ratios obtained for respectively 1 - and 4 -step-ahead forecast errors for the different specifications DV, VECM, WF and SCCF. Since the outcomes are presented relatively to $\mathcal{M}_{4}$, the horizontal line at 1 measures $\mathcal{M}_{4} / \mathcal{M}_{4}=1$. Consequently, lines above 1 (resp. below 1 ) indicate models that display, using the MSFE critcrion, less (resp. more) accurate point forecasts than $M_{4}$.

Concerning the one-step-ahead errors, it emerges from the different panels of Figure 6.2 that except DV which favors $\mathcal{M}_{4}$, other specifications show that $\mathcal{M}_{2}$ has the smallest MSFE: it is the case from horizon $H=1$ with the VECM and from horizon $H=3$ using the additional WF and SF restrictions. The performance of $\mathcal{M}_{1}$ is quite bad compared to other information sets except for forecast period $H=2$ and 3 under WF and SF restrictions. For 4-step-ahead MSFE, Figure 6.3 shows that $\mathcal{M}_{2}$ seems to largely encompass other models.

From this first visual analysis we sce that the empirical model we have used in Chapters 3 and 4 , namely a system with only consumption, income and output, gives the best results in terms of MSFE. While we expected these conclusions in comparison to $\mathcal{M}_{1}$ (sectorial employment account only for a small part of comovements with the GDP) and $\mathcal{M}_{3}$, it was not obvious a priori that this small model would "beat" the more complex ones that includes money, interest rates and inflation. These outcome may be due to bias in VAR estimates when the number of variables increases (Abadir et al., 1999; Gonzalo and Pitarakis, 1999), to the presence of very volatile variables or to non-constancies of different types.

\subsubsection{Comparing Specifications}

In this subsection we compare the four different specifications DV, VECM, WF and SCCF for each of the five models taken individually. We construct five figures (6.4 to 6.9) for respectively models $\mathcal{M}_{1}^{p=2}, \mathcal{M}_{1}^{p=6}, \mathcal{M}_{2}, \mathcal{M}_{3}$ and $\mathcal{M}_{4}$. Each of these figures consider separately 1-, 2-, 4- and 8 step ahead MSFE. It is therefore possible, for cach models, to compare the merits of the different restrictions.

Some comments are in order:

- For $\mathcal{M}_{1}^{p=2}$ (Figure 6.4), the model specified solely in first differences (DV) displays the best results in terms of MSFE, except. for forecast horizon $H=2$. VECM, WF and SF specifications behave similarly.

- For $\mathcal{M}_{1}^{p=0}$ (Figure 6.5), DV displays again the best results in terms of MSFE. There exist some minor differences between VECM, WF and SF. For instance, regarding 1- and 2-step ahead forecasts, the WF displays smaller crrors at the forecast horizon $H=4$ and 5 and worse outcomes for forecast horizons from 8 to 16 . For 4-step-ahead predictions, WF is 

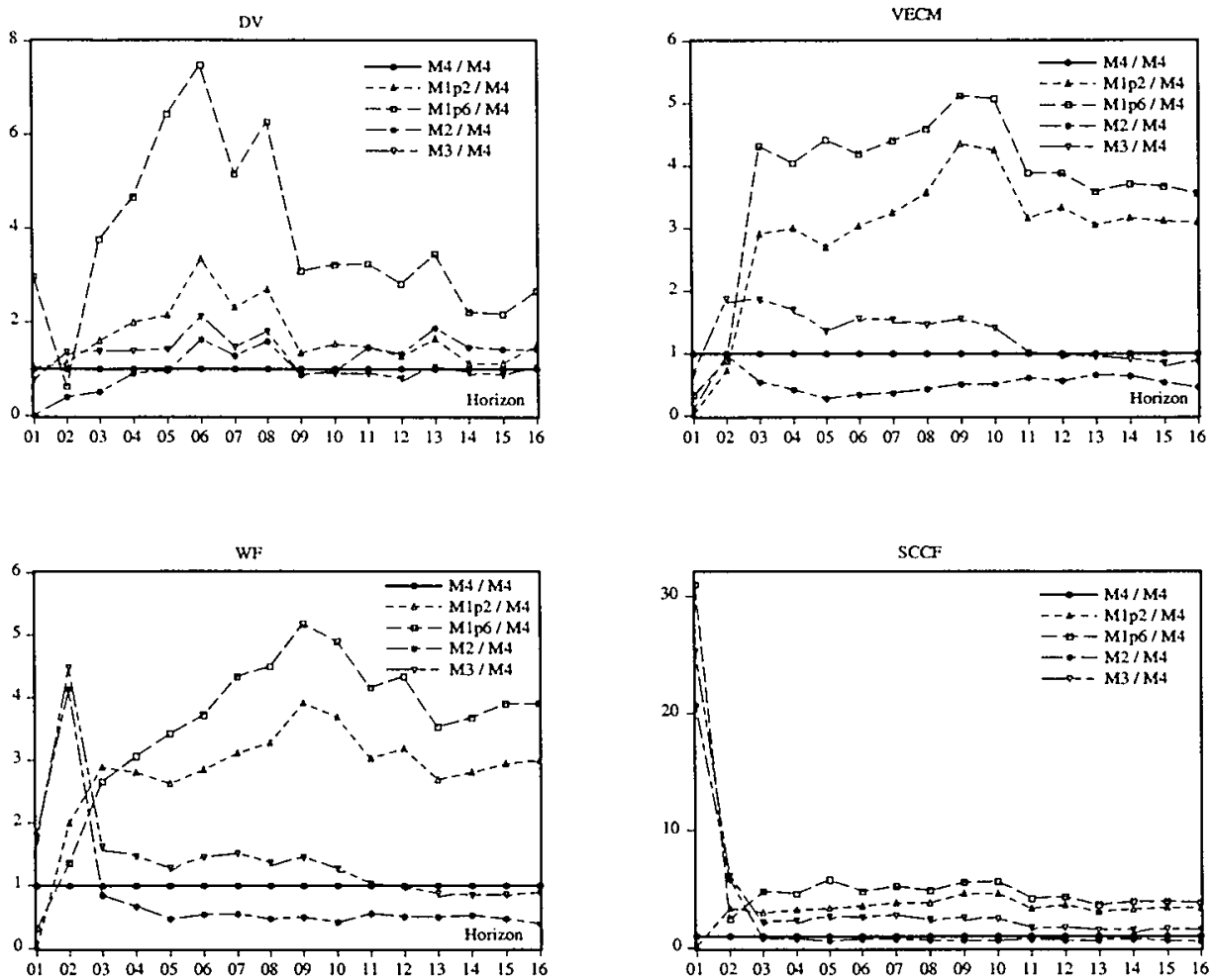

Figure 6.2: Model Comparison: 1-step ahead MSFE 

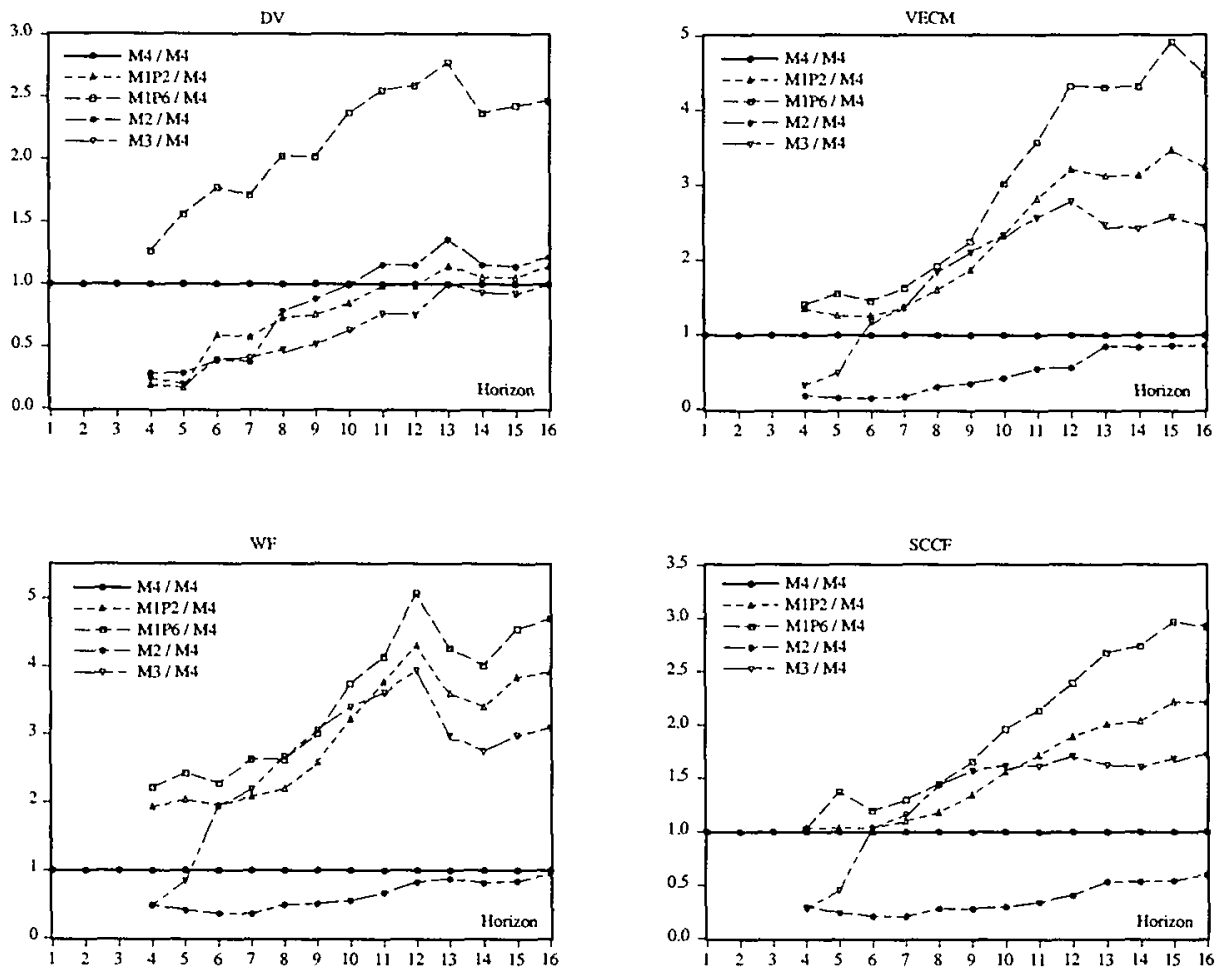

Figure 6.3: Model comparison, 4-step ahead MSFE 
better than both the SF and the VECM specifications, and for 8-step-ahead forecasts, the VECM seems to give better results than WF and SCCF.

- $\mathcal{M}_{2}$ (Figure 6.6) is very interesting. On Figures 6.2 and 6.3 we have seen that $\mathcal{M}_{2}$ secms to encompass rival models in terms of MSFE. Moreover, $\mathcal{M}_{2}$ is also the only case where DV does not have a smaller MSFE than the other specifications. From the analyses in Clements and Hendry $(1998,1999)$ we could infer that the error correcting specification is as good as the DV because long run relationships are more constant over the forecast period or more generally that the impact of parameter non-constancies is smaller than the consequences of the omission of error correcting terms. However, none of the specifications gives uniformly better results over the forecast period and/or over the $h$-step ahead point forecasts.

- For $\mathcal{M}_{3}$ (Figure 6.7), DV displays again uniformly the best results in terms of MSFE. Regarding 1- and 2-step-ahead forecasts, the WF and the VECM are quite close and give smaller errors than the SF. Notice that while a co-feature relationship statistically exists both under the SF and the WF, a test statistic has rejected the SF in favor of the WF. Hence, the WF does emphasize correct restrictions present in the VECM but the SCCF (or SF) is clearly misspecified.

- For $\mathcal{M}_{4}$ (Figure 6.8), DV gives again almost uniformly the best results in terms of MSFE. Concerning the other three specifications, the SF is better for the 1-step-ahead case, the VECM is better for the 2-step-ahead case and the WF is better for the 4 - and 8-step ahead case.

Consequently, except in the likely misspecified case of a SF structure for $\mathcal{M}_{3}$, we did not find clear evidence that imposing additional co-feature restrictions helps in forecasting the growth rate of the real gross domestic product. The performances of the different formulations are quite comparable and are summarized in Table 6.6 that reports the 1-, 2-, 4- and 8-step ahead MSFE for $H=16$, namely the last points on each graphs.

Note finally that we obtain the same conclusions by using multivariate forecast criteria like the TMSFE for instance. Table 6.7 illustrates this issue for $\mathcal{M}_{2}$.

\subsubsection{Using Full Sample Error Correction Terms}

Clements and Hendry $(1998,1999)$ have shown that forecasts obtained from a DV modeling may appear more robust with respect to some well defined parameter changes than those coming from a VECM . In this subsection we consider only one of the issues studied by these authors, namely the constancy of the long-run relationships during the forecast horizon.

We limit ourselves to the two models $\mathcal{M}_{2}$ and $\mathcal{M}_{3}$ and focus on the impact of the potential non-constancy of the cointegrating and of the co-feature vectors over the forecast period. We have chosen these two models because they seem to illustrate a case where the error correcting 

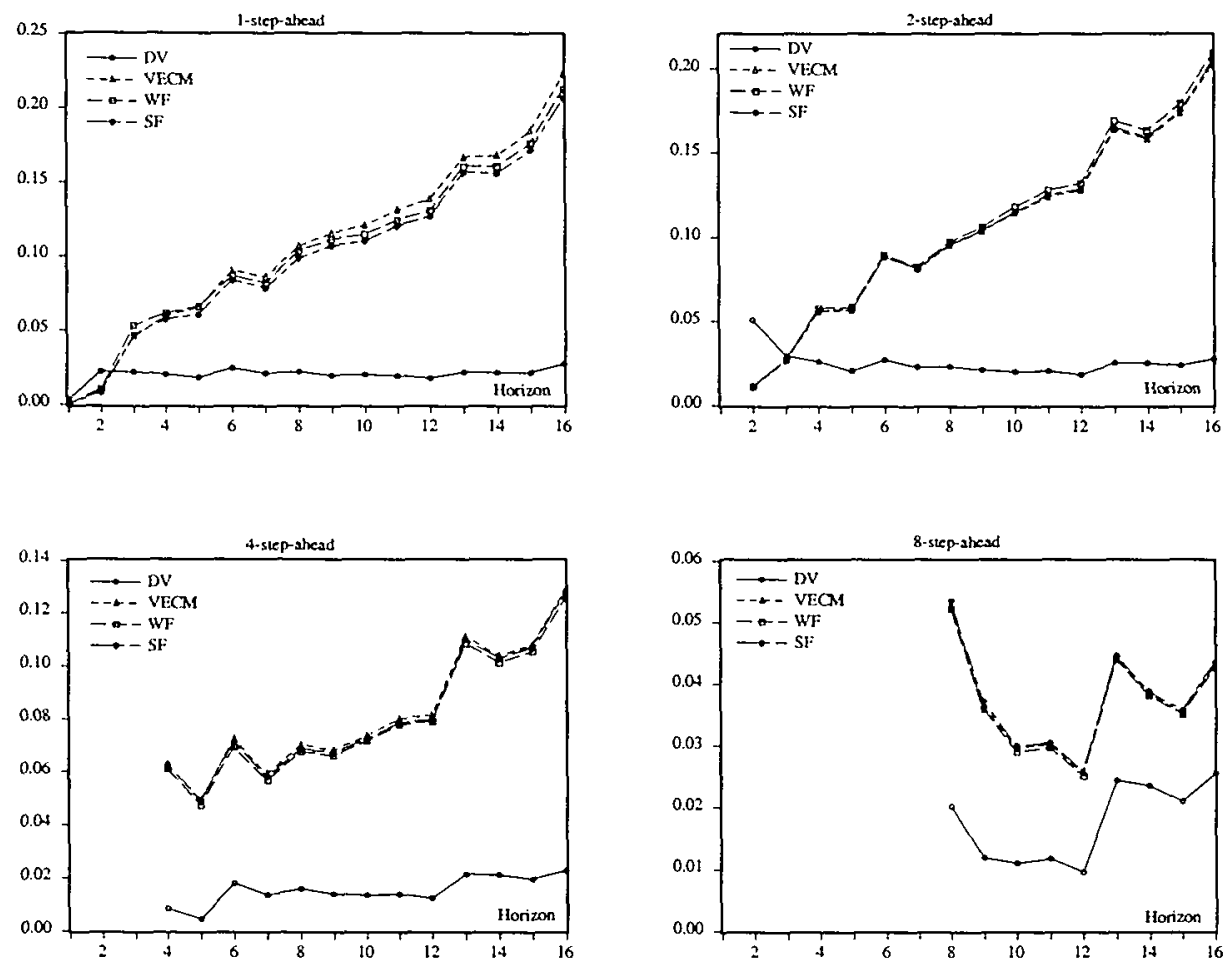

Figure 6.4: MSFE for Model 1 with $\mathrm{p}=2, \mathrm{H}=1$ to 16 

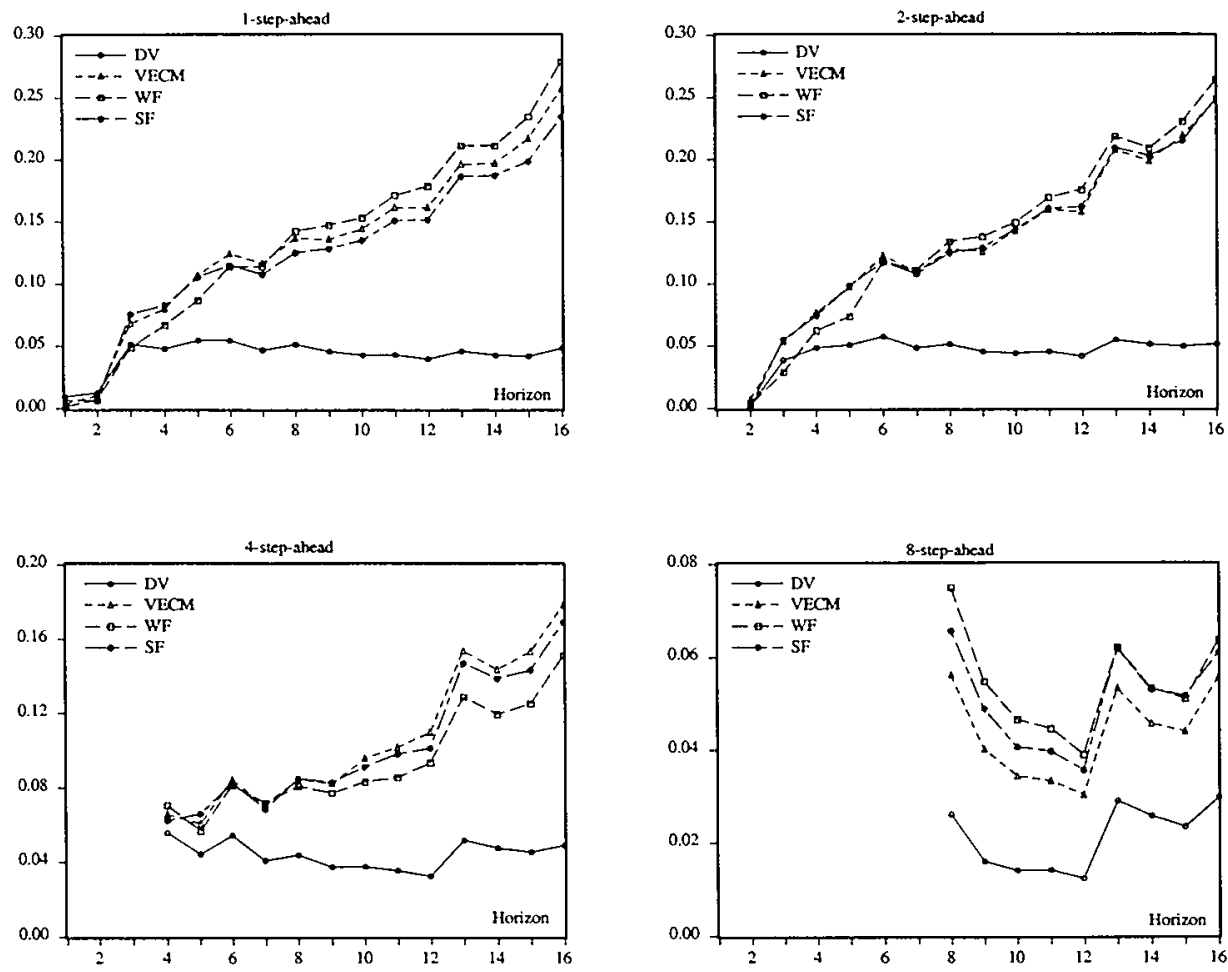

Figure 6.5: MSFE for Model 1 with $\mathrm{p}=6, \mathrm{H}=1$ to 16 

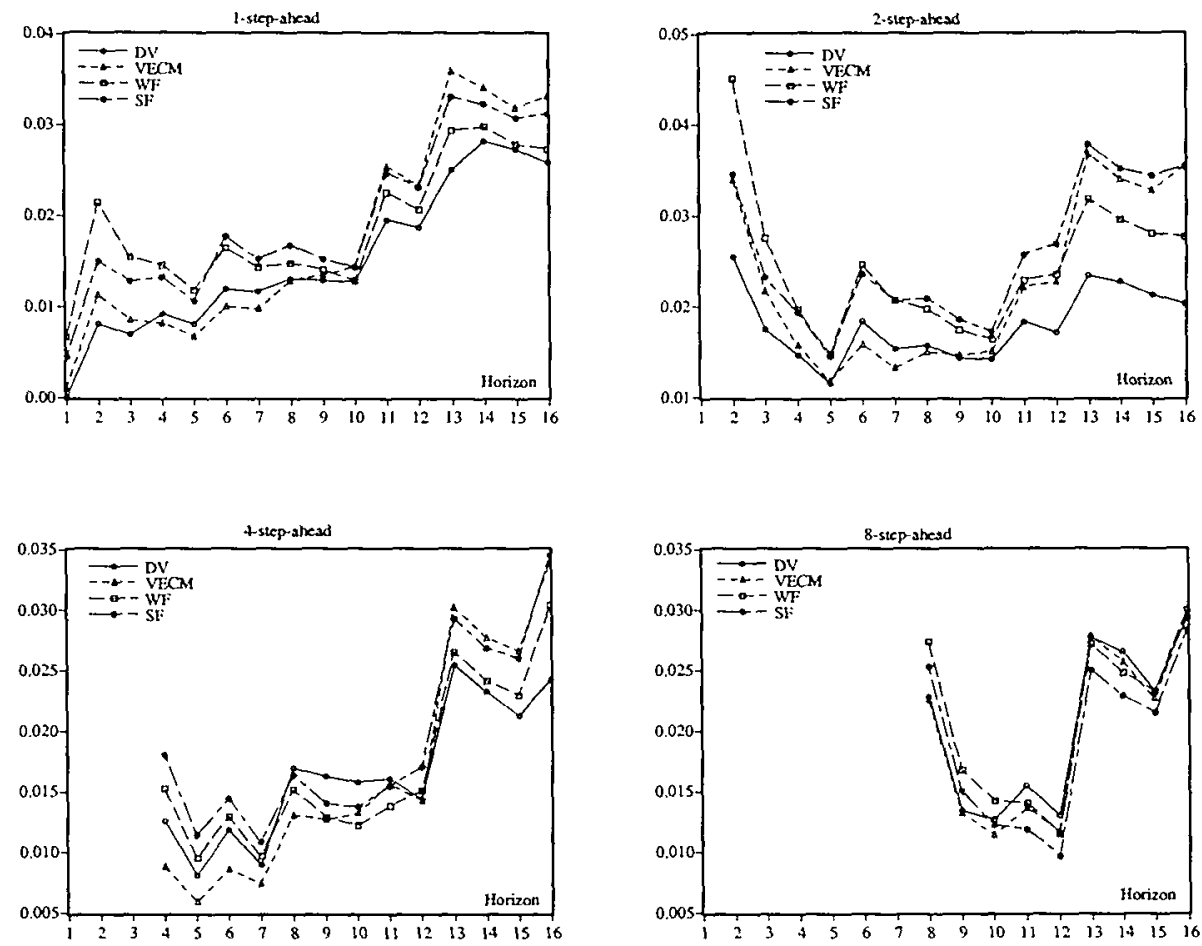

Figure 6.6: MSFE for Model 2, $\mathrm{H}=1$ to 16 

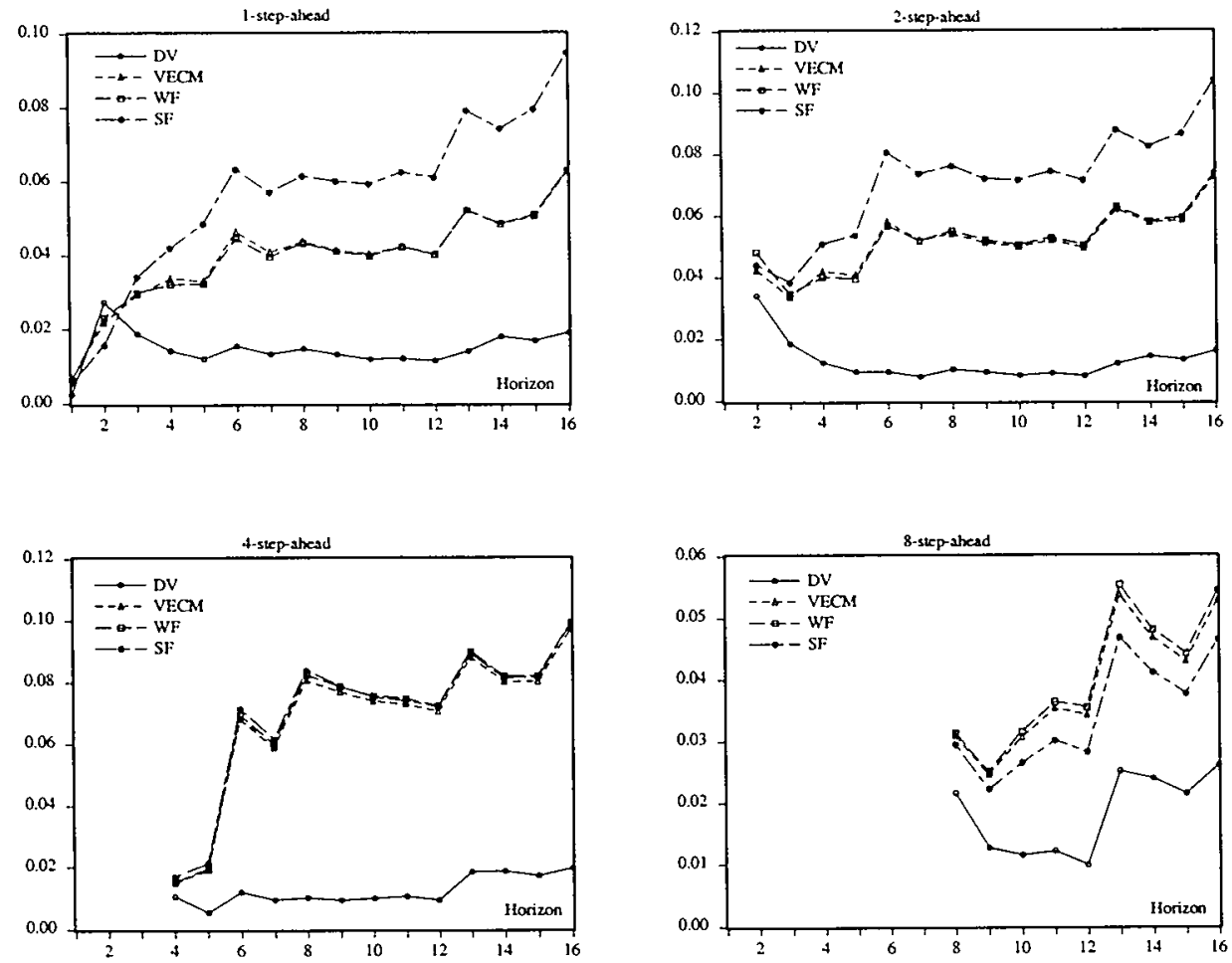

Figure 6.7: MSFE for Model 3, $\mathrm{H}=1$ to 16 

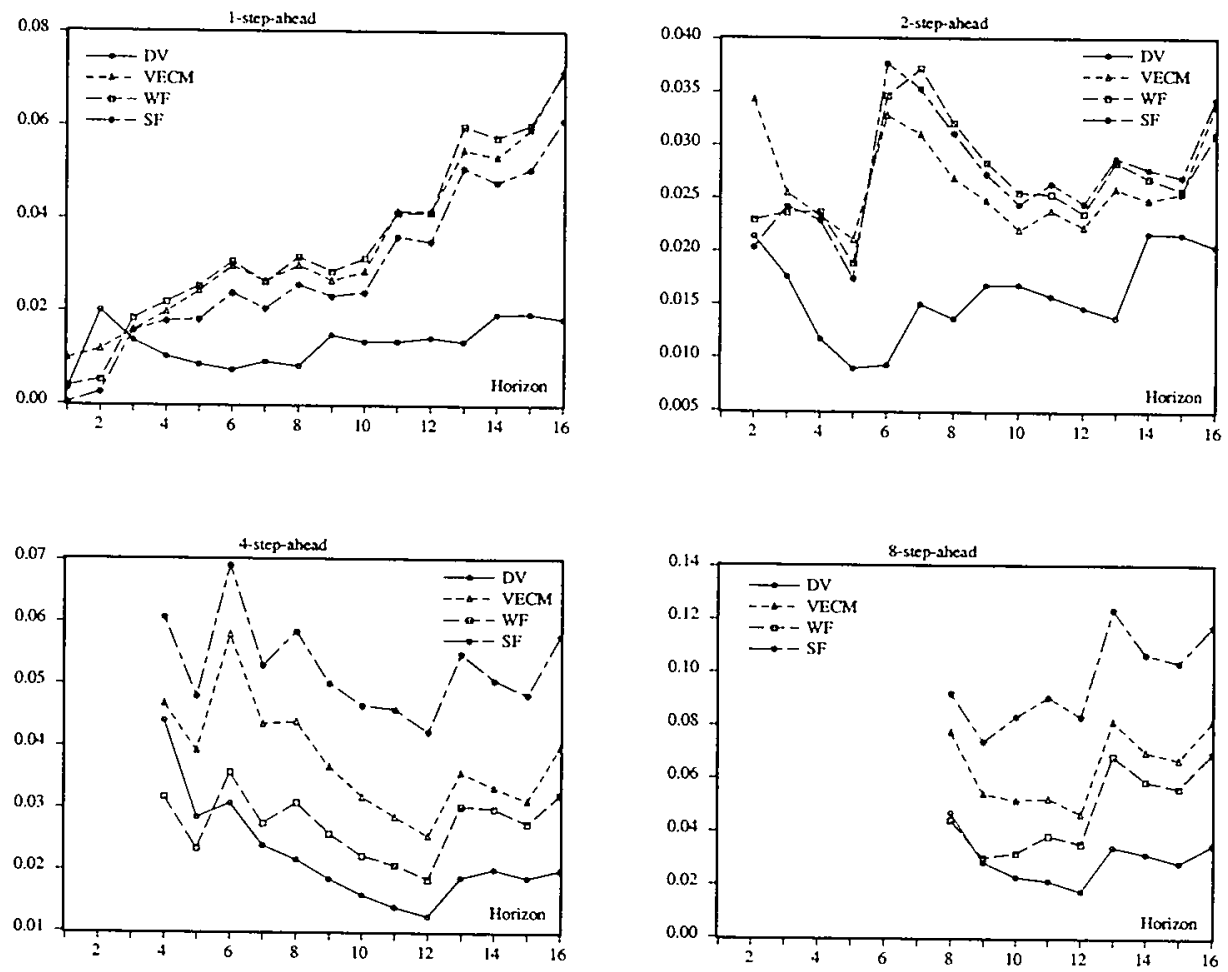

Figure 6.8: MSFE for Model 4, $\mathrm{H}=1$ to 16 

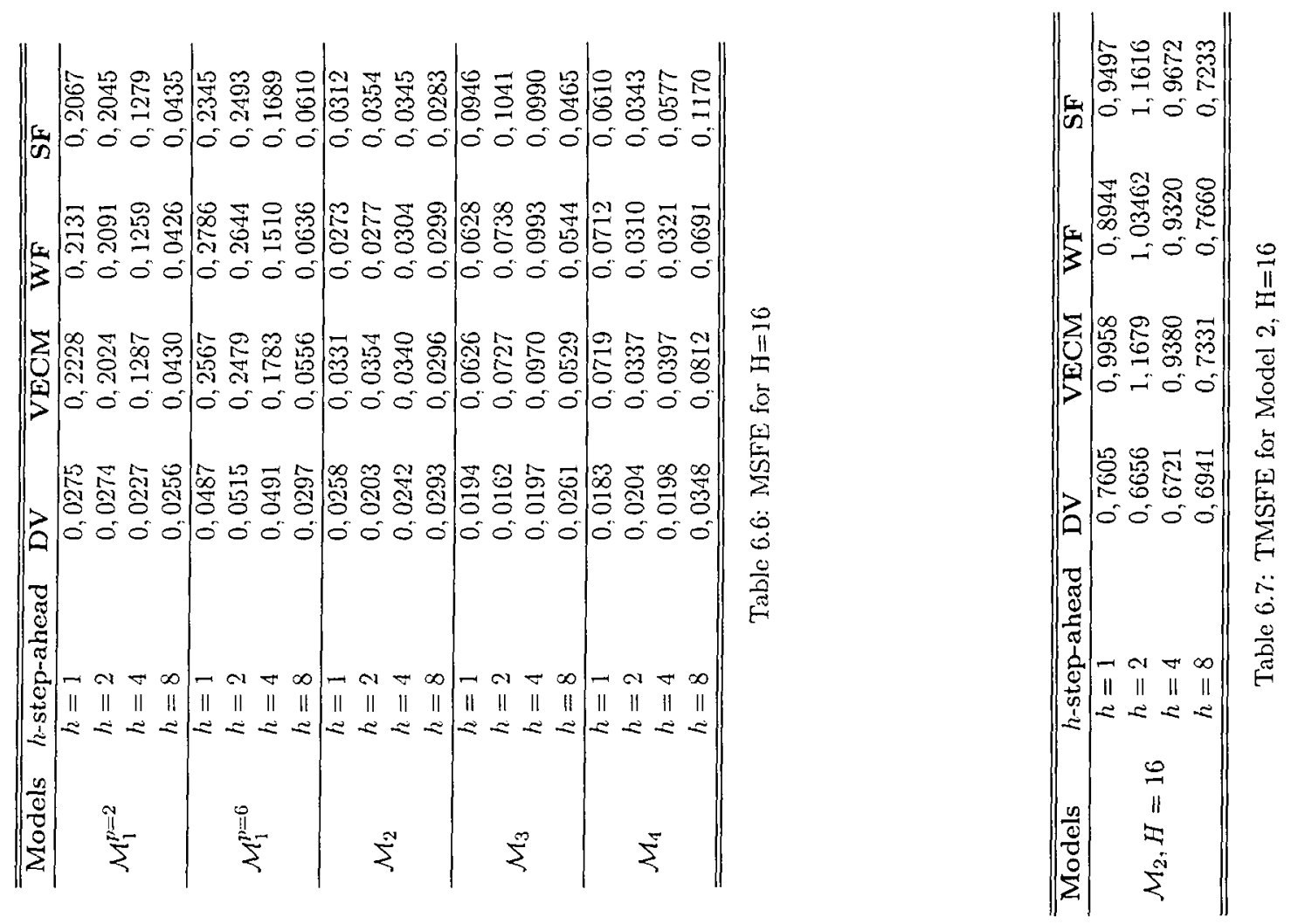
mechanism is constant on the forccast period $\left(\mathcal{M}_{2}\right)$ and a case where it is not $\left(\mathcal{M}_{3}\right)$. We compare the different specifications for the same forecast horizon (1995:Q1 to 1998:Q4) but with cointegrating and/or co-feature vectors estimated over the whole sample, namely from 1954:Q1+p until 1998:Q4. Four cases may show up, depending on the estimation period used for the estimation of the cointegrating and the co-feature relationships. These are summarized as follows:

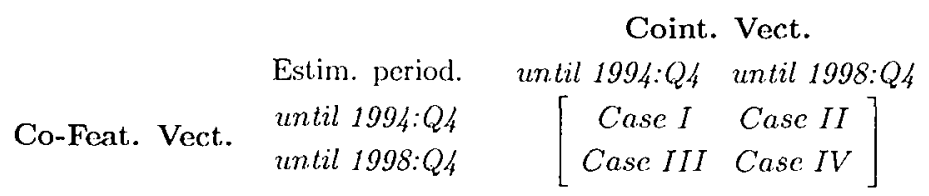

Case I has already been analyzed in the previous subsection and is used as a benchmark. We apply the same graphical approach as previously and we present the figures on the same scale (Y-axc). Figures 6.9 plot 1-step-ahead MSFE for cases I to IV for $\mathcal{M}_{3}$. It appears clearly that fixing cointegrating vectors to their whole sample values reduces significantly the MSFE after the forecast horizon $H=5$ or 6 . This indicates that the long run relationship is likely to be stable for a year or so. Note that the ranking of the different specifications previously observed in terms of the MSFE is maintained, i.e. DV $<$ VECM $\simeq$ WF $<S F$.

Estimating the common feature vector over the whole sample does not alter the forecasts. This indicates that co-feature vectors are likely to be stable during the forecast horizon. We obtain the same conclusions with 2-, 4- and 8-step-ahead MSFE but the graphs are not reported to save space.

The same analysis is performed for $\mathcal{M}_{2}$ : the model with consumption, investment and output. In contrast to the previous case, the two long-run relationships are quite stable over the forccast period. Extending the estimation period until 1998:Q4 does not alter the results with respect to the MSFE criterion. We do not report the figures where the co-feature vectors are estimated over the whole sample (cases III and IV) because those graphs are identical to the ones for case $I$ and II (sec Figure 6.10).

\subsubsection{Using Information Criteria}

An interesting strategy investigated by Vahid and Engle (1999) consists in determining simultaneously the lag order of the VAR $p$, (or more exactly $p^{*}=p-1$ in the VECM) and $s$ the number of SCCF vectors. In an exiensive Monte Carlo exercise, they show that information criteria (AIC, SCB and HQC) allow to choose reduced rank structures (SCCF) that are nonnested within the VECM. For example, due to the large number of variables it may come out that the minimum of an information criterion is reached at, say $p^{*}=3$. But if researcher allows for additional rank reduction in the short-run (common features) the model that would minimize the same information criteria is a structure with, say, $p^{*}=6$ and $s=2$. Vahid and Issler (1999) illustrates that using this approach, more accurate forecasts are obtained in terms of TMSFE or GFESM criteria. We apply this principle in this subsection for $\mathcal{M}_{2}$. 

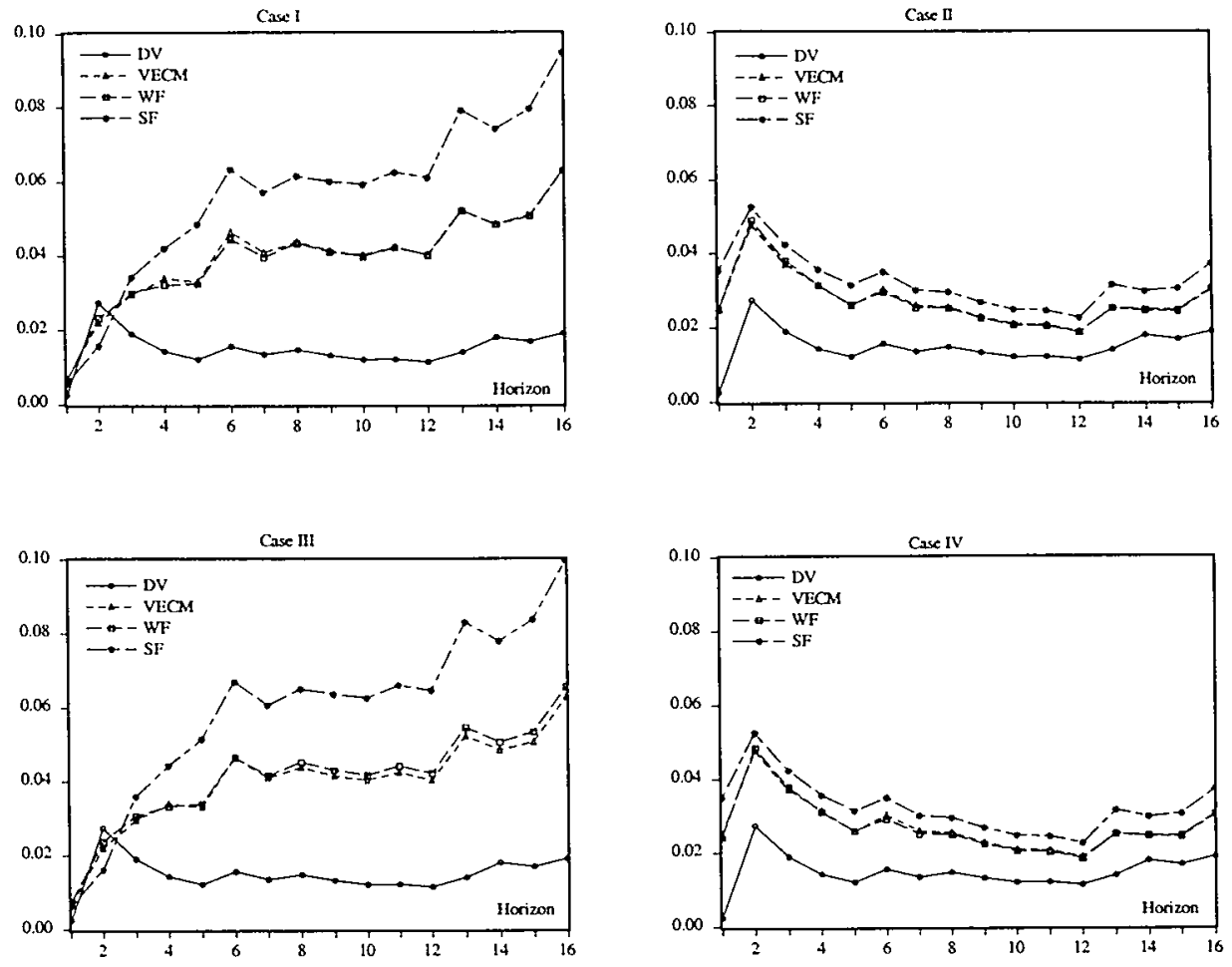

Figure 6.9: Stability Analysis / Model 3/ 1-step-ahead MSFE 

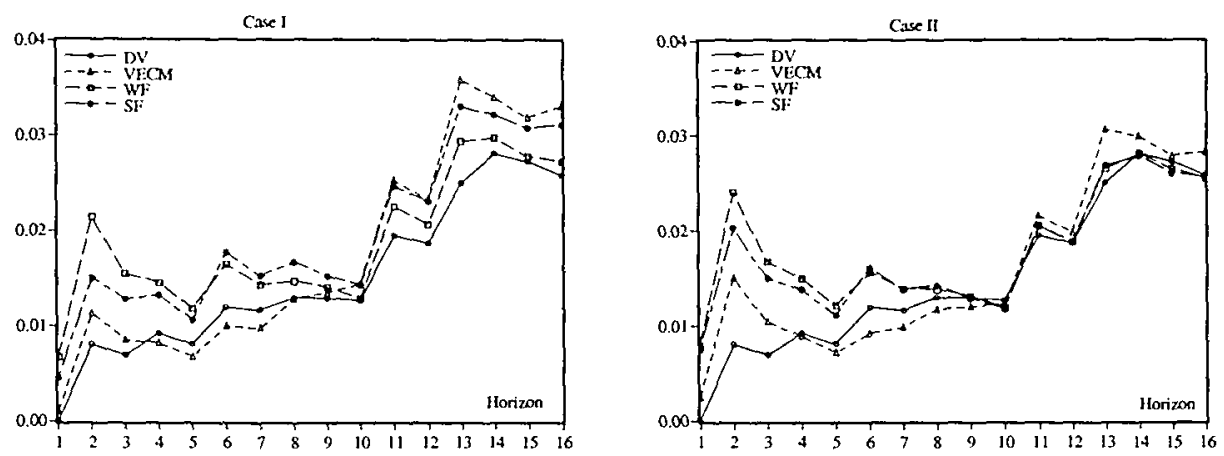

Figure 6.10: Stability Analysis / Model 2/1-step-ahead MSFE

Table 6.8 reports the values of two information criteria (AIC and HQC) for the SF, the WF and the VECM restrictions. The table also displays the value of the loglikelihood, the number of constraints and consequently the number of parameters, the different modeling imply. The VECM corresponds to the case where $s=0$, i $\mathrm{e}$. when there are not additional short-run restrictions. We have fixed the maximum lag order to 10 quarters, hence $p^{*}$, the maximum lag length in the VECM, varies from 1 to 9 . We estimate all systems over the same sample 1954:Q1+max $(p)$ to 1994:Q4, i.e. from 1956:Q3. For each $p^{*}$ we underline the value that minimizes information criteria both in the WF and the SF cases. The global minimum value is in bold.

From Table 6.8 it appears that minimizing the AIC, one would retain a VAR(5), i.e. a $\operatorname{VECM}(4)$, with two WF co-feature vectors. It would be a $\operatorname{VAR}(2)$, i.e. a $\operatorname{VECM}(1)$, using HQC with again two WF restrictions. Without any constraints on the short-run dynamics, both information criteria favor a VECM with $p^{*}=1$. We evaluate the forecast accuracy of these two models. Figure 6.11 reports the ratio of the MSFE of VAR(2)/VAR(5) with two WF restrictions. A value larger than 1 pleas in favor of the $\operatorname{VAR}(5)$. We notice that the $\operatorname{VAR}(5)$ outperforms the VAR(2) except if the forecast horizon $H$ is less than 6 quarters for the one-step-ahead predictions only. Note finally that the $\operatorname{VAR}(5)$ with two WF relationships for consumption, income and investment is not only the system we have analyzed in the empirical sections of this chapter but also the structure we have investigated in Chapters 3 and 4.

\subsection{Conclusion}

In this chapter we have tried to assess empirically the forecast performance of common cyclical feature restrictions in VAR with cointegration. We have considered the real private US domes- 


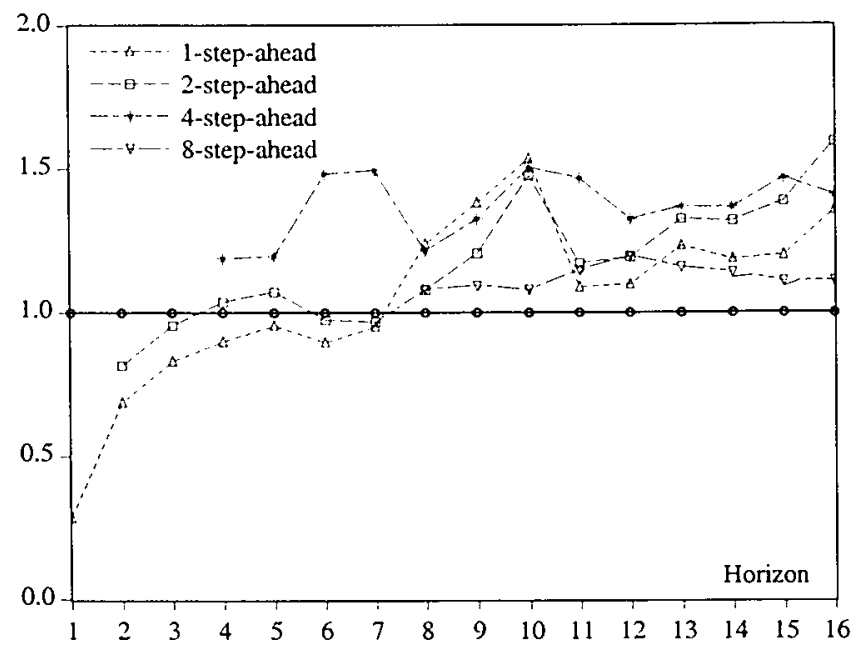

Figure 6.11: MSFE, Comparison of VAR(1) and VAR(5) under 2 WF

tic output and we have evaluated comovements with several macroeconomic variables. Some comments are in order:

First, it appears that there exists, in addition to cointegration, an important number of common cyclical feature relationships in all models. However while Monte Carlo experiments in Vahid and Issler (1999) have shown evidence in favor of imposing co-feature restrictions, the $\mathrm{SF}$ and the WF specifications do not uniformly provide more accurate point forecasts than the VECM without these type of restrictions imposed. This outcome is also pointed out in the literature about cointegration: while some theoretical results and Monte Carlo simulations show the merits of the VECM over the unrestricted VAR, empirical results are less clear cut.

Second, the small economic system restricted to consumption, income and investment seems to provide lower MSFEs than the rival models.

Third, with the exception of $\mathcal{M}_{2}$, non-constancies may be present in the forecast horizon because the difference in variables modeling is more robust than VECM, WF and SF structures. We have illustrated that a modification in the long-run equilibrium (in model 3) could partly be responsible for this situation. But the problem of parameter and equilibria non-constancies is not a trivial one. A complementary important issue that is worth considering in empirical analyses is the intercept correction approach as well as the updating of estimates. We have left these points for future analysis. 


\begin{tabular}{|c|c|c|c|c|c|c|c|c|c|c|c|}
\hline & & $\begin{array}{c}\text { SF } \\
\text { Loglik }\end{array}$ & $\begin{array}{c}\text { WF } \\
\text { Loglik }\end{array}$ & $\begin{array}{c}\text { SF } \\
\text { \# restr. }\end{array}$ & $\begin{array}{l}\text { WF } \\
\text { \# restr. }\end{array}$ & $\begin{array}{c}\text { SF } \\
\text { \# param.\# }\end{array}$ & $\begin{array}{l}\text { WF } \\
\text { param. }\end{array}$ & AIC_SF & AIC_WF & HQ_SF & HQ_WF \\
\hline \multirow[t]{4}{*}{$p^{\prime}=9$} & $s=0$ & 2179,11 & 2179,11 & 0 & 0 & 93 & 93 & $-27,0923$ & $-27,0923$ & $-26,3474$ & $-26,3474$ \\
\hline & $s=1$ & 2165,32 & 2169,3 & 27 & 25 & 66 & 68 & $-27,2639$ & $-27,2896$ & $-26,7352$ & -26.7449 \\
\hline & $s=2$ & 2144,56 & 2153,69 & 56 & 52 & 37 & 41 & $-27,3709$ & $-27,4375$ & $-27,0745$ & $-27,1091$ \\
\hline & $s=3$ & 2095,67 & 2111,93 & 87 & 81 & 6 & 12 & $-27,1386$ & $-27,2718$ & $-27,0905$ & $-27,1757$ \\
\hline \multirow[t]{4}{*}{$p^{*}=8$} & $s=0$ & 2175,09 & 2175,09 & 0 & 0 & 84 & 84 & $-27,1570$ & $-27,1570$ & $-26,4841$ & $-26,4841$ \\
\hline & $s=1$ & 2161,88 & 2165,8 & 24 & 22 & 60 & 62 & $-27,2971$ & $-27,3221$ & $-26,8165$ & $-26,8254$ \\
\hline & $s=2$ & 2144,11 & 2153,45 & 50 & 46 & 34 & 38 & $-27,4040$ & -27.4734 & $-27,1317$ & $-27,1690$ \\
\hline & $s=3$ & 2095,67 & 2111,87 & 78 & 72 & 6 & 12 & $-27,1386$ & $-27,2710$ & $-27,0905$ & -27.1749 \\
\hline \multirow[t]{4}{*}{$p^{*}=7$} & $s=0$ & 2170,18 & 2170,18 & 0 & 0 & 75 & 75 & $-27,2101$ & $-27,2101$ & $-26,6093$ & $-26,6093$ \\
\hline & $s=1$ & 2158,64 & 2164,5 & 21 & 19 & 54 & 56 & $-27,3330$ & $-27,3831$ & $-26,9004$ & $-26,9345$ \\
\hline & $s=2$ & 2143,08 & 2152,49 & 44 & 40 & 31 & 35 & $-27,4296$ & $-27,4999$ & $-27,1813$ & -27.2195 \\
\hline & $s=3$ & 2095,67 & 2111,66 & 69 & 63 & 6 & 12 & $-27,1386$ & $-27,2683$ & $-27,0905$ & $-27,1722$ \\
\hline \multirow[t]{4}{*}{$p^{*}=6$} & $s=0$ & 2169,17 & 2169,17 & 0 & 0 & 66 & 66 & $-27,3139$ & $-27,3139$ & $-26,7852$ & $-26,7852$ \\
\hline & $s=1$ & 2157,96 & 2163,95 & 18 & 16 & 48 & 50 & $-27,4021$ & $-27,4539$ & $-27,0176$ & $-27,0534$ \\
\hline & $s=2$ & 2142,75 & 2152,17 & 38 & 34 & 28 & 32 & $-27,4643$ & $-27,5347$ & -27.2400 & $-27,2783$ \\
\hline & $s=3$ & 2095,67 & 2111,74 & 60 & 54 & 6 & 12 & $-27,1386$ & $-27,2694$ & $-27,0905$ & $-27,1732$ \\
\hline \multirow[t]{4}{*}{$p^{*}=5$} & $s=0$ & 2163,42 & 2163,42 & 0 & 0 & 57 & 57 & $-27,3561$ & $-27,3561$ & $-26,8995$ & $-26,8995$ \\
\hline & $s=1$ & 2153,18 & 2158,9 & 15 & 13 & 42 & 44 & $-27,4179$ & $-27,4662$ & $-27,0815$ & $-27,1138$ \\
\hline & $s=2$ & 2139,46 & 2148,54 & 32 & 28 & 25 & 29 & $-27,4605$ & $-27,5265$ & $-27,2603$ & $-27,2942$ \\
\hline & $s=3$ & 2095,67 & 2111,86 & 51 & 45 & 6 & 12 & $-27,1386$ & $-27,2709$ & $-27,0905$ & $-27,1748$ \\
\hline \multirow[t]{4}{*}{$p^{*}=4$} & $s=0$ & 2161,32 & 2161,32 & 0 & 0 & 48 & 48 & $-27,4457$ & $-27,4457$ & $-27,0612$ & $-27,0612$ \\
\hline & $s=1$ & 2151,76 & 2157,96 & 12 & 10 & 36 & 38 & $-27,4774$ & $-27,5319$ & $-27,1890$ & $-27,2276$ \\
\hline & $s=2$ & 2138,65 & 2147,66 & 26 & 22 & 22 & 26 & $-27,4890$ & -27.5540 & $-27,3127$ & $-27,3458$ \\
\hline & $s=3$ & 2095,67 & 2111,81 & 42 & 36 & 6 & 12 & $-27,1386$ & $-27,2703$ & $-27,0905$ & $-27,1741$ \\
\hline \multirow[t]{4}{*}{$p^{*}=3$} & $s=0$ & 2151,62 & 2151,62 & 0 & 0 & 39 & 39 & $-27,4366$ & $-27,4366$ & $-27,1242$ & -27.1242 \\
\hline & $s=1$ & 2145,73 & 2148,66 & 9 & 7 & 30 & 32 & $-27,4770$ & $-27,4891$ & $-27,2367$ & $-27,2328$ \\
\hline & $s=2$ & 2135,3 & 2144,05 & 20 & 16 & 19 & 23 & $-27,4844$ & $-27,5461$ & -27.3322 & $-27,3619$ \\
\hline & $s=3$ & 2095,67 & 2111,95 & 33 & 27 & 6 & 12 & $-27,1386$ & $-27,2721$ & $-27,0905$ & $-27,1760$ \\
\hline \multirow[t]{4}{*}{$p^{*}=2$} & $s=0$ & 2139,84 & 2139,84 & 0 & 0 & 30 & 30 & $-27,4005$ & $-27,4005$ & $-27,1602$ & $-27,1602$ \\
\hline & $s=1$ & 2137,85 & 2138,55 & 6 & 4 & 24 & 26 & $-27,4526$ & $-27,4357$ & $-27,2603$ & $-27,2274$ \\
\hline & $s=2$ & 2128,25 & 2136,17 & 14 & 10 & 16 & 20 & $-27,4318$ & $-27,4827$ & $-27,3037$ & $-27,3225$ \\
\hline & $s=3$ & 2095,67 & 2112,02 & 24 & 18 & 6 & 12 & $-27,1386$ & $-27,2730$ & $-27,0905$ & $-27,1769$ \\
\hline \multirow[t]{4}{*}{$p^{*}=1$} & $s=0$ & 2137,46 & 2137,46 & 0 & 0 & 21 & 21 & $-27,4865$ & $-27,4865$ & $-27,3183$ & $-27,3183$ \\
\hline & $s=1$ & 2136,26 & 2136,88 & 3 & 1 & 18 & 20 & -27.5099 & $-27,4919$ & -27.3657 & $-27,3317$ \\
\hline & $s=2$ & 2127,77 & 2135,67 & 8 & 4 & 13 & 17 & $-27,4645$ & -27.5152 & $-27,3604$ & -27.3790 \\
\hline & $s=3$ & 2095,67 & 2112,03 & 15 & 9 & 6 & 12 & $-27,1386$ & $-27,2731$ & $-27,0905$ & $-27,1770$ \\
\hline
\end{tabular}

Table 6.8: Determination of $\mathrm{p}$ and s Simultaneoulsly using Information Criteria

Finally, a possible explanation for the absence of forecast improvement may be the relatively large number of observations we have used, i.e. $T \simeq 160$. Consequently, no gain in parameter estimates are found from imposing restrictions. Therefore, similarly to analyses using impulse response functions, common cyclical feature restrictions are likely to be more helpful to increase efficiency than to obtain better point forecasts.

It is also likely that some of the restrictions we have imposed are artefact because we have used seasonally adjusted series. We show in the next chapter how misleading infercnce in co-feature could be due to the presence of seasonally filtered variables. 
and

$$
\Delta_{1} y_{t}=\sum_{i=1}^{p} \Phi_{i} \Delta_{4} y_{t-i}+\varepsilon_{t}, \quad t=1 \ldots T
$$

where we assume without loss of generality that $y_{t}$ is measured quarterly. $y_{t}$ is a $n$-dimensional seasonal process with $\Delta=1-L$ and $\Delta_{4}=1-L^{4}$. As $\left(1-L^{4}\right)=(1-L)(1+L)\left(1+L^{2}\right)$, both models imply only one unit root at the zero frequency. Model (7.1) displays the usual deterministic pattern structure in which, for the first differenced series, the seasonal component is summarized by dummy variables. Representation (7.2) exhibits unit roots at all seasonal frequencies, so the seasonality is said to be stochastic (see Franses, 1996 for a survey). Notice that in order to simplify the analysis we have ruled out the presence of any cointegrating vectors both at zero and at seasonal frequencies.

Denote $\left[\Phi_{1}: \Phi_{2}: \ldots: \Phi_{p}\right]=\Phi_{n \times n_{p}}$, so that common cyclical features are present if the rank of $\Phi$ is smaller than $n$. In this chapter we analyze the sizc, i.c. when $\operatorname{rank}(\Phi)<n$, as well as the power, i.c. when $\operatorname{rank}(\Phi)=n$, of serial correlation common feature tests statistics introduced in Chapters 2 and 3. As we cannot ignore the presence of seasonal components we first analyze the case in which unadjusted $y_{t}$ has been filtered correctly by $(1-L)$ and four dummies or by the $\Delta_{4}$ operator. Then we compare those results with time series that have been adjusted by the Census X-11 filter to get rid of seasons. In this last situation we transform variables in levels by the linear approximation $y_{t}^{S A}=\Psi(\mathbf{L}) y_{t}$ where $\Psi(\mathbf{L})$ is a bloc diagonal $n \times n$ matrix whose elements are X-11 moving average filter $\Psi(L)=\sum_{i=-28}^{28} \Psi_{i} L^{i}$ applied to each of the $n$ time series. Such moving average filters are linear, symmetric and centered around the current observation, namcly $\Psi(L)=\Psi\left(L^{-1}\right)$ and $\Psi(1)=1$. The common feature analysis is then applied to the first or the fourth differences of seasonally adjusted data, i.e. on $\Delta y_{t}^{S A}$ or $\Delta_{4} y_{t}^{S A}$.

Proposition 7.1 The common feature space for the unadjusted data is not a common feature space for seasonally adjusted data.

Proof. Following inter alia Ericsson et al. (1994) it is useful to express $\Psi(L)=\Psi(1)+$ $\Psi^{*}(L) \Delta$, where $\Delta$ is the difference operator and $\Psi^{*}(L)$ is a two-sided linear filter with polynomial coefficients $\Psi_{i}^{*}$. For non cointegrated stationary time series, with for instance $z_{t} \equiv \Delta y_{t}$, we get $z_{\hat{t}}^{S A}=\Psi(L) z_{t}=\Psi(1) z_{t}+\Psi^{*}(L) \Delta z_{t}=z_{t}+\Psi^{*}(L) \Delta z_{t}$. Assume there exists a $n \times s$ matrix $\bar{\beta}$ whose columns span the cofeature space. Premultiplying both sides by $\tilde{\beta}^{\prime}$, we get $\tilde{\beta}^{\prime} z_{t}^{S A}=$ $\tilde{\beta}^{\prime} z_{t}+\tilde{\beta}^{\prime} \Psi^{*}(L) \Delta z_{t}$. $\tilde{\beta}^{\prime} z_{t}$ is a white noise by definition of $S C C F$. Note however that $\tilde{\beta}^{\prime} \Psi^{*}(L) \Delta z_{t}$ is a weighted sum of an invertible $M A(1)$ process, and hence $\ddot{\beta}^{\prime} z_{t}^{S A}$ will not be a white noise. ${ }^{1}$

Proposition 7.2 Inference on the common feature space conducted on unadjusted data differs from the one conducted on seasonally adjusted data.

\footnotetext{
${ }^{1}$ Consequently we end up with a non-synchronous common cycle as difined by Vahid and Engle (1997).
} 


\title{
Chapter 7
}

\section{Does Seasonal Adjustment Induce Common Cycles?}

\begin{abstract}
In this chapter we analyze via Monte Carlo simulations the behavior of common cyclical features test statistics when X-11 seasonal adjusted data are encountered. We emphasize both size and power distortions. We illustrate the analysis on Japanese consumption/income relationship and on European industrial production indexes.
\end{abstract}

\subsection{Introduction and Motivation}

Because official statistical agencies and international institutions often release seasonally corrected data, numerous papers have investigated the effect of seasonal adjustment on econometric inference and hypothesis testing (see inter alia Wallis, 1974, Ghysels and Perron, 1993, Ghysels et al., 1994 or Maravall, 1995). One of the most important messages is that estimates are no longer consistent when lagged dependent variables are present among the regressors. There is a notorious exception for cointegrating vectors that are not affected by scasonal filters even if the short-run dynamics is. Consequently, in this chapter we examine to what extent the common practice of seasonally adjusting data may yield spurious, or fails to reveal, common feature vectors.

We study the small sample impact of the well known X-11 linear filter within two different. models, namely

$$
\Delta y_{t}=\delta D_{t}+\sum_{i=1}^{p} \Phi_{i} \Delta y_{t-i}+\varepsilon_{t}, \quad t=1 \ldots T
$$

\footnotetext{
${ }^{\circ}$ Chapter 7 draws on Hecq (1998) with elements from Beine, Candelon and Hecq (2000) in Section 7.5. A related investigation has been independently undertaken by Cubadda (1999).
} 
and

$$
\Delta_{4} y_{t}=\sum_{i=1}^{p} \Phi_{i} \Delta_{4} y_{t-i}+\varepsilon_{t}, \quad t=1 \ldots T,
$$

where wo assume without loss of generality that $y_{t}$ is measured quartcrly. $y_{t}$ is a $n$-dimensional scasonal process with $\Delta=1-L$ and $\Delta_{4}=1-L^{4}$. As $\left(1-L^{4}\right)=(1-L)(1+L)\left(1+L^{2}\right)$, both models imply only one unit root at the zero frequency. Model (7.1) displays the usual deterministic pattern structure in which, for the first differenced series, the seasonal component is summarized by dummy variables. Representation (7.2) exhibits unit roots at all scasonal frequencies, so the seasonality is said to be stochastic (see Franses, 1996 for a survey). Notice that in order to simplify the analysis we have ruled out the presence of any cointegrating vectors both at zero and at seasonal frequencies.

Denote $\left[\Phi_{1}: \Phi_{2}: \ldots: \Phi_{p}\right]=\Phi_{n \times n p}$ so that common cyclical features are present if the rank of $\Phi$ is smaller than $n$. In this chapter we analyze the size, i.e. when $\operatorname{rank}(\Phi)<n$, as well as the power, i.c. when $\operatorname{rank}(\Phi)=n$, of serial correlation common feature tests statistics introduced in Chapters 2 and 3. As we cannot ignore the presence of scasonal components we first analyze the case in which unadjusted $y_{\ell}$ has been filtered correctly by $(1-L)$ and four dummies or by the $\Delta_{4}$ operator. Then we compare those results with time series that have been adjusted by the Census X-11 filter to get rid of seasons. In this last situation we transform variables in levels by the linear approximation $y_{t}^{S A}=\Psi(\mathbf{L}) y_{\iota}$ where $\Psi(\mathbf{L})$ is a bloc diagonal $n \times n$ matrix whose elements are X-11 moving average filter $\Psi(L)=\sum_{i=-2 \delta}^{28} \Psi_{i} L^{i}$ applied to each of the $n$ time series. Such moving average filters are linear, symmetric and centered around the current observation, namely $\Psi(L)=\Psi\left(L^{-1}\right)$ and $\Psi(1)=1$. The common feature analysis is then applied to the first or the fourth differences of seasonally adjusted data, i.c. on $\Delta y_{t}^{S A}$ or $\Delta_{4} y_{t}^{S A}$.

Proposition 7.1 The common feature space for the unadjusted data is not a common feature space for seasonally adjusted data.

Proof. Following inter alia Ericsson et al. (1994) it is useful to express $\Psi(L)=\Psi(1)+$ $\Psi^{*}(L) \Delta$, where $\Delta$ is the difference operator and $\Psi^{*}(L)$ is a two-sided linear filter with polynomial coefficients $\Psi_{i}^{*}$. For non cointegrated stationary time series, with for instance $z_{t} \equiv \Delta y_{t}$, we get $z_{t}^{S A}=\Psi(L) z_{\ell}=\Psi(1) z_{t}+\Psi^{*}(L) \Delta z_{t}=z_{t}+\Psi^{*}(L) \Delta z_{\ell}$. Assume there exists a $n \times s$ matrix $\tilde{\beta}$ whose columns span the cofeature space. Premultiplying both sides by $\tilde{\beta}^{\prime}$, we get $\tilde{\beta}^{\prime} z_{l}^{S A}=$ $\tilde{\beta}^{\prime} z_{t}+\tilde{\beta}^{\prime} \Psi^{*}(L) \Delta z_{t}$. $\tilde{\beta}^{\prime} z_{t}$ is a white noise by definition of $S C C F$. Note however that $\tilde{\beta}^{\prime} \Psi^{*}(L) \Delta z_{t}$ is a weighted sum of an invertible $M A(1)$ process, and hence $\tilde{\beta}^{\prime} z_{t}^{S A}$ will not be a white noise. ${ }^{1}$

Proposition 7.2 Inference on the common feature space conducted on unadjusted data differs from the one conducted on seasonally adjusted data.

\footnotetext{
${ }^{1}$ Consequently we end up with a non-synchronous common cycle as difined by Vahid and Engle (1997).
} 
Proof. Indeed, let us consider the VAR(p) (7.1) where $\Phi(L)=I-\sum_{i=1}^{p} \Phi_{i} L^{i}$. Applying the linear approximation of the seasonal filter yields $\Psi(L) \Phi(L) z_{t}=\Psi(L) \delta D_{t}+\Psi(L) \varepsilon_{t}$. Substituting $z_{t}$ by $z_{l}^{S A}+\left(z_{t}-z_{t}^{S A}\right)$ and using the previous filter we get ${ }^{2}$

$$
\begin{gathered}
\Psi(L) \Phi(L) z_{t}^{S A}+\Psi(L) \Phi(L)\left(z_{t}-z_{t}^{S A}\right)=\Psi(L) \varepsilon_{t}, \\
\Phi(L) z_{t}^{S A}=-\Psi(L) \Phi(L)\left(z_{t}-z_{t}^{S A}\right)+\Psi(L) \varepsilon_{t}-\Psi^{*}(L) \Phi(L) \Delta z_{t}^{S A} .
\end{gathered}
$$

Now, the crror process $\eta_{t}=-\Psi(L) \Phi(L)\left(z_{t}-z_{l}^{S A}\right)+\Psi(L) \varepsilon_{t}-\Psi^{*}(L) \Phi(L) \Delta z_{t}^{S A}$ is no longer an innovation and it is neither orthogonal to the regressors, which will induce inconsistency and inefficiency for the reduced rank test statistics.

\subsection{Test Statistics}

In the lines of the work by Tiao and Tsay (1989), Gouricroux and Peaucelle (1993), Ahn and Reinsel (1988), Velu et al. (1986) we test for zero canonical correlations between the $(T \times n)$ matrix $W_{1} \equiv \Delta Y_{l}=\left(\Delta y_{1} \ldots \Delta y_{T}\right)^{\prime}$ and the $T \times(n \times p)$ matrix $W_{2}=\left(\Delta Y_{t-1} \ldots \Delta Y_{t-p}\right)$. Note that for the non X-11 filtered case $W_{1}$ and $W_{2}$ have to be adjusted for their mean and seasonal components, i.e. both terms have been regressed on four centered dummies and the analysis is carried out on residuals. For stochastic seasonality the analysis is conducted between $\bar{W}_{1} \equiv$ $\Delta_{4} Y_{t}=\left(\Delta_{4} y_{1} \ldots \Delta_{4} y_{T}\right)^{\prime}$ and the $T \times(n \times p)$ matrix $\bar{W}_{2}=\left(\Delta_{4} Y_{t-1} \ldots \Delta_{4} Y_{t-p}\right)$ where both sets have been adjusted for their means. When the X-11 filter is used, we replace $W_{1}, W_{2}$ and $\bar{W}_{1}, \bar{W}_{2}$ by $\tilde{W}_{1}$ and $\tilde{W}_{2}$ where $\tilde{W}_{1} \equiv \Delta Y_{i}^{S A}=\left(\Delta y_{1}^{S A} \ldots \Delta y_{T}^{S A}\right)^{\prime}$ and the $T \times(n \times p)$ matrix $\tilde{W}_{2}=\left(\Delta Y_{i-1}^{S A} \ldots \Delta Y_{t-7}^{S A}\right)$. Notice that once the seasonal filter has been applied, it is numerically equivalent to first difference X-11 filtered data when the DGP is model (7.1) or to take the fourth differences of X-11 filtered data when the DGP is model (7.2). Consequently we do not report. both results. In practice, however, the statistical properties of raw data are usually not known. So, most of the time we will take first differences of non stationary scasonally adjusted data.

Test statistics are based on the null hypothesis that the sum of the $s$ smallest squared canonical correlations are zero. They are similar to those presented in previous chapters.

\subsection{The Data Generating Process and Simulation Results}

Under the reduced rank null hypothesis, we generate a stationary bivariate process in which the first as well as the fourth differenced variables follow a VAR of order 2 with a cofeature vector

\footnotetext{
${ }^{2}$ Notice that, if the sum of dummy variables coefficients is zero over a year, $\Psi(L) \delta D_{t}=0$ (Ericsson et al., 199.4).
} 
equal to $(1,-1)$ :

$$
\Phi_{1}=\left(\begin{array}{cc}
.2 & -.5 \\
.2 & -.5
\end{array}\right), \quad \Phi_{2}=\left(\begin{array}{cc}
-.32 & .16 \\
-.32 & .16
\end{array}\right), \quad \Omega_{\varepsilon}=\left(\begin{array}{ll}
9 & 3 \\
3 & 4
\end{array}\right), \quad \delta=\left(\begin{array}{cccc}
1 & 2 & -2 & 1 \\
-6 & 1.5 & -.5 & 5
\end{array}\right) .
$$

Under the alternative, it is casy to choose a DGP that would give a power of $100 \%$ in almost. all circumstances. To circumvent this problem, wo have determined an alternative hypothesis relatively close to the null by fixing $\Phi_{1.12}=\Phi_{2.21}=0$.

In the linear approximation of the X-11 filter we do not try to reconstruct the initial and the final observations in order to avoid the appearance of other problems such as nonlinearity (Maravall, 1997). Consequently we generate $52+28+T+28$ observations. We drop the first 52 to initialize the process and take the $T$ observations in the middle in each case. The weights of the linear 56th order moving average are often given (in Ghysels and Perron, 1993 for instance) up to 3 digits. In order to minimize rounding errors we recalculate the weights for eight decimals. Notice that in the simulations we exclude cointegrating relationships both at scasonal and at zero frequencies. We also assume that the deterministic seasonal pattern is constant through time.

Three sample sizes are used, that is $T=80,160,500$. The first two sample sizes mimic most economic time series for which we often get 20 or 40 years of quarterly data. We also add a larger sample size in order to analyze the behavior of test statistics when the sample increases. All the computations have been done in Gauss 3.14 with the RNDN generator process using 10,000 replications. The estimated model has successively $p=1,2,4,8$ lags. Table 7.1 to 7.3 give details of the simulations. Table 7.1 reports empirical sizes, the nominal one being $5 \%$, for the case in which the correct filters have been applied. Table 7.2 presents sizes when the X-11 filter has been used. For these two tables we present the rejection frequencics of the (truc) null hypothesis that the smallest eigenvalue is zero $\left(H_{0}: s \geq 1\right)$, the rejection frequency for the sum of the two eigenvalues being $100 \%$ in almost all cases. We also present the median of coefficient $\tilde{\beta}_{2}$ (called $\left.\tilde{\beta}_{.5}\right)$ for the normalized codependence vector $\left(1,-\tilde{\beta}_{2}\right)$, the true one being $(1,-1)$, as well as the interquartile range (spread) of $\tilde{\beta}_{2}$ as a parameter of dispersion. Table 7.3 reports the size-adjusted power ${ }^{3}$ for $T=160$ and $T=500$.

In the first case, the appropriate filter has been used. It emerges from Table 7.1 that there exist no bias nor size distortions in large samples. For smaller samples, we see a small size distortion if we overspecify the dynamics: empirical sizes go to $10 \%$ in the worst case when $T=80$ and $p=8$. A small bias also appears in the fourth difference case if one underspecifics the dynamies, that is taking $p=1$. The dispersion (measured by the spread) is smallest when the correct lag order is selected, that is when $p=2$. The power is also high under the true dynamic structure but decreases for small samples if we over-estimate the lag structure.

Once the data have been seasonally adjusted we observe huge size distortions yielding test.

\footnotetext{
${ }^{3}$ To get size-adjusted powers, we analyze frequencies of rejecting $H_{A}$ when $H_{A}$ is true with critical values corresponding to $5 \%$ empirical size levels calculated for each specific case.
} 


\begin{tabular}{|c|c|c|c|c|c|c|c|c|c|c|c|}
\hline$D G P$ & Filter & lags & $\begin{array}{l}\mathrm{T}=80 \\
s \geq 1\end{array}$ & $\tilde{B}_{.5}$ & $\tilde{\beta}_{.75-.25}$ & $\begin{array}{l}T=160 \\
s \geq 1\end{array}$ & $\tilde{\beta}_{.5}$ & $\bar{\beta}_{.75-.25}$ & $\begin{array}{l}T=500 \\
s \geq 1\end{array}$ & $\tilde{\beta}_{.5}$ & $\tilde{\beta}_{.75-.25}$ \\
\hline$\Delta y_{t}$ & $\Delta y_{t}$ & $p=1$ & 5.10 & .996 & .4253 & 5.20 & .998 & .2869 & 5.19 & 1.001 & .1559 \\
\hline \multirow[t]{3}{*}{$+S D$} & $+S D$ & $p=2$ & 5.70 & .993 & .3152 & 5.21 & .998 & .2172 & 4.96 & .999 & .1195 \\
\hline & & $p=4$ & 7.48 & .993 & .3411 & 5.98 & .998 & .2233 & 5.36 & .999 & .1211 \\
\hline & & $p=8$ & 10.76 & .993 & .3809 & 7.64 & .995 & .2366 & 5.67 & .999 & .1225 \\
\hline \multirow[t]{4}{*}{$\Delta_{4} y_{t}$} & $\Delta_{A} y_{t}$ & $p=1$ & 4.33 & .969 & .4085 & 4.80 & .984 & .2778 & 5.12 & .996 & .1558 \\
\hline & & $p=2$ & 4.81 & 1.005 & .3122 & 4.77 & 1.002 & .2147 & 4.82 & 1.000 & .1200 \\
\hline & & $p=4$ & 5.69 & 1.003 & .3321 & 5.03 & 1.002 & .2204 & 5.00 & 1.000 & .1204 \\
\hline & & $p=8$ & 8.03 & 1.003 & .3742 & 6.27 & .999 & .2351 & 5.08 & 1.000 & .1226 \\
\hline
\end{tabular}

Table 7.1: Empirical Sizes and Co-feature Vector Median and Spread for NSA

\begin{tabular}{|c|c|c|c|c|c|c|c|c|c|c|c|}
\hline$D G P$ & Filter & lags & $\begin{array}{l}\mathrm{T}=80 \\
s \geq 1\end{array}$ & $\tilde{\beta}_{.5}$ & $\tilde{\beta}_{.75-25}$ & $\begin{array}{l}\mathrm{T}=160 \\
s \geq 1\end{array}$ & $\tilde{\beta}_{.5}$ & $\tilde{\beta}_{.75-.25}$ & $\begin{array}{l}\mathrm{T}=500 \\
s \geq 1\end{array}$ & $\tilde{\beta}_{.5}$ & $\tilde{\beta}_{.75-.25}$ \\
\hline \multirow{4}{*}{$\begin{array}{l}\Delta y_{t} \\
+S D\end{array}$} & \multirow[t]{4}{*}{$\Delta y_{i}^{S A}$} & $\mathrm{p}=1$ & 8.62 & 1.157 & .5151 & 11.43 & 1.182 & .3549 & 16.83 & 1.192 & .1973 \\
\hline & & $\mathrm{p}=2$ & 19.62 & .976 & .3460 & 35.80 & .974 & .2321 & 82.82 & .974 & .1315 \\
\hline & & $\mathrm{p}=4$ & 41.46 & .955 & .4069 & 72.56 & .953 & .2611 & 99.95 & .952 & .1448 \\
\hline & & $\mathrm{p}=8$ & 67.29 & .952 & .4782 & 95.83 & .949 & .2931 & 100 & .949 & .1539 \\
\hline \multirow{4}{*}{$\Delta_{4} y_{t}$} & \multirow{4}{*}{$\Delta y_{t}^{S A}$} & $p=1$ & 94.03 & .549 & .3014 & 100 & .740 & .2637 & 100 & .555 & .1181 \\
\hline & & $p=2$ & 99.98 & .766 & .3257 & 100 & .763 & .2280 & 100 & .760 & .1243 \\
\hline & & $p=4$ & 100 & .831 & .3103 & 100 & .831 & .2161 & 100 & .831 & .1207 \\
\hline & & $p=8$ & 99.97 & .848 & .3330 & 100 & .847 & .2300 & 100 & .846 & .1259 \\
\hline
\end{tabular}

Table 7.2: Empirical Sizes and Co-feature vector Median and Spread for X-11 SA 


\begin{tabular}{|c|c|c|c|c|c|c|c|c|}
\hline$D G P$ & Filter & lags & $\begin{array}{l}T=160 \\
s \geq 1\end{array}$ & $\tilde{\beta}_{.5}$ & $\tilde{\beta}_{.75-.25}$ & $\begin{array}{l}T=500 \\
s \geq 1\end{array}$ & $\tilde{\beta}_{.5}$ & $\tilde{\beta}_{.75-.25}$ \\
\hline \multirow{4}{*}{$\begin{array}{l}\Delta y_{t} \\
+S D\end{array}$} & $\Delta y_{t}$ & $p=1$ & 49.69 & .145 & .2504 & 97.62 & .147 & .1344 \\
\hline & $+S D$ & $p=2$ & 95.02 & -.088 & .3906 & 100 & -.082 & .2012 \\
\hline & & $p=4$ & 87.00 & -.089 & .4024 & 100 & -.081 & .2016 \\
\hline & & $p=8$ & 70.66 & -.088 & .4214 & 99.96 & -.081 & .2053 \\
\hline \multirow[t]{4}{*}{$\Delta_{4} y}$, & $\Delta y_{l}^{S A}$ & $p=1$ & 0 & .165 & .1623 & 0 & .166 & .0899 \\
\hline & & $p=2$ & 7.69 & .059 & .2002 & 5.46 & .062 & .1086 \\
\hline & & $p=4$ & 16.14 & -.006 & .2246 & 24.66 & .000 & .1197 \\
\hline & & $p=8$ & 14.58 & -.019 & .2433 & 23.34 & -.014 & .1266 \\
\hline \multirow{4}{*}{$\begin{array}{l}\Delta y_{t} \\
+S D\end{array}$} & $\Delta y_{t}^{S A}$ & $p=1$ & 65.08 & .143 & .5346 & 99.4 & .138 & .2942 \\
\hline & & $p=2$ & 41.79 & -.154 & .6865 & 88.70 & -.162 & .3738 \\
\hline & & $p=4$ & 27.87 & -.127 & .6787 & 67.90 & -.1339 & .3511 \\
\hline & & $p=8$ & 19.79 & -.113 & .7578 & 49.66 & -.1351 & .3808 \\
\hline
\end{tabular}

Table 7.3: Empirical Sizes-Adjusted Power, Co-feature Vector Median and Spread

statistics that are too liberal i.e. the null hypothesis is too frequently rejected. Moreover, size distortions increase with the number of lags added in the estimated model and especially with the sample size. This results can be casily understood in terms of Propositions 7.1 and 7.2. Due to underlying MA structure, the MA parameters are consistently estimated when the sample size increases. Even if the bias is small in this case, it does not decrease when $T$ increases. The power of the test statistic strongly decreases for seasonally adjusted data even when the correct difference operator has been applied. Tests are still consistent in this case because $\lim _{T \rightarrow \infty} P_{H_{A}}($ Test $>c)=100 \%$. But the power is very small if we take the first difference of X-11 filtered data whilst the raw model has also unit roots at seasonal frequencies. Moreover, the consistency of test statistic is questionable with respect to the power evolution from $T=160$ to $T=500$. Unfortunately, taking the first difference of seasonally adjusted data is a natural practice in empirical studies.

The next two sections further illustrate the behavior of common feature test statistics when applied to seasonally adjusted series.

\subsection{The Japanese Consumption Function}

We now analyze the impact of seasonal adjustment in the consumption/income relationship. The raw as well as the seasonally adjusted data come from the Japanese national accounts and are recorded quarterly from $1955 \mathrm{Q} 2$ up to $1996 \mathrm{Q} 4{ }^{4}$ Consumption is the total consumption and

\footnotetext{
${ }^{4}$ The data may be found on the Japanese statistical office Web site http://www.stat.go.jp/19.htm.
} 


\begin{tabular}{cccc}
\hline & $\ln \mathrm{C}_{t}$ & $\ln \overline{\mathrm{Y}}_{t}$ & $\ln \left(\mathrm{C}_{t} / Y_{t}\right)$ \\
\hline lags & $1,3,4,5,6,8,10$ & $1,3,4,5,9$ & $1,2,3,6,7,10,11,12$ \\
det. part & $\mathrm{c}$, trend, SD & $\mathrm{c}$, trend, SD & $\mathrm{c}$, trend, SD \\
$\pi_{1}$ & -1.58 & -3.24 & -1.76 \\
$\pi_{2}$ & -.19 & .56 & -2.60 \\
$\pi_{3}$ & -2.63 & -2.16 & .10 \\
$\pi_{1}$ & -1.93 & -1.86 & -1.57 \\
$\pi_{3} \cap \pi_{4}$ & 5.26 & 4.11 & 1.25 \\
\hline
\end{tabular}

Table 7.4: HEGY Unit Root. Test. Statistic

\begin{tabular}{ccccc}
\hline$c_{t}=f\left(y_{t}\right.$, determ $)$ & & 0 frequency & semi-annual & annual \\
\hline \multirow{2}{*}{ Raw data } & lags & 1 to 5 & 1 to 7 & 1 to 8 \\
& det. part & c, trend & c, SD & c. SD \\
& Test Stat. & -4.60 & -2.64 & 4.38 \\
\multirow{5}{*}{ Season. adj. } & lags & 1 to 2 & - & - \\
& det. part & c, trend & - & - \\
& Test Stat. & -4.59 & - & - \\
\hline
\end{tabular}

Table 7.5: HEGY Seasonal Cointegration Tests

the income variable we retained is the GDP less government expenditures. The variables are in constant prices. Some seasonal unit root tests and cointegration analyses have previously been done on this data set by Engle et al. (1993) or Hall et al. (1997). As suspected by the last authors we also detect a shift in regime in 1974Q1, so we start our analysis in 1974Q2 in this illustrative example. The log levels of the data are drawn in Figure 7.1. As previous studies do not cover the same period, we also use FEGY (see Hylleberg et al., 1990) test statistics in order to analyze the roots at zcro or at scasonal frequencies. It is shown in Table 7.4 that the $\log$ of both unadjusted and seasonally adjusted data have a unit root at zero frequency, and that raw time series have also unit roots at all seasonal frequencies. Moreover, the vector $(1,-1)$ is not a cointegrating vector as it may be scen in the last column of Table 7.4.

Table 7.5 however shows the existence of a long run relationship at the zcro frequency but not at seasonal frequencies. The variables are stochastically cointegrated, e.g. are stationary around a deterministic trend, which partly measures the upward trend of the foreign balance. For the raw data the cointegrating relationship is $c_{1 t}=14.147+.010595 t r e n d+.637 y_{1 t}$, where $c_{1 t}=\left(1+L+L^{2}+L^{3}\right) c_{t}$ and $y_{1 t}=\left(1+L+L^{2}+L^{3}\right) y_{t}$, while it is $c_{t}=3.439+.002565$ trend $+.646 y_{t}$ with the seasonally adjusted data. Notice that both relationships present the same income elasticity as explained by Ericsson et al. (1994).

We may now conduct a common feature analysis of the variables written in their respective 


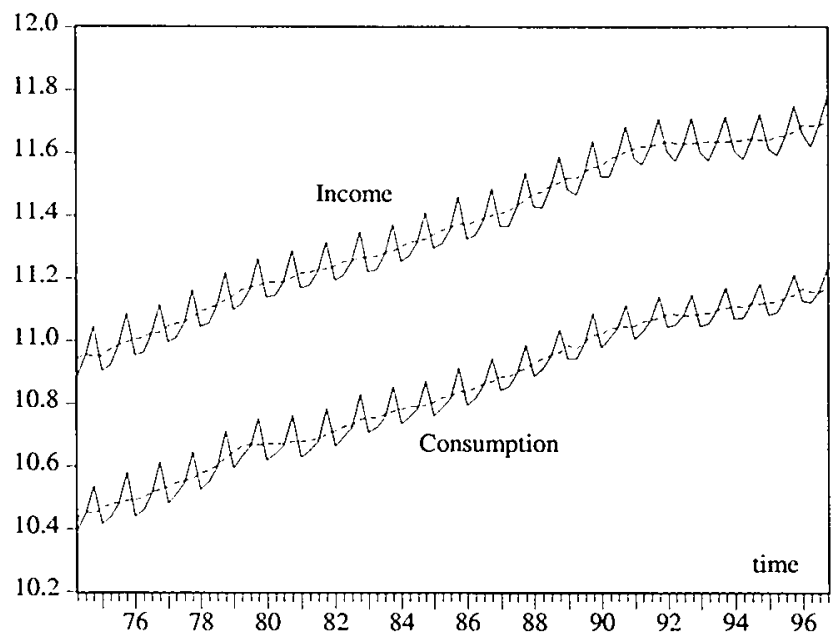

Figure 7.1: Japanese Consumption and Income at Constant. Prices (SA \& NSA)

ECM forms. ${ }^{5}$ VAR models which best characterize the covariance structure of the data are models with 1 or 6 lags for the fourth differences of the seasonally unadjusted model and 2,6 or 12 lags for the first differences of the seasonally adjusted model. Consequently we choose successively $p=2,6,12$ in order to examine the stability of results through $p$, having in mind that $p=6$ seems better for both models.

Table 7.6 gives the asymptotic WF test statistics for the raw as well as for the seasonally adjusted data set. In all cases the model that considers a linear combination of the first (or the fourth differenced data) corrected for long run effects instead of a combination of differenced variables only, has been retained with respect to likelihood ratio test statistics and information criteria.

It emerges from Table 7.6 that we cannot reject the hypothesis of one co-feature vector with seasonally adjusted data while we clearly rejected this hypothesis for the unadjusted data. In the light of the simulation outcomes, this result can be explained by a strong decrease of power for seasonally adjusted data. Also remark that we reject (results not reported) the presence of a common feature vector in the fourth differences of seasonally adjusted data. Indeed, because of unit roots both at zero and at seasonal frequencies, the power of SCCF test statistic is higher when the analysis is applied to fourth differenced adjusted data.

\footnotetext{
${ }^{5}$ With the $\left(1-L^{4}\right)$ difference operator and the function of $c_{1 t-1}, y_{1 t-1}$ for unadjusted time series and with the $(1-L)$ operator and the function of $c_{t-1}, y_{t-1}$ for the seasonaly adjusted data.
} 


\begin{tabular}{l|lllll|lllll}
\hline lags & $s \geqq 1$ & $5 \% \mathrm{cv}$ & $\begin{array}{l}\Delta_{1} \bar{l} t \\
s=2\end{array}$ & $5 \% \mathrm{cv}$ & $\tilde{\beta}_{2}$ & $s \geq 1$ & $5 \% \mathrm{cv}$ & $s=2$ & $5 \% \mathrm{cv}$ & $\tilde{\beta}_{2}$ \\
\hline & & & & & & & & & & \\
$p=2$ & 33.50 & $(7.81)$ & 146.52 & $(15.50)$ & .65 & 7.52 & $(7.81)$ & 22.11 & $(15.10)$ & .19 \\
$p=6$ & 60.93 & $(19.67)$ & 204.63 & $(36.41)$ & .71 & 16.78 & $(19.67)$ & 44.68 & $(36.41)$ & .67 \\
$p=12$ & 84.44 & $(35.17)$ & 259.76 & $(65.17)$ & .68 & 30.51 & $(35.17)$ & 74.03 & $(65.17)$ & 1.09 \\
\hline
\end{tabular}

Table 7.6: Common Feature Test Statistics for Japanese Consumption Function

\subsection{Assessing a Perfect European Optimum Currency Area}

Like it or not, the theory of optimum currency areas (OCA) remains the implicit reference framework to assess the real consequences of monetary integration. Initiated by Mundell (1961), the OCA approach has developed a set of criteria aimed at investigating whether a particular geographic zone will gain from adopting a single currency. These criteria may depend on labor mobility and trade openness (Mc Kinnon, 1963), industrial diversification (Kenen, 1969), wage and price flexibility (Blanchard and Muet, 1993) as well as fiscal federalism (Sachs and Sala-IMartin, 1992).

On the empirical side, the OCA approach has been applied to investigate whether Europe may be considered as an optimum currency area. There is nowadays a widespread consensus between economists to state that the answer to this question is negative and that only a subset of countries will gain from giving up their national currencies. However, the precise size and configuration of this restricted monetary union remains a matter of debatc. Indeed, direct applications of the above mentioned criteria turn out to be cumbersome because different empirical counterparts of the theoretical variables may yield quite different outcomes and consequently different implications for the composition of the monetary union.

It also comes out that this important literature faces some problems. One important problem concerns the use of bivariate statistical methods to determine the OCA which is, by definition, a multi-country concept even if Mundell (1961) gives a two country situation example in his seminal paper. Another crucial point concerns the distinction between the short-run and the long-run dynamics. We try to address these issues by analyzing in a multivariate $\operatorname{VAR}(p)$ both long-run and short-run comovements between economic indicators of a set of European countries. Economic theory often deals in this matter with long-run comovements via convergence analyses between the levels of GDP or other economic indicators. Those types of comovements have been largely studied in empirical work: ${ }^{6}$ through cointegration analysis. 'The analysis of the shortrun fluctuations has been a matter of fewer investigations. However, in the light of the OCA approach, it is more relevant to study whether a monetary union is sustainable on the basis of short-run relationships (sce Beine et al., 2000).

In this section, we investigate the existence of a core of countries within the European Com-

\footnotetext{
${ }^{6}$ See for instance Bernard and Durlauf (1995).
} 


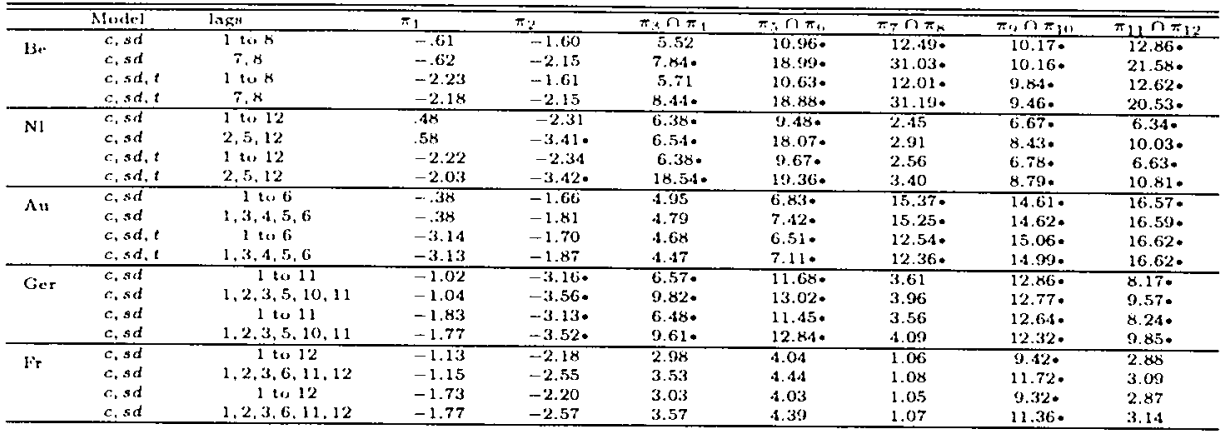

Table 7.7: Scasonal Monthly Unit Root Tests

munity using both raw and seasonally adjusted data. We first consider seasonally monthly unadjusted industrial production indexes for the period ranging from 1975:M1 to 1997:M4. Monthly industrial production indexes have already been analyzed within a codependence framework by Rubin and Thygesen (1996). However, their analysis relied on seasonally adjusted data alone, even if they claim to use raw time series and they carry out regressions with seasonal dummics. We consider a "potential core" of European countries made of Germany, Belgium, The Netherlands, Austria and France. Table 7.7 gives the results of monthly unit root tests both at the zero and the seasonal frequencies (sec Franses, 1991, 1996). We report unit root tests with a constant and seasonal dummies (c,sd) as well as those with a constant, seasonal dummies and a deterministic trend (c,sd,t). For these two regressions, Table 7.7 also gives unit root test statistics, with two ways for whitening the residuals. In the first one, we augment the regression with 1 to $p$ lags, where $p$ is the last significant lag using a $t$-statistic critcrion. We also present results for which we have dropped the non-significant. lags. A * means that the test statistic rejects at a $5 \%$ level the null hypothesis of a unit root at that specific frequency. It emerges from the table that all time series present a unit root at the zero frequency while they have different scasonal unit roots. Consequently, a seasonal cointcgration analysis turns out to be difficult, especially with monthly time series. We therefore assume here that there are no seasonal cointegrating vectors and wo filter the seasonal unit roots in order to study cointegration at the non-seasonal frequency. As $1-L^{12}=(1-L)(1+L)\left(1+L^{2}\right)\left(1+\sqrt{3} L+L^{2}\right)\left(1-\sqrt{3} L+L^{2}\right)\left(1+L+L^{2}\right)\left(1-L+L^{2}\right)$, we apply the filter $\left(1-\sqrt{3} L+L^{2}\right)$ for Germany and The Netherlands, $(1+L)$ for Belgium, $\left(1+L+L^{2}+L^{3}\right)$ for Austria and $\left(1+L^{3}+L^{6}+L^{9}\right)$ for France.

Formally, Johansen's ML statistics would retain only one cointegrating vector for those transformed data. However, both a graphical analysis and a study of the largest eigenvalues of the companion matrix conclude in favor of two vectors. The VAR model contains a linear trend constrained in the long-run as well as a constant, 11 seasonal dummies and outliers in $1984 \mathrm{~m} 6$, $1984 \mathrm{~m} 7$ and $1985 \mathrm{ml}$ unconstrained in the short-run. The lag length is chosen cqual to 10 according to the Hannan Quinn criterion. 


\begin{tabular}{ccc|ccc}
\hline$p=10$ & $\bar{H}_{0}$ & $p-$ value & $p=10$ & $H_{0}$ & $p$-value \\
\hline $77 \mathrm{~m} 1-97 \mathrm{~m} 4$ & $s \geq 1$ & $<.001$ & $83 \mathrm{~m} 3-97 \mathrm{~m} 4$ & $s \geq 1$ & $<.001$ \\
& $s \geq 2$ & $<.001$ & & $s \geq 2$ & $<.001$ \\
& $s \geq 3$ & $<.001$ & & $s \geq 3$ & $<.001$ \\
& $s \geq 4$ & $<.001$ & & $s \geq 4$ & $<.001$ \\
& $s=5$ & $<.001$ & & $s=5$ & $<.001$ \\
\hline
\end{tabular}

Table 7.8: WF Tests Statistics for Raw Series $(p=10)$

\begin{tabular}{ccc|ccc}
\hline$p=3$ & $I_{0}$ & $p-$ value & $p=10$ & $H_{0}$ & $p-$ value \\
\hline $83 \mathrm{~m} 3-97 \mathrm{~m} 4$ & $s \geq 1$ & $<.05$ & $83 \mathrm{m3-97m4}$ & $s \geq 1$ & $<.21$ \\
& $s \geq 2$ & $<.001$ & & $s \geq 2$ & $<.07$ \\
& $s \geq 3$ & $<.001$ & & $s \geq 3$ & $<.001$ \\
& $s \geq 4$ & $<.001$ & & $s \geq 4$ & $<.001$ \\
& $s=5$ & $<.001$ & & $s=5$ & $<.001$ \\
\hline
\end{tabular}

Table 7.9: WF Tests Statistics with SA Series $(p=3,10)$

Table 7.8 gives the p-values for WF common feature test statistics for the whole period (1977m1-1997m4 when we account for lags) as well as for a subperiod that starts in 1983m3, with the second phase of the EMS. One sees that we reject the presence of any weak form common feature vectors. ${ }^{7}$ Note that if (see Remark 3.7 of Chapter 3 ) we move the error correction terms from $t-1$ to successively $t-2 \ldots t-10$ we still do not find any cofeature vectors in this data set. Obviously there are infinitely many other ways to reparametrize the models and we have only considered the most common ones.

We use the X-11 procedure in Pc-Give to come up with seasonally adjusted data. We only report results for the second subsample since no differences emerge between SA and NSA series for the whole sample. While it was not obvious to discriminate between three and ten lags we have conducted the analysis with both specifications. We keep two cointegrating vectors that are estimated under each models. Table 7.9 illustrates again the potential pitfalls when using adjusted time series. With $p=3$ we do not reject the presence of one cofeature vector and two cofeature vectors arc now present with $p=10$.

\subsection{What can we do in Practice: Is there a Neat Solution?}

One of the main challenges in interpreting macroeconomic fluctuations would be to integrate in a coherent fashion all their components, namely to consider raw data and their comovements in the growth, the cyclical fluctuations and seasonal variations (see Ahn, 1997 and Cubadda, 1999, 2000). While there exists an increasing litcrature claiming that scasonal phenomena have to be fully considered and not relegate to noisy things, most of official statistical agencies still

\footnotetext{
${ }^{7}$ Notice that two non-synchronous relationships with a codependence order of 4 may be found (see Beine $e l$ al., 2000 for details)
} 
only release seasonally adjusted macro aggregates. Moreover, even if some time series start to be available in an unadjusted form, the past of the series is usually not.

When feasible, a complete analysis of long, short and seasonal comovements is worth considering (Cubadda 1999, 2000; Hylleberg and Engle 1996). But sometimes, we should say most of the time, raw time series are not available or are not releasable. Consequently, what can be done in practice? We are not going to give definitive answers to that difficult question but we stress situations where less anomalies could occur.

Assume a $\operatorname{VAR}(p)$ model for a $n$-dimensional $\mathrm{I}(1)$ vector time series $y_{t}$ with $r$ cointegrating vectors in a VECM form:

$$
\Delta y_{t}=\delta D_{t}+\alpha \beta^{\prime} y_{t-1}+\sum_{i=1}^{p-1} \Gamma_{i} \Delta y_{t-i}+v_{t}, \quad t=1, \ldots, T
$$

where $D_{t}$ represents seasonal dummies and where $v_{t}$ is a $n$-dimensional homoskedastic Gaussian mean innovation process relative to $\Im_{t}=\left\{y_{t-1}, y_{t-2}, \ldots, y_{1}\right\}$ with nonsingular covariance matrix $\Omega$. Further assume that the general regularity conditions are met. Serial correlation common features hold for the VECM $(7.3)$, if there exisis a $(n \times s)$ matrix $\tilde{\beta}$, whose columns span the cofeature space, such that $\tilde{\beta}^{\prime}\left(\Delta y_{t}-\delta D_{t}\right)=\tilde{\beta}^{\prime} v_{t}$ is a $s$-dimensional vector mean innovation process with respect to the information available at time $t$.

There are many ways to illustrate that the common feature space for the seasonally adjusted data is not a common feature space for raw data. The first way, along with Propositions 7.1 and 7.2 , is to express the elements of the filter such that $\Psi(L)=\Psi(1)+\Psi^{*}(L) \Delta$, with $\Psi^{*}(L)$ is a two sided linear symmetric filter with weight $\Psi_{i}^{*}$. Then $\tilde{\beta}^{\prime} \Delta y_{i}^{S A}=\tilde{\beta}^{\prime} \Delta y_{\ell}+\tilde{\beta}^{\prime} \Psi^{*}(L) \Delta^{2} y_{\ell}$. Another way is to do like in Wallis (1974), and replace $y_{t}^{S A}=\Psi(\mathbf{L}) y_{t}$ or $\Psi(\mathbf{L})^{-1} y_{t}^{S A}=y_{t}$ in the VECM (7.3) such that

$$
\begin{aligned}
\Psi(\mathbf{L})^{-1} \Delta y_{t}^{S A} & =\delta D_{t}+\Psi(\mathbf{L})^{-1} \alpha \beta^{\prime} y_{t-1}^{S A}+\Psi(\mathbf{L})^{-1} \sum_{i=1}^{p-1} \Gamma_{i} \Delta y_{t-i}^{S A}+v_{t} \\
\Delta y_{t}^{S A} & =\alpha \beta^{\prime} y_{t-1}^{S A}+\sum_{i=1}^{p-1} \Gamma_{i} \Delta y_{t-i}^{S A}+\Psi(\mathbf{L}) v_{t} .
\end{aligned}
$$

Remark that this holds if $\Psi(L)$ is a scalar filter, i.e. if the same filter is applied to each variables in $y_{t}$. Note again that $\Psi(\mathbf{L}) \delta D_{t}=0$ when the sum of dummy variables coefficients is zero over a year (Ericsson et al., 1994). This situation is relatively embarrassing. Indeed, if there exist a common feature matrix $\tilde{\beta}^{\prime}$ for raw data, the premultiplication by this matrix in (7.5) is no longer a $s$-dimensional white noise processes. The same structure however exists in the orthogonal complement of both long-run and short-run matrices. This hidden structure is the same but will not be uncovered duc to autocorrelation the seasonal adjustment has introduced.

Assuming that the adjustment filter is the same for all $n$ variables in $y_{l}$, the structure of the autocorrelation process is well known. For a bivariate system with one common feature vector 
we get an error process $u_{t}=\sum_{-m}^{m} \theta_{i} \tilde{\beta}^{\prime} v_{t+i}=\sum_{-m}^{m} \theta_{i} \varepsilon_{t+i}$ with $\tilde{\beta}^{\prime} v_{t}=\varepsilon_{t}$. It is well known that $u_{t}$ follows a finite $\operatorname{MA}(2 m+1)$ error process with $E\left(u_{t}\right)=\sum_{-m}^{m} \theta_{i} E\left(\varepsilon_{t+i}\right)=0, \operatorname{Var}\left(u_{i}\right)=\sigma^{2} \sum_{-m}^{m} \theta_{i}^{2}$, with $\sigma^{2}=\operatorname{Var}\left(\varepsilon_{t}\right)$ and

$$
\begin{aligned}
\gamma(h) & =\operatorname{Cov}\left(u_{t}, u_{t+h}\right), \\
& =\sum_{i=-m}^{m} \sum_{j=-m}^{m} \theta_{i} \theta_{j} E\left(u_{t+i}, u_{t+h+j}\right), \\
& =\left\{\begin{array}{ll}
\sigma^{2} \sum_{i=h-m}^{m} \theta_{i} \theta_{i-h} & \text { if } h \leq 2 m \\
0 & \text { otherwise }
\end{array} .\right.
\end{aligned}
$$

This structure has small positive autocorrelation coefficients at lags of $1-3,5-7$...quarters and larger negative correlations at lags 4,8 (sec Wallis, 1974). Consequently, test statistics may depend upon two effects: a size effect and a covariance effect.

\subsubsection{The Size Effect}

The first effect we call the "sample size effect" means that, with small samples sizes, some empirical autocovariances could be statistically non significant. To illustrate this well known point, let us apply the linear approximation of X11 seasonal adjustment to a white noise process. This is a centered moving average filter where the 57 weights are known. Hence, we can easily determine the significance of those autocovariances for the seasonally adjusted white noise. Figure 7.2 illustrates this point by plotting the $p$-values of the Ljung-Box statistics for the significance of the first 10 autocovariances. The size effect means that in practice, autocovariances are not significant with small sample sizes. However, the number of observations cncountered in a study with quarterly or monthly time series is usually relatively large.

\subsubsection{The Covariance Effect}

Under the null of common cyclical feature, first denote $\sigma^{2}=\operatorname{Var}\left(\varepsilon_{t}\right)=\operatorname{Var}\left(\tilde{\beta}^{\prime} v_{t}\right)$. We next consider the bivariate case and we assume a normalization of the cofeature vector on the first variable, i.c. $\tilde{\beta}=\left(1,-\tilde{\beta}_{2}\right)$. Consequently we have $\operatorname{Var}\left(\tilde{\beta}^{\prime} v_{t}\right)=\operatorname{Var}\left(v_{1 t}-\tilde{\beta}_{2} v_{2 t}\right)$. It turns out that $\sigma^{2}=\sigma_{v_{1 t}}^{2}+\tilde{\beta}_{2}^{2} \sigma_{v_{2 t}}^{2}-2 \tilde{\beta}_{2} \operatorname{Cov}\left(v_{1 t}, v_{2 t}\right)$. Even if this expression cannot be zero, first order 


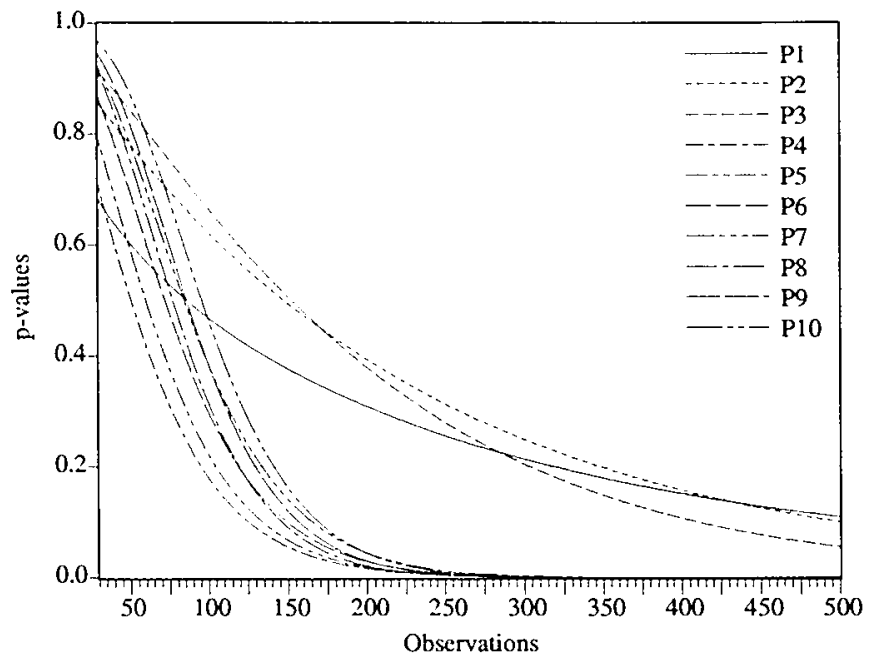

Figure 7.2: Theoritical p-values of Ljung-Box Statistics in a SA Whitc Noise

conditions for minimizing the variance $\sigma^{2}$ with respect to $\tilde{\beta}_{2}$ and $\sigma_{v_{1 t}}$ give:

$$
\begin{aligned}
\frac{\partial \sigma^{2}}{\partial \tilde{\beta}_{2}} & =2 \tilde{\beta}_{2} \sigma_{v_{2 t}}^{2}-2 \operatorname{Cov}\left(v_{1 \ell}, v_{2 t}\right)=0 \\
\frac{\partial \sigma^{2}}{\partial \sigma_{v_{1 t}}} & =2 \sigma_{v_{1 t}}-2 \tilde{\beta}_{2} \rho \sigma_{v_{2 t}}=2 \sigma_{v_{1 t}}-2 \tilde{\beta}_{2} \frac{\operatorname{Cov}\left(v_{1 t}, v_{2 t}\right)}{\sigma_{v_{1 t}}}=0 \\
& \Rightarrow \tilde{\beta}_{2}=\frac{\sigma_{v_{1 t}}}{\sigma_{v_{2 t}}}
\end{aligned}
$$

That means that size distortions are minimized when the estimated common feature vector we obtain is proportional to some parameters of the covariance matrix. Plugging in this expression in $\sigma^{2}$, we have:

$$
\sigma_{\min }^{2}=\sigma_{v_{1 t}}^{2}+\sigma_{v_{1 t}}^{2}-2 \sigma_{v_{1 t}}^{2} \rho=2 \sigma_{v_{1 t}}^{2}(1-\rho)
$$

Then, knowing that the common feature vector is close to the ratio $\frac{\sigma_{v_{11}}}{\sigma_{v_{2 t}}}$, the variance $\sigma^{2}$ is small when the covariance is high and $\sigma_{v_{1 \ell}}^{2}$ is small.

Let us illustrate this point throughout a small Monte Carlo experiment. We consider a bivariate cointegrated system with serial correlation common feature (of the strong type). Two 


\begin{tabular}{|l|ccc|ccc|ccc|ccc|}
\hline \hline & $S F$ & $T=$ & 80 & $S F$ & $T=$ & 160 & $W F$ & $T=$ & 80 & $W F$ & $T=$ & 160 \\
& $s \geq 1$ & $\beta_{.5}$ & $I R$ & $s \geq 1$ & $\beta_{.5}$ & $I R$ & $s \geq 1$ & $\beta_{.5}$ & $I R$ & $s \geq 1$ & $\beta_{.5}$ & $I R$ \\
\hline $\mathrm{COV}=1$ & & & & & & & & & & & & \\
$\rho=.25$ & 24.1 & 1.129 & .133 & 42.1 & 1.129 & .094 & 23.5 & 1.349 & .495 & 42.3 & 1.312 & .344 \\
$\rho=.5$ & 15.5 & 1.052 & .094 & 25.5 & 1.052 & .067 & 1.1 & 1.198 & .471 & 23.4 & 1.165 & .315 \\
$\rho=.75$ & 12.8 & 1.018 & .055 & 18.9 & 1.017 & .039 & 8.8 & 1.099 & .373 & 16.6 & 1.077 & .257 \\
$\rho=.95$ & 11.8 & 1.002 & .021 & 16.8 & 1.002 & .016 & 6.9 & 1.018 & .180 & 14.6 & 1.018 & .125 \\
\hline cov $=.5$ & & & & & & & & & & & & \\
$\rho=.25$ & 18.4 & 1.084 & .097 & 30.3 & 1.082 & .069 & 18.5 & 1.153 & .455 & 31.2 & 1.119 & .311 \\
$\rho=.5$ & 17 & 1.048 & .059 & 29.3 & 1.046 & .042 & 14.8 & .970 & .317 & 26.4 & .949 & .235 \\
$\rho=.75$ & 21.7 & 1.039 & .041 & 40.6 & 1.038 & .029 & 15.1 & .867 & .223 & 25.6 & .862 & .155 \\
$\rho=.95$ & 31.3 & 1.036 & .033 & 57.7 & 1.036 & .023 & 16.2 & .820 & .128 & 25.2 & .822 & .089 \\
\hline cov $=2$ & & & & & & & & & & & & \\
$\rho=.25$ & 33.6 & 1.174 & .146 & 59.7 & 1.171 & .106 & 31.4 & 1.459 & .515 & 56.7 & 1.422 & .350 \\
$\rho=.5$ & 24.2 & 1.107 & .142 & 41.1 & 1.106 & .100 & 21.2 & 1.410 & .507 & 36.8 & 1.376 & .353 \\
$\rho=.75$ & 18.3 & 1.026 & .118 & 29.5 & 1.028 & .084 & 12.9 & 1.389 & .548 & 19.7 & 1.360 & .378 \\
$\rho=.95$ & 23.4 & .967 & .082 & 40.6 & .969 & .057 & 9.95 & 1.538 & .619 & 23.5 & 1.480 & .419 \\
\hline \hline
\end{tabular}

Table 7.10: Empirical Sizes and Cofeature Vector Median and Interquartile Ranges for X-11 SA

practical sample sizes are analyzed, $T=80,160$, and 2000 replications are used. The DGP is given in the VECM (7.3) with the following coefficient matrices:

$$
\begin{aligned}
\alpha \beta^{\prime} & =\left(\begin{array}{cc}
-.5 & -1.0 \\
-.5 & -1.0
\end{array}\right), \quad \Phi_{1}^{*}=\left(\begin{array}{cc}
.2 & -.5 \\
.2 & -.5
\end{array}\right), \quad \Phi_{2}^{*}=\left(\begin{array}{cc}
-.32 & .16 \\
-.32 & .16
\end{array}\right), \\
\Omega_{\varepsilon} & =\left(\begin{array}{cc}
\sigma_{v_{1 t}}^{2}=\left(\frac{\operatorname{Cov}\left(v_{1 t}, v_{2 t}\right)}{\rho_{v_{2 t}}}\right)^{2} & \operatorname{Cov}\left(v_{1 t}, v_{2 t}\right) \\
\operatorname{Cov}\left(v_{1 t}, v_{2 t}\right) & \sigma_{v_{2 t}}^{2}=1
\end{array}\right), \quad \delta=\left(\begin{array}{cccc}
1 & 2 & -2 & 1 \\
-6 & 1.5 & -.5 & 5
\end{array}\right) .
\end{aligned}
$$

There cxist one cointegrating vector given by $\beta^{\prime}=(1,2)$ and one common feature vector $\tilde{\beta}^{\prime}=(1,-1)$. In the simulation experiments we fix without loss of generality $\sigma_{v_{2 t}}^{2}=1$, and we give three distinct values for $\operatorname{Cov}\left(v_{1 t}, v_{2 t}\right)$, i.e. $1, .5$ and 2 . Then we propose four values for the correlation, i.e. $\rho=.25, .5, .75$ and .95 . Given these inputs, we know the variance $\sigma_{v_{1 t}}^{2}$. Test statistics we use are based on a two step canonical correlation approach where the true value of the cointegrating vector is known. ${ }^{8}$ We analyze SCCF framework as well as the weak form reduced rank structure.

Table 7.10 reports for instance that in the first panel with $\operatorname{cov}=1, \frac{\sigma_{u_{11}}}{\sigma_{v_{2 t}}}=1$, and so size distortions decrease monotonically with $\rho$, i.e. when $\rho \rightarrow 1$. With cov $=.5$ size distortions are minimized when $\rho=.5$ because $\sigma_{v_{1 t}}=1$ in this case. Note that in general, distortions are smaller for the weak form than for the strong form. Also remark that sizes increase with $T$.

\footnotetext{
"Note that we also could rely the analysis on a super-consistent estimation of the cointegrating vector in the first step.
} 
Knowing that under the null, the error process is a finite order MA with known weights, one might expect that an autocorrclation consistent covariance matrix estimator (à la West, 1997 for instance) can be used in order to conduct inference on the reduced rank structure of the VECM's cocficient matrices. However this solution suffers of a scrious drawback, namely that it is necessary to make an a miori hypothesis on the nature of the seasonality of the series in order to correctly apply this approach. ${ }^{9}$ In fact, suppose that series $y_{t}$ have seasonal unit roots in place of deterministic seasonality. In this case, and assuming for the moment that there is no seasonal cointegration, equation (7.3) becomes

$$
\Delta_{4} y_{t}=\alpha \beta^{\prime} S(L) y_{t-1}+A^{*}(L) \Delta_{4} y_{t-1}+v_{t}
$$

where $S(L)=\left(1+L+L^{2}+L^{2}\right)$. Since $\Psi(L)$ is a scalar seasonal-adjustment filter applied to each of the variables in $y_{\ell}$, we can write $\Psi(L)=S(L) \Psi^{*}(L)$. This in turn implies

$$
\Delta y_{t}^{S A}=\Psi(L) \Delta y_{t}=\Psi^{*}(L) \Delta_{4} y_{t},
$$

hence, we finally see

$$
\Delta y_{t}^{S A}=\alpha \beta^{\prime} y_{t-1}^{S A}+A^{*}(L) \Delta y_{t-1}^{S A}+\Psi^{*}(L) v_{t}
$$

Note that equation (7.12) is similar to equation (7.5), but the error term has a different. antocorrelation structure. Clearly, there is no hope to take this decision by looking at the data once that these are seasonally adjusted.

The problem is even more serious if we allow for seasonal cointegration. In this case, we have

$$
\Delta y_{t}^{S A}=\alpha_{1} \beta^{\prime} y_{t-1}^{S A}+A^{*}(L) \Delta y_{t-1}^{S A}+\Psi^{*}(L) v_{t}+\Psi^{*}(L)\left(\alpha_{2} z_{2, t-1}+\alpha_{4} z_{A, t-2}\right),
$$

where $z_{2, t-1}$ and $\alpha_{4} z_{4, t-2}$ are the seasonal error correction terms. Hence, when cointegration occurs at a seasonal frequency, there is no common MA component with a known autocorrelation structure in the seasonally adjusted data.

\subsection{Conclusion}

We have seen, through Monte Carlo simulations, that the practice of using serial common feature test statistics on seasonally adjusted data is a perilous exercise. Actually, we are faced with the worst situation in which tests are not able to detect common features because of size distortions. Because of lack of power, they spuriously signal discovery of common features when these features do not exist. So, what could be done in empirical studies? It is easy to answer: use seasonally unadjusted data sets. Because the type of seasonality in raw data is crucial, we could at least

\footnotetext{
${ }^{9} \mathrm{I}$ ' $\mathrm{m}$ indebted to Gianlucis Cubadda for the stimulating discussion we have had on this issue.
} 
consider the statistical properties of the data if not from raw data, from previous studies. However it is well known that the power of procedures like HEGY is low in several situation like the presence of a shift in mean or in seasonal pattern, GARCH, nonlinearity.

Consequently, we would advice, when using scasonally adjusted data, to test for unsynchronous common cycle, that is a linear combination of a $\operatorname{VARMA}(p, 1)$ for instance which yields a MA(1) process, that is also a scalar component model of order $(0,1)$. The interpretation of the results is however different from Vahid and Engle (1997). In this case, cycles are not really non-synchronous because the vector corresponding to the $\operatorname{SCM}(0,1)$ may be a serial correlation common feature for the non-observable raw data. However, that is again only valid if we succeded in detecting the nature of the seasonality in raw data, otherwise we throw out the dynamics like we would throw out the baby with the bath water.

Note that similar considerations may apply to systems for variables which have been transformed to stationarity by using c.g. a Hodrick-Prescott. filter. 


\title{
Chapter 8
}

\section{Separation, Weak Exogeneity and P-T Decomposition in Cointegrated VAR Systems}

\begin{abstract}
The aim of this chapter is to study the concept of separability in multiple nonstationary time series displaying both common stochastic trends and common stochastic cycles. To restrict the dimension of the parameter space, searching for separability and common features is often required when modeling the dynamics of multiple time series for several entities such as countries, sectors, firms. For this purpose we introduce the concept of common feature separation and investigate the relationships between separation in cointegration and separation in serial correlation common features. Roughly speaking, we investigate whether a set of time series can be partitioned into subsets such that there are serial correlation common features in the sub-groups and that there do not exist linear combinations of the common cycles which are white noise. Conditions under which Permanent-Transitory decompasitions can be based on subsystem analyses only are investigated as well as the conditions for weak exogeneity under different forms of separation in both cointegration and common features. Likelihood ratio type tests for the different hypotheses under study are proposed. An empirical analysis of the link between economic fluctuations in the U.S. and Canada shows the practical relevance of the approach proposed in this chapter.
\end{abstract}

\subsection{Introduction}

When modeling multiple time series, it is often appropriate to cluster the series into groups of variables which have strong intragroup relationships and weak or no intergroup interactions. Since high dimensional analysis of multiple time series is subject to practical and theoretical

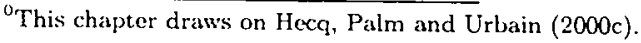


problems related to the number of parameters to estimate, such a grouping can be very appealing from both a theoretical and practical point of vicw. For example, Abadir, Hadri and Tzavalis (1999) and Gonzalo and Pitarakis (1999) show that an increase in the dimension of a cointegrated VAR model can lead to very undesirable properties for both the test statistics and usual estimators. Often however, empirical studies of permanent and transitory components in the field of macrocconomics or international finance focus on international comparisons aimed to detect (dis)similarities across countries. Practically speaking such international comparisons face the dimensionality problem once the study involves several variables and several countries. The solution that is usually adopted is to carry out a country by country analysis, e.g. extract the common stochastic trends components on a country by country basis. These "national" common stochastic trends are then compared to cach others in a later stage of the analysis - see for example Haffner, Kutan and Zhou (1997) and Hoffman (1999). While this seems the most practical solution given the problems mentioned above, this also raises some particular issues as to whether the extracted components and time series properties are affected by these marginalizations. Ideally, one would like to cluster or merge the series into groups of variables so that no loss of information occurs if the components extraction is based on a country by country analysis.

The concept of separation in cointegration provides a useful way of formally describing such a situation. Separation in cointegration, introduced by Konishi (1993), Konishi, Ramsey and Granger (1994), Konishi and Granger (1993) and later extended by Granger and Haldrup (1997) implies that common trends can be extracted from sub-systems of $I(1)$ time series. These authors consider situations where a subset of cointegration relationships exist between economic time series which have no variables in common. Examples considered by these authors are the presence of long-run relationships between real sector variables only, and between monetary variables only. Other examples arise in panel data for several countries when sets of variables for countries taken separately are cointegrated. Under complete separation, the common trends extracted from a sub-group by sub-system analysis do correspond to those that would have been extracted from the complete system. Useful conditions for empirical work under which this correspondence holds are given in Granger and Haldrup (1997). These authors however limit their attention to Permanent-Transitory (P-T) decompositions à la Gonzalo-Granger (1995) and are essentially interested in the extraction of the common stochastic trends components. In the presence of common stochastic cycles, see Engle and Kozicki (1993) or Vahid and Engle (1993), and/or when other P-T decompositions are considered, their results might need to be extended and generalized.

The major aim of this chapter is to study the concept of separability in multiple time series displaying both common stochastic trends and common stochastic cycles. For this purpose we introduce the concept of common feature separation and investigate the relationships between separation in cointegration and separation in serial correlation common features. We provide conditions under which a set of time series can be partitioned into subsets with subset-specific serial correlation common features and subset-specific common features only. Examples are again country-specific (i.e. country per country) business cycles in a set of panel data for several countries or separate monetary cycles and real cycles. 
More formally, consider two groups of $I(1)$ variables for a given country, $y_{1 t}$ and $y_{2 t}$ of dimension respectively $n_{1}$ and $n_{2}$. Under separation in cointegration the ranks of cointegrating spaces are $r_{1}<n_{1}$ and $r_{2}<n_{2}$ for sub-systems generating $y_{1 t}$ and $y_{2 \ell}$ while it is $r=r_{1}+r_{2}<$ $n=n_{1}+n_{2}$ in the system for $y_{t}=\left(y_{1 t}^{\prime}, y_{2 t}^{\prime}\right)^{\prime}$. In this case the $n_{1}-r_{1}$ and the $n_{2}-r_{2}$ common trends are not cointegrated. ${ }^{1}$ In a sinilar way we can also consider linear combinations of two subsets $\Delta y_{1 t}$ and $\Delta y_{2 t}$ which have no variable in common and yield white noise for the two subsystems individually. In this case, the common feature "sub-spaces" are of dimension $s_{1}$ and $s_{2}$ respectively and the dimension of the common feature space in the whole model is $s=s_{1}+s_{2}$. One can then extend the Konishi-Granger analysis by taking into account the reduced rank structures on both long-run and short-run matrices and decompose cach sub-group between their permanent. (P) and transitory $(\mathrm{T})$ components, such that

$$
y_{i t}=G_{i} y_{i t}^{P}+A_{i} y_{i t}^{T}, i \in\{1,2\}
$$

where $C_{i}$ and $A_{i}$ are of reduced rank $n_{i}-r_{i}, n_{i}-s_{i}$ respectively. This formulation is very general because at this stage we do not choose a particular form for the common trends and the common cycles (Beveridge-Nelson, Gonzalo-Granger, ...). Equation (8.1) only means that $y_{1 t}^{P}$ and $y_{2 t}^{P}$ are not cointegrated and that there are no common features between $y_{1 t}^{T}$ and $y_{2 t}^{T}$. If $y_{1 \ell}$ represents a set of time series for country 1, that means there does not exist a global stochastic trend for the two countries, nor does there exist a global cycle. Notice that the decomposition (8.1) might. hold for $y_{i t}$ adjusted for long-run components. Indeed, we will study the two different classes of cointegrated VAR models analyzed in Chapter 3 (WF and SF).

While complete separation provides conditions under which common trends and common cycles can be extracted on a sub-system basis, these conditions do not yet ensure that extraction based on these (marginal) sub-systems will be fully efficient. This latter point is also raised in this chapter where we provide a set of sufficient conditions under which valid inference can be conducted in marginal separated systems. Testing procedures and inferential procedures are developed and finally applied to a simple empirical illustration.

This chapter is organized as follows. Section 8.2 recalls the notion of separation in cointegration, shows the implications that separation in cointegration has in terms of common features and reversely. $A$ similar definition of separation for the common features or cofcature matrix is introduced. The conditions under which maximum likelihood inference within separated subsystems is fully efficient are analyzed in Section 8.3. Section 8.4 presents a set of likelihood ratio type statistics useful for testing particular separation hypotheses. In Section 8.5, an empirical analysis shows the usefulness of the concepts discussed in this study where we consider the presence of distinct common factors in US and Canadian consumption functions. Section 8.6 concludes.

\footnotetext{
${ }^{1}$ If there exists a cointegrating vector between these common trends, that implies there are $r>r_{1}+r_{2}$ cointegrating vectors in the full system and hence this contradicts the definition of separation.
} 


\subsection{Separation in Cointegration and in Cofeature}

\subsubsection{Definitions}

The notion of separation put forward by Konishi (1993), Konishi and Granger (1993) helps to identify sub-systems that could be investigated independently from each other, reducing thereby substantially the complexity and size of the modeling problem at hand. Let us consider again the VECM

$$
\Delta y_{t}=\alpha \beta^{\prime} y_{t-1}+\sum_{j=1}^{p-1} \Gamma_{j} \Delta y_{t-j}+\varepsilon_{t}, \quad t=1, \ldots, T
$$

Definition 8.1 Consider the $n$ dimensional cointegrated vector time series $y_{t}=\left(y_{1 t}^{\prime}, y_{2 t}^{\prime}\right)^{\prime}$ where $y_{1 t}$ and $y_{2 t}$ are distinct sub-vectors of dimension $n_{1} \times 1$ and $n_{2} \times 1$ respectively with $n_{1}+n_{2}=n$. Under the assumption of $r<n$ cointegrating vectors, the associated VECM is given by (8.2). If the matrix of cointegrating vectors $\beta^{\prime}$ can be factorized as:

$$
\beta^{\prime}=\left(\begin{array}{cc}
\beta_{11} & 0 \\
0 & \beta_{22}
\end{array}\right)^{\prime}
$$

where the sub-matrices $\beta_{11}$ and $\beta_{22}$ are respectively $n_{1} \times r_{1}$ and $n_{2} \times r_{2}$ full column rank matrices, then the system is said to be subject to separate cointegration.

In a recent paper, Granger and Haldrup (1997) further refined the idea of separation by considering other parameter restrictions that may arise on the remaining matrices of the systems. Assume the system (8.2) is subject to separate cointegration and consider the loading matrices and matrix polynomials which we partition conformably with the partitioning of the vector process $y_{t}$ :

$$
\alpha=\left(\begin{array}{ll}
\alpha_{11} & \alpha_{12} \\
\alpha_{21} & \alpha_{22}
\end{array}\right) \quad \text { and } \quad \Gamma(L)=\left(\begin{array}{ll}
\Gamma_{11}(L) & \Gamma_{12}(L) \\
\Gamma_{21}(L) & \Gamma_{22}(L)
\end{array}\right)
$$

The following definitions emerge:

Definition 8.2 If $\alpha_{12}=0, \alpha_{21}=0, \Gamma_{12}(1)=0, \Gamma_{21}(1)=0$ then the system is said to be completely separated in cointegration.

Definition 8.3 If $\alpha_{12} \neq 0$ and/or $\alpha_{21} \neq 0$ but $\Gamma_{12}(1)=0, \Gamma_{21}(1)=0$ then the system is said to be partially separated of Type A (or partially long-run separated of Type $A$ ).

Definition 8.4 If $\alpha_{12}=0, \alpha_{21}=0$ but $\Gamma_{12}(1) \neq 0$ and/or $\Gamma_{21}(1) \neq 0$ then the system is said to be partially separated of Type $B$. 
Note that Type B partial separation in (8.2) implies block diagonality of $\sum_{i=1}^{p} \Pi_{i}$ due to the block diagonality of $\alpha \beta^{\prime}=-\left(I-\sum_{i=1}^{p} \Pi_{i}\right)$. This does not, however, imply block diagonality of the $\Gamma_{j}$ 's since $\Gamma_{j}=-\sum_{k=j+1}^{p} \Pi_{k}$. In the sequel our common feature analysis will require an additional condition of short-run Granger non-causality $\Gamma_{12}(L)=0, \Gamma_{21}(L)=0$ so that we aiso define type $A+$ partial separation or short run separation of type $A$ :

Definition 8.5 If $\alpha_{12} \neq 0$ and/or $\alpha_{21} \neq 0$ but $\Gamma_{12}(L) \equiv 0, \Gamma_{21}(L) \equiv 0$ then the system is said to be partially separated of Type A+ (or short-run separation of Type A).

In the absence of common features, Granger and Haldrup (1997) show that complete separation in cointegration is required if the common trends have to be extracted from the separated marginal systems. We call a system completely separated iff it is partially separated of both Type A+ and Type B, i.e. if $\beta_{12}=0, \beta_{21}=0, \alpha_{12}=0, \alpha_{21}=0, \Gamma_{12}(L) \equiv 0, \Gamma_{21}(L) \equiv 0$. If in addition to complete separation, $\Omega_{12}=0$ the subsystems are independent. Once we allow for the existence of reduced ranks of the short run dynamic matrices, we may in analogy to the concept introduced above, define the concept of serial correlation common fcatures separation:

Definition 8.6 (Cofeature Separation) Consider a $s \times n$ cofeature matrix $\tilde{\beta}^{\prime}$ that satisfics either $\tilde{\beta}^{\prime} \Delta y_{t}=\tilde{\beta}^{\prime} \varepsilon_{t}$ or $\tilde{\beta}^{\prime}\left(\Delta y_{t}-\alpha \beta^{\prime} y_{t-1}\right)=\tilde{\beta}^{\prime} \varepsilon_{t}$ where $\tilde{\beta}^{\prime} \varepsilon_{t}$ is a s-dimensional vector mean innovation process with respect to the information set in $t-1, Y_{t-1}$. In terms of the partitioning of $y_{t}$ in $y_{1 t}$ and $y_{2 t}$, we say that the vector process $y_{t}$ has separate $S F$ cofeatures (or separate WF cofeatures) if the cofeature matrix can be written as

$$
\tilde{\beta}^{\prime}=\left(\begin{array}{cc}
\tilde{\beta}_{11} & 0 \\
0 & \tilde{\beta}_{22}
\end{array}\right)^{\prime}
$$

Notice that separation in common features as defined above only requires $\alpha$ and $\Gamma(L)$ to have a particular reduced rank structure. Since the goal of this chapter is, a.o., to find conditions under which distinct sub-systems might be analyzed separately for the purpose of common trend-common cycle decomposition, ${ }^{2}$ we will consider separation in cointegration as a maintained assumption in the sequel, we shall assume that the matrix of cointegration $\beta$ is block diagonal.

\subsubsection{Relation between Cointegration Separation and Cofeature Separation}

Writing VECM (8.2) conformably with the partitioning of $y_{t}$ :

$$
\left(\begin{array}{c}
\Delta y_{1 t} \\
\Delta y_{2 t}
\end{array}\right)=\left(\begin{array}{cc}
\Gamma_{11}(L) & \Gamma_{12}(L) \\
\Gamma_{21}(L) & \Gamma_{22}(L)
\end{array}\right)\left(\begin{array}{c}
\Delta y_{1 t} \\
\Delta y_{2 t}
\end{array}\right)+\left(\begin{array}{cc}
\alpha_{11} & \alpha_{12} \\
\alpha_{21} & \alpha_{22}
\end{array}\right)\left(\begin{array}{cc}
\beta_{11} & 0 \\
0 & \beta_{22}
\end{array}\right)^{\prime}\left(\begin{array}{l}
y_{1 t-1} \\
y_{2 t-1}
\end{array}\right)+\left(\begin{array}{l}
\varepsilon_{1 t} \\
\varepsilon_{2 t}
\end{array}\right.
$$

\footnotetext{
${ }^{2}$ Notice that Granger and Haldrup (1997) explicitely focus on the Gonzalo and Granger (1995) P-T decomposition. In this case the conditions $\Gamma_{12}(1)=0$ and $\Gamma_{21}(1)=0$ are sufficient for the extraction of the permanent components. This does however not extend to other decompositions like the multivariate Beveridge-Nelson-StockWatson decomposition that require $\Gamma_{12}(L) \equiv 0$ and $\Gamma_{21}(L) \equiv 0$ - see Section 8.2.3.
} 
under separation in conmon feature and under a WF reduced rank structure we have:

$$
\left(\begin{array}{cc}
\tilde{\beta}_{11} & 0 \\
0 & \tilde{\beta}_{22}
\end{array}\right)^{\prime}\left(\begin{array}{cc}
\Gamma_{11}(L) & \Gamma_{12}(L) \\
\Gamma_{21}(L) & \Gamma_{22}(L)
\end{array}\right)=0,
$$

while under a SF assumption we have in addition to (8.3)

$$
\left(\begin{array}{cc}
\tilde{\beta}_{11} & 0 \\
0 & \tilde{\beta}_{22}
\end{array}\right)^{\prime}\left(\begin{array}{ll}
\alpha_{11} & \alpha_{12} \\
\alpha_{21} & \alpha_{22}
\end{array}\right)=0 .
$$

These two sets of restrictions will form the basis of the analysis in this section. Note that in the absence of separation in cointegration, i.c. when $\beta$ is not block-diagonal, the conditions (8.3) and (8.4) would remain unchanged for separation in common features. In order to clarify the implications in terms of restrictions on the matrices of the VECM (8.2) of cofeature separation and the relation between cofeature and cointegration separation, we will first consider a simple cointegrated VAR(1) since this model is actually useful to illustrate the important characteristics we are interested in:

$$
\Delta y_{t}=\alpha \beta^{\prime} y_{t-1}+\varepsilon_{t}
$$

Provided of course that $\alpha$ is unrestricted, the maintained assumption of separation in cointegration implies (see also Hansen and Johansen, 1998) that the cointegration relations can be cxpressed as lincar combinations of $\beta_{11}^{\prime} y_{1 t-1}$ and $\beta_{22}^{\prime} y_{2 t-1}$, that is, there exists $\left(r \times r_{i}\right), i=1,2$ full column rank matrices $G_{i}, i=1,2$ such that $\alpha \beta^{\prime} y_{t-1}=\alpha^{*} \beta^{* \prime} y_{t-1}=\alpha^{*}\left[G_{1} \beta_{11}^{\prime} y_{1 t-1}+G_{2} \beta_{22}^{\prime} y_{2 t-1}\right]$ with

$$
\beta^{* \prime}=\left(G_{1}, G_{2}\right)\left(\begin{array}{cc}
\beta_{11} & 0 \\
0 & \beta_{22}
\end{array}\right)^{\prime}=G \beta^{\prime}, \quad \alpha^{*}=\alpha G^{-1},
$$

where $G=\left(G_{1}: G_{2}\right)$ is a $r \times r$ full rank matrix. The notation $\beta^{* \prime}$ is used to stress the blockdiagonality of the normalized cointegrating matrix. In (8.5), there exist by definition $n-r$ serial corrclation common feature vectors (or SF common features) given by the rows of $\alpha_{\perp}^{\prime}$ where $\alpha_{\perp}^{\prime}$ is the orthogonal complement ${ }^{3}$ of $\alpha$ - see for example Vahid and Engle (1993). By definition, SF common feature separation requires block diagonality of $\alpha_{\perp}^{\prime}$ :

$$
\alpha_{\perp}^{\prime}=\left(\begin{array}{cc}
\alpha_{11 \perp} & 0 \\
0 & \alpha_{22 \perp}
\end{array}\right)^{\prime}
$$

\footnotetext{
${ }^{3}$ We denote the orthogonal complement of any $n \times s$-dimensional matrix $B$, with $n>s$ and $\operatorname{rank}(B)=s$, by the $n \times(n-s)$ matrix $B_{\perp}$ such that $B^{\prime} B_{\perp}=0$ with $\operatorname{rank}\left(B_{\perp}\right)=n-s$ and $\operatorname{rank}\left(B: B_{\perp}\right)=n$.
} 
Since $\alpha_{\rfloor}^{\prime} \alpha=0$, common feature separation imposes rank restrictions on particular sub-matrices of $\alpha$ given by

$$
\operatorname{rank} \underbrace{\left(\alpha_{11}: \alpha_{12}\right)}_{n_{1} \times r}<r \text { and } \operatorname{rank} \underbrace{\left(\alpha_{21}: \alpha_{22}\right)}_{n_{2} \times r}<r
$$

Since $\operatorname{ranh}(\alpha)=r$ by assumption, these additional reduced rank restrictions on blocks of $\alpha$ imply that one may always find a full rank $r \times r$ square sub-matrix $D$ of $\alpha$ such that the loading matrix $\alpha$ can be written as the product of a $(n \times r)$ block diagonal matrix times a full rank $r \times r$ matrix:

$$
\alpha=\alpha^{*} D \text { with } \alpha^{*}=\left(\begin{array}{cc}
\alpha_{11}^{*} & 0 \\
0 & \alpha_{22}^{*}
\end{array}\right) .
$$

Consequently the VECM can be rewritten as

$$
\Delta y_{t}=\alpha^{*} D G^{-1} \beta^{* \prime} y_{t-1}+\varepsilon_{t}=\alpha \beta^{\prime} y_{t-1}+\varepsilon_{t}
$$

with $\beta^{\prime}=G^{-1} \beta^{* \prime}$ and $D G^{-1}$ is a $r \times r$ matrix with full rank $r$. It is clear that when $\alpha_{\perp}^{\prime}$ and $\beta$ are both assumed block diagonal, $\mathrm{II}(1)=\alpha \beta^{\prime}=\alpha^{*} D G^{-1} \beta^{* \prime}$ will not be block diagonal unless $D C^{-1}$ is block diagonal. On the other hand, it is easily seen that if complete separation in the long-run part is assumed so that

$$
\Pi(1)=\left(\begin{array}{cc}
\Pi_{11}(1) & 0 \\
0 & \Pi_{22}(1)
\end{array}\right)=\left(\begin{array}{cc}
\alpha_{11} & 0 \\
0 & \alpha_{22}
\end{array}\right)\left(\begin{array}{cc}
\beta_{1} & 0 \\
0 & \beta_{2}
\end{array}\right)^{\prime}
$$

then $D=G$. The implication for this simple model generalized to a $\operatorname{VAR}(p)$ model are summarized in the following propositions:

Proposition 8.7 In the $\operatorname{VECM}$ (8.2) under separate cointegration with a strong form reduced rank structure,

- Type B separation, and thus complete separation in cointegration implies scparation in common feature.

- Cofeature separation does in general not imply separation in cointegration of any type but only restrictions on some matrices, unless $D=G$.

- Partial separation in cointegration (Type A or $A+$ or B) is compatible with separation in common features but does generally not imply separate cofeatures, except in the VAR(1).

Under the assumption of a weak form reduced rank structure, it is straightforward to sce that. by following similar arguments, we have 
Proposition 8.8 In the VECM (8.2) under separate cointcgration with a weak form reduced rank structure,

- Type A+ separation or complete separation in cointegration with short run separation implies cofeature separation.

- Type B separation in cointegration does not generally imply separation in common feature.

- Common feature separation is compatible urth, but does not imply cointegration separation of any form.

It is important to observe that the above results hinge on a crucial assumption that was made earlier namely that the separation in cointegration and cofeature are related to the same partition of $y_{t}=\left(y_{1 t}^{\prime}, y_{2 t}^{\prime}\right)^{\prime}$. This assumption may be realistic in dynamic panel data. Of course, one can imagine cases where separation holds in cointegration for a given partitioning of $y_{t}$, while cofeature separation holds for another partitioning of the same $y_{t}$ vector. An example would be an analysis of 4 different times series related to real consumption and real income for two countries 1 and 2 (see the empirical applications below) where (i) cointegration separation holds so that consumption and income cointegrate only within a country, but (ii) separate cofcature relationships exist that relate the two changes in consumption or the changes in real income only. It is easily seen that in this situation, the results presented in the Propositions just mentioned no longer hold. In particular, under a WF reduced rank structure separation in cofeature is then only compatible with Type B partial separation in cointegration while separate cofeature in a VECM with a SF reduced rank structure is incompatible with cointegration separation of any form.

\subsubsection{Separation and P-T Decomposition}

After having clarified the relationships between separation in cointegration and separation in common features we now briefly reconsider the issue from a $\mathrm{P}-\mathrm{T}$ decomposition point of view and summarize the conditions under which common trends and common cycles may be extracted from separated sub-systems. Although such P-T decompositions are by definition arbitrary, most. of them take the form:

$$
y_{t}=G y_{t}^{P}+A y_{t}^{T}
$$

where $G$ and $A$ are of reduced rank in the case where we have both $n-r$ common stochastic trends and $n-s$ common stochastic cycles. $y_{t}^{P}$ is the common stochastic trend, $y_{t}^{T}$ the (possibly common if $s>0$ ) cyclical/ transitory component. Under the weak form mentioned above $y_{t}^{T}$ is made out of both a common cyclical part and "uncommon " transitory components (see Chapter 4). The matrices $G$ and $A$ are the corresponding loadings. 
Granger and Haldrup (1997) focus on the Gonzalo and Granger (1995) P-T decomposition which chooses $y_{t}^{P}=\alpha_{\perp}^{\prime} y_{t}$ and $y_{t}^{T}=\beta^{\prime} y_{t}$. In the presence of common features Vahid and Engle (1993) derive a common trend common cycle decomposition. This decomposition corresponds to the Gonzalo and Granger (1995) P-T decomposition (GG) analyzed by Granger and Haldrup (1997) under the condition that $s+r=n$, sce Proietti (1997). For the cases where $s+r \neq n$ one has to rely on a slightly modified version of the popular Beveridge-Nelson-Stock-Watson decomposition (BNSW), which, although related to the GG decomposition, exists under weaker conditions. For explicit analytical representations see Proietti (1997) and the Chapter 4 of this thesis..

From these decompositions and the results mentioned above a number of conclusions can be drawn:

- Complete separation in cointegration such as defined by Granger and Haldrup (1997) is a sufficient condition for sub-group common stochastic trend extraction based of GG. In the presence of common features, complete separation is also sufficient for sub-group common stochastic trend extraction based on BNSW if and only if the common features are of the strong form with $s+r=n$.

- Common feature separation, while necessary to ensure that the common cycles for each subgroup are linearly independent, is not sufficient to sustain sub-group extraction of common trends and common cycles (BNSW), sec the Section 8.5 for an illustration.

- In the presence of SF common features with $s+r<n$, necessary conditions for subgroup extraction of common trends and common cycles based on BNSW are given by the conditions of Granger and Haldrup (1997) to which we have to add the further condition of Granger Noncausality in both direction.

- In the presence of WF common features, necessary conditions for sub-group extraction of common trends and common cycles based on BNSW are again cointegration separation and Granger Noncausality in both directions (Type $\mathrm{A}+$ ).

To briefly summarize, when common features are present, common features separation cnsures that the common stochastic cycles are group-specific while cointegration separation ensures that the common trends are group-specific but to ensure that these only involve variables from the individual sub-groups, the condition of Granger Noncausality in both directions is the additional requirement we have to add which constitutes a rather strong requirement from a practical point of view, at least much stronger than the one derived in Granger and Haldrup (1997). While common feature separation implies the existence of independent common cycles between the two separated sub-groups it does however generally not imply that these can be derived independently from each other unless Granger Noncausality occurs. 


\subsection{Separated Cointegrated versus Partial Systems}

In the previous section we have pointed out that common trends and common cycles can only be extracted from a subsystem under rather stringent conditions on the dynamics of the complete system. These components, when based on a Gonzalo-Granger decomposition or on a Beveridge-Nelson-Stock-Watson decomposition, although expressed explicitly in terms of observable variables, require the efficient estimation of the different parameter matrices entering both components. Hence, while at the model representation level the aforenentioned conditions appear to be useful for the extraction on a group by group basis, they do not yct cnable us to assess whether this extraction can be done efficiently. Absence of weak exogeneity leads to a loss of efficiency and of power of tests and therefore may lead to the selection of a misspecified model. This efficiency issue is obviously closely related to (weak) exogencity conditions in this extended class of cointegrated models. It is important at this stage to once again recall that weak exogencity is specific to well-defined parameters of interest and is nothing but a statement about the parameter space of a given statistical model, sec Engle, Hendry and Richard (1983). Consequently, the standard conditions for weak exogeneity in cointegrated systems need to be adapted to cover VAR models with separate cointegration and common feature restrictions.

In their discussion of separation in cointegrated systems, Granger and Haldrup (1997, p.453) claim that the condition for Type $B$ partial separation in terms of the loading matrix $\alpha$ is actually equivalent to weak exogeneity of $y_{1 t}$ (resp. $y_{2 t}$ ) w.r.t. $\beta_{22}^{\prime}$ (resp. $\beta_{11}^{\prime}$ ), [see inter alia Johansen, 1992, 1995, Urbain, 1992], and hence that efficient ML inference based on the partial systems is actually achieved under complete separation in cointegration. This claim overlooks the model specific nature of weak exogeneity conditions. While there is an intuitive link between a completely separated cointegrated system in the sense of Granger and Haldrup (1997) and the weak exogencity, it should be noted that, as pointed out in Hansen and Johansen (1998), this is not true unless we have block-diagonality of the covariance matrix of the errors of the VECM (8.2). As one might expect, this naturally also carrics over to cointegrated systems with both separate common features and complete separation in cointegration. The central point of the analysis is to recognize that efficient estimation and inference in separated sub-systems will only be possible under stronger conditions than the weak exogeneity conditions usually considered in cointegration analyses (see inter alia, Johansen, 1992; Hendry and Mizon, 1993. Ericsson, 1995, Urbain, 1992). Indeed, under block-diagonality of the disturbance covariance matrix, complete separation implies that the likelihood function factorizes into the product of the likelihood functions of the marginal processes. This is naturally a very stringent condition.

Let us illustrate this issue with a simple VAR(2) model, results for higher model being casily derived from this simple case. We will first consider the case of a VECM with a WF reduced rank structure but without assuming any form of separation. This will enable to first derive a set of sufficient conditions for weak exogeneity from which the particular results that apply in the case of separation of various forms will be easily derived. Finally, conditions under which the conditional model reduced to a marginal model will be mentioned. 


\subsubsection{Weak Exogeneity in Cointegrated VAR with Common Features}

Let us first consider the case of cointegrated systems with common features restrictions. For this purpose let us partition $y_{t}=\left(y_{1 t}^{\prime}, y_{2 t}^{\prime}\right)^{\prime}$ where $y_{i t}$ are $\left(n_{i} \times 1\right), i=1,2$ variables with $n_{1}+n_{2}=n$. We make the following dimensionality assumption:

\section{Assumption (Rank) $n_{1} \geq \max (r, n-s)$}

which will appear essential to ensure that the cointegrating relationships are identified from the partial systems only. For the sake of simplicity of the notation and without loss of generality, ${ }^{4}$ we assume that the model is a VAR of order 2 which reads as:

$$
\begin{gathered}
\Delta y_{t}=\Gamma \Delta y_{t-1}+\alpha \beta^{\prime} y_{t-1}+\varepsilon_{t} \\
\left(\begin{array}{c}
\Delta y_{1 t} \\
\Delta y_{2 t}
\end{array}\right)=\left(\begin{array}{c}
\Gamma_{1} \\
\Gamma_{2}
\end{array}\right) \Delta y_{t-1}+\left(\begin{array}{c}
\alpha_{1} \\
\alpha_{2}
\end{array}\right) \beta^{\prime} y_{t-1}+\left(\begin{array}{c}
\varepsilon_{1 t} \\
\varepsilon_{2 t}
\end{array}\right),
\end{gathered}
$$

with

$$
\left[\begin{array}{l}
\varepsilon_{1 t} \\
\varepsilon_{2 t}
\end{array}\right] \sim N\left[\left(\begin{array}{l}
0 \\
0
\end{array}\right),\left(\left[\begin{array}{ll}
\Omega_{11} & \Omega_{12} \\
\Omega_{21} & \Omega_{22}
\end{array}\right]\right)\right] .
$$

We first study the presence of WF common feature vectors and reparametrize the VECM in terms of $\tilde{\beta}_{\perp}$, with $\tilde{\beta}_{\perp}^{\prime} \tilde{\beta}=0$, where $\tilde{\beta}$ denotes the cofeature matrix and $\tilde{\beta}_{\perp}$ denotes the orthogonal complement of $\tilde{\beta}$, and in terms of the common factors $f_{t}=C \Delta y_{t-1}$ where $C$ is a $(n-s) \times n$ full row rank matrix (sec Chapter 3 ). For the identification of $\tilde{\beta}_{\perp}$ and $C$ a normalization has to be imposed. Usually a $(n-s) \times(n-s)$ submartix of $C$ is set equal to $I_{n-s}$. Partitioning $\tilde{\beta}_{\perp}$ and $\alpha$ in accordance with the partitioning of $y / t$, we get:

$$
\begin{aligned}
\Delta y_{t} & =\tilde{\beta}_{\perp} C \Delta y_{t-1}+\alpha \beta^{\prime} y_{t-1}+\varepsilon_{t} \\
\left(\begin{array}{c}
\Delta y_{1 t} \\
\Delta y_{2 t}
\end{array}\right) & =\left(\begin{array}{l}
\tilde{\beta}_{1 \perp} \\
\tilde{\beta}_{2 \perp}
\end{array}\right) C \Delta y_{t-1}+\left(\begin{array}{c}
\alpha_{1} \\
\alpha_{2}
\end{array}\right) \beta^{\prime} y_{t-1}+\left(\begin{array}{c}
\varepsilon_{1 t} \\
\varepsilon_{2 t}
\end{array}\right) .
\end{aligned}
$$

We denote by $Y_{t-1}=\left\{y_{t-1}, y_{t-2}, \ldots, y_{0}\right\}$ the information set containing all information available up to period $t-1$. Conditions for weak exogeneity of a given set of variables require that the parameters of interest are specified. In the sequel, we shall assume that the parameters of interest. $\psi_{0}$ are given by the parameter matrices $\left(\alpha, \beta^{\prime}, \tilde{\beta}_{\perp}, C\right)$. From the joint model, the partial

\footnotetext{
${ }^{4}$ The generalization to the $\operatorname{VAR}(p)$ is straightforward by replacing $\Gamma$ by $\Gamma(L)=\sum_{i=1}^{p-1} \Gamma_{i} L^{i}$.
} 
(conditional) model for $\Delta y_{1, t} \mid \Delta y_{2, t}, Y_{t-1}, \theta_{c}$ is easily obtained from these equations:

$$
\Delta y_{1, t}=\pi_{0} \Delta y_{2, t}+\left[\tilde{\beta}_{1 \perp}-\pi_{0} \tilde{\beta}_{2 \perp}\right] C \Delta y_{t-1}+\left[\alpha_{1}-\pi_{0} \alpha_{2}\right] \beta^{\prime} y_{t-1}+\varepsilon_{11.2 t}
$$

where $\pi_{0}=\Omega_{12} \Omega_{22}^{-1}, \varepsilon_{11.2 t}=\varepsilon_{1 t}-\pi_{0} \varepsilon_{2 t}$. Sinilarly, the marginal subsystem for $\Delta y_{2, t}$ reads

$$
\Delta y_{2, t}=\tilde{\beta}_{2 \perp} C \Delta y_{t-1}+\alpha_{2} \beta^{\prime} y_{t-1}+\varepsilon_{2 t}
$$

The marginal model has parameters

$$
\theta_{m}^{\prime}=\left(\tilde{\beta}_{2 \perp}, C, \alpha_{2}, \beta^{\prime}, \Omega_{22}\right)
$$

while the conditional model has parameters

$$
\theta_{c}^{\prime}=\left(\pi_{0},\left[\tilde{\beta}_{1 \perp}-\pi_{0} \tilde{\beta}_{2 \perp}\right], C,\left[\alpha_{1}-\pi_{0} \alpha_{2}\right], \beta^{\prime}, \Omega_{11.2}\right),
$$

with $\Omega_{11.2}=\Omega_{11}-\Omega_{12} \Omega_{22}^{-1} \Omega_{21}$. It is seen that $\beta$ and $C$ cnter both the conditional and the marginal models so that, whether or not $\beta$ is block-diagonal, efficient estimation of the parameters of interest. from the conditional model can only be achicved under some rather strict conditions stated in the following proposition:

Proposition 8.9 In a VECM with WF reduced rank structure, then $y_{21}$ is weakly exogenous for the parameter of interest $\psi_{0}=\left(\alpha, \beta^{\prime}, \tilde{\beta}_{\perp}, C\right)$ if and only if $\alpha_{2}=0$ and $\tilde{\beta}_{2 \perp}=0$.

The proof follows the line of arguments presented in the case of a cointegrated system without common features and is omitted here to save space (see inter alia, Johansen, 1995, Urbain, 1992). Note that weak cxogencity of $\Delta y_{2 \ell}$ implies that $y_{2 t}$ is a random walk.

Consider now the same specification but with a SF reduced rank structure and assume for simplicity that $r+s \leq n$. The only difference in the analysis is that the loading (error correction) matrix $\alpha$ has the form $\alpha=\tilde{\beta}_{\perp} B$, with $B$ being a $(n-s) \times r$ full column rank matrix. Hence the standard condition for weak exogeneity of variables in a cointegrated system for the longrun parameters $\alpha_{2}=0$ may be equivalently be written as $\tilde{\beta}_{2 \perp} B=0$ which can only occur when $\tilde{\beta}_{2 \perp}=0$. Note that the parameters of interest are $\psi_{0}=\left(\alpha, \beta^{\prime}, \tilde{\beta}_{\perp}, C\right)$ or equivalently $\left(\beta^{\prime}, \tilde{\beta}_{\perp}, C, B\right)$ since the parameter $\alpha=\tilde{\beta}_{\perp} B$ under a SF. Hence:

Proposition 8.10 In a VECM with $S F$ reduced rank structure, if $\tilde{\beta}_{2 \perp}=0$, then $y_{2 t}$ is weakly exogenous for the parameter of interest $\psi_{0}=\left(\alpha, \beta^{\prime}, \tilde{\beta}_{\perp}, C\right)$.

\subsubsection{Weak Exogeneity and Separability}

Let us now consider a slightly modified analysis in which we may analyze the issue of weak exogeneity in relation to separation both in cofeature and in cointegration. We will first consider the WF and SF reduced rank structure cases under the condition that $n_{i} \geq r_{i}, i=1,2$, with 
$r_{1}+r_{2}=r$. The latter conditions being again needed to ensure that the cointegrating relationships might be identified and obtained from the sub-systems only. Under WF, the model (8.10) can be expressed as:

$$
\begin{aligned}
\left(\begin{array}{c}
\Delta y_{1 t} \\
\Delta y_{2 t}
\end{array}\right)= & \underbrace{\left(\begin{array}{cc}
\tilde{\beta}_{11 \perp} & \tilde{\beta}_{12 \perp} \\
\tilde{\beta}_{21 \perp} & \tilde{\beta}_{22 \perp}
\end{array}\right)}_{\tilde{\beta}_{1}, n \times(n-s)} \underbrace{\left(\begin{array}{cc}
C_{11} & C_{12} \\
C_{21} & C_{22}
\end{array}\right)}_{C_{1}(n-s) \times n}\left(\begin{array}{c}
\Delta y_{1 t-1} \\
\Delta y_{2 t-1}
\end{array}\right) \\
& +\underbrace{\left(\begin{array}{cc}
\alpha_{11} & \alpha_{12} \\
\alpha_{21} & \alpha_{22}
\end{array}\right)}_{\alpha_{2}(n \times r)} \underbrace{\left(\begin{array}{cc}
\beta_{11}^{\prime} & \beta_{12}^{\prime} \\
\beta_{21}^{\prime} & \beta_{21}^{\prime}
\end{array}\right)}_{\beta_{,}^{\prime},(r \times n)}\left(\begin{array}{c}
y_{1 t-1} \\
y_{2 t-1}
\end{array}\right)+\left(\begin{array}{l}
\varepsilon_{1 t} \\
\varepsilon_{2 t}
\end{array}\right),
\end{aligned}
$$

where $\tilde{\beta}_{i j \perp}$ is $n_{i} \times\left(n_{j}-s_{j}\right), C_{i j}$ is $\left(n_{i}-s_{i}\right) \times n_{j}, \alpha_{i j}$ is $n_{i} \times r_{j}, \beta_{i j}^{\prime}$ is $r_{i} \times n_{j}$. We again denote $\Omega_{12} \Omega_{22}^{-1}$ by $\pi_{0}$ so that the partial (conditional) model for $\Delta y_{1, t} \mid \Delta y_{2, t}, Y_{t-1}, \theta_{c}$ is easily found from these equations:

$$
\begin{aligned}
\Delta y_{1, t}= & \pi_{0} \Delta y_{2, t}+\left[\left(\tilde{\beta}_{11 \perp}-\pi_{0} \tilde{\beta}_{21 \perp}\right) C_{11}+\left(\tilde{\beta}_{12 \perp}-\pi_{0} \tilde{\beta}_{22 \perp}\right) C_{21}\right] \Delta y_{1 t-1} \\
& +\left[\left(\tilde{\beta}_{11 \perp}-\pi_{0} \tilde{\beta}_{21 \perp}\right) C_{12}+\left(\tilde{\beta}_{12 \perp}-\pi_{0} \tilde{\beta}_{22 \perp}\right) C_{22}\right] \Delta y_{2 t-1} \\
& +\left[\left(\alpha_{11}-\pi_{0} \alpha_{21}\right) \beta_{11}^{\prime}+\left(\alpha_{12}-\pi_{0} \alpha_{22}\right) \beta_{21}^{\prime}\right] y_{1 t-1} \\
& +\left[\left(\alpha_{11}-\pi_{0} \alpha_{21}\right) \beta_{12}^{\prime}+\left(\alpha_{12}-\pi_{0} \alpha_{22}\right) \beta_{22}^{\prime}\right] y_{2 t-1}+\varepsilon_{11.2 t}
\end{aligned}
$$

where $\varepsilon_{11.2 t}=\varepsilon_{1 t}-\Omega_{12} \Omega_{22}^{-1} \varepsilon_{2 t}$. Similarly, the marginal subsystem for $\Delta y_{2, t}$ reads as

$$
\begin{aligned}
\Delta y_{2, t}= & \left(\tilde{\beta}_{21 \perp} C_{11}+\tilde{\beta}_{22 \perp} C_{21}\right) \Delta y_{1 t-1}+\left(\tilde{\beta}_{21 \perp} C_{12}+\tilde{\beta}_{22 \perp} C_{22}\right) \Delta y_{2 t-1} \\
& +\left(\alpha_{21} \beta_{11}^{\prime}+\alpha_{22} \beta_{21}^{\prime}\right) y_{1 t-1}+\left(\alpha_{21} \beta_{12}^{\prime}+\alpha_{22} \beta_{22}^{\prime}\right) y_{2 t-1}+\varepsilon_{2 t} .
\end{aligned}
$$

From this specifications we are able to derive all the different cases of interest under the assumption of WF and SF reduced rank structures. Under a SF reduced rank structure $\alpha=\tilde{\beta}_{\perp} B$, which can be partitioned in the same way as $\Gamma=\tilde{\beta}_{\perp} C$ in (8.11). These different cases will depend on (i) the parameters of interest and (ii) the type of separation that underlies the model. Wo will now consider various cases of separability in cointegration and for each of these sufficient conditions will be derived. The results are summarized in Table 8.1. 


\begin{tabular}{|c|c|c|}
\hline Restrictions on VECM (8.11) & $\begin{array}{c}\text { Parameters of } \\
\text { interest } \Psi_{0} \\
\end{array}$ & $\begin{array}{l}\text { Conditions implying } \\
\text { weak exogeneity of } y_{2 t}\end{array}$ \\
\hline I. WF & $\alpha, \beta, \tilde{\beta}_{\perp}, C$ & $\begin{array}{l}\text { 1. } \alpha_{21}=0, \alpha \alpha_{22}=0 \\
\text { 2. } \tilde{\beta}_{211}=0, \tilde{\beta}_{221}=0\end{array}$ \\
\hline $\begin{array}{l}\text { II.S Fa nd } r+s \leq n \\
\left(\begin{array}{ll}\alpha_{11} & \alpha_{12} \\
\alpha_{21} & \alpha_{22}\end{array}\right)= \\
\left(\begin{array}{ll}\tilde{\beta}_{11 \perp} & \bar{\beta}_{12 \perp} \\
\tilde{\beta}_{211} & \tilde{\beta}_{22 \perp}\end{array}\right)\left(\begin{array}{ll}B_{11} & B_{12} \\
B_{21} & B_{22}\end{array}\right)\end{array}$ & $\alpha, \beta, \tilde{\beta}_{\perp}, C$ & $\tilde{\beta}_{2:}=0\left(\Rightarrow \alpha_{2}=0\right)$ \\
\hline $\begin{array}{c}\text { Ill.a. Scparation in cointegration } \\
\beta_{12}=0, \beta \quad \beta_{21}=0 \\
\text { and } \mathrm{WF}^{2}\end{array}$ & $\alpha_{11}, \beta_{11}, \tilde{\beta}_{\perp}, C$ & $\begin{array}{l}\text { 1. } \alpha_{21}=0, \alpha{ }_{12}-\Omega_{12} \Omega_{22}^{-1} \alpha_{22}=0 \\
\text { 2. } \tilde{\beta}_{21.1}=0, \tilde{\beta}_{22:}=0\end{array}$ \\
\hline $\begin{array}{c}\text { III.b. } \Leftrightarrow \text { (III.a) and Sep. type B: } \\
\alpha_{12}=0, \alpha_{21}=0\end{array}$ & $\alpha_{11}, \beta_{11}, \tilde{\beta}_{1 \perp}, C$ & $\begin{array}{l}\text { 1. } \Omega_{12}=0 \\
\text { 2. } \tilde{\beta}_{21 \downarrow}=0, \tilde{\beta}_{22 \alpha}=0\end{array}$ \\
\hline III.c $\Leftrightarrow(I I I . a)$ & $\alpha_{11,}, \beta_{11}, \bar{\beta}_{1 \perp}, C_{1}$ & $\begin{array}{l}\text { 1. } \alpha_{21}=0, \alpha,{ }_{12}-\Omega_{12} \Omega_{22}^{-1} \alpha_{22}=0 \\
\text { 2. } \tilde{\beta}_{21 ! 1}=0, \tilde{\beta}_{12::}-\Omega_{12} \Omega_{22}^{-1} \tilde{\beta}_{22:}=0\end{array}$ \\
\hline $\begin{aligned} \text { III.d. } \Leftrightarrow \text { (III.a) and Sep. type } \Lambda^{+} \\
\Rightarrow \bar{\beta}_{12 \perp}=0, \tilde{\beta}_{21 \perp}=0 \\
\\
C_{12}=0, C_{21}=0\end{aligned}$ & $\alpha_{11}, \beta_{11}, \bar{\beta}_{111}, C_{11}$ & $\begin{array}{l}\text { 1. } \alpha_{21}=0, \alpha_{12}-\Omega_{12} \Omega_{22}^{-1} \alpha_{22}=0 \\
\text { 2. } \Omega_{12}=0\end{array}$ \\
\hline $\begin{array}{l}\text { III.e. } \Leftrightarrow(I I I . a) \text { and } \\
\text { complete separation } \\
\alpha_{12}=0, \alpha_{21}=0 \\
\Gamma_{12}(L) \equiv 0, \Gamma_{21}(L) \equiv 0\end{array}$ & $\alpha_{11}, \beta_{11}, \tilde{\beta}_{11 \perp}, C_{11}$ & $\Omega_{12}=0$ \\
\hline $\begin{array}{l}\text { IV.a. } \Leftrightarrow \text { (II) and separation in } \\
\text { cointegration: } \beta_{12}=0, \beta_{21}=0\end{array}$ & $\alpha_{11}, \beta_{11}, \bar{\beta}_{-}, C$ & $\begin{array}{l}\text { 1. } \alpha_{12}=0 \\
\text { 2. } \tilde{\beta}_{211}=0, \hat{\beta}_{22}\left(\Rightarrow \alpha_{21}=0, \alpha_{22}=0\right)\end{array}$ \\
\hline $\begin{array}{l}\text { IV.b. } \Leftrightarrow(I V . a) \text { and separation of } \\
\text { Type B: } \alpha_{12}=0, \alpha_{21}=0\end{array}$ & $\alpha_{11}, \beta_{11}, \bar{\beta}_{11}, C$ & $\begin{array}{c}\beta_{21}=0 \Rightarrow \alpha_{21}=0 \text { and } \alpha_{22}=0 \\
\left(\Rightarrow \operatorname{rank}(\alpha)=\operatorname{rank}\left(\alpha_{11}\right)=r=r_{1}\right)\end{array}$ \\
\hline IV.c $\Leftrightarrow$ (IV.a) & $\alpha_{11}, \beta_{11}, \tilde{\beta}_{1 \perp}, C_{1}$ & $\begin{array}{l}\text { 1. } \alpha_{21}=0, \alpha{ }_{12}-\Omega_{12} \Omega_{22}^{-1} \alpha_{22}=0 \\
\text { 2. } \tilde{\beta}_{211}=0, \tilde{\beta}_{12}-\Omega_{12} \Omega_{22}^{-1} \tilde{\beta}_{22}=0\end{array}$ \\
\hline $\begin{array}{c}\text { IV.d. } \Leftrightarrow\left(\text { IV.a) and Sep. type } A^{+}\right. \\
\Rightarrow \bar{\beta}_{121}=0, \bar{\beta}_{21 \perp}=0 \\
C_{12}=0, C_{21}=0\end{array}$ & $\alpha_{11}, \beta_{11}, \tilde{\beta}_{11 \perp}, C_{11}$ & $\begin{array}{l}\text { 1. } \alpha_{21}=0, \alpha_{12}-\Omega_{12} \Omega_{22}^{-1} \alpha_{22}=0 \\
\text { 2. } \Omega_{12}=0\end{array}$ \\
\hline $\begin{array}{c}\text { IV.e. } \Leftrightarrow(\text { IV.a) and Complete Sep. } \\
\alpha_{12}=0, \alpha \quad \alpha_{21}=0 \\
\Gamma_{12}(L) \equiv 0, \Gamma_{21}(L) \equiv 0 \\
\end{array}$ & $\alpha_{11}, \beta_{11}, \tilde{\beta}_{11 \downarrow}, C_{11}$ & $\begin{array}{l}\text { 1. } \Omega_{12}=0 \\
\text { 2. } \hat{\beta}_{21 \perp}=0 \quad\left(\Rightarrow \alpha_{2}=0\right)\end{array}$ \\
\hline
\end{tabular}

Table 8.1: Weak Exogeneity Conditions 
A fow words of explanation are in order. In the first column of Table 8.1. the restrictions imposed on the VECM (8.12) are given. For the WF and SF, we first consider the model without and with separation in cointegration. Next, in addition to separation in cointegration, separation of Type $\mathrm{B}$ and $\mathrm{A}^{+}$and complete separation are considered respectively. ${ }^{5}$ Sufficient conditions for weak exogeneity of $y_{2 t}$ for the parameters of interest are given in the third column. The cases 1 and 2 correspond to the models considered in Proposition 8.9 and 8.10. These conditions follow in a straightforward way when the corresponding restrictions are imposed on the model in the form (8.12). The necessary and sufficient conditions for weak exogeneity given in Proposition 8.9 appear to be overly strong when only part of the cointegrating vectors, for instance are parameters of interest (for a similar analysis, abeit in a different set-up, see Hendry and Mizon, 1993; Ericsson, 1995; Ericsson, Hendry and Mizon, 1998). Under the conditions in (III.a), the marginal process for $\Delta y_{2 t}$ is a cointegrated VAR(1) with $r_{2}$ cointegrating relationships involving only the variables present in $y_{2, t}$. This implies that

$$
\underbrace{\bar{\beta}_{1}}_{n \times n-s}=\left(\begin{array}{c}
\underbrace{\tilde{\beta}_{11 \perp}: \tilde{\beta}_{12 \perp}}_{n_{2} \times n-s} \\
\underbrace{0}_{n_{1} \times n-s}
\end{array}\right)
$$

and hence $\tilde{\beta}_{\perp}$. musi be of full column rank $n-s$ since we assume that there are $s$ cofeature vectors. Consequently, we must not o:ly have $n_{1} \geq r_{1}$ (in order to be able to identify the $r_{1}$ cointegrating relationship from the sub-model) but also $n_{1} \geq n-s=n-s_{1}$.

Under the conditions presented in (III.c) the marginal model for $\Delta y / 2, t$ becomes a VECM of the form

$$
\begin{aligned}
\Delta y_{2, \ell} & =\tilde{\beta}_{22 \perp} C_{21} \Delta y_{1 t-1}+\tilde{\beta}_{22 \perp} C_{22} \Delta y_{2 t-1}+\alpha_{22} \beta_{22}^{\prime} y_{2 t-1}+\varepsilon_{2 t} \\
& =\tilde{\beta}_{22 \perp} C_{2} \Delta y_{t-1}+\alpha_{22} \beta_{22}^{\prime} y_{2 \ell-1}+\varepsilon_{2 \ell},
\end{aligned}
$$

while the partial model reads

$$
\begin{aligned}
\Delta y_{1, t} & =\pi_{0} \Delta y_{2, t}+\tilde{\beta}_{11 \perp} C_{11} \Delta y_{1 t-1}+\tilde{\beta}_{11 \perp} C_{12} \Delta y_{2 t-1}+\alpha_{11} \beta_{11}^{\prime} y_{1 t-1}+\varepsilon_{11.2 t} \\
& =\pi_{0} \Delta y_{2, t}+\tilde{\beta}_{11 \perp} C_{1} \Delta y_{t-1}+\alpha_{11} \beta_{11}^{\prime} y_{1 t-1}+\varepsilon_{11,2 t},
\end{aligned}
$$

where $C_{i}=\left(C_{i 1}: C_{i 2}\right), i=1,2$, so that no cross-equation restrictions remain. Remark that these are conditions concerning the efficiency of an analysis based on a conditional sub-system but do not yet enable one to consider separated marginal systems as $y_{1 t-1}$ and $y_{2 t-1}$ both appear in this sub-system. Also note that these are sufficient conditions and essentially show that the conditions in Proposition 8.9 are not necessary once we redefine the parameters of interest as being only

\footnotetext{
${ }^{5}$ Note that complete separation in cointegration (in the sense of Granger-Haldrup) does not fundamentally differ from separation of Type $B$ as far as the implications for weak exogeneity are concerned.
} 
sub-matrices of $\alpha, \beta^{\prime}$ and $\tilde{\beta}_{\perp}$. Alternative sets of conditions could be derived. For example, if $\Omega_{12}=0$,(conditional independence) then both $\alpha$ and $\tilde{\beta}_{\perp}$ have to be block diagonal. The cases (III.b), (III.d) and (III.e) summarize these implications. If in addition to complete separation in the Granger-Haldrup sense (III.c), $\Omega_{1.2}=0, \Gamma_{12}(L) \equiv 0, \Gamma_{21}(L) \equiv 0$, the block-diagonality of $\tilde{\beta}_{\perp}$ results and conditions for weak exogeneity (III.e) are satisfied.

Under the SF reduced rank structure assumption, the additional notational complication comes from the fact that the matrix of error correction terms takes the form

$$
\alpha=\tilde{\beta}_{\perp} B
$$

with $B$ being a $(n-s \times r)$ full column rank matrix, with here $n \geq s+r$. With the partitioning introduced earlier this yiclds

$$
\left(\begin{array}{ll}
\alpha_{11} & \alpha_{12} \\
\alpha_{21} & \alpha_{22}
\end{array}\right)=\left(\begin{array}{ll}
\tilde{\beta}_{11 \perp} & \tilde{\beta}_{12 \perp} \\
\tilde{\beta}_{21 \perp} & \tilde{\beta}_{22 \perp}
\end{array}\right)\left(\begin{array}{ll}
B_{11} & B_{12} \\
B_{21} & B_{22}
\end{array}\right) .
$$

The following results follow from the analyses developed in the preceding sub-sections. In particular from case (II) and (II.a), we see that if $\tilde{\beta}_{2 \perp}=\left(\tilde{\beta}_{21 \perp}: \tilde{\beta}_{22 \perp}\right)=0$, then $\alpha_{21}=$ $\tilde{\beta}_{21 \perp} B_{11}+\tilde{\beta}_{22 \perp} B_{21}=0$, and more generally $\alpha_{2}=\tilde{\beta}_{2 \perp} B=\left(\alpha_{21}: \alpha_{22}\right)=0$ so that under Type B separation $\alpha_{12}=\tilde{\beta}_{11 \perp} B_{12}+\tilde{\beta}_{12 \perp} B_{22}=0$ and $r=r_{1}$ (case IV.b) and we also have to require that $n_{1} \geq r, n_{1} \geq n-s$. Note that in this case the cointegrating relationships between the variables $y_{1 t}$ only appear in the model for the first group of variables.

\subsubsection{Partial vs. Marginal Subsystem}

The results presented in the preceding propositions and corollaries summarize conditions for efficient ML based estimation and inference on the parameters of interest within a partial model. It does not yet imply that the system may be split into two separated sub-systems, i.e. independent marginal systems that can be treated independently. Indeed, let us again rewrite the VECM by partitioning all matrices entering the VECM (8.11) under separation in cointegration as follows

$$
\begin{aligned}
\left(\begin{array}{c}
\Delta y_{1 t} \\
\Delta y_{2 t}
\end{array}\right)= & \left(\begin{array}{ll}
\tilde{\beta}_{11 \perp} & \tilde{\beta}_{12 \perp} \\
\tilde{\beta}_{21 \perp} & \tilde{\beta}_{22 \perp}
\end{array}\right)\left(\begin{array}{cc}
C_{11} & C_{12} \\
C_{21} & C_{22}
\end{array}\right)\left(\begin{array}{c}
\Delta y_{1 t-1} \\
\Delta y_{2 t-1}
\end{array}\right) \\
& +\left(\begin{array}{cc}
\alpha_{11} & \alpha_{12} \\
\alpha_{21} & \alpha_{22}
\end{array}\right)\left(\begin{array}{cc}
\beta_{1} & 0 \\
0 & \beta_{2}
\end{array}\right)^{\prime}\left(\begin{array}{l}
y_{1 t-1} \\
y_{2 t-1}
\end{array}\right)+\left(\begin{array}{l}
\varepsilon_{1 t} \\
\varepsilon_{2 t}
\end{array}\right)
\end{aligned}
$$

so that it now appears clearly that in general even under weak exogeneity of $y_{2 t}$, separation (here marginal systems in contrast to partial or conditional systems) will only cnable us to recover the parameters of interest in the case where $C$ is block-diagonal, e.g. when $C_{12}=0, C_{21}=0$ which corresponds to the case we introduced under the heading Type A+ separation in cointegration. In that case, if in addition to $\alpha_{21}=0, \tilde{\beta}_{21}=0$ and $\alpha_{12}-\Omega_{12} \Omega_{22}^{-1} \alpha_{22}=0$ for the weak form for 
example, then the common cyclical factors entering the partial, here marginal, system for $\Delta y_{1 t}$ are only made of linear combinations of lagged $\Delta y_{1 \ell}$ and $\beta_{1}^{\prime} y_{1 t-1}$.

These results have to be related to the general discussion of conditioning versus marginalization in dynamic econometric models. Valid marginalization is much more demanding than valid conditioning and actually requires that the joint likelihood of the complete system can be written as the product of the marginal likelihoods of the subsystems which entails not only weak exogeneity but also Granger-Noncausality (given common feature restrictions) and conditional independence. This last requirement of conditional independence is valid irrespectively of the presence or absence of common feature restrictions in a VECM as also pointed out by Hansen and Johansen (1998).

\subsection{Inference on Cofeature Separation and Modeling Strategy}

The results derived in Section 8.2 and 8.3 have obvious implications for the way in which separation should be tested in a VAR model in practice. The following sequence of increasingly restrictive hypotheses comes naturally to one's mind.

1. Within a complete VAR system first test for the number of cointegration relationships.

2. Second, test for separation in cointegration, e.g. using tests proposed by Konishi and Granger (1993).

3. Third, investigate whether $\alpha$ is block-diagonal (Type B partial separation).

4. Fourth, test the WF reduced rank structure.

5. Fifth, test the strong form. Note that under SF, type B separation implies common feature separation (sce Proposition 8.7).

6. Sixth, test for separation in common features, i.c. block-diagonality of $\tilde{\beta}$.

7. Seventh, if block-diagonality of $\tilde{\beta}$ is not rejected, check whether $\Gamma(L)$ is block-diagonal (type $A^{+}$separation). Notice that under WF block-diagonality of $\Gamma(L)$ implies common feature scparation (sec Proposition 8.8), whereas under SF, type $A^{+}$separation is compatible with common feature separation (sce Proposition 8.7).

8. Eight, test for weak exogeneity by checking the conditions given in Table 8.1.

9. Ninth, test for complete separation including the block-diagonality of $\Omega$.

The first three steps of our sequential testing strategy are standard and discussed in detail in a.o. Johansen (1995). The remaining steps need to be described in some details.

Let us first consider test statisties for weak form common features. Tests carried out in this chapter are again based on the sample canonical correlations of two sets of random vectors 
$\Delta Y=\left(\Delta y_{1}, \ldots, \Delta y_{T}\right), \Delta Z=\left(\Delta Y_{-1}^{\prime}, \ldots \Delta Y_{-p+1}^{\prime}\right)^{\prime}$, both corrected for deterministic components and coint.cgrating relationships $\beta^{\prime} Y_{-1}$, where $Y_{-1}=\left(y_{0}, \ldots, y_{T-1}\right)$ (see Chapter 3 for details). In the line of Nielsen and Rahbek (1998) and assuming that the deterministic component is simply specified as an unrestricted constant term, we can summarize the procedure as follows:

$$
\operatorname{Can} \operatorname{Cor}\left(\Delta Y, \Delta Z \mid 1, \beta^{\prime} Y_{-1}\right)
$$

In the same way as in the Johansen's ML approach for cointegration, squared canonical correlations are found as solutions of the usual eigenvalue problem $\left|\lambda I-S_{Y Y}^{*-1 / 2} S_{Y Z}^{*} S_{Z Z}^{*-1} S_{Z Y}^{*} S_{X X}^{*-1 / 2}\right|=0$, where for instance, $S_{Y Z}^{*}$ denotes the covariance matrix between the elements of $\Delta Y$ and $\Delta Z$ both corrected by a constant and long-run relationships. We call them $\Delta Y^{*}$ and $\Delta Z^{*}$. Note that in the usual SF model, the analysis consists in a canonical correlation analysis between $\triangle Y$ and $\Delta W=\left(\Delta Z^{\prime}, Y_{-1}^{\prime} \beta\right)^{\prime}$ both sets corrected for their mean only, i.e. $\operatorname{Can} \operatorname{Cor}(\Delta Y, \Delta W \mid 1)$. The computation of empirical squared canonical correlations $\lambda_{i}^{w}$ (for the WF), $\lambda_{i}^{s}$ (for the SF), allows us to test the null that the first $s$ linear combinations are white noises. Simple statistics $\xi_{W}=-T \sum_{i=1}^{s} \log \left(1-\lambda_{i}^{w}\right), s=1, \ldots, n$ and $\xi_{S}=-T \sum_{i=1}^{s} \log \left(1-\lambda_{i}^{i}\right), s=1, \ldots, n-r$ which have asymptotic $\chi^{2}$ distributions under the null, can be computed. The maximand of the likelihood is given by (sec Johansen, 1998):

$$
L R Q_{1}^{*}=-\frac{T}{2}\left\{\ln \left\{\operatorname{det}\left(S_{Y Y}^{*}\right)\right\}-\sum_{i=1}^{s} \ln \left(1-\lambda_{i}^{w}\right)\right\} .
$$

The corresponding expression for the strong form model $\left(L R Q_{1}\right)$ is immediate. To get intuitively into separation in common features let us reformulate the problem in terms of moment restrictions. The cxistence of a strong form common feature space means $H_{0} Q_{1}: E\left[\tilde{\beta}^{\prime} \Delta y_{t} \otimes \Delta W_{l}\right]=0$, where $\Delta W_{t}$ is a set of instruments composed of the $p-1$ lags of the $n$ variables of $\Delta y_{t}$ and the cointegrating vectors. In the WF framework the condition becomes $H_{0} Q_{1}^{*}: E\left[\tilde{\beta}^{\prime} \Delta y_{t}^{*} \otimes \Delta Z_{t}^{*}\right]=0$, where $\Delta Z_{t}^{*}$ is a set of instruments composed of the lags of all variables in $\Delta y_{t}$ only. Both $\Delta y_{t}^{*}$ and $\Delta Z_{t}^{*}$ have been concentrated on a constant and on the error-correction terms using superconsistent estimates of the cointegrating vectors obtained from a reduced rank regression in a first step. Now, under separation in common features we know that under the null the condition $H_{0} Q_{2}: E\left[\left(\tilde{\beta}_{1}^{\prime} \Delta y_{t}+\tilde{\beta}_{2}^{\prime} \Delta y_{t}\right) \otimes \Delta W_{t}\right]=0$ or $H_{0} Q_{2}^{*}: E\left[\left(\tilde{\beta}_{1}^{\prime} \Delta y_{t}^{*}+\tilde{\beta}_{2}^{\prime} \Delta y_{t}^{*}\right) \otimes \Delta Z_{t}^{*}\right]=0$, where $\tilde{\beta}_{1}$ and $\tilde{\beta}_{2}$ are common feature vectors for the mutually exclusive $n_{1}$ and $n_{2}$ sets, should also hold.

In order to determine whether $\tilde{\beta}^{\prime}$ is block-diagonal we need a test statistics to test different restrictions on common feature vectors. To do so, along the line of Johansen (1995). Konishi and Granger (1992) for cointegration a likelihood ratio test is computed using a switching algorithm for the restrictions:

$$
\tilde{\beta}=\left\{\tilde{\beta}_{1}, \tilde{\beta}_{2}\right\}=\left\{H_{1} \Psi_{1}, H_{2} \Psi_{2}\right\}
$$


where $H_{1}$ and $H_{2}$ are matrices of known constants respectively of dimension $\left(n \times n_{1}\right)$ and $\left(n \times n_{2}\right)$, while $\Psi_{1}$ and $\Psi_{2}$ are matrices containing unknown parameters of dimension $\left(n_{1} \times s_{1}\right)$ and $\left(n_{2} \times s_{2}\right)$.

Example 8.11 With $n_{1}=n_{2}=2$ and $s_{1}=s_{2}=1, r_{1}=r_{2}=1$, the situation we face in the cmpirical section of this chapter, these matrices can be parametrized such that:

$$
\begin{aligned}
& \tilde{\beta}_{1}=\left(\begin{array}{l}
\varphi_{1} \\
\varphi_{2} \\
0 \\
0
\end{array}\right), \quad \tilde{\beta}_{2}=\left(\begin{array}{l}
0 \\
0 \\
\varphi_{3} \\
\varphi_{4}
\end{array}\right), \quad H_{1}=\left(\begin{array}{ll}
1 & 0 \\
0 & 1 \\
0 & 0 \\
0 & 0
\end{array}\right), \quad H_{2}=\left(\begin{array}{ll}
0 & 0 \\
0 & 0 \\
1 & 0 \\
0 & 1
\end{array}\right), \\
& \Psi_{1}=\left(\begin{array}{l}
\varphi_{1} \\
\varphi_{2}
\end{array}\right), \quad \Psi_{2}=\left(\begin{array}{l}
\varphi_{3} \\
\varphi_{4}
\end{array}\right) .
\end{aligned}
$$

The maximand of the likclihood under common feature restrictions has been previously labelled $L R Q_{1}^{*}$ or $L R Q_{1}$. If convergence is achieved using a switching algorithm ${ }^{6}$, the log likelihood functions under separate restrictions on different common feature vectors are labelled as $L R Q_{2}^{*}$ or $L R Q_{2}$. In practice, alike Johansen for cointegration, we propose the following steps: ${ }^{i}$

Step 1. Choose an unrestricted vector $\tilde{\beta}_{1}$,

Step 2. Given $\tilde{\beta}_{1}$, solve $\operatorname{Can} \operatorname{Cor}\left(H_{2}^{\prime} \Delta Y^{*}, \Delta Z^{*} \mid \tilde{\beta}_{1}^{\prime} \Delta Y^{*}\right)$ to obtain $\tilde{\beta}_{2}$,

Step 3. Given $\tilde{\beta}_{2}$, compute $\operatorname{Can} \operatorname{Cor}\left(H_{1}^{\prime} \Delta Y^{*}, \Delta Z^{*} \mid \tilde{\beta}_{2}^{\prime} \Delta Y^{*}\right)$ to obtain a new $\tilde{\beta}_{1}$,

Step 4. Continue this process until convergence to the maximum of the likelihood function.

The maximum likelihood is given by

$$
L R Q_{2}^{*}=-\frac{T}{2}\left\{\ln \left\{\operatorname{det}\left(S_{Y Y}^{*}\right)\right\}-\sum_{i=1}^{s_{1}} \ln \left(1-\lambda_{i, \bar{\beta}_{1}}\right)-\sum_{j=1}^{s_{2}} \ln \left(1-\lambda_{j, \tilde{\beta}_{2}}\right)\right\}
$$

where $S_{Y Y}^{*}$ is defined above, $\lambda_{i, \bar{\beta}_{1}}$ and $\lambda_{j, \bar{\beta}_{2}}$ are the eigenvalues obtained after convergence under respectively the restrictions $\tilde{\beta}_{1}=H_{1} \Psi_{1}$ and $\tilde{\beta}_{2}=H_{2} \Psi_{2}$. A likelihood ratio test for separation in common features is then easily obtained by computing $L R^{s e p}=-2\left(L R Q_{2}-L R Q_{1}\right)$ for the strong form model or $L R^{s e p *}=-2\left(L R Q_{2}^{*}-L R Q_{1}^{*}\right)$ for the weak form. These statistics have asymptotically a $\chi^{2}$-distribution under the null. The number of degrees of frecdom is the number of zero restrictions (above the ones from normalization) separation imposes.

The last two steps are more straightforward. On the one hand weak exogencity analyses depend on the chosen parameters of interest but are easily conducted once the system (with cointegration and common features restrictions) is written in pseudo-structural form in terms of

\footnotetext{
${ }^{6}$ Note that due to the absence of cross-restrictions between the different common feature vectors, the convergence is very fast.

'We describe the algorithm for WF, the results for SF differ only by the fact that we do not concentrate on cointegrating vectors.
} 
the matrix $\tilde{\beta}_{\perp}$ and estimated by FIML. The last stcp, e.g. testing the block-diagonality of the covariance matrix could be investigated by standard variable addition tests. Note finally that a global LR test statistics can be easily conducted by comparing the log-likelihood of the complete model with the sum of the log-likelihoods of the two marginal models. Such a LR type test has a $\chi^{2}$ distribution under the null. It is a joint test of the null bypothesis that all the different sub-hypotheses jointly hold against the alternative that at least one of the sub-hypotheses is violated.

It is worth making a few remarks at this stage:

Remark 8.12 It is important to recognize that the sequential approach proposed above, while being appealing as we consider explicitly all the different restrictions sequentially, naturally raises the crucial issue of size distortion. Such distortions are likely to occur in any empirical application since the choice of the hypotheses tested is dependent on the outcome of the test of the previous hypothesis in the sequence. Hence for practical purpose it might be advisable to use rather low significance level to minimize the occurrence of size distortion and power loss.

Remark 8.13 The major drawback of the analysis we propose here relates to the need of specifying and estimating complete systems to assess the correctness (in terms of P-T decomposition) and efficiency of the sub-group based analysis. While the purpose of the aforementioned testing strategy is to sustain sub-systems analysis, it requires full system estimation to compute the different test statistics. This is naturally the most important drawback here but is not specific to the analysis we propose and it actually occurs in all (weak) exogencity and Granger Noncausality analyses. Hence, while feasible, our testing strategy implicitly supposes a low number of variables and sub-groups. An alternative, which is left for further research, would be to base the testing strategy on LM type tests.

Remark 8.14 An important final remark should be made concerning the restrictiveness of the specifications we investigate here. While the class of models we consider here may at the first sight appear overly restrictive, it should be noted that the restrictions we propose to investigate are actually systematically imposed without further investigation in the recent literature on panel data cointegration (see the surveys by Phillips and Moon, 1999; Baltagi and Kao, 2000) and panel common cycle analysis (see Chapter 9). While jew papers allow for a non-diagonal covariance matrix between the subgroups of the pancls, cointegration separation is indeed systematically imposed by excluding cointegration between members of a panel. With the exception of a few studies like Groen and Kleibergen (1999), Larsson and Lyhagen (1999) this restrictions is usually not explicitly discussed and studied. See also Hall, Lazarova and Urga (1999).

\subsection{Empirical Analysis}

To show the feasibility of the analysis we propose to illustrate empirically the testing strategy within a small dimensional problem that is also used in Chapter 9 where the notion of serial 
correlation common features is introduced in a nonstationary panel data context.

\subsubsection{The Economic Model}

We focus on the permanent income hypothesis versus the heterogenous consumer model proposed by Campbell and Mankiw $(1990,1991)$. These authors consider two groups of agents who receive respectively a disposable income $Y_{1 t}$ and $Y_{2 t}$ in fixed proportions of the total income $Y_{t}$, such that $Y_{1 t}=\lambda Y_{t}, Y_{2 t}=(1-\lambda) Y_{t}$ and $Y_{t}=Y_{1 t}+Y_{2 t}$. Agents in the first group are subject to liquidity constraints, consume their current income while agents in the second group consume their permanent income. We get the following systcm:

$$
\left\{\begin{array}{c}
C_{1 t}=Y_{1 t}=\lambda Y_{t} \\
C_{2 t}=Y_{2 t}^{P}=(1-\lambda) Y_{t}^{P} \\
Y_{1 t}=Y_{1 t}^{P}+Y_{1 t}^{T} \\
Y_{2 t}=Y_{2 t}^{P}+Y_{2 t}^{T}
\end{array}\right.
$$

where $C_{i t}$ is the consumption of agent $i$ and $Y_{i t}^{P}$ and $Y_{i t}^{T}$ are the permanent and transitory components of income of the agent $i$ and are assumed to be $I(1)$ and $I(0)$, respectively. Aggregating over agents we get $C_{t}=Y_{1 t}^{P}+Y_{1 t}^{T}+Y_{2 t}^{P}=Y_{t}^{P}+Y_{1 t}^{T}$, and thus:

$$
\left\{\begin{array}{c}
C_{t}=Y_{t}^{P}+\lambda Y_{t}^{T} \\
Y_{t}=Y_{t}^{P}+Y_{t}^{T}
\end{array}\right.
$$

which shows that aggregate consumption and income share a common trend $Y_{t}^{P}$. Note that because a fraction $\lambda$ of income accrues to individuals who consume their current income rather than their permanent income, this model has been labelled " $\lambda$ model" by Campbell and Mankiw (1990, 1991). It is also casily scen that if $\lambda=0$ we get the permanent income model. In order to stress the common cycle component let us take the first difference of the aggregate consumption $C_{t}=C_{1 t}+C_{2 t}$. By substituting the shares of income in the total incone we obtain $C_{t}=\lambda Y_{t}+(1-\lambda) Y_{t}^{P}$ and then:

$$
\Delta C_{t}=\lambda \Delta Y_{t}+(1-\lambda) \Delta Y_{t}^{P}
$$

Consequently, assuming that the permanent income is a martingale, the consumption function can be tested by using the regression $\Delta C_{t}=\lambda \Delta Y_{t}+(1-\lambda) \varepsilon_{t}$. However, $\varepsilon_{t}$ is a difference martingale which is not orthogonal by construction to $\Delta Y_{t}$. Therefore this equation cannot be estimated by OLS, but instrumental variables or FIML estimators are appropriate. Also notice that this model can be derived from maximization of a utility function but a more rigorous formalization is not. necessary for the sequel of the paper. Exception made of a few studies, including those of Vahid and Engle (1993) and Jobert (1995), the cointegrating vector is usually not considered as a valid 
instrument when testing equation (8.17) by instrumental variables method (IV), and are then subject to omission variable bias. Vahid and Engle (1993) also made the connection with the common feature literature which is the hypothesis that $\varepsilon_{t}$ is a white noisc ${ }^{8}$ with $[1-\lambda]$ the associate normalized common features vector. Empirical studies (see inter alia Campbell and Mankiw, 1990, 1991; Evans and Karas, 1993), have shown that $\lambda$ is usually significantly different from zero with coefficients in the range 0.3 to 0.5 for most countries.

\subsubsection{Empirical Results}

For illustrative purpose, we confine our analysis to the " $\lambda$ model" for the USA and Canada using annual data for the period from 1950 to 1992. The data are taken from the Penn World Tables Mark 5.6 (sce Summers and Heston, 1991) ${ }^{9}$ which due to their definition homogeneity are extremely useful and have been extensively used in cmpirical cross-country studies. It is interesting to note that Hoffman (1999)'s study points out that total permanent shocks seem to be relatively unimportant for output volatility for both Canada and US which motivates further an analysis of short run comovements between these two countries.

The data used are $Y=" R G D P L:$ Real GDP per capita (Laspeyres index) in 1985 international prices" and $C=$ " $C$ : Real Consumption share of GDP in 1985 international prices" $\times Y / 100$. This last operation is necessary to get the consumption in level and not in percentage of income. ${ }^{10}$ Although we may expect some lack of power with this small sample of annual data, the use of an annual frequency avoids the pitfalls that one would probably encounter with seasonally adjusted monthly or quarterly data (sec Chapter 7 and Cubadda, 1999).

A first descriptive analysis using standard unit root tests and both Engle and Granger (1987) and Johansen (1995) cointegration analyses reveals the existence of a single cointegrating vector for both countries taken separately. ${ }^{11}$ In both cases, the cointegrating vectors are close to the expected theoretical vectors, although the unit income elasticity is more plausible for the Canada (1.00) than for the US (1.15).

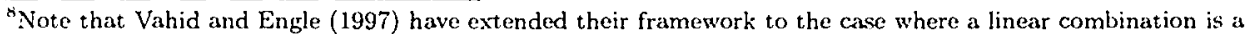
MA(q) process and not a white noise. They labelled this model non synchronous common cycle (see also Chapters 2 and 6$)$.

${ }^{9}$ The data may be downloaded via different internet sites such as http://www.nber.org/pwt56.html or http://datacentre.epas.utoronto.ca:5620/pwt.

${ }^{10}$ We did not consider a slightly different model in which real government expenditures are substracted from output. Indeed, as risised by Evans and Karas (1993), the " $\lambda$ model" should be extended to take care of the potential substitutability or complementarity between private and public goods. Without a fne distinction of the components of government expenditures, it might be desirable to extend the model to take into account a third variable. It is also possible to consider a simple alternative model where all the public goods are substitutable to private ones by substracting $G$ from $Y$.

${ }^{11}$ Detailed results are not reported to save space but some of them are reported in Chapter 9, Table 9.3.
} 


\section{Separation in Cointegration}

We first analyze cointegration separation at the level of the complete system for the two countries. The model which appropriately characterizes the covariance structure of this four dimensional data set is a VAR of order four in the level of time serics. Table 8.2 reports the $p-v a l u e s$ of different misspecification tests computed on the residuals of this VAR(4).

\begin{tabular}{lccc}
\hline Dep. Var. & $L M_{A R}(1)$ & $B J$ & $L M_{A I R C H}(2)$ \\
\hline$\Delta C a_{-} c_{t}$ & 0.901 & 0.085 & 0.883 \\
$\Delta C a_{-} y_{t}$ & 0.573 & 0.331 & 0.947 \\
$\Delta U S_{-} c_{t}$ & 0.204 & 0.740 & 0.765 \\
$\Delta U S_{-y_{t}}$ & 0.201 & 0.406 & 0.797 \\
\hline \hline
\end{tabular}

Table 8.2: Analysis of Residuals of a VAR(4) (p-values)

\begin{tabular}{cccccc}
\hline \hline & $\lambda_{i}$ & Max.Eig. Test & $\mathbf{9 5 \% \mathrm { cv }}$ & Trace Test & $\mathbf{9 5 \% \mathrm { cv }}$ \\
\hline$r=0$ & 0.71 & $48.64^{*}$ & 31.5 & $83.34^{*}$ & 63.0 \\
$r \leq 1$ & 0.43 & 21.98 & 25.5 & 34.70 & 42.4 \\
$r \leq 2$ & 0.22 & 9.87 & 19.0 & 12.72 & 25.3 \\
$r \leq 3$ & 0.07 & 2.85 & 12.2 & 2.85 & 12.2 \\
\hline \hline
\end{tabular}

Table 8.3: Johansen's ML Tests Statistics

These tests include a $F$-version of the LM test for 1 st order autocorrelation, the Bera-Jarque Normality test as well as a LM test for $\operatorname{ARCH}(2)$ in $F$-form. These results do not indicate any evidence of misspecification so that the VAR(4) will be retained for the subsequent analyses. We then apply Johansen's ML tests statistics with a deterministic linear trend restricted in the long-run. The results are reported in Table 8.3. Both the maximum eigenvalue and the trace test favor the existence of a single cointegrating vector. The smallness of our sample may however seriously affect the power of these tests so that the analysis is complemented by both a visual inspection of cointegrating vectors (Figure 8.1) and the analysis of the roots of $\left(\alpha \beta^{\prime}-I\right)^{12}$ that lead us to retain two cointegrating vectors.

The next step in our sequential analysis of to test for restrictions on the cointegrating space as well as on the loading matrix in order to analyze separation in cointegration. The results are presented in Table 8.4. The null hypothesis $H_{0}: \beta_{12}=\beta_{21}=0$ corresponds to the cointegration separation hypothesis that is tested using a LR test that is $\chi^{2}(3)$ distributed under the null of separation. ${ }^{13}$ The null $H_{0}: \beta_{12}=\beta_{21}=0 \& \alpha_{12}=\alpha_{21}=0$ corresponds to partial separation of Type $B$ while the two remaining hypotheses are joint hypotheses of cointegration separation and

\footnotetext{
${ }^{12}$ We get two pairs of complex roots whose modulus are respectively 1 and .207 .

${ }^{13}$ One should notice that the degrees of freedom of the different statistics are equal to the number of restrictions imposed under the different forms of seperation plus one since the linear trend coefficient is not significant in the Canadian long-run relationship.
} 

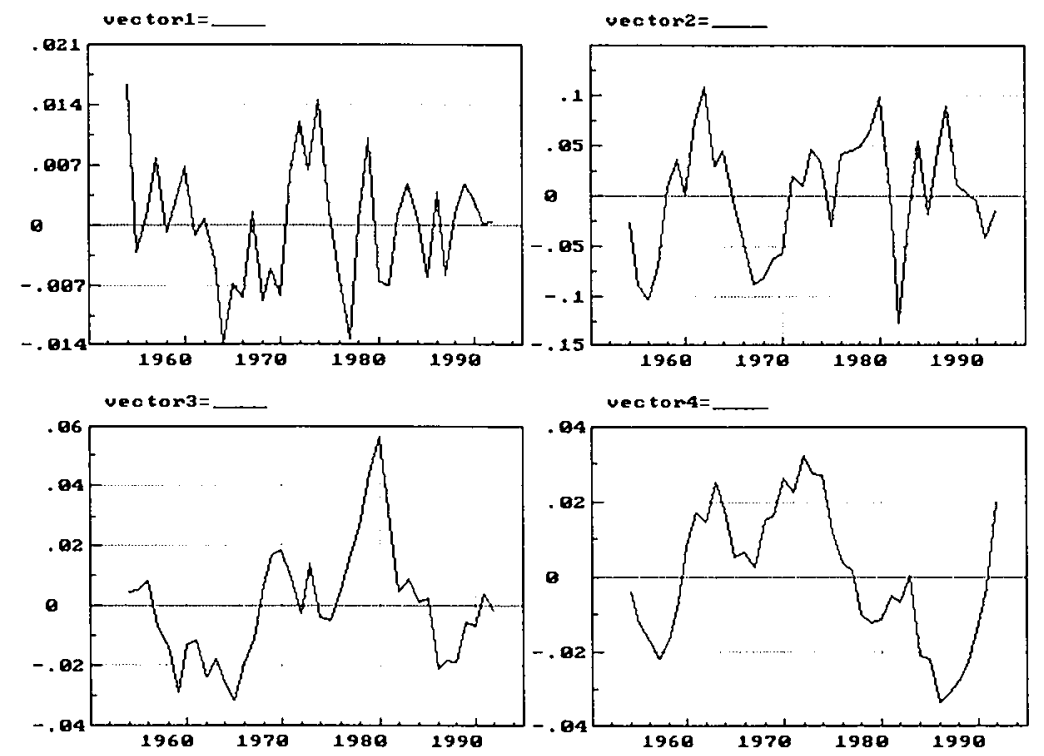

Figure 8.1: Cointegrating Relationships 
block-triangularity of the loading matrix. It appears that separation in cointegration cannot be rejected. On the contrary, we reject partial separation of Type B. Due to the small sample size we can however not clearly decide whether the loading matrix is block-triangular, i.e. whether $\alpha_{21}$ is zero or not but we guess that it is not. Indecd, the null hypothesis is clearly rejected when we do not restrict the trend to zero in the Canadian equation or we do not impose separation in cointegration.

\begin{tabular}{llll}
\hline \hline$H_{0}$ & Test stat. & Distr. & P-values \\
\hline$\beta_{12}=\beta_{21}=0$ & 0.762 & $\chi^{2}(3)$ & 0.858 \\
$\beta_{12}=\beta_{21}=0$ and $\alpha_{12}=\alpha_{21}=0$ & 42.58 & $\chi^{2}(5)$ & $<0.001$ \\
$\beta_{12}=\beta_{21}=0$ and $\alpha_{21}=0$ & 9.55 & $\chi^{2}(4)$ & 0.048 \\
$\beta_{12}=\beta_{21}=0$ and $\alpha_{12}=0$ & 22.19 & $\chi^{2}(4)$ & $<0.001$ \\
\hline \hline
\end{tabular}

Table 8.4: Hypotheses Testing

Assuming cointegration separation alone, c.g. only block diagonality of $\beta$, we obtain:

$$
\alpha \beta^{\prime} y_{t}=\left(\begin{array}{rr}
1.262 & -0.428 \\
-0.075 & 0.805 \\
1.280 & -0.554 \\
0.819 & -0.847
\end{array}\right)\left(\begin{array}{rrrrr}
-0.981 & 1.000 & 0.000 & 0.000 & 0.000 \\
0.000 & 0.000 & -0.923 & 1.000 & -0.004
\end{array}\right)\left(\begin{array}{l}
C a_{-} y_{t} \\
C a_{-} c_{t} \\
U S A_{-} y_{\ell} \\
U S A_{-} c_{t} \\
\text { trend }
\end{array}\right),
$$

which yiclds the following long-run consumption function, $c_{t}=0.98 y_{t}$ for Canada and $c_{t}=$ $0.92 y_{\ell}+.004 t r e n d$ for the USA. The estimated long-run income elasticity corresponds to that obtained from individual cointegration analyses in the case of Canada. The result is now more plausible for the USA. The possible explanation can be the omission of the trend and as well as Canadian variables in the static regression. This trend partially accounts for government. spending. Indced, it was seen in previous chapters that this deterministic linear trend was not necessary when we have considered real private output series, i.e. $y_{t}-g_{t}$ for variables in logs instead of $y_{t}$..

\section{Separation in Common Features}

Fixing the two cointegrating vectors to their estimated values and using test statistics based on canonical correlations proposed in Chapter 3 , we determine the dimension of the common feature space both in the WF and the SF model using the statistics $\xi_{W}$ and $\xi_{S}$ defined in Chapter 3 . Note that in the SF case the number of common feature vectors is bounded by $n-r$, i.e. by 2 in this analysis. Table 8.5 presents the results.

Under the SF model we do not retain any common feature vectors. In contrast, we obtain 


\begin{tabular}{|c|c|c|c|c|c|}
\hline$r=2$ & $\begin{array}{c}\lambda_{i}^{*} \\
\xi_{S} \quad \xi_{W}\end{array}$ & $\begin{array}{c}d f \\
\xi_{S} \quad \xi_{W}\end{array}$ & $\begin{array}{c}P b>\chi_{d f}^{2} \\
\xi_{S} \quad \xi_{W}\end{array}$ & $\begin{array}{l}L o \\
\xi_{S}\end{array}$ & $\begin{array}{r}g l l_{i} \\
\xi_{W}\end{array}$ \\
\hline$s \geq 1$ & - & - & - & 729.25 & 729.25 \\
\hline$s \geq 2$ & $0.401 \quad 0.217$ & 119 & $0.045 \quad 0.386$ & 719.245 & 724.467 \\
\hline$s \geq 3$ & $0.491 \quad 0.366$ & $24 \quad 20$ & $0.004 \quad 0.124$ & 706.056 & 715.552 \\
\hline$s \geq 4$ & $0.523 \quad 0.498$ & $39 \quad 33$ & $(<0.001) \quad 0.011$ & 691.588 & 702.084 \\
\hline$s=5$ & 0.817 & 56 & $(<0.001)<0.001$ & 658.378 & 376.215 \\
\hline
\end{tabular}

Table 8.5: Common Feature Tests

two weak form common features vectors. The normalized cofeature matrix is given by

$$
\tilde{\beta}^{\prime} \Delta y_{t}^{*}=\left(\begin{array}{rrrr}
1 & -0.671 & 0 & 0.257 \\
0 & 0.047 & 1 & -0.719
\end{array}\right)\left(\begin{array}{l}
\Delta\left(C a_{-} c_{t}\right)^{*} \\
\Delta\left(C a_{-} y_{t}\right)^{*} \\
\Delta\left(U S A_{-} c_{t}\right)^{*} \\
\Delta\left(U S A_{-} y_{t}\right)^{*}
\end{array}\right),
$$

where the subscript ${ }^{*}$ indicates that variables have been taken in the deviation to their (longrun) error-correction terms. The short run elasticities are quite plausible, namely 0.67 for Canada and 0.72 for the US. Under common feature separation, the switching algorithm proposed in the previous section yields a $\log$-likelihood value of $L R Q_{2}^{*}=714.29$ that should be compared with the value of the log-likelihood under two unrestricted common feature vectors, sec Table 8.5, namely 715.55. The LR test for separation in common feature, $\chi^{2}(2)$ under $^{14}$ the null, follows directly and yields a value of $L R^{s e p}=2.52$. Consequently we cannot reject the hypothesis of separation in weak form common features so that there apparently exist independent cycles in the USA and in Canada relative to the short run dynamics.

Finally, we estimate the complete system under the restrictions of separation in cointegration and separation in weak form common features. FIML estimates and associated asymptotic standard errors are given in Table 8.6. ${ }^{15}$ In the columns 2 and 4 , we find the estimates of the coefficients of the equations for consumption premultiplied by the common feature vectors which are $\left(\begin{array}{llll}1 & -0.475 & 0 & 0\end{array}\right)$ and $\left(\begin{array}{llll}0 & 0 & 1 & -0.682\end{array}\right)$ for Canada and the USA respectively. Under separation in common feature, the Canadian and US income elasticities are respectively 0.48 and 0.68 , namely slightly less than for the unrestricted model. In the columns 3 and 5 , the estimates of the error-correction form of the equations for income in resp. Canada and the USA are presented. Due to the significance of adjustment to error correction terms, c.g. a non diagonal $\alpha$ matrix, a P-T decomposition (whether GG or BNSW) cannot be extracted for each country independently (for more details sec Granger and Haldrup, 1997).

\footnotetext{
${ }^{14}$ Indeed the $4 \times 2$ normalized cofeature matrix contains two zeros. Separation in common feature adds two zero restrictions.

${ }^{15}$ The table reports FIML estimates under separation in cointegration and in weak form common features; asymptotic standard errors are reported in parentheses; $(\mathrm{Ca}-\mathrm{CI})_{t-1}$ denotes the cointegrating relationship found for the Canadian series, (ISSACI $)_{t-1}$ denotes the cointegrating relationship found for the US series.
} 
The test statistics for the hypotheses of block-diagonality of $\Gamma(L)$ are presented in Table 8.7. These statistics were computed under the maintained assumptions of separation in cointegration and separation in weak form common features. One can immediately observe that separation of type $\mathrm{A}^{+}$is rejeeted in all three cases. The finding is consistent with Proposition 8.8 that common feature separation does not imply type $A^{+}$separation.

In Tables 8.8-8.9, we finally report joint estimates of the coefficients of the system expressed in the common factor form

$$
\left(\begin{array}{l}
\Delta C a_{-} c_{t} \\
\Delta C a_{-} y_{t} \\
\Delta U S A_{-} c_{t} \\
\Delta U S A_{-} y_{t}
\end{array}\right)=\mu+\alpha \beta^{\prime}\left(\begin{array}{l}
C a_{-} c_{t-1} \\
C a_{-} y_{t-1} \\
U S A_{-} c_{t-1} \\
U S A_{-} y_{t-1}
\end{array}\right)+\underbrace{\left(\begin{array}{ccc}
\tilde{\beta}_{11 \perp} & 0 \\
\tilde{\beta}_{21 \perp} & 0 \\
0 & \tilde{\beta}_{32 \perp} \\
0 & \widetilde{\beta}_{42 \perp}
\end{array}\right)}_{\tilde{\beta}_{\perp}}\left(\begin{array}{l}
\text { Common Factor }{ }_{1} \\
\text { Common Factor }{ }_{2}
\end{array}\right)+\epsilon_{t} .
$$

The two common dynamic factors can be readily computed from the estimates in Table 8.9 . They are different linear combinations of the same set of lagged endogenous variables in first differences multiplied by the coefficient common to columns 2 and 3 and 4 and 5 respectively. We should point out that the results reported in Tables 8.5-8.9 were computed given estimates of the cointegration relationships obtained in a first step, so that the estimates of the short-run dynamics have standard asymptotic distributions. ${ }^{16},{ }^{17},{ }^{18}$

From the empirical results reported in Tables 8.5-8.9 and the conditions derived earlier it is immediately seen that none of the weak exogeneity conditions pointed out in Section 8.3 holds as both the hypotheses of diagonality of the error correcting matrix $\alpha$ and of the disturbance covariance matrix $\Omega$ are rejected at usual significance levels. As a by product, efficient inference requires estimation of the joint model and permanent-transitory decompositions may not be obtained separately for each country. Although the block-diagonality of the common feature matrix $\tilde{\beta}$ is not rejected by the data, so that the two common cycles appear to be country specific, the latter cycles may not be extracted country by country as they involve linear combinations of both Canadian and US dynamic.

\footnotetext{
${ }^{16}$ The two-step procedure was adopted to make sure that the parameter estimates of the matrix $\alpha$ and of the short run dynamics have standard asymptotic distributions. We also jointly estimated all parameters of the system by FIML. The value of the point estimates did not differ substantially from the two-step estimates and tests based on FML estimates led to the same conclusions as those using two-step estimates. These findings may be due to results obtained by Saikkonen (1995) for cointegrated systems who showed that FML estimates of the short-run dynamic coefficients have a standard asymptotic distribution.

${ }^{17}$ Table 8.8 reports FIML Estimates under Separation in Cointegration and in Weak Form Common Features of the Model in Common Factor Form; asymptotic standard errors are reported in parentheses; $(\mathrm{Ca} C \mathrm{CI})_{t-1}$ denotes the cointegrating relationship found for the Canadian series; (USA_CI $)_{-1}$ denotes the cointegrating relationship, found for the US series; $\left(C a_{-} C F\right)_{t-1}$ denotes the first common factor that only enters the Canadian equations; $\left(\text { l } S A \_C F\right)_{t-1}$ denotes the first common factor that only enters the US equations.

${ }^{18}$ Table 8.9 reports FIML Estimates of the common factors under Separation in Cointegration and in Weak Form Common Features of the Model in Common Factor Form: asymptotic standard errors are reported in parentheses.
} 


\begin{tabular}{|l|r|r|r|r|}
\hline \hline Dep.Var & $\Delta\left(C a_{-} c_{t}\right)$ & $\Delta\left(C a_{-} y_{t}\right)$ & $\Delta\left(U S A_{-} c_{t}\right)$ & $\Delta($ USA_y $)$ \\
& Coef.(Std.Err. $)$ & Cocf.(Std.Err. $)$ & Coef.(Std.Err.) & Coef.(Std.Err. $)$ \\
\hline$\Delta\left(C a_{-} y_{t}\right)$ & $.475(.107)$ & - & $-682(.103)$ & \\
$\Delta\left(U S A_{-} y_{t}\right)$ & - & - & & - \\
& & & & \\
Const & $-0.316(-)$ & $0.613(-)$ & $-0.010(-)$ & $0.606(-)$ \\
$\left(C a_{-} C I\right)_{t-1}$ & $-0.629(0.171)$ & $1.378(0.497)$ & $-0.148(0.076)$ & $1.355(0.394)$ \\
$\left(U S A_{-} C I\right)_{t-1}$ & $0.699(0.228)$ & $-1.075(0.551)$ & $-0.133(0.141)$ & $-1.012(0.502)$ \\
$\Delta\left(C a_{-} y_{t-1}\right)$ & & $1.132(0.374)$ & & $1.044(0.282)$ \\
$\Delta\left(C a_{-} y_{t-2}\right)$ & & $0.477(0.328)$ & & $0.767(0.271)$ \\
$\Delta\left(C a_{-} y_{t-3}\right)$ & & $0.405(0.308)$ & & $0.725(0.149)$ \\
$\Delta\left(C a_{-} c_{t-1}\right)$ & & $-0.745(0.285)$ & & $-0.939(0.231)$ \\
$\Delta\left(C a_{-} c_{t-2}\right)$ & & $-0.654(0.304)$ & & $-1.017(0.234)$ \\
$\Delta\left(C a_{-} c_{t-3}\right)$ & & $-0.627(0.319)$ & & $-0.642(0.245)$ \\
$\Delta\left(U S A_{-} y_{t-1}\right)$ & & $-2.514(0.583)$ & & $-2.165(0.459)$ \\
$\Delta\left(U S A_{-} y_{t-2}\right)$ & & $-1.616(0.477)$ & & $-1.321(0.403)$ \\
$\Delta\left(U S A_{-} y_{t-3}\right)$ & & $-.800(0.232)$ & & $-0.909(0.216)$ \\
$\Delta\left(U S A_{-} c_{t-1}\right)$ & & $2.833(0.610)$ & & $2.512(0.496)$ \\
$\Delta\left(U S A_{-} c_{t-2}\right)$ & & $2.104(0.547)$ & & $1.728(0.456)$ \\
$\Delta\left(U S A_{-} c_{t-3}\right)$ & & $1.253(0.268)$ & & $0.686(0.287)$ \\
\hline \hline
\end{tabular}

Table 8.6: FIML Estimates under Separation in Cointegration and in W F Common Features

\begin{tabular}{l|l|l|l|l}
\hline \hline Hypotheses testing & loglik & LR test & Dist. & $p$-values \\
\hline$H_{0}: \Gamma(L)$ is Block diagonal & 692.218 & 44.83 & $\chi^{2}(12)$ & $<.0001$ \\
$H_{0}: \Gamma(L)$ is lower triangular & 697.812 & 33.64 & $\chi^{2}(6)$ & $<.0001$ \\
$H_{0}: \Gamma(L)$ is upper triangular & 701.109 & 27.05 & $\chi^{2}(6)$ & $<.0001$ \\
\hline \hline
\end{tabular}

Table 8.7: Testing Short-Run Separation of Type At

\begin{tabular}{|l|r|r|r|r|}
\hline \hline Dep.Var & $\begin{array}{r}\Delta\left(C a_{-} c_{t}\right) \\
\text { Coef.(Std.Err) }\end{array}$ & $\begin{array}{r}\Delta\left(C a_{-} y_{t}\right. \\
\text { Coef.(Std.Err) }\end{array}$ & $\begin{array}{r}\Delta\left(U S A_{-} c_{l}\right) \\
\text { Coef.(Std.Err })\end{array}$ & $\begin{array}{r}\Delta\left(U S A_{-} y_{t}\right) \\
\text { Coef.(Std.Err) }\end{array}$ \\
\hline Comst & $-0.025(0.197)$ & $0.613(0.227)$ & $0.403(0.142)$ & $0.606(0.185)$ \\
$\left(C a_{-} C I\right)_{t-1}$ & $0.025(0.385)$ & $1.378(0.450)$ & $0.775(0.280)$ & $1.355(0.368)$ \\
$\left(U S A_{-} C I\right)_{t-1}$ & $0.189(0.492)$ & $-1.075(0.565)$ & $-0.823(0.356)$ & $-1.012(0.462)$ \\
& & & & - \\
$\left(C a_{-} C F\right)_{t}$ & $0.537(0.232)$ & $1.132(0.317)$ & - & - \\
$\left(U S A_{-} C F\right)_{t}$ & - & - & $0.712(0.190)$ & $1.044(0.259)$ \\
\hline \hline
\end{tabular}

Table 8.8: FIML Estimates of the Common Factor Representation 


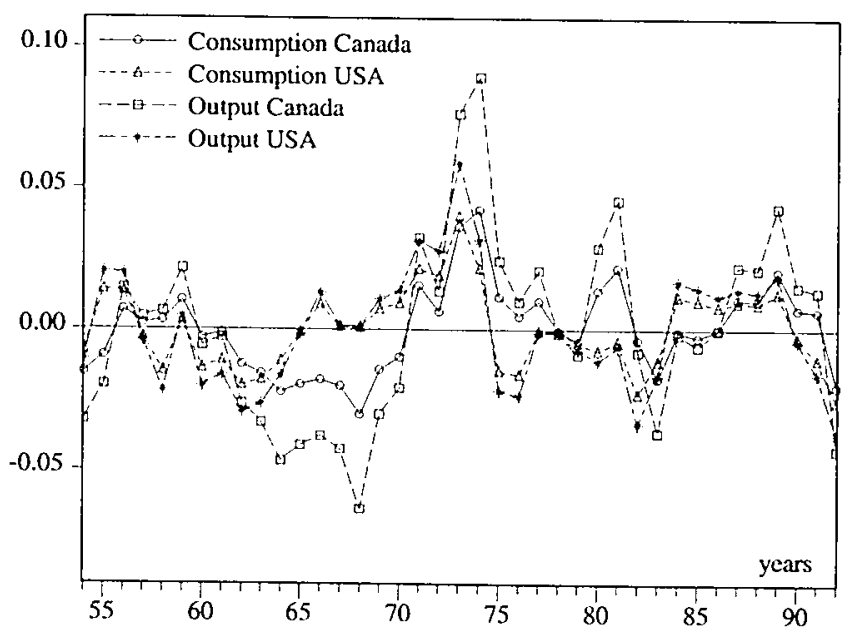

Figure 8.2: Full System, Decomposition of $y_{t}^{*}$

A final way to illustrate the relevance of our analysis and the importance of satisfying separability conditions can be based on the computation of P-T decompositions from both the full system and from the marginal sub-system. If all the different separability conditions mentioned in this paper hold, then both analyses should yield very similar decompositions up to slight difference that could arise from different normalizations. We therefore did compute the transitory components for each of the four series from both a complete system analysis and from marginal country by country analysis. As shown in Chapter 4 therc are different ways of presenting BNSW type P-T decompositions in the presence of WF common features. The first one is to investigate the decomposition of the variables in deviation from the long-run equilibrium (denoted by $y_{t}^{*}$ ) and hence to represent graphically the "common " transitory components, e.g. the components that are annihilated by the common features relations. Alternatively one may compute these BN type decompositions for the original series, denoted by $y_{t}$. In this case the transitory components are made of both a common part and a further transitory component that stems from the fact that the series in the common features analysis are taken in deviation from the long-run relationships.

Both decompositions are presented in Figures 8.2-8.7. Figure 8.2 and Figure 8.3 present the decomposition based on a full system analysis. Those reported in Figures 8.4-8.5 were obtained from the decomposition of the marginal sub-systems. The surprising differences in the graphical representation are clearly sufficient to highlight the importance of imposing valid separability 


\begin{tabular}{|l|r|r|}
\hline \hline Variable & $\left(C a_{-} C F\right)_{t}$ & $\left(U S A_{-} C F\right)_{t}$ \\
\hline$\Delta\left(C a_{-} y_{t-1}\right)$ & 1 & 1 \\
$\Delta\left(C a_{-} y_{t-2}\right)$ & $0.421(0.192)$ & $0.735(0.191)$ \\
$\Delta\left(C a_{-} y_{t-3}\right)$ & $0.357(0.159)$ & $0.694(0.158)$ \\
$\Delta\left(C a_{-} c_{t-1}\right)$ & $-0.658(0.123)$ & $-0.899(0.119)$ \\
$\Delta\left(C a_{-} c_{t-2}\right)$ & $-0.577(0.210)$ & $-0.974(0.235)$ \\
$\Delta\left(C a_{-} c_{t-3}\right)$ & $-0.554(0.187)$ & $-0.615(0.167)$ \\
$\Delta\left(U S A_{-} y_{t-1}\right)$ & $-2.221(0.384)$ & $-2.075(0.320)$ \\
$\Delta\left(U S A_{-} y_{t-2}\right)$ & $-1.427(0.359)$ & $-1.266(0.305)$ \\
$\Delta\left(U S A_{-} y_{t-3}\right)$ & $-0.707(0.223)$ & $-0.871(0.219)$ \\
$\Delta\left(U S A_{-} c_{t-1}\right)$ & $2.502(0.502)$ & $2.407(0.431)$ \\
$\Delta\left(U S A_{-} c_{t-2}\right)$ & $1.858(0.441)$ & $1.655(0.371)$ \\
$\Delta\left(U S A_{-} c_{t-3}\right)$ & $1.107(0.311)$ & $0.657(0.232)$ \\
\hline \hline
\end{tabular}

Table 8.9: Common Factors

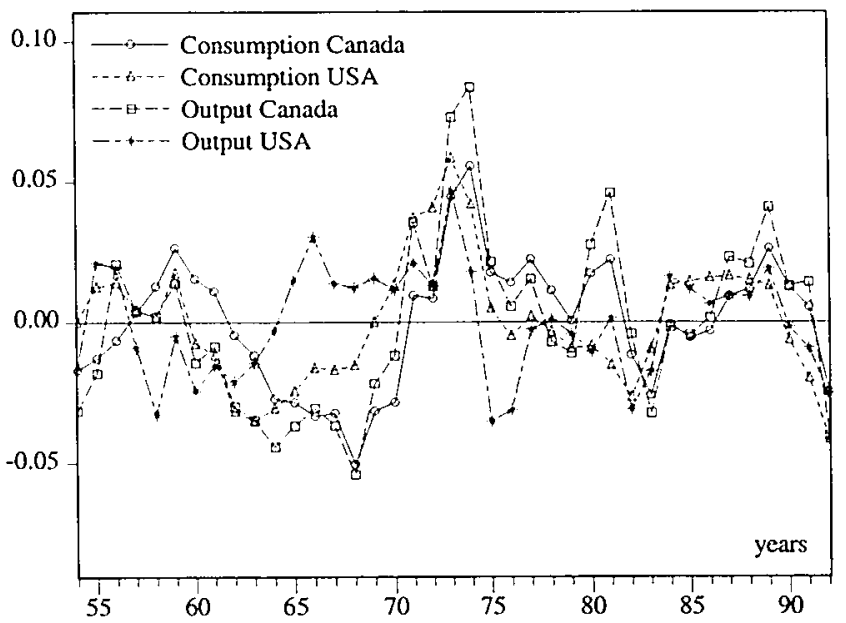

Figure 8.3: Full System, Decomposition of $y_{t}$ 


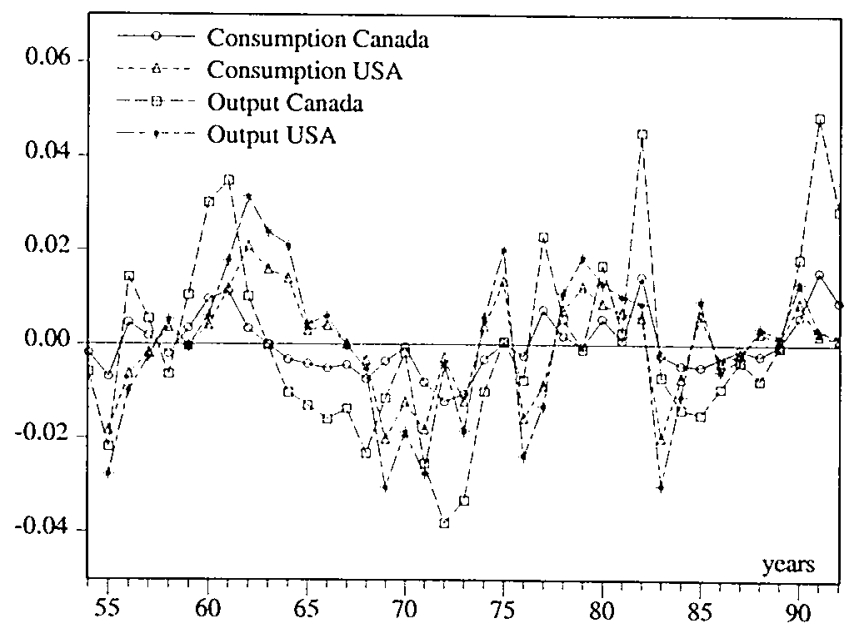

Figure 8.4: Marginal Systems, Decomposition of $y_{t}^{*}$

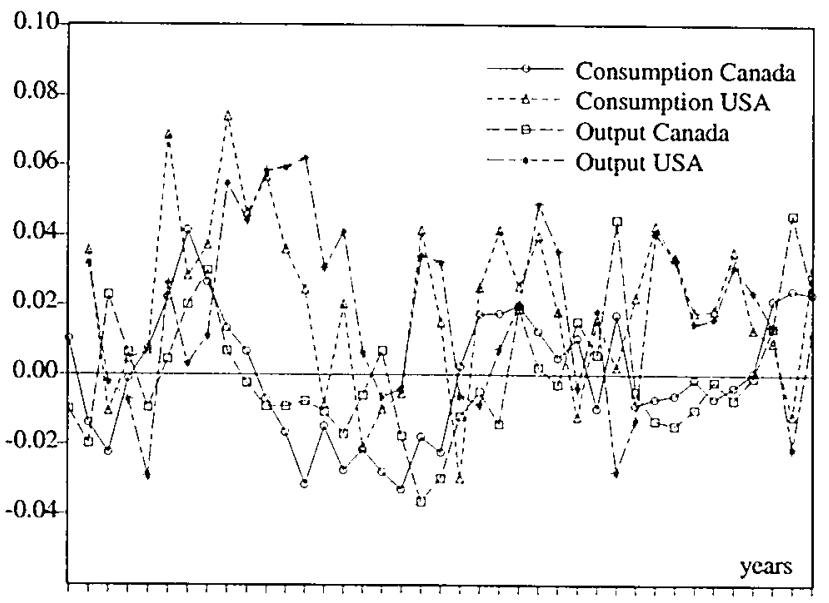

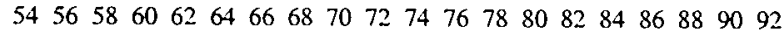

Figure 8.5: Marginal Systems, Decomposition of $y_{t}$ 


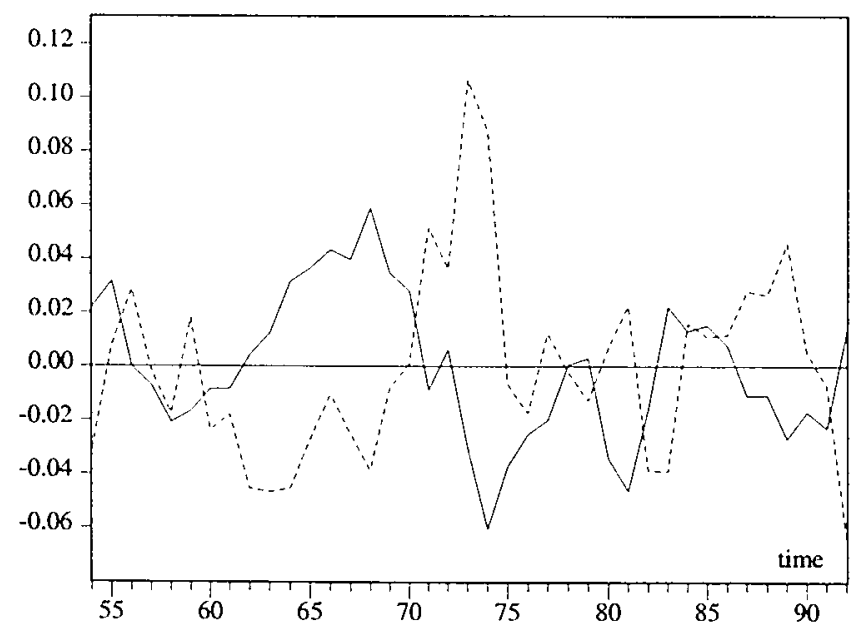

Figure 8.6: Common Cycles in the Full System

restrictions when computing P-T decompositions. Similar discrepancies in the common cyclical components can be observed from Figures 8.6-8.7 where it appears clearly that the two common cyclical components from the whole system (Figure 8.6) are substantially different from those that are obtained from the marginal systems (Figure 8.7).

\subsection{Concluding Remarks}

In this chapter, we have considered the issue of separation in cointegrated VAR models with common features by extending the analysis proposed by Granger and Haldrup (1997). In particular, we pointed out that their analysis needed to be extended to cover the case with common features. In this set-up, we have studied the relationships between various forms of separation in VAR models and the presence and form of common features. From this analysis, explicit testable parameter restrictions have been derived under which sub-group P.T analysis is equivalent to full system P-T analysis. These conditions entail both restrictions on the long-run matrices, on the loadings as well as more restrictive assumptions such as Granger Noncausality and conditional independence. We have also clarified the relationships between weak exogeneity and separability in this class of cointegrated VAR models and pointed out that the standard conditions of weak exogeneity (w.r.t. to the long-run parameters) usually considered in cointegrated VAR models need to be generalized in this class of models. 


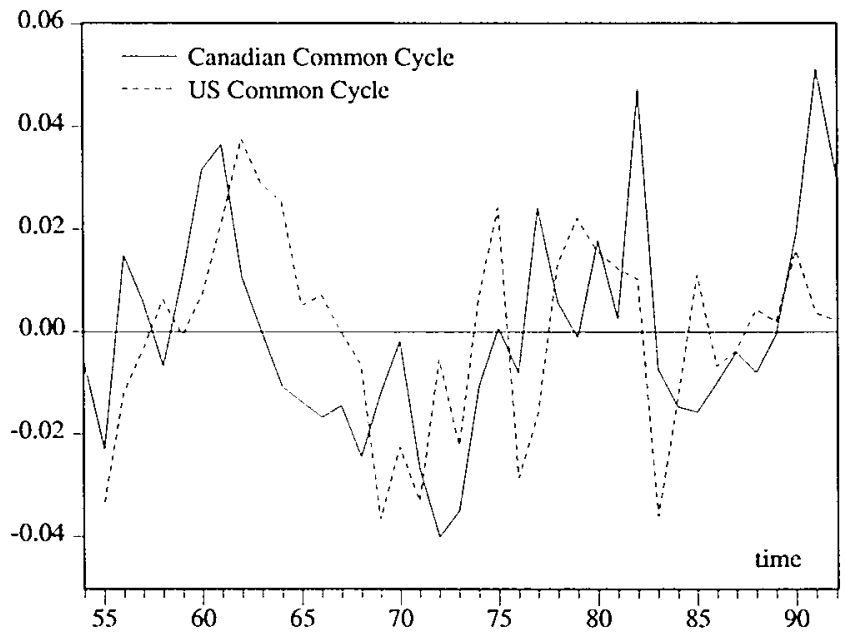

Figure 8.7: National Common Cycles in Marginal Systems

The separation restrictions considered arise in applications where sets of time series for several entities, houscholds, firms, sectors, regions or countries are analyzed. Not only do we point out the implications between the existence of common features and forms of separation, we also propose and adopt a modeling strategy that allows an investigator to test these implications in a systematic way and to finally obtain a model that is statistically validated and has restricted dynamics which can be interpreted in terms of economic theory. The analysis is illustrated using consumption and income series for both Canada and the USA which are found to satisfy separation in cointegration and which have two "common" cycles which appear to be linear combinations of lagged log-changes of the four series, but which are specific to each of the two countries so that a country by country BN decomposition of the series could lead to very misleading results.

A final important remark should be made concerning the restrictiveness of the specifications we have investigated in this chapter. While the class of models we consider here may at the first sight appear overly restrictive, it should be noted that the restrictions we proposed to investigate are actually those that are usually imposed without further investigation in the recent literature on panel data cointegration (see the surveys by Phillips and Moon, 1999; Baltagi and Kao, 2000) and panel common cycle analysis (see Chapter 9). While a very few numbers of papers allow for non-diagonal variance-covariance matrix between the subgroups of the panels, cointegration separation is indeed systematically imposed by excluding cointegration between members of a 
pancl although. With the exception of a few studies like Groen and Kleibergen(1999), Larsson and Lyhagen (1999) these restrictions are usually not explicitly discussed and studied. 


\title{
Chapter 9
}

\section{Testing for Common Cyclical Features in Nonstationary Panel Data Models}

\begin{abstract}
In this last chapter we extend the concept of serial correlation common features to panel data models. This analysis is motivated both by the need to develop a methodology to systematically study and test for common structures and comovements in panel data with autocorrelation present and by an increase in efficiency coming from pooling procedures. We propase sequential testing procedures and study their properties in a small scale Monte Carlo analysis. Finally, we apply the framework to the well known permanent income hypothesis for 22 OECD countries, 1950-1992.
\end{abstract}

\subsection{Introduction}

Serial correlation common features imply the existence of a reduced number of common dynamic factors explaining short-run comovements in economic variables. Also as for unit roots and cointegration tests, the power of common cyclical feature procedures may be low for small samples (Beine and Hecq, 1999 and Chapter 5). The power of tests might be increased by relying on panel data instead of using only time series data. Consequently, in this chapter we propose to extend these models by testing for serial correlation common features in a panel data framework. In order to avoid confusion, it is worth noticing that standard panel data models with common parameter structures obviously already imply a common feature structure, namely the one which allows to pool the bchavior of $N$ individuals. Notice that the assumption of poolability often made in panels may frequently be too strong. An investigator may want to test which poolability restrictions are supported by the data and which restrictions have to be rejected for the panel

\footnotetext{
${ }^{0}$ This chapter draws on Hecq, Palm and Urbain (2000b).
} 
data.

We propose to generalize the SCCF approach and apply it to search for common cyclical features in pancl data. In particular, we investigate whether there exist linear combinations of the variables for individual or entity $i$ which are white noise for all $i$, in other words, which weights in the linear combinations are identical across all entities. Developing a methodology to analyze and test common cyclical features in panel data is of theoretical and practical importance since common cyclical feature restrictions are less restrictive than the assumption of identical parameters across individuals usually made in panel data modeling.

Some purists might not speak about panel for this type of analysis. Indeed, in some situations we are interested in, $N$ will be relatively small compared to its value in usual panel data and $T$ is assumed large (with $T \rightarrow \infty$ asymptotics). Many macroeconomic studies deal with 15 to 50 annual observations for 20 to 100 countries, regions, industry levels or big firms. In those cases, the border between pure panel analysis $(N \rightarrow \infty)$ and pure time series analysis $(T \rightarrow \infty)$ is fuzzy. Far from impoverishing the panel data analysis, taking into account medium or large size time series raises new interesting issues such as testing for unit roots or cointegration in panel dala (see inter alia Levin and Lin, 1993; Pesaran and Smith, 1995; Evans and Karas, 1996a; Kao, 1997: Pedroni, 1997a; Phillips and Moon, 1999b, and Phillips and Moon, 1999a, for the asymptotic theory, and the recent special issue of Oxford Bulletin of Economics and Statistics, 1999).

This chapter is organized as follows. Section 9.2 illustrates serial correlation common features for the consumption function. Section 9.3 extends it to panel data. As we study differences and similarities in macroeconomic series for different countries, we concentrate our analysis on the fixed effects model (see Hsiao, 1986). Section 9.4 describes estimation procedures. In Section 9.5 simulation results are reported. In Section 9.6 we present an empirical analysis of the liquidity constraint consumption model for 22 OECD countries and the G7. Section $\mathbf{9 . 7}$ concludes.

\subsection{Common Features in Time Series}

In the context of time series analysis, SCCF means that there exist linear combinations of (stationary) economic time series which are white noise processes. Consider a cointegrated VAR of order $p=2$, written in its VECM form, for consumption and income, for $t=1 \ldots T$ :

$$
\left[\begin{array}{l}
\Delta c_{t} \\
\Delta y_{t}
\end{array}\right]=\left[\begin{array}{l}
\mu_{1} \\
\mu_{2}
\end{array}\right]+\left[\begin{array}{cc}
\lambda \phi_{21} & \lambda \phi_{22} \\
\phi_{21} & \phi_{22}
\end{array}\right]\left[\begin{array}{l}
\Delta c_{t-1} \\
\Delta y_{t-1}
\end{array}\right]+\left[\begin{array}{l}
\alpha \\
1
\end{array}\right]\left[\begin{array}{ll}
\beta_{1} & \beta_{2}
\end{array}\right]\left[\begin{array}{c}
c_{t-1} \\
y_{t-1}
\end{array}\right]+\left[\begin{array}{l}
\varepsilon_{1 t} \\
\varepsilon_{2 t}
\end{array}\right]
$$

where $\mu_{1}$ and $\mu_{2}$ are constant drift terms, $\left[\varepsilon_{1 t}, \varepsilon_{2 t}\right]^{\prime}$ is a bivariate white noise process with nonsingular covariance matrix $\Omega$. $\left(\beta_{2} / \beta_{1}\right)$ is the long-run income clasticity if one chooses consumption as normalized variable. The autoregressive coefficients matrix is of reduced rank and the system 
may be written as:

$$
\left[\begin{array}{l}
\Delta c_{t} \\
\Delta y_{t}
\end{array}\right]=\left[\begin{array}{l}
\mu_{1} \\
\mu_{2}
\end{array}\right]+\left[\begin{array}{l}
\lambda \\
1
\end{array}\right]\left[\begin{array}{ll}
\phi_{21} & \phi_{22}
\end{array}\right]\left[\begin{array}{l}
\Delta c_{t-1} \\
\Delta y_{t-1}
\end{array}\right]+\left[\begin{array}{l}
\alpha \\
1
\end{array}\right]\left[\begin{array}{ll}
\beta_{1} & \beta_{2}
\end{array}\right]\left[\begin{array}{l}
c_{t-1} \\
y_{t-1}
\end{array}\right]+\left[\begin{array}{l}
\varepsilon_{1 t} \\
\varepsilon_{2 t}
\end{array}\right] .
$$

In this simple bivariate model (9.1)-(9.2), the serial correlation common feature hypothesis may also be written in terms of moment conditions such as:

$$
\mathrm{E}\left[\left(\Delta c_{t}-\mu-\lambda \Delta y_{t}\right) \cdot W_{t}\right]=0
$$

where $\mathrm{E}[$.$] is the expectation operator and W_{t}=\left\{1, \Delta c_{t-1} \ldots \Delta c_{t-k}, \Delta y_{t-1 \ldots} \Delta y_{t-l}, z_{t-1}\right)$ is a set of instruments consisting of a constant term, the lags of both variables and the deviation from the long-run relationship $z_{t-1} \equiv c_{t-1}-\left(\beta_{2} / \beta_{1}\right) y_{t-1}$.

Adopting a two-step approach, there are two obvious ways to test for SCCF. The first way is, as in previous chapters, to carry out a canonical correlation analysis between consumption and income on the one hand and the set of instruments on the other hand. The nonsignificant squared canonical correlations reveal the existence of lincar combinations which yicld white noise processes. Alternatively, one can use generalized method of moments type estimators exploiting the moment condition (9.3). A test of overidentifying restrictions implied by (9.3) is a test of serial correlation common fcatures. The use of canonical correlation estimation has the advantage that results do not rely on the choice of the normalization of the moment conditions. Moreover, it is more convenient when we test for the number of common feature vectors. In this paper we treat the problem in a GMM framework for several reasons. Firstly, we have at most one common feature vector in a bivariate system. Secondly, this framework may be more easily extended to panel data models. Finally, normalization imposed on $I V$ by sclecting one variable as having a coefficient equal to one leads to an increase of the power of the test compared with those based on canonical correlations. ${ }^{1}$

\subsection{Extension to Panel Data Models}

Frequently, analyses comparing for instance the permanent income hypothesis (PIH) with " $\lambda$ model" (sec Chapter 8), concentrate on one country, very often the USA. In order to motivate the generality of the theory, some authors extend their empirical investigation to several countries (Campbell and Mankiw, 1991; Evans and Karas, 1996b). However it is difficult to claim that results for different countries are uncorrelated. Since it is not possible to construct a pure time series model with relatively few observations for a large number of individuals, such as a VAR model with $2 \times N$ endogenous variables in a bivariate case, alternatives must be found.

One solution would be to use the results of Chapter 8 and to analyze the system under separation in common features. Under separation in common features, the common feature matrix is

\footnotetext{
${ }^{1}$ See Anderson and Vahid (1996) for the connection between GMM and canonical correlation estimators.
} 


\begin{tabular}{|c|c|c|c|c|c|c|c|c|c|}
\hline \multirow{5}{*}{$N=2$} & & $\begin{array}{l}\text { Marg. } \\
\text { bias.5 }\end{array}$ & bias. $.5-.25$ & $x^{2}(2)$ & $\chi_{\mathrm{cor}}^{2}(2)$ & $\begin{array}{l}\text { Separ. } \\
\text { bias } 5\end{array}$ & bias $75-.25$ & $\chi^{2}(8)$ & $\chi_{c o r}^{2}(8)$ \\
\hline & $T=10$ & -.056 & 310 & 14.64 & 6.22 & -.040 & .441 & 70.98 & 12.8 \\
\hline & $T=25$ & -.026 & .155 & 7.56 & 5.20 & -.027 & .138 & 18.36 & 7.14 \\
\hline & $T=50$ & -.011 & .104 & 6.30 & 5.04 & -.013 & .090 & 10.16 & 6.16 \\
\hline & $T=100$ & -.005 & .068 & 4.86 & 4.42 & -.007 & .059 & 6.66 & 5.14 \\
\hline \multirow{5}{*}{$N=5$} & & $\begin{array}{l}\text { Marg. } \\
\text { bias.s.5 }\end{array}$ & bias $_{75-.25}$ & $x^{2}(2)$ & $\chi_{c o r}^{2}(2)$ & $\begin{array}{l}\text { Separ. } \\
\text { bias. }\end{array}$ & bias $_{.75}-.25$ & $\chi^{2}(14)$ & $\chi_{c o r}^{2}(14)$ \\
\hline & $T=10$ & -.061 & .299 & 14.14 & 5.86 & - & - & - & - \\
\hline & $T=25$ & -.025 & .152 & 7.82 & 5.44 & -.019 & .241 & 99.76 & 35.04 \\
\hline & $T=50$ & -.012 & .100 & 6.30 & 5.18 & -.011 & .087 & 62.88 & 15.26 \\
\hline & $T=100$ & -.006 & .069 & 5.58 & 5.04 & -.007 & .052 & 25.18 & 9.38 \\
\hline
\end{tabular}

Table 9.1: Monte Carlo Results: Separated vs. Marginal Systems

block-diagonal with blocks corresponding to one individual $i$ only. Treating the issue in the complete system with separation in common features avoids losing efficiency compared to an analysis of the marginal model for individual $i$ since separation does not require block-diagonality of the disturbance covariance matrix. This solution is however difficult to implement for more than two or three individuals. We illustrate this point via a small Monte Carlo experiment, of which the precise specification will be given in Section 9.5. Consider a DGP made out of bivariate systems similar to (9.2), with $\alpha=\lambda$ (SCCF hypothesis), for respectively two and five individuals. The only cross-sectional relations are due to a non-diagonal disturbance covariance matrix. Complete separation in cointegration, in common features as well as absence of bidirectional short-run Granger causality are thus maintained. Using a standard canonical correlation framework (see Chapter 3) we perform a serial correlation common feature analysis in the marginal model for the first individual, ignoring the disturbance cross-correlations. Alternatively, under separation in common features, we test the number ( $s=2$ or $s=5$ ) of common feature vectors for each individual in the complete system. We then constrain the common feature space to be block-diagonal and estimate the vector for the first individual.

In Table 9.1, we report for 5,000 replications the median and the sprcad (interquartile range) of the bias, $x^{2}$ test statistics for the overidentifying restrictions implied by the presence of common features as well as a small sample adjusted version (see Chapter 5). Although separation in common features holds at the level of the DGP, some efficiency loss, as measured by the spread, is observed in the marginal model compared to the full system for $T=25$ for $N=2$ and for $T=50$ for $N=5$. However the dispersion is too high for smaller sample size and test statistics reject too often the presence of respectivcly two and five common feature vectors.

These illustrative Monte Carlo results call for an extension to a (possibly nonstationary) panel common feature analysis. Let the subscript $i=1, \ldots, N$ indicate the different groups/entities/units, 
$t=1, \ldots, T$ denote the sample period and $j=1, \ldots, n$ denote the number of variables for each group/entity. We assume that the $n$-dimensional vector of observed $I(1)$ variables for entity $i$, $X_{i, t}$, is generated by a $p_{i}$-th order cointegrated VAR which can be expressed in error-correction form as follows:

$$
\Delta X_{i, t}=\mu_{i}+\gamma_{t}+\alpha_{i} \beta_{i}^{\prime} X_{i, t-1}+\sum_{j=1}^{p_{i}-1} \Gamma_{i, j} \Delta X_{i, t-j}+\varepsilon_{i, t}, \quad i=1, \ldots N, \quad t=1, \ldots, T,
$$

where $\mu_{i}$ denotes fixed individual effects, $\gamma_{t}$ denotes a vector of deterministic time effects, $\alpha_{i}$ and $\beta_{i}$ are $n \times r_{i}$ matrices of full column rank with $r_{i}$ being the cointegrated rank $\left(r_{i}<n\right)$ and $\varepsilon_{i, t}$ is a disturbance. The vector $\varepsilon_{t}=\left(\varepsilon_{1, t}^{\prime}, \ldots, \varepsilon_{N, t}^{\prime}\right)^{\prime}$ is an $n N \times 1$ dimensional homoskedastic Gaussian mean innovation process relative to $X_{-1}=\left\{X_{i, t-j}, i=1, \ldots, N ; j<t\right\}$ with nonsingular contemporaneous covariance matrix $\Omega$, the $(i, j)-t h$ block of which being $E\left(\varepsilon_{i, t} \varepsilon_{j, t}^{\prime}\right)=\Omega_{i, j}$. Notice that one could allow for random individual effects in expression (9.4). This would lead to an error-component structure of $\varepsilon_{i, t}$ similar to that used in the panel-data literature.

For system (9.4), we define a homogeneous SCCF panel model as follows:

Definition 9.1 A panel model is called an homogeneous panel common feature model if there exists, $\forall i=1, \ldots, N, a\left(n \times s_{i}\right)$ matrix $\tilde{\beta}_{i}=\tilde{\beta}_{j} \forall i, j=1, \ldots, N$, whose columns span the individual co-feature space, such that $\tilde{\beta}_{i}^{\prime} \Delta X_{i, t}=\tilde{\beta}_{i}^{\prime} \varepsilon_{i, t}$ is a $s_{i}$-dimensional white noise process for each individual.

This definition applies to the case where the individual co-feature matrices $\tilde{\beta}_{i}$, and hence their column ranks $s_{i}$, are the same across all individuals. A typical dynamic panel data model with fixed effects $\mu_{i}$ and deterministic time effects $\gamma_{t}$ arises as a special case of (9.4) when the parameters $\alpha_{i}, \beta_{i}, \Gamma_{i, j}$ and $\Omega_{i}$ are the same across entities $i$ (sec e.g. Hoogstrate, 1998). In order to clarify the nature of the hypotheses underlying the panel common feature restrictions, in the next subsection, following Groen and Kleibergen (1999) for panel cointegration, we consider a model resulting from sequentially testing and imposing restrictions to a high dimensional unrestricted VECM.

\subsubsection{A Panel VECM Representation}

We are interested in testing for cointegration and common serial features with respect to $n I(1)$ time series in vector $X_{i, t}$ within a dynamic model for $N$ individuals $i$. Without loss of generality, we consider a large VECM with one lag in the first differences, e.g. a VAR with two lags in levels. The generalization to high order dynamics is immediate by substituting $\Gamma_{i j}$ by $\Gamma_{i j}(L)$ in (9.5) but it makes the notation heavy. We consider the model without any time dummies for 
sake of simplicity. For $t=1, \ldots, T$ we may write the $n N \times 1$-dimensional system as:

$$
\Delta X_{t}=\left(\begin{array}{ccc}
\Pi_{11} & \ldots & \Pi_{1 N} \\
\vdots & \ddots & \vdots \\
\Pi_{N 1} & \ldots & \Pi_{N N}
\end{array}\right) X_{t-1}+\left(\begin{array}{ccc}
\Gamma_{11} & \ldots & \Gamma_{1 N} \\
\vdots & \ddots & \vdots \\
\Gamma_{N 1} & \ldots & \Gamma_{N N}
\end{array}\right) \Delta X_{t-1}+u_{t},
$$

where $\Delta X_{t}=\left(\Delta X_{1, t}^{\prime} \ldots \Delta X_{N, \ell}^{\prime}\right)^{\prime}, u_{\ell}=\left(u_{1, \ell}^{\prime} \ldots u_{N, \ell}^{\prime}\right)^{\prime}$ and $X_{t-1}=\left(X_{1, t-1}^{\prime} \ldots X_{N, t-1}^{\prime}\right)^{\prime}$ are vectors of dimension $n N \times 1$, or more concisely

$$
\Delta X_{t}=\Pi_{u r} X_{t-1}+\Gamma_{u r} \Delta X_{t-1}+u_{t}
$$

where $\Pi_{u r}$ and $\Gamma_{u r}$ are $n N \times n N$ matrices $^{2}$ and $u_{t}=\mu+\varepsilon_{t}, \mu=\left(\mu_{1}^{\prime}, \ldots, \mu_{N}^{\prime}\right)^{\prime}, \varepsilon_{t}=\left(\varepsilon_{1, t}^{\prime}, \ldots, \varepsilon_{N, t}^{\prime}\right)^{\prime}$ are $n N \times 1$ vectors with $\varepsilon_{t} \sim N(0, \Omega)$.

$$
\underset{n N \times n N}{\Omega}=\left(\begin{array}{ccc}
\Omega_{11} & \ldots & \Omega_{1 N} \\
\vdots & \ddots & \vdots \\
\Omega_{N 1} & \cdots & \Omega_{N N}
\end{array}\right)
$$

Without imposing any zcro block restrictions, the large unrestricted model (9.5) is not estimable in practice. Consequently, restrictions have to be considered. We first describe cointegrating restrictions before introducing serial correlation common feature restrictions.

\section{Cointegrating Restrictions in a Panel VAR}

We first consider restrictions on the long-run matrix $\Pi_{u r}$ in the unrestricted VECM. Two types (A and B) of sequences of hypotheses naturally arise in panel data. The hypothesis involved in a sequence can be tested either sequentially or jointly.

- A1: Absence of long-run Granger-Causality (see Granger and Lin, 1995) between the individual subgroups, i.e. $\Pi_{u r}$ is block-diagonal with elements $\Pi_{i j}=0$ for $i \neq j$.

- A2: Cointegration in absence of long-run Granger-causality, i.e. $\Pi_{i i}=\alpha_{i} \beta_{i}^{\prime}$, with $\alpha_{i}$ and $\beta_{i}$ being $n \times r_{i}$ matrices of rank $r_{i}, i=1, \ldots, N$.

- A3: Homogeneous panel cointegration, i.e. $\beta_{i}=\beta_{1}, i=1, \ldots, N ; r=N r_{1}$.

- B1: Cointegration, i.e. $\Pi_{u r}=\alpha \beta^{\prime}$, with $\alpha$ and $\beta$ being $n N \times r$ matrices of rank $r$.

- B2: Complete separation in cointegration (sec Granger and Haldrup, 1997), i.e. $\alpha$ and $\beta$ are block-diagonal with typical blocks $\alpha_{i}$ and $\beta_{i}$ respectively, of rank $r_{i}$, such that a typical block of $\alpha \beta^{\prime}$ is $\alpha_{i} \beta_{i}^{\prime}$ as defined in A2, and $r=\Sigma_{i=1}^{N} r_{i}$.

\footnotetext{
${ }^{2}$ When $\Pi_{u r}=0$, the system (9.6) is non-cointegrated. The approach presented can obviously be applied to non-cointegrated systems.
} 
- B3: Homogeneous panel cointegration, i.c. $\beta_{i}=\beta_{1} ; i=1, \ldots, N ; r=N r_{1}$.

When the first two sets of restrictions in either sequence hold, the following restricted structure arises.

$$
\Delta X_{t}=\left(\begin{array}{ccc}
\alpha_{1} \beta_{1}^{\prime} & 0 \ldots & 0 \\
0 & \ddots & 0 \\
0 & 0 \ldots & \alpha_{N} \beta_{N}^{\prime}
\end{array}\right) X_{t-1}+\left(\begin{array}{ccc}
\Gamma_{11} & \ldots & \Gamma_{1 N} \\
\vdots & \ddots & \vdots \\
\Gamma_{N 1} & \ldots & \Gamma_{N N}
\end{array}\right) \Delta X_{t-1}+u_{t}
$$

When it is appropriate to add a restricted trend in the cointegration space, we replace $X_{t-1}$ by $X_{t-1}^{*}=\left(X_{t-1}^{\prime}, t\right)^{\prime}$. For $N$ fixed, a likelihood ratio statistic for testing (9.8) versus (9.5) can be obtained using the sum of two different conditional likelihood ratio statistics to test the sets of restrictions $\{A 1, A 2\}$ or $\{B 1, B 2\}$. Next, homogencity of panel cointegration can be tested using a likelihood ratio test. A decomposition similar to $\{A 1, A 2\}$ is proposed by Groen and Kleibergen (1999). The main problen with this approach is that under A1, that is absence of long-run Granger-causality, the usual tests have an unknown asymptotic distribution, as the possible presence of cointegration interferes with the block-diagonality of $\Pi_{u \text { rr }}$. On the other hand, once the cointegrating rank in the unrestricted VECM has been fixed, a test statistic with separation as the null hypothesis has an $\chi^{2}$-asymptotic distribution. It is worthwhile to mention that although model (9.8) looks rather specific, it is less restrictive than the models used in the dynamic panel literature, where quite trequently, in addition to separation in cointegration, the same parameter structure is assumed to hold across individuals (see inter alia the overview in Phillips and Moon, 1999b). Occasionally, complete separation is relaxed to requiring $\beta$ to be block-diagonal leaving $\alpha$ unrestricted. (Larsson and Lyhagen, 1999).

\section{Common Feature Restrictions}

Imposing serial correlation common feature and short-run Granger-noncausality restrictions, system (9.8) becomes:

$$
\Delta X_{t}=\left(\begin{array}{ccc}
\tilde{\beta}_{\perp 1} \Psi_{1} \beta_{1} & 0 \ldots & 0 \\
0 & \ddots & 0 \\
0 & 0 \ldots & \tilde{\beta}_{\perp N} \Psi_{N} \beta_{N}
\end{array}\right) X_{t-1}+\left(\begin{array}{ccc}
\tilde{\beta}_{\perp 1} \Psi_{1}^{*} & 0 \ldots & 0 \\
0 & \ddots & 0 \\
0 & 0 \ldots & \tilde{\beta}_{\perp N} \Psi_{N}^{*}
\end{array}\right) \Delta X_{t-1}+u_{t}
$$

As for cointegrating restrictions, this model may be obtained by considering two of the next four hypotheses under (9.8).

- C1: Serial correlation common features: there exists a $(n N \times s)$ matrix $\tilde{\beta}$ such that $\tilde{\beta}^{\prime} \Delta X_{t}$ is an $s$ dimensional white noise, with $s=\sum_{i=1}^{N} s_{i}$. 
- C2: Absence of short-run Granger-causality between the individual subgroups: $\Gamma_{u r}$ is block-diagonal, i.e. $\Gamma_{i j}=0$ for $i \neq j$.

- C3: Separation in common features: the matrix $\widetilde{\beta}$ is block-diagonal with the $\left(s_{i} \times n\right)$ matrix $\tilde{\beta}_{i}$ being a typical block on the main diagonal, $s=\sum_{i=1}^{N} s_{i}$.

- C4: Homogeneity of common features: $\widetilde{\beta}_{i}=\widetilde{\beta}_{1} ; i=1, \ldots, N ; s=N s_{1}$.

Actually the hypothesis C2 is implicit when one stacks VECMs. Restriction C3 is developed in Chapter 8 for the SCCF as well as for the weak form structure. Here again a likelihood ratio for testing model (9.9) versus (9.8) can be obtaincd as the sum of two conditional likelihood ratio statistics to test either $\{\mathrm{C} 1, \mathrm{C} 2\}$ or $\{\mathrm{C} 2, \mathrm{C} 3\}$. This means that we can first test for common cyclical features under the maintained hypothesis of short-run Granger-noncausality C2. Alternatively, we can first test for absence of short-run causality and then test for SCCF since both sequences of restrictions imply separation in common features. This result is derived in Chapter 8 which states that under separation in cointegration and block-diagonality of this long-run matrix, the presence of common features implies that the co-feature matrix is block-diagonal.

\subsection{GMM Estimation}

To test for common features in a time series context, we have the choice between GMM estimators applied to a regression framework and a canonical correlation procedurc based on maximum likelihood (ML) estimation. Both methods have their advantages and drawbacks. The ML estimation is fully efficient and likelihood ratio tests are asymptotically most powerful. GMM estimators can be more easily implemented and especially more casily extended to our panel framework but they are in general not fully efficient.

Actually, since the beginning of this thesis, we have favored ML estimation through a canonical correlation analysis (except the Okun's law in Chapter 2). Indeed, we wanted to focus on the existence but also on the number of common cyclical feature relationships within a set of time series. But, the development of common feature test statistics in a panel data context raises a series of non trivial issues we leave for further research. For instance: how to "pool" systems of equations with a different number of variables and how to analyze in panel countries for which there exists a different cointegrating rank and/or a different number of common feature relationships?; what is the more appropriate way to take care of cross-correlation when we stack simultaneous equation systems?; is the SURE type estimator still efficient in this case and what are exactly the instruments we have to consider at each step?; how is it possible to obtain standard errors of the common feature vectors to construct confidence intervals? This latter problem is very important when we think that it is very recently, say 5 years, that standard errors are routinely implemented in econometric software when calculating cointegrating vectors in the Johansen's framework. Consequently, for the ease of our analysis and to overcome at this stage 
several unsolved issues, we take the road people have followed for the extension of unit roots and cointegration test statistics to panel data, namely estimators based on a regression approach. We are well aware that this choice can create some distortions if some of the assumptions underlying this approach do not hold, as it is the case for the Engle and Granger (1987) cointegration test. versus the Johansen's ML one.

We thinks however that this choice may be appropriate for the empirical application we use in this chapter. Indeed, our illustration considers only two variables, i.e. consumption and income, for a set of country. In this bivariate case we can get at most one cointegrating and one common feature vector. Moreover, there is a natural practice to take consumption as the dependent. variable.

Let us thus present a GMM estimator that will be applied in section 9.6. For each individual, let us split $X_{i, t}=\left(y_{i, \ell}, z_{i, t}\right)^{\prime}$ and let the bivariate DGP be

$$
\begin{aligned}
& \Delta y_{i, t}=\mu_{i}+\tilde{\beta}_{i}^{*} \Delta z_{i, t}+\eta_{i, t}, \\
& \Delta z_{i, t}=\alpha_{i}\left(y_{i}-\beta_{i}^{*} z_{i}\right)_{t-1}+\sum_{k=1}^{p_{i}-1} \delta_{i, 1 k} \Delta y_{i, l-1}+\sum_{k=1}^{p_{i}-1} \delta_{i, 2 k} \Delta z_{i, t-1}+\varepsilon_{i, t},
\end{aligned}
$$

where the second equation for $\Delta z_{i, t}$ is just one row of the VECM (9.8), with normalized cointegrating vector $\beta_{i}^{\prime}=\left(1,-\beta_{i}^{*}\right)$. Both the $y^{\prime} s$ and the $z^{\prime} s$ are autocorrelated as the disturbance depends on lagged values of $\Delta y_{i, t}, \Delta z_{i, t}$ and error correction mechanism. Under the null of serial correlation common features for individual $i, \eta_{i, t}$ is a white noise process and the normalized SCCF vector is given by $\tilde{\beta}_{i}^{\prime}=\left(1,-\tilde{\beta}_{i}^{*}\right)$.

In practice (Vahid and Engle, 1993, 1997), after the cointegration analysis in the first step, the GMM procedure proceeds as follows. Regress the explanatory variables $\Delta z_{t}$ on the whole set of instruments (i.e. lags of $\Delta X_{t}$ and cointegrating vectors) in order to obtain the best linear prediction $\Delta \hat{z}_{\ell}$. Then regress $\Delta y_{\ell}$ on a constant term and $\Delta \hat{z}_{\ell}$. This estimate gives the potential serial correlation common feature vector $\tilde{\beta}_{i}$. Finally, one tests for the validity of the overidentifying restrictions using Hansen's (1982) $\chi^{2}$ test (sce inter alia Hamilton.1994, p. 418421; Anderson and Vahid, 1998; Vahid and Engle, 1997 for a practical implementation ).

\subsubsection{Heterogeneous Independent Case}

When the observations on individuals are assumed cross-sectionally independent, a joint test for the existence of one individual-specific (heterogeneous) common feature vector can be obtained by computing the $\chi^{2}$-statistics for the SCCF restrictions for each individual $\left[\zeta_{i} \backsim \chi^{2}\left(\nu_{i}\right)\right]$, with the same number of variables for each $i$ but with the possibility of having a different dynamics and the presence or not of cointegrating vectors. The number of degrees of freedom is then given by $\nu_{i}=n\left(p_{i}-1\right)+r_{i}-(n-1)$ since $s_{i}$ equals one. Using the standard central limit theorem for 
$\operatorname{large} N$, we then have

$$
\frac{\sum_{i=1}^{N} \zeta_{i}-\nu}{(2 \nu)^{1 / 2}} \stackrel{a}{\sim} N(0,1) \quad \text { where } \nu=\sum_{k=1}^{N} \nu_{i}
$$

This procedure is however not appropriate in the presence of cross-correlation, a phenomenon pointed out inter alia by O'Connell (1998) in the case of panel unit root tests. The size distortions increase with $N$ and with the cross-correlation. While these distortions are DGP-dependent, we observe empirical sizes of about $20 \%$ (nominal size $=5 \%$ ) for $T=25$ and $N=10$ as well as for $T=50$ and $N=25$ using a Monte Carlo experiment similar to the one presented in Section 9.5. ${ }^{3}$

\subsubsection{Homogeneous and Heterogeneous Dependent Case}

In most cases disturbances across individuals $i$ will be at least contemporaneously correlated i.e. if some $\Omega_{i j} \neq 0$ for $i \neq j$, and/or for $\Omega_{i i}$ bcing non-diagonal for some $i$. For instance, when testing for PPP in panel data, contemporaneous disturbance correlation arises because one country must. serve as a benchmark. Also, for instance, for a given country consumption and income cannot be assumed independent. One way to deal with this cross-country correlation is to incorporate a common time dummy in the panel. This solution was pursued by Pedroni (1997b) in the contcxt of panel cointegration test, but it appears that time dummies do not capture all the correlation, see O'Connel (1998). Another solution we use here is to account for cross-correlation by using GLS or SUR type corrections. These corrections require that $T>N$ and the asymptotics we consider are mainly based on $T \rightarrow \infty$ while $N$ is fixed or at least grows at a lower rate than $T$.

Assuming that all the variables in levels are $I(1)$, we first test for each individual $i$ the existence of a cointegrating relationship using standard time series based procedures. In the case the null hypothesis of no-cointegration can be rejected, the cointegration vector(s) are then considered as known in the subsequent analyses. An alternative to the time series based cointegration analysis is to rely on a test procedure designed for cointegrated panel models, a procedure which could possibly be more powerful. The asymptotic arguments used in panel cointegration analysis are however mainly based on large $N$-asymptotics and independence across units while we are here dealing with fixed $N$ cases allowing for dependence across the units. Existing Monte Carlo simulations furthermore reveal (see inter alia McCoskey and Kao, 1998b, Pedroni, 1997b) the occurrence of some problems when cross-correlation exists. Moreover, the properties of common feature test statistics will be affected by the outcome of the cointegration analysis. Indeed, if one crroneously imposes an identical homogeneous cointegrating matrix $\beta_{i}^{*}$ for all $i$, while for some $j$ cointegration does not hold or holds with a cointegrating matrix different from $\beta_{i}^{*}$, the likelihood to reject the SCCF restrictions will tend to increase.

\footnotetext{
${ }^{3}$ Complete results are available upon request.
} 
Before presenting the GMM-estimator, we present the model under common features in general terms. Under the assumption of separation C3, the model (9.8) can be written as

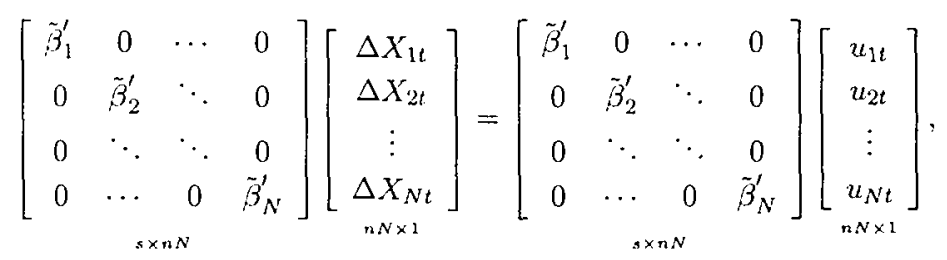

with $s=\sum_{i=1}^{N} s_{i}$ and $u_{t}=\left(u_{1 t}^{\prime}, u_{2 t}^{\prime}, . ., u_{N t}^{\prime}\right)^{\prime}$ being $\operatorname{IIN}(0, \Omega)$.

Under the homogeneity assumption $\mathrm{C} 4$, the model (9.13) specializes to become

$$
\left(I_{N} \otimes \tilde{\beta}_{1}^{\prime}\right) \Delta X_{t}=\left(I_{N} \otimes \tilde{\beta}_{1}^{\prime}\right) u_{t}
$$

As in (9.10) and (9.11), we partition the vector $\Delta X_{i t}$ as $\left[\Delta y_{i t}^{\prime}, \Delta z_{i t}^{\prime}\right]^{\prime}$, where $\Delta y_{i t}$ and $\Delta z_{i t}$ are $s_{i} \times 1$ and $\left(n-s_{i}\right) \times 1$ subvectors. The matrix $\tilde{\beta}_{i}^{\prime}$ is normalized (without loss of generality) as follows $\tilde{\beta}_{i}^{\prime}=\left[I_{s_{i}},-\tilde{\beta}_{i}^{* \prime}\right]$. Under this normalization, the system $(9.13)$ can be expressed as

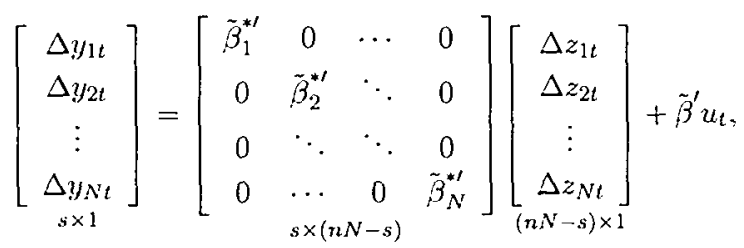

or more compactly

$$
\Delta y_{t}=B^{\prime} \Delta z_{t}+v_{t}
$$

with $\Delta y_{t}=\left\langle\Delta y_{1 t}^{\prime}, \ldots, \Delta y_{N t}^{\prime}\right]^{\prime}, B^{\prime}=\operatorname{diag}\left(\tilde{\beta}_{i}^{* \prime}\right), \Delta z_{t}=\left[\Delta z_{1 t}^{\prime}, \ldots, \Delta z_{N t}^{\prime}\right]^{\prime}, v_{t}=\tilde{\beta}^{\prime} u_{t}, \tilde{\beta}^{\prime}=\operatorname{diag}\left(\tilde{\beta}_{i}^{\prime}\right)$. Transposing (9.16) and writing the model for a sample of $T$ observations, we get

$$
\underset{T \times s}{\Delta Y}=\underset{T \times(n N-s)(n N-s) \times s}{\Delta Z}+\underset{T \times s}{V}
$$

or in vectorized form

$$
\underset{T s \times 1}{\Delta y^{*}}=\underset{T s \times\left(n s-\Sigma_{i} s_{i}^{2}\right)\left(n s-\Sigma_{i} s_{i}^{2}\right) \times 1}{\Delta Z^{*}}+\underset{T s \times 1}{v^{*}}
$$

with $\Delta y^{*}=\operatorname{vec}(\Delta Y), v^{*}=\operatorname{vec}(V), \Delta Z^{*}=\operatorname{diag}\left(I_{s_{i}} \otimes \Delta Z_{i}\right)$ with $\Delta Z_{i}=\left[\Delta z_{i t l}\right\}$, of dimension $T \times\left(n-s_{i}\right)$, with $t=1, \ldots, T, l=1, \ldots, n-s_{i}$; and $\delta$ being a vector with typical $i$-th subvector being equal to $\operatorname{vec}\left(-\tilde{\beta}_{i}^{*}\right)$. Under the homogeneity assumption $\mathrm{C} 4, \tilde{\beta}_{i}^{*}=\tilde{\beta}_{1}^{*}, i=1, \ldots, N, s=N s_{1}$, 
the system (9.18) specializes to become

$$
\Delta y^{*}=\Delta Z_{r}^{*} \delta_{r}+v^{*}
$$

with the $\left[T N s_{1} \times s_{1}\left(n-s_{1}\right)\right]$ matrix

$$
\Delta Z_{r}^{*}=\left[\begin{array}{c}
I_{s_{1}} \otimes \Delta Z_{1} \\
I_{s_{1}} \otimes \Delta Z_{2} \\
\cdots \\
I_{s_{1}} \otimes \Delta Z_{N}
\end{array}\right],
$$

and the $\left[s_{1}\left(n-s_{1}\right) \times 1\right]$ vector $\delta_{T}=\operatorname{vec}\left(-\tilde{\beta}_{1}^{*}\right)$.

The vector of parameters $\delta$ and $\delta_{r}$ can be estimated by GMM provided we have a (Ts $\left.\times k\right)$ matrix of instrumental variables $W$ such that $E W^{\prime} v^{*}=0$ and $k$ is equal to or larger than the number of unknown parameters in $\delta$ (or $\delta_{r}$ ).

The GMM estimator solving $W^{\prime} v^{*}=0$ using the weighting matrix $S$ is given by

$$
\hat{\delta}_{G M M}=\left[\Delta Z^{* \prime} W S^{-1} W^{\prime} \Delta Z^{*}\right]^{-1} \Delta Z^{* \prime} W S^{-1} W^{\prime} \Delta y^{*} .
$$

The optimal weighting matrix ${ }^{4}$ is $S=W^{\prime} \Sigma W$, where $\Sigma=E v^{*} v^{* \prime}=I_{T} \otimes \Sigma_{v}, \Sigma_{v}=\tilde{\beta}^{\prime} \Omega \tilde{\beta}$. When $\Sigma$ is unknown, it will have to be replaced by a consistent estimate. The asymptotic covariance matrix of $\hat{\delta}_{G M M}$ with optimal weighting matrix $S$ is given by

$$
\operatorname{Var}\left(\hat{\delta}_{G M M}\right)=\left[\Delta Z^{* \prime} W\left(W^{\prime} \Sigma W\right)^{-1} W^{\prime} \Delta Z^{*}\right]^{-1} .
$$

Under homogeneity $\mathrm{C} 4, \delta_{r}$ can be estimated by expression (9.20) replacing $\Delta Z^{*}$ by $\Delta Z_{r}^{*}$. When the number of instruments $k$ is strictly larger than the number of parameters $\delta$ (or $\delta_{r}$ ) to be estimated, these overidentifying restrictions can be tested using the well-known minimum distance criterion

$$
\min _{\delta}\left(v^{* \prime} W\right)\left(W^{\prime} \Sigma W\right)^{-1}\left(W^{\prime} v^{*}\right)
$$

which has an asymptotic $\chi^{2}$-distribution with the number of degrees of freedom being equal to $k$ minus the number of estimated parameters.

Some remarks on the choice of the instruments have to be made. We can determine the order $p_{i}$ of the VAR for each country $i$ using for instance information criteria. The lagged first differences of $\Delta X_{i \ell}, i=1, \ldots, p_{i}-1$, and the lagged long-run relations can be used to yield $n\left(p_{i}-1\right)+r_{i}$, instruments $W_{i}$ for $\Delta Z_{i}$ in $(9.16)$ and taking $W=\operatorname{diag}\left(\iota_{T s_{i}}, W_{i}\right)$ where $r_{i}$ is the cointegrating rank of individual $i$. As is well-known, the OLS estimator regressing $\Delta y^{*}$ on

\footnotetext{
${ }^{4}$ See e.g. Hamilton (1994, p.412-413), Anderson and Vahid, (1998) and Vahid and Engle (1997).
} 
$\Delta \widehat{Z}^{*}$, where the $\Delta \widehat{Z}^{*}$ are the projections of $\Delta Z^{*}$ on $W$, can be obtained as a GMM estimator by selecting $S=I_{T s}$ in $(9.20)$ and taking $W\left(W^{\prime} W\right)^{-1} W^{\prime}$ as instrument. Similarly, the GIS estimator regressing $\Delta y^{*}$ on $\Delta \hat{Z}^{*}=W\left(W^{\prime} \Sigma^{*-1} W\right)^{-1} W^{\prime} \Sigma^{*-1} \Delta Z^{*}$, with $\Sigma^{*}$ being the disturbance covariance matrix of the (multivariate) regression of $\Delta Z^{*}$ on $W$, can be obtained from $(9.20)$ by taking $S=\Sigma$ and using as instruments $W\left(W^{\prime} \Sigma^{*-1} W\right)^{-1} W^{\prime} \Sigma^{*-1}$ instead of $W$.

In the empirical analysis in Section 6.6, we consider a fixed effects model because in the macrocconomic application, we study the population and not a sample. Adding fixed effects to the model (9.18) for the case which we analyze, e.g. for $s_{i}=1, i=1, \ldots, N$ and $n=2$, yields

$$
\Delta y=Z_{\mu} \mu\left[+Z_{\lambda} \lambda\right]+\Delta Z_{r}^{*} \delta_{r}+v^{*}
$$

where $Z_{\mu}=\iota_{T} \otimes I_{N}$ and $Z_{\lambda}=I_{T} \otimes \iota_{N}$, with $\iota_{T}$ and $\iota_{N}$ being unit vectors of dimension $T$ and $N$ respectively. Let $J_{N}$ denote a $N \times N$ matrix of ones, so $Z_{\lambda} Z_{\lambda}^{\prime}=I_{\Gamma} \otimes J_{N}$ and the projection of $J_{N}$ on $Z_{\lambda}$ is $I_{T} \otimes \bar{J}_{N}$ with $\bar{J}_{N}=J_{N} / N$. This matrix averages over individuals. Also define time means by $Z_{\mu} Z_{\mu}^{\prime}=J_{T} \otimes I_{N}$ and the projection of $J_{T}$ on $Z_{\mu}^{\prime}$ is $\bar{J}_{T} \otimes I_{N}$. It is shown in Baltagi (1995, p28) that

$$
\widehat{\delta}_{r, G M M}=\left(\Delta \widehat{Z}_{r}^{* \prime} Q \Sigma^{-1} Q \Delta \widehat{Z}_{r}^{*}\right)^{-1} \Delta \widehat{Z}_{r}^{* \prime} Q \Sigma^{-1} Q \Delta y
$$

where $Q=I_{N T}-\bar{J}_{T} \otimes I_{N}$ for model with only individual effects and $Q=I_{T} \otimes I_{N}-\bar{J}_{T} \otimes$ $I_{N}-I_{T} \otimes \bar{J}_{N}+\ddot{J}_{T} \otimes \bar{J}_{N}$ when time dummies are present. The estimator (9.24) with $\Delta \hat{Z}_{r}^{*}=$ $W\left(W^{\prime} \Sigma^{*-1} W\right)^{-1} W^{\prime} \Sigma^{*-1} \Delta Z_{r}^{*}$ will be indicated as the GLS-LSDV (Least Squares Dummy Variables) estimator. When the matrix $\Sigma$ is replaced by the identity matrix, a less-efficient estimator arises which will be denoted as the LSDV estimator. The asymptotic covariance matrix of $\hat{\delta}_{r, G M M}$ with optimal weighting matrix $S$ is then given by

$$
\operatorname{Var}\left(\tilde{\delta}_{r, G M M}\right)=\left[\Delta \widehat{Z}_{r}^{* \prime} Q W\left(W^{\prime} \Sigma W\right)^{-1} W^{\prime} Q \Delta \widehat{Z}_{r}^{*}\right]^{-1}
$$

A test for the validity the overidentifying restrictions is obtained using (9.22) and is readily seen to be a test for the null hypothesis of $\mathrm{C} 4$, e.g. for the null of homogeneity of common features: $\widetilde{\beta}_{i}=\widetilde{\beta}_{1} ; i=1, \ldots, N$, with $s=N s_{1}, s_{i}=s_{1}=1, \forall i=1, \ldots, N$. In this specific case, the number of degrees of freedom for the overidentifying restrictions test (9.22) is given by $\left.\sum_{i=1}^{N}\left[n\left(p_{i}-1\right)+r_{i}\right)-(n-1)\right]+(n-1)(N-1)$ where $n, p_{i}, r_{i}$ are respectively the number of variables, the number of lags and the number of cointegrating relations for each $i$. Notice that the factor $(n-1)(N-1)$ arises as a consequence of the pooled estimation of the common feature vector. Imposing a common co-feature vector actually decreases by $(n-1)(N-1)$ the number of parameters to be estimated.

More generally, one could naturally extend the analysis (in the case $n>2$ ) and consider similar analyses for $s_{1}=1, \ldots, n-1$. Sequentially testing, for $s_{1}=1, \ldots, n-1$, the validity of the underlying overidentifying restrictions with (9.22), provides a direct way to test the number of common co-features in a GMM set-up, provided we first properly normalize the co-feature matrix 
as above. A somewhat similar use of GMM for the detection of the dimension of the common feature space, albeit in a pure time series context, is discussed in Vahid and Engle (1997).

In the next section, we evaluate the merits of this analysis (for $s_{i}=s_{1}=1, \forall i=1, \ldots, N$ ) in a small Monte Carlo experiment.

\subsection{Monte Carlo Simulations}

In this section we present some illustrative Monte Carlo evidence on the usefulness of the common feature test statistic (9.22) presented above for panel data. The data are generated as if there exists a huge VECM with both common feature and cointegrating restrictions. Under the null of reduced rank structures, the bivariate DGP for each of $N$ individuals assumes the existence of one cointegrating vector and of a single common feature vector. It has the form:

$$
\begin{aligned}
\left(\begin{array}{c}
\Delta y_{i, t} \\
\Delta z_{i, t}
\end{array}\right)= & \left(\begin{array}{c}
\mu_{1} \\
\mu_{2}
\end{array}\right)+\left(\begin{array}{c}
.25 \\
.5
\end{array}\right)\left(\begin{array}{ll}
1 & -1
\end{array}\right)\left(\begin{array}{l}
y_{1, t-1} \\
z_{1, t-1}
\end{array}\right)+ \\
& \left(\begin{array}{c}
.5 \\
1
\end{array}\right)\left(\begin{array}{ll}
.6 & .3
\end{array}\right)\left(\begin{array}{c}
\Delta y_{1, t-1} \\
\Delta z_{1, t-1}
\end{array}\right)+\left(\begin{array}{c}
\varepsilon_{i 1, t} \\
\varepsilon_{i 2, t}
\end{array}\right),
\end{aligned}
$$

where the $\mu$ 's are generated from uniform distributions $\mu_{1} \sim U(0, .3), \mu_{2} \sim U(-.25, .15)$ so that $E\left(\mu_{1}\right)=.15$ and $E\left(\mu_{2}\right)=-.05$. The normalized common feature vector is $\tilde{\beta}=(1,-.5)^{\prime}$ and the normalized cointegration vector is simply $\beta=(1,-1)^{\prime}$. For each individual $i,\left(\varepsilon_{i 1, t}, \varepsilon_{i 2, t}\right)^{\prime}$ is bivariate Gaussian with covariance matrix $\Omega_{i i}$. The cross-contemporaneous correlation matrices between individual $i$ and $j$ are all equal to $\Omega_{i j}$ so that the panel VECM covariance matrix is given by $(9.7)$ with

$$
\Omega_{i i}=\left(\begin{array}{cc}
1 & .8 \\
.8 & 1
\end{array}\right) \quad \Omega_{i j}=\left(\begin{array}{cc}
.7 & .6 \\
.6 & .75
\end{array}\right)
$$

We have considered in the Monte Carlo design the case where there exists some degree of hetcrogeneity between individuals. To simplify matters this structure is increasing with $N_{.}^{5}$

Figures 9.1 and 9.2 illustrate a realization of the DGP for 10 individuals and two variables and then they compare processes with (Figure 9.2) and without (Figure 9.1) this additional heteroscedasticity. From this DGP we sec that under the assumption of reduced rank the short run dynamic matrices (for cach $i$ ) are simply given by $\left(\begin{array}{ll}0.30 & 0.15 \\ 0.60 & 0.30\end{array}\right)$, while under the alternative

\footnotetext{
${ }^{5}$ The operation is the following. Consider a $N$ dimensional vector with increment four $g=(1,5,9 \ldots)^{\prime}$. We form a $n N \times n N$ matrix $G=g g^{\prime} \otimes R$ with $R$ a $n \times n$ matrix with all elements equal to 1 . Then the heteroskadasticity disturbance covariance matrix $\Omega^{*}$ is given by $\Omega^{*}=G \odot \Omega$, with $\Omega$ given in (9.7) and $\odot$ the elementwise product or Hadamard product.
} 


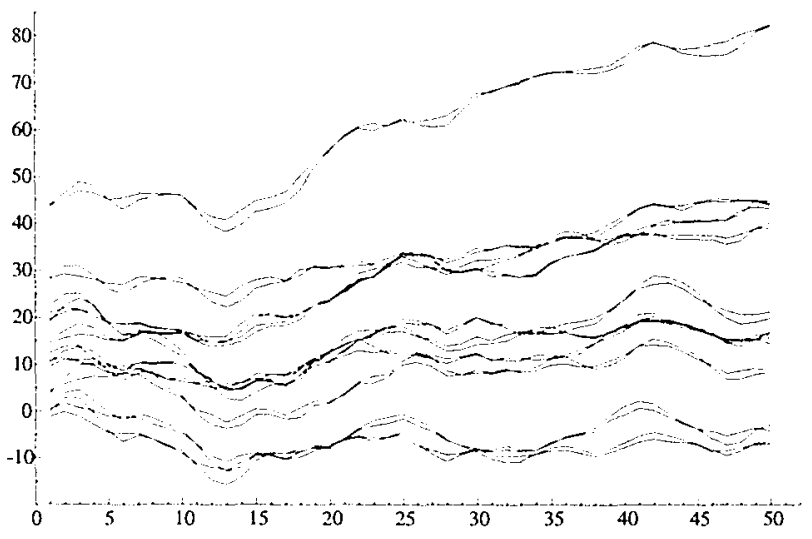

Figure 9.1: A Realization of the GDP for 10 Individuals

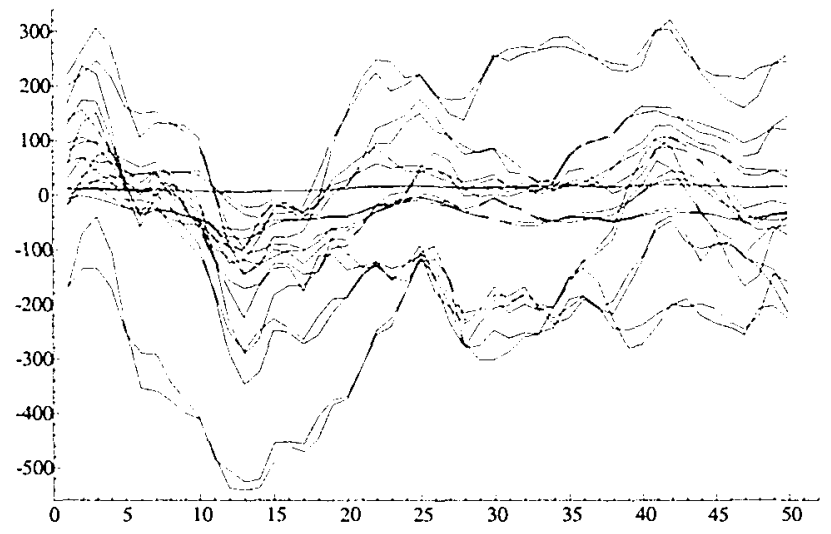

Figure 9.2: A Realization of the DGP with Additional Heteroscedasticity 


\begin{tabular}{|c|c|c|c|c|c|c|c|}
\hline \multirow{4}{*}{$N=1$} & & bias ${ }_{\text {Median }}$ & bias $_{Q 75-Q 25}$ & $\sigma\left(\widehat{\delta}_{r, G M M}\right)_{M e d i a n}$ & $\chi^{2} \mathrm{cv}$ & size & adj.power \\
\hline & $T=10$ & -.0123 & .2228 & .156 & (2) & 7.88 & 9.90 \\
\hline & $T=25$ & -.0101 & .1387 & .098 & (2) & 5.58 & 19.78 \\
\hline & $T=50$ & -.0067 & .0944 & .070 & $(2)$ & 5.54 & 34.68 \\
\hline \multirow[t]{3}{*}{$N=2$} & $T=10$ & -.0136 & .1817 & .106 & $(5)$ & 4.98 & 8.56 \\
\hline & $T=25$ & -.0069 & .1057 & .079 & $(5)$ & 6.18 & 16.58 \\
\hline & $T=50$ & -.0034 & .0726 & .057 & (5) & 5.72 & 31.52 \\
\hline \multirow[t]{3}{*}{$N=5$} & $T=10$ & -.0045 & .1409 & .067 & (14) & 3.96 & 7.26 \\
\hline & $T=25$ & -.0044 & .0751 & .060 & (14) & 5.68 & 12.52 \\
\hline & $T=50$ & -.0021 & .0460 & .047 & (14) & 5.74 & 24.82 \\
\hline \multirow[t]{2}{*}{$N=10$} & $T=25$ & -.0022 & .0658 & .046 & (29) & 4.70 & 11.00 \\
\hline & $T=50$ & -.0020 & .0377 & .038 & $(29)$ & 4.80 & 21.55 \\
\hline$N=25$ & $T=50$ & .0002 & .0398 & .029 & $(74)$ & 5.80 & 13.80 \\
\hline
\end{tabular}

Table 9.2: Monte Carlo Results: GMM Estimation and Test Statistics

we chose to arbitrarily fix one element to zero: $\left(\begin{array}{cc}0.30 & 0.00 \\ 0.60 & 0.30\end{array}\right)$.

We consider three sample sizes, i.e. $T=10,25$ and 50 , and five cases for the number of individuals, i.e. $N=1,2,5,10$ and 25 . We report the median and the spread (interquartile range) of the bias of the GMM panel estimator. We also report the median of the standard deviation of $\widehat{\delta}_{r, G M M}$. We report the empirical size (nominal being $5 \%$ ) as well as the empirical size-adjusted power for over-identifying restrictions test statistics. DF denotes the number of degrees of freedom. Due to the huge computational time required for these simulations, 5,000 replications were used for $N=1,2,5 ; 2,000$ for $N=10$ and 1,000 for $N=25$.

The results are presented in Table 9.2. One can directly observe that the bias is small and decreases when both $T$ and/or $N$ increase. The accuracy of estimates, measured both by the spread and the standard deviation of the estimate, also increases with $T$ and/or $N$. We interpret these illustrative findings as evidence in favor of the pooling estimator. No substantial size distortions are observed. Remark that the values of $N$ we have retained in these simulations are clearly too small to assess the validity of a central limit theorem based on large $N$ asymptotic. 


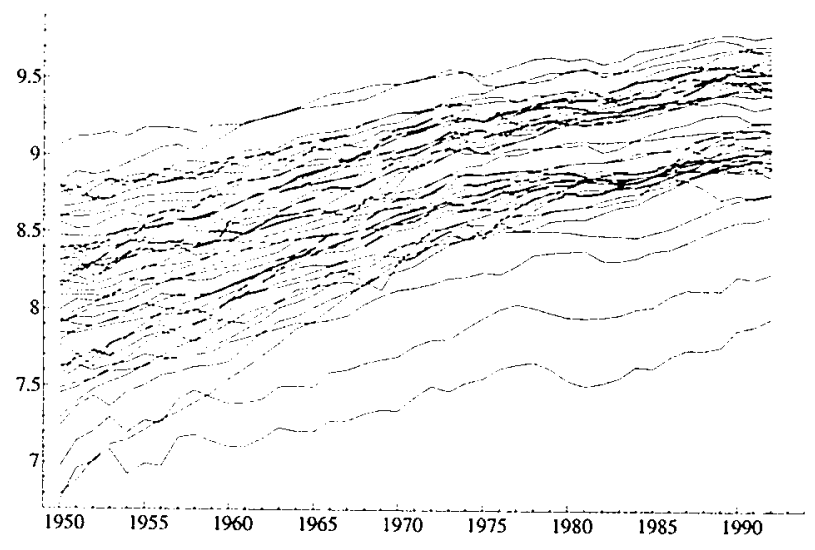

Figure 9.3: Consumption and Output Scries for the 22 OECD Countries

\subsection{Empirical Analysis}

The data we use are taken from the Penn World Tables Mark 5.6 (see Summers and Heston, 1991). ${ }^{6}$ Thanks to the homogeneity in their definition, these data are extremely useful and have been extensively used in the empirical literature. However the data are certainly not free of measurement errors because the price to pay for obtaining long series of homogeneous data for more than 150 countries is the reliance on a set of hypotheses, approximations and interpolations. Because of both the quality of the data as well as the underlying theoretical motivation. we limit our analysis to 22 OECD countries for the sample period 1950-1992 (up to 1991 for Greece and 1990 for Portugal). ${ }^{7}$ The data extracted are $Y=" R G D P L:$ Real GDP per capita (Laspeyres index) in 1985 intemational prices" and $C=" C:$ Real Consumption share of GDP in 1985 international prices" $\times Y / 100$. This last operation is necessary to get the consumption in lovel and not in percentage of income. ${ }^{8}$ Figure 9.3 plots the 44 series, namely consumption and income variables for the OECD countries. The picture also pleas in favor of disposing tools in order to modeling this information.

Table 9.3 reports time series statistics for each country. The first column of Table 9.3 lists in alphabetical order, the names of the countries as well as the date of joining OECD. ${ }^{9}$ Column

\footnotetext{
${ }^{6}$ See Chapter 8 , footnote 5 .

'Beciuse of computation facility, we have balanced the panel in this study and we did not consider either Greece and Portugal.

${ }^{8}$ See Chapter 8 , footnote 6 .

${ }^{9}$ Other countries joined the OECD. This was the case of the Czech Republic in 1995, Korea in 1996, Poland 1996 and Mexico 1994. We drop them because the ending year is 1992 in our data set. Also note that OECD
} 
2 gives the quality ranking of the data as presented in Summers and Heston (1991). It is seen that for the most part, the quality of the data is reasonable (a A means that the data are the most reliable). Columns 3 and 4 give the value of the Augmented Dickey-Fuller unit root test for respectively consumption and income. All tests are based on both a constant term and a trend. The number of lags necessary to whiten the residuals is given in parentheses. Columns 5 and 6 give respectively the value of the Engle-Granger Augmented Dickey-Fuller cointegrating test for each country separately and the long-run elasticity of consumption as a dependent variable. Column 7 gives the order of the $\operatorname{VAR}\left(p_{i}\right)$ in level, where $p_{i}$ is determined using multivariate Hannan-Quinn (HQC) criteria. These lags, as well as the presence of an error correcting mechanism term, will determine the instruments to be used in common features test statistics.

In Table 9.3 , a $" *$ indicates that individual unit root or cointegration test statistics reject the null at a $5 \%$ nominal level. It emerges that, except for Portugal, UK and Turkey, we cannot reject. the unit root hypothesis for consumption and income. Using the Engle-Granger cointegration test, the null hypothesis of non-cointegration is rejected for nine countries with long-run elasticity $\beta_{i}^{*}$ close to 1 . Consequently, we will use the cointegrating vectors as instruments in six different versions: four homogeneous cases and two hetcrogeneous oncs. We proceed in two steps. In the first step the cointegrating vectors are estimated. They are used as instruments in the second step to estimate the common feature vectors. The results are reported in Table 9.4.

The homogeneous cases refer to a panel estimation of a common cointegrating vector, that is parameters are assumed to be the same across countries and the contemporaneous disturbance correlation across countries and across variables for a given country is ignored. Absence of shortrun Granger-causality between countries is assumed throughout steps 1 and 2. Because most. panel cointegration test statistics assume independence across individuals, we cannot, strictly speaking, rely on these panel cointegration test statistics. However because the estimator of the cointegrating vectors is still consistent we use them to get estimates for four different cases.

- As Tables 9.3 shows cven when the absence of cointegration is not rejected, the elasticity is close to one. We first analyze a version in which we assume that there exists a homogeneous cointegrating relationship for all the countries with a coefficient $\beta^{*}$ equal to one (see upper panel of Table 9.4). Similar results are obtained using Johansen's MLE based procedure.

- A second panel cointegration test uses the group mean estimator (GM) of Pesaran et al. (1997). This means that we average cointegrating vectors over the 22 individuals.

- A third alternative uses the usual OLS estimator.

has its origin in the Organisation for European Economic Cooperation which grouped European Countries. This organisation was charged with administering United States aid, under the Marshall Plan, to reconstruct Europe after the World War II. Consequently, for countries that did not participate at the beginning in this project, homogeneity of cointegration and/or common features might be rejected for that reason. 


\begin{tabular}{|c|c|c|c|c|c|c|}
\hline & Qual.ranking & $\mathrm{ADF} c_{t}$ & $\mathrm{ADF} y_{t}$ & EG & $\widehat{\beta}_{i}$ & $\mathrm{HQC}$ \\
\hline Australia (1971) & $\overline{\mathrm{A}-}$ & $-1.21(4)$ & $-.93(2)$ & $-1.46(1)$ & .95 & 3 \\
\hline Austria (1961) & A- & $-.82(0)$ & $-1.25(2)$ & $-3.59(0)^{*}$ & 1.00 & 1 \\
\hline Belgium (1961) & $\mathrm{A}$ & $-1.43(1)$ & $-.74(1)$ & $-2.36(0)$ & .94 & 1 \\
\hline Canada (1961) & A- & $-1.50(1)$ & $-1.80(1)$ & $-3.89(1)^{*}$ & 1.00 & 1 \\
\hline Denmark (1961) & A- & $-.94(0)$ & $-.94(0)$ & $-3.69(0)^{*}$ & .82 & 1 \\
\hline Finland (1969) & A- & $-2.48(1)$ & $-.20(2)$ & $-1.69(3)$ & .98 & 4 \\
\hline France (1961) & A & $-.11(2)$ & $-.04(1)$ & $-1.96(0)$ & .98 & 2 \\
\hline Germany (1961) & A & $-2.18(2)$ & $-3.10(2)$ & $-1.69(2)$ & 1.07 & 2 \\
\hline Greece (1961) & A- & $-.58(0)$ & $.01(0)$ & $-.79(0)$ & .97 & 1 \\
\hline Iceland (1961) & $B+$ & $-2.64(1)$ & $-2.23(1)$ & $-4.52(0)^{*}$ & 1.04 & 1 \\
\hline Ireland (1961) & A- & $-2.54(1)$ & $-2.82(1)$ & $-3.76(2)^{*}$ & .81 & 1 \\
\hline Italy (1961) & A & $-.61(1)$ & $-.77(1)$ & $-1.86(1)$ & 1.09 & 4 \\
\hline Japan (1964) & A & $-.91(0)$ & $-.48(1)$ & $-4.75(1)^{*}$ & .92 & 4 \\
\hline Luxembourg (1961) & A- & $-1.45(1)$ & $-3.32(4)$ & $-2.16(4)$ & 1.34 & 4 \\
\hline Netherlands (1961) & $A$ & $-.71(2)$ & $-.20(2)$ & $-3.07(1)$ & 1.08 & 4 \\
\hline New Zealand (1973) & A- & $-2.26(0)$ & $-1.52(0)$ & $-5.93(0)^{*}$ & 1.02 & 1 \\
\hline Norway (1961) & A- & $-1.29(1)$ & $-1.76(1)$ & $-1.83(1)$ & .80 & 1 \\
\hline Portugal (1961) & A- & $-3.54(3)^{*}$ & $-2.95(3)$ & $-3.07(3)$ & .88 & 3 \\
\hline Spain (1961) & A- & $-1.25(0)$ & $-1.34(0)$ & $-2.99(0)$ & .94 & 1 \\
\hline Sweden (1961) & A- & $-.70(1)$ & $-.30(1)$ & $-3.58(1)^{*}$ & .81 & 2 \\
\hline Switzerland (1961) & $B-$ & $.03(4)$ & $-1.69(2)$ & $-3.28(0)$ & .92 & 2 \\
\hline Turkey (1961) & $\mathrm{C}$ & $-3.26(2)$ & $-3.48(0)^{*}$ & $-1.73(0)$ & .85 & 1 \\
\hline UK (1961) & A & $-3.61(1)^{*}$ & $-3.62(1)^{*}$ & $-2.13(0)$ & 1.04 & 3 \\
\hline USA (1961) & $\mathrm{A}$ & -1.75 & $-2.05(0)$ & $-4.08(0)^{*}$ & 1.15 & 2 \\
\hline
\end{tabular}

Table 9.3: Time Series Statistics: Individual Countries 
- The last one allows for intercept heterogeneity and is the usual LSDV estimator.

Notice that the pooled FM-OLS estimator proposed by Pedroni (1997a), which assumes independence across units, gives a point estimate of 0.971 for the 22 OECD countries and 1.021 for the G7 countries, the latter being not significantly different from one. Both results are very close to those obtained with the LSDV and OLS estimators so that the results of the common cyclical feature analysis obtained with Pedroni's FM-OLS estimator are not reported.

For the two heterogeneous cases we impose cointegration for the nine countries for which the Engle-Granger ADF test is significant. In step 2, we take as an instrument, cointegrating vectors for countries for which we reject the null. Notice that Phillips-Hansen Fully Modified OLS estimation was also used to test formally the assumption of unit long-run elasticity. The null of unit long-run elasticity was formally rejected in all cases of cointegration but for three (Austria, Canada and New-Zealand). Two different cases are considered:

- For the nine countries we take the estimated value of $\beta_{i}^{*}$ given by the long-run regression.

- We fix these values to 1 .

The maximum lag length for a country is four, so that $p^{*}=(p-1)=0,1,2$ or 3 for some countries. The following cases are considered:

- $p^{*}$ is fixed uniformly to respectively $1,2,3$

- $p^{*}$ is fixed to the value determined using the HQ criterion.

Note that over-estimating the lag length will certainly reduce the power of the test statistics (Beine and Hecq, 1999 and Chapters 2 and 5). The results of the two panel common feature statistics are presented. For the heterogeneous cases, the first two columns present the group mean estimates (denoted by $\widehat{\delta}_{r, G M}$ ) as well as the value of the Normal test statistics $\left(N_{G M}\right)$ in (9.12) which tests for the significance of one common feature vector. The next columns present the value of common feature elasticity for the homogeneous dependent case (denoted by $\widehat{\delta}_{r, G M M}$ and the associated standard errors denoted by $\sigma\left(\widehat{\delta}_{r, G M M}\right)$ ) as well as the value of the test of the overidentifying restrictions implied by the common feature vector and the associated $p$-values. Note that in the second step, we always assume the occurrence of nonzero contemporancous disturbances correlation.

It appears that the estimated coefficient $\widehat{\delta}_{r, G M M}$ and $\widehat{\delta}_{r, G M}$ are too high compared with a priori expectations. Moreover we reject the null of a panel common feature model for both test statistics. Table 9.5 presents the results for the G7. The results are similar to those for the panel of 22 countries. However in scveral situations we cannot reject the null of one homogeneous common features vector. In these cases, we imposed the unlikely hypotheses of an homogeneous cointegrating vector with a lag order uniformly fixed to $p^{*}=3$.

Finally, we want to notice the implications for empirical modeling that follow from a restriction between the number of variabies $n$ and the sum of cointegrated vectors and common features 
vectors. From Vahid and Engle (1993), Theorem 1, it follows that the common feature space and the cointegration space are linearly independent. This means that the sum of the number of common feature vectors $(s)$ and of the number of cointegrating vectors $(r)$ should be less than or equal to the number of variables $(n): r+s \leq n$. In a panel context under the absence of Granger long- and short-run causality, this has obvious but different implications depending on whether common features vectors and cointegrating vectors are homogencous or heterogencous. A misspecification of the number of homogeneous cointegrating vectors may for instance too heavily constrain the dimension of the homogeneous common feature space and lead to flawed inference regarding the existence of common features.

$A$ last remark seems in order. Although we can formally reject the existence of a common homogeneous co-feature relation in this OECD data set, one should be aware that our results do not per se imply the absence of SCCF for some of the countries taken individually.

\subsection{Conclusion}

In this chapter we extended the serial correlation common feature analysis to nonstationary panel data models. Concentrating upon the fixed effect model, we defined homogeneous panel common feature models. We give a scries of steps allowing to implement these tests. We then apply this framework when investigating the liquidity constraints model for 22 OECD and G7 countries. At a $5 \%$ nominal level, we reject the presence of a pancl common feature vector.

From the empirical analysis we can draw several (tentative) conclusions:

First, in a country by country analysis for approximately slightly less than $50 \%$ of the countries in the sample, there is evidence of cointegration between consumption and income. The cointegrating vector appears to be homogencous across these countries with a long-run consumption elasticity close to one.

Second, for the sample of 22 countries, the existence of one homogeneous SF (SCCF) common feature vector is rejected in most instances when using the test proposed in (9.12). For the sample of G7 countries, in several instances, the occurrence of a homogeneous SF common feature vector is not rejected. Notice that this rastriction is obviously less restrictive when it only applies to seven countries. However the $p$-values are quite low and the non rejection of the null hypothesis occurs when the model might be misspecified in particular because we have maintained a homogeneous lag length of 3.

Third, the overidentifying restrictions implied by the assumption of a homogeneous common feature vector are rejected in all instances in the sample of 22 countries. For the G7 countries, again there is occasionally evidence in favor of the overidentifying restrictions.

Again, it is not surprising to see that the assumption of homogenous common features is rejected more frequently than the assumption of homogenous cointegration. In the long-run consumption and income are closely linked to each other, short-run deviations are generally possible and can be realized through saving or borrowing. 


\begin{tabular}{|c|c|c|c|c|c|c|c|c|}
\hline \multirow{4}{*}{$\begin{array}{l}\beta_{i}^{*}=1 \\
(\forall i)\end{array}$} & & $\delta_{r, G M}$ & $N_{G M}$ & $\widehat{\delta}_{r, G M M}$ & $\sigma\left(\widehat{\delta}_{r, G M M}\right)$ & Test & $\mathrm{CV}$ & p_val \\
\hline & $p^{*}=1$ & .770 & 3.71 & .745 & .050 & 148.98 & 65 & $<.001$ \\
\hline & $p^{*}=2$ & .769 & 6.14 & .660 & .036 & 173.65 & 109 & $<.001$ \\
\hline & $p^{*}=3$ & .770 & 4.43 & .704 & .031 & 211.27 & 153 & .001 \\
\hline \multirow{5}{*}{$\begin{array}{l}\beta_{i}^{*}=\widehat{\beta}_{G M}^{*}=.979 \\
(\forall i)\end{array}$} & $p^{*}=p_{i}^{*}$ & - & - & .718 & .036 & 156.04 & 93 & $<.001$ \\
\hline & $p^{*}=1$ & .829 & 5.36 & .768 & .051 & 146.67 & 65 & $<.001$ \\
\hline & $p^{*}=2$ & .804 & 6.54 & .670 & .036 & 176.61 & 109 & $<.001$ \\
\hline & $p^{*}=3$ & .793 & 4.95 & .710 & .031 & 214.06 & 153 & $<.001$ \\
\hline & $p^{*}=p_{i}^{*}$ & - & - & .728 & .036 & 156.92 & 93 & $<.001$ \\
\hline \multirow{4}{*}{$\begin{array}{l}\beta_{i}^{*}=\widehat{\beta}_{O L S}^{*}=.939 \\
(\forall i)\end{array}$} & $p^{*}=1$ & .870 & 5.74 & .814 & .050 & 131.96 & 65 & $<.001$ \\
\hline & $p^{*}=2$ & .837 & 5.12 & .687 & .036 & 170.16 & 109 & $<.001$ \\
\hline & $p^{*}=3$ & .822 & 3.93 & .727 & .031 & 206.93 & 153 & .002 \\
\hline & $p^{*}=p_{i}^{*}$ & - & - & .738 & .036 & 145.01 & 93 & $<.001$ \\
\hline \multirow{4}{*}{$\begin{array}{l}\beta_{i}^{*}=\bar{\beta}_{L S D V}=.968 \\
(\forall i)\end{array}$} & $p^{*}=1$ & .855 & 6.03 & .782 & .051 & 142.93 & 65 & $<.001$ \\
\hline & $p^{*}=2$ & .821 & 6.25 & .677 & .036 & 175.97 & 109 & $<.001$ \\
\hline & $p^{*}=3$ & .804 & 4.94 & .715 & .031 & 213.50 & 153 & .001 \\
\hline & $p^{*}=p_{i}^{*}$ & - & - & .733 & .036 & 155.12 & 93 & $<.001$ \\
\hline \multirow{4}{*}{$\begin{array}{l}\beta_{i}^{*}=\widehat{\beta}_{j}^{*} \\
(\forall i, j \text { with } \\
\text { cointegration })\end{array}$} & $p^{*}=1$ & .814 & 6.89 & .782 & .053 & 138.45 & 52 & $<.001$ \\
\hline & $p^{*}=2$ & .726 & 6.16 & .647 & .036 & 158.74 & 96 & $<.001$ \\
\hline & $p^{*}=3$ & .755 & 4.46 & .696 & .031 & 210.03 & 140 & $<.001$ \\
\hline & $p^{*}=p_{i}^{*}$ & - & - & .707 & .037 & 146.50 & 80 & $<.001$ \\
\hline \multirow{4}{*}{$\begin{array}{l}\beta_{i}^{*}=1 \\
(\forall i \text { with } \\
\text { cointegration) }\end{array}$} & $p^{*}=1$ & .865 & 1.59 & .810 & .056 & 115.25 & 52 & $<.001$ \\
\hline & $p^{*}=2$ & .784 & 3.89 & .682 & .037 & 144.00 & 96 & .001 \\
\hline & $p^{*}=3$ & .775 & 2.72 & .734 & .033 & 192.33 & 140 & .002 \\
\hline & $p^{*}=p_{i}^{*}$ & - & - & .750 & .040 & 131.56 & 80 & $<.001$ \\
\hline
\end{tabular}

Table 9.4: Common Features within 22 OECD Countries 


\begin{tabular}{|c|c|c|c|c|c|c|c|c|}
\hline \multirow{5}{*}{$\begin{array}{l}\beta_{i}^{*}=1=\widehat{\beta}_{L S D V}^{*} \\
(\forall i)\end{array}$} & & $\delta_{r, G M}$ & $N_{C M}$ & $\delta_{r . G M M}$ & $\sigma\left(\hat{\delta}_{r, G M M}\right)$ & Test & $c v$ & p_value \\
\hline & $p^{*}=1$ & .866 & 2.47 & 1.042 & .087 & 32.83 & 20 & .035 \\
\hline & $p^{*}=2$ & .763 & 2.37 & .856 & .060 & 53.70 & 34 & .017 \\
\hline & $p^{*}=3$ & .755 & 1.81 & .872 & .052 & 67.05 & 48 & .036 \\
\hline & $p^{*}=p_{i}^{*}$ & - & - & .884 & .058 & 50.84 & 30 & .010 \\
\hline \multirow{4}{*}{$\begin{array}{l}\beta_{i}^{*}=\widehat{\beta}_{G M}^{*}=1.035 \\
(\forall i)\end{array}$} & $p^{*}=1$ & .893 & 1.64 & 1.021 & .082 & 31.51 & 20 & .048 \\
\hline & $p^{*}=2$ & .777 & 1.815 & .857 & .060 & 50.25 & 34 & .036 \\
\hline & $p^{*}=3$ & .766 & 1.49 & .878 & .052 & 62.75 & 48 & $.075^{*}$ \\
\hline & $p^{*}=p_{i}^{*}$ & - & - & .892 & .057 & 46.22 & 30 & .029 \\
\hline \multirow{4}{*}{$\begin{array}{l}\beta_{i}^{*}=\widehat{\beta}_{O L S}^{*}=1.023 \\
(\forall i)\end{array}$} & $p^{*}=1$ & .882 & 1.75 & 1.036 & .084 & 32.06 & 20 & .043 \\
\hline & $p^{*}=2$ & .771 & 1.89 & .856 & .060 & 51.11 & 34 & .030 \\
\hline & $p^{*}=3$ & .762 & 1.51 & .876 & .052 & 63.87 & 48 & $.062^{*}$ \\
\hline & $p^{*}=p_{i}^{*}$ & - & - & .890 & .057 & 47.84 & 30 & .021 \\
\hline \multirow{4}{*}{$\begin{array}{l}\beta_{i}^{*}=\widehat{\beta}_{j}^{*} \\
(\forall i, j \text { with } \\
\text { cointegration) }\end{array}$} & $p^{*}=1$ & .818 & 6.02 & .894 & .074 & 49.07 & 16 & $<.001$ \\
\hline & $p^{*}=2$ & .710 & 3.58 & .723 & .053 & 52.66 & 30 & .006 \\
\hline & $p^{*}=3$ & .737 & 2.13 & .787 & .047 & 64.46 & 44 & .024 \\
\hline & $p^{*}=p_{i}^{*}$ & - & - & .800 & .051 & 46.61 & 26 & .008 \\
\hline \multirow{4}{*}{$\begin{array}{l}\beta_{i}^{*}=\beta_{j}^{*}=1 \\
(\forall i, j \text { with } \\
\text { cointegration) }\end{array}$} & $p^{*}=1$ & .875 & 2.68 & 1.029 & .089 & 27.69 & 16 & .034 \\
\hline & $p^{*}=2$ & .753 & 2.60 & .859 & .062 & 47.49 & 30 & .022 \\
\hline & $p^{*}=3$ & .764 & 1.66 & .894 & .053 & 60.14 & 44 & $.053^{*}$ \\
\hline & $p^{*}=p_{i}^{*}$ & - & - & .917 & .061 & 43.97 & 26 & .015 \\
\hline
\end{tabular}

Table 9.5: Common Features within G7 Countries 
Our model representation is not stricto sensus a dynamic panel because only a part of the dynamies is common to all individuals. However it does part of the job. Indeed while no size distortions have been noticed in our Montc Carlo results, we can increase the power of test statistics, by going a step further towards dynamic panel data if the null hypothesis of panel common-cyclical feature model is not rejected. In the opposite case, it is not worth imposing further common restrictions if the null is rejected. This is a clue for considering less restrictive models like hetcrogeneous or group homogeneous models. A bootstrap procedure could certainly be undertaken to find the distribution. This is also perhaps the place to choose more flexible models like the non-synchronous common cycle model (Vahid and Engle, 1997) or the weak form common feature analysis developed in previous chapters. 


\section{Chapter 10}

\section{Postscript}

This thesis has discussed the issue of common fluctuations in macrocconomic time series and panel data. In multivariate analyses it is frequent to observe that one or more of the features detected in the single series are common to several variables and thus disappear by some suitable combination. Since most cconomic variables are usually non-stationary and autocorrelated we have focused on the interaction between long-run and short-run comovements. We have developed tools to analyze jointly these two characteristics of economic time series. As a result, one of the central point of this thesis has been the development in the Chapters 3 and 4 of a weak form reduced rank structure model. We have used this framework in numerous applications in subsequent chapters. The big advantage of this framework is that it allows to investigate economic systems with both a possible large number of cointegrating and of common cyclical feature relationships. Indeed, this was not possible in previous frameworks because the strong form scrial correlation common feature model of Vahid and Engle (1993) yields an upper bound on the possible number of common fluctuations. Consequently, the type of common cyclical features we have proposed allows to study econonic systens that are much more linked in the short-run than what we might expect a priori. The advantage of our approach is hence the possibility to extract sensible information on common fluctuations from multiple time series. In most situations this should help in avoiding to analyze comovements using a set of variables that have been detrended with univariate filters like the Hodrick and Prescott one for instance.

Clearly, due to the spurious regression issue, researchers are now convinced of the advantages of taking care of the possible presence of non-stationarity in the data. Analyses of long-run comovements are routincly applied in most empirical studies through a cointegration analysis whatever the method used.

Contrary to cointegration, the growth of common cyclical features literature is slower and much more confidential. Even if some empirical analyses exist (these are listed in Chapter 2), only few papers are released on new approaches, new theoretical developments or new questions. It appears that the stakes of taking into account the short-run fluctuations are not fully understood yet. We have done our best to contributing to fill in this gap. 
We believe that one of the important point of this monograph has been to stress that common cyclical features are present in a large number of applications. We found common cyclical features among key macrocconomic variables like output and consumption (Chapters 7, 8 and 9) or consumption, output and investment (Chapters 3,4 and 6). There also exist some co-features relationships among international stock price indexes (Chapter 4), among long and short-term interest rates (Chapters 3 and 6), among prices (Chapter 2), between unemployment and economic activity (Chapter 2), among employment time series (Chapter 5). among regional income variables (Chapter 5), among business economic indicators for different countries (Chapters 2 and 7). The outcome of these applications is summarized in Table 10.1. The first column indicates the series we have studied and column 2 refers to the chapter where these have been considered. Column 3 shows whether the analysis is carried out on a bivariate or on a multivariate basis. In this lat.ter case, the number of variables considered is within brackets. The fourth column points out the number of cointegrating vectors we have found. $r=0$ means that we have taken the first differences of $\mathrm{I}(1)$ variables becausc we did not find any long-run relationships. $r=n$ indicates that the series were stationary in levels. The last three columns emphasize the presence of common cyclical feature relationships. We consider separately the weak and the strong form. The number in brackets reports that different analyses gave different results as in Chapter 7 when we have considered both raw and seasonally adjusted data. The last column indicates that we tested for scalar component models and particularly for codependence when $p=0$, i.e. $\operatorname{SCM}\left(0, q_{1}\right)$. It emerges clearly that there exists in all applications an important number of common cyclical feature relationships and especially of the weak form type. In most applications, the number of common cycles is even lower than the number of common trends, indicating stronger short-run comovements than long-run ones.

After providing evidence of the existence of common cyclical features with a large spectrum of cmpirical analyses, we also think that these types of restrictions are useful both for an economic and a statistical point of view.

Taking into account of such strong relationships between stationary variables is interesting for an economic point of view for several reasons:

- First, because economic theory often predicts and explains such comovements. It allows to test for short-run elasticities from lincar combinations which are unpredictable from the past (or have shorter memory) like in rational expectation models, Okun's law (Chapter 2) or for the heterogenous consumer model (Chapters 8 and 9).

- In determining the short-run common factors within multiple time series, these tools are also useful for a multivariate descriptive data analysis purpose. For instance, the prescnce of a single common cycle between economic indicators of several countries indicates to policy makers that economies have already converged in the short-rum. Consequently, global strategies, perhaps at the European level, may be used without penalizing a particular country bccause it is known that no country will drift apart. 


\begin{tabular}{lcccccc}
\hline \hline Series & Ch. & Model & Coint & WF & SF & SCM $(p, q)$ \\
\hline Composite leading indicators & 2 & bivariate & $r=0$ & - & - & scm( $\left(0 . q_{1}\right)$ \\
Prices & 2 & multi.(7) & $r=7$ & - & - & sCM(p $\left.p_{1}, q_{1}\right)$ \\
Unemployment \& economic activity & 2 & bivariate & $r=0$ & - & - & scm( $\left(0, q_{1}\right)$ \\
Consumption, output and investment & 3 & multi.(3) & $r=2$ & $s=2$ & $s=1$ & - \\
Equity index, dividend, interest rates & 3 & multi.(4) & $r=2$ & $s=2(1)$ & $s=0(1)$ & - \\
Consumption, output \& investment & 4 & multi.(3) & $r=2$ & $s=2$ & $s=1(0)$ & - \\
International stock markets & 4 & multi.(5) & $r=2$ & $s=4$ & $s=3$ & - \\
US regional incomes & 5 & multi.(6) & $r=3$ & $s=5$ & - & - \\
Employment in Flemish provinces & 5 & multi.(6) & $r=2$ & $s=3$ & $s=2$ & - \\
Employment in Walloon provinces & 5 & multi.(6) & $r=3$ & $s=3$ & $s=2$ & - \\
Real output \& sectorial employment & 6 & multi.(6) & $r=2$ & $s=3$ & $s=2$ & - \\
Consumption, output and investment & 6 & multi.(3) & $r=2$ & $s=2$ & $s=1$ & - \\
Output and interest rates & 6 & multi.(3) & $r=1$ & $s=1$ & $s=1$ & - \\
Output, $C, I, M, R^{s}, R^{l}, \Delta P$ & 6 & multi.(7) & $r=4$ & $s=4$ & $s=2$ & - \\
Consumption and Output & 7 & bivariate & $r=1$ & $s=1(0)$ & - & - \\
Industrial production indexes & 7 & multi.(5) & $r=2$ & $s=1(0)$ & $s=0$ & - \\
Consumption and output for 2 countries & 8 & multi.(4) & $r=2$ & $s=2$ & $s=0(1)$ & - \\
Consumption and output for $n$ countries & 9 & multi. & $r=r_{i}$ & - & $s=s_{i}$ & - \\
\hline \hline
\end{tabular}

Table 10.1: Summary of Empirical Results

- Also the implications of common features in terms of impulse response analyses are important for policy makers. Indeed, it is possible to study the possible reduction in the delay of adjustment after the economies have been hit by a exogenous shock (instantaneous reaction such as in the SCCF or a shorter memory as for codependence).

- Since we believe that adjustment to long-run disequilibria is radically different from the effect of short-run variations, the weak form structure developed in the Chapters 3 and 4 may give additional insights on the role of short-run interactions. For example, let us consider two regional entities such that the Flemish and the Walloon regions of Belgium we have analyzed in the Chapter 5 . These regions have more or less now the same business cycle profile. However, due to historical, cultural or political backgrounds, it may be sensible to think that the adjustment process to long-run disequilibria are different from short-run variations and are for a while specific to these regions.

- The weak form specification could also be used to build a business cycle indicator from a set of time series since the presence of cointegrating relationships is not a problem anymore. This contrast sharply with the approaches recently developed by the NBER (see Stock and Watson, 1991 and the references therein) but also in dynamic factor models $\dot{a}$ la Reichling and co-authors, where all the I(1) time series are taken in first differences without taking into account of the presence of long-relationships.

Recognizing these common feature structures also presents numerous advantages from a sta- 


\section{tistical point of view:}

- Indeed, common features can be exploited to reduced the number of parameters to estimate and may increase efficiency since redundant factors can be removed. This parsimony principle has a long tradition in statistics and econometrics.

- The reduction process facilitates the analysis like in all factor models and may also improve forecast accuracy. We have seen in Chapter 6 however that the possible presence of non constancies during the forecast horizon may temperate this latter observation.

- Finally, the estimation of common feature can also avoid the spurious regression issue that can occurs with highly correlated variables.

We have shown the economic and the statistical interest of common features, we have found a lot of relationships using real data sets and we have developed new estimation strategies. In several chapters we have also considered, using Monte Carlo simulations, the effect of different. types of misspecification on the finite sample behavior of co-feature test statistics. In Chapter 2 we studied the issue of an incorrect identification of multivariate processes (a VAR or a VARMA instead of a VMA..), the impact of non-Gaussian errors and the omission of a cointegrating vector. Chapter 3 also explicitly takes into account the under or the overestimation of the number of cointegrating vectors. In Chapter 5 we evaluate a strategy consisting in considering, in an iterative way, cointegrating and common feature restrictions. A small sample size corrected version of the asymptotic $\chi^{2}$ test is also proposed. Chapter 7 deals with the important issue of estimating common cyclical movements using seasonally adjusted data. It appears that one can spuriously conclude in favor of co-feature relationships using adjusted data. When it is possible, or when statistical agencies release raw data, we strongly recommend to use these series.

In the last two chapters we have discussed the important issue which is to know what happens, and what to do, when the number of variables increases. Chapter 8 takes the road of separability and we have shown that the presence of common features can reconcile the P-T decomposition considered in Granger and Haldrup (1997) with the multivariate Beveridge-Nelson one (see also Chapter 4). Finally, Chapter 9 extends the serial correlation common feature model to panel data. In both Chapters 8 and 9 we have stressed the hypotheses that common features impose on parameter matrices while these assumptions are often implicit in this kind of literature. More specifically, Chapter 9 opens the door for new developments around panel macroeconomic models with both a large number of individuals and of obscrvations. In this context, the presence of non-stationarity and autocorrelated processes is unavoidable.

From the material gathered in this thesis, let us try to formulate a series of technical steps ${ }^{1}$ that could be followed in empirical work.

\footnotetext{
${ }^{1}$ It is obvious that economic theory can participate to all these steps.
} 
1. Given the number of variables the researcher wants to study, he has first to decide whether a system can be analyzed as a multiple time series model or whether a panel framework is more relevant. Alternatively, separation conditions can be imposed if it is not possible to test these restrictions in a latter stage.

2. The nature of the data must also be assessed. Are the data seasonally adjusted? In many cases it will be more sensible to work with raw series using procedures that take into account seasonal (co-)movements. Monte Carlo simulation have also revealed that common feature test statistics are sensitive to ARCH type processes and outliers. If aberrant values are frequently captured in an ad-hoc way by dummy variables, the analysis comovements in volatility, while beyond the scope of this thesis, is certainly an important issue.

3. Assuming that a $\operatorname{VAR}(p)$ characterizes the covariance structure of the data, the lag order $p$ is determined using standard LR statistics or information criteria. To ensure that the analysis makes sense, misspecification test statistics will be used to check whether this $\operatorname{VAR}(p)$ is a congruent, representation of the data.

4. Determine the presence and the number of cointegrating vectors. Notice that simulations have warned against the omission or the underestimation of these long-run relationships (sec Chapters 2 and 3).

5. These cointegrating vectors are then fixed to their estimated values obtained in the previous st.ep. Afterwards, we test for the number of weak and strong form common feature vectors (see Chaptcr 3), using perhaps a small sample version of the test statistics (sec Chapter 5). We can also use a switching approach (betwoen step 4 and 5 ) in the presence of a system with a small number of observations (sce Chapter 5).

6. Alternatively, one can determine jointly the lag order $p$ and the number of common feature vectors (see Vahid and Issler, 1999 and Chapter 6). But, doing so, we belicve that it would also be fair to jointly determine the cointegrating rank $r$ (Gonzalo and Pitarakis, 1999).

7. Evaluate the SF versus the WF as well as their implications for the mixed form (and may be for the NSCC model).

8. Extract the $n-r$ common trends and the $n-s$ (perhaps weak form) common cycles. Alternatively the $n$ trends and the $n$ cycles components may also be useful.

9. (Forecasting performance).

It appears clearly, for example from the Table 10.1, that we have privilege in this thesis the analysis of macroeconomic fluctuations, mostly for OECD countries and especially for US. There are several reasons for that. First, we wanted to avoid the additional difficult but important issue of the treatment of volatility (or perhaps common volatility) one may encounter with financial time series. Second, we have tried to consider countries whose national accounts and statistical 
data in general are the more reliable as possible ${ }^{2}$ even if we know that all time series are affected by measurement errors. Third, we have avoided the analysis of countries that have been subject to large structural breaks as it is the case for some South American countries for instance. Finally, except Okun's law (see Chapter 2) and the consumption function (see Chapter $7,8,9$ ) we have favored a multivariate descriptive data analysis without focusing on a particular obedience of the economic theory.

But we are well aware that other methods could be applied to the same data sets and/or that different variables could be considered and also that different interpretations could be given. Consequently the tools reviewed and developed in this thesis reflect one of the possible approaches for analyzing macrocconomic fluctuations and also our personal sensitivity and our interest to tackle these issues. We have followed this road because the ground was fertile for new developments, the methods used are relatively simple and intuitive: we search for a reduced number of channels for the transmission of the information contained in the past of the series. Morcover, most of the techniques are based on the linear model. Looking at programs or proccedings of international conferences on business cycles, it appcars obvious that the analysis of common cyclical features does not belong to the dominant paradigm. Regime switching, deterministic chaos, neural networks, threshold, non-parametric, asymmetric ... or more generally non-linear models are more fashionable. I think however that some non-linear models are very ad-hoc for business cycle analyses, require usually an important number of observation, most of them are not very intuitive, often lack economic meaning, are sometimes artefact ${ }^{3}$.... and there does not exist so much evidence that they do better than linear models for forecasting (Pagan, 1999). Consequently, simplicity is not a problem per se when it allows to bring new insights for understanding the interaction between economic variables without loosing too much information.

\footnotetext{
${ }^{2}$ In some developing countries, extrapolation methods are used to connect points between quinquennial surveys.

${ }^{3}$ Why is it important to analyze non-linear components in aggregate domestic output variables when seasonal adjustment is often responsible of this distorsion (see Maravall, 1997)?
} 


\section{Bibliography}

[1] Abadir, K.M., K. Hadri and E. Tzavalis (1999), The Influence of VAR Dimensions on Estimator Biases, Econometrica, 67, 163-181.

[2] Adler, M., ANd B. Dumas (1983), International Portfolio Choice and Corporation Finance: $A$ Synthesis, Journal of Finance, 38, 925-984

[3] Aнn, S.K. (1993), Reduced Rank Estimation of Vector Autoregressive Models with Cointegration and Codependence, mimeo.

[4] AHN, S.K. (1996), Common Cycles in Seasonally Cointegrated Time Series, Economics Letters, 53, 261-264.

(5) AHN, S. K. (1997), Inference o Vector Autoregressive Models With Cointegration and Scalar Components, Journal of American Statistical Association, 92, 350-356.

[6] Ahn, S. K. And G. C. Reinsel (1988), Nested Reduced Rank Autoregressive Models for Multiple Timc Scrics, Journal of the American Statistical Associntion, 83, 849-856.

[7] Anderson, T.W. (1984), An Introduction to Multivariate Statistical Analysis, 2nd Ed. (John Wiley \& Sons).

[8] Andersox, T.W. (1998), Estimation of Reduced Rank Regression, Technical Report. \#1998-4, Dept. of Statistics, Stanford University.

[9] Anderson, H.M. ANd F. Vamd (1998), Testing Multiple Equation Systems for Common Nonlinear Components, Journal of Econometrics, 84, 1-36.

[10] Appleby, A.B. (1979), Grain Prices and Subsistence Crises in England and France, 1590-1740, Journal of Economic History, 39, 4, 865-887.

[11] Arshanapalli, B., Doukas, J. and L.H. La.sg (1997), Common Volatility in the Industrial Structure of Global Capital Markets, Joumal of International Money and Finance; 16. 189-209.

[12] Bal, H. And S. Hall (1995), Testing for Common Features in the Main European Countries, Center for Economic Forecasting, Discussion Paper 26-95. 
[13] Baltagl, B. (1995), Econometric Analysis of Panel Deta, (John Wiley: New York).

[14] Baltagl, B. and C. Kao (2000), "Nonstationary Panels, Cointegration in Panels and Dynamic Pancls: A Survey," mimeo, Syracuse University.

[15] Basilevsky, A. (1994), Statistical Factor Analysis and Related Methods, Theory and Applications, (John Wiley: New York).

[16] Beine, M. AND A. HECQ (1997), Asymmetric Shocks Inside Future EMU, Journal of Economic Integration, 12, 131-140.

[17] Beine, M. And A. Hecq (1998), Codependence and Convergence, an Application to the EC Economies, Journal of Policy Modeling, 20, 403-426.

[18] Berne, M. And A. HeCQ (1999), Inference in Codependence : Some Monte Carlo Results and Applications, Annales d'Economie et de Statistique, 54, 69-90.

[19] Beine, M., Candelon, B. And A. Hecq (2000), Assessing a Perfect European Optimum Currency Arca: A Common Cycle Approach, forthcoming in Empirica.

[20] Bekaert, G., and R.J. Hodrick (1992), Charactcrizing Predictable Components in Excess Returns on Equity and Foreign Exchange Markets, Journal of Finance, 47, 467509 .

[21] Bernard and S. Durlauf (1995), Convergence in International Output, Journal of Applied Econometrics, 10, 97-108.

[22] Blanchard, O. And D. Quah (1989), The Dynamic Effects of Demand and Supply Disturbances, American Economic Review, 79, 655-673.

[23] Blanchard O.J. and D.T. Quah (1989), The Dynamic Effects of Aggregate Demand and Supply Disturbances, American Economic Review, 79, 655-673

[24] BoswiJk, H.P. And P.H. Franses (1992), Dynamic Specification and Cointegration, Oxford Bulletin of Economics and Statistics, 54, 369-81.

[25] BoswiJk, H.P. and P.H. Franses (1996), Common Persistence in Nonlinear Autoregressive Models, Econometric Institute Report 9702/A, Erasmus University Rotterdam.

[26] Breitung, J. and B. Candelon (2000), Common Cycles in the Frequency Domain, Humbolt University Discussion Paper.

[27] Brillinger, D.R. (1969), The Canonical Analysis of Stationary Time Series, in Multivariate Analysis-II, ed. P.R. Krishnaiah, New York: Academic Press, 331-350.

[28] Burns, A.F. And W.C. Mitchell (1946), Measuring Business Cycles, (New York, NBER). 
[29] Campbell, J.Y. and N.G. Mankiw (1990), Permanent Income, Current Income, and Consumption, Journal of Business \& Economic Statistics, 3, 265-279.

[30] Campbell, J.Y. And N.G. Mankiw (1991), The Response of Consumption to Income: A Cross-Country Investigation, European Economic Review, 35, 723-767.

[31] Candelon, B. (1998), Patlerns of Cyclical Variability, Ph.D. thesis, Université Catholique de Louvain, Ed. CIACO.

[32] Candelon, B. And A. Hece (1997), On the Power of Codependence Test Statistics, mimeo, Maastricht University.

[33] Candelon, B. And A. Hece (2000), Stability of the Unemployment-Activity Relationship in a Codependent System, Forthcoming in Applied Economics Letters.

[34] Candelon, B., HecQ A. And O. Lohest (2000), Labor Mobility in Belgium: An Empirical Analysis of the Relationship between Provincial Employment Dynamics and Migration, Maastricht University, RM 00/007.

[35] Caporale, G.M. (1997), Common Features and Output Fluctuations in the UK, Economic modeling, 14, 1-9.

(36) Carlino, G. and K. Sill (1998), The Cyclical Behavior of Regional Per Capita Incomes in the Postwar Period, Working Paper 98-11, Federal Reserve Bank of Philadelphia.

[37] Cheung Y.-W. and K.S. Lai (1993), Finite-sample Sizes of Johansen's Likelihood Ratio Tests for Cointegration, Oxford Bulletin of Economics and Statistics, 55, 313-28.

[38] Christoffersen, P. And F.X. Diebold (1998), Cointegration, and Economic Policy Cointegration and Long-Horizon Forecasting, Journal of Business and Economic Statistics, $16,4,450-458$.

[39] Clements, M.P. And D.F. Hendry (1998), Forecasting Economic Time Series, (Cambridge University Press).

[40] Clements, M.P. And D.F. Hendry (1999), Forecasting Non-stationary Economic Time Series, (MIT Press, Zcuthen Lecture Book Series).

[41] Costa, M., Gardini, A. and P. Paruolo (1997), A Reduced Rank Regression Approach to Tests of Asset. Pricing, Oxford Bulletin of Economics and Statistics, 163-181.

[42] Cubadda, G. (1999), Common Cycles in Seasonal Non-Stationary Time Series, Journal of Applied Econometrics, 14, 273-291.

[43] Cubadda, G. (1999), Common Serial Correlation and Common Business Cycles: A Calltious Note, Empirical Economics, 24, 529-535. 
[44] Cubadda, G, And A. Hecq (2000), Assessing Forecast Performance of Common Cyclical Features Restrictions in Cointegrated Systems, mimeo Maastrich University.

[45] Cubadda, G, Savio, G. and R. Zelli (1999), Seasonality, Productivity Shocks and Sectoral Comovements in a Real Business Cycle Model for Italy, mimeo DSEGS, Universita del Molise.

[46] Cubadda, G. (2000), Common Features in Time Series with Both Deterministic and Stochastic Seasonality, forthcoming in Econometric Reviews.

[47] Cuthbertson, K. (1996), Quantitative Financial Economics: Stocks, Bonds and Foreign Exchange, (John Wiley \& Sons, Series in Financial Economics and Quantitative Analysis).

[48] Dickey, D.A. ANd W.A. Fuller (1979), Distribution of the Estimators for Autoregressive Time Series with a Unit Root, Journal of the American Statistical Association, 74, 427-431.

[49] Diebold, F.X. (1986), Testing for Serial Correlation in the Presence of ARCH, Procedings of the ASA Business and Economic Statistics Section, 323-328.

[50] Diebold, F.X. (1998), Elements of Forecasting, (South-Western College Publishing).

[51] Eitrheim, $\oslash$., Huseb $\varnothing$, T.A. and R. Nymoen (1998), Error-correction versus Differencing in Macroeconomic Forecasting, Discussion Paper Norges Bank, Arbeidsnotat Series.

[52] Engle, R. F. and C.W.J. Granger (1987), Co-integration and Error Correction: Representation, Estimation and Testing, Econometrica, 55, 251-276.

[53] Engle, R. F. And S. Kozicki (1993), Testing for Common Features (with comments), Journal of Business and Economic Statistics, 11, 369-395.

[54] Engle, R. F. and J. V. Issler (1995), Estimating Common Sectorial Cycles, Joumal of Monetary Economics, 35, 83-113.

[55] Engle, R.F., Granger, C.W.J, Hylleberg, S. and H. Lee (1993), Seasonal Cointegration: The Japanese Consumption Function, Journal of Econometrics, 55, 275-98.

[56] Engle, R.F. and S. Hylleberg (1996), Common Seasonal Features : Global Unemployment, Oxford Bulletin of Economics and Statistics, 58, 615-630.

[57] Engle, R.F. And R. Susmel (1993), Common Volatility in International Equity Markets, Journal of Business and Economic Statistics, 11, 167-175.

[58] Engle, R.F. and M.W. Watson (1981), A One-Factor Multivariate Time Series Model of Metropolitan Wages, Journal of the American Statistical Association, 76, 545-565. 
[59] Engle, R.F. And B.S. Yoo (1987), Forccasting and Testing in Co-integrated Systems, Journal of Econometrics, 35, 143-159.

[60] Engle, R.F., D.F. Hendry and J.-F. Richard (1983), Exogeneity, Econometrica, 51, 277-304.

[61] Ericsson, N.E. (1995), Conditional and Structural Error Correction Models, Journal of Econometrics, 69, 159-171.

[62] Ericsson, N.E., D.F. Hendry and G.E. Mizon (1998), Exogeneity, Cointegration and Policy Analysis, Journal of Business Economics and Statistics, 16, 370-387.

[63] Ericsson, N., Hendry, D.F. And H-A Tran (1994), Cointegration, Scasonality, Encompassing, and the Demand for Money in the UK, in Nonstationary Time Series Analysis and Cointegration, (Ed. by Hargreaves, Oxford University Press).

[64] Escribano, A. AND D. PEÑa (1994), Cointegration and Common Factors, Journal of Time Series Analysis, 15, 577-586.

[65] Evans G.W. (1989), Output and Unemployment dynamics in the US 1950-1985, Journal of Applied Econometrics, 213-237

[66] Evans, P. and G. Karras (1996a), Convergence Revisited, Journal of Monetary Economics, 37, 249-265.

[67] Evans, P. and G. Karras (1996b), Private and Government Consumption with Liquidity Constraints, Journal of International Moncy and Finance, 2, 255-266.

[68] FAMA, E.F., AND K. R. FRENCH (1988), Permanent and Temporary Components of Stock Prices, Journal of Political Economy, 96, 246-273.

[69] Franses, P.H. (1996), Recent Advances in modeling Seasonality, Joumal of Economic Surveys, 10, 3, 299-345.

[70] Franses, P.H. And P. Boswijk (1996), Persistence in Nonlinear Autoregressive Models, USDC Disscussion Paper.

(71) Franses, P.H. And R.M. Kunst (1999), Testing Common Deterministic Seasonality, with an Application to Industrial Production. Econometric Institute Research Report. 9905/A.

172] Funke: M. And S. Hall (1995), Common Features in the Sterling/Deutschmark, the Sterling/Dollar and the Deutschemark/Dollar Exchange Rates, Center for Economic Forecasting Discussion Paper. 
[73] Funke, M. And S. Hall (1995), Common Output and Unemployment Persistence in Germany and the UK, Center for Economic Forecasting Discussion Paper.

[74] Gallo, G. AND H. KempF (1996a), Tendances communes et cycles communs : une application à la relation consommation-revenu sur données americaines, AFSE meeting.

[75] Gallo, G. and H. KempF (1996b), Cointegration, Codependence and Economic Fluctuations, University of Florence Discussion Paper.

[76] Geerkens. E. And A. HeCQ (1994), Correlation entre les prix sous l'Ancien Régime: application des tests de codependence, mimeo Université de Liège.

[77] Gervaz, C. (1997), Common Trends and Common Cycles in Belgian Sectoral GDP, Recherches Economiques de Louvain, 63, 79-102.

[78] Geweke, J. (1977), The Dynamic Factor Analysis of Economic Time Series, in: Aigner, D.J. and A.S. Goldberger (eds.) Latent Variables in Socio-Economic Models, (NorthHolland: Amsterdam).

[79] Ghysels, E., Lee, H.S. And P. Siklos (1993), On the (Mis)Specification of Seasonality and its Consequences: An Empirical Investigation with US Data, Empirical Economics, 18. 4, 191-204.

[80] Girysels, E. And P. Perron (1993), The effect of Seasonal Adjustment Filters on Tests for a Unit Root, Journal of Econometrics, 55, 57-98.

[81] Gonzalo, J. and C.W.J. Granger (1995), Estimation of Common Long-Memory Components in Cointegrated Systems, Journal of Business and Economics Statistics, 33, 27-35

[82] Gonzalo, J. and J.Y. Pitarakis (1999), Dimensionality Effect in Cointegration Analysis, Chapter 9 in Engle R. and H. White (Ed.), Cointegration, Causality, and Forecasting. A Festschrift in Honour of Clive W.J Granger, (Oxford University Press).

[83] Gouriéroux, Ch., A. Monfort and E. Renault (1991), A General Framework for Factor Models, INSEE Working Paper.

[84] Gouriéroux, Ch., A. Monfort and E. Renault (1993), Tests sur le noyau, l'image et le rang de la matrice des coefficients d'un modèle multivarié, Annales d'Economic et de Statistique, 32, 81-111.

[85] Gouriéroux, Ch. and I. Peaucelle (1989), Detecting a long-run Relationship, CEPREMAP Discussion Paper 8902.

[86] Gouriéroux, Ch. ANd I. Peaucelle (1993), Séries codépendantes : application à l'hypothèse de parité du pouvoir d'achat, in Macroéconomie, développements récents, (éd. Economica), 285-306. 
[87] Gourifiroux, Ch. And J. Jasiak (1999), Nonlinear Persistence and Copersistence, CEPREMAP Discussion Paper.

[88] Granger, C.W.J. and P. Newbold (1974), Spurious Regressions in Econometrics, Journal of Econometrics, 2, 111-120.

[89] Granger, C.W.J. And J.-L. Lin (1995), Causality in the Long Run, Econometric Theory, $11,530-536$.

[90] Granger, C.W.J. And N. Haldrup (1997), Separation in Cointegrated Systems and P-T Decompositions, Oxford Bulletin of Economics and Statistics, 59, 449-463.

(91) Granger, C.W.J. Hyung, N. and Y. Jeon (1998), Spurious Regressions with Stationary Scries, UCSD Discussion Paper 98-25.

[92] Greene, W.H. (1993), Econometric Analysis (MacMillan: New York).

[93] Groen, J.J. And F. Kleibergen (1999), Likelihood-Based Cointegration Analysis in Panels of Vector Error Correction Models, Discussion Paper TI 99-055/4, Tinbergen Institute, Erasmus University Rotterdam.

[94] Haffner, R.W., A.M. Kutan and S. Zhou (1997), Linkages in EMS Term Structures; Evidence from Common Trend and Transitory Components, Journal on International Money and Finance, 16, 595-607.

[95] Hall, S. (1986), An Application of the Granger and Engle Two-Step Estimation Procedure to United Kingdom Aggregate Wage Data, Oxford Bulletin of Economics and Statistics, $48,241-251$.

[96] Hald, S. (1987), Maximum Likelihood Estimation of Cointegrating Vectors: An Example of the Johansen Procedure, Oxford Bulletin of Economics and Statistics, 51, 213-218.

[97] Hall, S., Psaradakis, Z. and M. Sola (1997), Cointegration and Changes in Regime: The Japanesc Consumption Function, Journal of Applied Econometrics, 12, 151-68.

[98] Hall, S., S. Lazarova and G. Urga (1999) A Principal Components Analysis of Common Stochastic Trends in Heterogeneous Panel Data: Some Montc Carlo Evidence, Oxford Bulletin of Economics and Statistics, 61, 749-767

[99] Hannan, E.J. (1969), The Identification of VARMA Systems, Biometrika, 56, 1, 223-225.

[100] Hannan, E.J. (1971), The Identification Problem for Multiple Equation Syst.ems with Moving Average Errors, Econometrica, 39, 5, 751-765.

(101) Hamilton, J.D. (1994), Time Series Analysis (Princeton University Press: Princeton). 
[102] Hansen, L.P. (1982), Large Sample Properties of Generalized Method of Moment Estimators, Econometrica, 50, 1029-1054.

[103] Hansen, P.R. and S. Johansen (1998), Workbook on Cointegration, (Oxford University Press: Oxford).

[104] Harbo, I., Johansen, S., Nielsen, B. And A. Rahbek (1998), Asymptotic Inference on Cointegrating Rank in Partial Systems, Joumal of Business and Economic Statistics, $16,388-99$.

[105] Harvey, A.C. (1989), Structural Time Series Models and the Kalman Filter, (Cambridge University Press: Cambridgc).

[106] Harvey, D.I. And T.C. Mills (1999), Evidence for Common Features in G7 Macroeconomic Time Series, Economic Research Paper 99/9, Loughborough University.

[107] HecQ, A. (1995), Unit Root Tests with Level Shift in the Presence of GARCH, Economics Letters, 49, 125-130.

[108] HECQ, A.(1996), IGARCH effect on Autoregressive Lag Length Selection and Causality Tests, Applied Economics Letters, 3, 317-323.

[109] HeCQ, A.(1997), Common Features, Codependence \& Co., mimeo, Maastricht University.

[110] HecQ, A.(1998), Does Seasonal Adjustment Induce Common Cycles?, Economics Letters, 59, 289-297.

[111] HecQ, A. (1999), On the Uscfulness of Considering Common Serial Features and Cointcgrating Restrictions, University of Manstricht RM/99/017.

(112) HECQ, A. AND B. MAHY (1997), Testing for the Price- and Wage-Setting Model in Belgium using Multivariate Cointegration Tests, Labour, 11, 177-199.

[113] HecQ, A, F.C. Palm and J.P. Urbain (1998), Testing for Common Cycles in VAR Models with Cointegration, Maastricht University RM/97/031 (submited to Journal of Econometrics, $3^{\text {rd }}$ round).

[114] HecQ, A., F.C. Palm and J.-P. Urbain (1999), GMM-Based Estimation Procedures and Test Criteria for Reduced Rank Models, mimeo, Maastricht University.

[115] HecQ, A, F.C. Palm and J.P. Urbain (2000a), Permanent-Transitory Decomposition in VAR Models with Cointegration and Common Cycles, Oxford Bulletin of Economics and Statistics, (forthcoming). 
[116] Hecq, A, F.C. Palm and J.P. Urbain (2000b), Testing for Common Cyclical Features in Nonstationary Panel Data Models, in: B.H. Baltagi (editor), Advances in Econometrics: Nonstationary Panels, Pancl Cointegration and Dynamic Panels, Vol. 15. (JAI Press: New York), (forthcoming).

[117] HECQ, A, F.C. PALM AND J.P. Urbain (2000c), Separation and Weak Exogeneity in Cointegrated VAR Models with Common Features, mimeo, University of Maastricht.

[118] HecQ, A, F.C. Palm and J.P. Urbain (2000d), Comovements in International Stock Markets: What can we Learn from a Common Trend-Common Cycle Analysis, De Economist, 148, 1-12..

[119] HENDRY, D.F. (1995), Dynamic Econometrics, (Advanced Texts in Econometrics, Oxford University Press).

[120] Hendry, D.F. (1996), A Theory of Co-breaking, Institute of Economics and Statistics, Discussion Paper.

[121] Hendry, D.F. ANd G.E. Mizon (1993), Evaluating Dynamic Econometric Models by Encompassing the VAR, in: P.C.B. Phillips (editor), Models, Methods and Applications of Econometrics, (Basil Blackwell: Oxford).

[122] HENin P.Y. AND T. JobERT (1993), Chômage et écart conjoncturel: Une reformulation de la loi d'Okun, in La Persistence du chômage, (éd. Economica), 79-115.

[123] Hoek, H. and G. Romin (1996), The Estimation of Common Cycles in Vector Time Series Using the Box-Tiao Transformation, Tinbergen Institute Discussion paper.

[124] Hoffman, D.L. And R.H. Rasche (1996), Assessing Forecast Performance in a Cointegrated System, Journal of Applicd Econometrics, 11, 495-517.

[125] Hoffman, M. (1999), National Stochastic Trends and International Macrocconomic Fluctuations, European University Institute Florence Working Paper 99/26.

[126] Hoogstrate, A.J. (1998), Dynamic Panel Data Models: Theory and Macrocconomic Applications, Ph.D. Thesis, University of Maastricht.

[127] Hsiao, C. (1986), Analysis of Panel Data (Cambridge University Press: Cambridge).

[128] Hylleberg, S., Engle, R.F., Granger, C.W.J; . ayd S. Yoo (1990), Scasonal Integration and Cointegration, Joumal of Econometrics, 44, 215-38.

[129] Im, K.S., Pesaran, M.H. and Y. Shin (1997), Testing for Unit Roots in Hetcrogeneous Panels, mimeo, University of Cambridge. 
[130] Issler, J. V. AND F. VAhID (1998), Common Cycles and the Importance of Transitory Shocks to Macroeconomic Aggregates, EPGE Discussion Paper.

[131] Issler, J.V., Moreira, A.R., AND H. Lopes (1994), Using Common Cycles in Structural Identification of Multivariate Systems, mimeo.

[132] Jacobson, T., Vredin, A. And A. Warne (1998), Are Real Wages and Unemployment Related?, Economica, 65, 69-96.

[133] JoberT, T. (1995), Tendances et cycles communs à la consommation et au revenu : implications pour le modèle de revenu permanent, Economie et Prévision, 121, 19-38.

[134] Johansen, S. (1988), Statistical Analysis of Cointegrating Vectors, Joumal of Economic Dynamics and Control, 12, 231-254.

[135] Jonansen, S. (1992), Cointegration in Partial Systems and the Efficiency of SingleEquation Analysis, Journal of Econometrics, 52, 389-402.

[136] Johansen, S. (1995), Likelihood-Based Inference in Cointegrated Vector Autoregressive Models (Oxford University Press: Oxford)

[137] Johansen, S. (1999), A Small Sample Correction for Tests of Hypotheses on Cointegrating Vectors, European University Institute Florence Working Paper 99/9.

[138] KAO, C. (1999), Spurious Regression and Residual-Based Tests for Cointegration in Panel Data, Joumal of Econometrics, 40, 1-44.

[139] KASA, K. (1992), Common Stochastic Trends in International Stock Markets, Journal of Monctary Economics, 29, 95-124.

[140] Kaufman R.T. (1988), An International Comparison of Okun's Law, Journal of Comparative Economics, 12, 182-203.

[141] Kenen, P. (1969), The Theory of Optimum Currency Areas: an Eclectic View, in R. Mundell and A. Swoboda, eds, Monetary Problems in International Economy, (Chicago University Press).

[142] King, R.G., Plosser, C.I. And S. Rebelo (1988), Production, Growth and Business Cycles, II, Journal of Monctary Economics, 21, 309-341.

[143] King, R.G., Plosser, C.I., Stock, J.H. And M.W. Watso. (1991), Stochastic Trends and Economic Fluctuations, American Economic Review, 81, 4, 819-840.

[144] Konishi, T. (1993), Separation and Long-Run Non-Causality in a Cointegrated System, Ph.D. Dissertation, UCSD. 
[145] Konishi, T. and C.W.J. GRanger (1993), Separation in Cointegrated Systems, mimeo, UCSD.

[146] Konishi, T., Ramsey, V.A. And C.W.J. Granger (1994), Stochastic Trends and ShortRun Relationships between Financial Variables and Real Activity, mimeo, UCSD.

[147] Kugler, P. AND K. Neusser (1993), International Real Interest Rate Equalization : A Multivariate Time-Series Approach, Journal of Applied Econometrics, 8, 163-174.

[148] Kugler, P. And P. Schwendener (1992), Codependence in a VAR framework, mimeo, Universitat Bern.

[149] Kugler, P. And P. Schwendener (1993), Testing for an Equilibrium Relationship for Trend and Difference Stationary Series, mineo, University of Wien.

[150] Kunst, R. (1994), Seasonal Cointegration, Common Scasonals, and Forecasting Seasonal Scries, Empirical Economics,.18,4, 205-220.

[151] Kunst, R. AND K. Neusser (1990), Cointegration in Macroeconomic Systems, Journal of Applied Econometrics, 5, 351-365.

[152] LABrousse, C.E. (1933), Esquisse du mouvement des prix et des revenus en France au XVIII ${ }^{\text {mme }}$ siècle, (Paris, Librairie Dalloz, 2 tomes).

[153] LAhiri, K. AND J.G. Wang (1996), Interest Rate Spreads as Predictors of Business Cycles, in G.S. Maddala and C.R. Rao, eds, Handbook of Statistics, vol.14.

[154] Larsson, R. ANd J. Lyhagen (1999) Likelihood-Based Inference in Multivariate Pand Cointegration Models, SSE Working Paper 331, Stockholm School of Economics.

[155] Layard R. R. JaCkman and S. Nickeli. (1991), Unemployment, (Oxford University Press).

[156] Levin, A. And C.F. Lin (1993), Unit Root Tests in Pancl Data: Asymptotic and Finite Sample Properties, UCSD Discussion Paper.

[157] Lin, J.-L. AND R.S. TsAY (1996), Co-integration constraint and Forecasting: An Empirical Examination, Journal of Applied Econometrics, 11, 519-538.

[158] Lucke, B. (1994), Analysis of West German Macroeconomic Data Using Common Trends and Common Cycles, Discussion Paper 03/94 Free University of Berlin.

[159] Lumsdane, R.L. And E. Prasad (1997), Identifying the Common Components in International Economic Fluctuations, NBER Working Paper 5984.

[160] Lürkepohl, H. (1991), Introduction to Multiple Time Series Analysis, (Springer Verlag: Berlin). 
[161] Maravall, A. (1993), Stochastic Linear Trends: Models and Estimators, Journal of Econometrics, 56, 5-37.

[162] Maravall, A. (1995), Unobserved Components in Economic Time Series, in Handbook of Applied Econometrics.

[163] Maravall, A. (1997), Use and (mostly) Abuse of Time Series Techniques in Economic Analysis, paper presented at the 5th CEMAPRE Conference, Lisboa.

[164] MCCoskey, S., AND C. KaO (1998a), A Residual-Based Test of the Null of Cointegration in Panel Data, Econometric Reviews, 17, 57-84.

[165] MCCoskey, S., And C. KaO (1998b), A Monte Carlo Comparison of Tests for Cointegration in Pancl Data, mimeo.

[166] Mc Kinnon, R. (1963), Optimum Currency Areas, American Economic Review, Scptember, $657-665$.

[167] Mellander, E., Vredin, A. and A. Warne (1992), Stochastic Trends and Economic Fluctuations in Small Open Economy, Journal of Applied Econometrics, 7, 369-394.

[168] Mills, T.C. (1993), The Econometric Modelling of Financial Time Series, (Cambridge University Press).

[169] MiLls, T.C. (1998), Recent Developments in Modeling Nonstationary Vector Autoregressions, Journal of Economic Surveys, 12, 279-312.

[170] Mills, T.C. (1999), Great Ratios and Common Cycles: Do They Exist for the U.K.? Economic Research Paper 99/1, Loughborough University.

[171] Mills, T.C. And M.J. Holmes (1999), Common Trends and Cycles in European Industrial Production: Exchange rate Regimes and Economic Convergence, The Manchester School, 67, 557-587.

[172] Moosa, I.A. (1997), A Cross-country Comparison of Okun's Coefficient, Journal of Comparative Economics, 24, 335-355.

[173] Mundell, R. (1961), A Theory of Optimum Currency Areas, American Economic Review, $51,715-725$.

[174] Neusser, K. (1991), Testing the Long-Run Implications of the Neoclassical Growth Model, Journal of Monetary Economics, 27, 3-37.

[175] Nielsen, B. AND A. RAHBek (1998), Similarity Issues in Cointegration Analysis, Preprint, University of Copenhagen. 
[176] O'Connell, P. (1998), The Overvaluation of Purchasing Power Parity, Journal of International Economics, 44, 1-19.

[177] OKUn A.M. (1962), Potential GNP: Its Measurement and Significance, American Statistical Association. Proceedings of the Business and Economics Statistics Section, 98-104.

[178] Osterwald-Lenun, M. (1992), A Note with Fractiles of the Asymptotic Distribution of the Maximum Likelihood Cointegration Rank Test Statistics: Four Cases, Oxford Bulletin of Economics and Statistics, 54, 461-72.

[179] PaAp, R. ANd P.H. Franses (1999), Do the US and Canada Have a Common Nonlinear Cycle in Unemployment, Econometric Institute Report 9907/A, Erasmus University Rotterdam.

[180] Pagan, A. (1999), The Getting of Macrocconomic Wisdom, mimeo Australian National University.

(181) Pain, D. and R. Thomas (1997), Real Interest. Rate Linkages: Testing for Common Trends and Cycles, Bank of England Working Paper.

[182] Palm, F.C. AND J.M. Sneek (1984), Significance Tests and Spurious Correlation in Regression Models with Autocorrelated Errors, Statistical Papers, 25, 87-105.

[183] Palm, F.C. And J.-P. Urbain (1995), Common Trends and Transitory Components of Stock Price Volatility, mimeo, Maastricht University.

[184] PARker, E. (1997), Common Trends and Cycles and the Structure of Florida's Economy, Fedcral Reserve Bank of Allanta Economic Review, 3.

[185] Parnisari, B. (1999), Does Switzerland Share Common Business Cycles with other European Countries?, mimeo, Federal Office for Economic Development and Labour.

[186] Pastorello, S. And E. Renault (1994), Modèles à Facteurs en Finance, Chapter 7 in Modélisation ACRH, (Editions de l'Université de Bruxelles, A.S.U.).

[187] Pedroni, P. (1997a), Fully Modified OLS for Heterogeneous Cointegrated Panels and the Case of Purchasing Power Parity, Indiana University Working Paper.

[188] Pedroni, P. (1997b), Cross Sectional Dependence in Cointegration Tests of Purchasing Power Parity, Indiana University Working Paper.

[189] Pesaran, M.H. (1989), The Limits of Rational Expectations, (Basil Blackwell).

(190\} Pesaran, M.H. And R.P. Smith (1995), Estimating Long-Run relationships from Dynamic Heterogenous Panels, Joumal of Econometrics, 68, 79-113. 
[191] Pesaran, M.H., Shin, Y and R.P. Smitil (1997), Pooled Estimation of Long-Run relationships in Dynamic Heterogenous Panels, University of Cambridge Working Paper.

[192] PhilLtrs, P.C.B. (1986), Understanding Spurious Regressions in Econometrics, Joumal of Econometrics, 33, 311-340.

[193] Phillips, P.C.B. (1991), Optimal Inference in Cointegrated Systems, Econometrica, 59, 283-306.

[194] Philups, P.C.B. ANd H. Moon (1999a), Linear Regression Limit Theory for Nonstationary Panel Data, Econometrica, 67, 1057-1111.

[195] Phillups, P.C.B. AND H. Moon (1999b), Nonstationary Panel Data Analysis: An Overview of Some Recent Developments, Working Paper, Yale University.

[196] Proretri, T. (1997), Short Run Dynamics in Cointegrated Systems, Oxford Bullctin of Economics and Statistics, 59,405-422.

[197] Qunh, D. (1992), The Relative Importance of Permanent and Transitory Components: Identification and some Theoretical Bounds, Econometrica, 60, 107-118.

[198] RAo, C.R. (1973), Linear Statistical Inference and Its Applications, 2nd Ed, (John Wiley $\&$ Sons).

[199] RErNsel, G. C. (1993), Elements of Multivariate Time Series Analysis, (Springer Verlag: Berlin).

[200] Reinsel, G. C. AND S. K Ahn (1992), Vector Autoregressive Models with Unit Roots and Reduced Rank Structure : Estimation, Likelihood Ratio Tests, and Forecasting, Joumal of Time Series Analysis, 13, 353-375.

[201] Richards, A.J. (1995), Comovements in National Stock Market Returns: Evidence of Predictability, but not Cointegration, Joumal of Monetary Economics, 36, 631-654.

[202] Robinson, P.M. (1973), Generalized Canonical Analysis for Time Series, Joumal of Multivariate Analysis, 3, 141-160.

[203] Romisn, G. (1996), Common Trends and Cycles in the Dutch Construction Industry and the Macroeconomy : Empirical Evidence 1969-1994, Tinbergen Institute Discussion Paper.

[204] Rubin, J. And N. Thygesen (1996), A Cointegration/Codependence Analysis of Business Cycles in Europe : An Application to Monetary Union and Outsiders, Economie Appliquée, 3, 123-171. 
[205] Ruwer, J. (1966), Prix des céréales à Ruremonde, XVIII'me-XVIII ${ }^{\text {ème }}$ siècles, in Ruwer, Helin, Ladrier and Van Buyten, Marché des céréales à Ruremonde, Luxembourg, Namur ct Diest au XVII ime et XVIII ème siècles, (Université de Louvain, Recucil detravaux d 'histoire et de philologie, quatrième séric, fascicule 33 ).

[206] Sachs, J. and X. Sala-I-Martin (1992), Fiscal Federalism and Optimum Currency Areas : Evidence for Europe from the United States, CEPR Discussion Paper, no. 632.

[207] Saikkonen, P. (1995), Problems with the Asymptotic 'Theory of Maximum Likelihood Estimation in Integrated and Cointegrated Systems, Econometric Theory, 11, 888-911.

[208] Sharpe, W.F (1963), A Simplificd Model for Portfolio Analysis, Management Science, 9,1 .

[209] Sill, K. (1997), Regional Employment Dynamics, Working Paper 97/28 Federal Reserve Bank of Philadelphia.

[210] Singleton, K. (1980), A Latent Time Series Model of the Cyclical Behavior of Interest. Rates, International Economic Review, 21, 559-575.

[211] Sмiтн G. (1975), Okun's Law revisited, Quarterly Revicw of Economics and Business, $15,37-54$.

[212] STock, J.H. And M.W. Watson (1988), Testing for Common Trends, Journal of American Statistical association, 83, 1097-110\%.

[213] S'Tock, J.H. And M.W. Whison (1991). A Probability Model of the Coincident. Economic Indicators, in K. Lahiri and G.H. Moore (eds.), Leading Economic Indicators: New Approaches and Forecasting Records, ch. 4, 63-85, (New York: Cambridge University Press).

[214] Stoffer, D.S. (1999), Detecting Common Signals in Multiple Time Scries Using the Spectral Envelope, Joumal of American Statistical association, 94, 448, 1341-1356.

[215] Summers, R. And A. Heston (1991), The Penn World Table (Mark 5): An Expanded Set of International Comparisons, 1950-1988, Quarterly Joumal of Economics, 327-368.

[216] Söderlind, P. And A. VRedin (1996), Applied Cointegration Analysis in the Mirror of Macrocconomic Theory, Joumal of Applied Econometrics, 11, 363-81.

[217] Sørensen, N.K. (1996), Testing for Common Seasonal Features in Quarterly Time Series Among Countries of Western Europe, Discussion Paper, Danish Institute of Border Region Studies.

[218] TiaO, G.C. AND R.S. TSAY (1985), A Canonical Correlation Approach to Modeling Multivariate Time Series, in Proceedings of the Business and Economic Statistic Section, American Statistical Association. 
[219] Tiao, G. C. and R. S. Tsay (1989), Model Specification in Multivariate Time Series (with comments), Journal of Royal Statistical Society, Series B, 51, 157-213.

[220] Tiao, G.C.,. TSAY, R.S. And T. Wang (1994), usefulness of Linear Transformations in Multivariate Time Series Analysis, Empirical Economics, 18, 4, 11-37.

[221] TSAY, R.S. (1989), Parsimonious Parametrization of Vector Autoregressive Moving Avcrage Models, Journal of Business \& Economic Statistics, 4, 327-341.

[222] Urbain, J.-P. (1992), "On Weak Exogencity in Error Correction Models", Oxford Bulletin of Economics and Statistics, 54, 187-207.

[223] Vahid, F. And R. F. Engle (1993), Common Trends and Common Cycles, Journal of Applied Econometrics, 8, 341-360.

[224] VAhid, F. AND R. F. ENGle (1997), Codependent Cycles, Journal of Econometrics, 80, 199-221.

[225] VAhid, F. AND J.V. Issler (1999), The Importance of Common-Cyclical Features in VAR Analysis: A Monte-Carlo Study, paper presented at ESEM99.

[226] Variyam, J.N. (1996), Cointegration, Common Features, and Persistence in US Farm O-1tput, Empirical Economics, 459-473.

[227] Velu, R. P. , Reinsel, G. C. And D. W. Wichern (1986), Reduced Rank Models for Multivariate Time Series, Biometrika, 73, 105-118.

[228] WaLLis, K.F. (1974), Scasonal Adjustment and Relations Between Variables, Journal of the American Statistical Association, 69, 53-63.

[229] WEST, K.D. (1997), Another Heteroskedasticity and Autocorrelation Consistent Covariance Matrix Estimator, Joumal of Econometrics, 76, 171-191.

[230] Witt, R., Clarke, A. and N. Fielding (1998), Common Trends and Common Cycles in Regional Crime, Applied Economics, 30, 1407-1412. 


\section{Dutch Summary}

Veel economische variabelen vertonen een vergelijkbaar patroon wanneer zij worden waargenomen over een lange periode. Dit proefschrift. behandelt de analyse van gemeenschappelijke economische fluctuaties van, hoofdzakelijk, macro-economische tijdreeksen. Wanneer een univariaat tijdreeksen analyse, d.w.z. individuele reeksen analyse, is uitgebreid naar cen meerdimensionaal tijdrecksen analyse blijken vaak waargenomen kenmerken gemeenschappelijk te zijn. Geneenschappelijke kenmerken (common features) kumen seizoensbewegingen zijn, trends, volatiliteit, structurele breuken, uitschieters, ... Sinds vrijwel alle macro-economische tijdrecksen nict-stationair zijn, en zeker autocorrelatie vertonen, zullen we ons beperken tot de gezamenlijke analyse van zowel gemeenschappelijke trends en gemeenschappelijke cycli.

Hoofdstuk 1 geeft een algemene introductic aan deze monografie, terwijl hoofdstuk 2 gewijd is aan een overzicht en beschrijving van de begrippen en de bestaande methoden uit de literatuur. Hoofdstuk $3 \mathrm{cn} 4$ zijn centraal voor dit proefschrift. Daar introduceren wij een nieuwe klasse van restricties die we de zwakke vorm van gemeenschappelijke kenmerken noemen. In tegenstelling tot andere formuleringen uit de literatuur, zoals de formulering van Vahid en Engle (1993), hecft de zwakke vorm als voordecl dat we slechts een beperkt aantal gemeenschappelijke cycli kunnen extraheren. Onze aanpak heeft dus als voordeel dat we in staat zijn economische systemen t.e beschrijven die veel dieper zijn verbonden dan in het Vahid en Engle (1993) geval. Het is ook mecstal niet meer nodig gemeenschappelijke kenmerken te bestuderen op basis van "detrended" tijdreeksen.

Het belang van lange termijn gemeenschappelijke bewegingen is goed begrepen in de toegepaste econometrie literatuur. Gemeenschappelijke cycli zijn dit wat minder. We hebben dus ook getracht verschillende toepassingen van de methoden en technieken te bekijken. Hoofdstukken 7, 8 en 9 analyseren de relaties tussen nationale consumptic en nationaal inkomen; in de hoofdstukken 3,4 en 6 analyseren we consumptie, investeringen en inkomen. In hoofdstuk 4 bestuderen we mogelijke korte termijn relaties tussen aandeclmarkt indices; in de hoofdstukken 3 en 6 renten, in hoofdstuk 2 prijzen als ook economische groei. Tenslotte worden ook regionale inkomen en regionale werkgelegenheden reeksen beschouwd in hoofdstuk 5.

Alhoewel we dus in staat zijn empirisch de aanwezigheid van gemeenschappelijke cycli te tonen, kunnen we ons afvragen of economisch gezien gemeenschappelijke cycli van belang zijn. Theoretische modellen voorspellen in feite vaak korte termijn relaties. De analyse van gemeenschappelijke cycli geeft ons dus de gelegenheid alternatieve theoretische modellen te confronteren 
aan de hand van empirische uitkomsten. Het aantal gemeenschappelijke cycli kan bijvoorbeeld een indicatic van de convergentie tussen verschillende landen geven.

Statistisch gezien kunnen we de nauwkeurigheid van schatters verbeteren sinds het aantal onbekende parameters is verminderd. Dit heeft ook consequenties in termen van het interpreteren van de resultaten aangezien complexe bewegingen samengevat kunnen worden in een paar indices.

Verschillenden hoofdstukken uit dit proefschrift analyseren ook de kleine steekprocf statistische eigenschappen van de toetsen en de schatters. Hoofdstuk 2 bekijkt bijvoorbeeld het effect. van misspecificatic. In hoofdstuk 3 analyseren we via Monte Carlo simulaties het geval waar het aantal coïntegratie relaties over- of onder-schat is. Vervolgens wordt in hoofdstuk 5 een schattingstechniek voorgesteld waar zowel korte als lange termijn restricties expliciet gebruikt zijn. Het nut van gemeenschappelijke cycli restricties voor voorspelbaarheid is in hoofdstuk $6 \mathrm{~cm}-$ pirisch onderzocht. Hoofdstuk 7 is gewijd aan scizocnsbewegingen in de data en aan de mogelijke implicaties van standaard seizoenscorrectiemethoden. Uit onze analyse blijkt dat dergelijke correctiemethoden van invloed zijn op de eigenschappen van toetsen en schatters en kunnen als gevolg tot onjuiste economische interpretatics leiden.

Tenslotte behandelen we in de hoofdstukken 8 en 9 het geval waar het aantal variabelen te analyseren vrij groot is zodat ecn standaard VAR analyse bijzonder mocilijk wordt. In hoofdstuk 8 bespreken we dit probleem via de "separation" oplossing. In hoofdstuk 9 stellen we voor de analyse van gemeenschappelijke kenmerken uit te breiden naar panel data. 


\section{French Summary}

Cette thèse a pour objet l'étude des fluctuations communes, aussi appelées relations de coévolutions, au scin de séries temporelles macro-ćconomiques et en données de panel. En effet, lors du passage d'une analyse univariéc à une étude multivariée, il est fréquent que certaines caractéristiques observées sur ces séries individuclles soient partagées par plusicurs d'entre elles. Ces caractéristiques peuvent être de la saisonnalité, de la volatilité, des ruptures structurelles, des valcurs aberrantes. Cependant comme la plupart des variables macro-économiques sont nonstationnaires et autocorrélées, notre travail porte sur le traitement simultané des fluctuations communes de tendances et de cycles.

En conséquence, après le Chapitre 1 qui présente une introduction générale du contenu de cette monographic et le Chapitre 2 qui tente d'unifier les différentes approches rencontrées dans la littérature, les Chapitres 3 et 4 rassemblent un des points centraux de cette thèse. Nous y présentons une nouvelle modélisation, appelée forme faible de co-évolution, qui a l'avantage de pouvoir extraire un nombre réduit de tendances et de cycles communs. L'approche générale proposée par Vahid et. Engle (1993) ne permet pas ce traitement puisque l'hypothèse d’indépendance entre les relations de long terme et de court terme introduit une limite au nombre de fluctuations communes. Notre approche permet donc de mettre en évidence des systèmes économiques qui sont liés plus étroitement que dans celle de Vahid et Engle (1993) car il est possible d'analyser si un ensemble de pays ou de régions présentent un même profil conjoncturel. De plus, dans la plupart des cas, il n'est désormais plus nécessaire d'analyser des mouvements communs à partir de séries dont la tendence a éte filtrée grâce à des méthodes univariés telles le filtre d'Hodrick et Prescot.t.

Le problème des régressions fallacieuses et de l'importance des mouvements communs de long terme semble maintenant bien compris en économétrie appliquéc. Les cycles communs le sont moins. Nous avons done tenté de combler partiellement ce fossé en démontrant l'intérêt de cette approche dans un large éventail d'analyses empiriques: les Chapitres 7, 8 et 9 traitent de la relation entre la consommation et le revenu national, les Chapitres 3,4 et 6 de la relation entre la consommation, le revenu et l'investissement, le Chapitre 4 de la relation entre différents indices boursiers, les Chapitres 3 et 6 des taux d'intérêts, le Chapitre 2 des prix ainsi que de l'activité économique, le Chapitre 5 du revenu régional ainsi que de l'emploi, les Chapitres 2 et 7 de l'activité économique entre différents pays

Mais dès lors que l'existence empirique des cycles communs a été mise en évidence, il con- 
vient de s'interroger sur les avantages qu'elle procure au niveau théorique. D'un point de vue économique, la théoric économique prévoit et explique ce type de relations de court terme et cela permet de confronter certaines spécifications théoriques aux résultats que l'on obtient sur des données réelles. En outre, la présence d'un nombre restreint de cycles communs informe en soi sur le degré de convergence des économies. D'un point de vue statistique, la prise en compte de ces relations de court terme permet d'augmenter l'efficacité des estimateurs puisqu'on a réduit le nombre de paramètres à estimer en éliminant des facteurs redondants. Cette réduction facilite ensuite l'interprétation des résultats puisqu'on peut résumer un ensemble de mouvements complexes par quelques indicateurs. Cela peut enfin améliorer les prévisions, tout en remarquant comme dans le Chapitre 6, que le manque de stabilité des paramètres au cours de la période de prévision peut annihiler ce gain.

Après avoir mis en évidence l'utilité de l'étude des relations de co-évolution et après avoir illustré leur existence dans de nombreuses analyses empiriques, plusieurs chapitres s'attachent, à l'aide de simulations, à analyser les propriétés de diverses procédures dans le cas ou certaines hypothèses sont violées. Ainsi, le Chapitre 2 procure des éléments pour évaluer l'impact d'une mauvaise identification du modèle étudié. Le Chapitre 3 considère le cas où l'on omet ou on surestime le nombre de vecteurs de cointégration. Le Chapitre 5 évalue une stratégie consistant à itérer entre les contraintes de long et de court terme. Dans ce même chapitre, une correction pour petits échantillons des tests de co-évolution est proposée. Le Chapitre 7 s'intéresse au traitement des variations saisonnières par les instituts de statistiques. Cette opération de lissage des variables provoque des distorsions de taille et de puissance des tests de co-évolution et donc fausse l'interprétation économique.

Enfin les Chapitres 8 ei 9 abordent le traitement de systèmes qui contiennent un nombre élevé de variables et pour lesquels une analyse multivariée traditionnelle comme les VAR devient difficile. Le Chapitre 8 propose, par le biais de la "séparation", des solutions qui permettent une évaluation disjointe de systèmes, un par pays par exemple. Une seconde approche, peut être plus prometteuse, est proposée au Chapitre 9 dans lequel nous étendons l'analyse des fluctuations communes aux donnécs de pancl. 


\section{Curriculum Vitae}

Alain Hecq was born on September 28 $8^{\text {th }}, 1967$ in Liège, Belgium. From 1985 until 1989 he studied Economics (BA) at the Université de Liège, from 1989 until 1991 he did a Masters degree in Economics at the Université Catholique de Lowvain and from 1993 until 1996 a Diploma in Statistics and Econometrics from the Centre de Formation des Statisticiens-Economistes at the Université Libre de Bruxelles.

From October 1996 until October 2000, he was a Ph.D.-student at the Department of Quantitative Economics at Maastricht University. The research, on Common Cyclical Features, was supervised by Prof. dr. Franz Palm and dr. Jean-Pierre Urbain.

Before his AIO position at Maastricht University, he was research assistant (from March 1990 until June 1994) at the Center of Quantitative History, Université de Liège and econometrician (from August 1991 until October 1996) at the Department of Economic Studies and Statistics at the Ministry of the Walloon Region. 